\title{
Authentic Discourse and Model Exemplars in Japanese Language Textbooks: \\ A Case Study of Apologies and Requests
}

by

\section{Nobuko KATO}

A thesis submitted to the Victoria University of Wellington in fulfilment of the requirements for the degree of Doctor of Philosophy

Victoria University of Wellington 2015 


\section{TABLE OF CONTENTS}

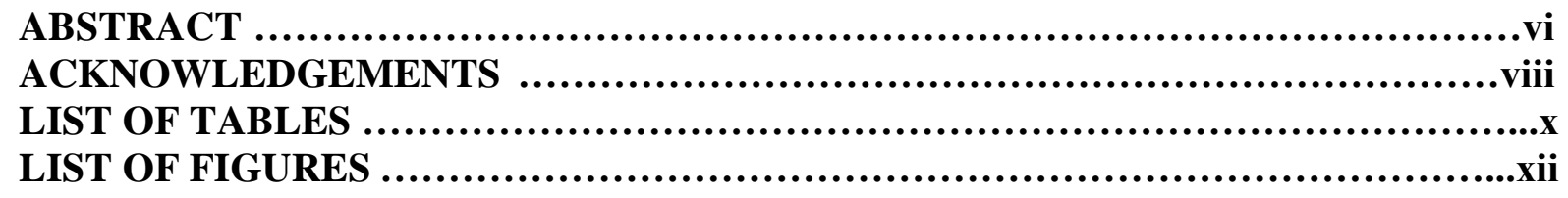

\section{CHAPTER 1 INTRODUCTION}

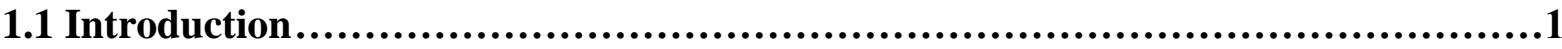

1.1.1 The Context of Textbooks

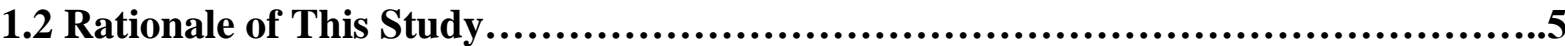

1.2.1 Cross-Cultural Communication

1.2.2 Politeness

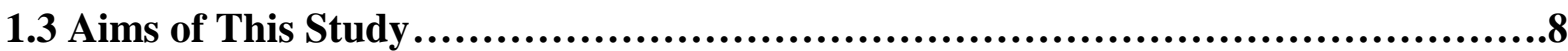

1.3.1 Earlier Studies

1.3.2 Aims of This Study

1.4 Organisation of This Study

\section{CHAPTER 2 LITERATURE REVIEW}

2.1 Textbooks

2.1.1 Introduction

2.1.2 Significance of Textbooks

2.1.3 Language Textbooks

2.1.4 Textbook Creation

2.1.5 Criticism of Language Textbooks

2.1.6 Method of Teaching and Learning in Second Language Acquisition

2.1.7 Summary

2.2 Discourses in Japanese

2.2.1 Introduction

2.2.2 Characteristics of Discourse in Japanese

2.2.3 Characteristics of Spoken Discourse in Japanese

2.2.4 Teaching Japanese Discourse

2.2.5 Summary

2.3 Politeness

2.3.1 Introduction

2.3.2 Politeness Theory

2.3.3 Politeness in Japanese Language

2.3.4 Criticism of Brown and Levinson's Politeness Theory

2.3.5 Cross-Cultural Communication

2.3.6 Summary

2.4.1 Introduction

2.4.2 Strategies of Apology

2.4.3 Expressions of Apology

2.4.4 Various Implications of Apology 


\subsubsection{Summary}

2.5.1 Introduction

2.5.2 Structure of Request in Japanese

2.5.3 Strategy of Request

2.5.4 Expressions of Request

2.5.5 Summary

\section{CHAPTER 3 METHODOLOGY}

3.1 Introduction.......................................................................107

3.2 Method of Data Collection........................................................107

3.2.1 Method

3.2.2 The CCSARP and Politeness Theory

3.3 Experiments

3.3.1 Outline of the Experiments

3.3.2 Some Limitations of the Experiments

\section{CHAPTER 4 APOLOGY}

4.1 Overview of Apology

4.1.1 Categorisation of Apologetic Utterances Based on the CCSARP

4.2 Analysis of Apology

4.2.1 Outline of Strategies of Apology

4.2.2 Head Acts of Apology

4.2.3 Supportive Moves of Apology

4.2.4 Structure of Discourse of Apology

4.3 Findings and Discussion.

4.3.1 Structure of Discourse of Apology

4.3.2 Expressions of Discourse of Apology

4.3.3 Order of Utterance

4.3.4 The Degree of Difficulties of Utterances by Learners

4.4 Summary

\section{CHAPTER 5 REQUEST}

5.1 Overview of Request

5.1.1 Categorisation of Requisitive Utterances Based on the CCSARP

5.2 Analysis of Request

5.2.1 Outline of Strategies of Request

5.2.2 Head Acts of Request

5.2.3 Supportive Moves of Request

5.2.4 Structure of Discourse of Request

5.3 Findings and Discussion.

5.3.1 Structure of Discourse of Request

5.3.2 Expressions of Discourse of Request

5.3.3 Order of Utterance 


\section{CHAPTER 6 SUMMARY}

6.1 Introduction.....................................................................297

6.2 Apology................................................................................297

6.3 Request...........................................................................300

6.4 Similarities and Differences in the Settings of Apology and Request................304

6.5 Textbooks......................................................................................................................306

6.5.1 Structure

6.5.2 Expressions

6.5.3 Model Discourses Created Based on Data Collected from the Present Experiments

\section{CHAPTER 7 CONCLUSION}

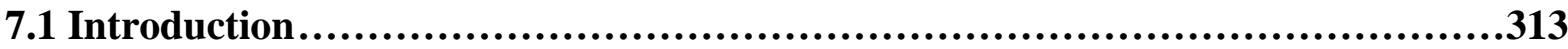

7.2 Overview of the Study..............................................................314

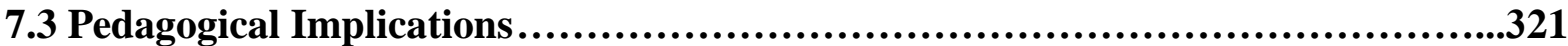

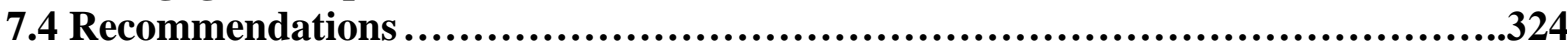

7.5 Considerations for Further Research................................................325

7.6 Concluding Remarks.............................................................326

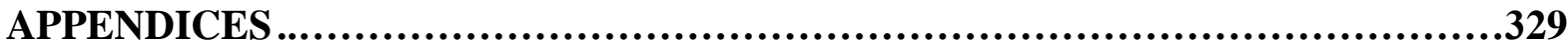

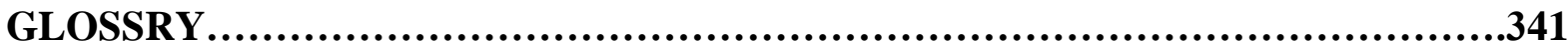

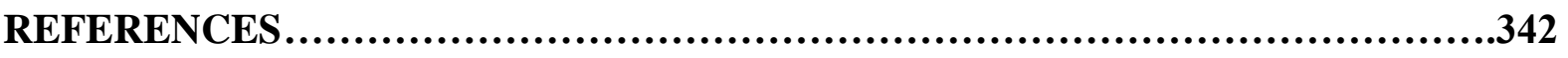




\begin{abstract}
In recent years, the communicative approach has prevailed in second language teaching, such that model dialogues are presented in textbooks for language learners. However, there has been little research on the extent to which such exemplars reflect authentic discourse in Japanese.

This thesis, therefore, explores the speech acts of Japanese language native speakers (NSs), non-native speakers who are learning Japanese (NNSs), and discourse model examples presented in Japanese language textbooks (TXs) in order to investigate their similarities and differences, and thereby enhance the contents of textbooks. In particular, this thesis focuses on the discourse structure and expressions.

The study takes a quantitative approach to the data analysis, using two different scenarios in settings of apology and requests. NSs pairs and NNSs pairs were requested to perform role-plays of two different scenarios in Japanese. The conversational data of the roleplays were transcribed, and the model exemplars of apology and request presented in the selected Japanese language textbooks were also analysed.

The analysis employs the framework of cross-cultural speech act realisation patterns (CCSARP) of Blum-Kulka et al. (1989) to categorise utterances based on the intentions of the speakers into two parts, namely: Head Acts (HAs) and Supportive Moves (SMs). Once the data were classified by the CCSARP, individual HAs and SMs were sorted according to Brown and Levinson's politeness theory (1987), which divides politeness strategies into four types: bald on record, positive politeness, negative politeness, and off record. By adopting both CCSARP and politeness theory, the characteristics of the discourse strategies and expressions of the three groups analysed in this thesis can be grasped.
\end{abstract}


The analysis produces several new findings by identifying various ways in which the model dialogues in Japanese language textbooks fail to reflect the natural discourse of Japanese native speakers. The thesis presents suggestions for improvement for the benefit of textbook authors in future. 


\section{ACKNOWLEDGEMENTS}

Since I enrolled in Victoria University of Wellington in the middle of 2010 from Christchurch, unimaginable natural disasters have occurred; catastrophic earthquakes in Christchurch and Japan were followed by another in Wellington, though it was not as severe as those in Christchurch. While I was in the office on Level 7, I heard the loud noise from the elevator as it hit the wall of the elevator hall and I was very horrified. Nevertheless, no matter how frightening the earthquakes in New Zealand, my determination to continue my research has been solid and unshakeable because my supervisor, Associate Professor Edwina Palmer, is here in New Zealand.

Firstly, without doubt, I would really like to express my deepest and sincere gratitude to my primary supervisor, Associate Professor Edwina Palmer, who has given me invaluable academic guidance, insightful comments in both the Japanese and English languages, and warm encouragement to pursue my research project on the Japanese discourses. Her guidance, wisdom, and enthusiasm supported me throughout the journey of my research. I feel extremely lucky to be one of her students. I am also grateful to my secondary supervisors, Associate Professor Stephen Epstein for his constant care, and Dr. Yushi Ito for his wholehearted involvement until his retirement last year.

Secondly, my special thanks go to the participants in my research. Some of them were my students of previous years and the others were students from other Japanese classes at Victoria University of Wellington and the University of Auckland, as well as Japanese exchange students, young working holiday makers and sojourners in Wellington. Without their dedication to perform the role-plays, this study would never have been completed. I am also indebted to Ms. Geraldine Murphy whom I have always depended on for her help in checking and correcting my very first drafts. 
The following members of the School of Languages helped me to keep a strong motivation toward my research. Dr. Mitsue Sandom and Ms. Yoshie Nishikawa who provided me with advice and experience in teaching Japanese whenever I needed to continue writing the thesis. Both Ms. Loveday Kempthorne and Ms. Sian Robyns, PhD student peers, were not only my writing teachers in English, but also good friends to share the laughter in our office, together with Ms. Mukta Dausoa, another PhD student colleague, in the weekends.

Lastly but most importantly, I would like to give big "thank you" to my family: my late husband, Nobuaki for his high spirits: my parents, Kōzō and Toyoko for their endless love, although my father is far from this world now: and family members in Kochi: Nodoka, Nanami, Chika and Kōtaro; and in Tokyo: Norito, Yumiko, Mizuki and Nanaho, whose big smiles and encouragement in the form of cheers have always been the greatest support. Their love enabled me to complete this research. 


\section{LIST OF TABLES}

\begin{tabular}{|c|c|c|}
\hline \multicolumn{2}{|c|}{ CHAPTER 2.1 LITERATURE REVIEW Textbooks } & \multirow{2}{*}{$\begin{array}{r}\text { Page } \\
24\end{array}$} \\
\hline Table 2.1.1 & Flow of Casual Request & \\
\hline \multicolumn{2}{|c|}{ CHAPTER 2.3 LITERATURE REVIEW Politeness } & \\
\hline Table 2.3.1 & Types of Speech Act and Intrusion upon Face & 60 \\
\hline \multicolumn{2}{|c|}{ CHAPTER 2.4 LITERATURE REVIEW Apologies } & \\
\hline Table 2.4.1 & Group of Apologetic Expressions & 82 \\
\hline Table 2.4.2 & Semantic Classification of Apologies in Japanese and English & 85 \\
\hline \multicolumn{2}{|c|}{ CHAPTER 2.5 LITERATURE REVIEW Requests } & \\
\hline Table 2.5.1 & Flow of Typical Discourse of Request & 89 \\
\hline Table 2.5.2 & Expressions of Addressing & 102 \\
\hline \multicolumn{2}{|c|}{ CHAPTER 3 METHODOLOGY } & \\
\hline Table 3.1 & Scenarios within and beyond the Scope of Present Reseach & 116 \\
\hline Table 3.2 & HA/SM Cross-tabulation (Apology: 3 groups) & 123 \\
\hline Table 3.3 & HA/SM Chi-Square Tests (Apology: 3 groups) & 123 \\
\hline Table 3.4 & HA/SM Cross-tabulation (Apology: 2 groups) & 124 \\
\hline Table 3.5 & HA/SM Chi-Square Tests (Apology: 2 groups) & 124 \\
\hline Table 3.6 & Politeness group Cross-tabulation (Apology: 3 groups) & 124 \\
\hline Table 3.7 & Politeness group Chi-Square Tests (Apology: 3 groups) & 125 \\
\hline Table 3.8 & Politeness group Cross-tabulation (Apology: 2 groups) & 125 \\
\hline Table 3.9 & Politeness group Chi-Square Tests (Apology: 2groups) & 125 \\
\hline Table 3.10 & HA/SM group Cross-tabulation (Request: 3 groups) & 126 \\
\hline Table 3.11 & HA/SM group Chi-Square Tests (Request: 3 groups) & 126 \\
\hline Table 3.12 & HA/SM group Cross-tabulation (Request: 2 groups) & 127 \\
\hline Table 3.13 & HA/SM group Chi-Square Tests (Request: 2 groups) & 127 \\
\hline Table 3.14 & Politeness group Cross-tabulation (Request: 3 groups) & 128 \\
\hline Table 3.15 & Politeness group Chi-Square Tests (Request: 3 groups) & 128 \\
\hline Table 3.16 & Politeness group Cross-tabulation (Request: 2 groups) & 129 \\
\hline Table 3.17 & Politeness group Chi-Square Tests (Request: 2 groups) & 129 \\
\hline \multicolumn{2}{|c|}{ CHAPTER 4 APOLOGY } & \\
\hline Table 4.1 & Proportion of Negative and Positive Politeness Strategies by Group & 177 \\
\hline Table 4.2 & Number and Percentage of Utterances by Group & 177 \\
\hline Table 4.3 & Characteristics of Head Acts by Group & 179 \\
\hline Table 4.4 & Proportion of Main Supportive Moves in Discourse by Group & 182 \\
\hline Table 4.5 & Proportion of Apologetic Expressions Uttered by Group & 188 \\
\hline Table 4.6 & Past Tense Used by Group & 191 \\
\hline Table 4.7 & Expressions of Past Tense & 191 \\
\hline Table 4.8 & Admission of the Facts Uttered by Group & 194 \\
\hline Table 4.9 & Proportion of Utterance of Chotto in Explanation & 198 \\
\hline Table 4.10 & Utterance of Promise by Group & 200 \\
\hline Table 4.11 & Characteristics of Expressions & 201 \\
\hline Table 4.12 & Utterance of Placation & 202 \\
\hline
\end{tabular}




\begin{tabular}{|ll|r|}
\hline Table 4.13 & Order of Utterances by Group & 203 \\
\hline Table 4.14 & Utterance Starting from Apology & 205 \\
\hline Table 4.15 & Utterance Starting from Admission of the Facts & 205 \\
\hline & & \\
\hline CHAPTER 5 REQUEST & 224 \\
\hline Table 5.1 & Typical Expressions of Bald on Record Strategy & 229 \\
\hline Table 5.2 & Typical Expressions of Positive Politeness Strategy & 236 \\
\hline Table 5.3 & Typical Expressions of Negative Politeness Strategy & 240 \\
\hline Table 5.4 & Typical Expressions of Off Record Strategy & 242 \\
\hline Table 5.5 & Combination of Politeness Strategies & 265 \\
\hline Table 5.6 & Number of Politeness Strategies Used by Group & 266 \\
\hline Table 5.7 & Number of Utterance per Discourse & 275 \\
\hline Table 5.8 & Ratio of Supportive Moves by Group & 282 \\
\hline Table 5.9 & Number of Head Acts per Discourse & 285 \\
\hline Table 5.10 & Nai Used in Positive Politeness Strategy & 286 \\
\hline Table 5.11 & Ka na Uttered in Positive Politeness Strategy & 288 \\
\hline Table 5.12 & Usage of Aposiopesis & \\
\hline & & 305 \\
\hline CHAPTER 6 & SUMMARY & 306 \\
\hline Table 6.1 & Summary of Findings of Major Differences among the Three Groups & 306 \\
\hline Table 6.2 & Features of Discourse of Apology & 310 \\
\hline Table 6.3 & Features of Discourse of Request & 310 \\
\hline Table 6.4 & Apology to a Senior Party & 311 \\
\hline Table 6.5 & Apology to an Equal Party & 311 \\
\hline Table 6.6 & Request to a Senior Party & \\
\hline Table 6.7 & Request to an Equal Party & \\
\hline
\end{tabular}




\section{LIST OF FIGURES}

\begin{tabular}{|c|c|}
\hline CHAPTER 2.3 LITERATURE REVIEW Politeness & Page \\
\hline Figure 2.3.1 Strategies for Doing FTAs & 59 \\
\hline \multicolumn{2}{|l|}{ CHAPTER 2.4 LITERATURE REVIEW Apologies } \\
\hline Figure 2.4.1 Classification Scheme of Accounts & 73 \\
\hline \multicolumn{2}{|l|}{ CHAPTER 4 APOLOGY } \\
\hline Figure 4.1 Number of Utterances and Sentences & 132 \\
\hline Length of Discourse & 132 \\
\hline Number of Utterances & 135 \\
\hline Proportion by Strategy & 135 \\
\hline Number of Head Acts by Group & 138 \\
\hline Apologetic Expressions by Group & 140 \\
\hline Apologies by Expression & 140 \\
\hline Proportion of Apologetic Expressions & 141 \\
\hline Past Tense Used by Group & 141 \\
\hline Figure 4.10 Proportion of Past Tense Used in Discourse & 142 \\
\hline Figure 4.11 Apologetic Expressions in Past Tense & 143 \\
\hline Figure 4.12 Past Tense in Apology & 143 \\
\hline Figure 4.13 Order of Appearance of Past Tense & 146 \\
\hline Figure 4.14 Number of Supportive Moves & 147 \\
\hline Figure 4.15 Reasons Explained by Group & 148 \\
\hline Figure 4.16 Reason Explained & 148 \\
\hline Figure 4.17 Reason for Being Late & 150 \\
\hline Figure 4.18 Reason for Being Late by Group & 150 \\
\hline Figure 4.19 Proportion of Reason for Being Late & 151 \\
\hline Figure 4.20 Chotto Uttered in Explanation by Group & 153 \\
\hline Figure 4.21 Proportion of Chotto Uttered in Explanation & 153 \\
\hline Figure 4.22 Number of Excuse in Explanation by Group & 156 \\
\hline Figure 4.23 Proportion of Excuse in Explanation & 156 \\
\hline Figure 4.24 Aposiopesis in Explanations by Group & 158 \\
\hline Figure 4.25 Proportion of Sentences of Aposiopesis & 159 \\
\hline Figure 4.26 Admission of the Facts by Group & 160 \\
\hline Figure 4.27 Proportion of Admission of the Facts & 160 \\
\hline Figure 4.28 Promise Uttered by Group & 162 \\
\hline Figure 4.29 Proportion of Promise Uttered & 162 \\
\hline Figure 4.30 Type of Placation & 167 \\
\hline Figure 4.31 Placation by Group & 167 \\
\hline Figure 4.32 Proportion of Placation & 167 \\
\hline Figure 4.33 Initial Utterance by Group & 169 \\
\hline Figure 4.34 Proportion of Initial Utterance & 170 \\
\hline Figure 4.35 Order of Utterances & 171 \\
\hline Figure 4.36 Order of Utterances by Group & 171 \\
\hline Figure 4.37 Proportion of Order of Utterances & 172 \\
\hline Figure 4.38 Number of Usage of Supportive Moves & 174 \\
\hline Figure 4.39 Number of Usage of Supportive Moves by Group & 174 \\
\hline
\end{tabular}




\begin{tabular}{|c|c|c|}
\hline Figure 4.40 & Proportion of Number of Usage of Supportive Moves & 174 \\
\hline Figure 4.41 & Number of Strategies Used & 178 \\
\hline Figure 4.42 & Number of Strategies Used by Group & 178 \\
\hline Figure 4.43 & Proportion of Politeness Strategies Used & 178 \\
\hline Figure 4.44 & Politeness Strategies in Supportive Moves & 182 \\
\hline Figure 4.45 & Politeness Strategies Used in Supportive Moves & 182 \\
\hline Figure 4.46 & Politeness Strategies of Supportive Moves by Group & 183 \\
\hline \multicolumn{3}{|c|}{ CHAPTER 5 REQUEST } \\
\hline Figure 5.1 & Number of Utterances and Sentences & 212 \\
\hline Figure 5.2 & Length of Discourse & 212 \\
\hline Figure 5.3 & Number of Utterances & 216 \\
\hline Figure 5.4 & Proportion of Utterance & 217 \\
\hline Figure 5.5 & Number of Utterances of Head Acts & 218 \\
\hline Figure 5.6 & Number of HA Utterances & 219 \\
\hline Figure 5.7 & Number of HA Utterances by Group & 219 \\
\hline Figure 5.8 & Proportion of Number of HA Utterances & 219 \\
\hline Figure 5.9 & Number of Strategies Used by Group & 221 \\
\hline Figure 5.10 & Number of Strategies Used & 221 \\
\hline Figure 5.11 & Proportion of Strategies by Group & 221 \\
\hline Figure 5.12 & Number of Bald on Record Strategy Used by Group & 223 \\
\hline Figure 5.13 & Expressions of Bald on Record Strategy & 224 \\
\hline Figure 5.14 & Expressions of Bald on Record Strategy by Group & 224 \\
\hline Figure 5.15 & Proportion of Expressions of Bald on Record Strategy & 225 \\
\hline Figure 5.16 & Number of Positive Politeness Strategy & 226 \\
\hline Figure 5.17 & Key Expressions of Positive Politeness Strategy & 228 \\
\hline Figure 5.18 & Key Expressions of Positive Politeness Strategy by Group & 228 \\
\hline Figure 5.19 & Proportion of Key Expressions of Positive Politeness Strategy & 228 \\
\hline Figure 5.20 & Nai in Positive Politeness Strategy by Group & 230 \\
\hline Figure 5.21 & Proportion of Nai in Positive Politeness Strategy & 230 \\
\hline Figure 5.22 & Nai in Expressions of Positive Politeness Strategy & 231 \\
\hline Figure 5.23 & Proportion of Nai in Expressions of Positive Politeness Strategy & 231 \\
\hline Figure 5.24 & $\mathrm{Ka}$ na in Expressions of Positive Politeness Strategy by Group & 232 \\
\hline Figure 5.25 & Proportion of Ka $n a$ in Expressions of Positive Politeness Strategy & 233 \\
\hline Figure 5.26 & Number of Negative Politeness Strategy & 234 \\
\hline Figure 5.27 & Expressions of Negative Politeness Strategy & 235 \\
\hline Figure 5.28 & Expressions of Negative Politeness Strategy by Group & 235 \\
\hline Figure 5.29 & Proportion of Expressions of Negative Politeness Strategy & 235 \\
\hline Figure 5.30 & Number of Off Record Strategy & 238 \\
\hline Figure 5.31 & Expressions of Off Record Strategy & 239 \\
\hline Figure 5.32 & Expressions of Off Record Strategy by Group & 239 \\
\hline Figure 5.33 & Proportion of Expressions of Off Record Strategy & 239 \\
\hline Figure 5.34 & Repeated Utterances of Head Acts by Group & 242 \\
\hline Figure 5.35 & Proportion of Repeated Utterances of Head Acts & 242 \\
\hline Figure 5.36 & Use of Different Politeness Strategies in Head Acts by Group & 243 \\
\hline Figure 5.37 & Proportion of Use of Different Politeness Strategies in Head Acts & 243 \\
\hline Figure 5.38 & Number of Supportive Moves & 245 \\
\hline Figure 5.39 & Grounder by Reason & 248 \\
\hline
\end{tabular}




\begin{tabular}{|ll|r|}
\hline Figure 5.40 & Grounder by Group & 248 \\
\hline Figure 5.41 & Proportion of Reasons for Grounder & 249 \\
\hline Figure 5.42 & Expressions of Minimiser & 250 \\
\hline Figure 5.43 & Minimiser by Group & 250 \\
\hline Figure 5.44 & Proportion of Minimiser & 251 \\
\hline Figure 5.45 & Expressions of Preparator & 253 \\
\hline Figure 5.46 & Preparator by Group & 253 \\
\hline Figure 5.47 & Proportion of Preparator & 254 \\
\hline Figure 5.48 & Reward by Group & 256 \\
\hline Figure 5.49 & Initiator of Reward & 256 \\
\hline Figure 5.50 & Expressions of Disarmer & 257 \\
\hline Figure 5.51 & Expressions of Disarmer by Group & 257 \\
\hline Figure 5.52 & Proportion of Disarmer by Expression & 258 \\
\hline Figure 5.53 & Order of Utterances & 260 \\
\hline Figure 5.54 & Order of Utterances by Group & 260 \\
\hline Figure 5.55 & Proportion of Order of Utterances & 260 \\
\hline Figure 5.56 & Number of Usage of Supportive Moves & 262 \\
\hline Figure 5.57 & Number of Usage of Supportive Moves by Group & 262 \\
\hline Figure 5.58 & Proportion of Usage of Supportive Moves & 263 \\
\hline Figure 5.59 & Proportion of Discourses by Politeness Strategy & 265 \\
\hline Figure 5.60 & Politeness Strategies Used & 267 \\
\hline Figure 5.61 & Politeness Strategies Used by Group & 267 \\
\hline Figure 5.62 & Proportion of Politeness Strategies Used by Group & 268 \\
\hline Figure 5.63 & Types of Politeness Strategies of Supportive Moves & 275 \\
\hline Figure 5.64 & Politeness Strategies of Supportive Moves by Group & 275 \\
\hline Figure 5.65 & Proportion of Politeness Strategies of Supportive Moves & 276 \\
\hline
\end{tabular}




\section{CHAPTER 1 INTRODUCTION}

\subsection{Introduction}

This thesis aims to explore the speech acts of Japanese language native speakers (NSs), non-native speakers who are learning Japanese (NNSs), and model exemplars presented in Japanese language textbooks (TXs) in order to improve the quality of Japanese language textbooks. The ultimate goal of this study is to present Japanese language learners with the best type of teaching materials so that they will be able to acquire natural and appropriate utterances for a given setting.

\subsubsection{The Context of Textbooks}

Various Japanese language textbooks have been published for decades. The objectives of these textbooks differ depending on target readers and their competence levels of these assumed readers. Out of the four language skills of reading, writing, listening, and speaking, the present study focuses on 'speaking'. The foci of my analysis are, firstly, native speakers of Japanese, secondly, upper elementary and intermediate levels Japanese language learners, and finally, textbooks.

Foreign language learning no longer means studying only vocabulary and grammar. Learning and comprehending the culture of a target language is regarded as important as acquiring the usage of words and their rules. It is essential to acknowledge the culture of the people who speak the target language, because cultural background influences people's speech acts, and speech acts in turn influence culture (Ide 2005: 6-7).

Japanese language education entails not only language teaching but also instruction in Japanese culture through the teaching of the language. Both the authors of textbooks and 
learners are, therefore, expected to be sensitive to the communication styles of native speakers. Many learners of foreign languages try to memorise model examples in textbooks because these models are considered to be widely used by native speakers. As learners outside Japan do not have the same chance to be exposed to the Japanese language environment, this tendency to memorise examples is more noticeable than for those learners based in Japan (Sei 2003: 18). The model discourse presented in textbooks, therefore, should be "natural", where natural conversation equates to conversation by native speakers.

Textbooks should list what teachers and learners can acquire from them. For instance, when introducing a conversational sentence, textbooks should include a description of the proper setting to be used in addition to the meaning. Also, more importantly, they should avoid presenting a sentence on its own divorced of a surrounding context (Terao 2000: 3-5). If a new sentence is used in conversation, such as in greetings, textbooks should display to whom and on what occasion it is appropriate to be used. This procedure is applicable to learners of any language. However, Japanese, in general, is more complicated than many other languages in terms of choosing appropriate linguistic forms of grammar and vocabulary, because these vary between the interlocutors.

Analysis of linguistic forms presented in Japanese language textbooks remind me of my personal experience as a Japanese language teacher. After obtaining a teaching certificate, I taught students from rudimentary to intermediate levels. The textbooks were mostly assigned by institutions where I was employed, and I taught students from them. These textbooks were published by Japanese publishing companies and designed commercially. Although I was interested in teaching, I was concerned about the contents of the textbooks I had to use.

In most cases, textbooks presented only a single or a few sentence(s) incorporating new grammatical items to show conversational exchanges. Apparently, as far as I could tell, 
the length of model conversations differed according to the competence level of learners. Once the learners had practised and memorised the new items, they could utter sentences, but I was doubtful whether they could adopt the sentences to comply with a given new setting, because the textbooks did not usually contain thorough explanations.

From my teaching experience, I noticed that not many textbooks described the features of conversational personae in the sentence patterns presented in them, or in what scenes they would utter them, etc. The detailed explanations and/or commentary notes in the textbooks seemed to be minimal for the students (and sometimes for teachers, too).

If students were eager to acquire Japanese through their own endeavours, they would open a textbook and memorise examples seriously. I do not deny memorisation as a way of learning, but memorising examples is different from understanding basic sociolinguistic rules. Nonetheless, it is understandable that a rote memory type of learning may prevail for some learners and memorising sentences patterns of a target language is often unavoidable. Yet, applicability of indiscriminate memorisation by students was the starting point for my questions and concerns about the contents of textbooks.

Here is an example of a lack of appropriate explanations. The greeting phrase, Ohay $\bar{o}$ [Mornin'] between friends is no problem in Japanese, and also when a teacher says it to students. ${ }^{1}$ But, the phrase on its own should not be used to a superior. Students might use it mistakenly to a senior unless an explanation about its usage in a textbook or a teacher informs them that, when greeting a superior, not Ohayō but Ohayō gozaimasu is appropriate. ${ }^{2}$

This is because a teacher-student relationship is not equal but hierarchical in Japanese culture.

2 In fact, I had an experience where one of my students when entering a classroom cheerfully said, Ohayō! It was not until then that I fully realised the importance of teaching the students that there were words/phrases to be used appropriately (or not to be used in order to avoid being regarded as uneducated or ill-mannered). 
When offering something to a guest of higher rank after dinner, a host may say, What do you want to drink? or Would you like something to drink?, which is not inappropriate in English. If this English sentence is literally translated into Japanese, it becomes Nanika (nani o) nomitai desu ka? which is not an appropriate expression in Japanese to be used to a superior, because asking a higher ranked person about his/her desire/preference is considered impolite, when the speaker's implication is to offer something to the senior and the host has at least already determined to offer something to drink. The appropriate expression, in this case, would be Nani o omochi shimashō ka? [What shall I bring to you?] or Nani ga yoroshii desu ka? [What is good for you?], although these sentences do not contain words directly asking the guest's preference of what to drink (Kabaya et al. 1998: 212-3).

The above examples show that word by word direct translation is not always appropriate, because a speaker's intention is, in general, transferred in a context (not in an individual word) and the context reflects its culture. Especially, if the cultural and linguistic distance between the two languages is wide, confusion may arise. As such, it is insufficient for learners to be taught only how to create a grammatically correct sentence. Okano (1990: $19,25)$ was also concerned about difficulty in teaching natural conversation, and she concluded that Japanese language teaching should focus on the practical functions of sentences in use, instead of the conventional teaching method of memorising sentence patterns.

When creating new textbooks, cultural information about appropriate use of discourse is essential, such as on greeting expressions when people first meet on the New Year's holiday (Kano 2002: 41, 49). A student once said to me at the first lecture after the New Year's holiday, Kinga shin'nen! [Happy New Year!]. I was very surprised at hearing him say this, for two reasons. One is that he used a very traditional and classical greeting phrase, and the other, more seriously is that the phrase kinga shin'nen is only applicable in written form, 
such as on a New Year's card, and is never used in speech. This incident made me realise that he must have learned the phrase from some written source that he had read, and exercised it proudly when he came into the classroom. He had not learned (or had not been taught) that this conventional phrase should not be used in spoken language. Whether this student had learnt this written-only phrase from a textbook or not is unknown, but the example demonstrates that the authors of textbooks and teachers should better explain the settings of model discourses in terms of the relationship between personae and what they say.

In some cases new expressions are introduced with no clear and explicit explanations about where to use them, let alone the cultural background behind them. A description in the textbooks is worthwhile, not only for students, but also for teachers who are native Japanese speakers, because sometimes it is not easy for teachers to see their own culture objectively, as it is too close to them (Terao 2001: 160-2).

These personal experiences of mine as a Japanese language teacher that occurred in recent years made me think about the quality of textbooks, and the value of appropriate textbooks. In particular, while conversing in Japanese, speakers have to be concerned about their relationship with their interlocutors because of numerous constraints in usage.

\subsection{Rationale of This Study}

\subsubsection{Cross-Cultural Communication}

The word culture has a broad meaning and the concept of culture is rather intuitive (Žegarac 2008: 49). Culture as described by Xu et al. (2012: 22) generally represents history, annual functions, food, clothing, literature, art, and it also includes a sense of values, language behaviour, and non-language behaviour. Hofstede et al. (2010: 5) regard it as patterns of thinking, feeling, and acting as software of the mind or culture, which includes the 
ordinary and menial things in life: greeting, eating, showing or not showing feelings, keeping a certain physical distance from others. The range of implications of culture is very wide. Nevertheless, it can be classified by its nature into two types: namely visible (or tangible) and invisible (or intangible).

With regard to Japanese culture, people may have in mind traditional ikebana (flower arrangement) or origami (Japanese art of paper folding); or for young people, ninja (spies in the feudal era) might be considered typically Japanese, most probably influenced by manga (comics) or anime (animated cartoons) or both. These sorts of things are elements of visible culture.

On the other hand, when people communicate in Japanese society, social hierarchy plays an important role in the choice of appropriate words and phrases. For example, gomen, which literally means "forgive me" when apologising, can be used to your equals. However, it is inappropriate to your seniors because, being grammatically a command form, it insults them. However, it can be used by a senior to a subordinate. When you have caused offence and have to apologise, a Japanese interlocutor (victim) would expect to hear the apology first followed by the reason for the offence, rather than the other way around. This is included in what Hofstede et al. referred to as software of the mind or culture, which is invisible.

Language cannot and should not be taught separately from culture. When studying a foreign language, it is important for learners to acknowledge the various backgrounds of the target language, including visible and invisible culture, such as social structure, geography, economy, etc. In addition, political or diplomatic ability may be required. For example, for a person referring to him/herself, a number of words are available in Japanese: watashi, watakushi, boku, ore, jibun, temae, and so on. The meaning of all of them is "I" in English. Which one is chosen depends on the setting (Miyahara, 2011: 22). As such, one of the characteristics of Japanese is the existence of sociolinguistic restrictions on vocabulary and 
expressions that are determined mostly according to the setting, including the relationship between the two parties. Thus it is important for learners to understand these subtleties, lest they cause offence.

The cultural backgrounds of learners vary. Even if a speech act meets the courtesy requirements of a learner's own culture, there is the possibility that it does not always suit in Japanese culture, and vice versa. Such problems may occur when uttering something based on the speaker's own linguistic and cultural norms and simply translating it into the target language, and the interlocutor may then receive the speaker's implication differently from what was intended. ${ }^{3}$ In my thesis, then, the definition of culture is invisible culture, and it can be interpreted as Japanese social hierarchy, because this affects the appropriateness of the words and phrases used when people speak.

\subsubsection{Politeness}

The way a conversation develops varies according to people and culture. Aside from individual personal differences, there may be cases where a conversation does not go smoothly or becomes awkward because the cultural backgrounds of the two parties are not close. In this study, therefore, the politeness theory of linguists Penelope Brown and Stephen C. Levinson will be adopted to examine the conversational data for this experiment claimed that politeness is universal. The core of their theory is that social language actions are uttered in order to establish a smooth personal relationship and maintain it. In this regard, they claim

3 For example, in Japanese, if higher rank guests are asked what they want to drink after dinner, Kōhì o nomitai desu ka [Do you want to drink coffee?], the guests may feel the host's speech is intrusive because of the word tai [want]. In practice, it is most socially acceptable not to ask one's preferences at all but to intuit what they prefer and serve that accordingly. 
that their politeness theory is universal in their book (1987), Politeness: some universals in language usage. ${ }^{4}$

Politeness theory centres on speakers' language strategy, with the view that it is mentioned to lubricate human relations. Thus, examining the speakers' choice of politeness strategy types ${ }^{5}$ may be a good educational means to envisage the differences and similarities among native speakers (NSs), non-native speakers (NNSs), and so-called model dialogues in textbooks (TXs). If differences are recognisable, it will enhance our understanding of these varied strategy choices.

As mentioned above, even though students may have memorised how to produce a grammatically correct sentence, if they utter it in an inappropriate situation, it may not be accepted well by the hearer. In other words, being able to generate a correct sentence in terms of grammar does not always mean that the speaker has 'acquired the language'. The key is whether textbooks (and teachers) can support students to reduce the differences.

\subsection{Aims of This Study}

\subsubsection{Earlier Studies}

There have been several comparative studies of conversational data between NSs and NNSs in the past (Akahori 2000, 36-45; Koike 2000, 62-68; Kumagai 2008, 27-32; Matsuda et al. 2008, 48-58). But there have been a small number of studies between NSs and TXs than between NSs and NNSs (Kawaguchi, Y. 2005, 1-6; Laohaburanakit 1995, 29-37).

\footnotetext{
4 Their theory is focused on the speaker' utterances of a sentence level in their book but not a discourse level (Usami 2002a: 226, Watts 2003: 85).

5 There are four types of politeness strategies: bald on record strategy, positive politeness strategy, negative politeness, and off record strategy (see Ch.2, Sec. 3 for further explanation).
} 
Nevertheless, to the best of my knowledge, no previous studies have compared the data of the three groups of NSs, NNSs, and TXs.

Furthermore, while comparative studies have examined individual expressions at a sentence level (Barnlund and Yoshioka 1990, 195-204; Nakada 1989, 194-202; Ogawa 1995, 39-51), only a few have considered larger discourse levels (Kumagai 1995: 23-9; Unosawa 2005, 144-169). In this regard, studying entire discourses is essential in order to comprehend the speaker's strategies and tactics in choosing expressions to develop the conversation.

\subsubsection{Aims of This Study}

The aims of this study are to explore similarities and differences in the speech acts of the three groups: NSs, NNS, and TXs. In order to achieve this, Brown and Levinson's politeness theory together with the work of Blum-Kulka et al., the Cross-Cultural Speech Acts Realization Project (CCSARP) ${ }^{6}$ will be applied as a framework. Textbooks are vital for language learners who rely on them all the time. Acknowledging and presenting the differences found in the present research, in particular differences between NSs and the others, will be significant elements for authors of Japanese language textbooks to improve their quality.

Recommendations for improving Japanese language textbooks will be an ultimate goal of this study. Based on my experiences as described in the previous section, utterances to a given setting may be grammatically correct but culturally inappropriate in the given context. This is not a problem of the speakers' linguistic ability, but a problem of teaching in a broad sense, including the teaching materials available.

6 The languages researched by Blum-Kulka et al. are American English, Australian English, British English, Canadian French, Danish, German, and Hebrew (Blum-Kulka et al. in Cross-cultural pragmatics: requests and apologies, Advances in discourse processes. 1989: 16). 
Hence, in order to rectify the situation, it is essential to grasp what types of differences occur among NSs, NNSs, and TXs, and this thesis will propose how to improve textbooks' quality so that learners may improve the appropriateness of their utterances. Accordingly, this study has three research questions.

Research question 1: What similarities and differences in discourse structures are revealed among the three groups: native speakers of Japanese, non-native speakers and model exemplars presented in the textbooks?

Research question 2: What similarities and differences in expressions are revealed among the three groups: native speakers of Japanese, non-native speakers and model exemplars presented in the textbooks?

Research question 3: In what way can Japanese language textbooks be improved in order to more closely reflect "authentic" native speaker discourse?

\subsection{Organisation of This Study}

This thesis is organised into seven chapters. Chapter 1 delineates the background and rationale for this study, outlines its aims, and presents three primary research questions. The collected data will be analysed from perspectives of the politeness theory of Brown and Levinson and the CCSARP of Blum-Kulka et al.

Chapter 2 is a review of the scholarly literature in this field to date, in which there are five subdivisions. Section 1 focuses upon textbooks, including creation of language textbooks as well as critiques of textbooks. Section 2 explores the characteristics of the Japanese language, especially spoken Japanese. This includes the relevance of the relationship between speaker and hearer. Section 3 treats Brown and Levinson's politeness theory, explaining what 
politeness theory is and how it relates to Japanese. Sections 4 and 5 analyse the literature on speech acts of apologies (Section 4) and requests (Section 5) in Japanese more specifically, since these situations are also problematic for non-native speakers to negotiate successfully.

In Chapter 3, the research method adopted and the data collection methods used for this study are explained. In terms of the conversational data, I drew on recorded role-play conversations of both NSs and NNSs. The procedures and information about the participants who took part in role-play conversations and limitations of the experiments are also included in Chapter 3.

In Chapters 4 and 5, the conversational data of apologies and requests are analysed. First they are categorised based on the CCSARP, then analysed in light of politeness theory. The results of analysis reveal the characteristics of each of the three groups studied in terms of structure and expressions. Then these findings are discussed not only in aggregate form as categories of apologies and requests but also comparing and contrasting the degree of similarity and differences in speech acts between apology and request.

Chapter 6 summarises all findings, discusses salient points that emerge from the analysis, and then makes recommendations for improving textbooks on the basis of the results of Chapters 4 and 5.

Finally, Chapter 7 concludes this study, by a broad overview of the findings, pedagogical implications, recommendations, and some implications for further study.

I hope that this study will contribute to expand the knowledge not only of the similarities and differences among NSs, NNS, and TXs, but also enable authors of textbooks to become more aware of the importance of thorough explanations of their model discourses, especially the factors that are necessary for them to sound natural and appropriate in given settings. These findings are relevant for authors of Japanese language textbooks, Japanese language learners, and their teachers. 
With this explanation of the background that provided impetus for the present study, and a description of the organisation of this thesis, we may proceed to Chapter 2, in which I review previous studies related to teaching materials, Japanese language, politeness, and speech acts of apologies and requests in Japanese. 


\section{CHAPTER 2 LITERATURE REVIEW}

In Chapter 2, I will explore prior research related to the present study. I divide this research into five relevant groups. Section 1 reviews studies about language textbooks, including the creation and criticism of textbooks. The purpose of Section 2 is to outline the characteristics of the Japanese language, in particular, the spoken language, with reference to its relevance to the teaching of Japanese as a second or other language. Section 3 focuses on the politeness theory established by Penelope Brown and Stephen C. Levinson in the 1980s, as it has been widely disseminated in the humanities and provides key aspects for the methodology of the present study. In Sections 4 and 5, I scrutinise studies on apologies (Section 4) and requests (Section 5).

\subsection{TEXTBOOKS}

\subsubsection{Introduction}

Textbooks generally take a central role in the teaching and learning environment (Hayashi 1996: 45, Nunan 1999: 81, Yokoyama 1995: 109), ${ }^{7}$ and they occupy a particularly important position when teachers have control over their curriculum (Furubeppu 2009, Okazaki 1989). Also, textbooks provide an effective support for teachers in their work (Tanaka 1988, Yokoyama 1995).

7 There may be cases when a textbook is not used as, for example, when learning a language through private tutoring. However, it is assumed here that a teacher is teaching a number of students a foreign language at school. 
It cannot be denied that teaching and learning depend greatly on the ability of teachers; however, since textbooks are widely used in the teaching of Japanese as a second or other language (JSOL), it is assumed that they also have an influence on the learning of students. Hence, it is essential for the present study to investigate the results of previous research regarding the efficacy of language textbooks and critical reviews of relevant language textbooks.

In the classroom, effective teaching materials are designed to be made the most of by students and teachers. They are important components of the curriculum, and are often the most tangible and visible component of pedagogy (Nunan 1991: 227), holding the prime place (Yokoyama 1995: 109). A textbook gathers relevant knowledge for course design (Hayashi 1996: 45), and it normally forms the basis of class work. There are two kinds of textbooks in terms of publication: those which are printed privately and used internally in a closed environment, such as by specific educational institutions, and others which are published in book form by a commercial or academic publishing company on the open market.

\subsubsection{Significance of Textbooks}

Print is the principal medium of educational communication (Davis 1962: 127), and arguably this has not changed despite technological innovations. A textbook-based class is still part of the mainstream in Japanese language teaching as a second or other language. Textbooks are thus necessary to supply indirect experience in large and well-organised amounts.

Kumar's (1986: 1309) view of textbooks is that they symbolise the authority under which the teacher accepts to work. They also signify the teacher's subservient status in the educational environment. The textbook is thus a major determinant of what is taught and how, 
because classes usually follow the order of content in a specified textbook (Furubeppu: 2009: 9-10, Okazaki 1989: 48-9). Duffy et al. (1989: 436) observe that the textbook lies at the core of the curriculum in most schools, and is typically the basis for creating shared knowledge between a teacher and students. Nunan (1988: 98) states, "Materials are essential elements within the curriculum, and do more than simply lubricate the wheels of learning..., they provide concrete models of desirable classroom practice."

Lester and Cheek (1997: 283) conclude that because of its immense influence on what is taught in the classroom, the textbook for any particular content area should be userfriendly to accommodate the student who will need to understand the information it contains. Conversely, from the teacher's point of view, Nunan (1991: 209) explains that commercial materials fulfil an important educational function of teachers, and remove much of the burden and time involved in creating materials from scratch. It is no easy task for teachers to determine what and how to teach, and a textbook is able to facilitate them in such decisions. Another role of good materials is to provide exemplars for teachers in developing their own supplementary materials.

The aforementioned scholars note how valuable and instrumental textbooks are with some useful comments, and how they have hitherto played a key role in the educational sector. Textbooks continue to occupy a prime position in the teaching and learning situation, even though various teaching tools have been introduced in recent years, including electronic resources.

No matter how substantial the role of textbooks in the teaching and learning environment is, insofar as it is merely a pedagogical tool, a textbook cannot be definitive. Rather, it should be suggestive in order to lead and support students to improve their basic skills and knowledge. In particular, language textbooks need to reflect real speech. This 
implies that they should have a degree of authenticity and convey the sociocultural context in which learners can comprehend the background of the target language (Nunan 1988: 99).

In the same way, Lester and Cheek (1997/98: 290) concede that it is unlikely that there will ever be a perfect textbook. They assert that textbook usage from the student's point of view provides implications of promoting a sense of ownership that can facilitate learning that is essential both inside and outside the classroom. The following two points are considered to be shortcomings: firstly, no textbook will be capable of catering for the diversity of learners' needs found in most language classrooms. Therefore, teachers will inevitably have to adjust or modify it in order to meet the requirements of their learners. Secondly, textbooks have not always kept pace with changing needs (Buckingham 1952: 244, Nunan 1991: 209, Okazaki 1989: 48-9, Yokoyama 1995: 109-10). These drawbacks are issues which have remained unresolved for years.

There is, of course, no faultless methodology of teaching and learning. Likewise, there is no perfect textbook, as Yokoyama (1995: 110) argues. As such, when teachers use a textbook that is available on the market, it might be necessary to adjust, add, or omit parts of it when using it in class so as to suit particular students' needs. In almost all circumstances of language education, textbooks have played and will continue to play the leading role on the teaching and learning stage - although it is almost impossible to find or to create all-purpose type textbooks.

\subsubsection{Language Textbooks}

In the Japanese language educational domain, along with inevitable changes which qualify society and the constant diversification of learners' purposes of study, students have been urged to acquire communicative skills, so that the authors of textbooks tend to focus on various strategies for conversations (Horiguchi 1997: 129). Ishida (1998: 42) notes that 
textbooks are mostly employed in class at the beginner and intermediate levels, whereas authentic materials are utilised more widely at the advanced level, such as newspaper articles and/or books on the market.

Until recently, teaching Japanese spoken language and written language has been largely divided according to the degree of proficiency of the learner. That is, the former has mainly been taught at beginner level and the latter from intermediate level. Sato (2000: 3-5) argues that the spoken language is also in need of being taught at the intermediate level in order for learners to expand their linguistic horizon towards the society of the target language. Kabaya (2011: 13) and Okano (1990: 18) agree with Sato that Japanese language pedagogy involves nurturing communicative ability with the aim of making learners become proficient in communication.

Several scholars have criticised Japanese language textbooks for emphasising a crammed teaching method that arguably leads to information overload. Noda (2009: $34-6)^{8}$ suggests that in order to avoid this, textbooks should be divided according to the four linguistic skills so as to set up realistic and attainable goals for learners, and to be flexible enough to comply with the objective(s) of individual students, ${ }^{9}$ because learners are not satisfied with mere memorisation of sentence patterns in textbooks (Furubeppu 2009: 9-10). Takagi (2011: 52) condemns Japanese language textbooks that do not provide learners with methods of self-expression and social participation, in spite of the fact that instruction in such methods is crucial for them, especially for those who have already arrived in Japan.

The model discourse presented in textbooks is expected to be natural where natural conversation equates to conversation by native speakers. Learners who mostly rely on a textbook often have limited language skills and vocabulary. Arguably, it is self-evident that

8 Noda (2009: 32-36) raises examples presented in Minna no nihongo shokyū 1.

9 There have been Japanese language textbooks designed to focus on each individual skill. However, Noda's point is to do this more thoroughly and completely. 
authors of textbooks must be careful not to present unnatural or unrealistic discourses in textbooks. In addition, since the context and the relationship between speakers determines the choice of speech level of utterances in Japanese, authors should provide detailed explanations of the usages of expressions and the relationships between conversational personae.

Liddicoat is concerned that language textbooks distort and oversimplify actual practices in conversation, which conceals the potential for difference in culture between the target language and that of the learners. He argues that it is beneficial for learners to have more opportunities to be exposed to culture-related items in the target language (Liddicoat 2000: 52).

\subsubsection{Language Textbooks and Culture}

Foreign language learning implies having a good grasp of the culture of the target language. If learners lack cultural awareness, there is a high likelihood they will experience incompatibility in communication, particularly in the case of learners whose native languages are distant from Japanese. Terao (2001: 159) asserts that it is now widely understood that smooth communication with people of different cultures only occurs when one has a good knowledge of the others' ways of life as well as their sense of values. This factor explains why descriptions of the target language's culture(s) are indispensable in language textbooks. Disappointingly, there are obvious cases where topics tackled in textbooks are obsolete, or an explanation is inappropriate, or there is no explanation at all. ${ }^{10}$

Terao (2001:158-9) argues that language textbooks must include descriptions about the culture of the target language and its background. Especially for teachers of non-native

10 For example, in Minna no nihongo shokyū II Honsatsu (1998: 140) the soroban [abacus] which is rarely used nowadays is presented in grammar exercises, and the textbook contains no explanation of the words nijikai [a party after a party] and bōnenkai [a year-end party] (ibid.: 118) (Terao 2001: 160). 
Japanese speakers, explanations of Japanese behavioural patterns, such as norms, discipline, official stances (and underlying motives), etc., are essential requisites to be described in JSOL textbooks. ${ }^{11}$ Ideno's (2011: 78) concern is that for teachers of non-native speakers outside Japan have few opportunities to be exposed to Japanese culture, let alone the students themselves. Therefore, the importance of conveying cultural information in JSOL textbooks should not be underestimated.

Worldwide over seventy per cent of Japanese language teachers are non-native speakers, and forty per cent of institutions that teach JSOL have no teachers who are native speakers. In addition, some teachers have never been to Japan, so their contact with Japanese culture is most likely to be through a textbook which is a familiar medium for them (Ideno: 2011: 68). Teachers who do not have a wide knowledge of culture and social norms have to depend mostly on textbooks. As a consequence, the role of the textbook for this purpose is magnified. In order to explain the cultural practices of the target language, the textbook should not focus on a single fragmented element of the target culture - as used to be the case in the past-but display it comprehensively, without failing to include core concepts of the culture.

Sentence-pattern practice seems to be indispensable when learning a foreign language. Frantzen (1998: 134-5), however, alleges that if practising sentence patterns is isolated from a context, learners will rarely exercise further, because these sentence patterns may not be applied to other situations. In other words, if contextualised exercises appear in realistic settings and are built around meaningful themes, they afford a natural context in which to practise grammar and vocabulary. Frantzen argues that form-focused activities with cultural

11 In 2012, around four million people were studying Japanese overseas, and the percentage of teachers who were non-Japanese native speakers was 77 per cent (Kokusai Kōryū Kikin [The Japan Foundation]. 2013. Kaigai no nihongo kyōiku no genjō [Survey report on Japanese]. pp. 5, 16-7). 
context and expansion of cultural activities with grammar practice move learners closer to the goal of integrating culture within the study of the target language structure. ${ }^{12}$ Furthermore, he argues that it is easier for learners to understand fully newly introduced words and/or phrases if they are found in a group — such as story or discourse — rather than being used in a single sentence, as an example. This applies to Japanese language education, too. For instance, when introducing new items of vocabulary, a new character should not be taught alone, but be employed in a sentence, since learners will better grasp when and how to use it through a context.

When teaching grammar, it is insufficient to teach by explaining the grammar alone. It is crucial to include the language and culture lying behind the grammar more holistically. As widely recognised, no matter what is being studied, it is easier for learners to remember when it is tied to a realistic context. Schmidt and Frota (1986: 306-7) assert that if language, including different morphological and syntactical forms, is practised in cultural contexts, content may serve as a device to help students recognise more easily how different what they produce is from the target. Frantzen (1998: 142) argues that grammar should not be taught separately from a likely setting, and Hadley (1993: 359-60) concludes that while acquiring sentence patterns and grammar is essential for learners, providing them also with the cultural knowledge that underpins them will be more effective.

Endorsing the findings of Frantzen and Hadley, Thomson and Otsuji (2009: 60) reveal dissatisfaction on the part of teachers and students with the Japanese textbooks used in Australian universities. ${ }^{13}$ The reasons were mainly lack of adequate explanations of the

12 One example explained by Frantzen (1998: 138-9) is that many textbooks have pictures of menus from actual restaurants that are used to practice new vocabulary. These same menus could be revisited later to practice various structures involved in carrying out the functions while exploring the cultural content.

13 The textbook is Getting down to Business Japanese for Business People. 
cultural and business background of the model discourses. In such cases, teaching and learning end up becoming a recitation of vocabulary and expressions, and it is hard for students to relate newly introduced items to their own interests.

To sum up, both students and teachers have high expectations that a language textbook will delineate social and cultural contexts for them, in addition to providing model discourses. This is particularly essential for non-Japanese teachers because they themselves may have insufficient familiarity with such contexts.

\subsubsection{Naturalness of Model Discourses}

Over the years, naturalness in model discourses has been sought by authors of textbooks, but creating 'natural' model discourses has proven challenging. Many researchers have been analysing the 'naturalness' of conversational examples, mostly comparing them with various authentic linguistic data as well as the flow of the context. The issues in generating naturalness in teaching materials are a common concern across language teaching.

In this present study, the definition of 'natural' or 'naturalness' is that the flow of conversation is plausible and reasonable in developing a discourse. For example, the discourse should run smoothly and there should be no sudden change without a plausible and

socially acceptable reason. Speakers (personae in model exemplars) talk about a topic coherently. In other words, the authors do not distort the discourse artificially for the purpose of introducing a new item of vocabulary or new grammatical sentence patterns.

\subsubsection{Textbook Creation}

Cross-cultural learning of the target language is an important aspect of foreign language study. Sawada (2001: 150-3) asserts that all topics related to Japanese ways of 
doing things, the relationships between people and the target language, such as greetings, must be explained in a JSOL textbook. Mori (2005: 281) argues that authors of language textbooks ought to include societal norms that have not been presented hitherto. Gondo and Iryo (2006: 152-6) agree with Mori, and stress that points to instruct students in include all cross-cultural elements, such as an appropriate attitude depending on the setting, the relationship with others, such as manipulating speech style appropriate to the interlocutor, and non-verbal linguistic factors, such as posture.

Fujii (2005: 329-30) argues that it is not necessary to present many grammatical items in textbooks for beginners, because the students' level of proficiency is too low for them to digest them in large numbers. Fujii prefers that an example of a conversation should form a discourse: a cluster of interactional sentences of two (or more) parties, and it should represent a context. As for context, Tomlinson (2010: 87-8), too, emphasises the importance of contextualisation of spoken texts for textbooks, and argues that many examples of discourses are also essential. He implies that the usage of a language is determined according to the given context. From the viewpoint of designing model discourses, Saito (1986: 5) is concerned about the plausibility, likelihood, and length of any scenario.

\subsubsection{Textbooks for Learners Located Overseas}

Textbooks for Japanese as second or other language (JSOL) can be categorised according to place of publication: in particular, those produced in Japan and those produced overseas. The most noteworthy difference between these locations is that in the case of the latter, authors and teachers are mostly non-native Japanese speakers who are often concerned about details of which most Japanese native speakers are not aware.

There have been several noteworthy projects for creating Japanese language textbooks overseas. Concerns about non-native Japanese teachers were raised to the design team of the 
textbook for high schools in China. They were requested to add an instruction manual with descriptions of the personae of the settings in the model discourses and additional teaching plans in order to pursue a communicative approach, which targets natural utterances (Kano 2002: 45-9). Similarly, Shinozaki et al. (2004: 93-9) outline their procedure for publishing Japanese textbooks for elementary school pupils, ${ }^{14}$ whereby they prepared a teachers' manual containing the principles of the textbook and a teaching methodology for using it. When the project of the textbook Indoneshia e yōkoso [Welcome to Indonesia] was underway, ${ }^{15}$ Evi et al. (2006: 124-6) paid careful attention to the needs of inexperienced JSOL teachers, so that they could simply follow the textbook's teaching manual.

Furthermore, providing clear explanations of grammatical points from a learner's viewpoint along with correct answers to questions and quizzes in the textbook ${ }^{16}$ were key when Miyachi and Sai (2009: 55-9) were producing their textbook in Taiwan. In contrast to many cases in Japan, ${ }^{17}$ they believed that it would help students engaged in self-study if the correct answers were included in their textbook.

What differentiates textbooks designed for teachers and learners in Japan and those outside Japan is the degree of depth. This is because primarily the level and needs of the learners differ. The characteristics of teachers-whether native Japanese speakers or nonnative speakers-need to be considered. It might not be a problem if suggested answers to the questions and quizzes are not supplied, when the textbook is sold and used in Japan, where the teachers are overwhelmingly native Japanese speakers. However, if textbooks are to be sold and used outside Japan, an explanation of topics presented in the textbooks and model

\footnotetext{
14 The textbook is 小学日語教材 [Xiao xue riyu jiaocai].

15 The project of 'Indoneshia e yōkoso' started 2001 and took five years.

16 The textbook is 日本語専門塾 [Ribenyu zhuanmenshu].

17 When a textbook is published in Japan, the correct answers are normally only included in the teachers' manual but not in the students' textbook.
} 
answers should be included, as these are more likely to be needed by the predominantly nonnative Japanese teachers, let alone their students.

\subsubsection{Textbooks for Learners Located in Japan}

Model dialogues have hitherto focused mostly on one speaker, but in order to establish a natural and smooth discourse, the response of the other party cannot be neglected. In a ground-breaking attempt, Gondo and Iryo $(2006: 154-6)^{18}$ devised a flow chart of model discourses for intermediate level university JSOL students. Their flow chart shows semantic speech acts and relevant model utterances. ${ }^{19}$ The purpose of this table was to enable the students of conversation classes to easily understand the structure of an individual discourse.

Table 2.1.1 Flow of Casual Request

\begin{tabular}{|l|l|}
\hline Semantic Speech Acts & Model Utterances \\
\hline 1 Broaching & A-san, anō chotto ii? [Mr. A, um, do you have time?] \\
\hline 2 Request & $\begin{array}{l}\text { V-te hoshii n' da kedo... [I'd like you to do V...] } \\
\text { V-te moraenai? [Can't I ask you to V?] }\end{array}$ \\
\hline 3 Closing & Arigatō [Thank you.] \\
\hline
\end{tabular}

(Adopted from Gondo and Iryo 2006:158)

Mizutani (1986: 74) argues for the importance of presenting a whole discourse for studying a conversation, rather than an individual sentence. There are indispensable elements of discourses that authors should consider from the viewpoint of contextualisation. His point was taken account of in Gondo and Iryo's work.

The textbook, Sōgō [Comprehensive] was created at Dōshisha University for its international and exchange students (Matsumoto et al. 2005: 84, Sao 2004: 79-80). The authors had recognised that the students either could not produce and develop appropriate phrases and sentences in response to a given setting, or could not necessarily make use of

18 They are members of Kurume University.

19 Their flow chart utilises symbols and callouts. English translations are not provided. 
them systematically or connect them to a given setting. As a result, student utterances were simplistic, clumsy, and unnatural. What Matsumoto et al. added in Sōgō was detailed information about the personae of the model discourses. With the perception of the characters as though in a play, the students could better understand when and how to use the expressions exemplified.

Another example of improving the quality of teaching materials occurred at Kumamoto University, where they had been using one of the best known and widely-used textbooks, Situational Functional Japanese, since 1995. Nonetheless, some teachers were dissatisfied and complained of the out-dated topics. So the teaching staff of the university created their own textbook with communication uppermost in the authors' minds (Katayama et al. 2008: 39-44). However, the freshness of the content could not be expected to last indefinitely, according to Katayama et al. ${ }^{20}$ What they did in order to comply with the teachers' requirements were: (1) removal of several expressions which they thought were not appropriate to be taught until intermediate level, ${ }^{21}(2)$ introducing new words and expressions based on highest frequency of usage, even though they may have more than one meaning, rather than introducing all meanings of a word at once, ${ }^{22}$ and (3) presenting a model discourse at the end of each lesson so as to focus on teaching more communicative skills.

20 The basic plan of creating Situational Functional Japanese was sometime in the late 1980s. Hence, during the 20 years after the first version was published, its content became obsolete to some extent.

21 They are, tsumori da [intend to V, will V], V-te hoshii [wish to V], rashii [seem to V, to be said], etc.

22 For example, they introduced only two meanings of the te-form, $V$-te $/ V$-te masu and $V$-te kudasai, rather than including temo ii desu [allowed to V] or te wa ikemasen [not allowed to V], etc. As for the juju dōshi, they introduced ageru [give] and morau [receive], but not kureru [give (a humble form of receive)]. 


\subsubsection{Criticism of Language Textbooks}

Conversational exemplars presented in language textbooks are crucial for learners as these model expressions can be their first phrases when they are confronted with a situation similar to that in the textbook. Critical views of language textbooks, in particular, conversational model expressions exist, however, as we will see in the following section. The following are some critiques of language textbooks.

\subsubsection{Insufficient Explanation and Limited Presentation in Model Discourses}

Japanese language textbooks on the market have been, and still are, designed based on grammar-oriented syllabi, although the communicative approach has become mainstream in teaching methodology (Yamaguchi 2008: 90-2). Model conversations presented in textbooks have drawn the attention of researchers because their quality so far does not seem satisfactory. Yamaguchi argues that, owing to the lack of sufficient explanation about the setting of the exemplars, learners might use them in inappropriate settings. ${ }^{23}$

Laohaburanakit (1995: 38) reveals that in 13 Japanese language textbooks only limited examples of refusals are presented. She discovered that most exemplars of refusal do not describe the relationship of the speaker to the interlocutor, nor the reason for refusal (e.g., time constraints, lack of ability on the part of the person requested, or the degree of demand

23 Yamaguchi raises questions about the conversational examples shown in Minna no nihongo shoky $\bar{u}$ where the expression Onegai shimasu [Please (do)] is used when requesting. What she points out is that the utterance Onegai shimasu is not always appropriate. For example, if you find a shoe you would like to try on, it may be awkward to use Onegai shimasu to ask the shop assistant to show you the other shoe that goes with it (Yamaguchi 2008: 92). Perhaps, in this case, Sumimasen ga, misete kudasai [Excuse me, may I take a look at it?] is more appropriate. Instead, Onegai shimasu may be said when you decide to buy them. 
of the requester). ${ }^{24}$ That information is relevant for learners, since the appropriate expressions and vocabulary will differ in Japanese depending on the setting.

Similarly, Matsushima (2003: 63-5) scrutinises 12 business-oriented Japanese language textbooks published in China. She observes that no single textbook that she examined explains necessary contextual information, such as the situation of the setting, in either the dialogues or the preface to the textbook. Information about the setting is necessary for learners, however, because the appropriateness of an utterance significantly depends on the occasion and the speaker's relationship with the interlocutor. She criticises the usefulness to learners of presenting only a model dialogue-with no explanation or information about the model discourses, because learners have difficulty in generating appropriate utterances without such information. So authors of textbooks should be aware of the importance of explanations and include at least the relationships between the personae in the discourses.

Model discourses in communicative-style language textbooks inevitably focus on dialogues that are presumably considered most relevant by the authors. Miyazaki (2005: 1922) examines model discourses in selected textbooks with attention to how verbally wrong expressions are rectified if a Japanese language learner makes a mistake. He notices that overwhelmingly an explicit adjustment is made in the textbooks. While other possible scenarios exist, such as implicit advice for adjustments, they are far fewer in number. ${ }^{25}$ Miyazaki notes critically that only easy-to-create model discourses were designed, which are eventually acquired by the learners rather effortlessly, whereas more difficult scenarios with

24 These factors include the mildness and pushiness of the requester, and the extremity of the request itself, such as asking to borrow a pen as opposed to $\$ 50,000$.

25 Other means of adjustment are: (1) The non-native Japanese speaker notices a wrong expression which he/she has uttered, and a Japanese native hearer corrects it, in 11 textbooks and 12 drills; (2) The Japanese native hearer notices and corrects it, in seven textbooks and nine drills; (3) The non-native Japanese speaker notices and corrects it, in one textbook and two drills (Miyazaki 2005:17). 
implicit advice seemed to be avoided. This avoidance may arise because it is not easy to create suitable examples of speakers noticing their errors by themselves and correcting them spontaneously.

Hashiuchi (1988: 47-8) argues that, in practice, speakers often do correct their own utterances as soon as they recognise a mistake, ${ }^{26}$ although this observation does not corroborate Miyazaki's claim that the number of model discourses was not balanced in the case of rectifying the utterance. A limited range of presentation is also recognised by Kawaguchi, S. (2005: 46). She claims that although the phrase, ka mo shirenai [may/might], possesses several different semantic functions, the five textbooks that she examines introduce only a few of them. ${ }^{27}$ There are various implications even for a single expression. As such, prioritising what to present in model discourses is significant for the authors of textbooks.

\subsubsection{Unnatural Model Discourses}

Several cases of unnatural model discourses appear in language textbooks. According to Hata (1988: 107), naturalness of model discourses should be reconsidered by authors of Japanese textbooks. He is concerned about oversights and a lack of thoughtfulness in the model dialogues presented, because each utterance in a dialogue should be coherent, which, he claims, is not always the case. ${ }^{28}$ The dialogues typically appear to have been designed

26 For example, a speaker says, Ano hito ni atta no wa mokuyō..., iya suiyō datta [When (I) met that person, (it was) Thursday. No, no, (it was) Wednesday].

27 The five textbooks are: Minna no nihogo shokyū II (1998), Bunka chūkyū nihongo I (1994), Shin bunka shokyū nihongo I (2000), Nihongo bunpō enshū (2003), and Topikku ni yoru nihongo sōgō enshī (2001).

28 Hata refers to a model dialogue in a Japanese language textbook for beginners, although he does not specify the title. The dialogue is as follows (106-7). The conversation between $\mathrm{X}$ and $\mathrm{Y}$ seems very unnatural and unrealistic.

$\mathrm{X}$ : Tadaima [I'm home.] Y: Okaerinasai [Welcome home]. Doko e ikimashita ka? [Where did you go?] 
primarily for practising new items of vocabulary and grammar in given sentences. As a consequence, the discourse in many cases sounds artificial, and the model utterances so presented may not be very useful for learners, as they may have little opportunity to use them.

Kawaguchi, Y. (2005: 5-6) defines conversation as an interactional activity of people who share a common topic and then talk about it. However, the model discourse which he points out does not meet this criterion. He criticises the authors of the textbook for not developing realistic and/or appropriate scenarios, and notes that they appear to have prioritised introducing new grammatical elements rather than presenting lifelike conversations. Such dialogue can fail to lead learners to understand the natural flow of discourse or actually prevent them from doing so.

Mori (2005: 268) focuses on sequences in which explanations or introductions to background to the utterance of requests are an issue. In the discourse presented in the textbook Genki I, she found fault with the dialogues, because they would not be likely to happen in real life, or even if they did, they could potentially damage a cordial relationship between the relevant parties. The reason for this is twofold. Firstly, in one dialogue (on p.128), ${ }^{29}$ a speaker does not give a specific reason before asking for money from a friend, which is not recommended as etiquette, no matter how artificial the scenario of the language textbook is; and secondly, in the same model discourse a friend asks for money in order to

$\mathrm{X}$ : A-san no uchi e ikimashita [I went to A-san's house.]

Y: Sō desu ka [Is that so?] Donna uchi desu ka? [What sort of house is it?]

X: Chiisai desu ga, atarashii uchi desu [It's a small but new house]. A-san no oku-san ni aimashita [I met his wife].

29 The dialogue presented in Genki $I$ is:

A: Sumimasen ga okane o karate mo ii desu ka? [Excuse me, but may I borrow money?]

B: Okane desu ka? Dōshite? [Money? Why?]

A: Ashita wa tomodachi no tanjōbi desu kara [Because tomorrow is my friend's birthday.]

B: Demo watashi mo okane ga arimasen. Ashita ryokō ni ikimasu kara. [But, I don't have any money either, because I'm going to travel tomorrow.] 
buy a present for another friend's birthday, which is not a socially acceptable justification in Japan. She recommends that the scenarios should be plausible and elaborated with care in accordance with or referring to real conversation.

In another comparative study between authentic conversations and model discourses, Lin (2010: 36-7) analysed the ending forms of sentences based on 13 hours of interview data (corpus) and four Japanese language textbooks. ${ }^{30}$ From a grammatical perspective, Lin notes that in spite of the fact that the masu and dictionary forms are very widely presented as the ending of sentences in Japanese textbooks, authentic conversational data showed that only 7.5 per cent of utterances of Japanese native speakers ended with the masu or dictionary forms. ${ }^{31}$ In other words, over ninety per cent of sentences in interactive spoken discourse end with other linguistic devices, such as sentence final particles, rather than the masu or dictionary forms which typically appear in textbooks. As a consequence, sentence patterns frequently appearing in textbooks were not actually used in daily conversations. Lin argues that what is taught in Japanese speaking classes should take natural conversation into consideration so as to minimise the gap between academic acquisition of the language and its real life usage.

Lin's findings are very similar to those of Jones and Ono (2005: 242), who analyse dialogues presented in seven Japanese language textbooks popular in the U.S.A. ${ }^{32}$ They find that authentic conversations are interactive and collaborative between speakers, while

30 They are: Minna no nihongo shokyū I (2002), Nihongo shoho I (1988), Shin nihongo no kiso I (1990), Shokyū nihongo Genki 1 (1999).

31 While a textbook presents, Robi ni terebi ga arimasu [There is a TV set in the lobby] which ends with the masu-form, in an authentic conversation, a native speaker of Japanese uttered not to use the masu-form but ended with other forms, in case of the conjunctive particle, it ends ... arimasu kedo...[(There) is (X)]. The sentence final particle, such as ne, yo, ga, kedo are added on masu/iru/aru forms instead of stopping at masu/iru/aru (Lin 2010: 28, 37).

32 The textbooks analysed are: Nakama 1 (1998), Nakama 2 (2000), Japanese: The spoken language, part 1 (1987), part 2 (1988), part 3 (1990). An integrated approach to intermediate Japanese (1994), and An introduction to modern Japanese (1997). 
exemplars are typically explanatory and informative for the speaker. Authentic conversation mostly comprises sentence fragments; therefore, one sentence (utterance) tends to be short. By contrast, complete sentences (not sentence fragments) most often appear in model discourses, which are obviously lengthy, and use of linguistic devices ${ }^{33}$ is infrequent in the textbooks. Jones and Ono argue that "textbook dialogues do not reflect the ways in which real talk is produced in actual interactions" (252). They suggest that when textbooks are used in the classroom, activities should be planned in reference to authentic or realistic conversations.

In a nutshell, Hughes (2010: 221) concludes that the challenge of dealing with spontaneous speech is crucial, as many teaching materials have not yet met this need in a systematic way.

\subsubsection{Method of Teaching and Learning in Second Language Acquisition}

In second language acquisition, the question arises as to which method is more suitable, either implicit teaching and learning or explicit teaching and learning. Ellis (2009: 3, 2012: 275) and Ellis and Shintani (2014: 337-8) define that implicit L2 (second language) learning proceeds without making demands on central attentional resources, and learners remain unaware of the learning that has taken place. Implicit instruction aims to attract learners' attention to exemplars of linguistic forms, and directs at enabling learners to infer rules for themselves without any awareness of what they are learning. Explicit L2 learning, on the other hand, typically involves memorising a series of successive facts and thus makes heavy demands on working memory; learners are aware that they have learned something and can verbalise what they have learned. Explicit instruction directs learners not just to attend to grammatical forms but also to develop conscious mental representations of them.

33 Linguistic devices include repetition, repair, final particles, back-channelling, fillers, etc. 
Athough few studies seem to favour implicit instruction when teaching grammar and/or vocabulary of L2. Winitz (1996: 40) confirms that the American university students of an implicit instruction group achieved significantly higher average scores of Spanish grammar than those of an explicit instruction group. He suggests that the implicit instructional strategies may be an important consideration in the development of foreign language educational programmes. Schmitt (2008: 333) argues that the more proactive approach by students is very important, because an adequate lexis will no longer simply be picked up from exposure to language tasks. Also, he points out that if the students willingly learn implicitly over a certain period of time, they tend to attain substantial vocabulary size regardless of the quality of instruction.

The effect of explicit instruction, on the other hand, is supported by scholars such as Scott (1990: 785), Dekeyser (1994: 192), Hernandez (2008: 671-2), Culman et al. (2009: 29), Ellis and Shintani (2014: 104-12). They claim that explicit instruction helps learners develop explicit knowledge of grammatical features and vocabulary. To sum up their findings, explicit learning, in particular the grammar of L2, was effective. This suggests that some distinguishing features of Japanese, perhaps especially keigo [honorifics], should be taught explicitly to L2 learners.

It is common for learners to make various kinds of errors while they are studying. By being corrected by others, learners' language ability may be raised. When a teacher corrects a student's error, however, it is necessary to consider the learner's readiness to learn and the most effective way of correction. While the corrective feedback could be explicit, it is not always necessary to use direct and explicit correction. There is no 'best' way of conducting oral corrective feedback (Ellis and Shintani 2014: 280-1).

With regard to scenario design of textbooks, Miyazaki (2005: 21-2) studied the types of error correction presented in the model discourses in Japanese language textbooks. He 
discovered that almost 90 per cent of both textbooks and drills used explicit correction of a speaker's (learner's) errors by a hearer (Japanese native speaker). This result conforms to Ellis and Shintani's (2014) argument that explicit correction was, in general, more effective for the learners and more likely to occur in real life.

\subsubsection{Summary}

The core function of language textbooks, which was claimed by scholars decades ago, has remained largely unchanged. However, it is nowadays considered vital for language textbooks that cultural matters of the target language be incorporated, not in isolated single sentences, but in a whole and appropriate context.

Various on-going issues remain with textbooks published for general sale. These are not impossible to resolve. What is necessary, however, is that authors add clear explanations to the model discourses on why the personae utilise (utter) a particular sentence in a given setting. Obviously, these must be understood not only by teachers but also by students. The explanations should be included and be explicitly written.

Achieving naturalness in spoken Japanese should not be very hard for textbook authors, as long as they take a close look at authentic conversations and absorb their essence and characteristics. In all, it is apparent that the role of the textbook is to connect learners with target languages; and that textbook content has to consider cultural matters because language and culture are inseparable. 


\subsection{Discourses in Japanese}

\subsubsection{Introduction}

The previous section critiqued problems with so-called model discourses in textbooks for Japanese as a second or other language. The primary purpose of this section is to explore in greater depth the essential meaning of the word 'discourse'-danwa in Japaneseespecially in spoken language. Discourse analysis relates to any phenomena pertaining to the expressions and structures of language. The study of discourse analysis began last century; in particular after the 1960s, its speed of progress accelerated. The following will therefore provide an overview of scholarly findings related to discourse in general and to Japanese discourse in particular.

John L. Austin (1962: 12-24) developed a theory of speech acts, in which he examined meanings of language expressions from the viewpoint of the speaker's intention. He focused on the meaning of utterances through the context, connecting the setting with the speech act. A speech act in his theory is composed of three subordinate levels: (1) a locutionary act is an act of uttering something with a meaning; (2) an illocutionary act is an act of utterance which conveys the speaker's intention; and (3) a perlocutionary act is the effect of an illocutionary act. When we say 'speech act', in general, it indicates the illocutionary act of Austin. These three speech components are related to the present study, especially illocutionary acts and perlocutionary acts. ${ }^{34}$

34 One utters Pen wasureta [I forgot my pen]. The interpretations of the three speech acts based on this are as follows. (1) Locutionary act: a straightforward statement of the facts [I've forgotten a pen]. (2) Illocutionary act: The utterance Pen wasureta implies the speaker's intention of making a request. (3) Perlocutionary act: The utterance Pen wasureta conveys to the hearer an unspoken psychological effect, such as gaining the hearer's sympathy, expressing selfdeprecation, conveying informality, etc., to get the hearer to understand what the speaker wants him/her to do. 
John Searle (1969: 54-71) claims that a speech act has to be satisfied with a felicity condition $^{35}$ which consists of circumstance, setting, and participants. He also proposes the concept of the indirect speech act, which is essential for discourse analysis. An indirect speech act appears, for example, when one says, "It's rather stuffy here", and the reply is, "I'll open the window" instead of a direct response, "Yes, it is." The first utterance states only the condition of the room as a form of declarative sentence, but the speaker indirectly asks the interlocutor to do something to keep the room cool. This type of conversation requires contextual knowledge and an inference from previous utterances. Searle argues that ordinary conversations are not usually bounded by the superficial meanings of utterances; rather the real intention of the speaker is hidden within. As we shall see later, this is an important aspect of discourse in Japanese. ${ }^{36}$

How these intentions of utterances are expressed and understood was investigated and explicitly explained by Grice (1989: 26-30). His explanation was that if speakers want the conversation to succeed, they tend to speak cooperatively, according to a theory called "Cooperative Principles". ${ }^{37}$ Grice also coined the phrase conversational "implicature", which refers to a thought contained in an utterance but not overtly uttered. Once the thought is generated, it is understood through the interlocutor's inference. In the present study, this intuitive cooperation is regarded as overlapping with Brown and Levinson's "off record strategy" (see Ch. 2, Sec. 3) and will henceforth be referred to as such.

35 J. L. Austin (1962: 12-24) in Lecture II of How to do things with words: Oxford: Clarendon Press and John R. Searle (1969: 54-71) in Chapter 3 of Speech acts: an essay in the philosophy of language. London, Cambridge U.P, explained that a performative utterance. "Requests" are "felicitous" so long as (1) the speaker (requester) believes the hearer (requestee) can perform the requested act; however, (2) it is not sure that the hearer would perform the requested act (p. 66).

36 The utterance "It's rather stuffy here" may be regarded as an example of Brown and Levinson's off record strategy (see Ch. 2, Sec. 3). This type of utterance appeared in the present experiments. 37 Cooperative Principles have four maxims of conversation: Maxim of Quantity, Maxim of Quality, Maxim of Relation, and Maxim of Manner (p. 26). 
The indirect speech act and conversational implicature claimed by Grice closely relate to theories of politeness. Brown and Levinson's Politeness: Some Universals in Language Usage (1987) is in many ways representative of theories pertaining to politeness. Their view is that 'politeness' is a speech act intended to build and maintain a good relationship with others. They argue that when expressing politeness to others, universal rules or principles of communicative strategies function regardless of language and location. Brown and Levinson's theory rests upon the basis of a face-threatening act. 'Face' here is a synonym for the self-esteem held by everyone. One of their politeness strategies, 'off-record', violates Grice's maxim; however, the intention of the speaker is conveyed by conversational implicature (1987: 2)

The fundamental notion of the politeness theory that Brown and Levinson established is that, in discourse, the speaker and hearer tend to prioritise keeping a good relationship over efficacy of information transmission. This concept has most influenced Japanese scholars researching the field of spoken language, in particular, discourse analysis.

\subsubsection{Characteristics of Discourse in Japanese}

Language reflects culture, and vice versa. Communication rules are both culturally and contextually bound, according to Porter and Samovar (1997: 23-4), who argue that cultures develop rules that govern human interaction in specific contexts, which range from high to low. ${ }^{38}$ Andersen (2012: 298) explains that in high context cultures, information is integrated from the environment, context, situation, and subtle nonverbal cues that give

38 In Porter and Samovar's typology, Japanese sits at the end of the high-context culture, while German-Swiss is at the end of the low-context culture in the opposite. Arabic comes after Japanese followed by Greek, Spanish, Italian, English, French, American English, the Scandinavian languages, German, and finally German-Swiss (Porter and Samovar 1997: 24). 
messages meaning not available in explicit verbal utterances. These imply that Japanese people seem not to need, or do not like, to speak explicitly.

The word danwa [discourse] has been defined by many Japanese scholars, with each producing slightly different interpretations. Discourse is a cluster of coherent speeches or written forms of more than one sentence, including both speech acts and non-speech acts, such as gestures (Minami \& Tanaka 1983: 1-6, Hinata and Hibiya 1988: 1). Hashiuchi (1999: 211) observes that danwa can include (1) a language unit larger than a sentence, (2) a usage of speech, and (3) utterances, and (4) text. Oki (2006: 66) argues that discourse does not require more than one word as, for example, So? [(Is that) so?], Hontō? [Really?], or Uso! [(lit.) a lie, I can't believe it!], which are independent one-word sentences. She asserts that the thought by which these single words are understood to be sentences has already become the mainstream. ${ }^{39}$

According to Hayashi et al. (1996: 558), characteristics of Japanese discourse include that speakers do not want to accentuate themselves and even avoid having a perceptible standpoint when opposing the other. Eloquence is not always appreciated in Japanese, and speakers tend to make use of apologetic expressions even though they may have done nothing wrong. Usami (1999: 40-3) asserts that the study of discourse of the spoken language, for instance, comprises an investigation of the rules of language use and interactional functions of conversation.

Maynard (2004: 277-80) points out an advantage of conducting discourse analysis. She postulates that by analysing the Japanese language, the whole picture of the language becomes clear: not only the phenomena of vocabulary and grammar, but also the correlation between the speaker and his/her speech act. This is because the thought and feeling of the speaker often appear in the speech act, especially at the end of the sentence.

39 These words work as sentences from the viewpoint of semantics. 
Kashiwazaki (2011: 70) agrees with Maynard that it is insufficient and even difficult to envisage an entire discourse if the scope of the discourse analysis is limited to only a sentence. In the learners' cognitive process in a target language, for instance, the important thing for them is whether or not they can produce sentences that are cohesive and coherent on the topic concerned. As such, to grasp and understand the structures of discourse is to apprehend how the discourse is comprised. It is meaningful, therefore, to analyse discourse as a whole.

\subsubsection{Developing Discourse}

Scholars have researched discourse from the aspect of sociolinguistics and/or culture. The main purpose of developing a discourse between two (or more) parties is to maintain the relationship of the parties involved. Successful maintenance is often more important than merely exchanging information. In a broad sense, this is one of the politeness strategies, as it focuses on attempting to keep and maintain a good relationship with others (Hayashi 2008: 78). Moro (1999: 20) argues that study of discourse does not mean a study of the language alone, but should encompass the process of the learners' cognition of language in a societal frame.

From the viewpoint of discourse analysis, Maynard (1997: 11-3, 2004: 31-2, 227-8) defines discourse as speech act that conveys sizeable and congruous information. She argues that only by analysing an entire discourse does it become possible to comprehend the whole picture of the discourse, which could also possibly depend on the intention of the speaker and his/her relationship with the interlocutor. The way the speaker employs words and phrases reflects the relationship between the two. Maynard claims that from the speaker's way of employing these, one may recognise what kinds of expressions are preferred or not preferred, and when and how these are utilised. 
Another characteristic of Japanese discourse which Maynard highlights is the speaker's modality, which mostly emerges at the end of a sentence owing to the usage of sentence-final particles (SFP). Because SFP can control the level of the relationship between the speaker and an interlocutor and how (im)politely the speaker thinks of the other. She argues that SFP is not only applicable at the sentence level, but at the discourse level. The speaker's thoughts and feelings are disclosed not at the starting point of the discourse in Japanese, but towards the end of it, as well as at the end of the individual sentences. Maynard's point of argument is, therefore, that the characteristics of Japanese discourse cannot be grasped unless the whole discourse is analysed.

\subsubsection{Order of Utterances}

The hearer's impression varies according to the order of appearance of utterances even though the components may be the same (Kashiwazaki 2010: 38, 2011: 74). Kashiwazaki emphasises the importance of comprehending the meaning, which is the speaker's real intention, from the pragmatic viewpoint. The significance of the order of appearance and emphases of the utterances are underlined by Kato (2004: 263), who argues that the order of appearance displays the intention of the speaker.

\subsubsection{Courtesy in Human Relations}

Social factors such as the social distance, age and rank between the speaker and the hearer determine the manner of speech acts. What language in a certain setting can or should be used is determined almost mechanically in the Japanese language environment (Mizutani 1991: 26-8). Miyake (2011: 15) supports Mizutani's view by affirming that the speech acts of the Japanese language can easily be formalised socially. She raises examples of apologetic situations, in which an apologiser uses standardised expressions frequently, such as 
sumimasen [I am sorry], and the apologiser does not greatly diverge from these patterns. The reason for this is that it is safe as long as the speakers utilise the standard patterns. In particular, if the interlocutor is not close to the apologiser, they tend to adopt a standardised speech act.

Kato (2004: 9, 116) regards the teinei-sa [politeness] of the Japanese as arising from avoidance of confrontation with others, as well as attentiveness to and consideration for the other people in the conversation. When responding to an offer of going out for a cup of coffee, i.e. Kōhī nomi ni ikanai?, [Why don't we have a cup of coffee?], an invitee is far more likely to respond with something like Kore kara jugyō ga aru n'da [I am going to a class now] instead of answering, Uun ikanai or Ikenai [No, I don't go or I can't go], in order to avoid a negative or unsympathetic answer. This is a typical example of displaying consideration towards the other. ${ }^{40}$ Coulmas (2005: 321) argues that teinei-sa in an interrogative sentence is canonically related to a question, but in many languages it is used in a derivative way to make polite requests. Utilising an interrogative sentence, in particular together with a negative form, makes the sentence sound more polite than an affirmative form pragmatically. In Japanese discourse, courteousness and attentiveness are key elements in maintaining a smooth relationship with others.

\subsubsection{Characteristics of Spoken Discourse in Japanese}

Complicated sentence structures rarely appear in spoken Japanese compared with the written language, while ellipsis and anastrophe are often used (Kokuritsu Kokugo Kenkyūjo [The National Institute for Japanese Language and Linguistics] 1989:26). Mizutani (1988:

40 In this case, the respondent who says I'm going to a class now expects the hearer to surmise the speaker's inability even though the speaker did not clearly mention it. In other words, the speaker believes the hearer's guess-work (implicature) from what the speaker said. 
98-103, 1993: 4) asserts that when Japanese native speakers converse with each other, the number of complete sentences is less than that of incomplete sentences or aposiopesis [iisashi bun]. For example, sentences often terminate in the te-form of verbs, nebo shite...[(I) overslept...]; or particles such as kara... [so, because, as, since], Densha ga okureta kara... ['Cause the train was late...]; kedo... [but, however, although], Hayaku neta kedo...[I went to bed early, but...], etc. ${ }^{41}$ The effect of aposiopesis is to articulate the speaker's hesitancy. Takasaki (2008: 10) supports Mizutani in her explanation that an aposiopesis presents the speaker's feeling to the hearer about how irresolute the speaker is to utter something —often something not actually spoken.

Hashimoto (2000: 26-7) highlights attentiveness to others as a key principle of the spoken language of Japanese. In the case of a request, if a speaker utters the topic indirectly or ambiguously, the addressee may feel find it easier to reject or decline than a more direct request. The speaker strategy of attentiveness to the other party allows the interlocutor a wider choice of responses. Hayashi (2008: 71-6) observes that hedging is also considered an effective conversational strategy to mitigate the impact on the hearer, such as $X$ toka [something like X], X demo [about X], chotto [a little bit]. ${ }^{42}$

There are customarily-used speech styles in social norms (Shibuya 2008: 22). When a speaker has to choose either the desu/masu-form (polite/formal) or the da-/dictionary form (plain/casual), the speaker relies on the suitability for the setting. Hence, on almost all occasions, the speaker's choice is determined routinely. Japanese native speakers unconsciously acquire this skill and use it with native speaker intuition, which may not be the case for learners.

\footnotetext{
41 The word kedo reveals a speaker's feeling of hesitancy (Kanasugi 2008: 55-6).

42 For example, Chotto enpitsu toka, pen demo motte tara kashite yo [If you have something like a pencil or pen, can you lend me it?]
} 


\subsubsection{Indirectness and Ambiguity}

One of the strategies of speakers in Japanese is to utter what they say indirectly or ambiguously in order to avoid exposing their real intention. Tsuda (1999: 19-22) argues that in Japanese discourse there is no guarantee that the hearer can correctly understand the speaker's thought, because meanings are frequently unclear owing to the indirectness or ambiguity of the utterances. In such cases, the hearer has to surmise the speaker's intention. In a sense, the speaker even expects that the hearer should intuit what he/she wants to say extraneous to the words. Indirect or ambiguous expressions are often used in settings where these is a large difference in social rank between the two parties, or when they are unfamiliar with each other, and seem to maintain an appropriate distance. Tsuda affirms that these expressions are employed to prevent one from appearing pushy, for example, when making a request.

Indirect expressions work to lighten the speaker's disadvantage, or a negative or unpleasant situation (Jinnouchi (2006: 123-6), Kawakami (1993: 49), Makino (1996: 66-7) and Yasui (1983: 95-6)). For example, instead of saying, Kono ryōri wa mazui nā [This cooking tastes awful], a speaker might say Mō onaka ga ippai da [I'm really full already]. This is a kind of consideration through trying not to be too close, and therefore too blunt, to the hearer. Using such an indirect expression is a negative politeness strategy. The indirectness can, therefore, avoid confrontation with others.

Business meetings in Japanese do not seem to go straightforward fashion, nor does the political world. Torikai (2005:29) stresses that there are typical expressions used by Japanese politicians, such as Zensho shimasu [I'll deal it with adequately] and Maemuki ni kangaemasu [I'll consider (it) in a forward-looking manner]. These indirect expressions are also one of the features of Japanese discourse styles for declining requests. She agrees there is 
a cultural influence on the speech acts of Japanese people, particularly when they avoid direct terms due to the possibility that they may threaten to insult the other's face.

Haugh (2003: 170-2) argues that the longer non-Japanese people have lived in Japan, the less they observe that the Japanese communication style is ambiguous and indirect, although Japanese communications are generally characterised by the belief that Japanese is more vague and indirect than English and they are more subjective, emotional and nonlogical, polite, tacit, and non-verbal. However, according to his study, neither the majority of Japanese respondents, nor of non-Japanese, held this sort of belief.

Experiments by Jones (1993: 71-2) and Spees (1994: 239, 248-9) respectively yielded results similar to Haugh's, with cases where the Japanese clearly spoke without hesitation when contradicting others. Spees's observation from her research of 30 each of Japanese and American students as to why the Japanese expressions are often regarded as indirect is because the Japanese frequently used minimisers, such as chotto [a little bit] in their discourses. By adding softer words while conversing, she perceives that the illocutionary force of the utterance is weakened. Yet she confirms that the head acts - the key elements of the sentence containing the speaker's real intention-were uttered explicitly, not ambiguously or indirectly.

\subsubsection{Attenuation}

Research into the minimiser chotto [a little bit] by Matsumoto (1985: 149-151, 157, 2001: 3-6) affirms that the word's function is to decrease the weight of the speaker's performance of the utterance, and at the same time reduce the obligation that would be required of the addressee for the success of the speech act. The word chotto is often used in a request or refusal in a negative situation, and serves to attenuate the impact of the utterance and mitigate the presumed rudeness, because the speaker may intend his/her attenuated stance to be soft and less of an imposition on the addressee. Matsumoto surmises that there are 
various possible reasons for mitigation, mostly rooted in the speaker's hesitation in performing a certain speech act.

Nevertheless, she argues that when apologising or promising, chotto is not compatible and the speaker has to present unambiguously. In these cases, utterances must employ explicit performative verbs. Speakers should not use a minimiser like chotto in such sentences. For example, when apologising, the utterance chotto sumimasen [I'm sorry a little bit] is incompatible and unacceptable.

Hidasi's study (2007: 82-7) presents an example of chotto which lessens the negative impact on the addressee. The example she raises is of someone asking for an eraser and the addressee replying, Keshigomu wa chotto arimasen nē [(lit.) There is no eraser a little bit. (I don't have it)]. The word chotto does not semantically fit in this sentence, although what the speaker (requestee) who was asked for an eraser wants to tell the interlocutor (requester of the eraser) is easily understood. Hidasi explains that the speaker uses chotto out of generosity because the speaker could not comply with the request, and is trying to play down the requestee's advantage over the requester.

\subsubsection{Aposiopesis}

In spoken Japanese, aposiopesis performs more than merely conveying a message to the hearer, in order to communicate smoothly, because the speaker's intention and feeling appear at the end of a sentence (Cho 2000: 27, Morita: 1996: 249, Ogiwara 2000: 27). From a grammatical viewpoint, Mihara (1995: 79) defines aposiopesis as an utterance in which, although there is a dependent clause, the last part of it is left unspoken, and a subjective clause is omitted. Mizutani (1993: 5-6) affirms that Japanese native speakers' conversation frequently ends in the form of an unfinished sentence-aposiopesis, particularly using the teform or the conjunctive particle kedo. 
The effect of aposiopesis is that the speaker's feeling is expressed in a circumlocutory way. Therefore, when the speaker reluctantly has to utter something delicate to an interlocutor in settings of, for example, request, complaint, and dissenting opinion, aposiopesis is often employed, such as X nan' desu kedo... [but, however, (I think/am) X...]. Moreover, speakers even expect that the hearer will correctly conjecture what they want to say. Ikeda (1995: 128-9) is concerned that it is necessary for Japanese language learners to be taught discourse grammar more consciously, because if learners use nothing but complete sentences (i.e., do not use aposiopesis), their utterances are discomfiting for Japanese native speakers. ${ }^{43}$

The basic sentence patterns of expressing a reason(s) for an incident employ $V$-te, no de, kara, and so on. Research into the te-form, no de, kedo, kara, was conducted by Shirakawa (1991:40, 1996: 9-10, 16, 2009: 143, 194-5), ${ }^{44}$ who demonstrated the differences among them. The te-form in aposiopesis is most frequently used when explaining a thing/incident. Although the sentence is grammatically incomplete, it works in the same way as a complete sentence with no da/desu at the end. Kara works in presenting a fact that a hearer does not yet know, and the hearer is expected to decide what to do, while kedo does not have this kind of implication. Shirakawa asserts that kedo is neutral in terms of the hearer's knowledge about the matter. Therefore, kedo sounds modest and reserved.

43 This is because it may sound like a statement or even a declaration. For example, when declining an offer of another, Mō onaka ga ippai de... [I'm really full already, so...] sounds more polite than Mò onakaga ippai de taberaremasen [I'm really full already, so can't eat any more].

44 When explaining the reason for being late, Densha ga okureta kara desu is better than densha ga okureta mono/mon' desu kara [The train was late], according to Shirakawa's finding. 


\subsubsection{Excuses}

Some particles and phrases are used especially for explaining reasons or causes. Boku (2010: 229-32) discovered that learners at the intermediate level concentrate on using one of the te-forms, kara, or kedo when explaining a reason. While the use of the te-form declined at more advanced levels of proficiency, kedo came to be utilised more; and in the further advanced level (super-advanced), kedo was used much more. On the other hand, the frequency of use of no de by students of the advanced/super-advanced levels remained less than half of that of the native speakers. She deduces from this that the order of ease of acquisition is the te-form>kara $>$ kedo $>$ no de. Mizutani and Mizutani (1984: 26-7) claim that kara at the end of a sentence connotes more of an imposition than no de, since the former has connotations of the speaker trying to justify the reason for his/her response more than in the latter case.

Subordinate clauses with the phrase mono da kara also explain a cause or a reason for an incident, but expose the negative attitude of the speaker. ${ }^{45}$ The phrase mono da kara usually appears in spoken language, and sentences in which it is used often finish in a form of aposiopesis (Kokuritsu Kokugo Kenkyūjo 1989: 26, 54-6). Tamamura (1993: 364) argues that the phrase is used when a speaker has recognised his/her acts as a fault or failure, and explains a reason or an excuse. Examples he presents are (1) a reason: Awatete uchi o deta mono da kara, saifu o wasurete shimatta [Because I left the house so quickly, I forgot my wallet], (2) an excuse-like reason: Hajimete na mono desu kara, umaku dekiruka dō ka wakarimasen. [As this is my first time, I don't know If I can do it well]. ${ }^{46}$ As a consequence, because of the use of mono da kara, it sometimes sounds as if the utterance were an excuse.

45 For example, Kōtsüjiko ga atta mono da kara, kaigi ni okureta [Because of the traffic accident, I was late for the meeting].

46 The sentence Hajimete na mono desu kara, umaku dekimasen deshita [As this is my first time, I failed] sounds like an excuse rather than an explanation. 
Mine $(1995 ; 74)$ argues that $n$ 'da kara sounds as though the speaker is criticising the other; it may, therefore, seem overtly defensive. ${ }^{47}$

\subsubsection{Expression at the End of Sentence}

In most Japanese sentences, the predicate is located at the end of a sentence together with sentence final particles, and it reflects the speaker's feeling through the form of the speech-style, for instance, the desu/masu-form (polite/formal) or the $d a /$ dictionary-form (casual/informal) (Ijuin 2004: 14-7). She reports that young Japanese university students use the da-/dictionary form even in their first meeting with others whom they do not know well. This implies, according to Ijuin, that native speakers adopt a positive politeness strategy to some extent.

However, on the other hand, the native speakers in her study tended to apply negative politeness strategy with fellow Japanese. ${ }^{48}$ For example, they also elaborated by adding various particles/phrases to the end of the sentence, such as kedo... [but, however], kara... [because, as], because aposiopesis can maintain a distance between the two parties. Furthermore, the particles $n e^{49}$ and $y o,{ }^{50}$ etc., generally considered to strengthen and intensify friendliness as positive strategy, were not used very much when conversing with Japanese. As a result, comparing a contact setting with a discourse between two native Japanese speakers, the negative strategy ${ }^{51}$ was used much more than the positive politeness strategy.

47 The phrase, n' da kara, is a casual form of no da kara. For example, Kimi ga itta n' da kara kasa o motte ikanakatta n' da. Demo ame ga futta yo [Because you told me, I didn't bring the umbrella, but it rained.]

48 See Ch2, Sec. 3 Politeness. It is generally believed when Japanese people meet the first time, they use the desu/masu-form (polite/formal).

49 The particle $n e$ is to ask the hearer for agreement softly.

s0 The particle yo indicates certainty, emphasis, etc.

51 See this Chapter, Section 3 Politeness. 
Yanabu (1984: 225-9) argues that if there is a common understanding between the speaker and the hearer, the utterance can be terminated before completing the utterance and left as an aposiopesis with particles of the te-form, kara, kedo, etc., at the end, as long as the following unspoken part can be predicted by the hearer. Mizutani (1991: 28-9, 1993: 5-6) also emphasises that the reasons for aposiopesis are that speakers have the expectation that the hearer will understand what has been left unspoken. Learners may tend to think the complete sentence is more polite than aposiopesis, which it seems difficult for them to acquire. As a result, their speech may sound unnatural, albeit grammatically correct.

\subsubsection{Juju dōshi [Verbs of Giving/Receiving]}

In Japanese, verbs of giving and receiving are called juju dōshi. They distinguish between kureru "giving to the speaker", ageru "giving from the speaker", and morau "receiving", and thereby they express the direction of the movement when one provides something with some kind of favour, which may be a thing or an act (Kindaichi et al. 1989: 174). Typically, therefore, the juju dosshi, kureru [give to the speaker] and morau [receive], are employed when a speaker asks someone for something.

Kataoka and Ide (2002: 14-5) note that the use of juju doshi [verbs of giving/receiving] and sentence final particles play a key role in creating harmony with others, which is one of the basic concepts of Japanese culture. Sakamoto (2001: 17-8) argues that expressing consideration to others comes not only through using honorifics. In her analysis, a sentence such as Kite kurete arigatō [Thank you for coming] can convey a speaker's appreciation more than Irasshatte arigato [Thank you for coming], ${ }^{52}$ in spite of the fact that the latter uses irassharu [(lit.) an honorific form of 'come/go']. The difference arises because

52 The word irassharu [come/go] is a polite form of kuru. From the viewpoint of pragmatics, irasshatte+kudasatte (juju dōshi)+arigatō is appropriate. The sentence kite+kurete (juju $d \bar{o} s h i)+$ arigatō is appropriate. The difference is whether juju dōshi is employed or not. 
if the speaker expresses his/her feeling as a beneficiary of someone's action kite kureru [(X) come (for my benefit)] by using juju dōshi, the sentence shows more consideration than by employing an honorific (irasshatte). Sakamoto's point is that the forms of V-te kureru [(X) does (for me)] and V-te morau $[(I)$ receive (from $X)]$ work to express more sincere consideration than honorifics. Yamada (2011: 4) agrees with Sakamoto, and adds a further description of kureru [give] and morau [receive], as not only do these verbs express an action of giving/receiving something, but they also have the function of displaying an act of benefit. Whereas the subject of kureru is the person giving, and that of morau is the recipient, the latter displays more of the appreciative nuance of a beneficiary.

In their research into the use of juju dōshi in a requisitive setting, Kabaya, et al. (1998: 136) conclude that the level of requisitive expressions chosen depends on the relationship between the two parties. They arrange requisitive expressions in the order of casual to formal as follows: moraeru $\uparrow^{53}>$ moraemasu $k a>$ itadakemasu ka > itadakemasu desho ka. ${ }^{54}$ Iori's study (2011: 51) affirms that negative expressions tend to be more polite than positive expressions: for example, the order of increasing politeness in interrogative sentences would be, kuremasu $k a>$ kuremasen $k a>$ kudasaimasu $k a>$ kudasaimasen $k a$.

It thus becomes clear that: (1) the functions of juju dōshi are to bring about a feeling of appreciation in the beneficiary; (2) among the verbs of juju dosshi, there is a recognisable order of polite expressions; (3) negative expressions (in the case of requisitive sentences) are considered more polite than positive expressions, and (4) it can be possible to show a feeling of appreciation to a speaker without using honorifics by employing juju dōshi.

\footnotetext{
$53 \uparrow$ indicates a rising tone.

54 Itadaku is a humble form of morau. The difference between itadakemasu $\mathrm{ka}$ and itadakemasu deshō $k a$ is to imply a speaker's conjecture/presumption by the word, deshō, [I wonder].
} 


\subsubsection{Teaching Japanese Discourse}

Discourse is composed of both speaking and listening skills, and the interlocutor's response takes on a significant role in a conversation. As Horiguchi (1997: 130) indicates, appropriate responses in the case of Japanese, as well as when and how often they are generated by the interlocutor, make the conversation proceed smoothly.

Some research suggests that conventional pedagogical methods do not seem to be effective for teaching discourse. Okazaki (1987: 165-8) envisages that learners sometimes have an issue with competence in discourse, because studying in class often means that they lack the opportunity to take part in real conversations. He alleges that the Japanese language demands a great deal of an interlocutor, such as for example, back-channelling and nodding by the interlocutor, because these are key elements of authentic conversation. These nonvocalised interactions should, therefore, be taught as a part of discourse. Ohta (2001: 120) argues that expressions of acknowledgement and alignment by an interlocutor are indispensable for Japanese language learners in order to acquire the speech skill of natural conversation.

Ide, R. (1998: 527) researched discourse, employing a combined interactional and ethnographic perspective. She studies usage of the word sumimasen [I'm sorry], and argues that contextual meanings emerge through each individual situational usages, and are enforced through the process of socialisation and the everyday practice of pragmatic usage. ${ }^{55}$

From the viewpoint of inter-personal relations, both Kumai and Liddicoat are concerned with learners' lack of pragmatic understanding of the language rather than grammar. Kumai (1992: 72) claims that when a learner of Japanese has poor speaking skills, and makes mistakes grammatically or pragmatically, native Japanese speakers are usually

55 Ide discusses the various usages of sumimasen: greeting, leave taking, requesting, conversational ratification and attention getting, thanking, demonstrating, politeness, and apologising. 
generous and forgiving. However, once a learner's proficiency level is raised, a Japanese interlocutor may react differently if the speaker's utterance is not appropriate to the setting.

Liddicoat's (2008: 278) research also demonstrates that people are less tolerant of pragmatic errors than those of grammar. Hori et al. (2006: 170) report similarly that English native speakers do not mind so much erroneous usage of vocabulary and grammar made by Japanese students, but they feel uneasy when they make errors and misunderstandings owing to a lack of societal knowledge when building up a relationship with other people.

The studies summarised above confirm that comprehending and applying the appropriate way to approach the establishment of a good relationship in Japanese and in English respectively is as important as knowledge of these languages to convey a message, and is perhaps even more significant than the speech itself. They contend that focusing only on conveying a verbal message to others - something which has been emphasised for many years now in language teaching - is insufficient.

Competency in discourse cannot be acquired only by knowledge of the target language's grammar and vocabulary. Rather, it demands knowledge of interactional speech acts which must be taught appropriately, and conducted in a given setting. These practices do not seem to have been studied, or to be of concern, let alone implemented appropriately in model conversations in textbooks.

\subsubsection{Summary}

There is an idiom in Japanese, Ishin denshin which may be loosely rendered as Understand each other with no words, as if we could read each other's minds. In Japanese culture, loquaciousness is not a preferred or admired quality. The speaker has the expectation that the hearer will grasp his/her message, primarily because of the speaker's wish to avoid conflict, by using a covert utterance. In particular, when the speaker's situation is not good, 
i.e. he/she has to say something unfavourable to the hearer, there is a tendency for this type of strategy to be employed. Hence, a Japanese conversation is often vague, ambiguous, indirect, and circumlocutory. Hedging expressions, too, are utilised.

From a viewpoint of grammar, the speaker's feeling appears towards the end of the utterance, so the speaker sometimes terminates it before the end of the sentence and aposiopesis is used. Yet, even without the last part of the sentence, the essence of the speaker's message can be conveyed to the hearer, but accordingly, it sounds ambiguous.

The significance of teaching grammar is obvious and undeniable. As for communicative teaching/learning, displaying a series of individual simple sentences is insufficient and inappropriate for students of Japanese as a second or other language. They need to be shown conversational exchanges as a whole between a speaker and a hearer, so that they can comprehend the entire flow of the conversation along with when and what to utter in the discourse. This way of teaching can be very useful and effective, in particular, for those learners who come from an environment culturally distant from that of the Japanese. For those who are from a distance in terms of not only geography but also languages and cultures, teaching materials can be good sources for the learners to notice the differences (and the similarities) in the Japanese way of thinking and living in real life. This point should be taken more into consideration when designing new and innovative teaching materials. 


\subsection{POLITENESS}

\subsubsection{Introduction}

The primary purpose of learning a foreign language is ostensibly to communicate with people of the target language. In the early stage of learning, learners may have a look of delight on their faces when they can make themselves understood in the newly acquired language. It is natural that the more advanced their level becomes, the more they focus on becoming a native-like speaker. With any language, no matter how faultless the grammar of the learner, this does not necessarily mean "real" communication with people of the target language has been achieved. The extent of communication may only be the same as a tourist's conversation that comprises a few limited nouns and verbs.

Language has roots deep in culture. Conversation is a verbal exchange between people, and for a conversation to proceed appropriately a speaker needs to register an interlocutor's reaction. For learners of Japanese, the acquisition of speaking skills in settings that necessitate same negotiation — not a speech-act of correct grammar and vocabulary, but the procedure of conversation development-is required for making conversation naturally. In particular, in difficult or socially sensitive settings, such as apologies and requests, the speaker must pay attention to his/her own utterances. Such settings more so than others particularly demand careful consideration.

Language bears the culture of the people who speak it. Practising cross-cultural study through language entails that at least two different cultures be compared with each other by utilising two different languages. The real meaning of words is found in the context of speaking, not in an individual word. When studying two different languages, there must be deliberate concern with each culture, for culture is inherent in language (Ide, S. 2005: 6-7). True understanding of another culture, as Ide claims, demands going as deeply as possible into a speaker's language practice. 
A speaker's perception of difficulty in apology and request, for example, lies in that understanding and acceptance by the other party is not obtained unless the speaker intrudes on the psychological space (or territory) of the interlocutor. Having entered into the other party's territory, a polite and sincere approach is required, and all the more so when the speaker's cultural and/or linguistic background is different from that of the other party.

It is unquestionably essential in any culture to be polite towards the other party while conversing. The English word, politeness, is defined by the Oxford English Dictionary as "courtesy, good manners, behaviour that is respectful or considerate of others". ${ }^{56}$ More definition of politeness here, however, how to express politeness and at what point it should be expressed vary according to the language and culture. The fundamental concept of politeness is arguably universal, but it is important to recognise that the way of expressing it may vary depending on the language. This is normally a matter of course for a native speakers of any language. Nevertheless, 'a matter of course' must be acknowledged in crosscultural circumstances (Hori: 2006).

\subsubsection{Politeness Theory}

Given the topic of this thesis, it is important here to examine theories of politeness in language from the act. Two leading scholars, Penelope Brown and Stephen C. Levinson, advocated their framework of politeness theory as "universal" in their book Politeness. Since then, it has attracted a great deal of attention worldwide. Their work has been very influential in the fields of linguistics, psychology, sociology, language education, etc., hundreds of articles and books related to their politeness theory have been published since their first article in 1978 (Fraser 2005: 65). For the present study, although works by other scholars

56 Cited by http://www.oed.com/view/Entry/146882?redirectedFrom=politeness\#eid (accessed on April 5, 2012). 
about politeness theories are referenced, Brown and Levinson's politeness theory is central, because it has been broadly accepted and become established.

Brown and Levinson set up a framework of politeness which they argued is universal, and, by definition, this should of course include Japanese. The Japanese language is conspicuous for its complex and elaborated honorific scheme known as keigo [honorifics]. In Japanese, this is a generic name for a scheme of speech (and written) acts to show respectful feeling towards the addressee and/or the referent. Many Japanese people associate the English word, politeness, with keigo (Takiura 2008: 50). Brown and Levinson, however, made little reference to it. Is the politeness theory of Brown and Levinson truly inclusive of Japanese? If so, what kinds of speech acts are uttered in Japanese to express the speaker's politeness towards the interlocutor?

\subsubsection{The Politeness Theory of Brown and Levinson}

In Politeness: some universals in language usage, Brown and Levinson set forth the universality of human intention to be polite in any given circumstance through speech acts. Since then, the theory of politeness has been widely accepted and influenced scholars. ${ }^{57}$ Their research is based on analysis of three distant and disparate languages, i.e. English, Tzeltal in Mexico, and Tamil in India. Based on their analyses, they assert the commonality of people's consideration for others in speech acts.

The substance of Brown and Levinson's politeness theory is centred on 'facethreatening acts' (FTAs). The meaning of face primarily comes from Chinese and Japanese ideologies of honour, dignity, or prestige. Goffman (1967: 5), to whom they dedicate their work, defines face as "the positive social value a person effectively claims for himself by the

57 Such as Arundale 2010, Dobeta 2001, Fraser 1990, Jucker 1988, Kanasugi 2008, Kitao and Kitao 1988, Kwarciak 1993, Locher et al. 2005, Matsui 2001, Sanada 1992, and Werkhofer 2005 among others. 
line others assume he has taken during a particular contact". Brown and Levinson explain face as an individual's self-esteem and the public self-image that every member wants to claim for himself; thus face-threatening acts intrinsically undermine the other's self-esteem (1987: 2, 12, 61), and their politeness theory ranges from sociolinguistics to pragmatics.

Brown and Levinson categorise FTAs into four types of strategy: (1) bald on record, (2) positive politeness strategy, (3) negative politeness strategy, and (4) off record. ${ }^{58}$ They argue that these strategies appear widely not only in English but also in unrelated languages, such as Tzeltal and Tamil. Thus, they claim that "It is legitimate to project from a careful three-way experiment in three unrelated cultures to hypotheses about universals in verbal interaction..." (ibid. 59). They conclude that there are parallels in the expression of politeness in the above three unrelated languages. The following are the politeness strategies that Brown and Levinson formulate.

\section{(1) Bald on record}

A bald on record action is one in which a speaker expresses his/her thoughts straight out without considering the other's feelings. The message of the speaker is conveyed forthrightly, but it is simultaneously an FTA, so this is a case where the relationship between the two parties might be damaged. Brown and Levinson (ibid. 95) illustrate bald on record as "in general, whenever S[peaker] wants to do the FTA with maximum efficiency more than he wants to satisfy H[earer]'s face, even to any degree, he will choose the bald on record strategy". An example of a bald on record action is when speakers demand, "Open the window", when the room feels hot to them.

58 There is a fifth strategy, which is 'Do not do the FTA' (Brown and Levinson 1987: 69). Owing to its nature, this paper excludes it. 
(2) Positive politeness

In examples of positive politeness, the speaker tries to lessen the distance between speaker and hearer because of the speaker's wish to be regarded as amiable. Brown and Levinson's explanation is that 'Positive politeness is redress directed to the addressee's positive face, his perennial desire that his wants (or the actions/acquisitions/ values resulting from them) should be thought of as desirable" (ibid. 101). Such an act includes an offer or promise. In the case of room temperature, for example, one might say, "Shall I open the window?"

\section{(3) Negative politeness}

In the case of negative politeness, the speaker tries to keep a distance in the relationship between speaker and hearer because of the speaker's wish not to enter into the other's personal territory, nor to be bothered by the other. Brown and Levinson (ibid. 129) assert that negative politeness is redressive action addressed to the addressee's negative face: his want to have his freedom of action unhindered and his attention unimpeded. This strategy is shown when a speaker apologises, because negative face is the desire to remain at or keep a distance. Hedging expressions are typical in order to avoid possible conflicts, including statements like examples, "Would you mind opening the window, please?"

\section{(4) Off record}

Off record is an indirect strategy, in which a speaker does not express his/her intention directly in words, and hence the hearer has to infer it. This strategy contains a communication risk in that the hearer might not receive the appropriate hint. On the other hand, neither party loses face, as the FTA is minimal. Brown and Levinson delineate off record acts as those in which "the actor leaves himself an 'out' by providing himself with a 
number of defensible interpretations; he cannot be held to have committed himself to just one particular interpretation of his act" (ibid. 211). An example of an off record strategy is, "It's rather stuffy here, isn't it?" meaning "Can't you open the window?"

Maximising the efficacy of conveyance of information inversely minimises the consideration to others. Comparing the off record strategy with the bald on record strategy, the former pays a high level of consideration to others, invades the other's territory least, and ultimately the efficiency of transmissibility is poorest. By contrast, the latter is the complete opposite, because while the speaker wants to express a message explicitly, at the same time he/she does not want to threaten the other's face.

The off record strategy leaves responsibility of recognition of the message to the addressee, so that the speaker is divested of taking responsibility. It is obvious that strategy choice depends on culture. Although there are differences in terms of the degree of usage by individual politeness strategies, Takiura (2008: 32, 45-7) argues that Brown and Levinson's politeness strategies overall have been applied to all languages. Their contribution has been to establish a politeness theory that can be used as a framework for the speech acts of any language..$^{59}$

59 Here are four different politeness strategies when a speaker (requester) wants to borrow a pen from a friend. (1) Bald on record strategy: Pen kariru yo [I'll borrow a pen.], (2) Positive politeness strategy: including a praising phrase. Ii pen da ne, kashite kureru? [It's a nice pen, can you lend me it?], (3) Negative politeness strategy: It contains expressions of apology and the subjunctive mode. Mōshiwake nai n' da kedo, motteru nara pen kashite kurenai? [Sorry to bother you, but if you have, can't I borrow your pen?], and (4) Off record strategy: It does not include explicit words/phrases of request. Shimatta, pen wasurete kichatta [Ooops, I forgot a pen.] Adopted from Takiura (2005: 150-1). 


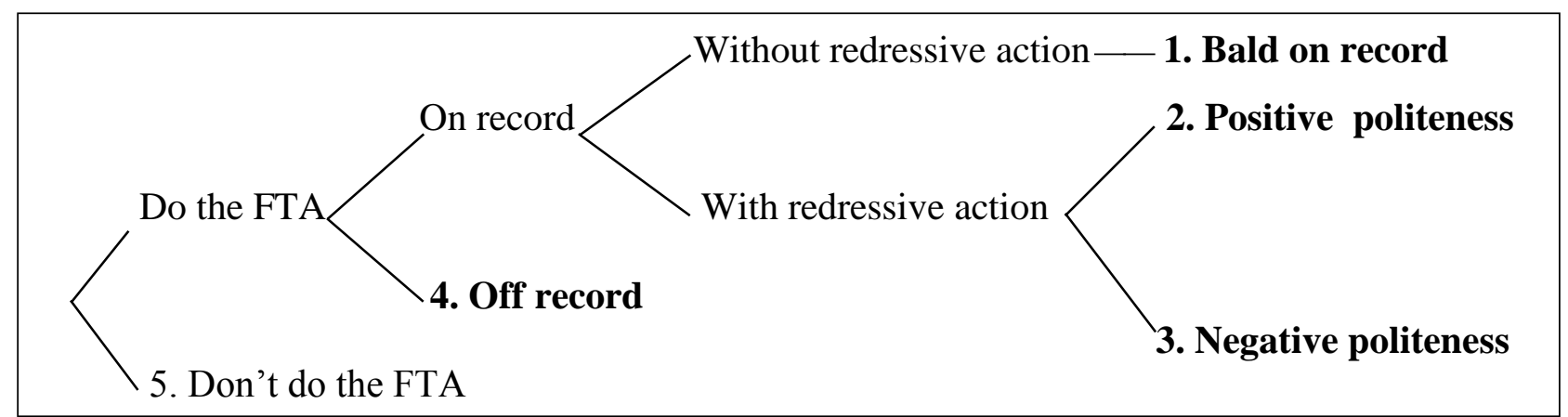

Figure 2.3.1 Strategies for Doing FTAs

(Adopted from Brown and Levinson 1987: 69)

\subsubsection{Face-threatening Acts}

People tend to want to secure an appropriate distance between themselves and others physically as well as psychologically (Kamio 2002: 7). It is unavoidable, however, that some speech acts will intrude on the other person's and sometimes even on the speaker's own face. As the speaker's linguistic consideration, a polite act enables the speaker to adjust the distance from the other person appropriately, in order to lighten or redress this intrusion on the other's face from having invaded his/her territory.

One of the salient points of Brown and Levinson's theory is the formula of FTA which indicates the degree of politeness that the speaker recognises when conducting a speech act. The formula consists of three elements: distance (D)-the level of distance between the speaker $(\mathrm{S})$ and the hearer $(\mathrm{H})$; power $(\mathrm{P})$-the level of the hearer's psychological power to influence the speaker; and the ranking of imposition (R)-the level of imposition from the speaker to the hearer, which may vary according to the particular culture. They calculate the weightiness (Wx) of an FTA in the following formula:

$$
\mathrm{Wx}=\mathrm{D}(\mathrm{S}, \mathrm{H})+\mathrm{P}(\mathrm{H}, \mathrm{S})+\mathrm{Rx}(\mathrm{S}, \mathrm{H}) \quad \text { (Brown and Levinson 1987: 74-6) }
$$

The politeness strategy directly relates to face risk. If speakers want to convey their message unambiguously or even baldly to a hearer, the face risk is high; on the other hand, if 
speakers do not want to take the risk of committing an FTA, the certainty of conveying the message is low but the risk of an FTA is also low. The face risk and the certainty of communicability are incompatible, so that the speaker has to opt for the best politeness strategy in any given situation by weighing up the advantages and disadvantages (ibid. 68-74). Hashimoto (2001: 47-9) perceives that the motives of circumlocutory expressions that sacrifice the efficiency of communicability-for example, utterances shown in negative politeness - are one of the core factors of Brown and Levinson's politeness theory.

The table below indicates the relationship between speech acts and face, as to when a speaker's and/or a hearer's face is intruded upon or damaged by speech acts of promise, request, apology, and criticism.

Table 2.3.1 Types of Speech Act and Intrusion upon Face

\begin{tabular}{|l|l|l|}
\hline Face & Negative face & Positive face \\
\hline Speaker & $\begin{array}{l}\text { Promise } \\
\text { (A speaker's negative face is } \\
\text { intruded upon by his/her promise.) }\end{array}$ & $\begin{array}{l}\text { Apology } \\
\text { (A speaker's positive face is damaged } \\
\text { by his/her apology.) }\end{array}$ \\
\hline Hearer & $\begin{array}{l}\text { Request } \\
\text { (A hearer's negative face is intruded } \\
\text { upon by the speaker's request.) }\end{array}$ & $\begin{array}{l}\text { Criticism } \\
\text { (A hearer's positive face is damaged by } \\
\text { the speaker's criticism.) }\end{array}$ \\
\hline
\end{tabular}

(Adopted from Takiura 2008: 30)

\subsubsection{Politeness in Japanese Language}

It can be said that politeness is at the centre of the Japanese discourse domain which highly values relationships with others. It involves social distancing, so some Japanese expressions can appear indirect, circumlocutory, and vague or dissembling, especially for non-native speakers. For example, as an indirect refusal to cooperate, Kangaete okimasu [I'll think about it], is an expression used when speakers wish the hearer to sense that they are declining something the other party requests, without saying so outright. Another expression is Muzukashii desu nee [It is difficult], which does not directly reject the other's request, but 
it is hoped the hearer will take the hint and withdraw the request of his/her own accord. These two expressions do not contain words of rejection, but both sentences connote hesitancy, reluctance, and thus refusal.

Most Japanese native speakers who hear these expressions in context do not take them literally but understand that they are indirectly and equivocally being refused. They recognise immediately that by saying I'll think about it, the speaker has no intention of cooperating with the other party, whereas culturally uninitiated learners may interpret this response at face value. Japanese discourse can be characterised as being highly inferential of indirect phrases. Articulating politely in Japanese implies ways of conscious, thoughtful consideration, and refraining from confrontation with others. Thus, the real meaning of words is found in the context of speaking, not in the individual words, and Japanese people even have an expectation that others are also able to understand the unarticulated communication.

This is a characteristic feature of languages of high context culture, such as Japanese (Andersen 2012: 299, Porter and Samovar 1997: 23-4). Kato (2004: 105-6) explains that in awkward settings of "request-rejection", for instance, a person who is asked by another tries to avoid a clear rejection lest it cause offence. Vagueness is, therefore, acceptable or even preferable, and often uttered according to the setting. Japanese discourse can be characterised as being highly inferential of equivocal phrases.

Japanese employs an elaborate system of 'honorific' speech which is known as 'respect language' (keigo), as noted in this chapter section 3.2. It is important to understand that 'respect' depends entirely on the relation in the social hierarchy between the speakers, and that this is socially prescribed. In other words, it has little to do with any feelings of respect on the part of the speakers. For example, one may feel little respect for one's interlocutor, but must still use appropriate honorific language if the interlocutor is one's senior. On the other hand, one may feel enormous respect for another to whom one is 
particularly close, and yet have no need to use honorific speech. In this sense, the framework of respect language in Japanese is a little different from Brown and Levinson's concept of politeness based entirely on face-threatening acts. In other words, the appropriate usage of keigo obviates the speech act becoming a face-threatening act. However, failure to use keigo in the appropriate specific context may in and of itself comprise an FTA.

\subsubsection{Understanding Brown and Levinson's Politeness Theory}

Brown and Levinson's achievement is that they established a framework that enables us to compare languages with elaborated normative grammar and politeness schemes, such as Japanese, with languages which have more freedom or fewer pragmatic constraints for the speaker. Furthermore, they built up the scale that allows us to map politeness strategies on an equal footing (Takiura 2001: 30).

Jary (1998: 2) observes that their theory covers pragmatic strategies, which primarily focus on reducing FTAs in order to hold and maintain polite communications. As Usami (2002c: 109-12) summarises, the theory of Brown and Levinson comprises key concepts of FTAs, a formula for calculating the weightiness of an FTA, strategies of politeness that a speaker applies, and the surrounding situational context.

The serial studies of Usami (2001a: 21-6. 2001b: 23-4, 2002b: 98-112, 2002c: 10912) elucidate that several scholars have critiqued their theory in ways which are relevant to the present study. Brown and Levinson considered not only meanings and functions linguistically, but also the relationship between the speaker and the hearer, the psychological social distance between them, and the level of burden on the hearer, because these elements powerfully influence the speaker's choice of a politeness strategy. Usami's point is that Brown and Levinson's politeness theory claims that the mechanisms for the selection of speech acts in order to produce smooth communication are universal. As such, Usami 
criticises some other scholars who undervalue Brown and Levinson's work for the reason that it contains little description of the peculiar characteristics of specific languages, e.g., keigo [honorifics] of the Japanese language.

\subsubsection{Criticism of Brown and Levinson's Politeness Theory}

It appears to be a universal rule that a relationship with others demands consideration for them, which includes, for example, usage of appropriate language. However, the customs of other people and how to be considerate towards them vary depending on individual languages and cultures (Hori 2006: 241, Kitao and Kitao 1988: 53, 60-4). It is apparent that the Japanese apply their own strategic styles of language use, which are not usually noticed by native speakers of Japanese.

With regard to polite expression, the function and usage of keigo are conspicuous. Kanasugi (2008: 50) affirms that keigo taikei [the honorific scheme] is much more elaborate in Japanese than in other languages; and the use of keigo while conversing can place and/or maintain an addressee at a distance. Matsui (2001: 52) describes keigo as language that is used to a senior party. When people use keigo, they constantly gauge the other's social status so that they maintain an appropriate verbal distance from that person. However, with people of the same rank or a junior party, there is less concern about the form of speech.

Using keigo is a constraint in Japanese society. Takiura's $(2008: 13,38)$ explanation of the linguistic meaning of keigo is that since it has been woven deeply into Japanese grammar, using it properly is not just a matter of language but has the character of a social institution. He asserts that the preserved ways of speech represented by keigo equate with the negative politeness strategy of Brown and Levinson's politeness theory (as opposed to the 
positive strategy). Tameguchi [casual speech between peers], on the other hand, is regarded as positive politeness strategy because it does not show social distance.

Usami (2002c: 110-2) clearly differentiates between keigo and Brown and Levinson's politeness theory: while keigo belongs to normative politeness, Brown and Levinson's theory focuses on pragmatic politeness, which prioritises the effectiveness of speech acts. Therefore, she does not agree that they are equivalent. She argues that even if keigo is used, there are cases which are polite on the surface (of the utterance) but are actually contemptuous. On the other hand, even though tameguchi and jokes are sometimes used to a superior, such speech is not necessarily impolite, because these casual speech acts may shorten the distance between the two parties and create a feeling of in-group solidarity. ${ }^{60}$

If the social rank of the hearer is higher than that of the speaker and there is no familiarity between the two, using keigo is obligatory (Okamoto 2002: 133-4). In other words, if keigo is not used in such circumstances, the junior's speech acts will be regarded as rude and the speaker may be seen as a person of poor behaviour. Okamoto raises another example of the over-polite expression which, on the contrary, becomes ironic or sarcastic. For instance, an overly honorific question to a flatmate such as, Mōshiwake gozaimasen ga, dōka mado o shimete itadakenai deshō ka? [Would you mind if I asked you to keep the window closed, please?] would be judged to be more ironic than appropriately polite or would be used to convey sarcasm. ${ }^{61}$

Usami (2003: 126) points out that two types of politeness exist: one is absolute politeness and the other is relative politeness. ${ }^{62}$ As in the past, the word "politeness (or

60 This is undeniable; however, the speaker (the junior) must be careful of the relationship with the superior and his/her personality when applying tameguchi.

61 In English, too, an embarrassingly over-polite expression is regarded as a critical/sarcastic remark.

62 Absolute politeness: Utilising keigo is typical of absolute politeness. To a senior, a speaker uses 
polite)' stands for absolute politeness. However, she claims that politeness must not be treated at single sentence level but at discourse level, since using keigo does not always imply 'polite' (as mentioned above). Even tameguchi can be polite in a certain setting, where it is obvious that the level of politeness of tameguchi is low from the viewpoint of linguistics, but it works to increase a harmonious feeling. ${ }^{63}$ Therefore, she claims that the practical result of politeness should be measured as a whole discourse.

Hori (2006: 58, 240-1) argues that being polite in the Japanese language is synonymous with exhibiting courtesy. However, as noted above, a polite and courteous attitude in Japanese society does not include the positive politeness strategy of Brown and Levinson. This means that as long as there is no common basis between the two parties, the speaker keeps a distance and tries to maintain it, because these kinds of behaviours are regarded as modest and polite. Showing intimacy actively is deemed undue familiarity, and it does not fall into the category of politeness in Japan: if the hearer is not particularly close to the speaker, it is impolite and fails in propriety. Therefore, in general, polite behaviours and speech acts in Japanese fall into the negative politeness strategy.

In order to facilitate daily life smoothly and harmoniously, indirect expressions are often utilised in Japanese (Tsuda 1999: 20-1, 39). That is, to be reserved and/or to speak in a circumlocutory manner is utilised so as to gauge and to confirm the other's response while speaking. Indirect expressions are also exercised if the other party is either socially higher than the speaker or unfamiliar. Tsuda interprets these speech acts as defending the speaker's position, which is the negative politeness strategy of Brown and Levinson's politeness theory.

the word irassharu [go/come] instead of the plain word $i k u$ [go/come]. Relative politeness: A speaker uses tameguchi [casual talk, peer language] to a senior in order to express his/her feeling of closeness to the senior, even though casual talk to the senior violates the formal language norm in Japanese.

63 For example, teenage students might say to their teacher very casually, Sensei no sono kutsu, meccha suteki! [Your shoes are super cute!] in an excited voice and with a showy gesture. 
Japanese people, who often use indirect expressions, primarily expect a speaker's hidden/unspoken intention to be perceived by the hearer instead of having to utter it overtly.

Keigo connotes formality and the discernment [wakimae] of the speaker, who understands his/her relationship with an addressee. An utterance exercised with keigo indirectly reveals the speaker's sense of social position. Using keigo is normative and expresses the speaker's formality, and it is not simply a question of the speaker's personal preference or internal feelings (Okamoto 1999: 69-70). In other words, keigo does not necessarily bear any relationship to the speaker's feelings of respect or disrespect for the interlocutor. Okamoto finds it questionable that a politeness rule of one language can be applied to other languages as a universal rule. In addition, rules and moods have to be considered in context, because as the context changes, a speaker's choice might also change. She argues that politeness is a strategy of speech-style used in a specific social and historical context.

In sum, on the surface, both keigo and politeness look similar. However, they do not seem to equate exactly. While keigo has sophisticated and very complicated rules which a speaker is expected to discern and utilise according to social and linguistic norms, Brown and Levinson's politeness theory is elaborated based on a speaker's spontaneous (emotional or voluntary) utterance rooted in the FTA. Although both come from a speaker's intention to build up or maintain a good relationship with an interlocutor, the fundamental concepts of keigo and politeness do not necessarily overlap.

\subsubsection{Cross-Cultural Communication}

There is no doubt that different politeness strategies exist in Japanese and English because the individual speech act appearing on the surface differs by language. Nevertheless, 
universality as a strategy of being polite in order to have a good relationship with others should exist in any language (Usami 2003: 120). Likewise, as we have seen, for an expression to be regarded as polite depends on the culture and society of the speaker and hearer (Tanaka and Tanaka 1996: 138), and a speaker's choice of appropriate utterance is largely controlled by cultural values and social customs. Usami (2003: 120) asserts that positive politeness strategy is more problematic than negative politeness strategy in crosscultural communication, owing to different cultural understandings.

Cultural difference is affirmed by Kitao and Kitao's study (1988: 60-1). The weightiness of an imposed burden is differently perceived according to culture, and they researched the effect of weightiness in the case of similar requests with Japanese and American subjects. For the Japanese, once a request was made of them, it became hard for them to reject it. The Japanese felt the request was burdensome. Therefore, they tried to avoid requests as much as possible. But, if they had to make one, they used negative politeness strategy and attempted to reduce the burden on the addressee. However, if the relationship with the hearer was very close, they seemed to make a request rather more easily, even if the request was significant. On the other hand, Americans tended to reject requests more casually, so that a speaker could make a request with less hesitation. They used the positive politeness strategy frequently, or no polite strategy was used, and they used the negative politeness strategy less, if at all. They could plainly reject the request—which means the addressee did not feel an obligation to accept it—even though their relationship was very close.

Azuma (2001: 69-70) carried out experiments through role-plays with three pairs of Japanese and three pairs of Americans. In the role-play scenario, the relationship between the pair is of a senior and a junior in an office. The senior complains about the frequent late arrival of the junior. The result of the experiments was that the Americans expressed complaints openly, because they judged that conveying their discontent should be more 
important than concern with the other's face. In contrast, the Japanese adopted the off-record strategy, so that the efficiency of conveying their dissatisfaction to the junior might have been less but at least the other's face would not have been severely damaged. Azuma names the latter the 'others-centred independence' style. This suggests that when two parties of different cultural backgrounds communicate, their conversation does not always go smoothly, but rather may be attended by difficulties.

In Azuma's experiments, another distinguishing difference was recognised. The Americans, both seniors and juniors, often uttered words of appreciation, i.e. adopted a positive politeness strategy. In the case of the Japanese, however, among whom wakimae [discernment ${ }^{64}$ prevails, this expression of appreciation occurred only from the juniors to the seniors, and not the other way around. Azuma's interpretation of this phenomenon is that it is not necessary to take the positive strategy from the socially higher to the lower, which is based on the traditional social norm of wakimae. Moreover, the Japanese juniors used apologetic expressions frequently to their seniors, but this phenomenon was very rare among the Americans. The speech act of frequent apologies by the Japanese juniors thus appears to be based on the social norm of wakimae, which they observed in order to maintain the relationship, while the Americans' politeness is based on friendliness (Azuma 1997: 134).

In each different culture factors influence the use of politeness strategies, such as how to weigh individual factors when determining the politeness strategy, including social rank or the closeness of the relationship. Americans tend not to see so much difference in social rank and try to be equal in their speech to reduce the social gap between the ranks of the two if it does exist. Therefore, even if there is a rank difference, it has no substantial influence on the use of politeness strategies. For the Japanese, conversely, it is apparent that a speaker uses negative strategy towards a person who is unfamiliar. Positive politeness strategy is reserved

64 Discernment of which level of speech and which vocabulary is appropriate in the given situation. 
for a person who is close, or when the speaker's social rank is higher than that of the hearer, or he/she does not use a polite strategy at all in the latter case. If the two parties are socially equal and very close, either the positive strategy or no strategy is employed. As culture is the basis of the speech act, it influences the exercise of appropriate politeness strategy (Kitao and Kitao 1988: 60-1).

In normative Japanese culture, merely giving one's opinion to an inquiry by saying yes or no, for example, could be regarded as a highly face-threatening act. Therefore, Japanese often prefer to take negative and/or off record strategies. Hori (2006: 30-1) goes so far as to claim that Japanese culture takes the opinion of the speaker into account almost as though it were a part of his/her personality. When Japanese express an opinion by using the off record strategy to people from a different culture, it can lead to misunderstandings. Conversely, Japanese people, in general, are not accustomed to being spoken to directly; therefore, they may have a feeling of discomfort or unpleasantness if someone speaks in a very straight fashion, particularly when expressing a dissenting opinion. These discrepancies come from the different cultural backgrounds of speakers, and people tend to take for granted that their own culture overrules others. The speaker's own criteria do not always suit those of others from the viewpoint of second language learning pedagogy. In cross-cultural settings, people should be aware of others' speech acts which come from their own respective cultural background.

\subsubsection{Summary}

The politeness theory advocated by Brown and Levinson is often said to be universally applicable. However, some scholars, as a result of some individual examples of particular languages including Japanese, do not accept that politeness theory is universal. This dissent is especially pronounced in the case of Japanese, since the keigo scheme has 
been well established for centuries and plays a significant role as a norm of the spoken language. Such scholars insist that Brown and Levinson's politeness theory is not entirely applicable to Japanese because their theory was based on the face-threatening acts rather than sociolinguistic norms.

Without doubt, because of the sociolinguistic norm of keigo, the register of speech the speaker has to utter is almost mechanically determined by the setting, not the emotions the speaker. Keigo, in which appropriate polite words and phrases are utilised, does not entirely accord with Brown and Levinson's concept of face. In other words, the speaker merely activates his/her skilful command of the Japanese language including keigo. This implies that a core of politeness theory is the speaker's sense of closeness or distance from an interlocutor. The latter is the same as the function of keigo at a point in order to maintain the distance with an interlocutor.

From the aspect of the ultimate goals of constructing and keeping a harmonious relationship with others, using keigo and the fundamental concept of Brown and Levinson's politeness theory can be said to be similar to a certain degree. Keigo is the method for keeping an appropriate distance from the interlocutor, which is Brown and Levinson's negative politeness strategy; while on the contrary, tameguchi is used to shorten the distance between the speaker and the interlocutor, which is Brown and Levinson's positive politeness strategy. In this way, the functions of the Japanese language's keigo and tameguchi schemes, which nonetheless do not derive from the concept of FTAs, are similar to Brown and Levinson's politeness theory. 


\subsection{APOLOGIES}

\subsubsection{Introduction}

An apology is an expressive illocutionary speech act (Leech 1983: 124). It is pronounced by a person who has committed an offence which has a harmful influence on another party (the victim). An apology is defined as what the offender utters to the victim in order to restore the relationship. The Japanese language dictionaries Kokugo Daijiten (1988: 1207) and Köjien (1991: 1195) contain the words shazai (n.) and wabi (n.) as equivalents to the English word "apology". Shazai is described as apologising for a crime or an error because the offender feels sorry for getting someone into trouble and asks for that person's forgiveness, and wabi is begging a person's pardon. From the viewpoint of Western scholars, Leech (1983) concisely explains apology as follows:

Apologies express regret for some offence committed by the s[peaker] against the h[earer] - and there is no implication that $s$ has benefited from the offence.... If the apology is successful, it will result in $h$ 's pardoning or excusing the offence....[i]f we commit an offence against someone, we talk of owing that person an apology, thereby treating the apology as in some sense an expiration of the offence (124-5).

As a presupposition of apology, an offender has violated social and ethical norms, which the offender and the victim understand in common. Fraser (1981: 262) succinctly observes that there are two things necessary when apologising: one is to take responsibility for an offensive act, and the other is to express regret for the offence commited. Schlenker \& Darby (1981: 276) claim, “Apologies were by far the favored strategy for dealing with the predicament, the accounting tactics of excuse and justifications were not endorsed with a high likelihood of occurrence". Although this favouring seems to be true, it is also a matter of degree. 
Across cultures it is a common understanding that strategies of apology depend on social and situational factors (Bataineh, et al., 2006: 1921). It is also widely recognised that the more severe the offence, the more elaborate and earnest are the apologies. This point suggests that complex strategies of apologetic discourse are indispensable and can include not only uttering the apology but other aspects. If the offence is very serious, the importance of uttering an apology becomes comparatively less significant, and other approaches, such as offering reparation or promising never to do it again, etc. are to be used, because an apology alone does not necessarily reduce the anger of the victim (Kasugawa 2010: 66, Ohbuchi et al. 1989: 220-6). Kumagai (1993: 4-5) clarifies that an apology is an act to adjust a relationship with the other party socially, so that an apologiser has to admit his/her error, take responsibility for the damage to the other, and beg the victim's forgiveness. In a broad sense, then, the speech act of apology in discourse involves a response from the other party.

Itoi et al. (1996: 930) describe that, from the offender's perspective, apologies are motivated by the desire to alleviate the anger of the victim. Further distinctions may be made if the speaker accepts responsibility, his/her speech act is regarded as an "apology". But, when the offender does not take responsibility, the criterion for the offence is understood as either "justification" or "excuse". Justification and denial are chiefly driven by the motive to avoid punishment. If the speaker does not admit that the conduct was inappropriate, his/her speech tends to be perceived as "justification", and if a speaker does not take responsibility for the conduct, it tends to be recognised as an "excuse". 


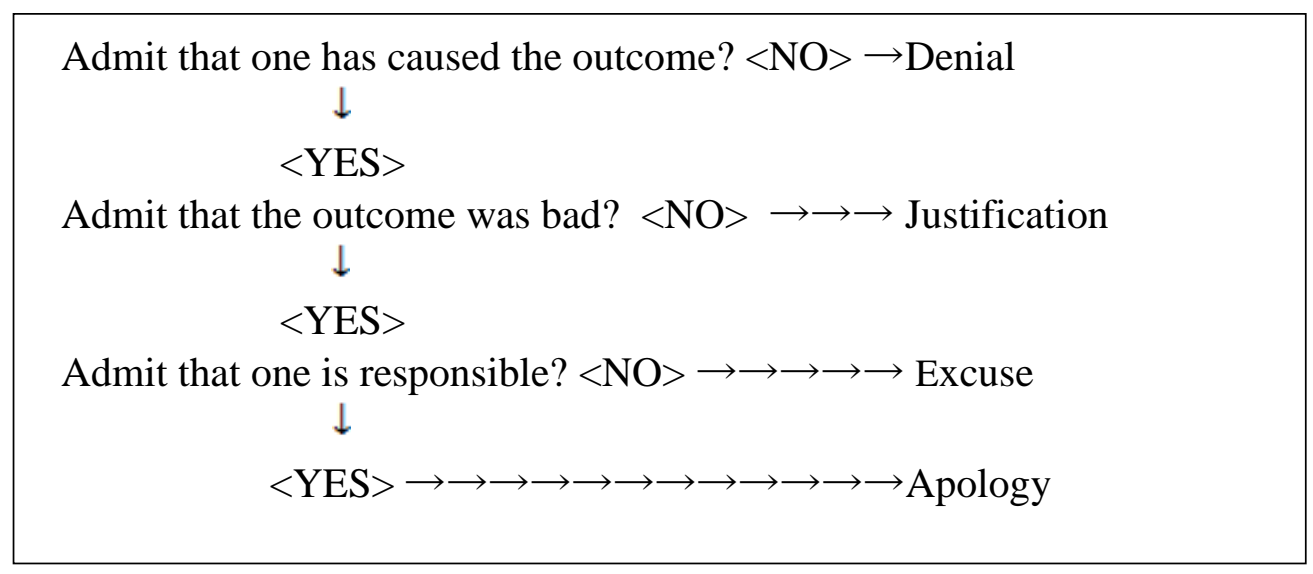

Figure 2.4.1 Classification Scheme of Accounts

(Adopted from Itoi, Ohbuchi \& Fukuno 1996: 914)

From the viewpoint of "face", Holmes (1989: 198, 1990: 159) defines apology as a speech act addressed to the victim's face-needs that is intended to remedy an offence for which the apologist takes responsibility, and restores equilibrium between apologiser and victim. She affirms this point by referring to Brown and Levinson's work $(1978: 65,192)$ in which apologies are described as generally aimed at face redress associated with facethreatening acts (FTAs) or an offence which has damaged the addressee's face in some way. Olshtain's (1989: 157) interpretation is that an apology is basically a speech act that is intended to provide support for the hearer (victim) who was actually or potentially badly affected by a violation $\mathrm{X}$ carried out by the offender. The speaker (apologiser) is willing to humiliate him/herself to some extent and to admit to fault and responsibility for X. Hence, the act of apologising is face-saving for the hearer and face-threatening for the speaker. Coulmas (1981: 83) argues that for Japanese people, breaches of conduct, violations of a social rule, etc. involve, first of all, loss of the offender's face due to his/her misconduct followed by an apology, but at the same time, the victim's face, too, is damaged. Kumagai (1993: 10) likewise depicts apology as an act of respect to the other party's face, although it may simultaneously threaten the apologiser's face. Maintenance of face, in particular the victim's face, is one of the central values governing interactions in Japan. 
For the present research, an apology is defined as the speech act uttered by a person (offender) who has said or done something wrong to the other party (victim), which has caused him/her disadvantage and unpleasantness, a fact that the offender recognises. The equilibrium of the relationship of the two parties has been damaged, as a consequence of which the victim holds advantage over the offender. The offender tries to restore their relationship in order to recreate the previous state. The interactions between the apologiser (offender) and the addressee (victim) until the victim accepts (or rejects) are comprehensive of the discourse of apology. This study will primarily examine the speech acts of the apologiser.

\subsubsection{Strategies of Apology}

The strategies of apology that an offender employs when apologising have been studied by many scholars. The motivation for an apology comes from the offender's wish to restore the relationship between the offender and the victim, since their relationship has been damaged. A question arises: are there globally efficacious strategies of apology, or only those that are practicable for a given culture? Or, conversely, are there strategies that are not appreciated or even abhorred by a particular culture or nation?

A scholar of the Japanese language, Kindaichi (1987: 79-82) claims in Japanese culture, justification, account, and explanation for the incident by the apologiser are not regarded as favourable strategies of apology. He observes that many Japanese seem to apologise straightway if trouble happens even if there is only a possibility that they might have been the possible cause. He further presumes that, in such a case, the real motivation for an unprompted apology by the Japanese is not because of a repentant feeling, but to minimise the deterioration of the relationship between the offenders and victims. 
Kumagai (2008: 27-30) explains the mechanism of face loss for the Japanese offender. Owing to the fact that the offender utters the apology, the offender's (apologiser's) position becomes lower than the victim's (addressee's). This lowering destroys the equilibrium between the two parties. As the offender's position has been lowered vis-à-vis the victim, the latter might think of assisting the apologiser by offering a word of acceptance. Such a response might be the victim's inward intention and can be said to be an elaborated interaction between the two parties. However, this interaction would not be feasible if one of the two does not engage with the other party's insight, or if the victim does not want to accept the apology because of the scale of damage, for example. Furthermore, if the cultural distance between the two parties is very great, this scheme may not always work, as Kumagai explains, because it can only function appropriately if the two parties share a common understanding, which is determined primarily by culture.

In a cross-cultural study, Ikeda (1993: 16-7) conducted an experiment of a discourse by Japanese and Americans respectively in which one party arrives thirty minutes late in order to identify whether there are cultural difference in apologies. Eighty per cent of each language group articulated apologies, such as sumimasen and gomen by the Japanese, and I'm sorry by the Americans. As a distinguishing difference, however, the Americans used strategies of account, explanation, and reparation more often than the Japanese so as to rebuild the relationship, the Japanese were dominant in uttering an apology alone or combining an apology and taking responsibility. The Japanese avoided strategies of account, explanation, and justification, in particular if the addressee was their superior.

Another difference between Japanese and Americans that arose was that the Americans used a wider variety of strategies as the severity of the offence rose. This variation was not applicable for the Japanese. In other words, the Japanese were earnestly and exclusively intent on uttering apologies. Ikeda ponders, from a perspective of 'face', whether 
the Americans appear not only to have respect for the other party's face but also attempt to minimise their own loss of face. Ultimately, this concern increases the number of usages of apologetic strategies by the Americans. Her finding supports Kindaichi's claim that the Japanese mostly focus on apology, and not on account, explanation, and justification.

Among various methods of researching strategies of apology, Itoi et al. (1996: 929) used movie scenarios for analyses of their empirical study. One hundred and sixty-nine Japanese and 174 American students read the scenarios, and they responded to questions on the correlation of the severity of the offence and the apology. From the results of this study, Itoi et al. found that uttering an apology was favoured more by the Japanese than the Americans, but, on the other hand, justification were the opposite. Itoi et al. concluded that the Japanese tend to avoid a conflict, since they feel harm more strongly than the Americans, and therefore, the Japanese offender takes a means of mitigation to the other (victim). They concluded that the research results arose from the collectivism of the Japanese, who are more concerned about relationships with others than the Americans; whereas the individualism of the Americans allows them to be more assertive and justify their behaviour.

Sugimoto (1997: 357-70) examined key factors in apologetic strategies among 181 Japanese and 200 American university students by measuring their responses to an openended questionnaire that included a scenario of being delayed. The result was that the Japanese applied more elaborate apologies and promises, in particular, when arriving one hour late; they uttered promises seven times more often than the Americans did, ${ }^{65}$ and then they asked for forgiveness three times more frequently than the Americans. One of the findings of her research was that receiving the offender's verbal apologies could be as satisfying for the victim as actual remediation, which implies that the apology has to be overtly uttered, because it may function as relief and indemnification for the victim.

65 The percentage of promise is $16.7 \%$ for Japanese and $2.2 \%$ for Americans (Sigimoto 1997: 357). 
Kumagai (2008:27-31) investigated the difference in the discourse structure of apology between native speakers and non-native speakers of Japanese. ${ }^{66}$ Her comparative study reveals that Japanese people utter the apology itself first, followed by accounts and/or explanations; in contrast, non-Japanese in her study tended to take the reverse order, i.e. accounts and/or explanations came first followed by the apology, if they thought such apology was necessary. Kumagai argues that the structure applied by the Japanese focuses on expressing responsibility for the incident, which puts the speaker in a disadvantageous position. ${ }^{67}$

On the other hand, the strategy of the non-Japanese was to explain the incident first, and if the speaker (offender) senses that the hearer (victim) would understand and accept a not-yet-uttered apology, the offender would subsequently utter an apology. Moreover, Americans explain the situation with more animation if the incident were serious; conversely, the Japanese did this less. For Japanese, negating the victim's feeling is more important than explaining the background of the incident, and they wish to avoid a possible risk of worsening the victim's feeling by justifying the offender-self. The tendency towards preferred apologetic strategies arises based on individual cultural background (Kumagai 2008; Sugimoto 1997).

Schlenker \& Darby (1981: 271) found that as the severity of a social predicament increases, so did both the use of non-perfunctory apologies and the number of components, such as expressing remorse, offering to help the victim, and explicitly requestng forgiveness. Kumatoridani (1993: 29-30) discovered that apologies, promises (e.g. that it would not happen again), accounts, and compensations are commonly used by Japanese and British. A

\footnotetext{
66 The non-native speakers of Japanese are Brazilian, French, Korean, American, and Vietnamese, all of whom live in Japan. As for Japanese native speakers, they live in either Japan or in the following five countries, Brazil, France, Korea, USA, and Vietnam.

67 For example, to utter Watashi ga warukatta [I was wrong].
} 
difference between them, however, only the British ask for an acceptance of the apology, Please accept my apology, or state the apology as an offer, I would like to offer my apology. Obviously, these sentences can be expressed in Japanese linguistically, too. However, they may not be recognised as a practice of sincere apology by Japanese, because they do not fit in Japanese language pragmatics. He observes that it is customary for a Japanese offender to apologise first, prior to someone (typically, the victim) initiating a conversation, because the essence of an apology is to rebuild the relationship and, therefore, the offender has to display his/her willingness to apologise first. In this sense, the utterance, I would like to offer my apology [(lit.) Watashi no owabi (no kimochi) o teiji shimasu], is not considered strong enough in the Japanese social context to express the offender's feeling of remorse. ${ }^{68}$

Holmes (1990: 167, 192) examines the remedial interchanges of 183 New Zealand university students of European descent. She argues that although the correlation of three elements - the cost or ranking of the imposition on the victim, the social distance between them, and power relations-determines the apology's level, ${ }^{69}$ the correlation appears curvilinear because the degree of remedial work by the offender is not always in proportion to the severity of the offence. In her experiment, the most elaborated apology was taken by the students and applied to close friends. This indicates that the offender is most concerned about relationships involving those with whom they will continue to associate. Another notable finding shows quite a low number of utterances of promise, as low as 1.7 per cent. Holmes recognises that the social group to which the apologiser and the victim belong controls the method of apology. She deduced this point because if the relationship between

68 There is in essence no such an expression as I would like to offer my apology in Japanese. Therefore, the direct translation sounds unnatural.

69 The three elements conform to Brown and Levinson's calculation formula of weightiness for an FTA (see Ch. 2, Sec. 3). 
the New Zealand university students was very close, the apology seemed to be uttered very carefully and discreetly.

An ultimate goal of apology is to maintain a good relationship with others. Otani (1999: 52-3) claims that there is a unique strategy among Japanese vitims in response to the offender's apology. The victim may choose to lower him/herself when, for instance, an offender (latecomer) apologises, Gomen, osokunatte [Sorry, I'm late]. The victim may also respond, Uuu, jitsu wa watashi mo okurechatte sa, ima kita toko na no [No problem, as a matter of fact, I was late, too, and just got here]. This victim's words, which disclose she has just arrived ${ }^{70}$ whether true or not, adjust the imbalance between the two parties. The victim in Otani's example does not wish to leave the relationship unbalanced, so the victim displays her own disadvantage to the offender, regardless of whether she has been delayed. Otani points out that the Japanese have a strong tendency to attempt to maintain a relationship when it has deteriorated.

Being late is a typical offence in any place, but the degree of felt severity may vary according to culture. The Japanese are concerned about punctuality, as seems to be the case with Germans, British, and Americans (Yabuuchi, 2004: 47). ${ }^{71}$ How much one is late is not of primary importance. Rather, it appears that the reason why one is late is more important than how late one actually is. For many Japanese, whether the offender arrives ten minutes or thirty minutes late is not very significant.

Interpreting a single word in other languages is easier than the translation of sentences and or much larger chunks of discourse. The influence of the mother language and culture on

70 In this example, the victim is using a typical female language, such as na no.

71 Japanese generally expect that trains keep schedule, so if it is delayed by even a minute or so, conductors announce an apology to the passengers, particularly, in metropolitan areas. The extreme tragedy of a train crash occurred in 2005 near Osaka, where the morning commuter train had been delayed for 90 seconds and the driver tried to make up the time. Over100 lives were lost. (Cited from http://www.mlit.go.jp/fukuchiyama/index.html, accessed on 15/12/2012). 
language learners is undeniable. For learners, the significance of foreign language learning demands grasping the entire structure of the conversational interaction with others.

If the learners' competency in the target language is insufficient, hearers may not comprehend the intention of the speaker, especially at the discourse level, although the individual words may be translated correctly. If, however, speakers have no trouble uttering sentences in the target language, but structure the discourse in the style of their native language, they may be interpreted by the people of the target language as arrogant or impertinent at worst. Teaching other languages has long focused on a close-up view of items, such as words and sentences, but we should not exclude macroscopic consideration of view, such as discourse level conversations.

\subsubsection{Expressions of Apology}

As seen above, apologies are generally classified as a form of politeness or remedial facework in the large body of research on the social action of maintaining a good relationship with others. Spoken discourse studies of apologies have largely focused on how they are produced by speakers (Chang \& Haugh: 2011: 411). When an apology is necessary, as we have seen in most cases, people use formulaic expressions, such as I'm sorry or Excuse me in English, and sumimasen [I'm sorry] or gomen nasai [Excuse me], etc, in Japanese. ${ }^{72}$

Kumagai (2008: 29-30) highlights that in a discourse of apology between Japanese people, there are not only explicit apologies, such as mōshiwake arimasen [It's inexcusable, I'm very sorry], but also other speech acts appear, such as, explanations, Densha ga okureta no de [Because the train was late]; taking responsibility, (Watashi ga) warukatta [(I) was in the wrong]; offers of compensation, Ichijikan bun no kyūryō o hiite kudasai [Please cut my

\footnotetext{
72 An English translation of the Japanese expressions is put here only comprehensively. Therefore, there may be cases where excuse me is more appropriate for sumimasen, and so is I'm sorry for gomen nasai because it depends on the context of a setting.
} 
pay by one hour (as I was late)]; and promises, mò nido to shimasen [(I) won't repeat the same mistake again]. Although it is possible for a single word to constitute an apology, such as gomen [Sorry], explanation, admitting responsibility, compensation, promise, etc. are generally key components of forming a discourse of apology in Japanese.

\subsubsection{Formulaic Expressions of Apology}

An apology is usually uttered through formulaic words or expressions. Ogawa (1995:48-50) collected Japanese apologetic expressions with a questionnaire to 221 Japanese females aged between 20 and 80. She categorised the data into four basic groups with variations: gomen nasai $G$ (group), sumimasen $G$, mōshiwake $G$, and shitsurei $G$ [(lit.) I lose/lost courtesy]. In the case of being late at a meeting place, she indicates that the mōshiwake $G$ is widely used by all ages, especially if the other party is the superior (teacher, higher up in the workplace, and so forth). The moshiwake $G$ is also preferred by older speakers if an incident is serious. The higher the age of the apologiser, the more sumimasen $G$ seems to be employed, especially by those in their 70s and 80s. By contrast, those aged in their 20s and 30s never used this group if the other party was a friend (an equal). While elders are inclined to use shitsurei $G$ more often than any other age group, conversely they use gomen less. Ogawa ascertains that the criteria for choosing apologetic expressions depends on the social distance between the two parties, the severity of the incident, and the age of the apologiser.

Collecting TV and movie scenarios is now a widely-accepted means of analysing speech acts. Yamamoto (2004: 24-6) and Sato (2011: 29-36) examined Japanese TV dramas and movies. Yamamoto collected scenarios from eight TV shows and 25 movies, and Sato from 91 TV shows. Apologetic expressions were grouped into five categories by Yamamoto, and four by Sato. Three expressions were common in both: namely, gomen $G$, sumimasen $G$, 
and mōshiwake G. While Yamamoto added warui [(I'm) in the wrong] and miscellaneous, Sato separated gomen and gomen nasai more explicitly within the gomen $G$. The majority of apologetic expressions in Japanese include only three broader groups that overlap.

Table 2.4.1 Group of Apologetic Expressions

\begin{tabular}{|l|l|}
\hline Yamamoto & Sato \\
\hline \multirow{2}{*}{ gomen $G$} & gomen \\
\cline { 2 - 2 } & gomen nasai \\
\hline sumimasen $G$ & sumimasen $G$ \\
\hline mōshiwake $G$ & mōshiwake $G$ \\
\hline warui & - \\
\hline Others & - \\
\hline
\end{tabular}

Yamamoto affirms that if an apologiser is the junior in a scenario, he/she uses sumimasen $G$ most to the superior. In contrast, a superior uses gomen $G$ and warui, instead of sumimasen G. Sato's result is very similar to that of Yamamoto. He concludes that while gomen is widely used by males between the ages of 10 and 30 and applied to equals or juniors, gomen nasai is female language; the sumimasen $G$ is used by both genders towards superiors. The mōshiwake $G$ is uttered most often if the addressee is a superior; also it is mainly used by males over 50 years of age. Although both sumimasen $G$ and mōshiwake $G$ contain similar implications, Sato argues that mōshiwake arimasen is more polite because of its length of expression. It can be understood from this point that if an apologiser thinks that his/her offence is serious, the expression of apology should be longer. It is also recognised that preference for items of vocabulary to be used in apology varies based on gender and age (Sato 2011: 29-33).

Apologetic expressions were categorised according to intensity by Coulmas (1981: 88) gomen is a very casual apology used in an intimate setting, while sumimasen $G$ and shiturei $G$ are apologies often used on insignificant occasions. By contrast, Oyurushi kudasai [Please forgive me], Owabi itashimasu [I apologise], or Mōshiwake arimasen [It is inexcusable] communicate a more dramatic sense of apology, and are reserved for occasions 
when something serious has happened. Long (2010: 1074) observes a difference in the use of sumimasen and gomen nasai, the latter being less formal than sumimasen. He corroborates that more formal apology is used with non-intimates and superiors.

\subsubsection{Order of Utterances of Apology in Japanese}

Mizutani and Mizutani (1987: 49) suggest that giving some reason for being late, such as Densha ga okuremashite [The train was late], is alright. But it is regarded as proper to utter the apology first and then follow it by explanations. They assert that the briefer the explanation the better. They also describe that in actual conversations, the apologysumimasen or gomen nasai-comes first, very commonly with the -te form at the end of the utterance, for example, Sumimasen, osoku natte [Sorry for being late]. This order is more appropriate than the other way around, Osoku natte sumimasen [For being late, sorry], when the speaker has hurried and is out of breath. In short, saying something in this order, i.e. apology first, then an explanation or admission of the fact/situation, sounds more expressive than otherwise.

\subsubsection{Past Tense of Apology}

A particular feature of gomen $G$ is that it does not have a past tense, unlike other expressions. Kindaichi (1987: 77-82) argues that words without the past tense can hardly be employed to a superior as they are not suitable unless the speaker is a child. ${ }^{73} \mathrm{He}$ delineates the different usage of present and past tenses when apologising as follows: the past tense in the - $t a$ form of verbs is perceived to be more polite than the present tense, because the apologiser acknowledges and appreciates that he/she is benefiting from the addressee's

73 As an exception, there may be a case where an offender is a male teenager who bluntly apologises to his parent(s), saying gomen. 
(victim's) favour of accepting his/her apology (as the victim accepts). This connects to the degree of apologetic feeling in the present tense, i.e. in the -masu form (present tense), which admits the apology less than when using the past tense first. In other words, while the present tense expresses only the apology, by contrast, the past tense includes not only the apology but also the appreciation that the apology has been accepted.

Here is an example of comparing the present tense with the past tense: Mōshiwake arimasen [I'm very sorry] (present tense) versus Mōshiwake arimasen deshita (past tense). Kindaichi (1987: 77-8) suggests that the latter is more polite than the former, since the past tense includes not only the immediate behaviour but also that of the past. ${ }^{74}$ Hence, the degree of regret conveyed by the past tense is deeper than that of the present tense. In addition, the etymological meaning of gomen combines the honorific prefix go and men for pardon/ forgive/release. ${ }^{75}$ In apologies it is frequently used in the imperative form gomen nasai [Pardon/forgive me]. As a consequence, gomen $G$ is regarded as a light polite word among other expressions of apology since it has no past tense.

\subsubsection{Utterance of Offending Behaviour}

Another characteristic feature of apology in Japanese is depicted by Sakuma (1983: 58) and Oki (2006: 347). Offenders (apologisers) utter their offense, which itself turns into the apology. For instance, a latecomer utters Okuremashita [I was late] to the hearer (victim) who has been waiting. The word contains no apologetic meaning at all, and it literally only describes the present situation of the speaker. Oki claims that this type of utterance, in which speakers (offenders) describe their own immediate behaviour, i.e. 'being late', provokes a nuance of apology to the addressee. Nevertheless, in order for this type of expression to be

\footnotetext{
74 The implication of 'past' here is just a few minutes/seconds before.

75 Cited from http://gogen-allguide.com/ko/gomen.html accessed on 16/12/2011.
} 
recognised as an apology, it must be uttered at the very outset, such as the initial moment of the encounter when the two parties meet in the case of being late.

\subsubsection{Various Implications of Apology}

Referring to Cohen and Olshtain's semantic classification, ${ }^{76}$ Nakada (1989: 57) compared 400 utterances of apologetic expressions presented in Japanese and American TV, radio, and movies in each language respectively. Nakada categorised them by connotations, since the meanings of apologetic expressions vary. The following is the classification adopted.

Table 2.4.2 Semantic Classification of Apologies in Japanese and English

\begin{tabular}{|c|c|c|c|c|}
\hline $\begin{array}{l}\text { Categorised by } \\
\text { semantics }\end{array}$ & Japanese & Total & English & Total \\
\hline $\begin{array}{l}\text { Expression of } \\
\text { regret }\end{array}$ & $\begin{array}{l}\text { sumimasen (157) } \\
\text { kyōshuku desu, osore } \\
\text { irimasu (4) } \\
\text { menboku nai, ohazukashii } \\
\text { kagiri desu (2) }\end{array}$ & 163 & $\begin{array}{l}\text { I am sorry (271) } \\
\text { I feel guilty/badly (2) } \\
\text { how awful (1) }\end{array}$ & 274 \\
\hline Offer of apology & $\begin{array}{l}\text { ayamarimasu (15) } \\
\text { owabi shimasu (11) }\end{array}$ & 26 & $\begin{array}{l}\text { I apologise, my } \\
\text { apologies, I owe you an } \\
\text { apology (17) }\end{array}$ & 17 \\
\hline $\begin{array}{l}\text { Request for } \\
\text { forgiveness }\end{array}$ & $\begin{array}{l}\text { gomen nasai (100) kannin/ } \\
\text { kanben shite kudasai (13) } \\
\text { yurushite kudasai (5) } \\
\text { kibun waruku shinai de } \\
\text { kudasai (1) }\end{array}$ & 119 & $\begin{array}{l}\text { excuse me }(68) \\
\text { forgive me (27) } \\
\text { pardon me (15) } \\
\text { no hard feelings (1) }\end{array}$ & 111 \\
\hline Excuse & $\begin{array}{l}\text { warugi ga atte shitanja nai } \\
\text { (2) }\end{array}$ & 2 & $\begin{array}{l}\text { I didn't mean it (9) } \\
\text { no offence (2) }\end{array}$ & 11 \\
\hline $\begin{array}{l}\text { Acknowledgement } \\
\text { of responsibility }\end{array}$ & $\begin{array}{l}\text { mōshiwake arimasen (45) } \\
\text { warui (39) } \\
\text { shitsurei shimashita (37) } \\
\text { sekinin wa watashi ni (2) }\end{array}$ & 123 & $\begin{array}{l}\text { my fault (2) } \\
\text { I was at fault (1) }\end{array}$ & 3 \\
\hline Total & & 433 & & 416 \\
\hline
\end{tabular}

(Adopted from Nakada (1989: 195). The numbers in parentheses are the frequency of appearance out of 400 utterances in each language. As some expressions contain more than one implication, the total comes to over 400 .

76 Cohen, A. D., \& Olshtain, E. (1981). Developing a Measure of Socilcultural Competence: The Case of Apology. Language Learning, 31(1), 119. 
The most common apologies are sumimasen and I'm sorry, expressions of regret in both languages. The frequency of the sentence I'm sorry is noteworthy. The acknowledgement of responsibility, on the other hand, reveals quite an opposite tendency, i.e. mōshiwake arimasen and similarly Japanese occur over 100 times, while, in English, "It was my fault" and "I was at fault" total only three. It is clear that both nations have widely used apologetic expressions of regret. While Japanese are typically inclined to take responsibility, Americans concentrate on saying I'm sorry, as an expression of regret. This suggests that different varying strategies of apology might come from differing cultures.

The expressions of apology in Japanese hold another implication. Nakada (1989: 2012) emphasises that the characteristics of Japanese apologetic words not only express the offender's regret, but also embrace a feeling of appreciation when the offender is advantaged and the hearer (addressee) is disadvantaged. In the latter case, the offender utters apologetic expressions that contain a feeling of thankfulness. ${ }^{77}$ In particular, the sumimasen $G$ is often uttered to show appreciation to a person who is of help to the speaker. ${ }^{78}$

\subsubsection{Summary}

The four most frequently used apologetic word groups and their variants in Japanese are: gomen $G$, sumimasen $G$, mōshiwake $G$, and shitsurei $G$. The criteria for choosing them by the apologiser are determined by three key factors: (1) the relationship of the two parties,

77 For example, two miners have been trapped in an underground pit. Only one has a bottle of water, which has little left. The other is so thirsty that the one who has the bottle is urging it on him. The recipient says said, Suman [I'm sorry]. In this case, the recipient uses the apologetic expression as appreciation (Nakada 1989: 201-2).

78 This usage arises because sumimasen literally means "unfinished", i.e., the speaker has unfinished business with the interlocutor, such as "I am indebted to you". Therefore, it may double as both "sorry" and "thank you". 
(2) the apologiser's age and gender, and (3) the severity of the offence. Some apologetic words are not appropriate to use in a certain setting, in particular, to a superior. Despite the variety of Japanese apologetic expressions, it is nevertheless desirable to minimise selfjustification unless absolutely necessary.

Another noteworthy feature of Japanese native speakers' apologies is that apologetic expressions are repeated many times. It is necessary to explain the reason(s) and/or the background of the offence, but many excuses may not be accepted very favourably by the hearer. Rather, apologising sincerely many times is typically better received than excuses. Furthermore, taking responsibility is also crucial. It is apparent that Japanese people express responsibility for the offence more frequently than English native speakers.

Some apologetic expressions have not only a form of the present tense but also of the past tense. The use of the past tense occurs when the apology has been recognised and accepted by the hearer and the apologiser then opts to utilise the past tense. It is thought that the past tense comes from the apologiser's feeling of appreciation towards the hearer (for having accepted the apology). An apology in the present tense, on the other hand, is literally asking the hearer for acceptance. Verbalising the fact of the offence without using apologetic words or phrases is also considered an apology if it is spoken at the very outset of the apology.

Learners of the Japanese language would do well to be aware of these above conditions when using apologetic words and expressions, because even though some of the Japanese apologies might also commonly be used in the environment of their native language, it does not always mean that the equivalent word in Japanese is used as often as, or in a similar setting to. 


\subsection{REQUESTS}

\subsubsection{Introduction}

The meaning of the Japanese word irai (n.) is usually translated as request in English, and vice versa. ${ }^{79}$ In Japanese language dictionaries, irai is described as an act of a person (speaker) who asks someone to do something (Kokugo daijiten 1988: 186, Köjien 1991: 184). From the discipline of linguistics, Searle (1969: 66) sets out the rules on the felicity conditions of the illocutionary act of request in his book, Speech Acts: An Essay in Philosophy of Language, as follows:

Propositional content: Future act A of $\mathrm{H}$ [earer].

Preparatory: $\quad 1 . \mathrm{H}$ is able to do A. S[peaker] believes $\mathrm{H}$ is able to do A.

2. It is not obvious to both $\mathrm{S}$ and $\mathrm{H}$ that $\mathrm{H}$ will do $\mathrm{A}$ in the normal course of events of his own accord.

Sincerity: $\quad \mathrm{S}$ wants $\mathrm{H}$ to do A.

Essential: $\quad$ Counts as an attempt to get $\mathrm{H}$ to do A.

Okamoto (1988: 8) identifies three determining elements which go to make up an expression of request: the attributes of the requester, the relationship between the speaker (requester) and the hearer (requestee), and the speaker's recognition of issues around the act that he/she is going to request, for example, the hearer's capability of executing the request and obstacles lying before and throughout its execution.

Regarding another aspect of irai, Sasaki (1995: 61) describes it an instructive act in which the requester can receive benefit from the requestee who has the option of whether or not to accept it. She acknowledges that it is not easy to distinguish the speech act of irai from adjoining speech acts in the Japanese language, such as meirei [order], yōkyu [demand], sasoi [invitation], and so forth. For example, the sentence, To o shimero [Shut the door] is clearly an order in the imperative form of the verb shimeru [shut, close]. On the other hand, the

79 The translation of a word into another language depends on the context; therefore, there are cases where the word irai can be translated into other English words, such as reliance, etc. 
following two sentences, To o shimete kure [Please shut the door] and To o shimete kurenai ka? [Can't you please shut the door?] are similar in terms of the decreed result. It is perceptible; however, that the former is a strong request and the latter is a weaker entreaty.

From the perspective of politeness theory, Kumagai (2008: 28-31) argues that the act of a request means a speaker has recognised that his/her positive face might be adversely affected when making a request. Nevertheless, the requester's damaged face could be saved by the requestee's acceptance of the request. In Japanese interactional behaviour, what is to be respected and/or to be recovered is the other's face. Kindaichi et al. (1989: 613) affirm that when a requester attempts to be polite to an interlocutor, longer expressions and more diversified strategies tend to be employed, regardless of the usage of keigo [honorifics].

\subsubsection{Structure of Request in Japanese}

The structure and flow of requests in the Japanese language is outlined by Kokuritsu Kokugo Kenkyūjo [The National Institute for Japanese Language and Linguistics] as follows (cited by Nakamichi and Doi 1995: 89):

Table 2.5.1 Flow of Typical Discourse of Request

\begin{tabular}{|l|l|l|}
\hline Narrator & In the Case of Acceptance & In the Case of Rejection \\
\hline S(peaker) & Request H to do X \\
\hline H(earer) & (Ask for an explanation) $)^{80}$ & \multicolumn{2}{|l|}{} \\
\hline S & Explain the reason for requesting \\
\hline S & (Present a proposal) & \multicolumn{2}{|l|}{} \\
\hline H & Understand and accept the request & Reject the request \\
\hline S & (Thank H) & $\begin{array}{l}\text { (Present a proposal again: Demand } \\
\text { acceptance or withdraw the request) }\end{array}$ \\
\hline H & Offer the action & Reject the request \\
\hline S/H & Close the discourse & Close the discourse \\
\hline
\end{tabular}

(Adopted from Nakamichi and Doi 1995: 89)

80 The actions in parentheses stand for non-essential conditions for a requisitive context. 
Requests may be divided into two groups, according to acceptance or rejection. Both start from broaching and explaining the reason for the request by the speaker. If the interlocutor accepts the request, the speaker thanks him/her. If the hearer rejects the request, the speaker may make the request again by attaching conditions or modifying the request in order for the interlocutor to accept it more readily. If there is no sign of acceptance from the interlocutor, the speaker has to withdraw the requests (see Table 2.5.1). Requests may thereby involve a degree of negotiation.

Nakamichi and Doi (1995: 89) suggest that showing the entire flow of discourse structure - the conversational exchanges between the two (or more) parties - is effective for learners of Japanese to deepen their awareness of the ways of communication, especially in order to acquire the corresponding procedure and style of the target language.

Uttering explicitly what a speaker wants an interlocutor to do X, such as Mado o akete yo [Open the window] is not the only way of uttering a request. Kumatoridani (1995: 17) argues that another style of request only mentions what the speaker wants implicitly. A typical example of such an utterance is Kono heya wa mushiatsui ne [This room is stuffy, isn't it?] ${ }^{81} \mathrm{He}$ asserts that indirect expressions of request contain the speaker's wish in the context which, subsequently, is asking the interlocutor to respond. The aforementioned sentence states only the condition of the room, and it does not contain any requisitive expressions. However, if uttered, the hearer may have a hunch that the speaker is indirectly requesting permission to open the window or asking the hearer to open the window.

Whether or not a request is successful depends on the interlocutor. A speaker's act of request brings benefit to the speaker, only if the interlocutor accepts it and executes it. As a result, the interlocutor may receive damage or suffer disadvantage by acting on the speaker's

81 Politeness theory advocated by Brown and Levinson (1987) deals with this type of indirect request (implicature) as "off record" strategy. 
wish (request). Hence, in some cases part of the speech act of request includes the restoration of the relationship between the two parties by offering a reward to the requestee.

The following sentence retains three elements of a speech act of request in one single speech: apology, explanation, and request. Warui kedo, saifu wasureta n' de, sukoshi kashite kurenai? [Sorry, but I forgot my wallet, so can't you lend me a little money?]. ${ }^{82}$ This illocutionary act represents at first a form of apology warui kedo [(I'm) sorry, but], followed by an explanation of the reason for the request saifu wasureta n' de [(I) forgot my wallet], and lastly the request sukoshi kashite kurenai? [(Can't you) lend me a little?]. This sentence contains all the essential elements of a requisitive sentence (Kumatoridani 1995: 18).

Conventional speech patterns of request, as analysed by Hashiuchi (1988: 47-8), start smoothly with a passable topic followed by pseudo-apologetic sentences, such as Chotto shitsurei desu ga [Sorry for being a bit rude, but] or Warui n' desu ga [Sorry for being bad, but]. Izaki (2000: 86-7), who supports Hashiuchi's observation, affirms from her experiment that in Japanese native speaker conversation in the setting of request, prior notice is explicitly given to the interlocutor before the request is made. ${ }^{83}$ In other words, the Japanese usually do not make requests bluntly or abruptly, but choose rather an indirect and implicit style, as they expect the interlocutor to conjecture the requester's thought or wish.

In a discourse of request, requesters should not behave (speak) as if they have the right of decision, because the decision must be taken by the requestee; otherwise it becomes an order. Kumai (1992: 78-9) raises the example of an inappropriately uttered request between a Japanese language teacher and an international student who asks the teacher to lend a book.

82 The interlocutor of this example must be a close friend or a junior party of the speaker because of its casual style of speech.

83 This strategy is opposed to that of French native speakers, who prefer to ask the other party's situation first (Izaki 2000: 87). 
Student: Doyō kurai wa kashita hō ga ii desu [On Saturday, at least, it would be better if you lend me the book.]

Teacher: Jā, kyō kashite agetara, raishū no suiyōbi ni wa kaeshite kureru? [Well, if I lend you the book today, can you return it next Wednesday?]

Student: Hai, ii desu [(lit.) It is good. Yes, it is all right.]

The problem of the student's speech act is that while requesting, the student (requester) uses the judgemental phrases, V-ta hō ga ii [(You'd) better V], and ii desu [It is all right], in spite of the fact that the speaker is the requester. The first sentence would have been improved if the student had said Kashite kudasaimasen ka? [Couldn't you lend me?] so as to concede the decision entirely to the teacher. The student's second sentence should have been Wakarimashita [I understood] in order to show understanding. By doing so, the requester could avoid the judgemental utterance of $i$ [good]. Kumai (1992) stresses that this example of discourse reveals inappropriate sentence patterns of request, such as the wrong choice of vocabulary $i$ being used ${ }^{84}$, and not using aposiopesis. ${ }^{85}$ These usage may result in offence to the interlocutor.

Kumai (1992) argues that with communication in foreign language pedagogy it is important to teach whole discourses, rather than just isolated sentences. Kashiwazaki (1993: 60 ), too, claims that from the aspect of Japanese language pedagogy, it is necessary to teach the requisitive patterns at the discourse level, rather than expressions of request specifically, because if learners start a request abruptly without presenting the topic, it can surprise the interlocutor, or the message will not be adequately conveyed.

84 The reason why the usage of $i i$ by a student is inappropriate in the context is that the student should not utter a judgemental word, such as $i i$ when being asked by a superior. An appropriate reply would be, for example, hai wakarimashita [Yes, I understood].

85 Aposiopesis is explained in Ch. 2, Sec. 2, and it is demonstrated in Chapters 4 and 5, too. 
Kumagai and Shinozaki (2006: 20-2) claim that a concern of the speaker in the setting of request is to elicit a means of approach to the interlocutor in the discourse. This includes the order of utterances, the way of broaching the topic, the explanation about the background of the request, the expression of request, etc. Through these activities, the requester's seriousness is revealed. If a discourse is lengthy, it is due to the various strategies the requester has employed.

\subsubsection{Strategy of Request}

The purpose of requisitive behaviour is to get the hearer to do something for the speaker. This conduct can interfere with the requestee's negative face, which touches upon the feeling of not being concerned with his/her rights or freedom. It seems, therefore, that the requester tends to be concerned with the flow of conversation when developing a discourse of request. There is a normative flow in Japanese when developing a requisitive discourse in the following sequence: broaching the topic, getting permission to talk, giving prior notification of a request, making the request, and explaining the reason, and thanking (Sakamoto 2001: 17-8).

The Japanese attitude towards a person who is depending on them is generally sympathetic. Lebra (1976: 81) depicts the persuasive appeal of a humble posture combined with empathy on the part of Japanese people, who are moved by the sight of a helpless person. It seems that the negotiator (requester) succeeds by causing the other party pleasure and at the same time guilt for keeping the requester in such a humble posture. Lebra's explanation is applicable to Kumagai's notion that by exposing his/her weakness the requester lowers his/her position, a strategy that is highly efficacious in Japanese society, because the Japanese tend to think of reciprocal face-support patterns. As a result, the two parties can uphold each other's face. 
Since requests do not work positively in favour of the speaker's face, his/her face has been damaged by uttering something disadvantageous to the speaker (such as exposing a weakness). The reciprocal face-support function of the interlocutor works, because as long as the interlocutor is aware that what is to be respected, protected, and restored is both his/her own face and the other's face, the requester's behaviour which has put him/her in an unfavourable position is understandable and worthwhile when making a request (Kumagai and Shinozaki 2006). ${ }^{86}$

An indirect speech act is also adequate in order to communicate the request. Nakamura et al. (2004: 112-3) assert that the more indirect a speech act is, the more likely a request will be accepted, the lower the negative impression of the requester becomes, and the more the intimacy with the requester is increased. For this reason, interlocutors (requestees) tend to take the request into consideration, because they want to show attentiveness. If the cost of the request is small, indirectness works. If the cost is significantly larger, however, speech acts of desire are accepted most frequently as, for example, the utterance of $V$-te hoshii n' desu ga... [wish to V], which implies that the requestee's sense of burden is lessened by the strength of the wish on the part of the requester.

\subsubsection{Expressions of Request}

\subsubsection{Politeness and Requisitive Sentences}

The level of politeness in a requisitive sentence depends on the relationship between the requester and the requestee (Kabaya et al. 1993: 68). The degree of politeness is shown in

86 A typical utterance when requesting contains five strategies including the sub-strategy:

Sumimasen [Excuse me (apology)], Chotto [A little bit (softening/miniming)], Kore kara yōji aru n' de [Having an errand (reason)], Chotto kono hen mawatte kuru n' de [Going around here a little (detailed reason)], Kono nimotsu chotto okasete moraitai n' da kedo [I'd like to leave this luggage a little while (request)], Onegai shimasu [Please do me a favour (request)] (Kumagai and Shinozaki 2006:51-2). 
Japanese by an affirmative interrogative sentence or a negative interrogative sentence by using juju dōshi, such as V-te kudasaimasu ka? [Please would you do me the favour of V?] and V-te kudasaimasen ka? [Please wouldn't you do me the favour of V?]. When the speaker and the interlocutor are equal in status or the interlocutor is subordinate, the negative interrogative yet casual forms, V-te kurenai? [Please not V?] and V-te moraenai? [Please could not I get you to V?], is very often applied. When the interlocutor is a superior and the cost of the request is small, the utterance is either direct V-te kudasai [please would you V], or an affirmative interrogative sentence, V-te kuremasu ka? [Please would you V?] or V-te moraemasu ka? [Please could I get you to V?] is used. If the cost is great, the negative interrogative sentences $V$-te kuremasen ka? [Please would you not V?], V-te moraemasen ka? [please could I not get your to V?], V-te itadakemasen ka? [Please could I not receive the favour of V?], or polite patterns with desire, V-te hoshii n' desu [I want you to V] or V-te itadakitai n' desu [I should like you to V], are applied (Okamoto 1988: 10-11).

In short, the distance between the requester and the requestee and the impact of the burden on the requestee determine the speech level of the request, and juju dōshi [verbs of giving/receiving] are used when requesting very much. In addition, the more polite the expressions are, the more negative forms are employed (Ueno 1983: 49).

Kim (2000: 42) conducted an experiment on 300 Japanese native speakers in order to grasp if usage differs depending on whether an interlocutor is an inner-group member or an outer-group member. The expressions analysed are divided into four groups: the V-te kureru [(lit.) to give] group, the $V$-te kudasaru [(lit.) to give (polite)] group, the $V$-te morau [(lit.) to receive] group, and the $V$-te itadaku [(lit.) to receive (polite)] group. ${ }^{87}$ The results showed that the kureru (affirmative) group was the most used expression to anyone in an in-group,

87 For example, the kureru group has many variations, such as kureru $\uparrow$, kurenai $\uparrow$, kure, kurenai $k a$, kurenai ka na, etc.; the kudasaru group has kudasai, etc.; the morau group is moraeru etc.; and the itadaku group has itadakenai deshō ka, etc. 
typically to family members. The kurenai (negative of kureru) was used to junior parties of outsider-groups, and the itadaku group was used to senior parties of outsider-groups. The morau group followed the kureru group in all cases. The youngest respondents in Kim's study, were university students of whom a remarkably low number used the itadaku group, which is one of the most polite forms. Conversely, the oldest respondents aged between 53 and 66 years preferred the kudasaru form. These expressions are closely related to the usage of honorifics. Such results demonstrate that Japanese native speakers differentiate between requisitive expressions depending on whether the interlocutor is in an in-group or an outsider group.

A strong sense of in-group solidarity demonstrates that the young generation of Japanese university students leans towards positive rather than negative politeness strategy, compared with British counterparts. Obviously, the harder the content of the request, the more varied are the politeness strategies used by both subjects (Fukushima 1996: 686-7). ${ }^{88}$ Fukushima notes that overall Japanese subjects tended to use more direct expressions to their fellow students than the British participants did, including positive or even bald on record strategies. This directness seems to arise because the feeling of solidarity is high if the speaker and the interlocutor are in-group members.

On the other hand, when a request is made to out-group members, the requester may have a fear of conducting face-threatening acts and infringing on the other's personal territory, because the distance between the two is highly valued. Thus, the requester may choose negative politeness or off record politeness strategies with mitigating devices, and this choice

88 The request (which is predetermined by the researcher) is that the subject has to make a request to a (fictional) flatmate asking if the subject's friend can stay over in the flatmate's room because the subject will have another friend who will stay in his/her own room and there is no space for the second friend. The questionnaire was administered in a written form, and the subjects generated responses to the fictional flatmate in their first language. 
may depend on the scale of the imposition of the request. If the request is at the high end of the imposition scale, it is likely to threaten the requester's face, but requests with a low degree of imposition may not do so, and this difference is reflected in the choice of strategies employed to make the request.

Unlike young Japanese, the generation of their parents, and in particular their fathers, tend to use more indirect strategies. This outcome was deduced from research conducted by Sato (1997: 228-30). The subjects of her study were nine male and nine female university students along with their parents making a total 36 Japanese native speakers. Several settings of request were studied, ${ }^{89}$ Sato observes that the fathers were more socialised from having worked in business fields where they would have had greater opportunity to meet people they do not know very well. From this, she concluded that the distance in the relationship between the two parties and the length of social experience, too, influences speech style. In particular, the latter may place the requester on the safe side in order to avoid risk while conversing.

On the whole, it is noteworthy that all three groups used positive politeness strategy more often than negative; the ratio was approximately six to four. The closer the relationship with the interlocutor, the more positive politeness strategy increased. This does not necessarily mean, however, that the number of usages of positive strategy increases, but that the proportion differs in a single utterance. Almost certainly within Japan, utilising the politeness strategy differs to some extent by region, age, gender, class, and so forth.

\subsubsection{Order of Utterance of Request in Japanese}

Conventional speech patterns of request, Hashiuchi (1988: 47-8) explains, generally start smoothly with a passable topic followed by pseudo-apologetic sentences, such as Chotto

89 The settings of requests were, to ask to turn down the volume of music, to change the date of a meeting, to extend the deadline of submitting a paper, and to lend some money (Sato 1997: 226). 
shitsurei desu ga [Sorry for being rude] or Warui n' desu ga [Sorry for being bad]. Izaki (2000: 87), similar to Hashiuchi, recognises that in Japanese native speakers' conversation in the setting of request, a prior notice is explicitly given to the interlocutor before the request is made. In other words, they do not request directly or straightforwardly, but choose an indirect and implicit style as they expect the interlocutor to interpolate the requester's thought. Kimura (1987: 60-1) affirms that if a sentence is used in the pattern of sumimasen+chotto $+V$ te [(lit.) Sorry $+a$ bit $+V]$, the level of politeness increased, and ultimately it functions to reduce the impression of a request.

Whether or not requesters explain the reason for the request before making it depends on the social distance between them and the speaker (Unosawa 2005: 168). Unosawa's research involved four English and nine Japanese native speakers who were asked to create a scenario of request to the other party in a close relationship. ${ }^{90}$ Two types of processes appeared in their scenarios.

(1) Explanation of a reason $\rightarrow$ Request $\rightarrow$ (accept)

(2) Request $\rightarrow$ Explanation of a reason $\rightarrow$ (accept)

All English native speakers with only one exception and some Japanese participants followed process 1 . Process 2 was followed only by the Japanese. Unosawa interprets that in a close relationships, the speaker (requester) is inclined to use process 2 , i.e., the request coming before the explanation. She does not assert that Japanese are more forthright when making a request; rather she is cautious in drawing stereotypical views of different languages and/or cultures from this experiment because the number of participants was small and the data used had not been collected in real life.

Kyi (2004: 88-92) draws a chart of the conversational flow of requests in settings where a requestee cannot easily comply. Kyi states that in such a case, groundwork on the

90 The scenario of request was to ring at 5.30 a.m. on the morning of travelling overseas. 
part of the requester is feasible and effective by grasping the interlocutor's (un)availability and gauging how the requestee may perceive the validity of the request. If groundwork cannot be carried out prior to the request, asking the other's situation and conditions while making the request allows the requester to negotiate by adjusting the degree or contents of the request.

\subsubsection{Aposiopesis}

Aposiopesis is a distinguishing characteristic of the Japanese language. It is well recognised that Japanese conversation does not always comprise linguistically complete sentences (utterances) (Ogiwara: 2008: 5). Kumai (1992: 73) found from her experiments that there are different ways of ending an utterance. She compared requisitive expressions uttered by five Japanese native speakers and 14 international students who were studying Japanese. While all Japanese native speakers ended their speech act of request with an unfinished sentence, such as no da+ke(re)do(mo)... [(lit.) the fact is that...but...], only two learners out of 14 did so. This indicates that almost all learners produced a completed sentence at the end of their speech. Aposiopesis is, according to Kumai, an indispensable, sophisticated feature of the requisitive, because it creates an atmosphere of leaving the outcome to the discretion of the interlocutor (requestee). On the other hand, the completed sentence might give the impression of a declaration or demand to the interlocutor, even though the actual words convey a request. A sentence with aposiopesis mitigates or lessens the possibility of conflict that might arise with the interlocutor because of the request. Mihara (1995: 83) argues that the expressions of kedo... and desu ga... contain the implication that the speaker wants to know the hearer's intention/comment even though these sentences do not form direct interrogatives. Shimizu (2009: 37) affirms the effectiveness of aposiopesis desu ga.../desu 
kedo..., which avoids giving an order-like impression, and solicits the interlocutor's favourable response.

Likewise, Kashiwazaki (1993: 59-60) describes learners' awkwardness when they request. She observes that such awkwardness comes from their use of complete sentences, such as $V$ masu $[V]$ instead of using aposiopesis, such as (n') desu kedo... [V, but...] and desu $g a \ldots[V, b u t \ldots]$. Aposiopesis is a distinctive feature of the Japanese language, and was exercised by many Japanese native speakers when requesting. It is socially and pragmatically inappropriate if an interlocutor (requestee) might perceive the speech act of the speaker $(\text { requester })^{91}$ as impertinent or impolite because of the absence of aposiopesis by the speaker, although the requester might believe that it is "good" in terms of grammar and may be unaware of having breached etiquette.

From these experiments, Koike (2000: 67-8) recognises that there are differences between native Japanese speakers and non-native speakers' speech. Her points of difference are (1) NNSs' lack of consideration towards the other party, (2) NNSs' lack of lightening the imposition on the other party, and (3) NNSs' lack of negotiation upon receiving the other party's reaction because they (the non-native speakers) tend to repeat the same phrases if an interlocutor does not accept the request instantly. These elements negatively affected the Japanese native participants who performed the counterpart in role-plays. These subtle linguistic differences, i.e., $\underline{\text { arimasu }}$ vs. aru n' desu ga..., affected the sensibilities of the native speakers. This is something which can easily be overlooked, because it is too slight to recognise. This delicate and faint sound " $n$ " has a considerable influence on hearers, and it is possible for the requester to avoid being offensive if it is explicitly taught. ${ }^{92}$

\footnotetext{
91 In Kashiwazaki's case, they were international students learning Japanese.

92 The learners are all in their 30s, while the Japanese native speakers are in their 20s, and teachers of the Japanese language. The difference in social rank might have affected the students who employed the masu-form, as it is more polite.
} 
It is a common understanding that the more advanced the learners' competency is, the more their speech acts approach those of native speakers. Samejima (1998: 82-3) observes that advanced learners' use of the requisitive expression te kudasai [V please] decreases, and the phrases n' desu kedo... $\left[V\right.$, but...] and $n^{\prime}$ desu ga... $[V, b u t \ldots]$ are increased in comparison with learners of lower proficiency. He notes that even if the sentence ending form is appropriately acquired by learners when they have developed speaking skills, once they have to apply it in real life, they often struggle to broach a topic of request and/or to close the conversation other than by saying arigato [thank you] to the interlocutor.

In other words, their knowledge of the Japanese language is fragmented, and individual knowledge remains disconnected-perhaps owing at least in part to methods of teaching and learning. Few textbooks for beginners and intermediate levels pay attention to the structure of entire discourse. Instead, they deal with each expression piecemeal. Samejima suggests presenting a whole discourse to learners, in such a way that they will not cause offence when they want to bring up a request or close the conversation with the other party.

\subsubsection{Apologetic Expressions}

Apologetic expressions, such as in particular, sumimasen [I'm sorry] seem to be inseparable from broaching a request in Japanese. Kumagai and Shinozaki (2006: 52) used a questionnaire on 431 Japanese native speakers in order to investigate the process of approach to an interlocutor. They observed that the word of apology sumimasen was very frequently uttered when raising a request, in particular by the young generation. This implies not only appealing to the other party to pay attention to the speaker by uttering sumimasen, but also conveying the speaker's feeling of indebtedness prior to the request. They argue that when approaching others using a phatic phrase such as sumimasen, the word works to initiate good communication. On the other hand, mature respondents utilised words expressing their 
feelings of gratitude or obligation, such as Osore irimasu ga [Excuse me / I'm very sorry] to realise their request.

Kashiwazaki (1993: 56-62) studied ways of conveying requests to the other party. The words of address uttered by students who were Japanese native speakers were compared with those of international students. The participants made a request to academics and administrators at a counselling room or a reception office of a university. The Japanese students employed sumimasen (ga) [I'm sorry, but] (42\%) followed by anō [um...] (22\%), shitsurei shimasu/desu ga [excuse me] (14\%), kon'nichiwa [hello] (6\%), and chotto [(lit.) a moment, say] (4\%); while the international students used sumimasen (ga) (28\%) followed by kon,nichiwa (26\%), anō (19\%), chotto (16\%), and shitsurei shimasu/desu ga (2\%).

Table 2.5.2 Expressions of Addressing

\begin{tabular}{|l|r|r|}
\hline Expressions at Addressing & Japanese students & International students \\
\hline sumimasen(ga) [I'm sorry, but] & 42 & 28 \\
\hline anō [well] & 22 & 19 \\
\hline shitsurei shimasu/desu ga [excuse me] & 14 & 2 \\
\hline Kon'nichiwa [hello] & 6 & 26 \\
\hline chotto [(lit.) a moment, say] & 4 & 16 \\
\hline Others & 12 & 9 \\
\hline Total & $\mathbf{1 0 0 \%}$ & $\mathbf{1 0 0 \%}$ \\
\hline
\end{tabular}

(Adapted from Kashiwazaki, 1983: 56)

The contrast in these findings is noteworthy. The Japanese rarely used the greeting phrase kon'nichiwa or the typical casual addressing word chotto to a senior party at rather formal places. By contrast, the international students less frequently said sumimasen ( $\mathrm{ga}$ ) and shitsurei shimasu/shitsurei desu ga in which an apologetic feeling is conveyed when starting a topic of request. Kashiwazaki discusses why many Japanese used apologetic expressions such as sumimasen (ga) and shitsurei shimasu/shitsurei desu ga, which were typically followed by the phrase $X$ no koto nan' desu kedo... [I'm here about topic X]. She deduced that the Japanese preferred to disclose their concern about the burden (to be imposed) on the 
interlocutor. In addition, she observed that this kind of consideration can help connecting with the other party and smoothly lead to the main topic of the request.

The international students, on the other hand, applied friendliness, with the greeting kon'nichiwa. They did not, however, explicitly state the main topic of their upcoming request, but got into the details from the beginning. Therefore, the interlocutor had to guess, which imposed a burden on the other party. Kashiwazaki's research result offers good advice to Japanese language teachers - teaching sentences individually does not support learners in understanding what phrases to use appropriately and when. Ideally, introducing new sentences in the classroom should be carried out together with a description of a scenario they are likely to encounter.

Gagné (2010: 10-2) demonstrates that the Japanese apologetic feeling when making a request comes from being culturally normalised in the Japanese social context. Thus, those who fail to recognise it or do not display apologetic feelings and attitudes may cause social discomfort, and they might be perceived as offensive and violating cultural common sense. Li (2005: 11-2), too, confirmed that 70 per cent of 104 Japanese university students used apologetic expressions when making requests. They also used aposiopesis or off-record (hint) strategy in which the speaker expects the other party to comprehend what the speaker wants him/her to do. These characteristics did not appear in the requests made by Chinese students in her study.

In sum, requisitive sentences of the Japanese language have some distinctive features, such as apologetic expressions or aposiopesis, which are not necessarily seen in requests in other languages, and these features are very frequently employed as if they were pragmatically established rules when requesting. These specific characteristics have been cultivated based on Japanese culture, which is typically concerned about the other party's feelings. Needless to say, when making a request, it is not very difficult for tourists visiting 
Japan who are not sure of their route simply to ask for help from someone in pointing out their destination on the map. However, the foregoing discussion highlights that in the Japanese language teaching environment, it is advisable that the Japanese language features of requisitive sentences be distinctly taught to students together with an indicative conversational flow of the discourse of request.

\subsubsection{Summary}

There are distinctive features in a discourse of request in Japanese including rules in normative structure and order of appearance of utterances. If a request is turned down, the requester gives up on another attempt or makes a request a second time. It does not mean, however, that the requester literally repeats the same phrase. A different approach may be taken the second time, instead of repetition. There are several approaches to persuade the addressee. One is to emphasise the seriousness or importance of the request, or if the first attempt is not successful, the imposition might be reduced. Furthermore, it can also be an effective strategy to express a feeling of being sorry since this may appeal to the Japanese people's emotions. Yet, it is not wise to approach too assertively.

Regarding the characteristics of request utterances, first, there is the use of juju dosshi to be polite, and among these there is an order in terms of politeness. When using juju dōshi in requests, the sentence is always in either an affirmative or negative interrogative form, and the latter is regarded as more polite than the former, because the addressee would feel less pressure if he/she is asked but wants to decline the request. Indirectness is also one of the characteristics. It is not always necessary to employ indirect utterances, but rather depends on the relationship between the two parties and the magnitude of the imposition. In the case of a light request to a close friend, a casual form can be employed. 
Aposiopesis too is utilised frequently since it suggests the speaker has a reserved attitude. It is important that speakers express their apologetic (or sorrowful) feeling because the addressee will have to take on a burden if they accept. This decision is at the discretion of requestees, so that the requesters lower their position compared to the requestee.

In a cross-cultural setting, misunderstandings and/or errors easily occur between the two parties if the cultural distance is wide. Viewed as a whole, the above review of scholarly literature on the topic suggests that, it is vital for Japanese language learners not to be taught individual short sentences, but to be presented with whole discourses to comprehend the structure, strategies, and expressions according to the circumstances. However, the foregoing discussion highlights that in the Japanese language teaching environment it is advisable that the language features of requisitive sentences be taught distinctively to students together with the conversational flow of the discourse of request.

In Chapter 2, I have reviewed previously conducted studies from the five different aspects, and then discussed the characteristics of (1) textbooks, (2) discourses in Japanese, (3) politeness theory, (4) speech acts of apologies, and (5) speech acts of requests. In Chapter 3, I outline the experiments that form the core of the present study, participants' profiles, the textbooks used for the experiments, and some limitations of the experiments. 


\section{CHAPTER 3 METHODOLOGY}

\subsection{Introduction}

This research aims to identify similarities and differences in discourse by Japanese native speakers (NSs) and Japanese language learners (NNSs), and discourse model exemplars presented in Japanese language textbooks (TXs), in order to contribute to the creation of more efficacious Japanese language textbooks. In this chapter, the purpose of this study is to identify the similarities and differences among the three groups, NSs, NNSs, and TXs. In order to achieve this goal, a quantitative approach is applied. The chapter is organised in the following order: firstly, research aims; secondly, the rationale for the method of data collection; thirdly, experiments; and lastly, an outline of the experiments.

\section{Aims of the present research:}

(1) To elucidate discourse structures of the three subjects, NSs, NNSs, and TXs: similarities to, or differences from one another, and their extent.

(2) To elucidate expressions used by the three subjects, NSs, NNSs, and TXs: similarities to, or differences from one another, and their extent.

(3) To clarify factors to improve the quality of textbooks.

\subsection{Method of Data Collection}

The aim of discourse analysis is to reach a better understanding of natural spoken discourse. This process involves close examination of the forms and patterns of speech acts and consideration of whether anything can be taught to help language learning (McCarthy: 1991). The method of examining and analysing discourses depends on the purpose of the 
individual study. A quantitative approach comparing the above three subjects, the NSs, the NNSs, and the TXs, will be used for obtaining results from the experiments For the present research, which aims to facilitate the production of more effective textbooks.

\subsubsection{Method}

Conversational data may be collected by such means as recording naturally occurring conversation, discourse completion tests (DCTs), or role-plays. The reasons for utilising roleplays for this research are as follows.

Naturally occurring discourses are constructed conversational exchanges between at least two people. Apparently, these are the most ideal authentic resources for discourse analyses, but, needless to say, it is unrealistic and impractical to collect a sufficiently large volume of data by this method to fulfil the research purpose.

The discourse completion test (DCT) was used by Blum-Kulka et al. (1989: 13) who developed the Cross-Cultural Speech Act Realization Project (CCSARP) coding scheme ${ }^{93}$ to compare the speech acts of native and non-native speakers. They argue that the CCSARP is appropriate to investigate similarities and differences in the realisation patterns of speech acts between native and non-native speakers of a given language. The DCT also enables researchers to collect sizeable amounts of standardised data in a short period. There is, however, a drawback in that these data have not occurred spontaneously; rather, participants in DCT fill out a blank field with scripted prompts. Therefore, participants' freedom in response is limited. Furthermore, in studying the characteristics of discourse, the whole picture of any one discourse cannot be ignored, but if much of the discourse is provided by

93 Blum-Kulka, Shoshana, Juliane House, and Gabriele Kasper. 1989. The CCSARP Coding Manual. In Cross-Cultural Pragmatics: Requests and Apologies, edited by S. Blum-Kulka, J. House and G.

Kasper. Norwood, New Jersey: Ablex Publishing Corporation. In this study, not all coding schemes presented in the CCSARP have been utilised because, for instance, the CCSARP covers "threat" as one of the aggravating supportive moves, which was not relevant to the "request" setting of this study. 
the researcher, the order of utterance, for example, is prescribed and therefore cannot be analysed.

Role-play is, on the other hand, the acting out of a scenario by participants who take on the role of given characters engaging in a whole and spontaneous discourse. It cannot be denied that compared with natural conversation role-plays are still a kind of performance. However, as long as the players act out an impromptu scenario, extemporise, and perform immediately upon hearing what the other party says, with no script previously prepared, the utterances can be regarded as being close to naturally occurring conversations. Nishimura (2011: 97), citing Gass \& Houck (1999: 7), states that "open role plays seem to be the closest to what we might expect to reflect naturally occurring speech events", and she conducts open role-plays to collect the conversational data for her research. Moreover, collecting verbal data by role-plays is far easier and more feasible than collecting conversational data in real life.

\subsubsection{The CCSARP and Politeness Theory}

\subsubsection{The CCSARP by Blum-Kulka et al.}

The cross-cultural investigation of speech act (CCSARP) by Blum-Kulka et al. involves comparing across languages the patterns in two speech acts of requests and apologies (Blum-Kulka and Olshtain: 1984, 196). The general goal of the CCSARP investigation is to investigate the similarities and differences in the realization patterns of given speech acts between native and non-native speakers of a given language, and relative to the same social constraints (Blum-Kulka et al: 1987, 13). A more specific goal for BlumKulka et al. is to establish patterns of request and apology realisations under different social constraints across a number of languages and cultures, including both native and non-native varieties. Both their aim and that of the present research are to grasp similarities and 
differences between native speakers and non-native speakers under the same setting. Hence, in this study, the CCSARP method is partially utilised for the analyses. ${ }^{94}$ The difference is that while Blum-Kulka et al. focus on the sentence level, the present study concerns with a larger level of discourse.

The data collected in the present study are categorised in accordance with the coding manual of the CCSARP: first, each discourse is separated into Head Act (HA) and relevant Supportive Moves (SMs). The Head Act is the most significant sentence in a discourse in revealing the speaker's ultimate intention. The supporting Moves are divided into subcategories. In apologies, these are: (1) Explanation; (2) Admission of the fact; (3) Promise; and (4) Placation. In requests, these are: (1) Preparator; (2) Grounder; (3) Disarmer (4) Promise of Reward (Reward); and (5) Imposition Minimiser (Minimiser). These are the relevant parts of the CCSARP Coding Manual (287-94), which the researcher applied to the present study.

94 The CCSARP Coding Manual includes an Alerter which is described as, "an opening element preceding the actual request, such as a term of address or attention getter" (Blum-Kulka, et al. 1989: 275-6). For this research, Alerter was omitted because it is not substantially relevant in comparing the substance of the utterances of the NSs and the NNSs. 


\subsubsection{Reasons for Not Using the CCSARP to Analyse Head Acts}

The CCSARP coding scheme was designed primarily based on characteristics of the English language (and other Indo-European languages, as well as Hebrew). ${ }^{95}$

According to the CCSARP of request, there are nine categories in the Head Acts. ${ }^{96}$ Some of these, however, are not appropriate when analysing Japanese discourse, because sentence structure and lexical usage in English differs from Japanese. Often an English word can be translated into several words in Japanese. For instance, the want statement is defined in the CCSARP as "[t]he utterance [which] expresses the speaker's desire that the event denoted in the proposition [will] come about," and the example presented in the CCSARP is, "I'd like to borrow your notes for a little while" (279).

However, this single sentence can be translated into Japanese in several ways: (1) an explicit and even blunt utterance depending on the situation by using the verb misete [show (me)], for example, Nōto misete [Show me your notes]; (2) clearly expressing the speaker's desire but with an expression containing the speaker's hesitant feeling, Nōto o misete kurenai?[Can't you show me your notes?]; (3) Furthermore, the speaker may use an expression that pushes his/her hesitation to the forefront, so that although the sentence uttered is not a complete sentence in terms of grammar, the hearer is able to understand the intention,

95 CCSARP categorises the additional case of Can I borrow your notes? as Preparator (280). The Japanese translation of this may be, karitemo ii desu ka (Explicit Performative) or karirareru kana (Suggestory Formula) if the CCSARP is applied for analysis. As the definition of Preparator shows, "[t]he utterance contains reference to a preparator condition for the feasibility of the request, typically one of ability, willingness, or possibility, as conventionalized in the given language" (280), and both Japanese translations display interrogative forms. However, the implications of the two differ from each other. Hence, the former is in the Explicit Performative and the latter is in the Suggestory Formula of the CCSARP coding respectively.

96 The nine subcategories of HA, as defined by Blum-Kulka et al. are, (1) Mood derivable, (2) Explicit Performative, (3) Hedged Performative, (4) Locution Derivable, (5) Want Statement, (6) Suggestory Formula, (7) Preparator, (8) Strong Hint, and (9) Mild Hint. These are included in the CCSARP Coding Manual (Blum-Kulka, et al. 1989: 278-80). 
as in, for example, Nōto o misete kureru to arigatai n'da kedo... [I'd appreciate it if you could show me your notes...].

These differences in Japanese phrases are not contained within a single instance of the want statement of the CCSARP, because all the uttered sentences are a sort of "want statement" but the degree of intensity of each sentence differs significantly in Japanese. This is one of many examples that could be cited indicating that the use of the CCSARP to analyse the HAs of discourses in Japanese is inadequate.

\subsubsection{Reasons for Using the Politeness Theory of Brown and Levinson to Analyse}

\section{Head Acts}

The politeness theory of Brown and Levinson is deemed more appropriate for the present research to analyse the HAs in detail, because it focuses on the language behaviour employed by speakers to establish and maintain a smooth relationship with others. Brown and Levinson established the concept of the "face-threatening act" (FTA) which they analyse in terms of a combination of the two parties' (speaker and hearer) distance, difference in social rank, and the degree of burden incurred by the topic. They framed four strategies based on the level of politeness. ${ }^{97}$ In other words, the politeness strategies of Brown and Levinson define an utterance based on the speaker's intention of how much he/she wants to shorten (or keep) a distance from the hearer or avoid intruding on the hearer's psychological territory. This definition implies that politeness strategies are not necessarily categorised by lexical or grammatical forms. For example, the previously mentioned three Japanese sentences (see $\mathrm{Ch}$ 3, Sec. 2) involving requests to see notes would be categorised by Brown and Levinson's

\footnotetext{
97 Politeness theory has a fifth group, which is defined as the strategy of not uttering, when a speaker suppresses his/her intention and does not express it Therefore, the fifth strategy may or may not be recognised by an interlocutor. For the purpose of role-play in the present study, this strategy was deemed superfluous.
} 
politeness theory as (1) bald on record, (2) positive politeness, and (3) negative politeness. In this regard, then, Brown and Levinson's politeness theory is more appropriate and feasible than the CCSARP coding scheme for analysing the HAs in Japanese discourse.

\subsubsection{A Combined Approach: Reasons for Using the CCSARP by Blum-Kulka et al. and Politeness Theory by Brown and Levinson}

From the perspective of discourse analysis, it is essential to grasp discourse structure macroscopically. The CCSARP is suitable for categorising a discourse that encompasses a speaker's individual utterances. Each group categorised by the CCSARP has a unique characteristic. For example, in the case of "promise" when a speaker is apologising, the apologiser promises to the victim not to make the same mistake again. The speaker's intention is to restore the distance that was widened between the two parties because of the apologiser's misconduct, and he/she wants to restore favour with the other party. A hidden intention in uttering a promise is to narrow the distance between the two, along with the speaker's wish to restore the relationship. Such a strategy exemplifies how Brown and Levinson's positive politeness is used.

In a setting of request, requesters may attempt to make their imposition as light as possible for the hearer, so a minimiser is used. In such cases, requesters want the requestee to accept their request in a light-hearted manner, and some items of vocabulary are used to minimise the impression of a request. They come from the speaker's intention to reduce the invasion of the other's psychological territory. This kind of speech act is included under the negative politeness of Brown and Levinson.

It is therefore deemed more appropriate for the present study to first classify utterances in Japanese broadly according to the CCSARP and then applied the politeness theory of Brown and Levinson. 


\subsection{Experiments}

For the present study, impromptu conversations in Japanese were elicited by means of role-plays performed by a pair of speakers of the same native language in order to collect conversational data. The subjects were instructed to carry out performances in two different settings: apology and request. The native speakers of Japanese were the control group for the present experiments.

In the scenario of apology devised, one of the two speakers plays a part-time student worker at a busy café, and the other acts as the 40-year old café owner. The part-time worker is expected to show up at 10 a.m.; however he/she comes thirty minutes late to work without any prior notice. Their social rank differs insofar as it involves workplace boss-subordinate relationship.

The other scenario involves request. The two participants are instructed to play university students in the same class, so their social rank is the same. In the scenario they have an assignment, which is due the next day. One of the two has finished it and the other has not even started. The latter wants some help from his/her fellow student.

Participants were given only the outline of the situation explained above by the researcher (see Appendices 1 and 2, Information Sheet of Experiment) and within it they were expected to generate dialogue spontaneously. Their role-play was video recorded. I did not instruct the respondents, for example, to apologise or explain the reason(s) for his/her delay, or in the case of request, to appeal for the hearer's help. What was observed here are the conversational structure, strategies, approaches and expressions used spontaneously by the players. Once the first performance of a scenario finished, the pair swapped roles. They performed four plays in total, in opposite roles in the two settings. 


\subsubsection{Outline of the Experiments}

The present research has been conducted based on the authentic conversational roleplay data of pairs of native speakers of Japanese (NSs) and of Japanese language learners (NNSs), along with exemplars of model discourses as presented in Japanese language textbooks (TXs). The focal points to be examined in what follows are firstly, the conversational structure of the apologies and requests in the role-plays, particularly from the viewpoints of the CCSARP and Brown and Levinson's politeness theory, and secondly, the expressions commonly and frequently used among the three groups, as well as expressions distinctively used only by a particular group(s), but not by all. The resulting discourses are structured according to the order of utterance, for instance, opening (broaching), the main topic, and closing.

Although there are innumerable circumstances in which speakers may interact, the scenarios examined in this research involve acquaintances and are: (A) in the setting of apology, (i.e., one is an apologiser who is late for work and the other is a victim as he/she had to wait) and (B) in the setting of request when (a requester wants to raise his/her main topic of asking for help).

In Scenario A, the second party is most likely to become an interlocutor as soon as the two meet because both have already been affected by the purported scenario, but in Scenario $\mathrm{B}$, the other is unaware that a request is imminent and is not yet primed to become an interlocutor.

In daily conversation, a setting such as that in Scenario A is frequently encountered, i.e. the second party becomes an interlocutor because the two share the same information. Both of them know each other, so the speaker can broach the main topic quickly and smoothly. In other words, the speaker does not need to present any cues. In the case of Scenario B, on the other hand, even though the other party is an acquaintance, the speaker has 
to establish the basic setting of the conversation for the other party because he/she is not yet prepared to become an interlocutor on this topic (Horiguchi, 8-15).

Table 3.1 Scenarios within and beyond the Scope of the Present Research

\begin{tabular}{|l|l|l|}
\hline Setting & Apology & Request \\
\hline Acquaintance & $\begin{array}{l}\text { Within scope: Being late for an } \\
\text { appointed time. }\end{array}$ & $\begin{array}{l}\text { Within scope: Asking a classmate } \\
\text { for help in writing an essay. }\end{array}$ \\
\hline Stranger & $\begin{array}{l}\text { Beyond scope: Apologising for } \\
\text { treading on the toes of a stranger on } \\
\text { a train. }\end{array}$ & $\begin{array}{l}\text { Beyond scope: Asking a stranger } \\
\text { the way to the library. }\end{array}$ \\
\hline Remarks & $\begin{array}{l}\text { Immediately becomes an } \\
\text { interlocutor when the speaker } \\
\text { broaches the topic. }\end{array}$ & $\begin{array}{l}\text { Unprepared to become an } \\
\text { interlocutor when a speaker } \\
\text { broaches the topic. }\end{array}$ \\
\hline
\end{tabular}

(Adapted from Horiguchi 1997: 7)

The discourse comprises three sections: the opening section, the main topic, and the closing section. Horiguchi defines the conversation as follows:

\section{(1) Opening section}

Broaching is seeking the other party's attention in order for him/her to listen to the speaker. The content is primarily to be something related to the other party. The statement of broaching varies with the degree of acquaintance and the preparedness of the interlocutor to engage in dialogue.

(2) Main topic

Once the topic has been introduced, the speaker and the interlocutor proceed with the conversation by taking turns at speaking. Sometimes, however, the speaker keeps talking and the interlocutor only listens. In such a case, the interlocutor's role is minimal and he/she may be only back-channelling or similar. 
(3) Closing section

The conversation must be concluded in due course and appropriately. It cannot be satisfactorily stopped abruptly, and when closing the conversation, one party must convey a feeling of finalising the conversation. Upon the other receiving that feeling, and accepting it, the two close the conversation.

The present experiment is limited to cases in which both parties know each other, but because the interlocutor's readiness differs in cases $\mathrm{A}$ and $\mathrm{B}$, the development of the conversation is likely to differ accordingly. I have omitted cases when the other party is not an acquaintance because if the two parties are strangers in settings of apology and request, the realistic scenarios are more limited. Such settings include, for example, a person bumping against another on a street corner in the case of apology, or a person asking for an application form from a receptionist at a city office in the case of request. Such scenes might require only a short conversation; as the speakers would not need to develop the conversation elaborately, they were considered unsuitable for the present experiments.

The purpose of the present experiment is to compare and contrast authentic conversational data of two different competency level groups together with exemplars of Japanese language textbooks. I considered that the analysis of the conversational data did not have to be so precise as to measure the length of pauses, etc. as the order of utterances and their contents have greater relevance. The transcriptions of the video recorded role-plays are written in a mixture of kanji and kana characters (kanji-kana majiri bun) as used in modern standard Japanese. 


\subsubsection{Participants}

It was essential to obtain approval from the Human Ethics Committee of Victoria University of Wellington prior to starting the experiments, which granted towards the end of 2010. Some funding was provided to facilitate the research. The experiments started in May 2011, and the data collection was completed about one year later. Participants in this research were attracted through posters placed in the campuses of universities (for Japanese language learners) and English language schools in Wellington (for Japanese native speakers). In addition, advertisements were placed in free magazines for short-term Japanese visitors to New Zealand, such as working-holiday visa holders, because their ages are generally close to those of university students.

This study involved 80 native speakers of Japanese (NSs) present in New Zealand, and 80 university students at the intermediate level of Japanese language courses (NNSs) who had either never been to Japan or who had stayed for less than one year. There were 21 males and 59 females among the Japanese native speakers; and 36 male and 44 female students in the Japanese language courses. The Japanese native speakers consisted of university students, working holiday visa holders, and housewives, and their ages ranged between 18 and 41. The median age was 24. Students of Japanese were between 17 and 27 years, with a median age of 20 , and came from both Victoria University of Wellington and the University of Auckland. They had been studying the Japanese language for 5.2 years on average, including study at school and university. Fifty-nine students out of 80 had visited Japan at least once, having taken a trip with their secondary schools or a family trip, and had stayed for 62 days (an average of 80 NNSs) or 84 days in the case of excluding those who had not yet visited Japan. The first language of the NNSs were English (64), Chinese (9), Korean (4), and others (3). 


\subsubsection{Selection of the Japanese Language Textbooks}

The library of the Japan Foundation is the most extensive known depository of Japanese language textbooks, so I accessed that library in order to select the textbooks for the study. It is possible that the Japan Foundation Library did not hold all published textbooks, but their collection is considered as representative of those widely available. I assumed that there would be a number of model discourses of apology and request in Japanese textbooks, but once the conditions of the experiments, such as the year of publication, the settings of the senior-junior relationship of apology and the equal relationship of request were set, the number of relevant textbooks was reduced.

The reference book Nihongo kyōzai risuto [Guide to Japanese language teaching materials] shows that around 200 textbooks are on the market in Japan. ${ }^{98}$ Since there was a very wide range of teaching materials available as of 2011 , the criteria for the selection of textbooks for the present study were as follows:

(1) Textbooks published in Japan, commercially available, and held in the Library of the Japan Foundation, Saitama Prefecture, as of the end of 2011.

(2) Teaching materials that a university originally developed locally but had never been on the market were excluded.

(3) Textbooks published more than 25 years earlier were likewise excluded.

(4) The selected model exemplar(s) of discourse from the textbooks were scenarios of apology and/or request similar to those of the present research.

(4-1) The reason for the apology had to be lateness, in order to ensure maximum comparability with the role-play scenarios. The relationship between the two parties in textbooks was not, however, limited to a senior-junior relationship, for there were some cases

98 This includes not only comprehensive textbooks but also those for specific purposes, such as textbooks for the business environment, or for learners who are small children, and textbooks focusing on reading or conversation, and so forth. 
where the relationship was the same, such as both being work colleagues. Regardless of their relationship, the salient point was that the apologiser was late for an appointment. This situation was chosen because late arrival is an occurrence that relationship, the salient point was that the apologiser was late for an appointment. This situation was chosen because late arrival is an occurrence that can happen to anybody due to causes beyond one's own control and also due to one's own fault.

(4-2) The reason for the request in the role-plays had to be asking for some help with homework (assignment of writing an essay) from the other, since this is likely to be experienced at some time by many learners of a foreign language.

Under the above-mentioned conditions, the Japan Foundation Library collection contained only 12 model examples of 'apology' in 12 textbooks and 13 model examples of 'request' in 11 textbooks. The textbooks used for the experiments are listed in Appendices 4 and 5 .

\subsubsection{Some Limitations of the Experiments}

Ideally, it would have been most desirable for both NSs and NNSs to be similar in age, and for the gender balance of the subjects to be 50 per cent each. The higher the number of subjects the better, in particular because descriptive statistics were to be applied. However, the maximum number of subjects was set at around one hundred owing to time constraints on the transcription of all the discourses, as well as due to the time cost for the volunteer subjects. As a result, the data which I was able to use for the experiments amounted to 80 participants per group. 


\subsubsection{Limitations of the Participants}

\section{Gender differences}

There were relatively few male NS respondents aged in their early 20 s in NZ when the data were collected, so the number of male NSs was only one-third that of female NSs. Ideally the ratio of males to females overall should be the same, but this did not turn out to be practicable. This may have somewhat skewed the results, since there is a gender difference in vocabulary choice in the Japanese language, and this difference is easily recognised at the end of sentences. This kind of gender difference in utterance was probably under-represented in the results of the present experiments, perhaps because of the young ages of the subjects.

\section{Age difference}

The NSs were four years older than the NNSs on average. Despite this age difference between the two groups, I doubt that it affected the results of the present experiments.

\section{First language difference}

Among the $80 \mathrm{NNSs}$, the first language for the majority was English (64 NNSs) followed by nine with Chinese, four with Korean, and three others with Dutch, German, and Indonesian, respectively. It cannot be denied that the way of developing the discourses by the Chinese and Korean NNSs was likely influenced by their own cultural background, because of the similarity to Japanese culture compared with that of English. No obvious differences according to the first languages of the NNSs were noticed, however.

\section{Previous learning experience}

The NNSs had been studying Japanese 5.2 years on average before entering university, so those participants must have started studying Japanese at around 14 or 15 years of age. 
However, data were not collected regarding what kind of textbook(s) they used or what teaching method their teachers applied before they entered university. Unlike in Japan, there is no standardised school textbook in use in NZ. Thus, it is very difficult to grasp the influence of previous usage of Japanese textbooks on the NNSs. In addition, as many as 59 NNSs had spent time in Japan and their average period of stay was 84 days. Their utterances would have been influenced by their stay in Japan, but the influence of this was not measured in this experiments.

\subsubsection{Inferential Statistics}

The present study was analysed mostly through descriptive statistics, which displayed the data in actual numbers. This was because the number of sentences in the model exemplars in the textbooks was too small to use for inferential statistics, compared with the utterances of the participants who performed role-plays, even though as many model exemplars as possible in the Japan Foundation Library were collected.

Firstly, the three kinds of data, NSs, NNSs, and TXs, were calculated using inferential statistics to see the correlation among them. However, because of the small number of examples presented in the textbooks, the textbooks' data were excluded, and two data sets, NSs and NNSs, were calculated. Thus, for consistency for the purpose of the present study, descriptive statistics were employed in order to clarify the similarities and differences among the three groups. Analyses by descriptive statistics were presented in Chapter 4 (Apology) and Chapter 5 (Request).

The following section is to demonstrate the outputs of inferential statistics of the two settings: apology and request. These are (1) Apology by CCSARP, (2) Apology by Politeness, (3) Request by CCSARP, and (4) Request by Politeness. 


\section{(1) Apology (CCSARP: HA/SM)}

There was insufficient evidence to reject the null hypothesis of no difference between the three groups $\left(\chi^{2}(2)=5.295, \mathrm{p}=0.071\right)$, but there was a trend toward statistical significance. (See Table 3.3).

Table 3.2 HA/SM group Cross-tabulation (Apology: 3 groups)

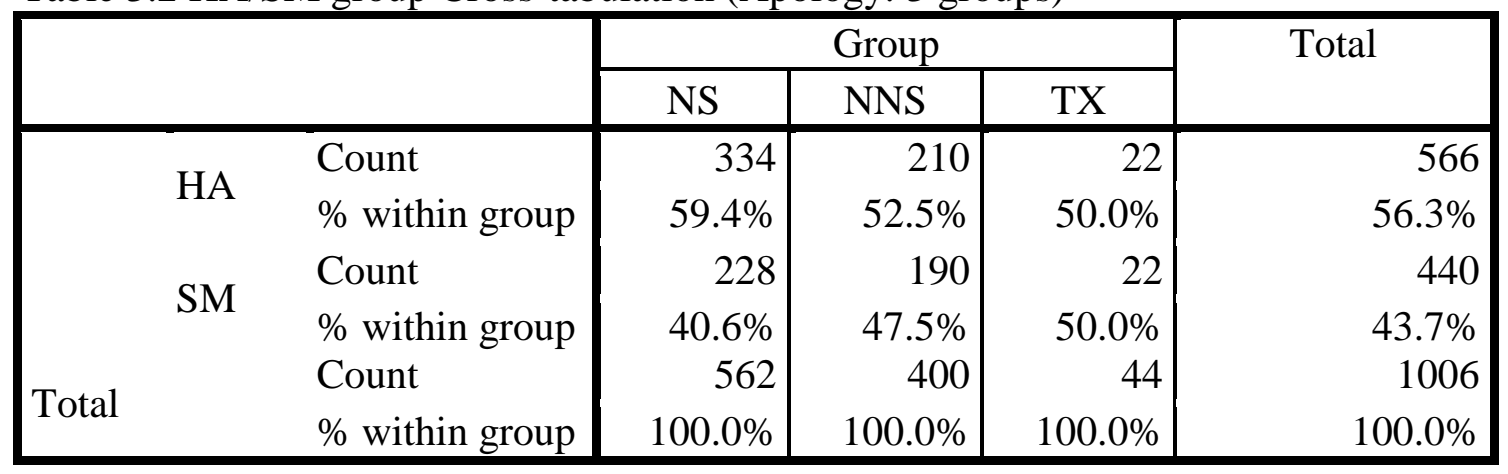

Table 3.3 HA/SM Chi-Square Tests (Apology: 3 groups)

\begin{tabular}{|l|r|r|r|}
\hline & \multicolumn{1}{|c|}{ Value } & \multicolumn{1}{c|}{ df } & Asymp. Sig. (2-sided) \\
\hline Pearson Chi-Square & $5.295^{\mathrm{a}}$ & 2 & .071 \\
Likelihood Ratio & 5.291 & 2 & .071 \\
Linear-by-Linear & 5.058 & & 1 \\
Association & 1006 & & .025 \\
N of Valid Cases & & \\
\hline
\end{tabular}

a. 0 cells $(0.0 \%)$ have expected count less than 5 . The minimum expected count is 19.24.

Removing the TX data because of its small group counts (number of sentences), the data for the two groups, NSs and NNSs, show that there was a statistically significant difference in the proportion of Head Acts and Supportive Moves $\left(\chi^{2}(1)=4.568, p=0.035\right.$ (see Table 3.5). This means that the HA/SM utterances for apology are significantly different between the NSs and NNSs group. 
Table 3.4 HA/SM group Cross-tabulation (Apology: 2 groups)

\begin{tabular}{|c|c|c|c|c|c|}
\hline & \multicolumn{2}{|c|}{ Group } & \multirow[t]{2}{*}{ Total } \\
\hline & & & NS & NNS & \\
\hline & \multirow[b]{2}{*}{ HA } & Count & 334 & 210 & 544 \\
\hline & & $\begin{array}{l}\% \text { within } \\
\text { group }\end{array}$ & $59.4 \%$ & $52.5 \%$ & $56.5 \%$ \\
\hline & \multirow[b]{2}{*}{$\mathrm{SM}$} & Count & 228 & 190 & 418 \\
\hline & & $\begin{array}{l}\% \text { within } \\
\text { group }\end{array}$ & $40.6 \%$ & $47.5 \%$ & $43.5 \%$ \\
\hline \multirow{2}{*}{\multicolumn{2}{|c|}{ Total }} & Count & 562 & 400 & 962 \\
\hline & & $\begin{array}{l}\% \text { within } \\
\text { group }\end{array}$ & $100.0 \%$ & $100.0 \%$ & $100.0 \%$ \\
\hline
\end{tabular}

Table 3.5 HA/SM Chi-0Square Tests (Apology: 2 groups)

\begin{tabular}{|c|c|c|c|c|c|}
\hline & Value & $\mathrm{df}$ & $\begin{array}{l}\text { Asymp. Sig. } \\
\text { (2-sided) }\end{array}$ & $\begin{array}{l}\text { Exact Sig. } \\
\text { (2-sided) }\end{array}$ & $\begin{array}{l}\text { Exact Sig. } \\
\text { (1-sided) }\end{array}$ \\
\hline Pearson Chi-Square & $4.568^{\mathrm{a}}$ & 1 & .033 & & \\
\hline Continuity Correction $^{\mathrm{b}}$ & 4.290 & 1 & .038 & & \\
\hline Likelihood Ratio & 4.563 & 1 & .033 & & \\
\hline Fisher's Exact Test & & & & .035 & .019 \\
\hline $\begin{array}{l}\text { Linear-by-Linear } \\
\text { Association }\end{array}$ & 4.563 & 1 & .033 & & \\
\hline $\mathrm{N}$ of Valid Cases & 962 & & & & \\
\hline
\end{tabular}

a. 0 cells $(0.0 \%)$ have expected count less than 5. The minimum expected count is 173.80 .

b. Computed only for a $2 \times 2$ table

\section{(2) Apology (Politeness: Positive/Negative)}

There was insufficient evidence to reject the null hypothesis of no difference between the three groups $\left(\chi^{2}(2)=2.308, p=0.315\right.$, see Table 3.7).

Table 3.6 Politeness group Cross-tabulation (Apology: 3 groups)

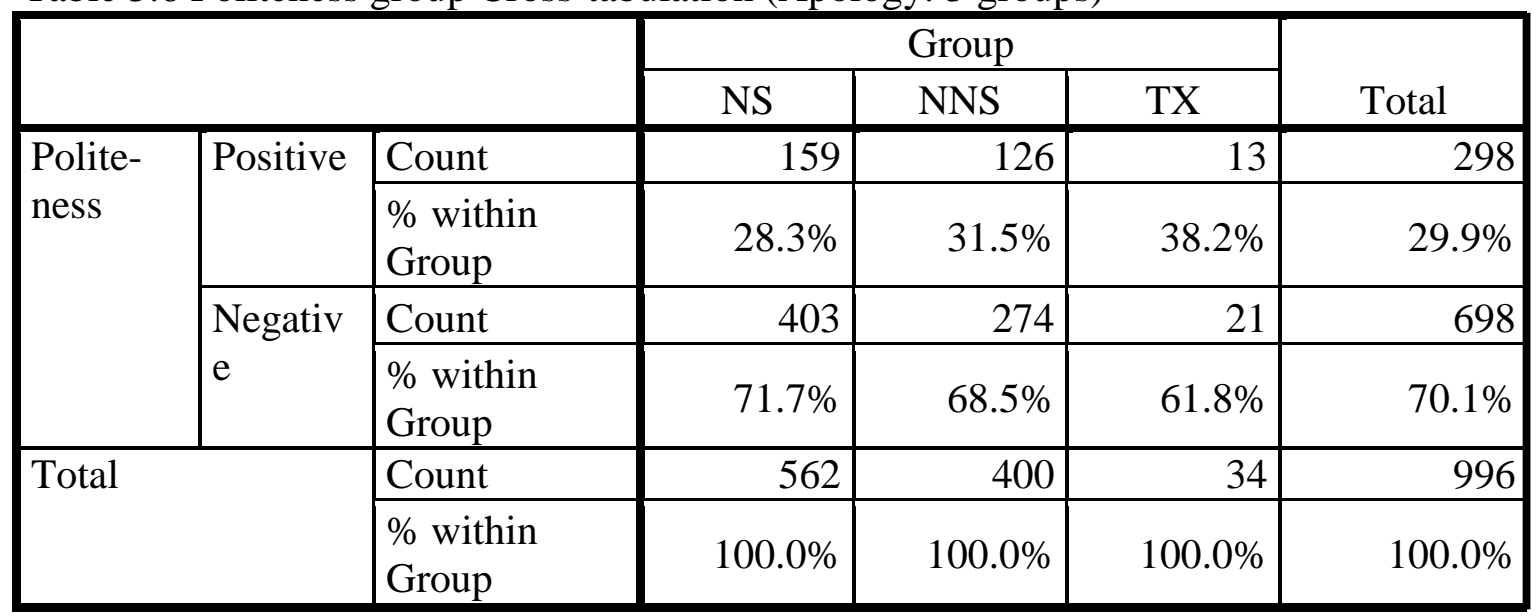


Table 3.7 Politeness group Chi-Square Tests (Apology: 3 groups)

\begin{tabular}{|l|r|r|r|}
\hline \multicolumn{1}{|c|}{ Value } & $\mathrm{df}$ & Asymp. Sig. (2-sided) \\
\hline Pearson Chi-Square & $2.308^{\mathrm{a}}$ & 2 & .315 \\
\hline Likelihood Ratio & 2.261 & 2 & .323 \\
\hline Linear-by-Linear Association & 2.162 & 1 & .141 \\
\hline N of Valid Cases & 996 & & \\
\hline
\end{tabular}

a. 0 cells $(.0 \%)$ have expected count less than 5 . The minimum expected count is 10.17 .

Again the TX data were removed because of small group counts. For the other two groups (NSs and NNSs), there was insufficient evidence to reject the null hypothesis of no difference between the two groups, NSs and NNSs $\left(\chi^{2}(1)=0.283, p=0.284\right.$, see Table 3.9).

Table 3.8 Politeness group Cross-tabulation (Apology: 2 groups)

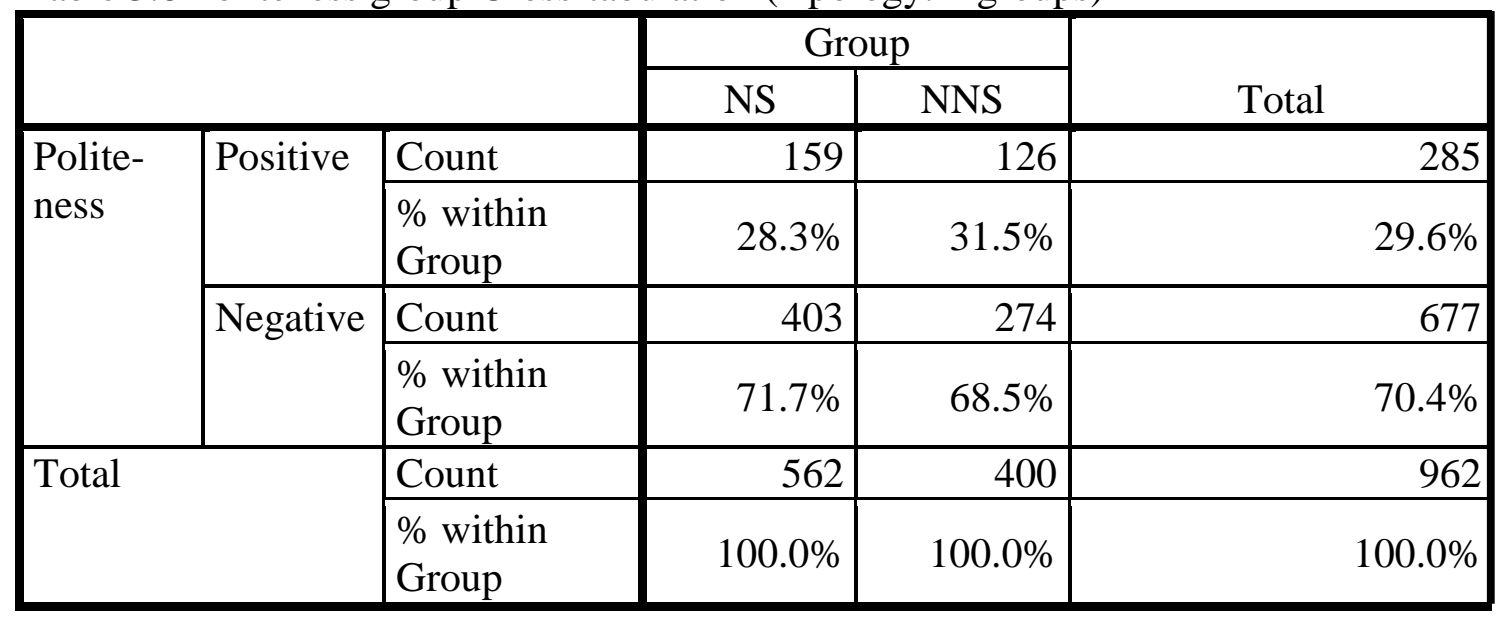

Table 3.9 Politeness group Chi-Square Tests (Apology: 2 groups)

\begin{tabular}{|c|c|c|c|c|c|}
\hline & Value & $\mathrm{df}$ & $\begin{array}{c}\text { Asymp. } \\
\text { Sig. (2- } \\
\text { sided) }\end{array}$ & $\begin{array}{l}\text { Exact Sig. } \\
\text { (2-sided) }\end{array}$ & $\begin{array}{l}\text { Exact Sig. } \\
\text { (1-sided) }\end{array}$ \\
\hline Pearson Chi-Square & $1.154^{\mathrm{a}}$ & 1 & .283 & & \\
\hline Continuity Correction $^{b}$ & 1.005 & 1 & .316 & & \\
\hline Likelihood Ratio & 1.150 & 1 & .284 & & \\
\hline Fisher's Exact Test & & & & .284 & .158 \\
\hline $\begin{array}{l}\text { Linear-by-Linear } \\
\text { Association }\end{array}$ & 1.152 & 1 & .283 & & \\
\hline $\mathrm{N}$ of Valid Cases & 962 & & & & \\
\hline
\end{tabular}

a. 0 cells $(.0 \%)$ have expected count less than 5 . The minimum expected count is 118.50 .

b. Computed only for a $2 \times 2$ table 


\section{(3) Request (CCSARP: HA/SM)}

There was insufficient evidence to reject the null hypothesis of no difference between the three groups $\left(\chi^{2}(2)=5.099, \mathrm{p}=0.078\right.$, see Table 3.11).

Table 3.10 HA/SM group Cross-tabulation (Request: 3 groups)

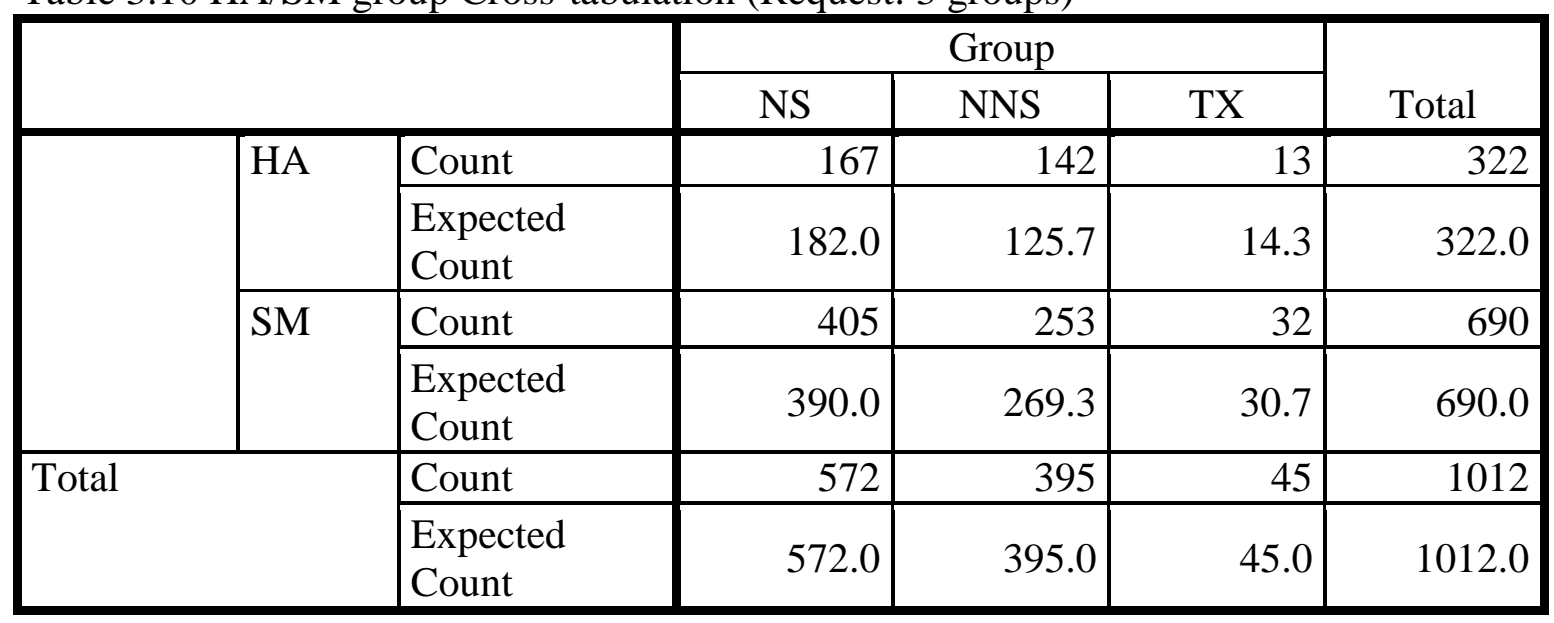

Table $3.11 \mathrm{HA} / \mathrm{SM}$ group Chi-Square Tests (Request: 3 groups)

\begin{tabular}{|c|c|c|c|}
\hline & Value & $\mathrm{df}$ & Asymp. Sig. (2-sided) \\
\hline Pearson Chi-Square & $5.099^{\mathrm{a}}$ & 2 & .078 \\
\hline Likelihood Ratio & 5.064 & 2 & .079 \\
\hline $\begin{array}{l}\text { Linear-by-Linear } \\
\text { Association }\end{array}$ & 2.516 & 1 & .113 \\
\hline $\mathrm{N}$ of Valid Cases & 1012 & & \\
\hline
\end{tabular}

a. 0 cells $(.0 \%)$ have expected count less than 5 . The minimum expected count is 14.32 .

Again the TX data were removed because of small group counts. There was a statistically significant difference in the proportion of Head Acts and Supportive Moves between the two groups, NSs and NNSs $\left(\chi^{2}(1)=4.901, p=0.030\right.$, see Table 3.13). This means that the HA/SM utterances for request are significantly different between the NSs and the NNSs groups. 
Table 3.12 HA/SM group Cross-tabulation (Request: 2 groups)

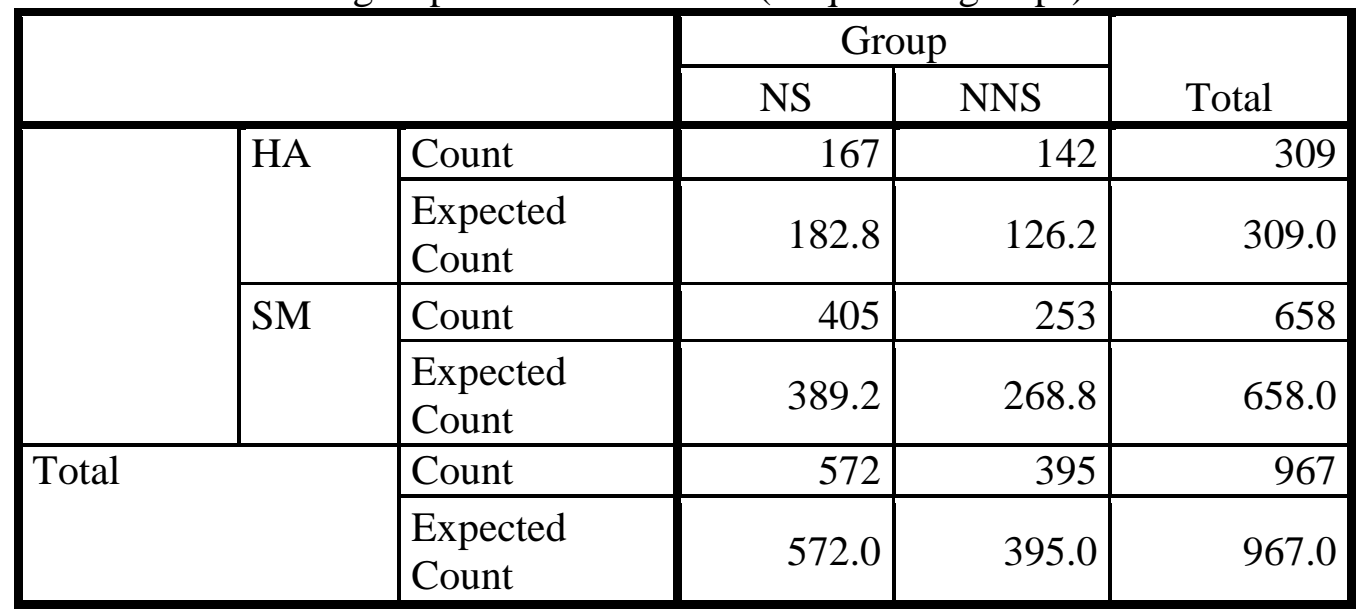

Table 3.13 Chi-Square Tests (Request: 2 groups)

\begin{tabular}{|c|c|c|c|c|}
\hline & Value & df & $\begin{array}{l}\text { Asymp. Sig. } \\
\text { (2-sided) }\end{array}$ & $\begin{array}{l}\text { Exact Sig. (2- } \\
\text { sided) }\end{array}$ \\
\hline Pearson Chi-Square & $4.901^{\mathrm{a}}$ & 1 & .027 & \\
\hline Continuity Correction $^{\mathrm{b}}$ & 4.596 & 1 & .032 & \\
\hline Likelihood Ratio & 4.875 & 1 & .027 & \\
\hline Fisher's Exact Test & & & & .030 \\
\hline $\begin{array}{l}\text { Linear-by-Linear } \\
\text { Association }\end{array}$ & 4.896 & 1 & .027 & \\
\hline $\mathrm{N}$ of Valid Cases & 967 & & & \\
\hline
\end{tabular}

a. 0 cells $(.0 \%)$ have expected count less than 5 . The minimum expected count is 126.22 .

b. Computed only for a $2 \times 2$ table

\section{(3) Request (Politeness: Bald on record, Positive, Negative, Off record)}

There was sufficient evidence to reject the null hypothesis of no difference between the three groups. The politeness strategies for requests were significantly different between the NSs and the NNSs groups $\left(\chi^{2}(6)=62.697, \mathrm{p}<0.000\right.$, see Table 3.15). 
Table 3.14 Politeness group Cross-tabulation (Request: 3 groups)

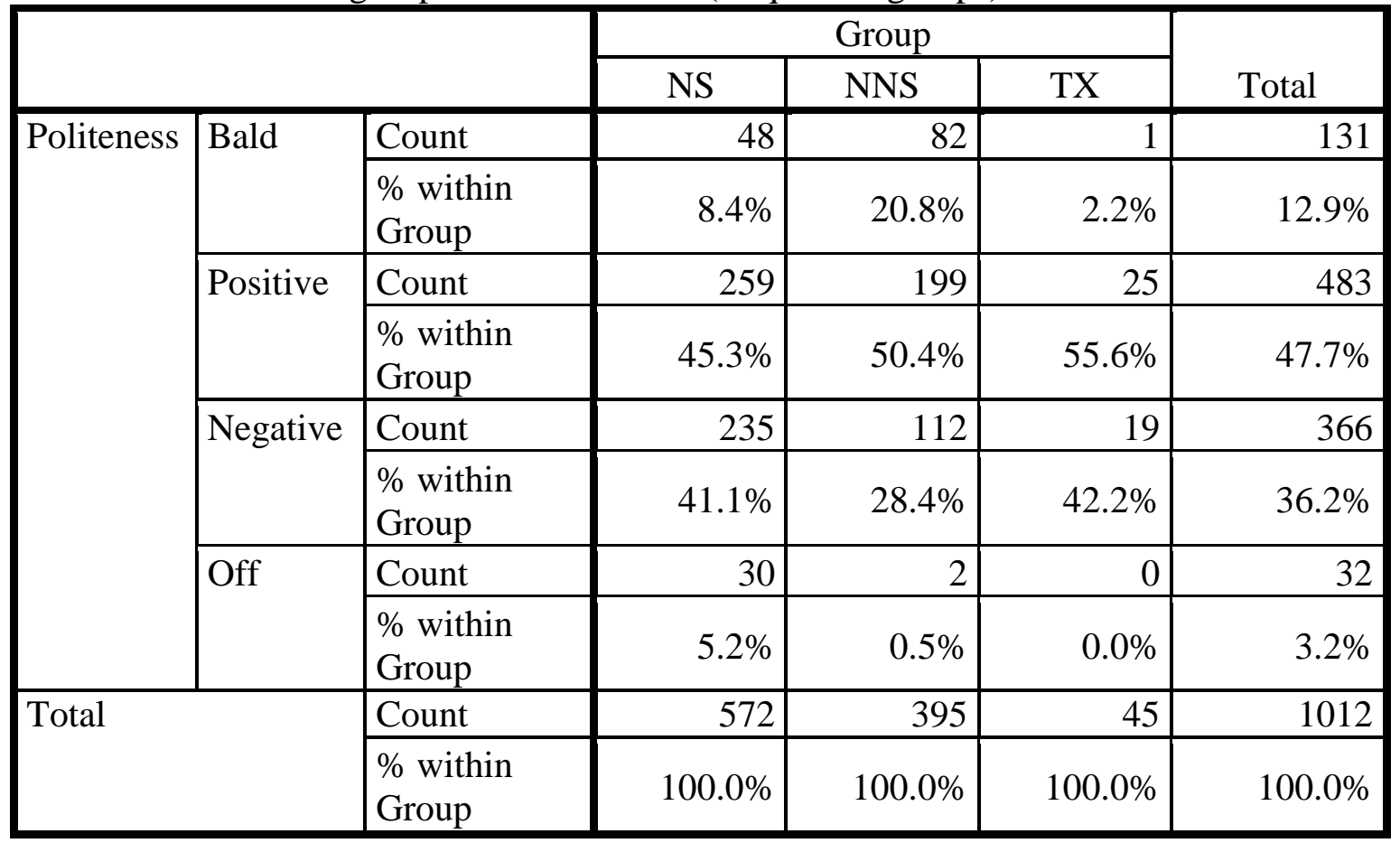

Table 3.15 Politeness Chi-Square Tests (Request: 3 groups)

\begin{tabular}{|l|r|r|r|}
\hline & Value & df & Asymp. Sig. (2-sided) \\
\hline Pearson Chi-Square & $62.697^{\mathrm{a}}$ & 6 & .000 \\
\hline Likelihood Ratio & 68.509 & 6 & .000 \\
\hline Linear-by-Linear Association & 29.544 & 1 & .000 \\
\hline N of Valid Cases & 1012 & & \\
\hline
\end{tabular}

a. 1 cells $(8.3 \%)$ have expected count less than 5 . The minimum expected count is 1.42 .

There was sufficient evidence to reject the null hypothesis of no difference between the two groups. This evidence was statistically significant $\left(\chi^{2}(3)=54.272, p<0.000\right.$, see Table 3.17). 
Table 3.16 Politeness group Cross-tabulation (Request: 2 groups)

\begin{tabular}{|c|c|c|c|c|c|}
\hline & & & Gro & & \\
\hline & & & $\mathrm{NS}$ & NNS & Total \\
\hline Politeness & Bald & Count & 48 & 82 & 130 \\
\hline & & $\begin{array}{l}\% \text { within } \\
\text { Group }\end{array}$ & $8.4 \%$ & $20.8 \%$ & $13.4 \%$ \\
\hline & Positive & Count & 259 & 199 & 458 \\
\hline & & $\begin{array}{l}\% \text { within } \\
\text { Group }\end{array}$ & $45.3 \%$ & $50.4 \%$ & $47.4 \%$ \\
\hline & Negative & Count & 235 & 112 & 347 \\
\hline & & $\begin{array}{l}\% \text { within } \\
\text { Group }\end{array}$ & $41.1 \%$ & $28.4 \%$ & $35.9 \%$ \\
\hline & Off & Count & 30 & 2 & 32 \\
\hline & & $\begin{array}{l}\% \text { within } \\
\text { Group }\end{array}$ & $5.2 \%$ & $0.5 \%$ & $3.3 \%$ \\
\hline Total & & Count & 572 & 395 & 967 \\
\hline & & $\begin{array}{l}\% \text { within } \\
\text { Group }\end{array}$ & $100.0 \%$ & $100.0 \%$ & $100.0 \%$ \\
\hline
\end{tabular}

Table 3.17 Politeness Chi-Square Tests (Request: 2 groups)

\begin{tabular}{|l|r|r|r|}
\hline & \multicolumn{1}{|c|}{ Value } & \multicolumn{1}{|c|}{ df } & Asymp. Sig. (2-sided) \\
\hline Pearson Chi-Square & $54.272^{\mathrm{a}}$ & 3 & .000 \\
\hline Likelihood Ratio & 58.258 & 3 & .000 \\
\hline $\begin{array}{l}\text { Linear-by-Linear } \\
\text { Association }\end{array}$ & 51.554 & 1 & .000 \\
\hline N of Valid Cases & 967 & & \\
\hline
\end{tabular}

a. 0 cells $(.0 \%)$ have expected count less than 5. The minimum expected count is 13.07.

Above I have elucidated the methodology of the present study, including reasons for using a combination of two frameworks for my analysis. I have also illustrated the profiles of the subjects of the experiments, criteria for selecting which textbooks would be used, and the reason for the small number of model exemplars in the selected textbooks. In addtion, the outputs of inferential statistics were included to justify using descriptive statistics. In the next two chapters, I will report on the experiments, and discuss my findings concerning speech acts of apologies in Chapter 4, followed by requests in Chapter 5. 


\section{CHAPTER 4 APOLOGY}

\subsection{Overview of Apology}

In Chapter 4, I will compare and contrast the collected data of the discourses of apologies of the three individual groups in order to discover the similarities and differences. The discourse setting of the two parties is a senior-junior relationship. The numerical data of the individual groups are displayed in graphs. I will then discuss the results of the analyses.

Analyses of the present study were carried out based on two types of conversational data, with one being the discourse transcription of spontaneous role-plays performed by participants who comprised a pair of the same language. There were 40 pairs in each group of native Japanese speakers (NSs) and non-native Japanese speakers (NNSs). The other consisted of 12 exemplars presented in the selected 12 Japanese language textbooks (TXs) which were published between 1990 and 2008 in Japan.

In the setting of the role-plays, one player who was a young part-time worker had come late to the workplace, a café, and apologised to the other player, who played the café owner who was aged 40 years (see Appendices 1 and 2). The model exemplars presented in the TXs were similar in concept to the role-plays: for example, an apologiser, such as a student or an office worker, apologises to a superior who is far apart in terms of social seniority, i.e. the personae in discourse exemplars were teachers in eight scenarios and managers or seniors in their workplaces in four scenarios.

Five hundred and sixty-two utterances by NSs, 400 utterances by NNSs, and 44 exemplars of discourses presented in the Japanese language textbooks were collected for apology analysis. The Japanese native speakers in the role-plays spoke almost twice the number of utterances compared to those in the TXs: the average number of utterances per 
discourse was 7.0 for the NSs, 5.0 for the NNS, and 3.7 in the TXs. The average length of a discourse was 43 seconds for the NS pair and 53 seconds for that of the NNSs. It is apparent from this that the NSs took 3.1 seconds per sentence and the NNSs took 5.3 seconds; the latter taking around 70 per cent longer than the NSs to utter a sentence.
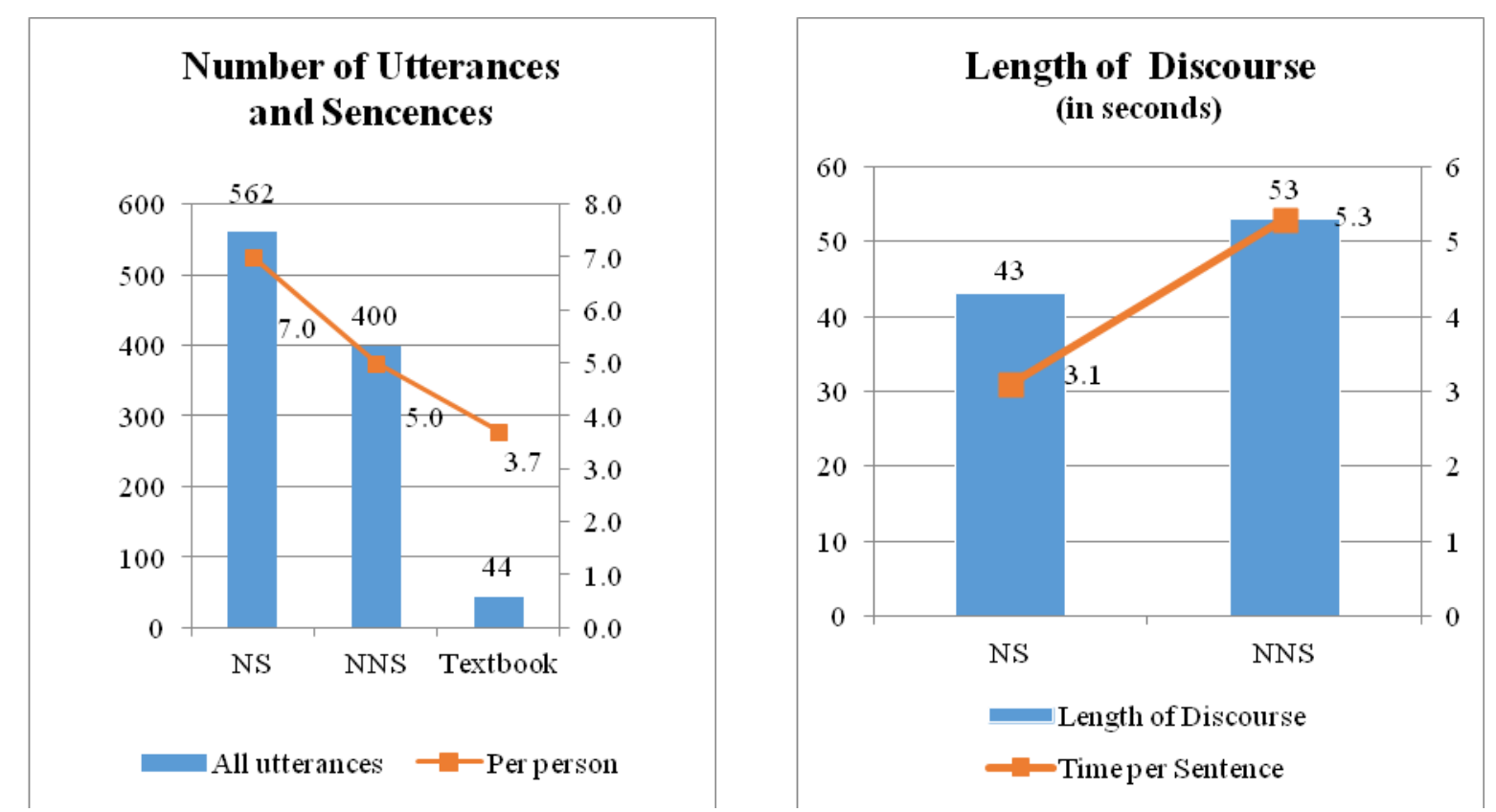

Figure 4.1 Number of Utterances and Sentences

\subsubsection{Categorisation of Apologetic Utterances Based on the CCSARP}

The CCSARP places the discourse components of apology in five main categories: (1) Illocutionary Force Indicating Devices (IFIDs), including expressions marked for register, such as I apologise..., (2) Taking on Responsibility, (3) Explanation or Account, (4) Offer of Repair, and (5) Promise of Forbearance. ${ }^{99}$ In the present research, owing to the particular characteristics of utterances in Japanese that are not described in the CCSARP Coding

99 The CCSARP Coding Manual in Cross-Cultural Pragmatics (1989: 289-94). 
Manual, the categorisation has been modified by adding other standpoints. ${ }^{100}$ These are Admission of the Facts ${ }^{101}$ and Placation. In addition, the items of classification have been modified, and a subcategory under Placation has been created. In a nutshell, the categorisation of the present research is as follows.

\section{Head Act(s)}

(1) Illocutionary Force Indicating Devices, such as sumimasen [I'm sorry]

\section{Supportive Move(s)}

(2) Explanation, such as Nebō shimashita [I overslept],

(3) Admission of the Facts, such as Okuremashita [I was late],

(4) Promise, such as Kondo hayaku nemasu [I'll go to bed early next time],

(5) Placation

- Pledge, such as Ganbarimasu [I'll do my best],

- Repair, such as Kyūyo katto shite kudasai [Please dock my pay],

- Responsibility, such as Watashi no sekinin desu [It's my responsibility],

- Beseeching, such as Kubi ni shinaide kudasai [Please do not sack me].

100 Hou (2005:80-1) defines that there are expressions recognised as apology semantically in Japanese, such as sumimasen [I'm sorry], gomen [pardon me], mōshiwake nai [inexcusable], etc. These are clearly expressions of apology even without the context. On the other hand, sentences such as Kongo ki o tsukemasu [I'll be more careful from now on], Benshō shimasu [I'll compensate for it], Sekinin o torimasu [I'll take responsibility] may not be recognised as apology if there is no appropriate context.

101 "Admission of the Facts" is included as a subcategory (lower category) of "taking on responsibility" in the CCSARP, defined as: "The speaker does not deny his or her involvement in the offensive act but abstains from openly accepting responsibility" (292). In this experiment, however, over 80 per cent of participants of both groups articulated Okuremashita [I was late] after or before uttering an apology. It is understood that the implication of this Japanese sentence is that the speaker explicitly admits to having been late and also implicitly accepts it was his/her responsibility. Therefore, unlike the CCSARP, "admission of facts" was treated as a distinct category rather than as a subcategory of "taking on responsibility". 
In analysing the data of apology, IFID are the primal expressions according to the CCSARP Coding Manual, which gives such examples as, sorry, excuse me, I apologise, forgive me, pardon me for..., and so forth (290). Although the CCSARP does not name the IFIDs as Head Acts, ${ }^{102}$ for the purposes of this present experiment they are defined as Head Act(s) in settings of apology, and other components of the discourse are regarded as Supportive Moves (SMs), as in the case of request. ${ }^{103}$

\subsection{Analysis of Apology}

\subsubsection{Outline of Strategies of Apology}

The Head Acts (HAs) and the Supportive Moves (SMs) of apology ${ }^{104}$ of Japanese native speakers (NSs), those of non-native speakers (NNSs), and the personae in the model dialogues in textbooks (TXs) were compared by extracting them from the components of their discourses. The items analysed were:

(1) The total number of utterances and utterances per person/persona

(2) The proportion of utterances of the three groups (NSs, NNSs, and TXs)

(3) Structure of discourse

102 The CCSARP Coding Manual describes Illocutionary Force Indicating Devices as they are, and does not go so far as to name them as the Head Acts.

103 The analysis of request will follow in Chapter 5.

104 The Head Acts and the Supportive Moves are deemed to be strategies which were taken by speakers. 


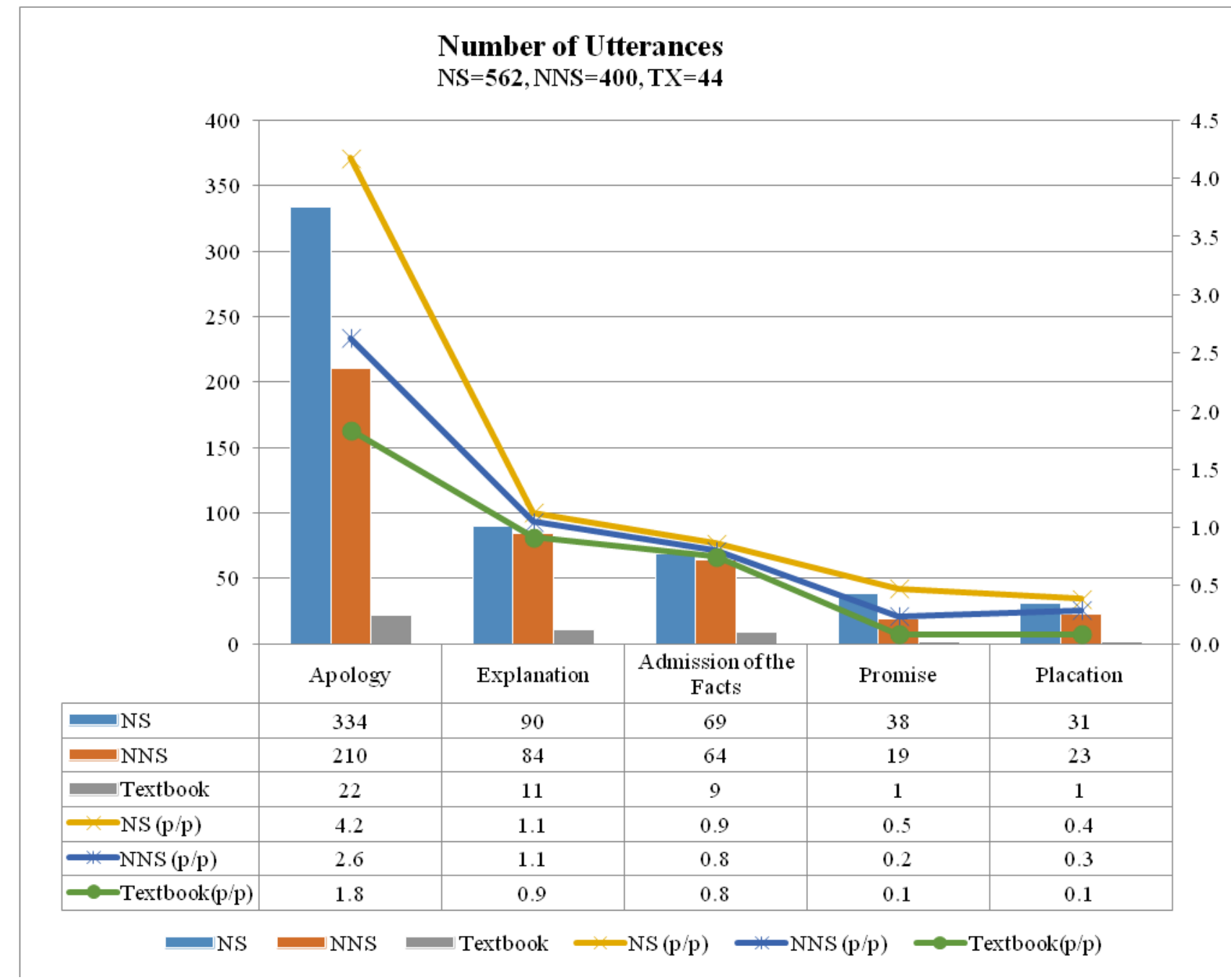

Figure 4.3 Number of Utterances
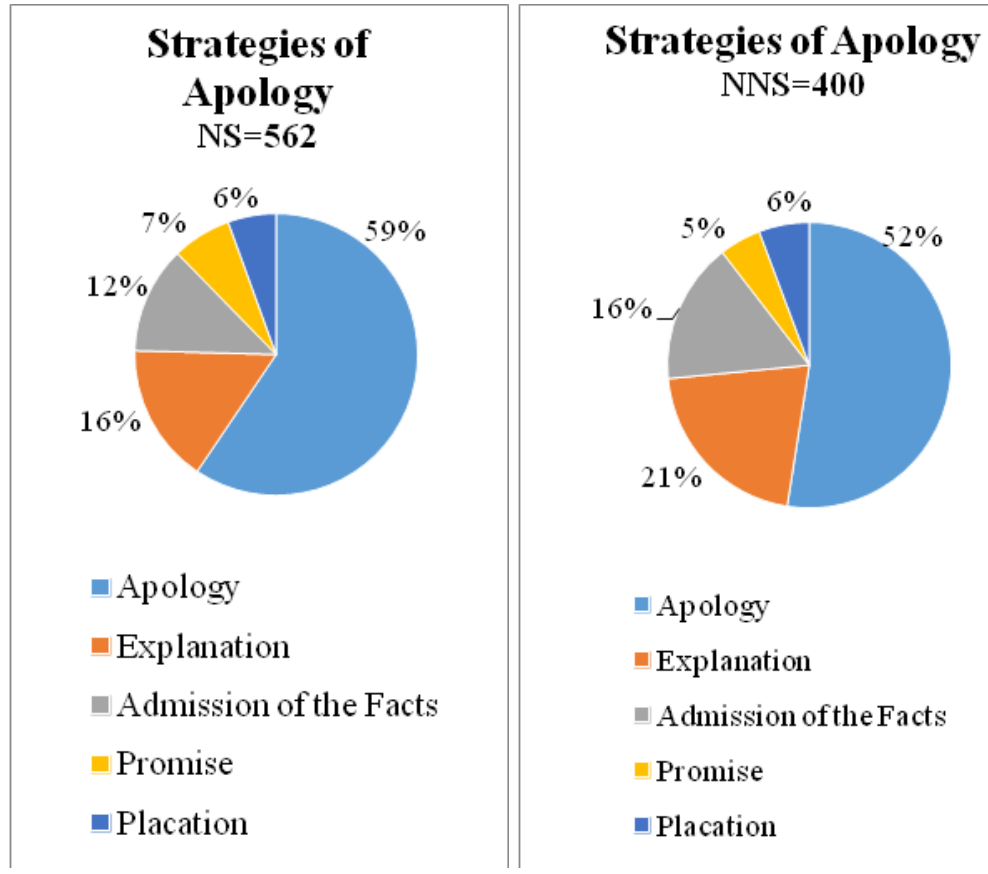
Strategies of Apology
Textbook $=44$

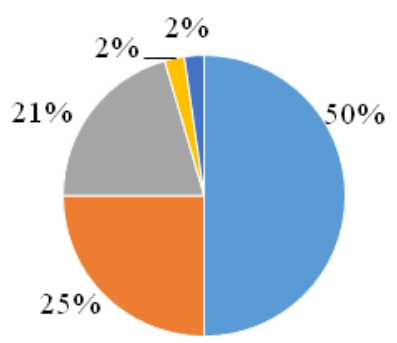

Ap ology

- Explanation

Admission of the Facts

Promise

- Placation

Figure 4.4 Proportion by Strategy 
The number of HAs (apologetic expressions) occupied more than half the total utterances of the NSs (59\%) and the NNSs (52\%), and just half of those of the TXs (50\%). The number of the two main supportive moves - explanations for being late and admission of the facts (of being late) - followed that of the apologetic expressions. The proportion of the two supportive moves of the NSs in relation to their apologetic expressions was only 48 per cent, but in the case of the NNSs, it was 71 per cent, and 92 per cent for the TXs. ${ }^{105}$ This means that among the NSs, the NNSs, and the TXs, the NSs prioritised the SMs least: by contrast, the authors of the TXs handled the SMs and the apologetic expressions rather equally compared with the NSs and the NNSs. The NSs, in general, focused on apologetic utterances more weightily than the others.

The explanation for being late was uttered more than once per participant, and in the case of the TXs, almost all in the model exemplars. The admission of the fact refers to the presence of a clear statement of the speaker's lateness to the interlocutor, in this case the café owner. This occurred less frequently than an explanation, a little less than 1.0 time per discourse by the participants and personae of the TXs. The conspicuous characteristic of the model dialogues is that there are few appearances of promise and placation in comparison with those of the NSs and the NNSs.

In the real conversations from the impromptu role-plays, especially those of the NSs, the apology was far more frequent than explanation or admission of the facts, since the proportion of the former was more than double the two SMs. The NNSs were between the NSs and the TXs. The apologisers among the NSs appear to have concentrated on the act of sincerely seeking forgiveness when performing their role-plays, while the TXs displayed a contrary trend. It may be inferred that this is probably because the textbook authors aimed

105 The formulae for these are $(16+12) / 59=48 \%$ (NSs); $(21+16) / 52=71 \%$ (NNSs); $(25+21) / 50=92 \%$ (TXs). 
primarily at introducing apologetic expressions, which they considered the main segments of the discourse of apology, to the readers of the TXs (non-native learners). These findings demonstrate that the model exemplars do not always accurately portray the natural discourse of NSs.

\subsubsection{Head Acts of Apology}

The HAs of the NSs were compared with those of both the NNSs and the TXs by extracting them from the individual discourse.

\subsubsection{Number of Head Acts}

Explicit and intelligible apologetic expressions were pronounced by all performers as apologisers except one NS. ${ }^{106}$ The total number of apologies was 334 utterances by the NSs, 210 by the NNSs, but only 22 in the TXs, which means that on average there were 4.2 apologies per discourse uttered by the NSs, 2.6 by the NNSs, and 1.8 in the TXs. This indicates that the NSs apologised 1.6 times and 2.3 times more frequently than the NNSs and the TXs respectively.

The HAs of the NSs averaged 4.2 utterances per person per discourse, and those of the NSs and the TXs were respectively 2.6 and 1.8. This indicates that the NSs apologised more than four times repeatedly in a discourse, while the NNSs did so at least twice, but the exemplars in the TXs did so less than twice on average. In the utterances of apology by the NSs and the NNSs they either repeated the same phrase or used different phrases. It might be surmised that the TXs produced the smallest number because of the limitations of page space,

106 The one NS who did not utter any apologetic sentence admitted the fact of his delay by saying Osoku narimashita [I was late], followed by the reason for being late, which was that the train had not arrived on time. 
and perhaps the authors might have deliberately avoided the repetition of similar or the same phrases.

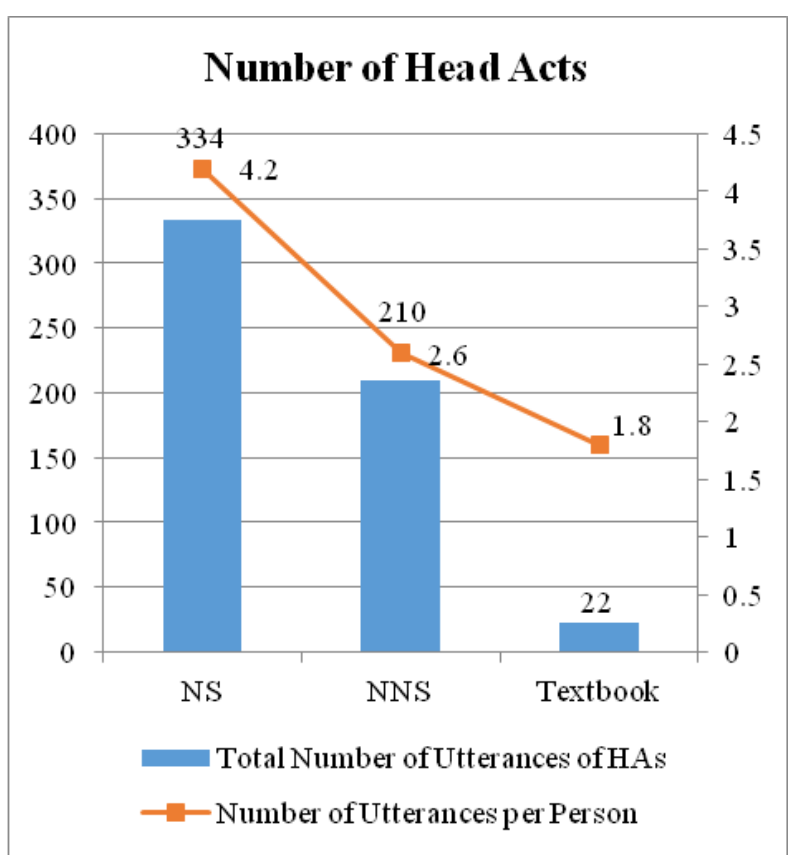

Figure 4.5 Number of Head Acts by Group

\subsubsection{Expressions of Apology}

In the setting of apology (during the role-plays) where the apologiser clearly admitted his/her offence to the interlocutor, the apology was plainly expressed without faltering. Sumimasen was the most utilised expression, used by 82 per cent of the NSs, by 66 per cent of the NNSs, and 73 per cent of the TXs. In other words, the NSs uttered sumimasen 3.4 times when apologising, the NNSs 1.7 times and the TXs 1.3 times. ${ }^{107}$ This implies that the NSs made use of sumimasen twice as often as the NNSs and 2.6 times as frequently as the TXs. In contrast to sumimasen, gomen nasai was favoured more by the NNSs, with 27 per cent using it, than by the NSs, of whom only 10 per cent used it. In extreme contrast, none of the TXs used gomen nasai.

107 The formulae for these are $334 \times 0.82 / 80=3.4$ (NSs); $210 \times 0.66 / 80=1.7$ (NNSs); $22 \times 0.73 / 12=1.3$ (TXs). 
Mōshiwake nai (or arimasen) was used by 7 per cent of both the NSs and the NNSs, but by 27 per cent of the TXs, which is almost four times more than the NSs and the NNSs. In addition to these, there were other expressions of apology uttered only by the NSs, such as Gomeiwaku o okake shimashita [I put you to trouble], and so on.

Among the three most frequent apologetic expressions, mōshiwake nai (or arimasen) sounds more formal and official, and gomen nasai is more casual than the other two. From the viewpoint of grammar, gomen nasai (being a command form: 'forgive me') does not have a past tense, unlike the others. As seen earlier (see Ch2, Sec. 4), the past tense ending in the ta-form contains the speaker's appreciation of his/her benefit, whereas the degree of appreciation of the speaker who uses the present tense is less (Kindaichi 1987: 76-7).

As Kindaichi claims, the range of uses of gomen nasai is more limited than the other two; it is preferable not to use it to a superior. For example, it is hard to imagine that a small child would apologise by saying sumimasen or mōshiwake arimasen. Instead, they are expected to say gomen nasai when they have done something wrong. On the other hand, a superior may say gomen [I am sorry] to a junior rather than sumimasen or mōshiwake arimasen. This means that particular items of vocabulary are limited to certain users. Although the function of these three apologetic expressions is the same, it can be said that the appropriate vocabulary when making an apology is to some extent determined based on who the apologiser is and the relationship of the apologiser to the hearer. 


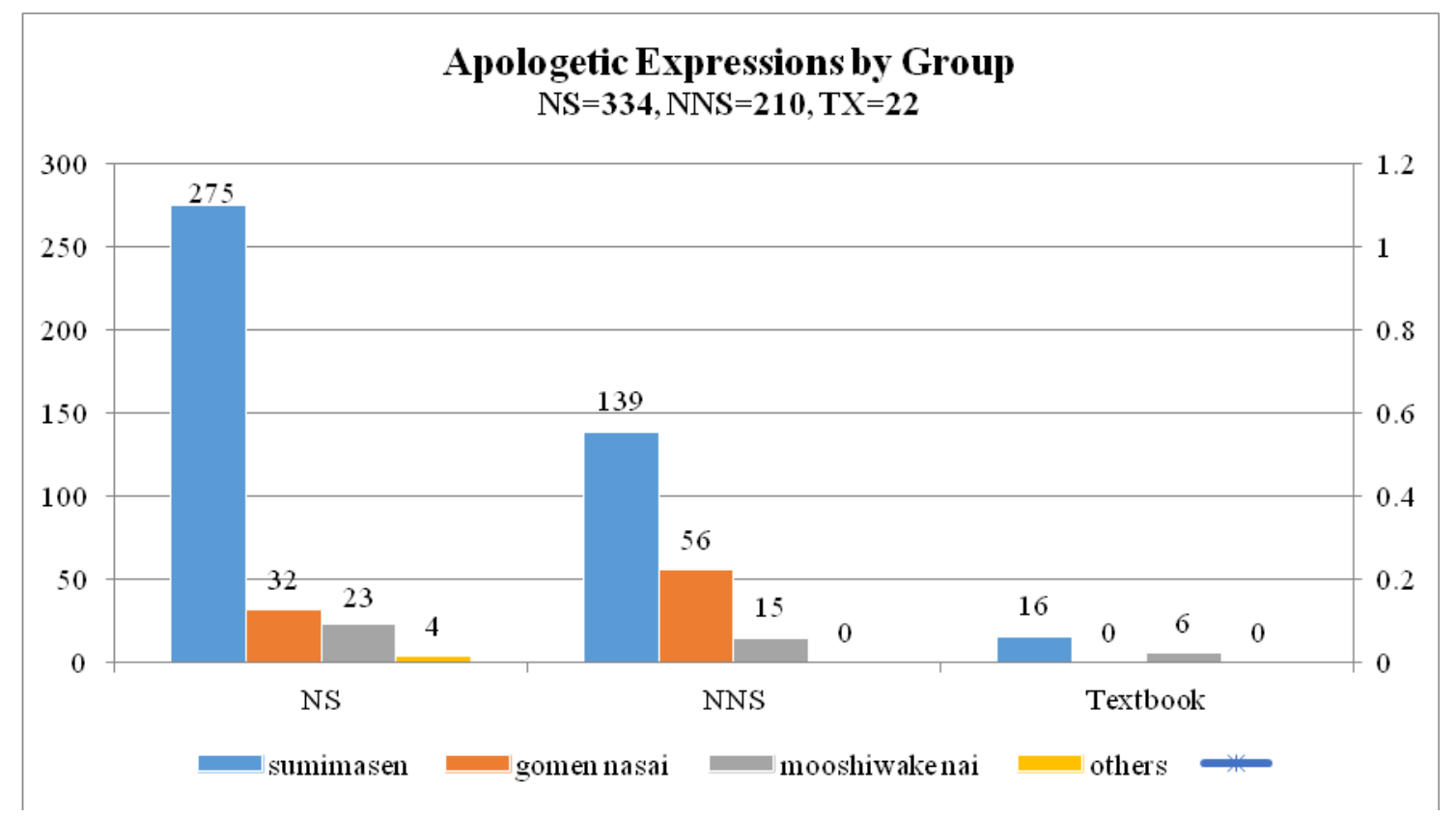

Figure 4.6 Apologetic Expressions by Group

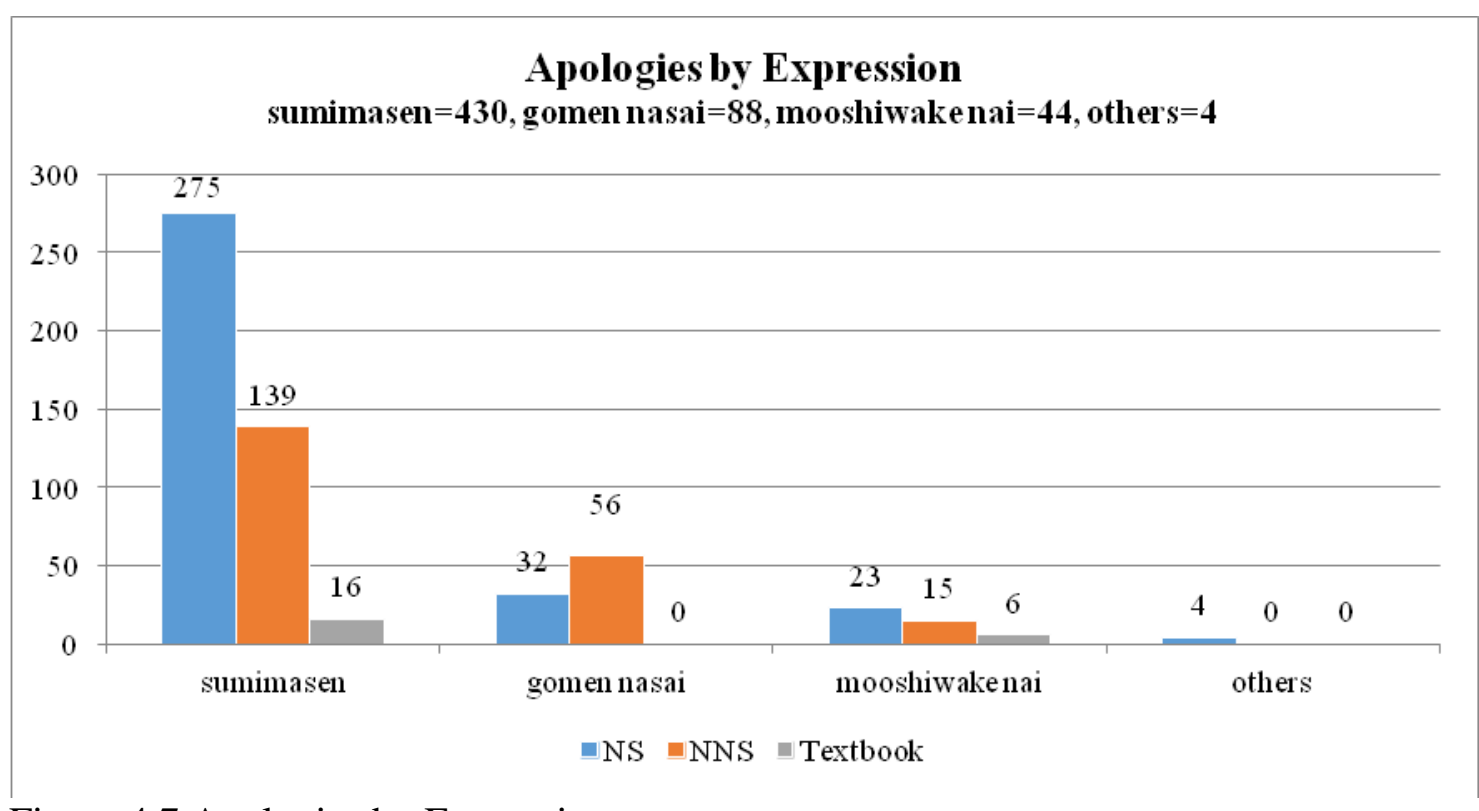

Figure 4.7 Apologies by Expression 


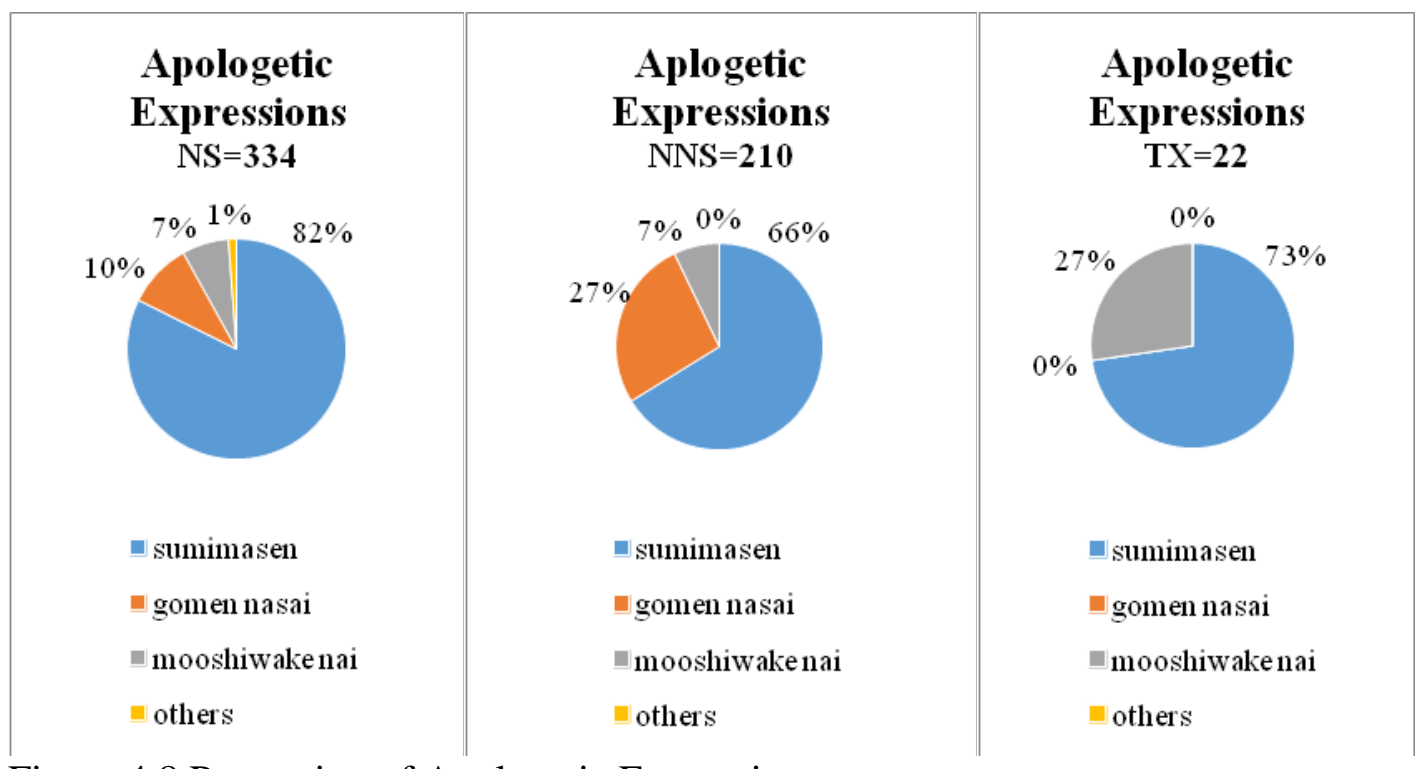

Figure 4.8 Proportion of Apologetic Expressions

\section{Use of the Past Tense}

When apologising in Japanese, there is the possibility of using one or the other of the following: the present tense only, the past tense only, or a combination of both. In the present experiment, 34 NSs (42\%), 13 NNSs (16\%) out of 80 participants in each group, and five out of 12 TXs (42\%) used a combination of the present tense and the past tense at least once or more in their discourse. By contrast, 46 NSs (58\%), 67 NNSs (84\%) participants, and seven TXs (58\%) used the present tense only.

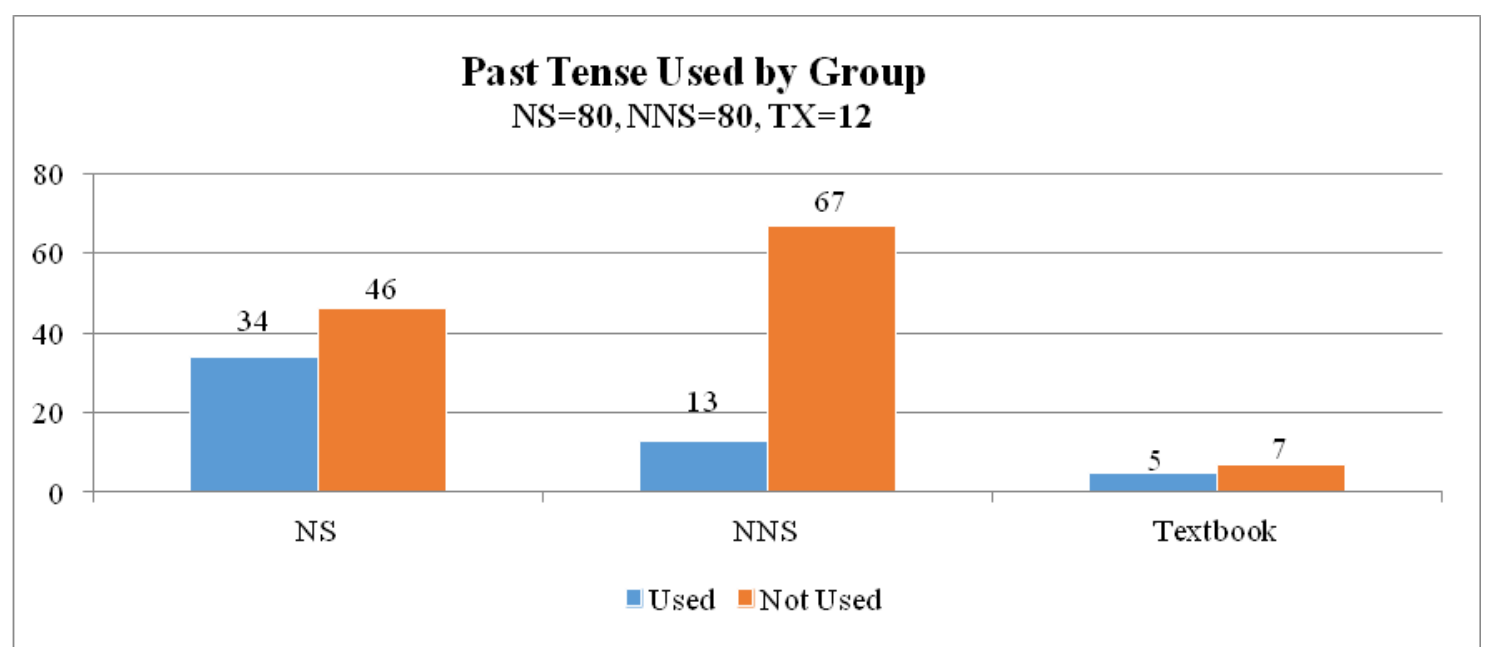

Figure 4.9 Past Tense Used by Group 


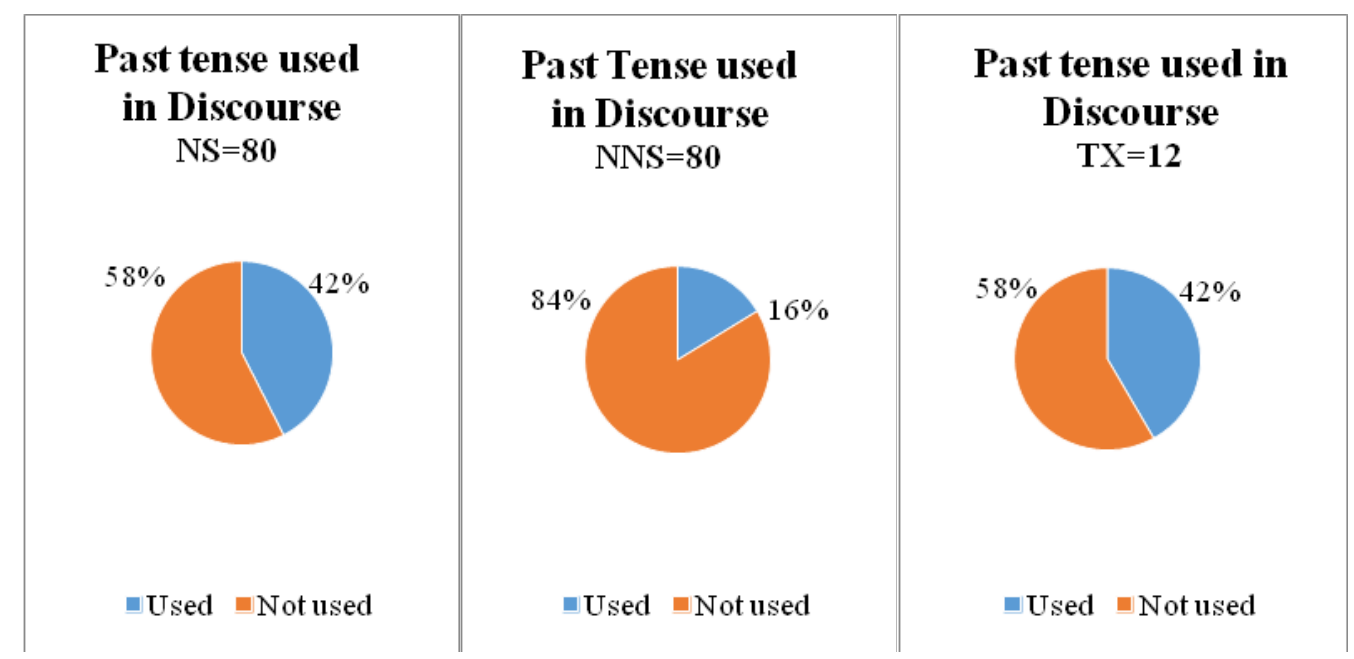

Figure 4.10 Proportion of Past Tense Used in Discourse

The frequency of use of the past tense out of the total apologetic expressions was 50 utterances $(15 \%)$ by the NSs, 18 utterances $(9 \%)$ by the NNSs, and five exemplars $(23 \%)$ in the TXs. ${ }^{108}$ Almost all of the past tense expressions were sumimasen deshita (past tense of sumimasen) [I was sorry]. The use of the other two expressions, mōshiwake arimasen deshita [it was inexcusable], and meiwaku o okake shimashita [I put you to trouble] was minimal.

Judging from this, 42 per cent of the NSs and the TXs used the past tense, but the proportion of the past tense to the total number of apologetic utterances was 15 per cent and 23 per cent respectively. If the total number of apologetic expressions is large, the proportion in the past tense, if uttered, is comparatively small, but it was uttered at least once. This means that the past tense was not uttered so many times as the present tense was. For instance, the TXs contain relatively few examples, with the result that the past tense was used relatively more frequently than by NSs and NNSs.

The NSs quite often uttered the present tense of apology and also included the past tense. Nevertheless, the use of the past tense was 1.5 utterances per apologiser among the

108 The formulae for these are 50/334=15\% (NSs); 18/210=9\% (NNSs); $5 / 22=23 \%$ (TXs). 
NSs who used it. ${ }^{109}$ The NNSs also showed a similar frequency of use of the past tense, which was 1.4 utterances per apologiser who used it. ${ }^{110}$ There were five apologetic expressions of the past tense in the five different TXs, so the average is 1.0 utterance. This indicates that the authors of the TXs did not repeat the past tense more than once on average in the scenario of apology—noticeably less than in the simulated 'real life' conversations.

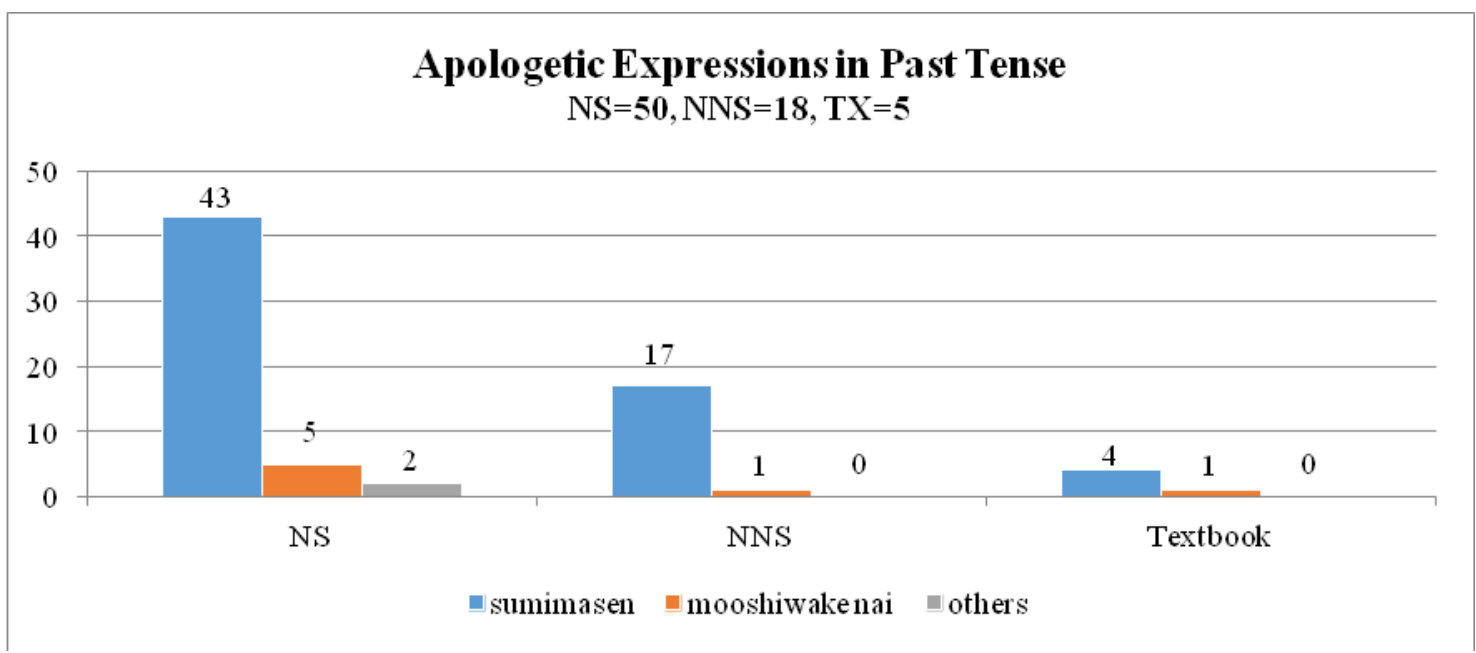

Figure 4.11 Apologetic Expressions in Past Tense

\begin{tabular}{|c|c|c|}
\hline $\begin{array}{c}\text { Past Tense in } \\
\text { Apology NS=334 }\end{array}$ & $\begin{array}{c}\text { Past Tense in } \\
\text { Apology NNS }=210\end{array}$ & $\begin{array}{c}\text { Past Tense in } \\
\text { Apology } \\
\text { TX }=22\end{array}$ \\
\hline $\begin{array}{l}\square \text { sumimasen } \\
\text { mooshiwakenai } \\
\square \text { others } \\
\text { not used }\end{array}$ & 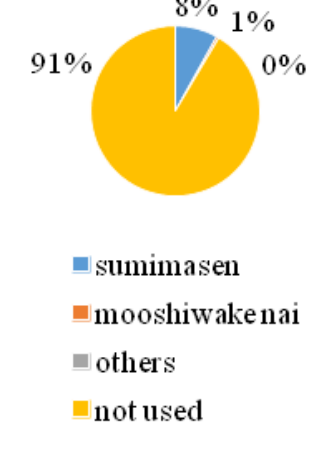 & $\begin{array}{l}\text { sumimasen } \\
\text { mooshiwakenai } \\
\text { mothers } \\
\text { not used }\end{array}$ \\
\hline
\end{tabular}

Figure 4.12 Past Tense in Apology

109 Thirty-four NSs used the past tense and the number of past tense apologies was $50(50 / 34 \doteqdot 1.5)$.

110 Thirteen NNSs used the past tense and the number of past tense apologies was $18(18 / 13 \doteqdot 1.4)$. 


\section{Order of Appearance of Past Tense}

The order of appearance of the past tense differed among the three data sets. None of the NSs who used the past tense in a discourse uttered it first when apologising. The most typical example was their use of sumimasen [I am sorry] first, then sumimasen deshita [I was sorry], which was mostly uttered at the end of the discourse. Five out of 34 NSs who used the past tense uttered the present tense even after the past tense. For example, the order was, sumimasen (present tense) > sumimasen deshita (past tense) > mōshiwake arimasen (present tense and more formal). Moreover, once the past tense of mōshiwake arimasen deshita was uttered, none of the NSs went back to sumimasen (present tense).

It may be inferred that this was because these speakers apologised more than three times in a discourse, unlike those who uttered apologies only once or twice. Secondly, the order of utterance could somewhat reflect the degree of the speaker's sense of regret (present tense) and feelings of gratitude (past tense). Arguably, the past tense of apology could be tinged with more politeness than the present tense, because when most apologisers used the past tense, it was after the interlocutor had accepted the apology. Therefore, the apologiser acknowledged the benefit bestowed on them by the interlocutor. Besides, the phrase mōshiwake nai/arimasen (deshita) contains more implications of courtesy than sumimasen (deshita) (Kindaichi 1987: 76-7). ${ }^{111}$

The pattern of usage of the past tense was as follows: initial apologies tended to be in the present tense, and were uttered repeatedly, but no matter how many times present tense apologies were uttered by a given native speaker, the speaker's final apology tended to be in the past tense. This means that the more frequently a speaker apologised, the lower the

111 The past tense of mōshiwake nai (desu) may also be mōshiwake (nakatta desu). However, this was not uttered in the present experiment. Instead of using nai after mōshiwake, arimasen is more polite. Its past tense is mōshiwake arimasen deshita. Therefore, mōshiwake arimasen deshita was especially preferred by the NS participants when apologising. 
proportion of apologies there were in the past tense. Since the textbook exemplars typically included fewer utterances of apology in total, a higher proportion of these were in the past tense.

From the utterances in the present experiment, it may be argued that the present tense of moshiwake arimasen is more polite than the past tense of sumimasen. In order of increasing politeness, the expressions of apology as they were utilised by the NSs are:

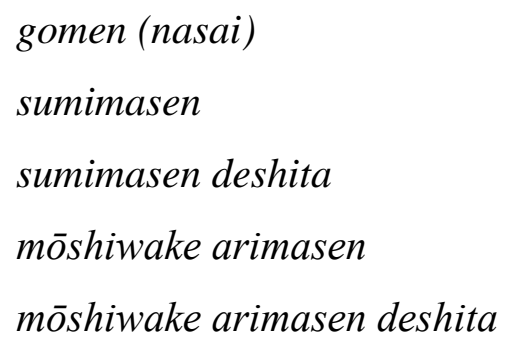

It was observed that mōshiwake arimasen deshita only ever occurred at the end of the discourse. It appears that the NS apologisers selected this more polite apology in order to finalise the discourse. On the other hand, eight out of 13 NNSs (62\%) uttered the past tense from the beginning. Their apology typically appeared only once in the discourse, or even if more than once, the second apology was repeated in the past tense.

Similarly to the NNSs, who did not utter an apology as many times on average as the NSs, the TXs presented either one type of apologetic expression (eight TXs) or two different types (four TXs) in the model discourse. ${ }^{112}$ The appearance of the past tense in the case of the TXs was limited to only five exemplars, but four out of the five (80\%) appeared in the past tense as the second apology.

112 Eight textbooks presented one type of apology; seven of them used sumimasen, and one used mōshiwake nai. The four textbooks presented two different types of apologies; all of them applied a combination of sumimasen and mōshiwake nai. 


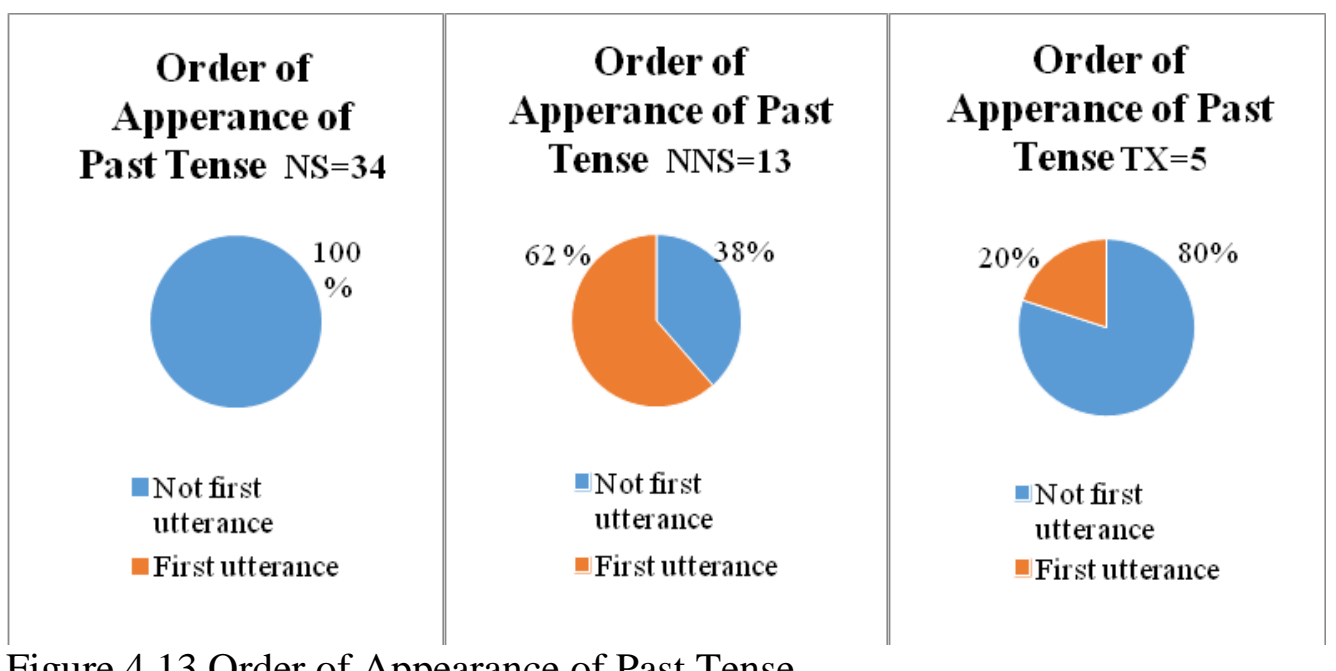

Figure 4.13 Order of Appearance of Past Tense

A distinct discrepancy in the usage of the past tense was observed between the NSs/TXs and the NNSs. In the former-the NSs and the TXs - there appeared to be a higher proportion of use and frequency of the past tense compared with the NNSs. Likewise, the order of appearance in the two also showed differences. The NSs and the TXs tended to use the past tense at the end of the discourse, but the NNSs did not.

There may be several reasons why the NNSs did not seem to use it as often as the NSs and the TXs, in spite of the fact that there were several model exemplars in the TXs, and these are:

(1) They did not grasp the flow and significance of using the past tense in a discourse of apology, regardless of having been taught it or not.

(2) Around 80 per cent of the NNSs participants were English native speakers, and in English the past tense is not used when apologising, ${ }^{113}$ suggesting influence from their own linguistic background, whereas in Japanese it is not unnatural to use it. Hence, the majority of the NNS participants might not have thought of using the past tense.

113 When a speaker (an apologiser) explains the scene of his/her apology in the past, the past tense, such as I was sorry instead of I am sorry. 


\section{2. 3 Supportive Moves of Apology}

In the setting of apology in the present experiment, the following four major supportive moves (SMs) were uttered by the participants or presented in the model exemplars of the TXs. The SMs were Explanation, Admission of the facts, Promise, and Placation. Items analysed were:

(1) The number and proportion of utterances of SMs.

(2) The order of utterances of SMs.

\subsubsection{Number of Supportive Moves}

The total number of the four different SMs uttered by NSs was 228 (an average of 2.9 times per participant), 190 by NNSs (an average of 2.4 times per participant), and 22 by the TXs (an average of 1.8 times per exemplar). The number of SMs uttered by NSs was 1.2 times and 1.5 times more than that of NNSs and the TXs respectively.

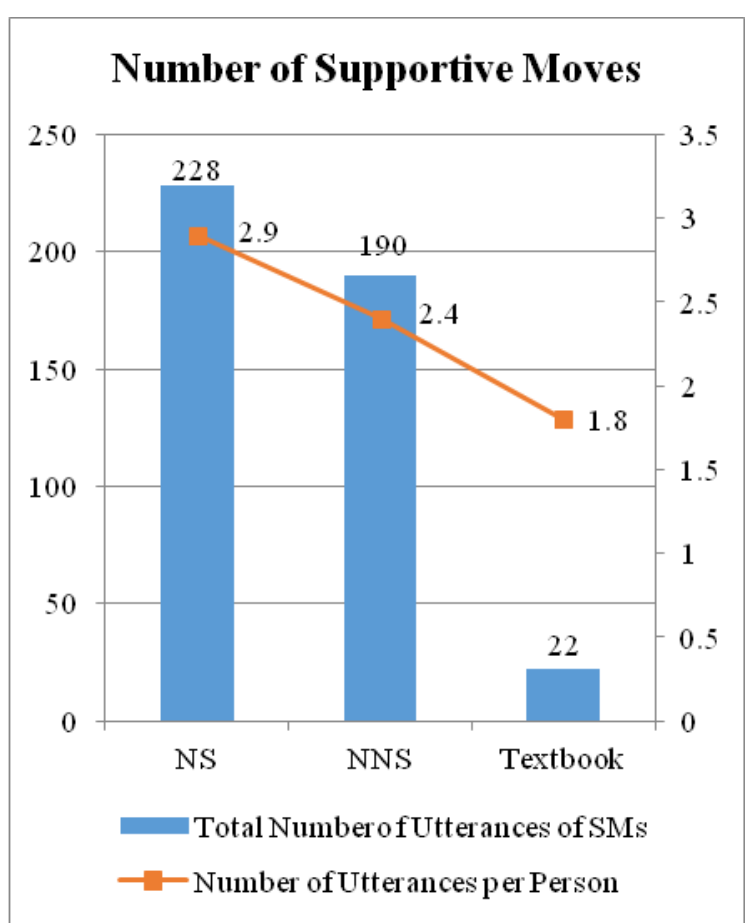

Figure 4.14 Number of Supportive Moves 


\section{Explanation (Positive politeness)}

In the present study, the collected discourses were categorised into four supportive moves, other than apologetic expressions which are Head Acts. ${ }^{114}$ The most frequently uttered supportive move was an explanation by an apologiser as to why he/she was late, which was uttered at least once on average in a discourse by participants and appeared in the model exemplars. Nevertheless, five NSs (6\%), eight NNSs (10\%), and one TX (8\%) gave no explanation.

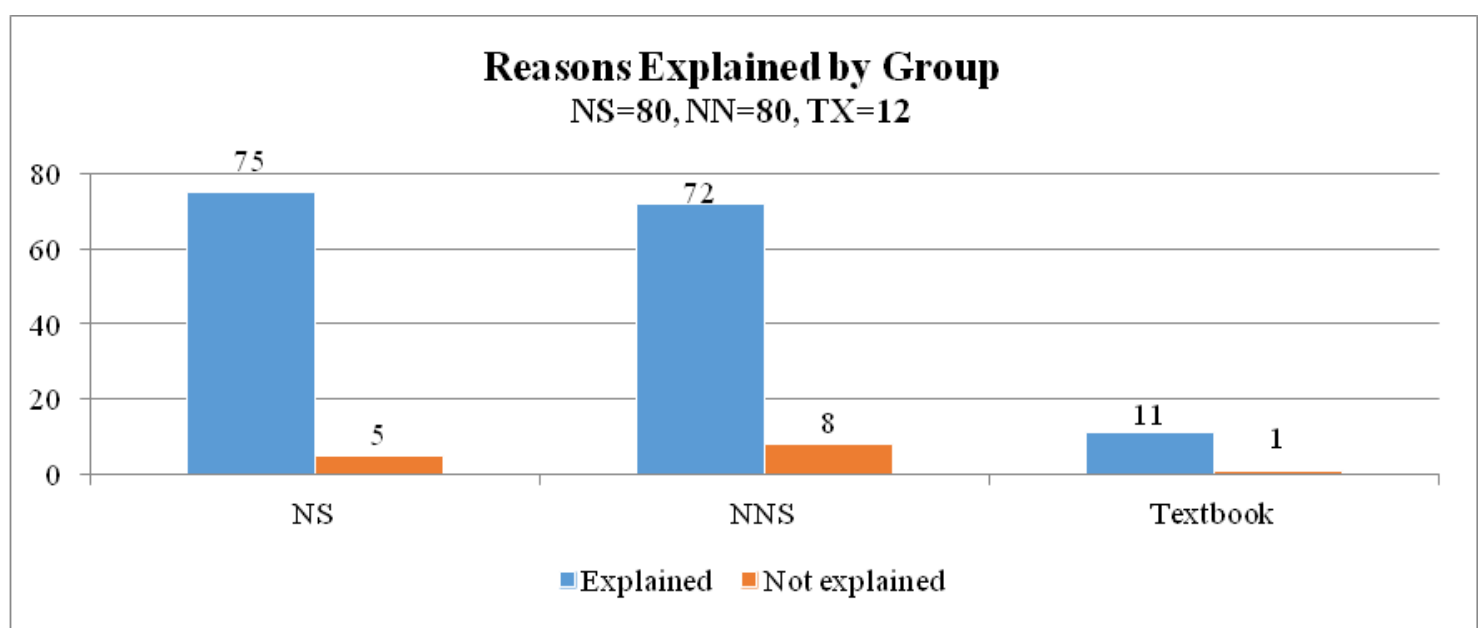

Figure 4.15 Reasons Explained by Group

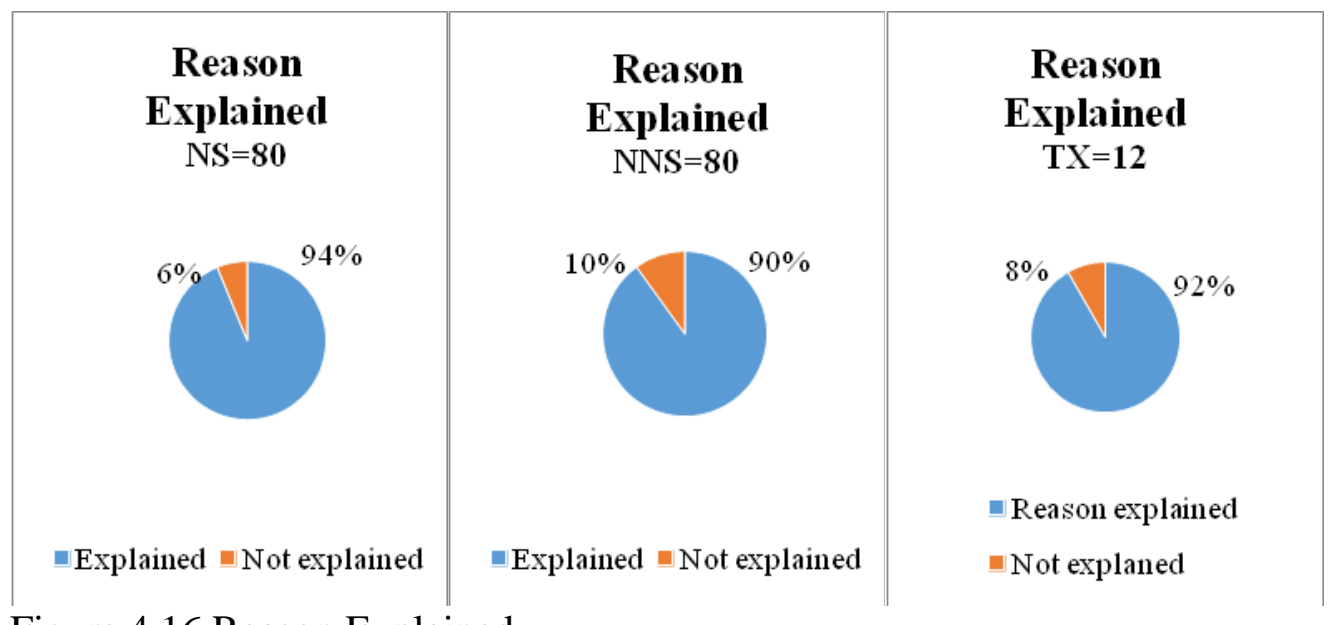

Figure 4.16 Reason Explained

114 They are explanation (including excuse as a subcategory), admission of the facts, promise, and placation (containing pledge, repair, responsibility, and beseeching as subcategories). 


\section{Reasons for Being Late}

The total number of explanations was 90 utterances by the NSs (16\%), 84 utterances by the NNSs $(21 \%)$, and 11 utterances in the TXs $(25 \%) .{ }^{115}$ Personal reasons, transport, and school (classes) were the three major reasons cited for being late, which were uttered by 75 NSs, 72 NNSs, and 11 in the TXs. The personal reason was the most common, appearing in 52 per cent of the NSs, 56 per cent of the NNSs, and 59 per cent of the TXs. Most of them said that they had overslept. The second reason, claimed by 39 per cent of the NSs, 26 per cent of the NNSs, and 25 per cent of the TXs, was that public transport had not come on time. A school matter provided three per cent of the NSs, and eight per cent each of the NNSs and the TXs with reasons, such as Zemi ga nagaku natta n' desu [A seminar got protracted].

The reason for being late because of public transport showed the largest discrepancy among the three groups. The NSs were most inclined to attribute their lateness to trains or buses, and of the reasons for being late, only public transport was beyond the speakers' control. In other words, it contains a nuance of evading responsibility. ${ }^{116}$

Something else noteworthy, other than the wide discrepancy between the NSs and the NNS/TXs, was the similarity between the NNSs and the TXs. This is perhaps because the authors of the TXs might have taken into consideration learners outside Japan where the people do not necessarily expect public transport always to come on time, or where

115 The percentage in the parentheses shows the ratio to the total numbers of utterances including apologetic expressions (Head Acts). The formulae for explanation are, 90/562=16\% (NSs); $84 / 400=21 \%$ (NNSs); 11/44=25\% (TXs).

116 Two of the NSs even tried to show (in the role-play) a certificate of lateness issued by a railway company. Usually railway companies in Japan issue these if a train has arrived later than the timetable by a certain number of minutes. As long as it is presented to an employer, a person who has come late for that reason would be excused as not being late. 
punctuality is not a sort of de facto standard. Likewise, the NNSs might not think of the late arrival of public transport as being a persuasive explanation. ${ }^{117}$

Another reason the NNSs did not refer to transport as often as the NSs might be that not all of them were sufficiently acquainted with the situation with Japanese transport services. They would be unlikely to give an explanation for more than what they knew, unless it had been explained in the TXs or by the teachers from whom they had learnt. Hence, for the NNSs, transport would not necessarily provide a hint of a plausible explanation for the lateness.

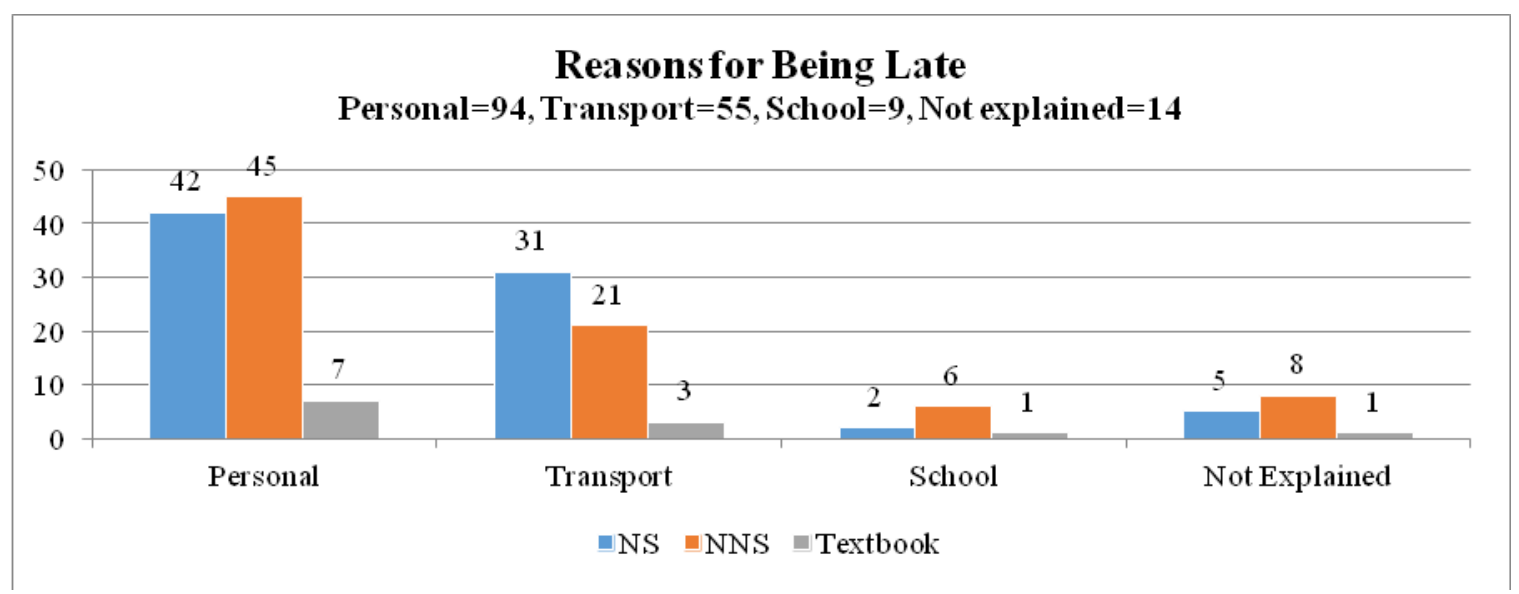

Figure 4.17 Reasons for Being Late

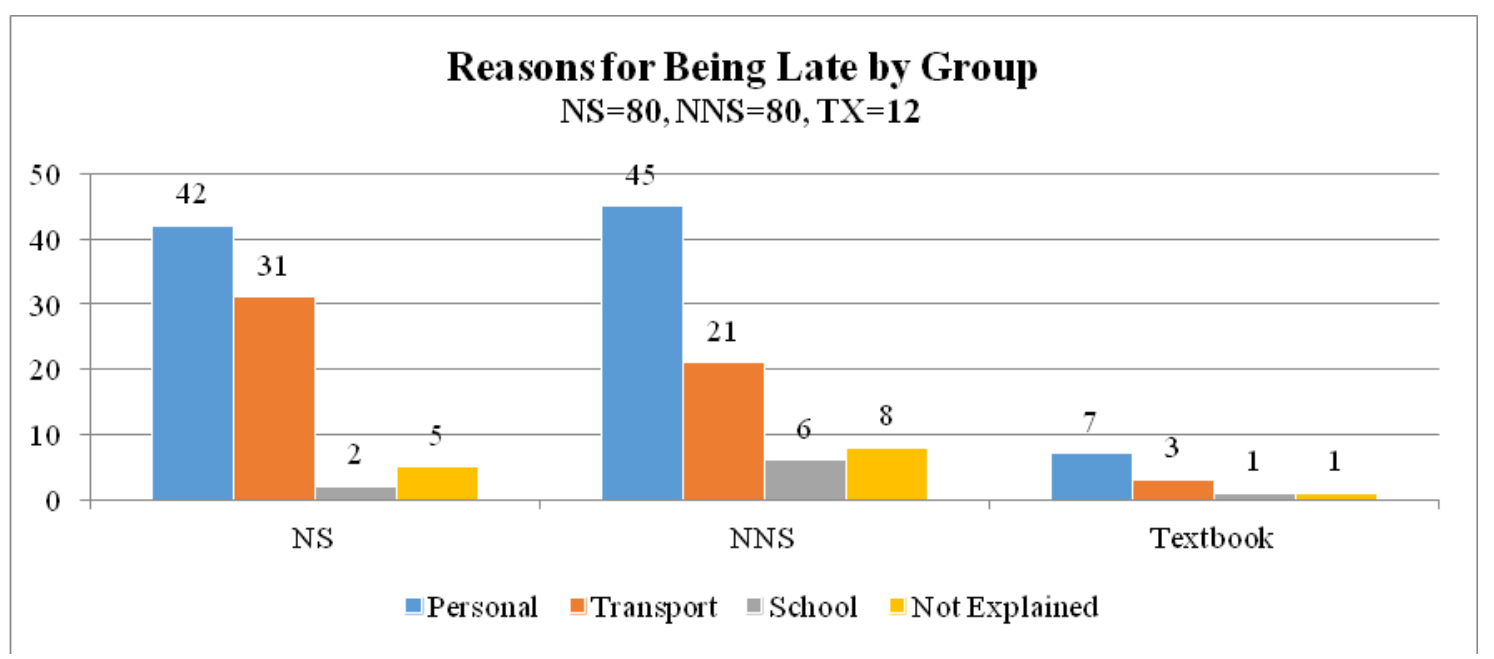

Figure 4.18 Reason for Being Late by Group

117 The NNSs might have thought that if it were not taken for granted that transport would run on time, the speaker should have included that possibility into their time calculations, and set off earlier. 


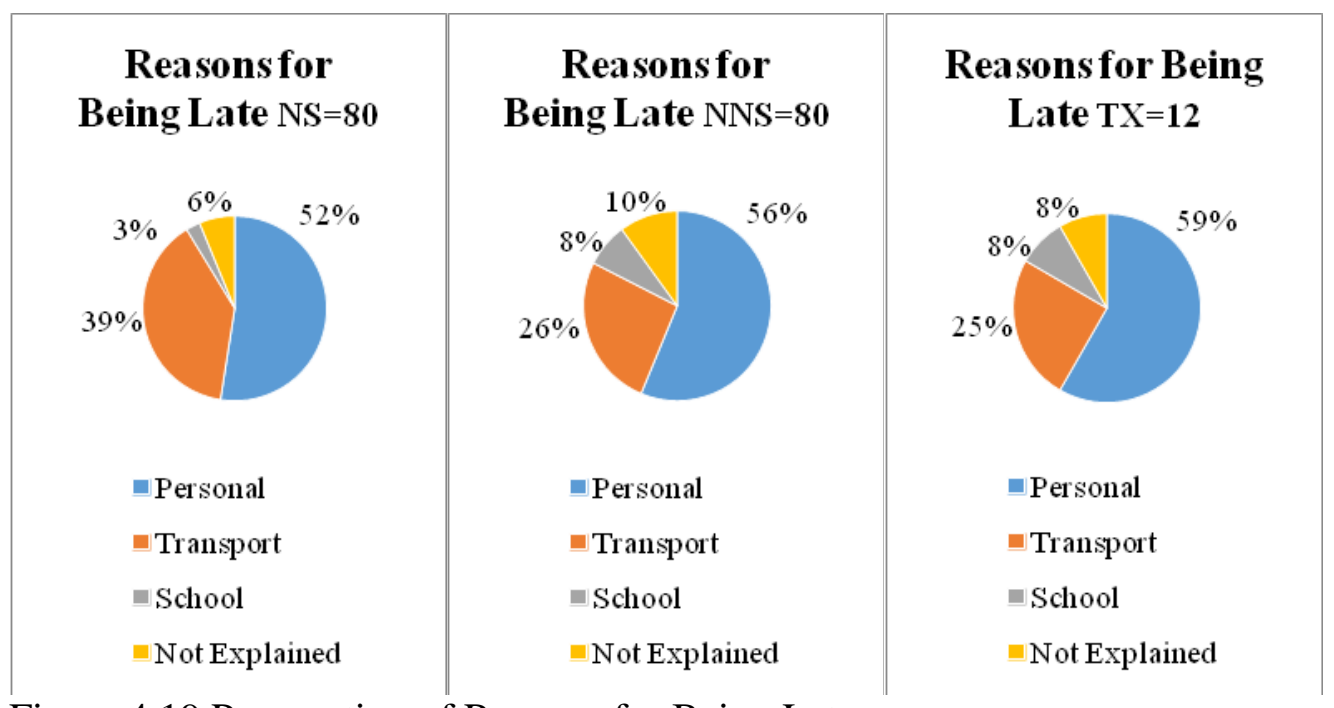

Figure 4.19 Proporation of Reasons for Being Late

\section{Attenuation}

Attenuation is one of the methods of expression devised by the speaker in order to avoid giving a pushy impression to the hearer. ${ }^{118}$ When explaining the reason for their lateness to the interlocutor, the NSs uttered the minimiser word chotto [a little bit] very frequently, for example, Chotto nebō shichatte [I overslept a bit] or Chotto densha ga konakatta n' de ['Cause the train did not come (on time)]. The word chotto was used in the explanations of fifty-six out of 75 NSs $(75 \%)$ who articulated the reason to the interlocutor. In contrast, only 13 NNSs (18\%) used it out of 72 explanations, and merely one out of 11 exemplars of the TXs (9\%) used chotto in the explanation, such as Chotto wasuremono ni ki ga tsuite tori ni modotta mono desu kara ['Cause I just noticed there was something I'd forgotten to bring].

118 Refer to Chapter 2, as Kindaichi et al. described, when making a request, women tend to choose less pushy expressions than men, such as chotto [a little bit], dekireba [If possible], moshi yokattara [If it's all right with you] (1989: 560). In Japanese, this is frequently achieved by the use of chotto, for example. 
What this means is that the majority of the NNSs and the exemplars in the TXs did not use the word chotto in their discourses, in strong contrast to the NSs' very frequent use of it. It may be inferred that the reason the NSs uttered chotto very often in their explanation was that they wanted to minimise the impression of offending by being late for the appointed time. In spite of its being short and easy to use for the NSs, the reason chotto was rarely utilised by the TXs may be owing to the casualness implied by chotto-perhaps it was considered too casual by TX writers to be represented in a model discourse, although little space is necessary for the word chotto when creating a scenario-and so the NNSs might not have used or even recognised it because the word was rarely presented in this context in the model discourses from which they learn. Or else, they just overlooked it without recognising the significance of the word.

From the above, it can be seen that the majority of NSs attempted to alter the impact of their offence by using the minimiser chotto. In striking contrast to this, the majority of NNSs used no minimiser, and it appeared in only one of the exemplars. It may be inferred that the writers of the TXs tend to regard chotto as a casual expression for use in a model discourse rather than as the more widely used minimiser that it is. Its lack of inclusion in exemplars may account for or contribute to the large discrepancy in usage between native speakers and non-native learners. This is a clear case in which exemplars fail to represent real-life discourse. 


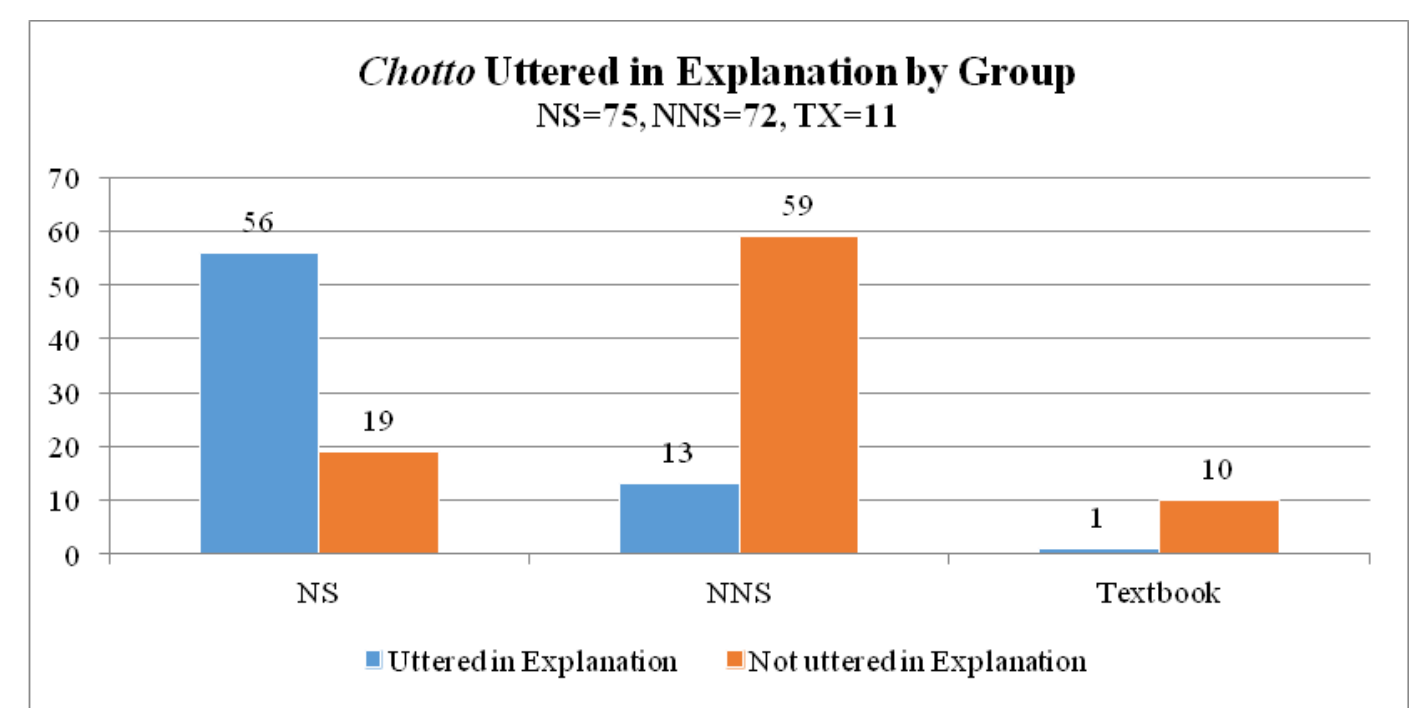

Figure 4.20 Chotto Uttered in Explanation by Group

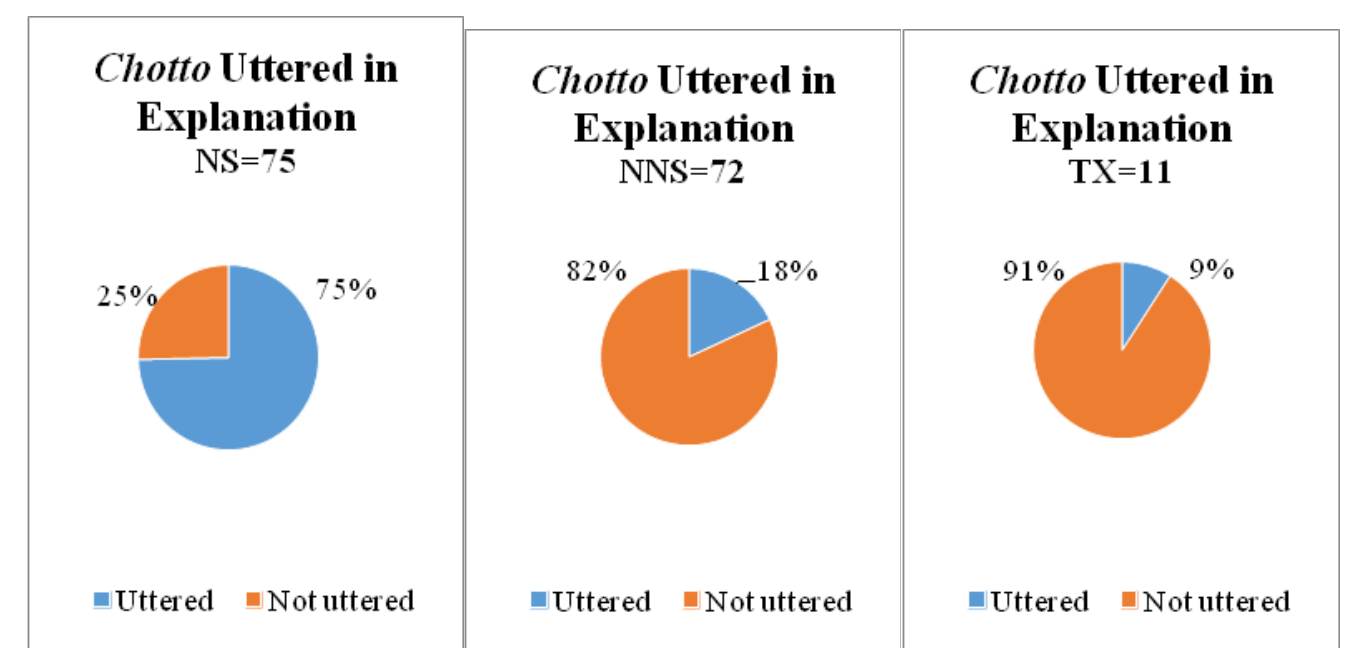

Figure 4.21 Proportion of Chotto Uttered in Explanation

\section{Excuse}

An excuse was defined in the present study as occurring when an apologiser added a conjunction such as kedo [however, but], demo [but], or mono desu kara [because (it is)] at the end of or during the explanation, or the apologiser repeatedly defended his/her behaviour 
regardless of the reason, and when such utterance was deemed to constitute a justification for the offence. ${ }^{119}$

Twenty-eight out of 90 explanatory utterances by NSs (31\%), nine out of 84 by NNSs (11\%), and five out of 11 presented in the TXs (45\%) were recognised as excuses when explaining the reason(s) for being late to the superior. Among these, the number of uses of kedo by the NSs was 19 out of 28 excuses, by the NNSs it was three out of nine, and there was none in the TXs, which, however, had five exemplars of using mon(o) desu kara in explanation.

Typical utterances of the NSs were Denwa shiyo to omotta n' desu kedo [I meant to ring you, but...], and kedo followed by a reason, such as Chien shömeisho o kubatteta n' desu kedo, chikoku suru to omotte [They were handing out certificates of lateness at the station, but I thought I would be late (if I waited for one)] or Arämu o kaketa n' desu kedo [I set my alarm clock, but...]. For the NNSs, Okita kedo, Uerinton no densha wa itsumo okurete imasu [Although I got up on time, the Wellington trains are always behind schedule] ${ }^{120}$ or Okāsan chotto byōki na n' desu kedo [My mother is a bit sick].

Other utterances without using kedo by the NSs were Denwa suru jikan ga nakatta n' desu [I didn't have time to call you], or Densha no naka toka datta n' de keitai tsukaenakute

119 According to Mihara (1995: 86) and Mizutani (2001: 115), a sentence ending with a conjunctive particle kedo and its variants function to provide an interlocutor with information as to what the speaker has done, and the sentence contains the implication of a judgement being left up to the discretion of the interlocutor. An utterance, such as Mezamashi kaketa n' desu kedo... [Though, I set up the alarm...], gives a soft and gentle impression while informing the speaker's intension or idea to the interlocutor. Nevertheless, at the same time, it sounds like a justification of the speaker's behaviour.

120 Regarding the punctuality of train services, there is a perception gap between the NSs and the NNSs. The basic understanding of the former is that the train is a means of transport which is bound to come on time, while for the others it is not. 
[I was on the train, so I couldn't use my mobile]. ${ }^{121}$ For the NNSs, for example, explanations included Okureru tsumori wa arimasen deshita [I didn't intend to be late], Kinō wa watashi no tanjōbi da [Yesterday was my birthday (so that I overslept)], or Demo konshū tesuto ga arimashita [But, I had an exam this week].

In the TXs, although there were only 11 explanations, five of them employed the phrase, mono desu kara [because], which was used neither by the NSs nor the NNSs. Examples in the TXs were Demo densha ga okureta mono desu kara [But, the train was late] or Yoshū fukushū no jikan ga nakanaka torenai mono desu kara [Because I don't have enough time for preparation and revision (for my Japanese class)]. While the phrase mono desu kara is more formal and polite than kara, it sounds stronger than kara and even defiant and like an excuse. ${ }^{122}$ Thus it is questionable whether it is appropriate to use this expression when apologising to a superior. Its over-representation in TXs calls into question their role as exemplars.

121 In terms of etiquette, talking on a mobile phone while on the train/bus is regarded as inappropriate and offensive behaviour in Japan. However, the former apology is not an entirely convincing explanation, and the latter apologiser could have called the superior as soon as the train arrived at the station.

122 Tamamura, Fumio, and Kaigai Gijutsusha Kenshū Kyōkai [The Association for Overseas Technical Scholarship]. 1993. Nihongo jitsuō jiten [The AOTS Nihongo Dictionary for Practical Use]: Surīei Nettowāku (p.364). 


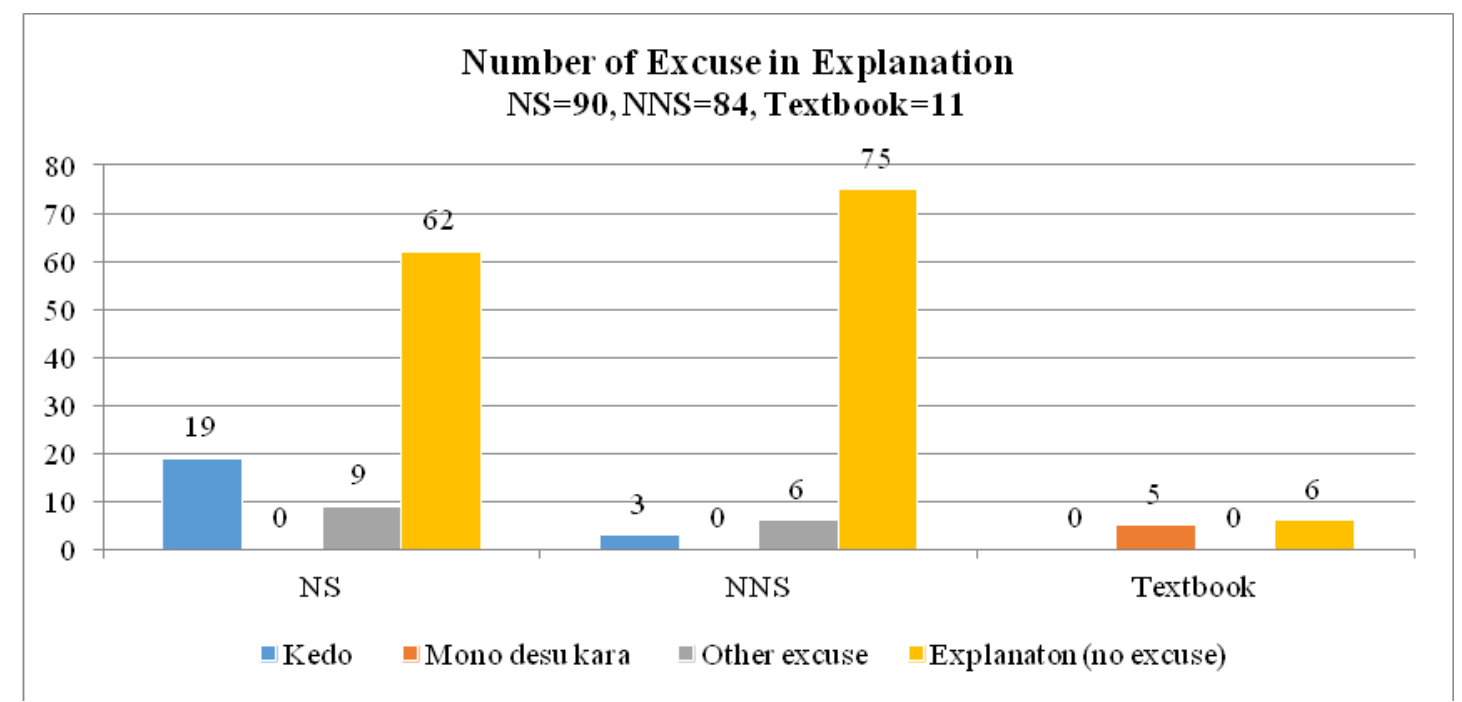

Figure 4.22 Number of Excuse in Explanation by Group

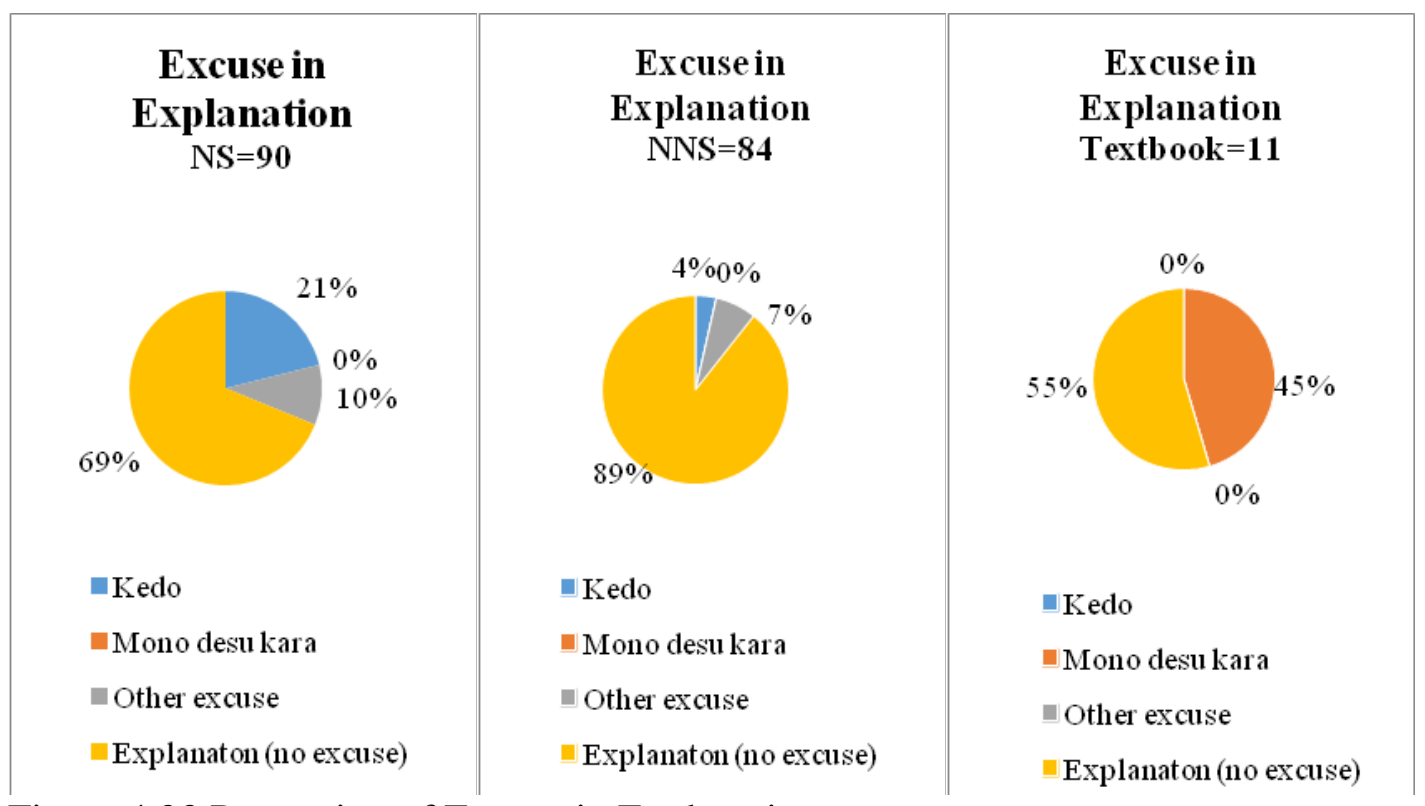

Figure 4.23 Proportion of Excuse in Explanation

\section{Aposiopesis}

The definition of aposiopesis here is as follows in reference to Shirakawa (1996: 10, see Chapter 2). A speaker ends a sentence after uttering a subordinate clause, so that there is no main clause in the utterance. The omitted clause can easily be assumed by the hearer from the previous context. In other words, the speaker's intention is conveyed to an interlocutor, albeit not explicitly. Aposiopesis used by an apologiser when explaining the reason for being late could emphasise the speaker's sense of sorrow and regret. In particular, the 
sentence ending in the te-form is uttered with the intention of apology, so it is a favoured sentence pattern when apologising in Japanese (Shirakawa 1991: 42). This is because leaving the sentence open-incomplete as no other clause follows-leaves an interlocutor to complete the apologiser's sentence in his/her own mind. As a consequence, the interlocutor, who has been upset by the apologiser, might be persuaded to accept the apology, although this depends on the situation.

Significant differences were discovered among the three groups. Three-quarters of the NSs' explanations (including the excuses) used unfinished sentences. Forty-seven (52\%) of the explanations comprised the te-form, for example, Densha ga okurete... [The train was late so...]. An example of using the word kedo [but] was Isoide kita n' desu kedo...[I hurried here, but...], which could prompt thoughts, such as Yappari okurete shimaimashita [Yet, I was late]. Because the unuttered clause was very obvious to both the apologiser and the interlocutor, it was unnecessary to enunciate it repeatedly. On the contrary, if it were uttered, it might be thought that the speaker was excessively and inappropriately persistent.

It appears to have been difficult for the NNSs to generate sentences using aposiopesis since only nine $(11 \%)$ of them did so. Of these, a mere four (5\%) used a te-form sentence. In the case of 11 exemplars in the TXs, four of them (36\%) did not use aposiopesis (there were no unfinished sentences); in other words, the authors of these exemplars used only complete sentences. The distinguishing characteristic of the TXs was the use of the phrase, mono desu kara [Because (this is the reason)], which was applied at the end of the sentence only in the exemplars. Five exemplars out of 11 explanations (46\%) presented this phrase, and all of them formed aposiopesis. Furthermore, as noted earlier, the phrase mono desu kara was used by neither the NSs nor the NNSs in the role-plays.

Frequently uttered aposiopesis by the participants was Densha ga okureta n' de... [The train was late so...]. When this is compared with the sentence ending mono desu kara, 
for example, Densha ga okureta mono desu kara [Because the train was late], the latter sounds as if the speaker is trying to justify him/herself. ${ }^{123}$ The former, by contrast, although it states the real cause of the trouble, is an unfinished sentence, whereby the speaker leaves the ending to the discretion to the interlocutor. It makes the speaker's position lower (and therefore more polite) because the judgement (of the justification) is left to the discretion of the interlocutor. This mechanism seems to work in particular for the NSs. It may be that the apologiser applies this negative strategy in order to widen the emotional distance between the two parties.

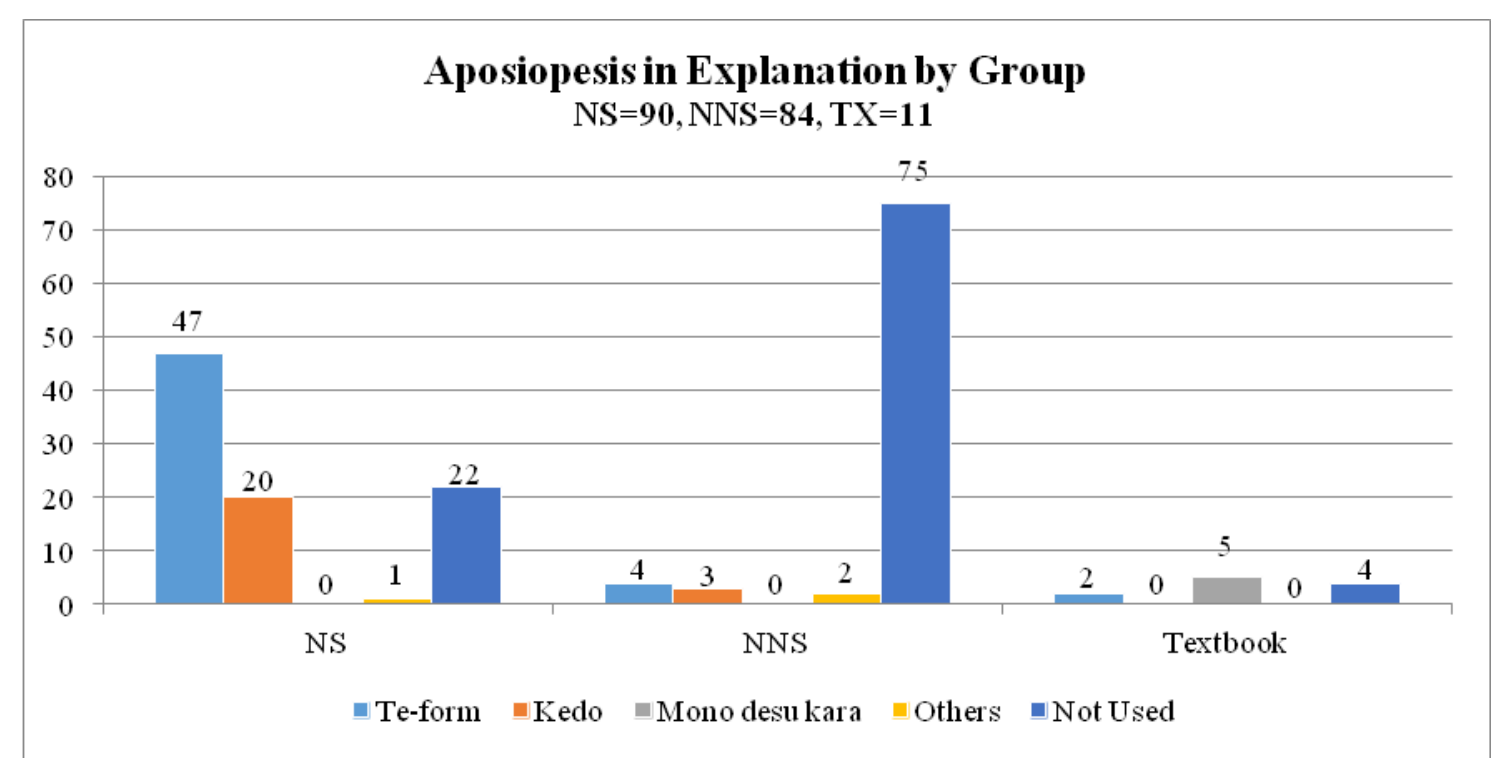

Figure 4.24 Aposiopesis in Explanations by Group

123 The word kedo cannot be used in this context because it does not make sense. The utterance, Densha ga okureta n' desu kedo..., implies that the speaker was nevertheless in time for the appointment. 


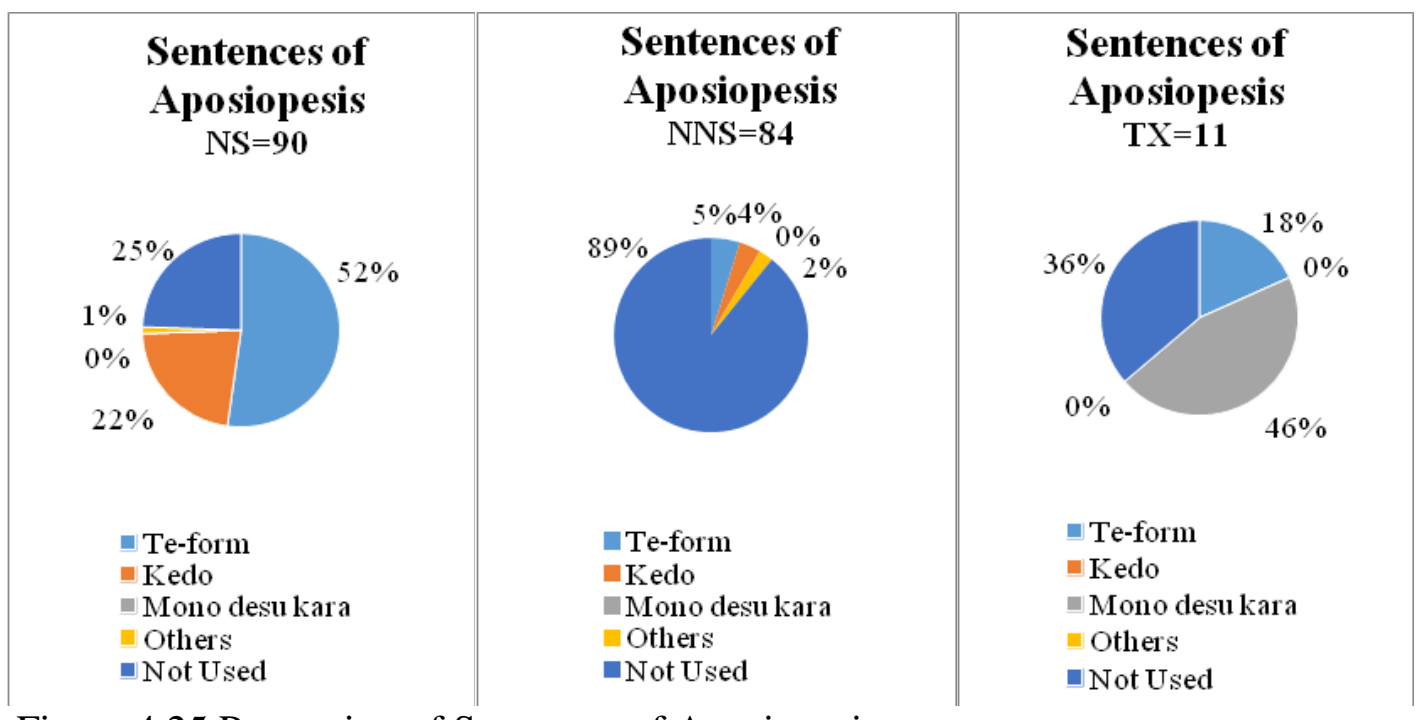

Figure 4.25 Proportion of Sentences of Aposiopesis

\section{Admission of the Facts (Negative politness)}

The definition of admission of the facts, in this present study, is that an apologiser explicitly states or confesses the fact to the interlocutor either before or after the apologetic expression. ${ }^{124}$ A typical utterance is: Osoku narimashita [I was late] or Sanjuppun chikoku shimashita [I was 30 minutes late]. All sentences admitting the facts contain words related to lateness, such as osoku [late], okurete [delay], chikoku [be late for], etc., although both the apologiser and the interlocutor are already aware of the lateness. An explicit utterance of the fact, which should be articulated in the past tense, and mostly the $t e$-form either followed by or following the apologetic word, can therefore be recognised as part of the strategies of apology. ${ }^{125}$

124 In the CCSARP, "Admission of facts" is one of eight subcategories of "Taking on responsibility", which is defined as "The speaker does not deny his or her involvement in the offensive act but abstains from openly accepting responsibility" (292). In this experiment, however, all participants who played the role of late-comers basically accepted responsibility. Hence, "Admission of facts" was separated from "Taking on responsibility".

125 Osoku narimashita [I was late] is a conventional fixed form expression of apology under these circumstances (Sakuma 1983: 58). 
The number of utterances of admission of the facts was 69 NSs (86\%), 64 NNSs $(80 \%)$ out of 80 participants in both groups, and nine out of 12 TXs (75\%). Those who used admission of the facts uttered the apology at the same time, with only one exception. This is evidence that apologetic expressions in Japanese tend to co-occur with a sentence of admission of the facts. The present findings suggest that textbook exemplars tend to underrepresent this discourse phenomenon.

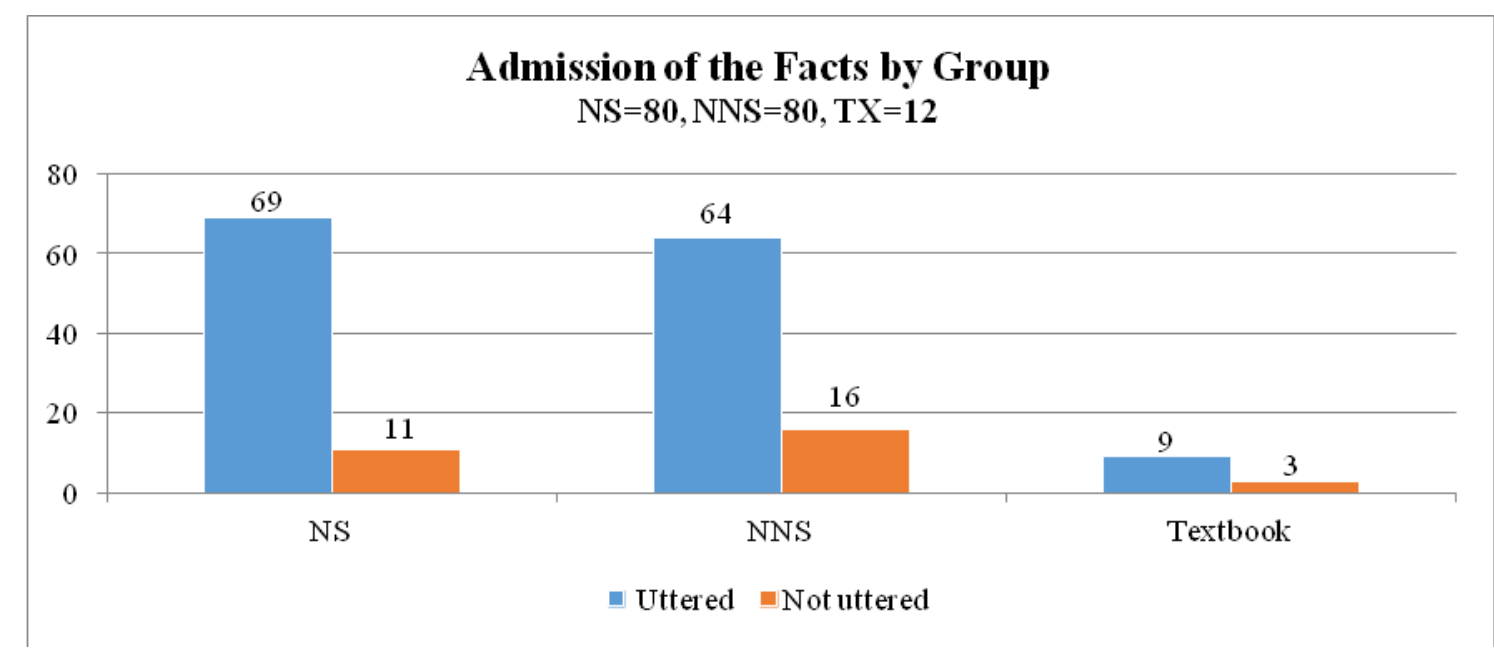

Figure 4.26 Admission of the Facts by Group

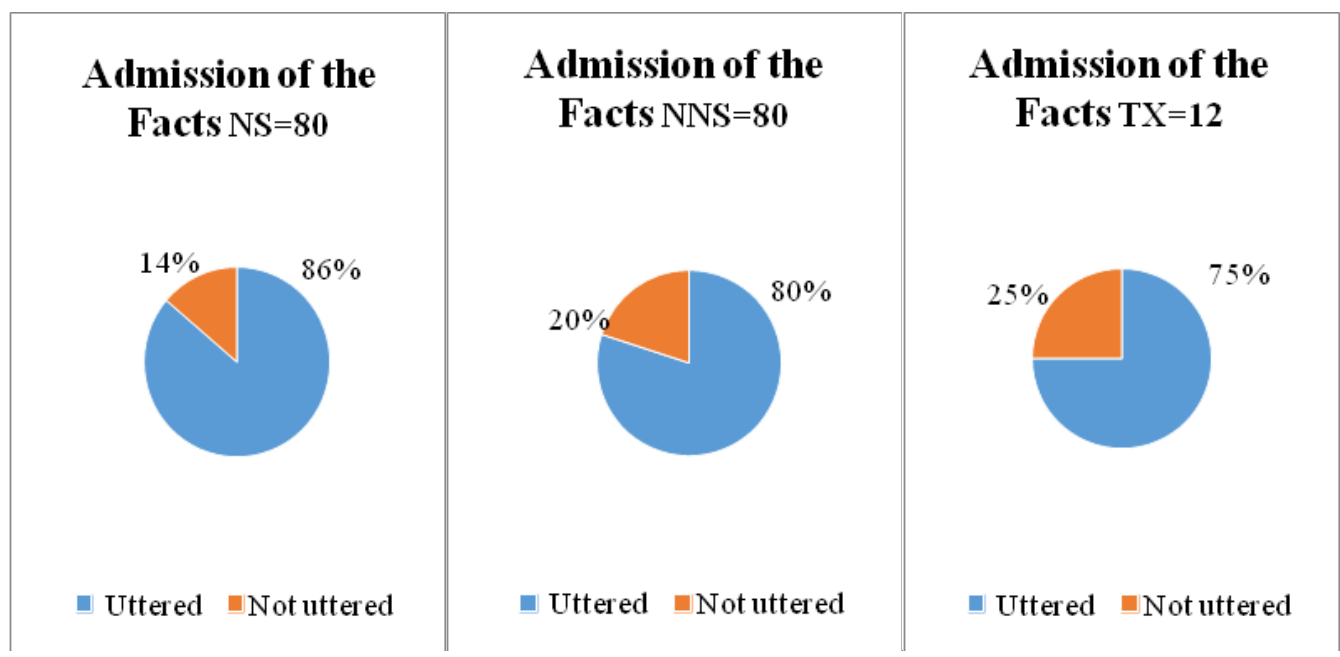

Figure 4.27 Proportion of Admission of the Facts

\section{Promise (Positive politeness)}

The promise not to repeat the same mistake again was uttered by 38 NSs (48\%) and 19 NNSs (24\%) out of 80 participants in each group, and one exemplar out of 12 TXs (8\%). 
In the case of the NSs, where the delay occurred because of public transport (which, unlike having overslept, was not their own fault), as many as 13 promises were uttered out of 31 cases of public transport delays. It can be interpreted that even though the apologiser could not take responsibility for the delay in the trains or buses, he/she apologised and promised to the superior to be more careful to take the possibility of delays into account next time. What they said was, Kondo kara ki o tsukemasu [I'll be more careful from now], Kongo nai yō ni shimasu [It won't happen again (meaning not being late)], Tsugi kara wa okurenai yō ni shimasu [From next time, I'll make sure I'm not late], and so on. For the NSs, uttering a promise regardless of the cause of the delay was regarded as important, as almost half of them did so.

By contrast, only one NNS (5\%) out of 19 made a promise similar to or the same as the NSs, as the apologiser said Tsugi chotto hayai densha ni noru tsumori desu [I'll try to catch an earlier train next time]. The reasons for being late in the remaining 18 utterances were not public transport, but the speaker's own fault. As such, the NNSs presumably felt it necessary to promise not to repeat the same mistake again because of their personal fault. The only exemplar in the TX was the apologiser uttering to his teacher, Kondo kara okurenai yo ni shimasu [From now on, I'll make sure not to be late] at the very end of the discourse.

A distinctive characteristic of the promise was that the NSs were inclined to promise regardless of the cause of the trouble, to show their positive attitude towards solving a problem in order to assuage the interlocutor's anger, at least as an attenuating effect. Again, it was strikingly clear from the present data that exemplars under-represent this feature of Japanese discourse.

The fault with the exemplars of the TXs was that they tended not to see the apology from a broader cultural, rather than a simply linguistic, perspective. The proportion of 
promises by the NSs was seven per cent of the entire discourses, which is not very large; however, that of the TXs was as low as only two per cent. ${ }^{126}$

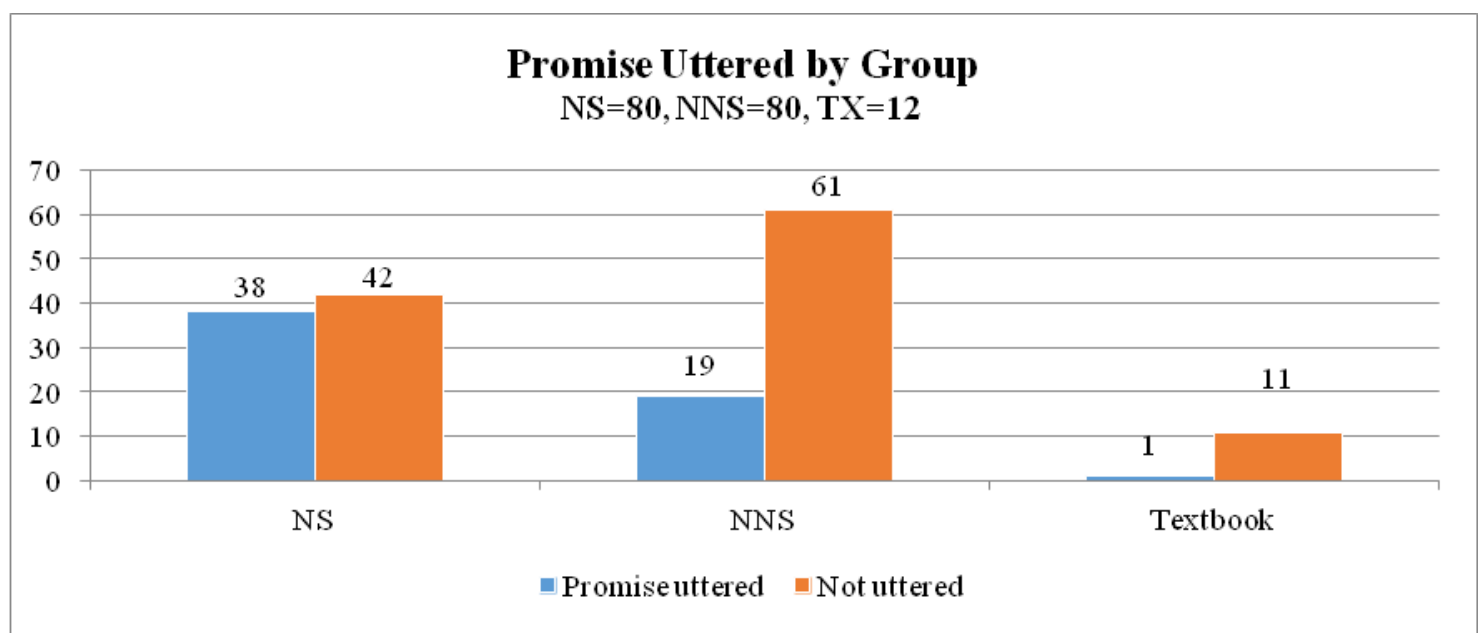

Figure 4.28 Promise Uttered by Group

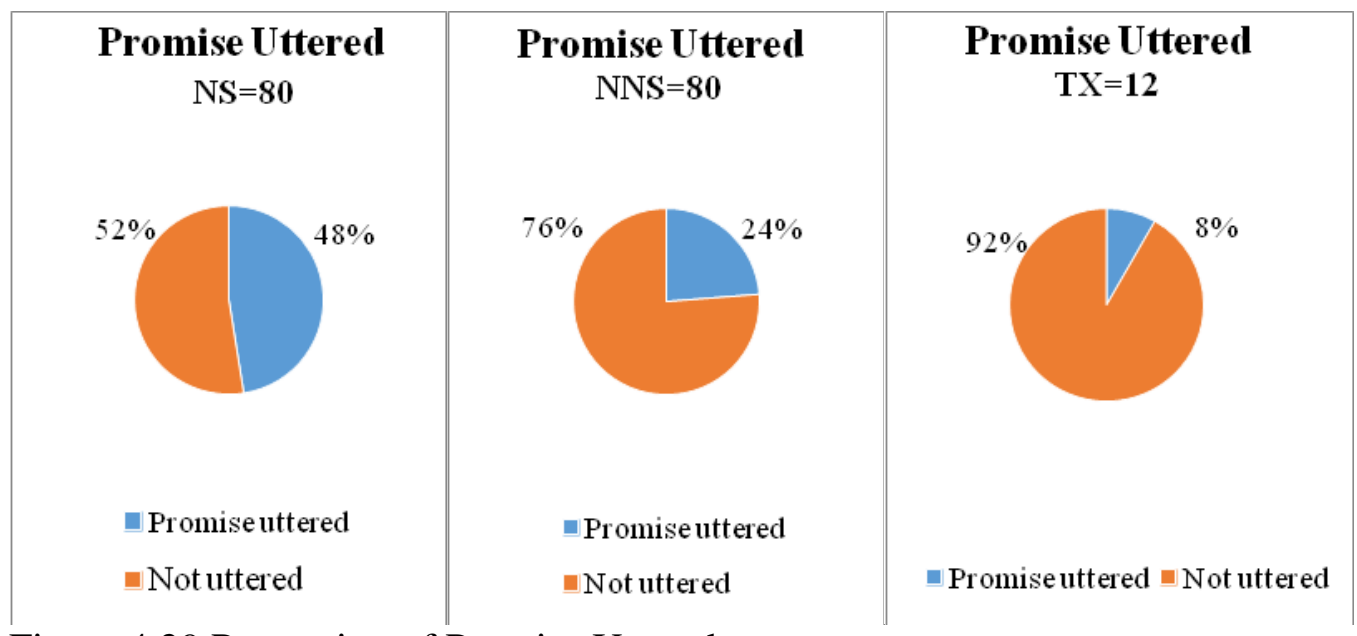

Figure 4.29 Proportion of Promise Uttered

\section{Placation (Positive politeness)}

Once the apologisers had uttered apologetic expressions, explanations for being late, admission of the facts, and sometimes promises, some of them tried to reduce the interlocutors' anger by pointing out their strong enthusiasm for work (pledge), offering

126 It may be speculated, however, that the authors of the TXs imagined that the feeling of an interlocutor would be considerably different depending on whether the apologiser promised or not. 
reparations (repair), stating his/her responsibility for unacceptable behaviour (taking responsibility), or beseeching a favour from the interlocutor (beseeching). The character of these four types was similar and functioned to allay the vexation of the interlocutor; however, their expressions differed. They are dealt with here together as utterances of "placation."

There were 31 placatory utterances by the NSs and 23 by the NNSs, which were uttered by 24 NSs and 20 NNSs players. Some participants made use of more than one measure of placation to the interlocutor. But there was only one exemplar presented in a TX.

\section{Pledge}

A pledge was uttered by $19 \mathrm{NSs}, 14 \mathrm{NNSs}$, and there was one exemplar in the textbook. Typical expressions of a pledge were Ganbarimasu [I'll try as best I can] and Isshō kenmei hatarakimasu [I'll work as hard as I can]. While a promise commits the speaker to a specific action, such as catching an earlier bus next time, a pledge emphasises more generally the positive feelings and attitude of the apologiser.

\section{Repair}

Six NSs and three NNSs adopted the strategy of repair. It is noteworthy that the NSs mainly focused on time, while the NNSs focused on money. For example, the expressions of five out of six NSs related to overtime, such as Nokorimasu [I'll stay longer] and Sanjuppun yokei ni hatarakimasu [I'll work 30 minutes longer]. Only one NS who mentioned the wages said, Kyūryō katto shitemo ii no de yurushite kudasai [Please forgive me as I do not mind if you dock my wages]. Although only three NNSs uttered repair, two out of the three said, Kyo okane harawanakutemo ii yo [You don't have to pay me today] and Kyō okane yasuku nareba 
ii yo [I do not mind if you reduce my pay today $]^{127}$ The repair of one NNS was Ashita hayai arubaito ni kimasu [I'll come and do part-time work earlier tomorrow].

\section{Responsibility}

Taking responsibility was uttered only by the NSs. Their typical expressions were Jibun no sekinin desu [It's my fault] and Hontō ni watashi no fuchüi desu [Definitely it's my carelessness]. Their distinctive feature was the use of the first person pronoun, watashi or jibun $[\mathrm{I}]$ in the sentences of responsibility, in spite of the fact that, while conversing with others in general, the Japanese do not usually utter the first person pronoun unless the speaker wants to emphasise it for a specific purpose. Because of this, the NSs who uttered responsibility for his/her mistake fostered an atmosphere in which the apologiser repented.

By contrast, none of the NNSs or the TXs mentioned that the lateness was their own responsibility. If an apologiser explicitly uttered his/her responsibility with the first person pronoun, it can be judged that the apologiser was heartily apologising. It is not surprising that the NNSs did not utter responsibility because it does not appear in the TXs. Furthermore, the implications of a delay of thirty minutes may vary depending on culture. It is possible that the NNSs might not have thought to state responsibility, as their misbehaviour was "only" a thirty-minute lateness.

One of the reasons why the TXs do not include responsibility in their exemplars might be because of a limitation of space, because the average number of apologisers' utterances in the TXs was 3.8 where, in most cases, apologetic expression, explanation, and admission of the facts were already included. In some exemplars, however, 'excuse' could be recognised, whereby apologisers used the phrase mono desu kara, which is a comparable defensive

127 The sentence is inappropriate pragmatically, but the meaning of this is that the speaker does not mind the payment being reduced. 
expression depending on the scenario. Therefore, it is important for the authors to distinguish the differences between 'excuse' and 'apology'. As Itoi et al. formulate (see Figure 2.4.1 in Ch.2, Sec.4) in the scheme of accounts, if an apologiser does not utter anything related to his/her responsibility but repeats explanations of the cause, there will be a risk in Japanese that the apologiser's words will be regarded as excuses.

\section{Beseeching}

Regarding beseeching measures, there was only one NS who used this in uttering straightforwardly, ...na n' de yurushite kudasai [As such, please forgive me] in response to the interlocutor. In this discourse, the apologiser had made an offer to the interlocutor to work thirty minutes longer and then the café owner counter-offered by offering not to pay wages for those thirty minutes. The apologiser agreed to the counter-proposal of the café owner, and then uttered, na n' de yurushite kudasai. Judging from the circumstance of the situation, which falls into the negative politeness strategy, the expression of the apologiser contains a nuance not only of beseeching but also of a conclusion to the negotiations with the interlocutor, who was expected thereby to acquit the apologiser of his/her debt. ${ }^{128}$

Six NNSs used a beseeching measure. Their utterances were, for example, Kubi ni shinaide kudasai [Please do not sack me], Konkai wa yurushite kudasai yo [Please forgive me this time], and Watashi wa sutaffu desho, sutaffu wa kazoku mitai deshō [I'm a staff member, and staff are like members of the family, right?]. These were uttered after various types of apologies had been pronounced and then forgiveness begged. According to Brown

128 This applies also to the NNSs' Kubi ni shinaide kudasai [Please do not sack me], and Konkai wa yurushite kudasai yo [Please forgive me this time], since these expressions beg the interlocutor's forgiveness for the debt (see Brown and Levinson 1989: 189). 
and Levinson, "begging forgiveness" is regarded as negative politeness. ${ }^{129}$ The utterances of beseeching by the NNSs were more directly begging forgiveness. ${ }^{130}$

In particular, even the in-group identity marker [I'm a staff member, and staff are like members of the family, right?], which is a positive politeness strategy, ${ }^{131}$ was uttered in the role-play as aforementioned. This type of utterance, which emphasises a speaker him/herself as one of the in-group, is one of the means of asking for acceptance. This, however, did not appear in the utterances of any of the NSs nor in the TXs. This discrepancy may arise from the cultural difference between Japanese participants along with the authors of the TXs, and non-Japanese participants who were mostly native English speakers. In other words, NNSs may tend to use positive politeness in situations where Japanese use negative strategies.

Here utterances of beseeching appeared to be using both negative and positive politeness strategies, albeit only one or a few. In order to obtain forgiveness from the interlocutor, the most common type of beseeching was to beg forgiveness using negative politeness, and was overtly articulated.

In this experiment, placation was the least used strategy by the apologisers. Nevertheless, it is noteworthy that there was no exemplar presented in any of the TXs. It is understandable that the TXs would place the least priority on placation most probably because of the limits of space as well as the fact that the authors may have prioritised individual apologetic expressions over the structure of discourse. Judging from the number of apologetic expressions and explanation of the offence or admission of the facts, it was apparent that these were more important and significant than placation to the authors of the TXs.

\footnotetext{
129 Examples in English are, Please forgive me if..., or I hope you'll forgive me if....

130 Unlike the case of the NS, there was no clear negotiation between the apologiser and the interlocutor.

131 Brown and Levinson 1989: 107.
} 


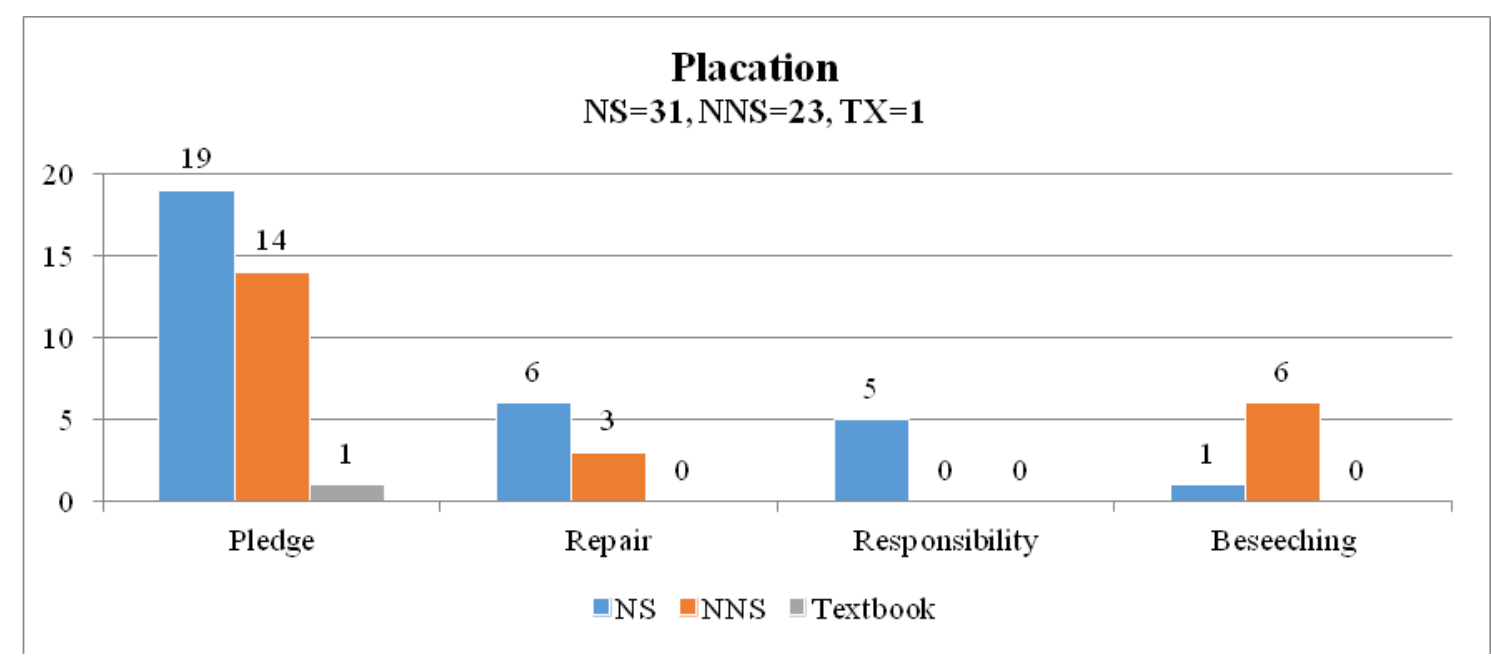

Figure 4.30 Type of Placation

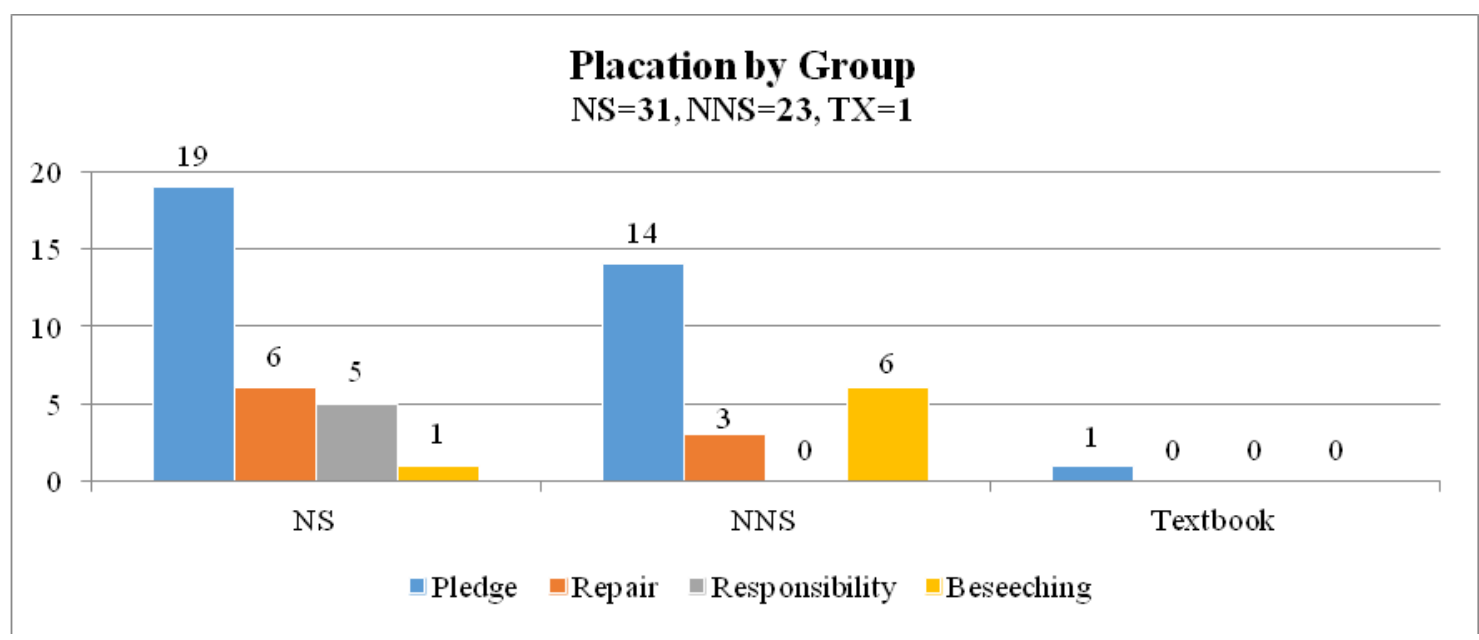

Figure 4.31 Placation by Group

\begin{tabular}{|c|c|c|}
\hline $\begin{array}{c}\text { Placation } \\
\mathrm{NS}=\mathbf{8 0}\end{array}$ & $\begin{array}{c}\text { Placation } \\
\text { NNS }=80\end{array}$ & $\begin{array}{c}\text { Placation } \\
T X=12\end{array}$ \\
\hline $\begin{array}{r}6 \\
6 \% \\
1 \%\end{array}$ & $17 \%$ & $8 \% \quad 0 \%$ \\
\hline$\square$ Pledge & $\square$ Pledge & $\square$ Pledge \\
\hline Repair & Repair & $\square$ Repair \\
\hline Responsibility & Responsibility & Responsibility \\
\hline Beseeching & Beseeching & Beseeching \\
\hline & No Placation & No Placation \\
\hline
\end{tabular}

Figure 4.32 Proportion of Placation 
The discrepancy in the distribution of the subcategories of the NSs and the NNSs is marked. The biggest difference is in beseeching, employed by only three per cent of the NSs but 26 per cent of the NNSs. On the other hand, responsibility was uttered by 16 per cent of the NSs but by none of the NNSs or in the model exemplars. Pledges showed the same proportion, 61 per cent, in groups of the NSs and the NNSs. There were various strategies to placate the anger of the interlocutor, who was the workplace superior of the apologiser. Nonetheless, preferred strategies differentiated one group from the other.

\subsubsection{Structure of Discourse of Apology}

\subsubsection{Initial Utterance}

In the setting of apology, the apologiser's purpose in the discourse is clear, which is to obtain the interlocutor's forgiveness for his/her offence and acknowledge and express regret for the mistake and the offence caused to the interlocutor. The order of utterance reflects the speaker's recognition of the degree of seriousness of the offence. This varies, however, depending on the distance and the hierarchy between the two parties and the level of offence. Nevertheless, in the present experiment, these were already pre-conditioned, so that the differences in the two language groups and the Textbook exemplars - if any-are relatively easily observed. It is worthwhile identifying what and how different the discrepancies are, in order to recognise structural differences in the progression of the discourse. This can be utilised in teaching Japanese by comprehending the differences among the three parties.

Eighty performances by each of the NSs and NNSs, along with 12 TXs, started with one of either apology, admission of the facts, or explanation. There were large differences among them in initial utterances. Seventy-six NSs (95\%) started with apologetic expressions, such as sumimasen [I'm sorry]; on the other hand 29 NNSs $(36 \%)$ out of 80 broached the 
topic by admitting the facts, such as osokunatte [I am late]. The other, explanation before any other utterances, appeared only once and was in a textbook.

Ninety-five per cent of the NSs started the discourse by uttering an apologetic expression. This absolute majority implies that when speakers had admitted their offence to themselves and considered apologising to an interlocutor, they thought that an apologetic word or phrase should come at the very beginning of the discourse. For the NSs, apologising must have been most important because, regardless of the cause of delay, it was irrefutable that the apologiser had inconvenienced the interlocutor. Hence, uttering the apology first in the discourse can be interpreted as expressing his/her feeling of compensation for incurring a nuisance.

On the other hand, the possible reason the NNSs were inclined to some extent to utter admission of the facts first compared with the NSs could be the influence of the TXs, in which the admission of the facts occupied an overall ratio of one-third of the entire initial utterances. A typical sentence of the admission of the facts is okuremashita [I was delayed] and its variations, such as okurete [I am late]. These phrases are statements of the facts followed by the apology. The masu-form and te-form which were employed in these phrases of admission of the facts are basic vocabulary for beginners. As such, it seems to be easy for the NNSs to use them.

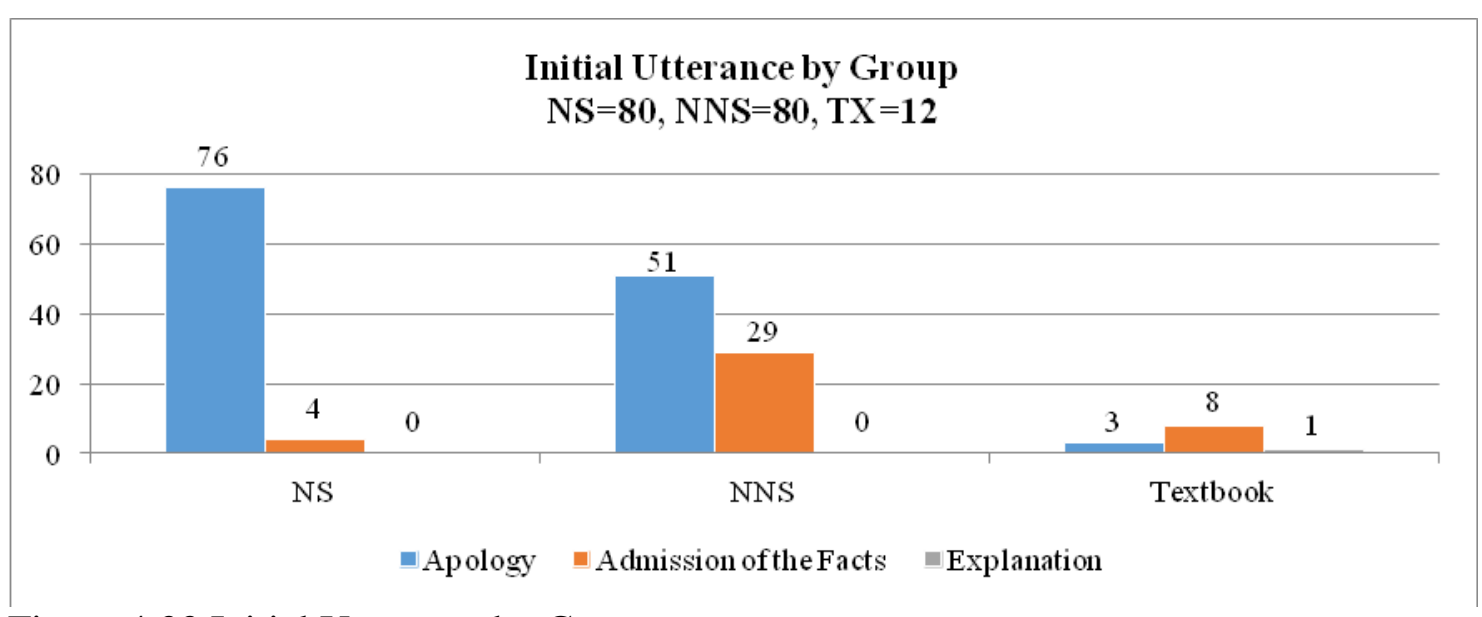

Figure 4.33 Initial Utterance by Group 


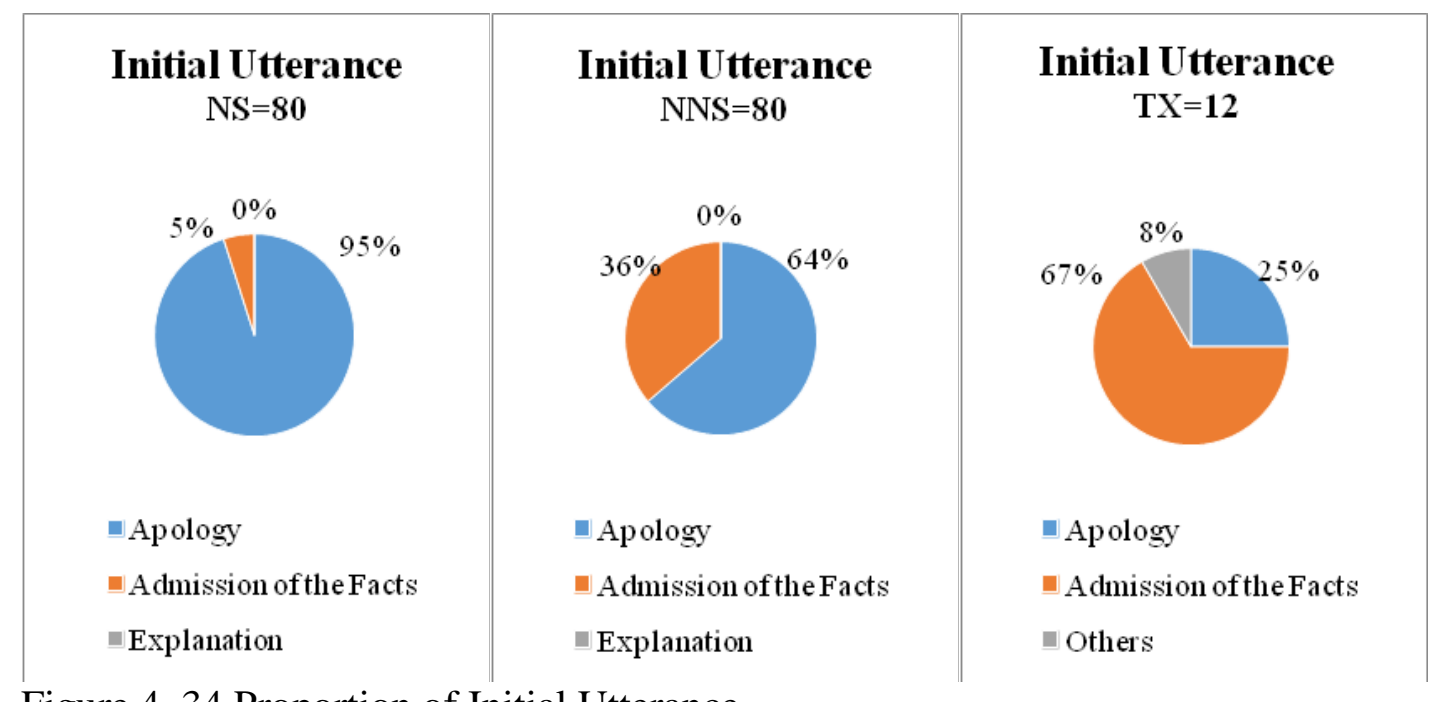

Figure 4. 34 Proportion of Initial Utterance

\subsubsection{Order of Utterance}

\section{Starting from Apology}

The order of utterances starting from the apology was divided into two groups based on the specific utterances that followed the apology. One was an apology followed by admission of the facts, such as Sumimasen, osoku narimashita [I'm sorry, I was late]. There was a considerable discrepancy between the NSs and the NNS in choosing the second sentence after uttering the apology, as the proportion of the NNSs was only about a half of the NSs. Of eighty utterances by the NSs, $63(79 \%)$ were followed by admission of the facts, while among the NNSs, there were 33 such utterances (41\%).

The other group consisted of an explanation uttered immediately after the apology. Thirteen utterances by the NSs $(16 \%)$ and 18 by the NNSs (23\%) fell into this group. A typical example was Sumimasen, nebō shimashite [I'm sorry. I have overslept]. Compared to those who uttered an admission of the facts following an apology, the difference in terms of the number of utterances by the two language groups was not very large in the case of an explanation following the apology. By contrast, the TXs were apt to start from the admission of the facts and not from the apology, so that their results were different from 
those of the NSs and NNSs. Although there may be room for argument, it can be said that the TXs do not really reflect authentic discourse.

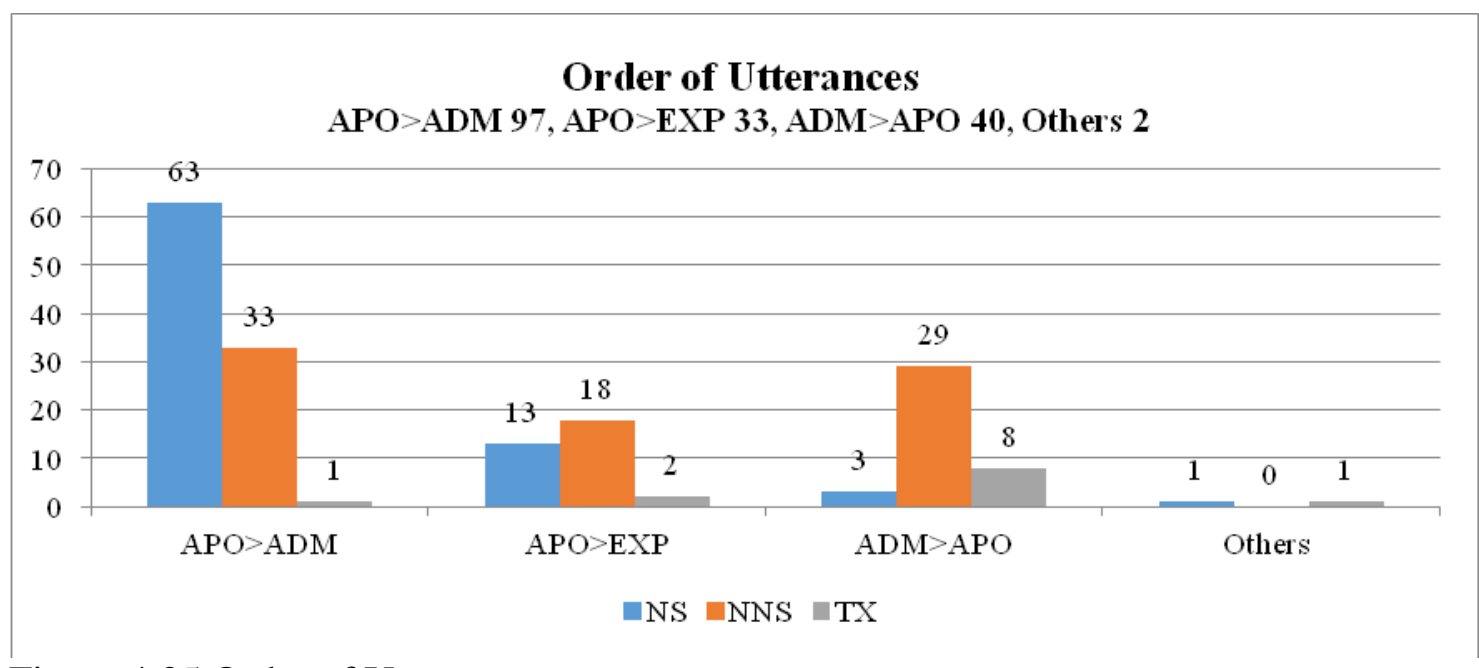

Figure 4.35 Order of Utterances

$\mathrm{APO}=\mathrm{Apolog}, \mathrm{ADM}=\mathrm{Admission}$ of the Facts, $\mathrm{EXP}=$ Explanation

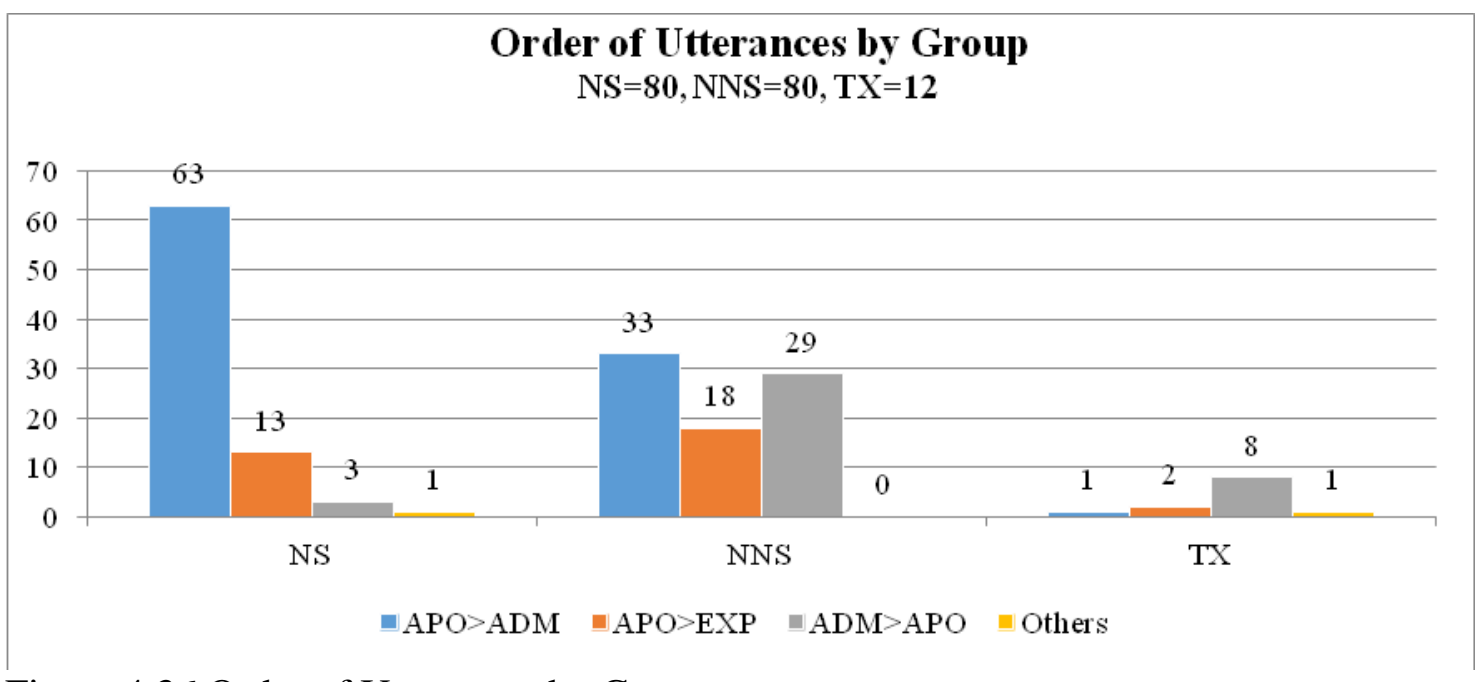

Figure 4.36 Order of Utterances by Group 


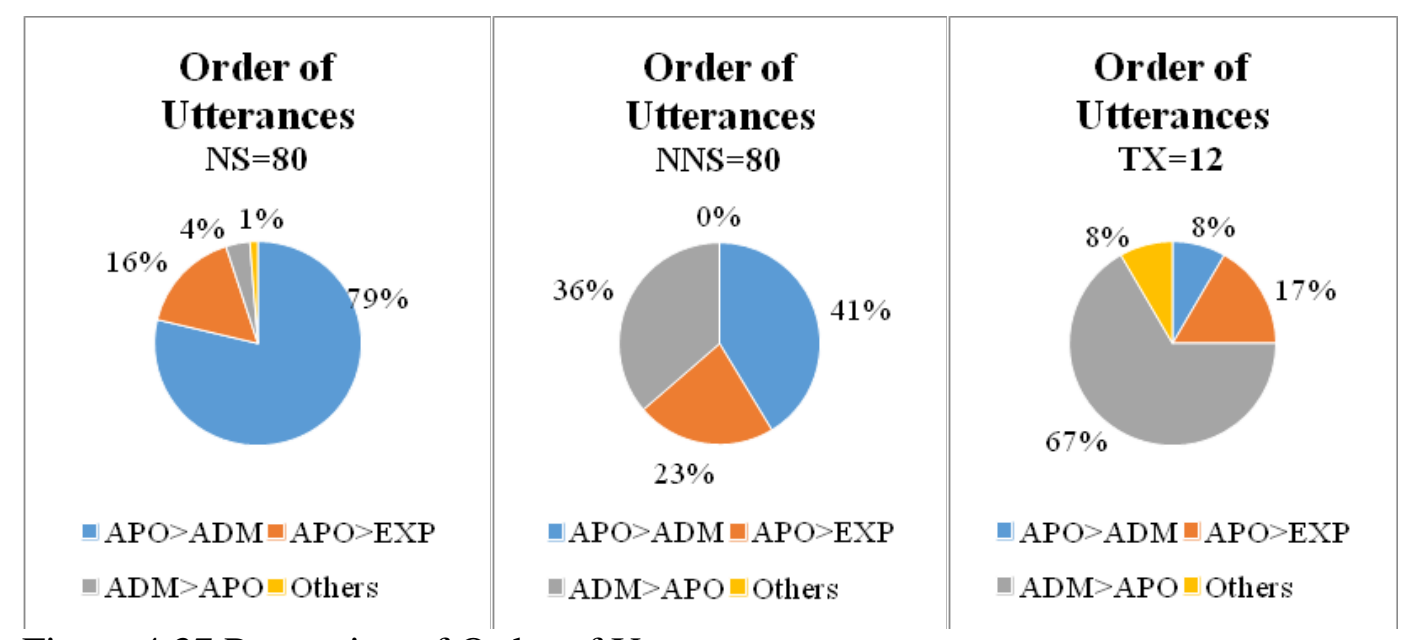

Figure 4.37 Proportion of Order of Utterances

\section{Starting from Admission of the Facts}

A notable difference in the three groups was identified. Twenty-eight NNSs (35\%) uttered an apology after admission of the facts, but the NSs rarely took this strategy as there were only three such utterances $(4 \%)$ by them. A typical utterance by the NNSs was Okurete gomen nasai [I was late, I am sorry]. In contrast to the NSs and the NNSs, the exemplars' proportion of starting with the admission of the facts was as high as 67 per cent; i.e. twothirds of the entire discourse exemplars of the TXs in which the personae started with an admission of the facts was followed by an apology, such as Osokunatte sumimasen [I was late, I am sorry]. The admission part took the te-form, and all exemplars starting with admission of the facts utilised the $t e$-form in their scenarios.

Generating the $t e$-form and completing the sentence was presumably easier for the NNSs than using aposiopesis as many NSs did, such as Sumimasen, okurete...[I am sorry. I was late...]. Although aposiopesis, too, took the te-form by the participants in this present experiment, the difference between aposiopesis and the te-form is that the speaker of the former ends his/her turn by leaving the sentence open, while the speaker of the latter finishes the utterance by completing the sentence, such as Okurete gomen nasai [I'm late. I'm sorry]. 
This might be one of the reasons for more than one-third of the NNSs uttering admission of the facts in the very first broaching. It may be surmised that those who are still studying Japanese might have used the te-form they had already acquired followed by the apology, since they might find it linguistically difficult to leave a sentence unfinished.

The proportion of the NNSs who broached the conversation by means of admission of the facts (35\%) was about half that of the TXs (67\%). Nevertheless, it might be that the exemplars influence the order of utterance of the NNSs. If the NNSs broached the apologetic discourse with admission of the facts followed by an apology under the influence of the textbooks, there is a possibility that a Japanese native-speaker interlocutor may feel a little uncomfortable. This may be inferred because there was a big difference between the NSs and the NNSs/TXs, since only four per cent of the NSs employed an admission as the dialogue starter.

\section{Starting from Others}

There were a few uncommon cases and the breakdown of these is as follows. One each of the NSs and NNSs uttered admission of the facts followed by a promise, one NNS uttered apologies only, and one exemplar started with an explanation first. These were the lowest numbers in each group, so they may be regarded as exceptional.

\subsubsection{Usages of Different Supportive Moves}

Owing to the nature of apology, a sizable number of NSs and NNSs uttered the apologetic expression - the HA — at the very beginning of the discourse. Even if an apology was not uttered at first, the apology followed second, after admission of the facts. In a nutshell, most apologetic expressions were uttered either in the very first sentence or the 
second sentence. Hence, the frequency of usage of supportive moves before uttering the first HA was either HA only or one SM+HA.

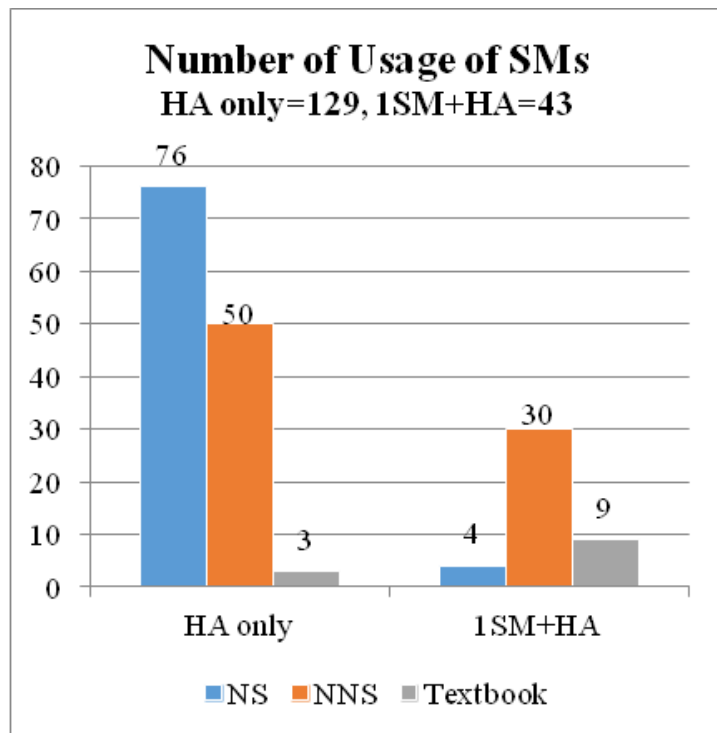

Figure 4.38 Number of Usage of SMs

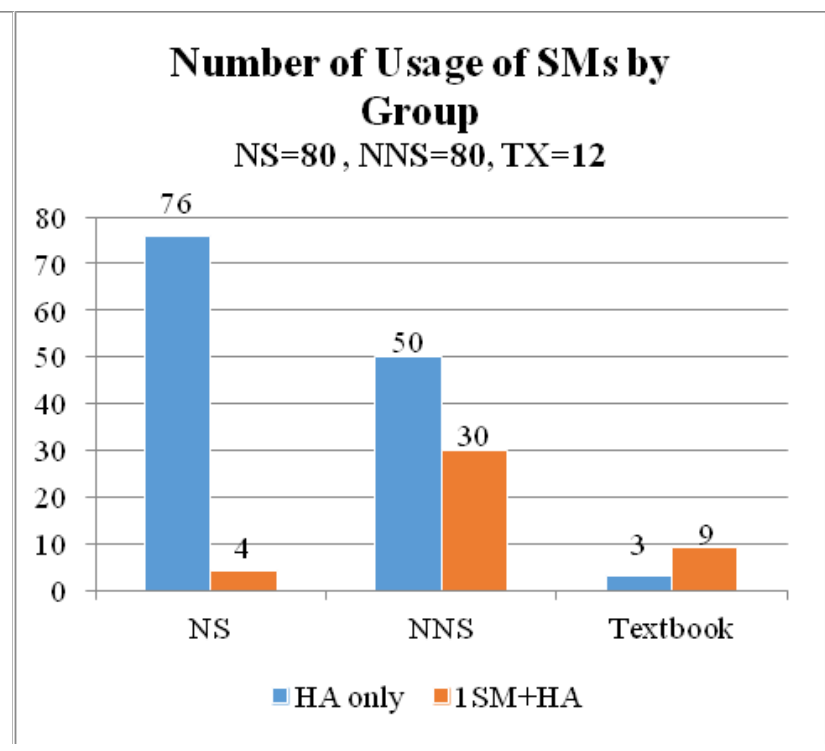

Figure 4.39 Number of Usage of SMs by Group

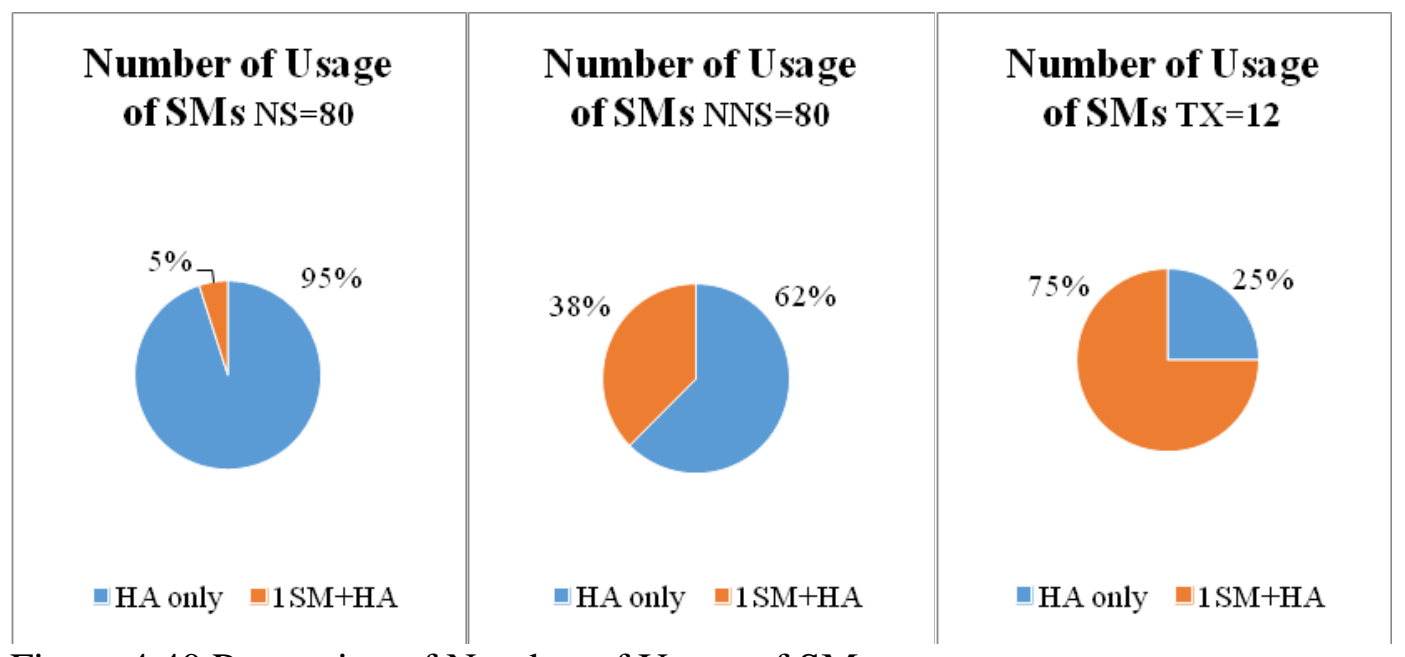

Figure 4.40 Proportion of Number of Usage of SMs 


\subsection{Findings and Discussion}

In this chapter, in order to answer the research questions posed in Chapter 1, the results of analyses are discussed from the aspect of the similarities and differences in the experimental data of native speakers of Japanese (NSs), Japanese language learners (NNSs) and exemplars of the textbooks (TXs) based on Brown and Levinson's politeness theory. The discussion is particularly focused on discourse structures and expressions, followed by discussion including key factors in order to improve the quality of Japanese language textbooks.

\section{Research questions}

To remind the reader, the research questions addressed in this chapter are:

1. What similarities and differences in discourse structures of the settings of apology are perceived among the three groups? The participants/personae are native speakers of Japanese, Japanese language learners, or model exemplars in Japanese language textbooks?

2. What similarities and differences in expressions of the settings of apology are perceived among the three groups? The participants/personae are native speakers of Japanese, Japanese language learners, and model exemplars in Japanese language textbooks?

3. In what way can Japanese language textbooks be improved in order to more closely reflect "authentic" native speaker discourse?

\subsubsection{Structure of Discourse of Apology}

The present research examined the extent to which negative and positive strategies were used in each of the three groups, the NSs, the NNSs, and the TXs. None of the participants in the experiments or the personae in the examples in the TXs employed either 
the bald on record strategy or the off record strategy. They only utilised either the positive politeness strategy or the negative strategy or both in their speech acts. The findings from the analyses of the present study are that there was a similar usage of two strategies, positive and negative, by the three groups. This corroborates Bataineh \& Bataineh's research (2006) which claims that the strategy of apology tends to be close if social and situational factors are close. However, minor differences occurred in the individual sentences and phrases which are categorised by the cross-cultural speech act realization project (CCSARP) into Head Acts (HAs: expressions of apology) and Supportive Moves (SMs). The sentences and phrases categorised were then examined according to Brown and Levinson's politeness theory.

On the other hand, the remaining three SMs, explanation, promise, and placation, are regarded as positive strategies, because these utterances express the feeling that the offender wants the situation of his/her error to be understood and even wants to be liked by the victim or at least wants not to be disliked. For example, an offender might explain the situation to a victim with the intention and hope that the background of the behaviour will be comprehended, and then promise not to repeat the same error again in order to express his/her commitment. Furthermore, the offender tries to conciliate the victim and performs an act to restore the relationship which has been damaged. These actions all emerge from the aim of becoming closer once again to the victim ("repair"). Brown and Levinson argue that discourse of apology is in itself overall a negative politeness strategy.

Nevertheless, when the Japanese discourse in the present experiments was examined at a deeper level, such as through the sentences actually employed, not only was the negative politeness strategy discovered but also positive politeness strategy was employed by some apologisers. This, therefore, does not entirely accord with Brown and Levinson's assertions. What is noteworthy here is that there are no significant differences among the participants and personae of the three groups in terms of the strategies employed in discourses. When all 
sentences and phrases are analysed, on the whole, negative politeness strategy accounted for more or less 70 per cent across the three groups. This means that the positive politeness strategy amounted to nearly a third of the discourses of apology, in contrast to Brown and Levinson's politeness theory for which they claim universality.

Table 4.1 Proportion of Negative and Positive Politeness Strategies by Group

\begin{tabular}{|c|c|c|c|}
\hline Strategies by Group & $\mathbf{N S}$ & NNS & TX \\
\hline $\begin{array}{l}\text { Proportion of Negative } \\
\text { and Positive strategies }\end{array}$ & $\begin{array}{l}71 \% \text { Negative } \\
\text { politeness }(403 / 562)\end{array}$ & $\begin{array}{l}68 \% \text { Negative } \\
\text { politeness }(274 / 400)\end{array}$ & $\begin{array}{l}68 \% \text { Negative } \\
\text { politeness }(31 / 46)\end{array}$ \\
\hline
\end{tabular}

The majority of apologisers, with a few exceptions, delivered both negative and positive strategies in discourses. In detail, the percentage of the negative politeness strategy employed by the NSs was 71 per cent ( 29 per cent positive), and those by the NNSs and the TXs were 68 per cent (32 per cent positive) respectively. In other words, there was little difference overall in terms of the ratio of positive to negative politeness strategy of the structure. In the breakdown of the negative politeness strategy used by the NNSs, it appears that the ratio of the HAs was higher by four points than that of the TXs. This means that the NNSs used HAs more often than the TXs did, although both show the same proportion of the positive and negative strategies at a high level of aggregation (see Table 4.2). It may perhaps be deduced from this that the NNSs were somewhat influenced by the TX discourses.

Table 4.2 Number and Percentage of Utterances by Group

\begin{tabular}{|ll|cc|cc|cc|}
\hline \multicolumn{2}{|l|}{ Politeness Strategy } & \multicolumn{2}{|c|}{ NS } & \multicolumn{2}{|c|}{ NNS } & \multicolumn{2}{c|}{ TX } \\
\hline HA & Negative & 334 & $(59 \%)$ & 210 & $(52 \%)$ & 22 & $(48 \%)$ \\
\hline SM & Negative & 69 & $(12 \%)$ & 64 & $(16 \%)$ & 9 & $(20 \%)$ \\
\hline Subtotal & Negative & 403 & $(71 \%)$ & 274 & $(68 \%)$ & 31 & $(68 \%)$ \\
\hline SM & Positive & 159 & $(29 \%)$ & 126 & $(32 \%)$ & 15 & $(32 \%)$ \\
\hline Total & & $\mathbf{5 6 2}$ & $\mathbf{( 1 0 0 \% )}$ & $\mathbf{4 0 0}$ & $\mathbf{( 1 0 0 \% )}$ & $\mathbf{4 6}$ & $(\mathbf{1 0 0 \%})$ \\
\hline
\end{tabular}

132 Formula: Total number of utterances of negative strategy/Total utterances 


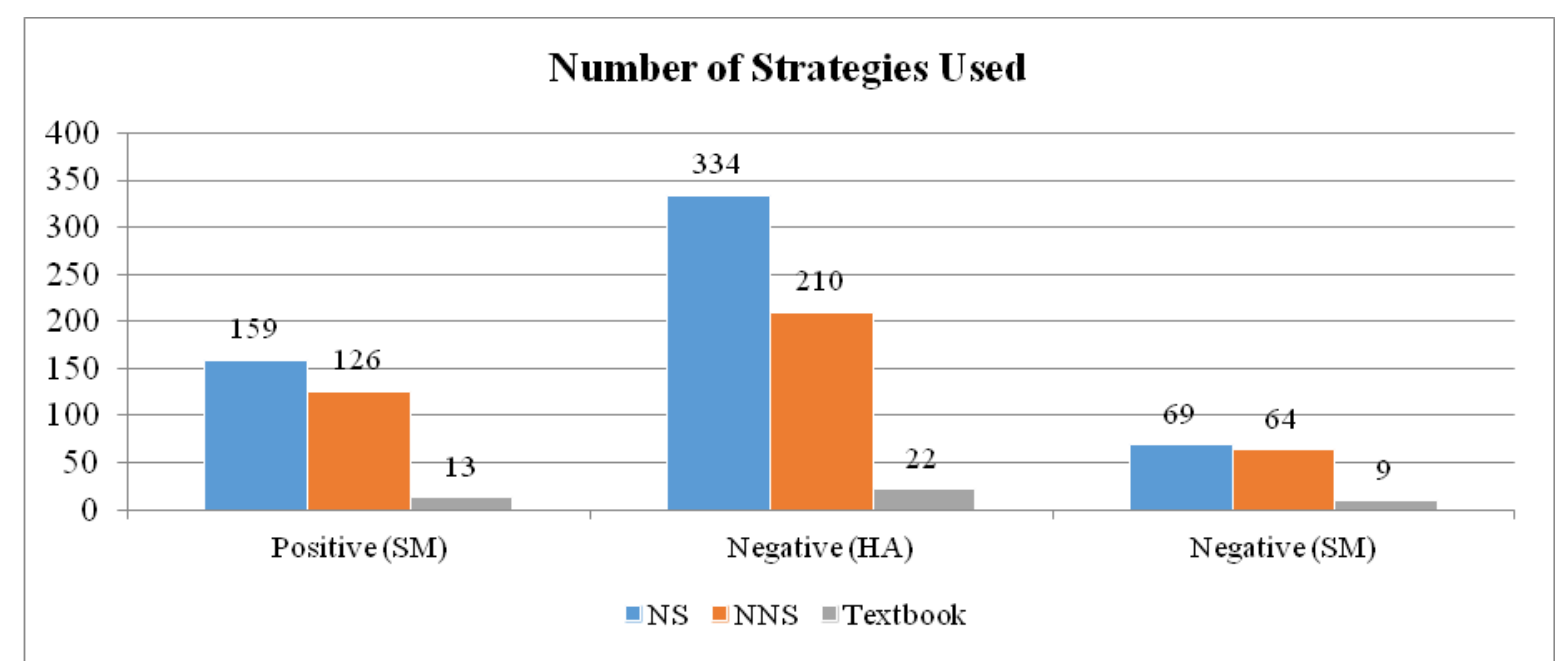

Figure 4.41 Number of Strategies Used

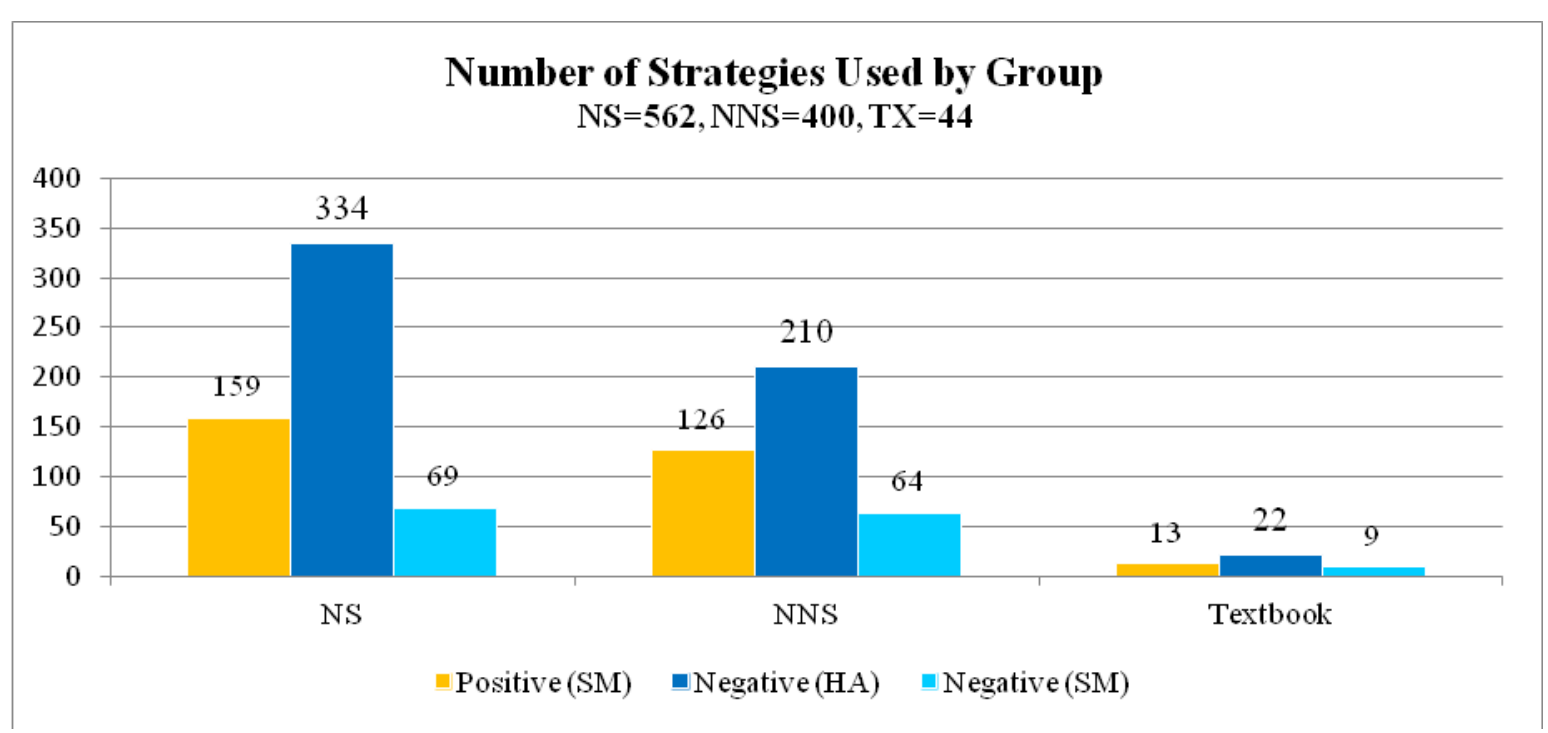

Figure 4.42 Number of Strategies Used by Group

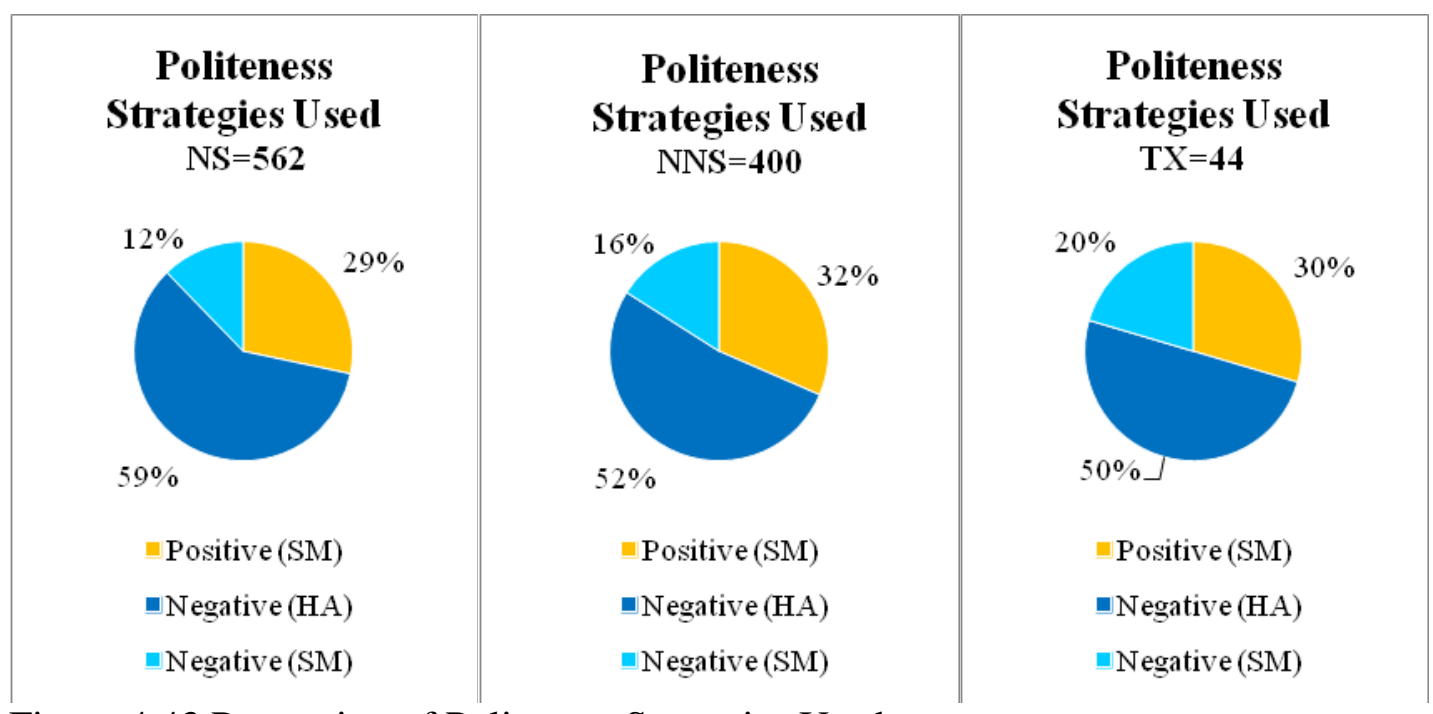

Figure 4.43 Proportion of Politeness Strategies Used 


\subsubsection{Structure of Head Acts}

As was noted earlier in this chapter, an utterance of apology is regarded as the Head Act. A distinguishing characteristic of the NSs in the present study is that, on average, they uttered an apology (HA) 4.2 times in a discourse which is much higher than the 2.6 times of the NNSs and the 1.8 times of the TXs. This finding supports the claims of scholars such as Coulmas (1981), Ikeda (1993), Itoi et al. (1996), Kindaichi (1987) and Sugimoto (1997) that Japanese people apologise more often than others.

Why then, is there a large difference between the NSs and the TXs in terms of the number of utterances of apology, considering that the authors of TXs are all native speakers of Japanese? As Saito (1986: see Ch. 2, Sec. 1) indicates, this might have arisen from a concern of the authors over the length of model discourses. If it were very long in order to get as close as possible to authentic discourse, ${ }^{133}$ learners might find it too difficult to comprehend and lose motivation to study. Furthermore, there is some limitation in producing textbooks, such as pressure of page space exerted by publishers on authors.

On the basis of the present findings, it is evident that in Japanese, if an offender recognises his/her fault and admits misconduct, it is vital to apologise at the beginning of the utterance and repeat the apology many times. This is an issue of pragmatics rather than vocabulary acquisition, of which the authors of textbooks and teachers need to be more aware.

Table 4.3 Characteristics of Head Acts by Group

\begin{tabular}{|l|l|l|l|}
\hline Head Acts & \multicolumn{1}{|c|}{ NS } & \multicolumn{1}{c|}{ NNS } & \multicolumn{1}{c|}{ TX } \\
\hline $\begin{array}{l}\text { Proportion of apologetic utterances (HAs) } \\
\text { in a discourse }\end{array}$ & $\begin{array}{l}59 \% \\
(34)\end{array}$ & $\begin{array}{l}52 \% \\
(210 / 400)\end{array}$ & $\begin{array}{l}50 \% \\
(22 / 44)\end{array}$ \\
\hline $\begin{array}{l}\text { Average number of times HAs occurred in } \\
\text { a discourse }\end{array}$ & 4.2 times & 2.6 times & 2.0 times \\
\hline
\end{tabular}

133 Based on the result the present experiments provided, if a writer of a TX intends to create a new scenario of apology of a similar length to the authentic discourse as possible, the TX would have to be extended to be around 84 per cent longer than the current TXs used for this research.

134 Formula: The total number of Head Acts/total number of utterances. 


\subsubsection{Structure of Supportive Moves}

As Ohbuchi et al. (1989) and Kasugawa et al. (2010) argue (see Ch. 2, Sec. 4), the function of SMs is critical, especially when the victim's annoyance may not be quelled by utterances of apology alone. With regard to the proportion of SMs in a discourse, it was 41 per cent by the NSs, comprising 29 per cent positive politeness strategy and 12 per cent negative politeness strategy, i.e., admission of the facts. Likewise, the SMs of the NNSs comprised 48 per cent (32 per cent positive politeness strategy and 16 per cent negative politeness strategy), and those of the TXs were 50 per cent ( 30 per cent positive politeness strategy and 20 per cent negative politeness strategy).

It is noteworthy that the proportion of the SMs of the TXs comprised just half of all the sentences. This, therefore, equates the total number of HAs, and marked less than those of the NSs and the NNSs. The authors of the TXs seem to recite the possible sentences to be uttered in an apology setting with no prioritisation of them, instead of reconstructing a scene which would actually occur in real discourse.

Apology itself is regarded as a negative politeness strategy, according to Brown and Levinson. However, discourses of apology as a whole have been scrutinised in this research, and the uses of positive politeness strategy, too, have been detected. How much an apologiser cares about the victim, apart from the number of utterances of apology, is examined by grasping the use of SMs in the entire discourse. Supportive moves are used to underpin the intention of the apologisers, especially when it is not easy to convince the victim and obtain an acceptance of the apology. Therefore, by examining the main SMs-explanation and admission of the facts - the apologiser's real thoughts can be revealed.

Among SMs, as previously mentioned, only admission of the facts is classified as negative politeness strategy, and the remaining three are categorised as positive politeness strategy. The result of analyses reveals that unlike the HA, SMs tend towards positive 
politeness strategy, since around 70 per cent of the SMs of the NSs, 66 per cent of those of the NNSs, and 59 per cent of those of the TXs were categorised as positive politeness strategy. Positive politeness strategy in the whole of the discourses analysed in this research is secondary, and far behind the negative politeness strategy in terms of usage. This is because of the high proportion of utterances of apology, which are categorised as negative politeness strategy.

Possible reasons for using positive politeness strategy in SMs may be suggested, together with taking the order of appearance into consideration. After apologisers adopt the negative politeness strategy by uttering an apology (HA, negative) repeatedly, and acknowledge their own misconduct by uttering admission of the facts (SM, negative), they may feel that they have gone too far towards the negative and may then adopt a positive politeness strategy in order to counterbalance the relationship with the victim.

It might be perceived that a high frequency of utterances of apology gives the impression that the whole discourse of apology is very negative in terms of politeness strategy. Moreover, for Japanese native speakers, apologising many times induces the victim (hearer of Japanese) to think: "I should accept the apology now because he/she is seriously and deeply apologising." There seems to be a commonly understood attitude by Japanese native speakers that if an apology is said thoroughly and repeatedly, the victim (hearer) would be hard put not to forgive the apologiser, as Kumagai (2008) argues (see Ch. 2, Sec. 4). Needless to say, it is important for the apologiser to explain the background of the misconduct logically to the victim. Even so, it seems to be effective to apologise repeatedly in the case of Japanese, as this may arouse the sympathy of the victim-insinuating "I/apologiser apologise to you/victim very much"- since it expresses how heartily and fully the apologiser regrets his/her offensive action. 
Table 4.4 Proportion of Main Supportive Moves in Discourse by Group

\begin{tabular}{|l|l|l|l|}
\hline Supportive Moves & \multicolumn{1}{|c|}{ NS } & \multicolumn{1}{c|}{ NNS } & \multicolumn{1}{c|}{ TX } \\
\hline $\begin{array}{l}\text { Proportion of SMs } \\
\text { in discourse }\end{array}$ & $41 \%(228 / 562)$ & $48 \%(190 / 400)$ & $50 \%(22 / 44)$ \\
\hline $\begin{array}{l}\text { (1) Proportion of } \\
\text { Explanation/HAs }\end{array}$ & $\begin{array}{l}27 \%(90 / 334) \\
1 \text { Explanation/3.7 HAs }\end{array}$ & $\begin{array}{l}40 \%(84 / 210) \\
1 \text { Explanation/2.5 HAs }\end{array}$ & $\begin{array}{l}50 \%(11 / 22) \\
1 \text { Explanation/2.0 HAs }\end{array}$ \\
\hline $\begin{array}{l}\text { (2) Proportion of } \\
\begin{array}{l}\text { Admission of the } \\
\text { facts/HAs }\end{array}\end{array}$ & $\begin{array}{l}21 \%(69 / 334) \\
1 \text { Admission/4.8 HAs }\end{array}$ & $\begin{array}{l}30 \%(64 / 210) \\
1 \text { Admission/3.3 HAs }\end{array}$ & $\begin{array}{l}41 \%(9 / 22) \\
1 \text { Admission/2.4 HAs }\end{array}$ \\
\hline $\begin{array}{l}\text { Proportion of two } \\
\text { main SMs/HAs }\end{array}$ & $48 \%(27 \%+21 \%)$ & $70 \%(40 \%+30 \%)$ & $91 \%(50 \%+41 \%)$ \\
\hline
\end{tabular}

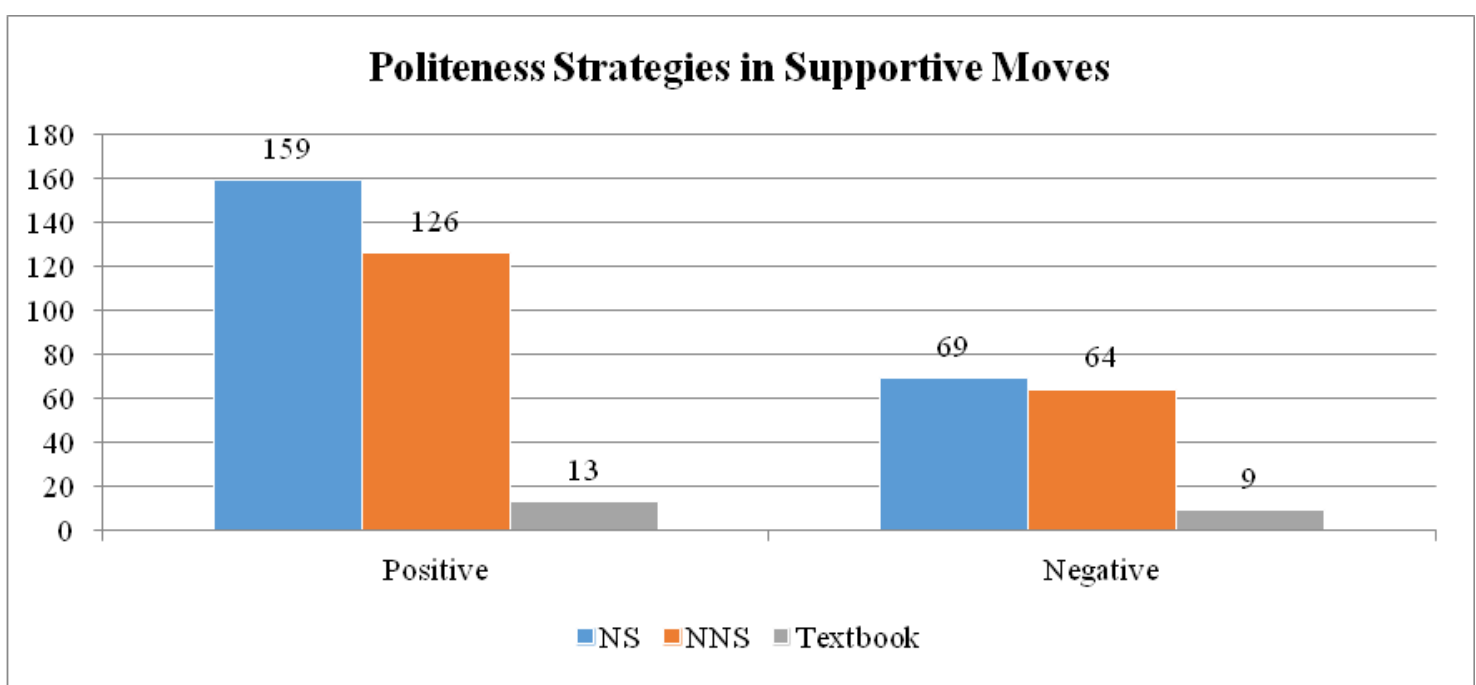

Figure 4.44 Politeness Strategies in Supportive Moves

Positive: Explanation, Promise, Placation, Negative: Admission of the Facts

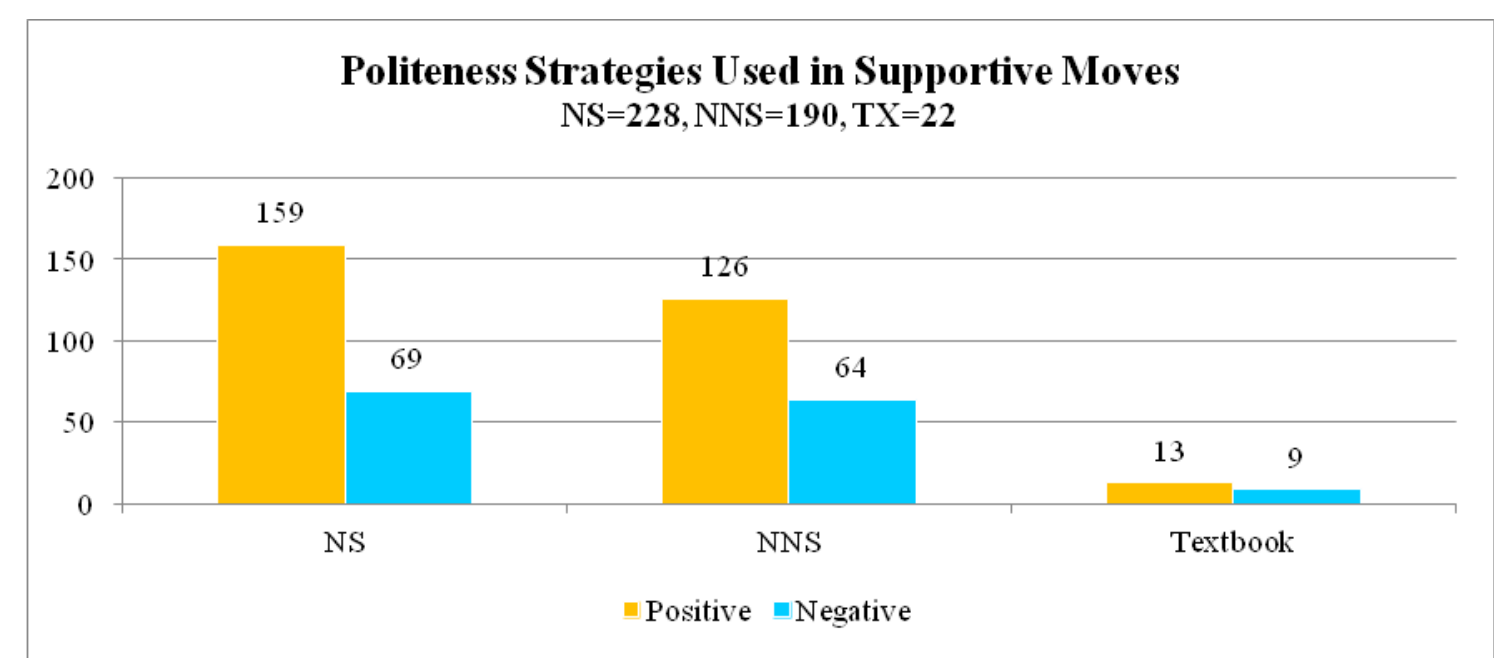

Figure 4.45 Politeness Strategies Used in Supportive Moves 


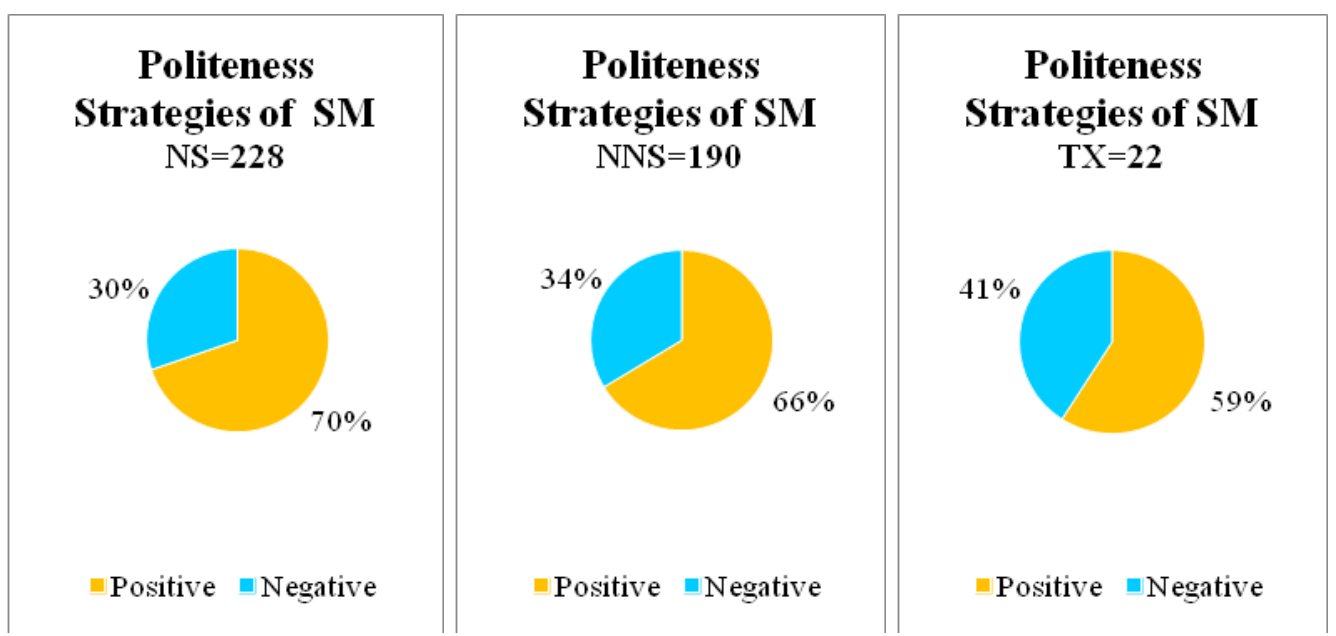

Figure 4.46 Politeness Strategies of Supportive Moves by Group

\section{Admission of the Facts (Negative politeness)}

With regard to admission of the facts which is the only negative politeness strategy among the SMs, the NSs used it 12 per cent of the time in discourses, while it was 16 per cent for the NNSs, and 20 per cent in the TXs. ${ }^{135}$ The proportion of admission of the facts presented in the TXs is thus considerably higher than that of the other two. There does not seem to be much difference in the three groups in terms of the proportion of positive and negative strategies given that they all used negative politeness strategy in around 70 per cent of the entire sentences and phrases. Nevertheless, there were differences in the use of the HAs and admission of the facts. The NSs mostly focused on the HAs; by contrast, the TXs made greater use of admission of the facts. The NSs employed admission of the facts proportionately the least of the three groups, while the TXs used the HAs least. There is a different preference by participants and personae here, in that "model" discourses in the TXs do not reflect accurately the speech acts of native speakers.

Admission of the facts is the second most popular supportive move, but few NSs uttered it in a discourse; only 21 per cent did so compared to 30 per cent of the NNSs, and 41

135 NS: $69 / 562=12 \%$, NNS: $64 / 400=16 \%$, TX: $9 / 44=20 \%$ 
per cent in the TXs. In short, the NS uttered one admission of the facts while they apologised 4.8 times in apologetic utterances; in the case of the NNSs, they uttered it 3.3 times in apologetic utterances, and the TXs presented it 2.4 times. The proportion of these main supportive moves in apologetic utterances is 48 per cent for the NSs, 70 per cent for the NNSs, and 91 per cent for the TXs. From this, it becomes clear that the NSs mostly focused on the apology itself, whereas this occurred in a conspicuously low proportion of apologetic utterances in the TXs. The NNSs fell between the NSs and the TXs. In this respect too, therefore, the TXs diverged from native speakers' discourse.

\section{Explanation, Promise and Placation (Positive politeness)}

Three types of supportive moves were employed in the present study as the positive politeness strategy. These were explanation, promise, and placation. With regard to the use of explanation (e.g. for being late), in the discourses by the NSs, it accounted for 27 per cent of SMs, by the NNSs 40 per cent, and in the TXs 50 per cent. The low usage by the NSs is noteworthy, whereas the TXs presented explanations almost double the number of times that the NSs did. As Itoi et al. (1996), Kindaichi (1987), and Mizutani and Mizutani (1987) (see Ch. 2. Sec. 4) assert, the low use of explanation by the NSs is that the Japanese are generally apt to utter less explanation and more apologies, in marked contrast to the NNSs and the TXs. The reason for this might be that the authors of TXs were preoccupied with introducing sentence patterns, which is an orthodox teaching method, rather than being concerned with the natural flow of the discourse. This corroborates what Kawaguchi, Y. (2005) has already pointed out (see Ch. 2, Sec. 1). In order to improve the content of Japanese language textbooks, presenting an entire coherent discourse to learners must be one of the significant factors for a conversational model, not just several lines of sentences for the purpose of introducing new grammar points. 
Other SMs are promises and placation. The order of frequency is that the NSs demonstrated the highest usage of promise, followed by the NNSs and the TXs. This means that promises were used by the NSs who employed them twice as frequently as the TXs. Placation by the NNSs comprised 11 per cent of their apologetic utterances, while those of the NSs and the TXs were nine per cent and five per cent respectively.

The higher use of placation by the NNSs is their way of trying to shorten the distance or rebuild the relationship between the apologiser (speaker-self) and the victim (hearer). Placation is little used by the NSs, who prefer a self-focused and different means of positive politeness strategy, such as promises. Both promise and placation are regarded as positive strategies in Brown and Levinson's politeness theory, but the concepts behind their use by the participants of the present research seem different. Promising reveals the speaker's intention while placation, on the other hand, directly approaches the hearer, i.e. is other-focused. These different preferences by the NSs and the NNSs are presumably a result of their different cultural and social backgrounds.

It has become clear that preferences in SMs differ among the three groups. In general, the NNSs are located between the NSs and the TXs. While the NSs preferred to employ promise over placation, the NNSs preferred the converse and tended to use placation instead of promise. The TXs, however, exemplified neither in the present study. In other words, it can be surmised that authors of the TXs were more concerned with the contribution of explanation and admission of the facts, as these two main supportive moves play key roles when apologising compared to promise and placation. 


\section{Supportive Moves: Positive Politeness Strategy in the Textbooks}

With regard to the TXs, there is a high proportion of admission of the facts (negative politeness strategy) $)^{136}$ and almost no repeated utterances of apology, as a consequence of which the proportion of positive politeness strategy in SMs is the lowest among the three groups. No assertive means of recovering the relationship between the apologiser and the victim is exemplified in the TXs.

It is understandable that, owing to publishing constraints, the model exemplars cannot display apologies repeatedly in a discourse as frequently as authentic utterances. However, the difference in the number of apologies between the NSs and the TXs is so wide that it cannot be overlooked. The TXs mostly present apologetic expressions only once in a model discourse. If a NNS learner is able to voice an apology correctly in Japanese, but utters it only once or twice at the most, the Japanese victim (hearer) would tend to feel sincerity is lacking or be unsatisfied with the apology, because for Japanese people, one apology alone does not indicate a sincere apology. This point should be explained overtly in textbooks to learners.

\subsubsection{Expressions of Discourse of Apology}

\subsubsection{Expressions of Head Acts}

It has become clear that virtually only three kinds of apologetic expressions were used for head acts (HAs) — sumimasen, gomen nasai, and mōshiwake arimasen - in the present study. As Ogawa (1995, see Ch. 2, Sec. 4) points out, apologetic expressions are regarded as conventional phrases, so learners should recognise their appropriate usage. The usage of the three expressions depends on the setting, mainly the relationship between the speaker

136 Admission of the fact in the TXs took up on average 20 per cent of a discourse and 41 per cent of SMs. 
(apologiser) and the hearer (victim) and the level of damage. Of the three groups, the NSs uttered HAs most frequently, that is, 1.6 times more than the NNSs and 2.3 times more than the TXs.

The preference in apologetic expressions differs according to the group. While the NSs used sumimasen the most, the NNSs preferred gomen nasai, and the TXs mōshiwake arimasen. The TXs differed considerably from the NSs and the NNSs in that they used mōshiwake arimasen four times more often than the NSs and the NNSs, and this is the most polite vocabulary of the three HAs. The TXs take every opportunity to emphasise courtesy. Thus, there is a demonstrably large gap in politeness between the TXs' first choice of apologetic expression and that of the NSs and the NNSs. The reason for the difference may be that the authors were anxious that learners should not cause offence, and therefore considered that a somewhat exaggerated unnaturally polite expression should be learnt by the learners.

The expression sumimasen was the most frequently uttered in this research: 82 per cent in all apologies by the NSs, followed by 73 per cent in the TXs and 66 per cent by the NNSs. It is noteworthy that 27 per cent of the NNSs used gomen nasai, whereas as few as ten per cent of the NSs did so, and there was no use of gomen nasai in the TXs. Another feature discovered here is that the use of the politest expression mōshiwake arimasen by the TXs was as high as 27 per cent, but accounted for only seven per cent of both the NSs and the NNSs. It is notable that the courteousness exemplified in the TXs is easily enunciated by the NNSs, and the NSs came in between the NNSs and the TXs. Regarding the choice of apologetic expressions in the scenario of being late for an appointment, it became apparent that the NSs used a common expression of apology, the NNSs used a simple and plain apology, while the TXs used a careful and polite apology. 
The question now arises as to why mōshiwake arimasen appeared so much more frequently in the TXs, in a quarter of them. By contrast, the NSs scarcely used it at all. This may be because the TXs overemphasised courteousness in the model dialogues. In addition, owing to limited space, they may have preferred to be on the safe (more polite) side as Ogawa asserts (see Chapter 2.4) if presenting apology in a model discourse less than twice.

The function of politeness in Japanese is to create a distance between the relevant parties, i.e. the apologiser and the victim. The distance created by use of the word mōshiwake arimasen is the longest among the three expressions. Gomen (nasai) is the shortest; hence, the speakers who may use this word are restricted, ${ }^{137}$ and it sounds casual (Coulmas: 1981, see Ch.2, Sec. 4). Technically, a superior can choose gomen nasai/gomen yo/gomen ne, ${ }^{138}$ etc. Nevertheless, at present it is difficult to imagine a situation in which a male senior would use gomen nasai to a subordinate.

Table 4.5 Proportion of Apologetic Expressions Uttered by Group

\begin{tabular}{|l|r|r|r|}
\hline Apologetic Expressions & \multicolumn{1}{|c|}{ NS } & \multicolumn{1}{|c|}{ NNS } & \multicolumn{1}{|c|}{} \\
\hline sumimasen & 82 & 66 & 73 \\
\hline gomen nasai & 10 & 27 & 0 \\
\hline mōshiwake arimasen & 7 & 7 & 27 \\
\hline Others & 1 & 0 & 0 \\
\hline Total (\%) & $\mathbf{1 0 0}(\boldsymbol{\%})$ & $\mathbf{1 0 0}(\boldsymbol{\%})$ & $\mathbf{1 0 0}(\boldsymbol{\%})$ \\
\hline
\end{tabular}

\section{Past Tense}

Using the past tense when apologising, such as sumimasen deshita rather than sumimasen (present tense) expresses a speaker's deep emotion of appreciation to the victim,

137 While gomen (nasai) and mōshiwake (arimasen) contain the feeling of regret of an apologiser, sumimasen includes not only this feeling but sometimes appreciation. Moreover, sumimasen implies that the apologiser (speaker) recognises the disadvantage of the victim (hearer) because of the apologiser's offence (Kindaichi: 1987, 78).

138 The phrases, gomen (nasai)/gomen yo/gomen ne, can be uttered by both genders except gomen yo which is normally male speech. 
who has forgiven the apologiser (Sakuma 1983, Kindaichi 1987, see Ch. 2, Sec. 4). It works to shorten the distance which has been widened between the two parties, or to restore it to its original condition. It appears almost always at the end of the discourse in the present experiments. From such a point of view, as Sato (2011) and Yamamoto (2004) affirm (see Ch. 2, Sec. 4), gomen nasai is not appropriate to use to a senior, as Kindaichi (1987) claims (see Ch. 2, Sec. 4), because it does not have a past form (being grammatically a command), although 56 utterances (27\% out of all apologies among the NNSs) contained it.

Thirty-four out of 80 NSs (42\%) used the past tense, as did 13 out of 80 NNSs $(16 \%),{ }^{139}$ and five exemplars in 12 TXs $(42 \%)$. There are three different apologies forming the past tense, i.e., sumimasen deshita, mōshiwake arimasen deshita, and shitsurei shimashita. Sumimasen deshita was uttered far more often than mōshiwake arimasen deshita: 86 per cent of the NSs, 94 per cent of the NNSs, and 80 per cent of the TXs used the past tense of sumimasen out of the total past tense apologies. Shitsurei shimashita was uttered only twice by the NSs. ${ }^{140}$

According to Kindaichi (1987: 78), shitsurei suru [(lit.) 'lose courtesy/good manners, being impolite'] is the weakest among the options in terms of the apologiser's feeling of remorse, repentance, and compensation. Even shitsurei suru/shimasu (present tense) does not necessarily contain the nuance of apology. Instead, it is usually uttered, for example, to the other when one encroaches on the other's space, or in the case of shitsurei shimashita (past tense), it is used when one leaves the other's space where a conversation has been carried out. Owing to these characteristics of shitsurei suru/shimasu (present tense) and shitsurei

139 In the English language, an apology of the past tense, for example, I was sorry is pragmatically inappropriate when apologising at the time of the misconduct. On the other hand, it is appropriate to use the past tense when the apologiser apologises for his/her misconduct which happened in the past and he/she has recalled it.

140 The use of the word Shitsurei shimashita is regarded as an exceptional case in this study, because the number of appearances was minimal and it was used only by the NSs. 
shimashita (past tense), it is inappropriate to use shitsurei suru/shimasu/shimashita when a sincere apology is needed. This is doubtless the reason why the authors of the TXs did not employ this expression in the exemplars of apology.

The frequency of utterance of the past tense was 1.5 times per NS, 1.4 times per NNS, and once per model discourse in the TXs. The similarity in the frequency of use of the past tense by the three groups seems to mean that once they had uttered the past tense, they did not do it again because they thought once was enough. This is evident from the findings on the order of appearance of the past tense in the discourses, particularly in the case of the NSs. All thirty-four NSs who employed the past tense uttered it after the first or subsequent apologies in the present tense, and three out of five exemplars $(60 \%)$ in the TXs presented the past tense in the second apology. ${ }^{141}$ From the results of the use of the past tense by the NSs, it may be inferred that since none of them uttered it in the first apology, it might be that the past tense of an apologetic phrase is not considered appropriate at the beginning of the apology. This result corresponds to Sakuma's and Kindaichi's claim (see Ch. 2, Sec. 4). By contrast, it is notable that eight NNSs out of $13(62 \%)$ used the past tense in the first apology. It may be deduced that those who employed the past tense apology from the outset did not comprehend the implications of the past tense or had not been explicitly taught it.

As Nakada (1989, see Ch. 2, Sec. 4) indicates, a feeling of appreciation on the part of the apologiser is contained in the past tense, and it was found in the present research that all NS participants uttered it only after the victim had accepted the apology. Apology is categorised as negative politeness strategy by Brown and Levinson's politeness theory; however, by using the past tense after the victim has accepted the apology, the past tense apology becomes a positive politeness strategy because it shortens the distance between the two. Therefore, an apologetic expression may not always be classified as negative politeness

141 There was no third apology. 
strategy because, even though it is the same expression, it sometimes works positively in Japanese, depending on the context.

Meanwhile, the NNSs tended to use gomen nasai, which does not have a past tense, rather than the other two apologetic expressions. This may also be one of the reasons they did not use (or could not generate) the past tense as much.

Table 4.6 Past Tense Used by Group

\begin{tabular}{|l|c|c|c|}
\hline The past tense used & NS & NNS & TX \\
\hline $\begin{array}{l}\text { Number of past tense uses by } \\
\text { participant/exemplar }(\%)^{142}\end{array}$ & $34(42 \%)$ & $13(16 \%)$ & $5(42 \%)$ \\
\hline $\begin{array}{l}\text { Number of utterances per } \\
\text { participant/exemplar (times) }\end{array}$ & 1.5 times & 1.4 times & 1.0 time \\
\hline $\begin{array}{l}\text { Appearance order not from the first } \\
\text { apology } 143\end{array}$ & $34(100 \%)^{144}$ & $5(38 \%)$ & $3(60 \%)$ \\
\hline
\end{tabular}

Table 4.7 Expressions of Past Tense

\begin{tabular}{|l|c|c|c|}
\hline Expressions of the past tense & NS & NNS & TX \\
\hline sumimasen deshita (number of use: \%) & $43(86 \%)$ & $17(94 \%)$ & $4(80 \%)$ \\
\hline mōshiwake arimasen deshita & $5(10 \%)$ & $1 \quad(6 \%)$ & $1(20 \%)$ \\
\hline shitsurei shimashita & $2(4 \%)$ & 0 & 0 \\
\hline Total & $\mathbf{5 0}(\mathbf{1 0 0 \% )}$ & $\mathbf{1 8}(\mathbf{1 0 0 \%})$ & $\mathbf{5 ( 1 0 0 \% )}$ \\
\hline
\end{tabular}

The two past tenses of the TXs appearing as the first apology are as follows: one exemplar presents the first apology (in the past tense) Omatase shite sumimasen deshita [I was sorry for having kept you waiting], then the second apology Hontō ni mōshiwake arimasen [Indeed it is inexcusable of me]. Although the second apology uses the present tense, it employs the adverb, hontō $n i$ [indeed, really] in order to emphasise the apologetic expression of Mōshiwake arimasen. This means that since mōshiwake arimasen is recognised as being more polite than sumimasen, as Long (2010) and Sato (2011) point out (see Ch. 2, Sec. 4), this type of apology can be thought of as not impolite or contrary to the social norm

\footnotetext{
142 The past tense used by all participants (all exemplars).

143 Although the participants used the past tense, they did not utter it in the first apology.

144 Formulae: NS; 34/34=100\%, NNS; 5/13=38\%, TX; 3/5=60\%
} 
even if the present tense of möshiwake arimasen is used after the past tense of sumimasen deshita.

Generally speaking, as discussed above, shitsurei shimashita has a weaker nuance of apology. Nonetheless, Kindaichi (1987: 79) argues that by adding the adverb taihen [very, extremely], Taihen shitsurei shimashita [Very/extremely out of courtesy] is even more reparative than Mōshiwake arimasen. If Kindaichi's argument is taken into consideration, the above exemplars in the TXs in which Mōshiwake arimasen deshita (past tense) comes prior to Hontō ni mōshiwake arimasen (present tense), should not necessarily be deemed to be poorly designed model discourses, although none of the NSs uttered the past tense first in the present experiments. In the other example of the TX which started with the past tense followed by the present tense, ${ }^{145}$ it appears to contravene actual speech for no obvious reason.

To summarise, at most there were three apologetic expressions (HAs) used in the present research. The politeness levels of these HAs differ from one another. The individual words, therefore, should be appropriately uttered according to the setting, especially the relationship between the two parties along with the level of imposition. While the politest expression was extensively presented in the TXs, the NNSs depended heavily on a weaker expression of apology. The NSs employed the widely used apology sumimasen, and their usage of the past tense has considerable instructive value, especially for learners. For the NNSs, it is vital to be encouraged to utter the appropriate words for the setting, not only more frequent use of HAs, but also the use of the past tense as a final stage in apologising.

145 Banno, Eri, Yutaka Ohno, Yoko Sakane, Chikako Shinagawa, and Kyoko Tokashiki. 1999. Genki: An Integrated Course in Elementary Japanese II. Tokyo: The Japan Times. (p. 70). 


\subsubsection{Expressions of Supportive Moves}

\section{Admission of the Facts (Negative politeness)}

The proportion of use of admission of the facts was nearly the same in the three groups. Eighty-nine per cent of the NSs, the highest among the three, 80 per cent of the NNSs, and 75 per cent of the TXs used admission of the facts, if pronouncing the fact of the misconduct by an offender overtly, for example, Okuremashita [I was late], is regarded as an apology, as Sakuma (1983) and Oki (2006) affirm (see Ch. 2, Sec. 4).

The scenario of the present research was designed based on the offender admitting what he/she had done was not right, and the victim was the superior whom the offender knew well. Because of the given conditions, "admission of the facts" should have been more easily uttered than in the case where, for instance, the offender did not know the victim, such as having stepped on the foot of a stranger in a crowded train. On such an occasion, it is unlikely that the offender would utter admission of the facts in Japanese, Ashi o fumimashita [I stepped on your foot]. Another example is that if someone has accidentally broken a friend's tea cup in front of him/her, the offender would not utter admission of the facts, such as Kappu o kowashimashita [I broke the tea cup] to someone who has seen what happened, instead of simply uttering the apology of sumimasen.

Admission of the facts, in the case of Japanese, therefore, may not be uttered if the victim is present at the incident, because it is so obvious for both the offender and the victim. In the case of coming late, almost by definition in the present experiments, the offender was not in the victim's presence when the offender should have arrived at the appointed time. Likewise, if a person spilled coffee on a borrowed book and the lender of the book was not present, when the offender returned the book, he/she might acceptably say, Kōhi o koboshite shimai, hon o yogoshite shimaimashita [I spilled coffee on your book and soiled it] as admission of the fact before or after the apologetic expression. 
From these examples, it appears that whether or not admission of the facts is uttered mostly depends on the situation. Admission of the facts seems to be reserved for when the two parties are not in the same proximity when the offence takes place. The high percentage of admission of the facts in the present study would come from the fact (scenario) that the victim was not present at the time.

Moreover, uttering both apology (HA) and admission of the facts (SM) together-as both are negative strategies_ could emphasise the apologiser's feeling of regret more firmly than repeating apologetic expressions alone. Some NSs uttered admission of the facts more often than others and can be recognised as "apology-intensive", because they uttered both apologetic expression (HA) and admission of the facts (SM) most.

Table 4.8 Admission of the Facts Uttered by the Group

\begin{tabular}{|l|c|c|c|}
\hline Admission of the Facts & NS & NNS & TX \\
\hline Uttered & $69(86 \%)$ & $64(80 \%)$ & $9(75 \%)$ \\
\hline Not uttered & $11(14 \%)$ & $16(20 \%)$ & $3(25 \%)$ \\
\hline Total & $\mathbf{8 0}(\mathbf{1 0 0 \%})$ & $\mathbf{8 0}(\mathbf{1 0 0 \%})$ & $\mathbf{1 2}(\mathbf{1 0 0 \%})$ \\
\hline
\end{tabular}

\section{Explanation (Positive politeness)}

\section{Aposiopesis}

Aposiopesis is verbal behaviour in which the speaker stops the utterance in the middle of a sentence, so that the sentence is incomplete from a grammatical point of view. The hearer, however, can surmise the speaker's intention, as in Grice's 'implicature'. There were significant differences in the use of aposiopesis among the three groups: 75 per cent of the NSs and 69 per cent of the TXs utilised sentences of aposiopesis when they were explaining the reason for the delay. On the other hand, only 11 per cent of the NNSs did so. Aposiopesis did not appear in the utterances of promise or placation because of the nature of aposiopesis. The reason for the frequent use of aposiopesis, as Yanabu (1984) and Mizutani (1988, 1991, 1993) indicate (see Ch. 2, Sec. 2), is because it is one of the salient characteristics of the 
conversation of Japanese people, and her argument was corroborated by the present findings. It may be inferred that since the apologiser is reluctant when explaining the reason for the misconduct, his/her hesitancy appears in the utterance in the form of aposiopesis.

A typical expression applying aposiopesis is to use the te-form, such as nebo shite... [I overslept]. While 55 per cent of the NSs and 31 per cent of the TXs used the te-form as aposiopesis, only five per cent of the NNSs did so. The te-form is usually introduced to learners at beginners' level, so the NNSs were familiar with this grammatical form. However, judging from the results of the present study, its use for deliberate purposes of aposiopesis did not seem to have been fully acquired yet by the learners. There is also the probability that it had not been taught explicitly, as in spite of the fact that the exemplars in the TXs use aposiopesis - finishing the sentence by using the $t e$-form totalled 31 per cent in the TXs-it hardly appeared at all in the NNSs' results. Another possible reason is that generating a complete sentence is much easier than aposiopesis for the NNSs, perhaps as a 'direct translation' from their native language.

The authors of nine TXs employed aposiopesis, accounting for 69 per cent. Four exemplars out of nine in the TXs used the te-form, and the other five exemplars used the expression mono desu kara.... The expression mono desu kara presented in the TXs forms aposiopesis. For example, Mezamashi ga naranakatta mono desu kara... [Because my alarm clock did not go off...]. This did not appear in the results of either the NSs or the NNSs. In spite of its being aposiopesis which is usually likely to convey the speakers' hesitancy, however, mono desu kara connotes an excuse-like sentence.

In the Japanese language, an apologiser must avoid the risk of being misunderstood as giving an excuse rather than an explanation of apology (see Itoi et al. Figure 2.4.1 in Ch. 2, Sec. 4). The phrase mono desu kara has two points to be concerned with when using it as an 
explanation of a speaker's misconduct. One is the use of kara [because/since/as], and the other is the use of mono desu [(lit.) thing is]. ${ }^{146}$

The word mono can be used when a speaker (apologiser) explains the situation and/or circumstance of a nuisance in the case of the setting of apology, and the word kara describes the reason subjectively. Kara contains a nuance of the speaker's implication that the reason for the trouble is a matter of course, so that there is a risk of its being interpreted as an excuse or self-defence by a hearer. An example is, Amari oishii mono da kara, tabesugite shimaimashita [It was so delicious that I ate more than enough] (Tamamura 1993: 364) and Mine (1995) in Ch. 2, Sec. 2). In this example, the speaker is the same person as the victim. Therefore, the sentence would not be taken as an excuse to the hearer, who is not a victim. ${ }^{147}$ Owing to these usages, the combination of mono desu and kara in the explanatory sentence has self-assertive and intrusive implications, or at least connotes denial of personal responsibility. As the authors of the TXs are Japanese native speakers, they may have wanted to introduce the phrase mono desu kara as a grammatical structure. However, as Kawaguchi (see Ch. 2, Sec.1) criticises, there are some inappropriate examples such as this case. The

146 The word mono may be used when explaining a reason for something, such as Konkai koko wa shimin ni kaihō shita mono desu [This place was opened to the public this time] and it may also contain a speaker's feeling/wish to be indulged by a hearer, such as Boku wa ikanakatta mono, shiru wake ga nai yo [Since I didn't go, I cannot possibly know, can't I?]. The latter connote something being 'natural' so that it's beyond one's control. These examples were cited and modified from Sanseido kokugojiten [Sanseido Japanese language dictionary] edited by Hidetoshi Kenbo. Tokyo: Sanseido. (1992: 1207).

147 N.B. The word no de functions similarly to kara. The difference between no de and kara is that the implication of no de is an objective one. An example of using no de is Kaigichū datta mono de, odenwa ga osokunatte sumimasen [I'm sorry I'm late phoning, but I was in a meeting] (Tamamura 1993: 364). This dialogue explicitly describes the situation of the speaker (apologiser) who was not able to make a call because of the meeting. It was an unavoidable situation for the apologiser as it was beyond their control, but they still apologised as if it were their responsibility. 
exemplars used for the present experiments are correct from the viewpoint of grammar; however, it can also be said that they are poor examples from the viewpoint of pragmatics.

\section{Attenuation}

It has become clear from the results of the experiments that attenuation in order to mitigate or reduce the bad impression of what a speaker had done (being late for an appointment) was used overwhelmingly more frequently by the NSs than by the NNSs and the TXs. As many as 75 per cent of the NSs employed the word chotto [a little bit] when explaining the reason for lateness; by contrast, its use by the NNSs and the TXs was 18 per cent and only nine per cent respectively. The speaker expects consciously (or even unconsciously) that chotto will work to minimise the negative impact on the hearer, as Matsumoto (1985) and Hidasi (2007) affirm (see Ch. 2, Sec. 2). It is the intention of the speaker to be favoured — or to avoid being reviled—by the hearer. The apologiser admits his/her misconduct, but at the same time wants the fact (misconduct) to be regarded as being as insignificant as possible. By uttering chotto, the apologiser would have been trying to reduce the distance which has been widened in the relationship between the two. This attempt to reduce the distance is one of the positive strategies according to Brown and Levinson (101).

The large gap in the frequency of the word chotto between the NNSs on the one hand and the TXs on the other might be because of the writers of the latter not recognising the effectiveness of the word chotto. They might have not used it in the exemplars because chotto is so often unconsciously uttered by native speakers that they overlooked it, especially if their focus was on grammar and syntax. The authors are likely to have underestimated the efficacy of chotto in apologies and treated it as adjunctive and fragmental. The findings of the present research demonstrate that the NSs employed it very often so that it could work effectively to convey feelings of remorse, and this supports studies by Matsumoto and Hidasi respectively. 
In Japanese, the impression given by the sentence Nebō shimashita [I overslept] compared with Chotto nebō shimashite... [I overslept a little bit...] is quite different, because the latter contains the speaker's feeling of hesitation and even guilt, but the former sounds more like a bald and perhaps even uncaring declaration with a cavalier attitude.

To summarise, the explanation for being late is categorised as positive politeness strategy, and on scrutinising the data, one particular word chotto was found to be utilised mostly by the NSs but was rarely used either by the NNSs or the TXs. Since chotto cannot be used for HAs, ${ }^{148}$ its function is to mitigate a victim's anger, and TXs should pay closer attention to this.

Table 4.9 Proportion of Utterance of Chotto in Explanation

\begin{tabular}{|c|c|c|c|}
\hline Utterance of chotto & NS & NNS & $\mathbf{T X}$ \\
\hline Explanation uttered in discourse ${ }^{149}$ & $75(94 \%)$ & $72(90 \%)$ & $11(92 \%)$ \\
\hline Chotto uttered in explanation ${ }^{150}$ & $56(75 \%)$ & $13(18 \%)$ & $1(9 \%)$ \\
\hline
\end{tabular}

\section{Promise (Positive politeness)}

Promises, such as Ki o tsukemasu [I'll be more careful] remind the victim of the apologiser's strong resolution to amend their behaviour in future. It is noteworthy that 38 out of 80 NSs (48\%) employed promises, whereas only 19 NNSs (24\%) did so. What stands out is that there is only one example of promise presented in the TXs (8\%). This fact may contribute to the low rate of the NNSs' utterance of a promise, as it may be surmised on the basis of this that learners had not been taught to make a promise when apologising.

According to Sugimoto (1997), the Japanese uttered promises about 7.6 times more frequently than Americans (16.7\% vs. $2.2 \%$ ). The data of the present study show that the NSs

\footnotetext{
148 For example, people should never say Chotto gomen nasai [I'm sorry a little bit] when apologising because chotto denies his/her apology.

149 Formulae: NS; 75/80=94\%, NNS; 72/80=90\%, TX;11/12=92\%

150 Formulae: NS; $56 / 75=75 \%$, NNS; $13 / 72=18 \%$, TX; $1 / 11=9 \%$
} 
uttered promises twice as often as the NNSs, and six times more often than the TXs respectively. Although the result of the present study is a much smaller number than Sugimoto's, it shows the NSs tended to utter promises more than the others (see Sugimoto (1997) and Holmes (1990), Ch. 2, Sec. 4).

A promise is a positive politeness strategy according to Brown and Levinson's politeness theory, ${ }^{151}$ so by using this, the NSs were trying to narrow the distance between the two which had been widened by one's transgression. To make a promise means that a speaker (apologiser) expresses firm determination and restriction of his/her future behaviour. Obviously, promises were uttered after apologetic expression(s) and the NSs anticipated forgiveness in return.

Three different types of expressions of promises were recognised through the experiments. These were:

(1) Promises resulting from a decisive intention, which were frequently uttered especially by the NSs, such as Ki o tsukemasu [I'll be more careful].

(2) Promises of countermeasures, such as Hayaku nemasu [I'll go to bed early].

(3) Uttering explicitly the word of promise, Yakusoku shimasu [I promise].

Not much difference appeared between the NSs and the NNSs in the proportion of usage of these three, with only one exception which was that none of the NSs used the particular word yakusoku when they were promising, but three NNSs did so.

For the NSs, uttering determined and concrete expressions or specific countermeasures seems to be more important than explicitly using the word of promise yakusoku. This is because the expressions of promise observed in the present experiments, such as Ki o tsukemasu [I'll be more careful], Hayaku nemasu [I'll go to bed early], implicitly contain the speaker's firm

151 Positive politeness, Strategy 10: Offer, promise (p. 126). 
promise. It is possible to say, however, Yakusoku shimasu [I promise] after having spoken the sentence of resolve, such as Hashitte kimasu, yakusoku shimasu [I'll run here, I promise] uttered by the NNS. ${ }^{152}$

Another aspect of the expressions of promise examined was the type of expressions uttered, either abstract or specific (not abstract). The abstract promise is, for example, Nido to shimasen [I won't do it again] which can be uttered in any circumstances of apology. On the other hand Kondo hashitte kimasu [I'll run here next time] is limited and specific in its use and may be appropriate only when the apologiser's misconduct was a late arrival. It was revealed in the proportions of these two types of apology that the NSs and the NNSs adopted the opposite usage. Seventy per cent of the NSs preferred abstract promises, but by contrast 68 per cent of the NNSs chose specific promises. The probable reason in the NNSs' minds was that specific promises are more suited to convincing the victim than abstract promises, or they might not have been explicitly taught what to say when promising in Japanese, or both. This might be because the TXs do not include examples or explanations.

Table 4.10 Utterance of Promise by Group

\begin{tabular}{|l|lc|ll|rr|}
\hline Promise & \multicolumn{2}{|c|}{ NS } & \multicolumn{2}{|c|}{ NNS } & \multicolumn{2}{c|}{ TX } \\
\hline Uttered & $38(48 \%)$ & 19 & $(24 \%)$ & 1 & $(8 \%)$ \\
\hline Not uttered & $42(52 \%)$ & 61 & $(76 \%)$ & 11 & $(92 \%)$ \\
\hline Total & $\mathbf{8 0}(\mathbf{1 0 0 \%})$ & $\mathbf{8 0}(\mathbf{1 0 0 \%})$ & $\mathbf{1 2}(\mathbf{1 0 0 \%})$ \\
\hline
\end{tabular}

${ }^{152}$ Even if the NSs did not use the word yakusoku, the speaker (apologiser) would have thought that a hearer could recognise the firm determination of the speaker from the context, such as hayaku nemasu. Perhaps, if the apologiser used yakusoku openly, it might have the opposite effect, particularly in a scene of apology. Because the apologiser apparently wants the hearer (victim) to forgive him/her, if the word yakusoku was added on top of the apologetic expression, it would become rather lengthy and even repetitive in terms of meaning. As a consequence, it sounds as if the speaker is forcing the hearer to permit early forgiveness. 
Table 4.11 Characteristics of Expressions

\begin{tabular}{|c|c|c|c|c|}
\hline Characteristics & Expressions & $\mathbf{N S}$ & NNS & TX \\
\hline Abstractive & $\begin{array}{l}\text { Ki o tsukemasu } \\
\text { [I'll be more careful.] } \\
\text { Nido to shimasen } \\
\text { [I won't do it again.] }\end{array}$ & $28(70 \%)$ & $6(31 \%)$ & $1(100 \%)$ \\
\hline $\begin{array}{l}\text { Specific } \\
\text { (Countermeasure ) }\end{array}$ & $\begin{array}{l}\text { Hayaku nemasu } \\
\text { [I'll go to bed early.] } \\
\text { hashitte kimasu [I'll run.] }\end{array}$ & $12(30 \%)$ & $10(53 \%)$ & 0 \\
\hline $\begin{array}{l}\text { Use a word } \\
\text { yakusoku [promise] }\end{array}$ & $\begin{array}{l}\text { Okinai koto o yakusoku } \\
\text { shimasu } \\
\text { [I promise it will not happen } \\
\text { again.] }\end{array}$ & 0 & $3(16 \%)$ & 0 \\
\hline Total & & $\begin{array}{r}40 \\
(100 \%)^{153}\end{array}$ & $19(100 \%)$ & $1(100 \%)$ \\
\hline
\end{tabular}

\section{Placation (Positive politeness)}

Placation was not a prominent feature of the present findings, as 31 NSs, 23 NNSs, and only one TX presented it. Nevertheless, placation is a useful strategy to rebuild the relationship of the two parties positively. Placation was divided into four subcategories: pledge, repair, responsibility, and beseeching.

The typical utterances of pledge according to the present findings were: Ganbarimasu [I'll do my best] and Isshō kenmei hatarakimasu [I'll work as hard as I can]. The former is often used by anyone in any place, whenever the speaker wants to show his/her strong enthusiasm. The phrase ganbarimasu was presumably familiar not to only the NSs (including the authors of one TX), but also to the NNSs. As such, 14 out of 23 NNSs (61\%) who utilised placation (pledge) uttered it. Likewise, 19 out of 31 NSs (61\%) did so.

In order to repair the relationship, six NSs and three NNSs made a self-sacrificing offer, such as doing overtime work, Zangyō shimasu [I'll work overtime]. As Fraser (1981) noted (see Ch. 2, Sec. 4), it is important when apologising to take responsibility for the

153 Several NSs uttered promises more than once. Therefore, the total number was 40, although the number of the NSs who uttered promises was 38 . 
incident, but there was not much utterance of responsibility among the present responses; however, five NSs uttered sentences which clearly enunciated his/her responsibility Jibun no sekinin desu [It's my responsibility]. Because of the apologiser's lateness, it was apparent whose responsibility it was even without their saying so. Yet utterance of responsibility by the apologiser seemed to work positively, at least for the Japanese people. It is noteworthy that none of the NNSs uttered responsibility.

On the contrary, the NNSs used different means, such as beseeching. For example, they begged Konkai wa yurushite [Please forgive me this time]. Six NNSs used beseeching, but only one NS did so. Perhaps reflecting NS usage, the TXs did not include placation at all. As mentioned earlier in Fraser (1990) and Tomlinson (2010) (see Ch. 2, Sec.1), above all, the authors of the TXs did not seem to focus on a discourse-level approach. Placation, which was a type of peripheral or secondary supportive move in this study, was not presented in the model discourses used for the present research.

Table 4.12 Utterance of Placation

\begin{tabular}{|c|c|c|c|}
\hline Placation & $\mathbf{N S}$ & NNS & TX \\
\hline Pledge: Ganbarimasu [I'll do my best.] & $19(24 \%)$ & $14(17 \%)$ & $1(8 \%)$ \\
\hline $\begin{array}{l}\text { Repair: Kyūryō o henjō shite mo ii desu } \\
\text { [I don't mind giving you back my wages.] }\end{array}$ & $6(8 \%)$ & $3(4 \%)$ & 0 \\
\hline $\begin{array}{l}\text { Responsibility: Watashi no sekinin desu [It's my } \\
\text { fault.] }\end{array}$ & $5(6 \%)$ & 0 & 0 \\
\hline $\begin{array}{l}\text { Beseeching: Kubi ni shinaide kudasai } \\
\text { [Please do not give me the sack.] }\end{array}$ & $1(1 \%)$ & $6(8 \%)$ & 0 \\
\hline No placation & $49(61 \%)$ & $57(71 \%)$ & $11(92 \%)$ \\
\hline Total & $80(100 \%)$ & $80(100 \%)$ & $12(100 \%)$ \\
\hline
\end{tabular}

\subsubsection{Order of Utterance}

The order of utterance of apologetic expressions among the NSs corroborated claims of Kindaichi (1987), Kumatoridani (1993, 1995), and Mizutani (1987) (see Ch. 2, Sec. 4). They posit that if an apologiser admits his/her offence, the apology must be presented first. It was conspicuous that the first utterance of 75 NSs (95\%) was an apologetic expression. 
Likewise, 51 NNSs (64\%) also started with an apologetic sentence. The result of the present experiments also accorded with Kumagai (2008) (see Ch. 2, Sec. 4) who argued that the order of appearance in an apology by Japanese native speakers was for the apologetic utterance to come first followed by an explanation. She also found that the order among non-Japanese native speakers is the converse. In this respect, the data of the present study differ from Kumagai's to some extent, because even though proportionally fewer NNSs than NSs uttered the apology first, still about two-thirds of them did so.

The majority of sequences of the NSs, which comprised 79 per cent of the total, started with an apology, then went to admission of the facts. The second most used sequence was apology followed by explanation, which comprised 16 per cent. In the case of the NNSs, apology followed by admission of the facts was 41 per cent, and the reverse sequential order of admission of the facts followed by apology was 36 per cent. Unlike the NSs and the NNSs, the TXs broached admission of the facts first followed by the apology.

Table 4.13 Order of Utterances by Group

\begin{tabular}{|l|rr|rc|rc|}
\hline Order of Utterance & & NS & & NNS & & TX \\
\hline Apology > Admission & 63 & $(79 \%)$ & 33 & $(41 \%)$ & 1 & $(8 \%)$ \\
\hline Apology > Explanation & 13 & $(16 \%)$ & 18 & $(23 \%)$ & 2 & $(17 \%)$ \\
\hline Admission > Apology & 3 & $(4 \%)$ & 29 & $(36 \%)$ & 8 & $(67 \%)$ \\
\hline Others & 1 & $(1 \%)$ & 0 & $(0 \%)$ & 1 & $(8 \%)$ \\
\hline Total & $\mathbf{8 0}$ & $\mathbf{( 1 0 0 \% )}$ & $\mathbf{8 0}$ & $\mathbf{( 1 0 0 \% )}$ & $\mathbf{1 2}$ & $\mathbf{( 1 0 0 \% )}$ \\
\hline
\end{tabular}

Quite unlike either the NSs or the NNSs, the TXs put weight on admission of the facts rather than the apologetic expressions. A possible reason for this could be that they were concerned with the proficiency level of the students. If admission of the facts comes first, followed by apology, learners need only acquire the te-form: okurete+gomen nasai [being late+I am sorry] rather than the other way around, i.e., gomen nasai+okurete [I am sorry+being late]. The problem with the latter is that learners must generate a form of aposiopesis, which, as was seen earlier, is probably not easy for them: teachers are unlikely to have taught how to generate aposiopesis, and textbooks usually do not explain aposiopesis 
either, even when the exemplars employ it. It can be said, therefore, that aposiopesis holds the key to determining the order of utterance of apology. If learners are able to utter an apologetic expression followed by admission of the facts, their utterance is close to that of the NSs and their speech sounds acceptably natural.

The present study concludes that it is most important for the authors of the TXs to unambiguously note that the apologetic part should be foremost in an apology, but this explanation is currently absent in the TXs. The naturalness of the speech of the learners could thereby be improved in the event that they are involved in an incident requiring their apology. Scholars such as Kashiwazaki (2010, 2011) Kato (2004) (see Ch. 2, Sec. 2), and Mizutani (1987) (see Chapter 2, Sec. 4) postulate that the order of utterance indicates the speaker's feeling and many NSs apologised very frequently in their discourses. So the difference in the order of utterance between the NSs and the authors of the TXs may be, as mentioned earlier, because the writers were sensible of the readers' level of proficiency. This is in spite of the fact that if aposiopesis were uttered, it could provide the hearer (victim) with a humble impression which would be more suited to the setting of apology. In this respect, the TXs fail to reproduce authentic native speaker discourse patterns.

\subsubsection{The Degree of Difficulties of Utterances by Learners}

The following two tables are based on the present findings. They consist of only two main elements of apology: an apologetic expression and admission of the facts. One starts with the apology followed by admission of the facts, and the other starts from admission of the facts followed by the apology. These two tables suggest the degree of difficulty for learners when generating an apology. Detailed comparison reveals that numbers $1,2,5$, and 6 may be evaluated as either "easy" or "rather easy" when being uttered by learners. Neither 1 
nor 5 use the $t e$-form nor aposiopesis, so those sentences are formed in basic expressions which the learners must have already studied. In both 2 and 6 , on the contrary, the te-form and aposiopesis are used. Although using the te-form would not be very difficult for the learners of the present research, employing aposiopesis when apologising would not be easy for them or they may not have even imagined its use.

Table 4.14 Utterance Starting from Apology

\begin{tabular}{|l|l|l|l|}
\hline te-form & $\begin{array}{l}\text { Aposio- } \\
\text { pesis }\end{array}$ & Sequence of utterance & $\begin{array}{l}\text { Probability of utterance by } \\
\text { the NNSs }\end{array}$ \\
\hline 1. Not used & Not used & $\begin{array}{l}\text { sumimasen (chotto) } \\
\text { osokunarimashita. }\end{array}$ & Easy \\
\hline 2. Used & Used & sumimasen (chotto) osokunatte... & Rather easy \\
\hline 3. Used & Not used & $\begin{array}{l}\text { sumimasen (chotto) } \text { osokunatte } \\
\text { shimaimashita. }\end{array}$ & Not easy due to shimaimashita \\
\hline 4. Used & Used & $\begin{array}{l}\text { sumimasen (chotto) } \\
\text { osokunarimashite... }\end{array}$ & Difficult due to narimashite \\
\hline
\end{tabular}

Table 4.15 Utterance Starting from Admission of the Fact

\begin{tabular}{|l|l|l|l|}
\hline te-form & $\begin{array}{l}\text { Aposio- } \\
\text { pesis }\end{array}$ & Sequence of utterance & $\begin{array}{l}\text { Probability of utterance by } \\
\text { the NNS }\end{array}$ \\
\hline 5. Not used & Not used & $\begin{array}{l}\text { (chotto) osokunarimashita. } \\
\text { Sumimasen. }\end{array}$ & Easy \\
\hline 6. Used & Used & (chotto) osokunatte..., sumimasen. & Rather easy \\
\hline 7. Used & Not used & $\begin{array}{l}\text { (chotto) } \text { osokunatte shimaimashita. } \\
\text { Sumimasen. }\end{array}$ & Not easy due to shimaimashita \\
\hline 8. Used & Used & $\begin{array}{l}\text { (chotto) } \text { osokunarimashite... } \\
\text { sumimasen. }\end{array}$ & Difficult due to narimashite \\
\hline
\end{tabular}

Number (1): This was the most frequently used form of utterance by the NNSs, so it appears to have been the easiest for them, presumably because neither the $t e$-form nor aposiopesis needed to be used. For a shortcoming, it sounds as if it were a declaration which hardly conveys a feeling of apology.

154 Shimau means to 'put away'. When used as an auxilary verb after a verb in the te form, it indicates that the te form action has been completed; and depending on the context it might convey the speaker's adverse feelings of regret, failure, annoyance, etc. E.g., Tabete shimaimashita means either "[He] ate it all up", or "He went and ate it [to my annoyance]". 
Number (2): The te-form as in (2) osokunatte (or okurete) appears to have been taught to intermediate level learners already, and perhaps aposiopesis, too, has been introduced. However, even if learners have been taught how to create aposiopesis, it appears difficult for them to employ it, because it tends to elicit a question, such as "why" from the hearer, which the NNS apologiser may be linguistically challenged to reply to, unless the following word (or sentence) after aposiopesis has been determined by the apologiser.

In other words, if the apologiser stops his/her apology in the middle of the sentence, i.e., the sentence remains unsaid (aposiopesis), the victim would expect some words/sentences to follow. For example, Sumimasen osokunatte...nebō shita no de [I'm sorry, I'm late... 'cause I overslept]. In particular, the scenario of the present study is that the victim is a boss; therefore, the superior might ask the reason for being late if the apologiser does not continue the utterance, for example, mentioning the reason for the cause. Because of having nothing to say to fill in the moment, the atmosphere of the place has worsened and the apologiser becomes uncomfortable.

Number (5): As with (1), there is no te-form or aposiopesis in (5). So, it is apparently not difficult for learners to employ this order. However, it is inevitably heard by the victim as a declaration rather than as apology.

Number (6): This is similar to (2) as both the te-form and aposiopesis are being used. The difference between (2) and (6) is only in the utterance order of the two words. In (6), as soon as aposiopesis is used, such as osokunatte, the apology can immediately follow, and the apologiser need not think of what should be uttered next because this forms the complete sentence.

Utterances of (3), (4), (7) and (8) are either not easy or difficult for learners, these are, therefore, not to be explored further in the present study. 
On the basis of the above, it is unclear whether or not the authors of the TXs consciously introduced admission of the facts before the apology itself even though native speakers uttered it the other way around. In sum, the acquisition (or teaching) of aposiopesis appears to be one of the main selecting factors for order of utterance (or introduction of apology in model discourses).

\subsection{Summary}

Through the analysis of the data from the three groups of NSs, NNSs, and the TXs, the characteristics of the individual groups have become clear. First, the NSs uttered more sentences than the NNSs per discourse, and the NSs' time per sentence on average was about 40 per cent shorter than that of the NNSs, i.e., the NNSs spoke 1.7 times longer per sentence than the NSs. ${ }^{155}$

The tendency to employ politeness by the three groups was very similar: all of them commonly shared the ratio of positive and negative politeness at around 3 (positive) to 7 (negative). It can also be recognised that the ratios of HA to SM were roughly 6 to 4 (NSs) and 5 to 5 (NNSs/TXs). The uses of four SMs were close to one another with the two main SMs, explanation and admission of the facts especially, showing no remarkable differences. As for making a promise, however, half of the NSs unambiguously declared a promise to the interlocutor, such as intending to leave home earlier in future etc., but the numbers for the NNSs/TXs were far fewer. Likewise, regarding responsibility, 16 per cent of the NSs referred to his/her failure, whereas none of the NNSs/TXs did so.

By contrast, most of the differences were revealed in the expressions employed. The frequent use and the number of utterances of apology varied among the groups. While the

155 The formula is 5.3 seconds/sentence by NNSs/3.1(NSs) $\doteqdot 1.7$ 
NSs were likely to utter sumimasen, the authors of the TXs definitely preferred mōshiwake arimasen to gomen nasai which was uttered by the NNSs the most. The total number of apologies by the NNSs was less than two thirds that of the NSs. Other points of difference were that the past tense of apologetic expressions was used by the NSs/TXs, but not by the NNSs; and there was a huge difference in the use of the attenuating word chotto which was used by the NSs, but not by the NNSs/TXs. Another unique feature of the TXs was that they presented the phrase mono desu kara in the exemplars, which was applied by neither of the other two.

By leaving the end of the sentence unfinished in terms of grammar, aposiopesis conveys the speaker's unspoken implication of remorse and guilt to the interlocutor. The main users of this function were the NSs, followed by the TXs, but the NNSs rarely made use of this. The te-form was the most common way of generating aposiopesis in the three groups, and although it was likely to have already been introduced to the NNSs, only five per cent of them used it.

The very first utterance to a victim is important when apologising. The order of utterances of the three groups could be divided into two groups, the NSs/NNSs and the TXs. Almost all of the NSs concentrated on uttering the apology first, and two-thirds of the NNSs did so, but on the contrary, the first utterance in the TXs was admission of the facts. The order of utterances may not have stood out very much in comparison with vocabulary or expressions in the model exemplars, but the approach to a hearer by the TXs was very different.

In Chapter 4, I analysed the collected data of experiments conducted for apologies. One of the main findings is that only three apologetic expressions were used in the responses, 
and each group preferred different expressions. The different order of appearance of apologetic expressions by each group was also noteworthy. In Chapter 5, I will continue to analyse the data but this time the targeted discourses are the speech acts of requests. 


\section{CHAPTER 5 REQUEST}

\subsection{Overview of Request}

In Chapter 5, similarly to Chapter 4, I will analyse the collected data of the speech acts of requests, and discuss the results. The data will also be illustrated by graphs. The setting of requests, unlike that of apologies, is that the two role-play partners are in an equal relationship.

The experiment of request was carried out, in the same way as that of apology, based on two types of conversational data of role-plays performed by 40 pairs of native Japanese speakers (NSs) and another 40 pairs of non-native Japanese speakers (NNSs). The other consisted of 13 exemplars presented in the selected eleven Japanese language textbooks (TXs) which were published between 1988 and 2008. All textbooks were published in Japan (see Ch. 3).

In the setting of the role-plays of request, both personae were university classmates, so that their relationship was close and socially equal. One of them has not yet started writing an assignment, which is due the following day, while the other has already finished it. The student who has not started the assignment wants some help from the other. Neither of them knows whether or not the other has finished the assignment (see Appendices 1 and 2).

Thirteen model exemplars presented in the TXs were similar to the topic of the roleplays. The relationship of the personae in the exemplars was either that of friends (nine exemplars) or fellow co-workers in a workplace (four exemplars), so their social status was the same. However, the purpose of making a request was slightly different. In seven exemplars, students asked for notes, or some help with homework, etc., while in the case of the other six exemplars of co-workers, the requester asked that a manuscript which he/she 
had written be checked or asked the requestee to be a conversational partner in his/her native language. While these were a little different from the scenario of the role-plays, the act of asking someone of equal social status to help the requester with something which was not a heavy burden was similar.

Five hundred and seventy-two utterances by the NSs and 395 utterances by the NNSs, and 45 utterances of discourses presented in the TXs were collected for analysis of the request. The average number of utterances per discourse was 7.2 by the NSs requesters, 4.9 by the NNSs, and 3.5 in the TXs. The Japanese native speakers who performed the role-plays uttered around 47 per cent more than the NNSs, and about twice as many as the TXs. The average length of a discourse was 62 seconds for the NS pair and 67 seconds for the NNS pair. Of this, the NSs took 4.3 seconds per sentence/requester and the NNSs took 6.8 seconds. This means that the NNSs took about 58 per cent longer to utter a sentence than the NSs.

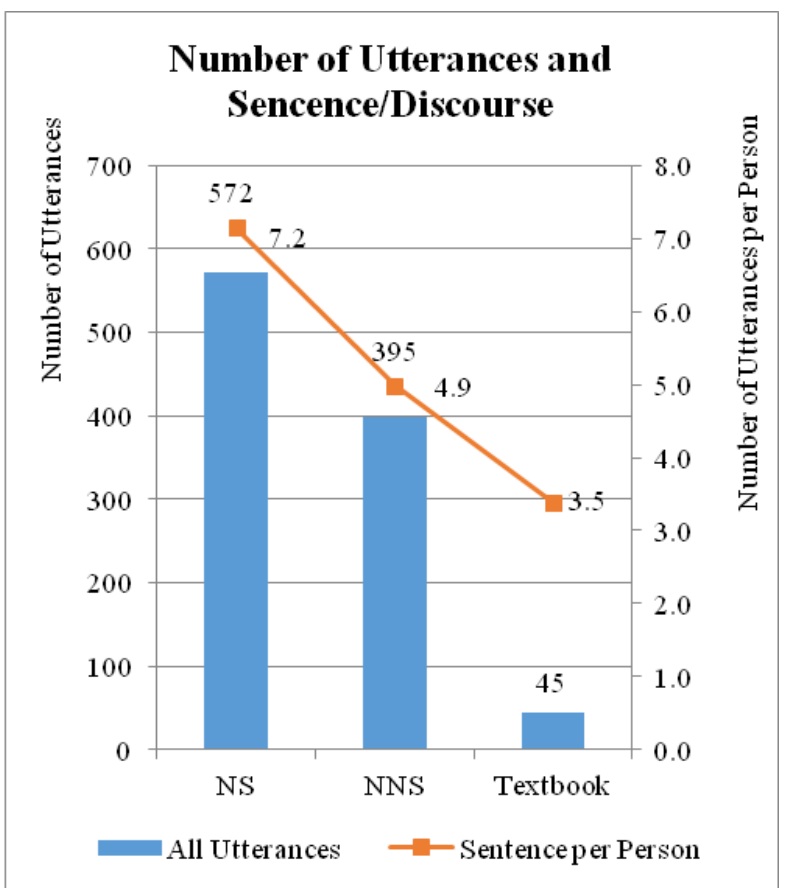

Figure 5.1 Number of Utterances and Sentences

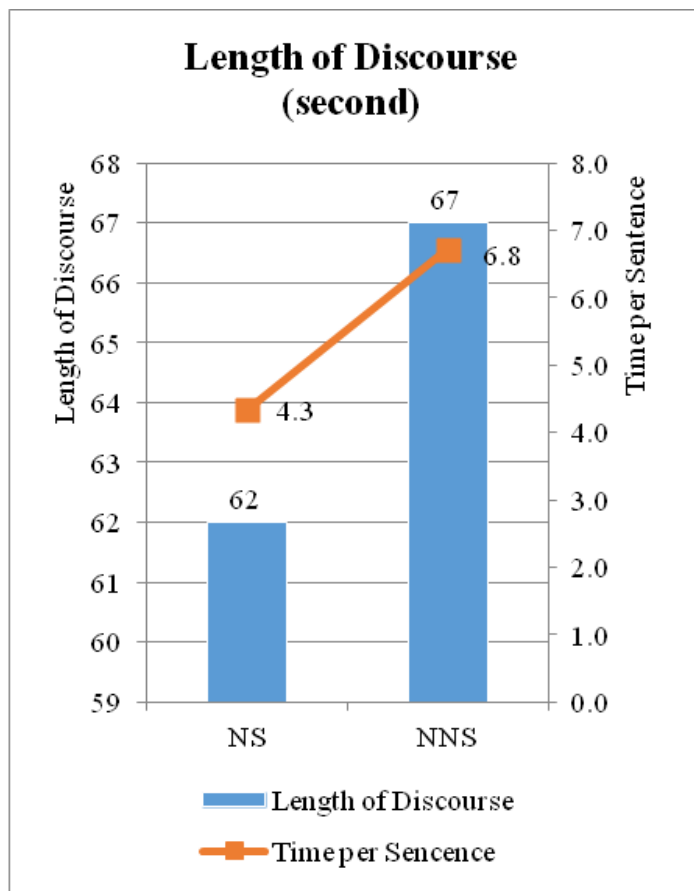

Figure 5.2 Length of Discourse 


\subsubsection{Categorisation of Requisitive Utterances Based on the CCSARP}

As applied to the discourses of apologies (see Ch. 4), the CCSARP separates the head acts (HAs) and the supportive moves (SMs) within large frames. If the discourse study is only focusing on either HAs or SMs, this is insufficient to comprehend the entire picture of the discourse, because in the study of discourses, it is imperative to consider the entire interaction between the speaker and the interlocutor. Primarily, based on the relationship between the two parties and the topic of conversation, the speaker instantaneously determines what kinds of vocabulary and expressions should be used at the time of utterance.

With reference to requests, according to the CCSARP,Blum-Kulka et al. identify a Head Act as "the minimal unit which can realize a request; it is the core of the request sequence" (1989: 275). The example presented in the CCSARP is, John, get me a beer, please. I'm terribly thirsty. ${ }^{156}$ In the present experiment, Nōto misete [Show me your notes], corresponds to the CCSARP as an example to meet these conditions.

The CCSARP deems that the SMs when occurring on their own can be raised to the status of a requisitive Head Act. In other words, it is not necessary for a requester to utter any clear requisitive words when requesting, and the contextual information relevant to the performance of a request can itself assume the status of a request. For example, instead of making a request explicitly, such as Could you clean up this mess? a requester could say, I'm having some friends over for dinner tonight, which is a typical implicit request, namely, Grice's implicature (1989: 276). A similar example in Japanese might be that when urging the return of a book, instead of using a direct expression, Ano kashita hon kaeshite yo [Return that book which I lent you], the sentence Ano hon mada yonderu no? [Are you still reading that book?] implies the speaker's wish for the return of the book, although there are no explicit requisitive words/phrases in the utterance.

156 In the CCSARP Coding Manual (275). 
In the present experiments, therefore, the entire dialogue of each discourse was categorised functionally according to the CCSARP method into the HAs and the SMs. On the other hand, because Brown and Levinson's politeness theory analysed the feeling of the speaker when uttering the main topic, this theory was utilised for the analyses of the HAs. By combining both approaches, it is possible to grasp the structure of the whole discourse within a large frame and the speaker's process in developing the discourse.

In the CCSARP, there is another part, described as Alerter. This is an element in the discourse which alerts the hearer, such as a name, title, pronoun, etc., to what will likely follow. For the setting of the present experiments, this part was excluded because it was deemed irrelevant in the scenario where the conversational partners were friends/co-workers of the same social status.

As seen earlier, the Supportive Move is a unit external to the request, but which, however, modifies its impact (1989: 276). Not all SM categories in the CCSARP were utilised for the present study because some of them, such as insult, threat, etc., were not relevant to the present experiments.

The categorisation used in this study was as follows:

Head Act:

(1) Request, such as Misete kurenai? [Wouldn't you let me see it?].

Supportive Moves: ${ }^{157}$

(2) Preparator (Preparator in this study), such as Mó yatta? [Have you finished it yet?],

(3) Grounder (Grounder), such as Mada yatte nai n' da [I haven't started yet],

(4) Disarmer (Disarmer), such as Tomodachi darō? [You're my friend, aren't you?],

(5) Promise of reward (Reward), such as Ranchi ogoru yo [I'll buy you lunch],

(6) Imposition minimiser (Minimiser), such as Chotto dake [Only a little bit].

157 The order of Supportive Moves here conforms to the CCSARP. 
In the CCSARP, a preparator means that the speaker's intention is to get an interlocutor mentally prepared for dealing with something that the speaker is going to say. In the case of a request, the speaker may specifically ask something that will indicate whether or not the interlocutor is capable of accomplishing the request, but the speaker does not impart the contents or the essence of the request. Examples of preparators presented in the CCSARP are, I'd like to ask you something [Kikitai/tazunetai/onegai shitai koto ga aru n' desu ga] or May I ask you a question? [Shitsumon shite mo ii desu ka?].

Although the CCSARP separates 'getting a precommitment' from 'preparator'. 'Getting a precommitment' is an utterance prior to making a request to test whether or not there is a possibility that the interlocutor may refuse it. A sample sentence of 'getting a precommitment' presented in the CCSARP is Could you do me a favour?, and its translation in Japanese is [Onegai ga aru n' desu ga]. There does not seem to be any difference in terms of the way of thinking for both categories in Japanese, so 'getting a precommitment' is included in 'preparator' in this study. Once the sentence, Onegai ga aru n' desu ga [Could you do me a favour] is uttered as 'getting a precommitment', the interlocutor should recognise it as the CCSARP's preparator and get ready to listen to the speaker's next utterance in the case of Japanese. In consequence, there is no difference in Japanese between getting the interlocutor to prepare for listening to the speaker's next utterance and confirming the interlocutor's possibility of refusing a request. Unless it was amalgamated, it would have been hard to separate Onegai ga aru n' desu ga that the participants uttered. Hence, in this study, the supportive move of 'getting a precommitment' is merged into the preparator. 


\subsection{Analysis of Request}

\subsubsection{Outline of Strategies of Request}

The Head Acts (HAs) and the Supportive Moves (SMs) of request of native speakers (NSs), those of non-native speakers (NNSs) and personae in model exemplars in textbooks (TXs) were compared by extracting them from the components of their discourses.

The items analysed were:

(1) The total number of utterances and utterances per person/persona

(2) The proportion of utterances of the three groups (NSs, NNSs, and TXs)

(3) Structure of discourses

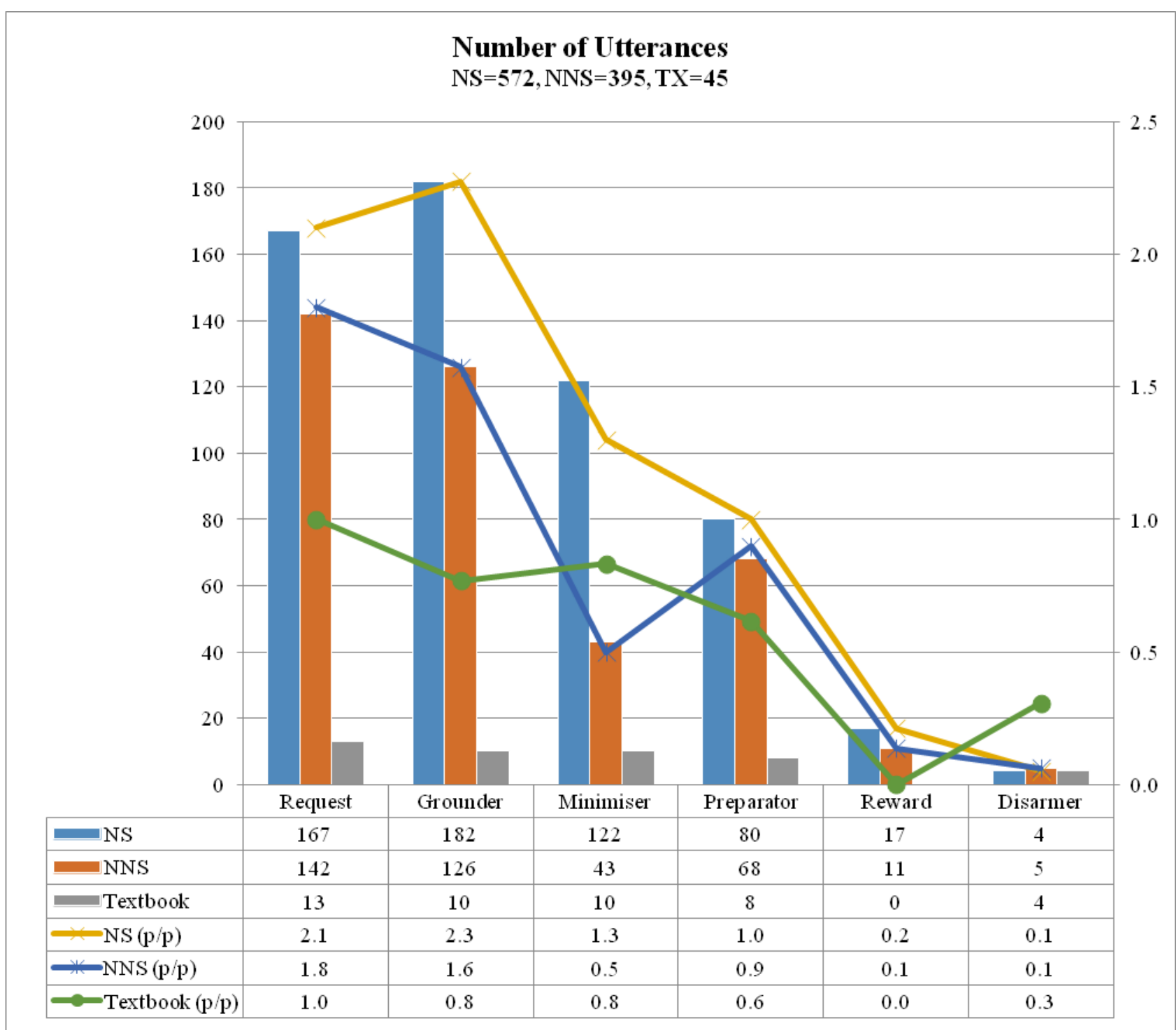

Figure 5.3 Number of Utterances 


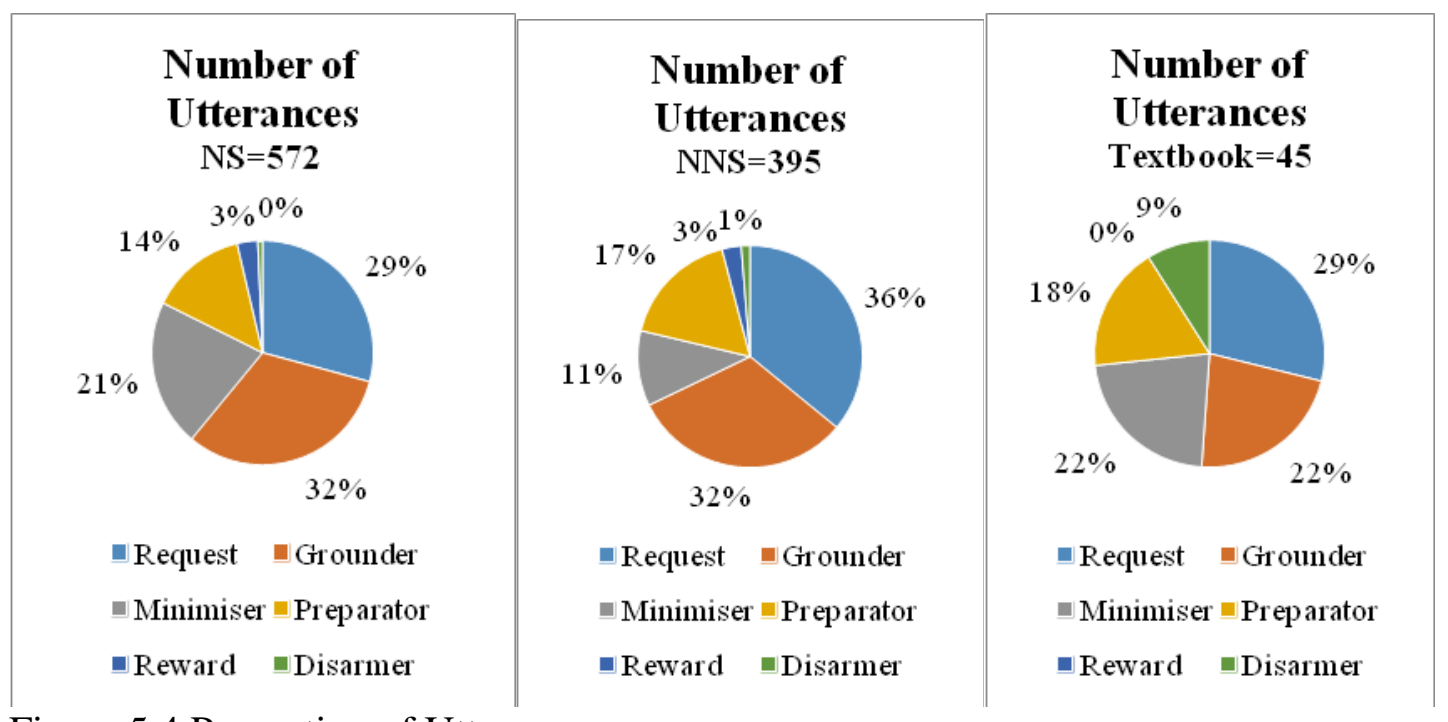

Figure 5.4 Proportion of Utterance

\subsubsection{Head Acts of Request}

The Head Act of the NSs was compared with that of the NNSs and of the exemplars of the TXs by extracting it from the individual discourse.

\subsubsection{Number of Head Acts}

There were 322 requisitive utterances as HAs in total. Their details were: 167 by the NSs (an average of 2.1 times per requester), 142 by the NNSs (an average of 1.8 times per requester), and 13 in the TXs (an average of 1.0 times per persona). The HAs per NNS were about 15 per cent less than those of the NSs. 


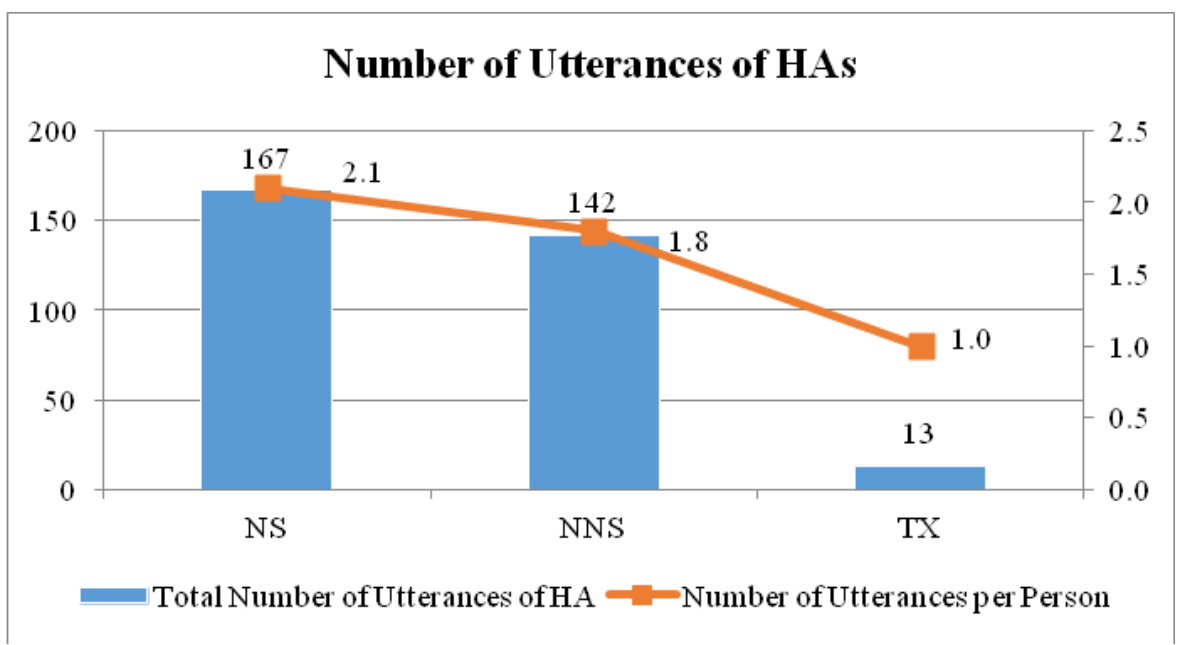

Figure 5.5 Number of Utterances of Head Acts

Regarding the number of utterances of requisitive sentences (HA) in a discourse, analysis reveals that 28 NSs (35\%) uttered HAs twice and 23 NSs (29\%) did so only once, although the average use by the NSs was 2.1 times. Hence, around two-thirds of the NSs (64\%) uttered either one or two requisitive sentences. Twenty-five NSs (31\%) uttered more than three times, in contrast with four NSs who did not utter the HA. ${ }^{158}$

Among the NNSs the average number of HA utterances was 1.8 times in a discourse. Forty-four NNSs (55\%) uttered the HAs only once, and 22 NNSs $(27 \%)$ did so twice. Those who uttered the HA once or twice reached 82 per cent of all NNSs, and 13 NNSs (17\%) uttered the HAs more than three times. One NNS did not employ a HA. The biggest difference between the NSs and the NNSs in their frequency of the HA utterances is that, while the number of the NSs who uttered one HA was only about half that of the NNSs, the number who uttered the HA more than three times was about double that of the NNSs. By contrast, none of the personae in the TXs uttered more than one HA. The majority of the NS and the NNS participants, as well as the model exemplars presented in the TXs, employed one or two requisitive sentences in a discourse. Nevertheless, it is conspicuous that around one-third of the NSs (31\%) uttered them more than three times.

158 They only uttered SMs (no HA), yet developed the discourse of request. 


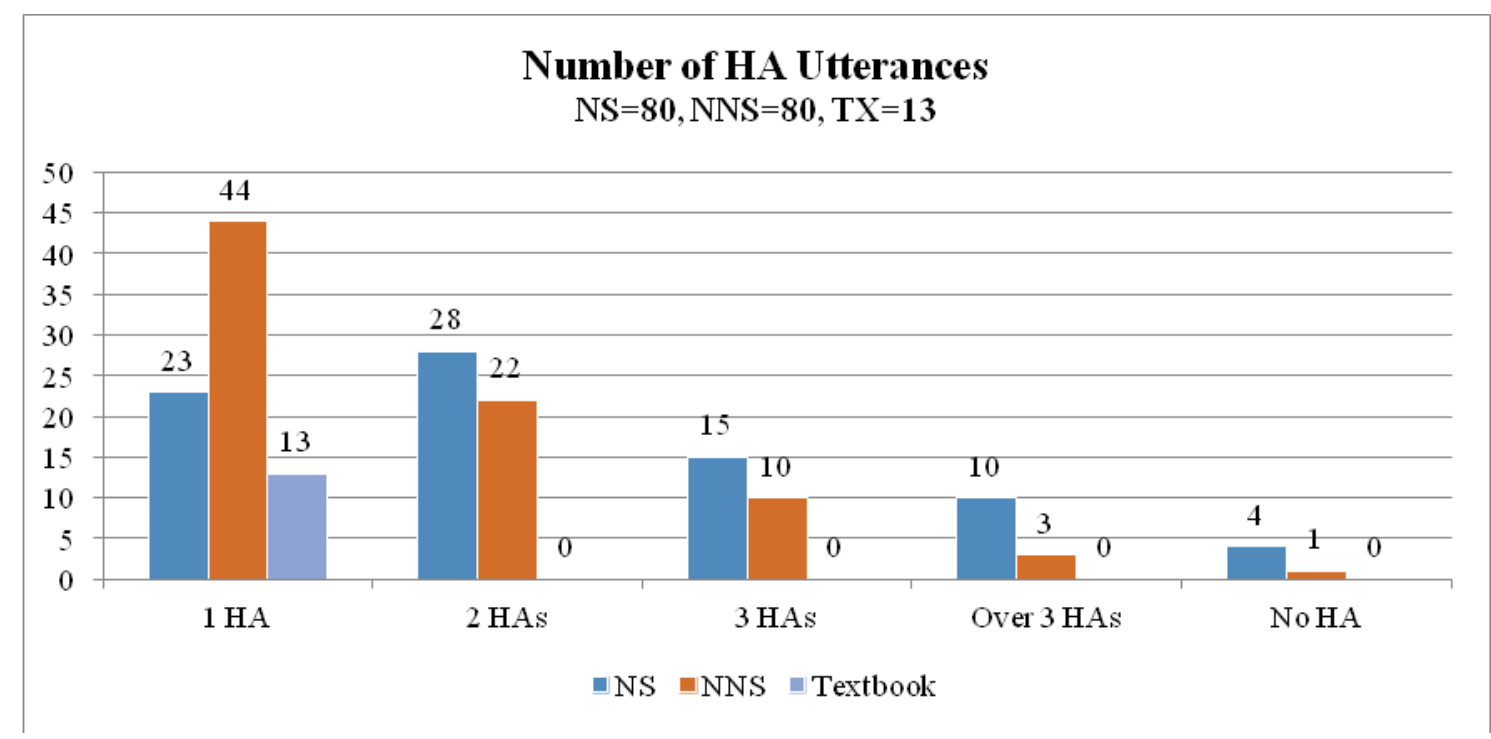

Figure 5.6 Number of HA Utterances

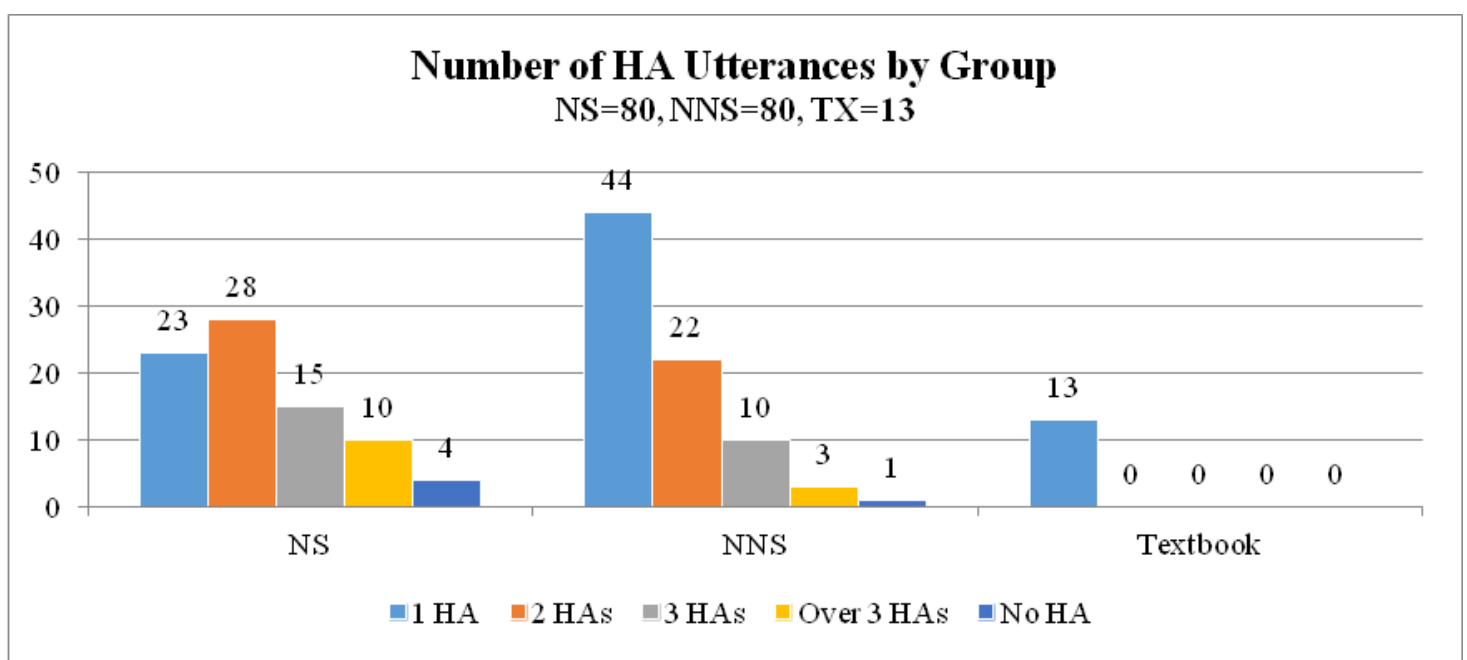

Figure 5.7 Number of HA Utterances by Group

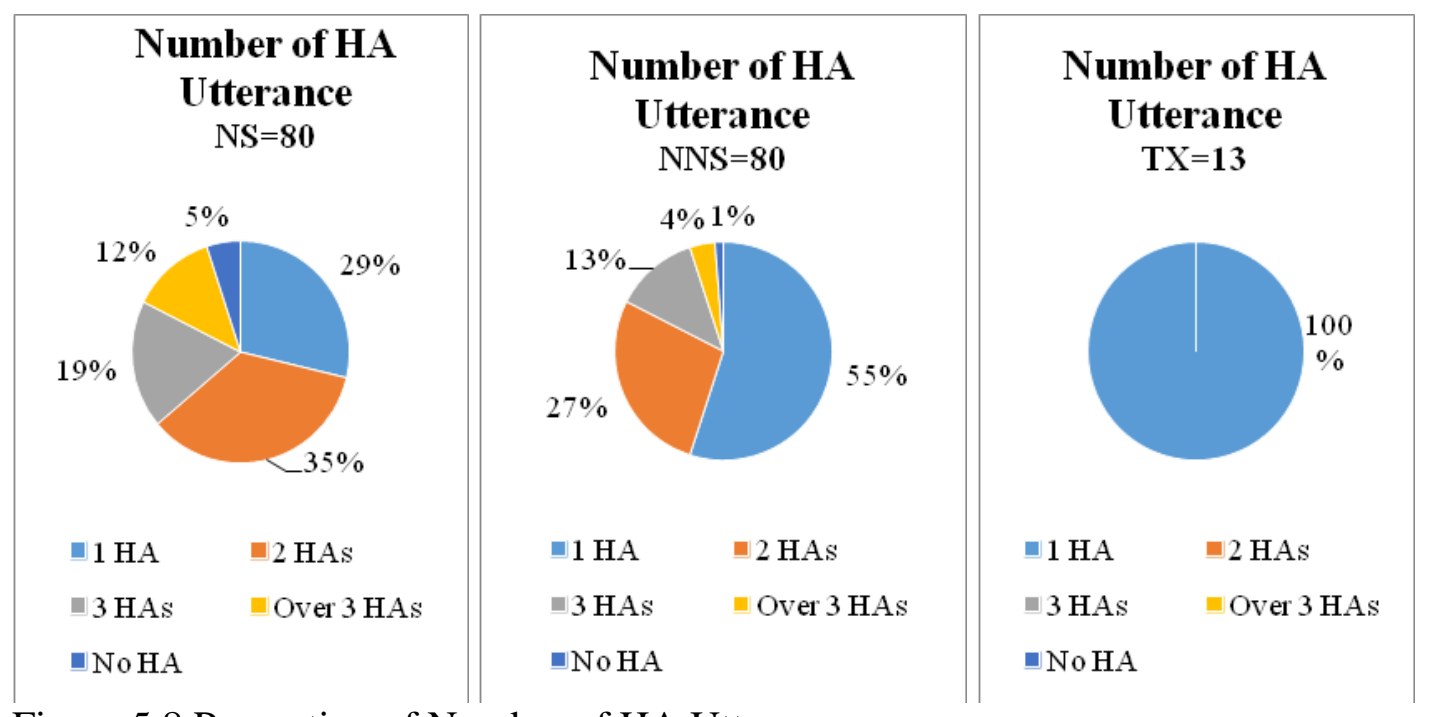

Figure 5.8 Proportion of Number of HA Utterances 
Regarding the use of the politeness strategy, the NSs made use of all four politeness strategies rather uniformly. ${ }^{159}$ These were bald on record strategy (29\%) together with positive politeness strategy $(33 \%)$ which together occupied 62 per cent out of the four strategies. This was followed by negative strategy (20\%) and off record strategy (18\%). Almost all NNSs used either bald on record (58\%) or positive politeness strategies (40\%), and the other two strategies, negative politeness and off record strategies, were very rarely used, their combined total was two per cent. The TXs showed a different type of deflection of applying politeness strategies. The TXs employed mostly positive politeness strategy (84\%), eight per cent each of bald and negative politeness strategies, and no off record strategy. These extreme polarisations in different usage among the three groups were noteworthy.

What is obvious from this is that there were really only two strategies adopted by the NNSs, bald on record strategy and positive politeness strategy. It may be inferred that these two strategies contained comparatively easier expressions for learners to acquire than the others. On the other hand, the authors of the TXs mostly preferred positive politeness strategy followed by bald on record and negative politeness strategies, but they did not seem to consider off record. The lack of coverage of off record strategy might have resulted from the essential nature that foreign language textbooks have, namely, that they are expected to present appropriate examples of discourses, the model examples should be easy to understand and be acquired by learners. In this sense, the examples employing off record strategy would not be suitable for the textbooks.

159 Definitions of the four different politeness strategies are described in this Ch. 2, Sec. 3. 


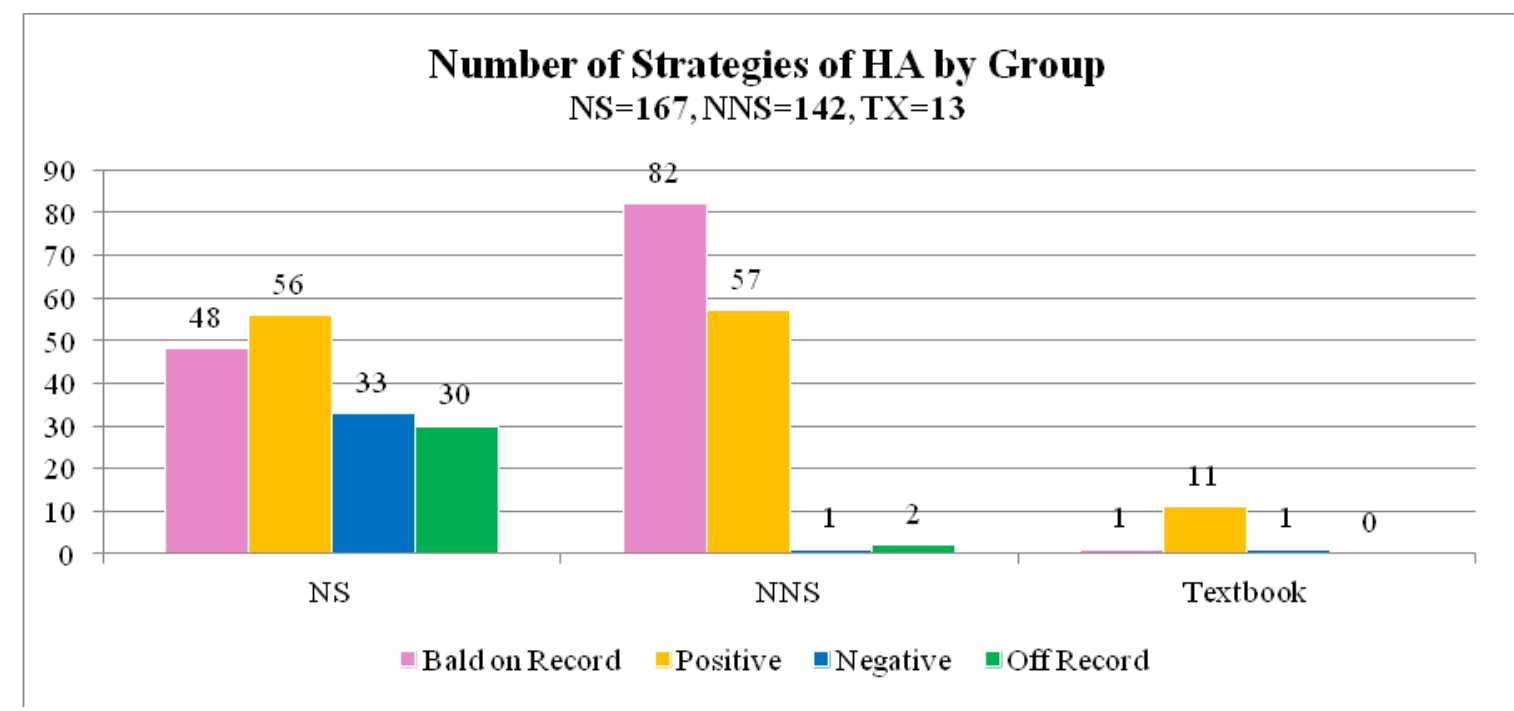

Figure 5.9 Number of Strategies Used by Group

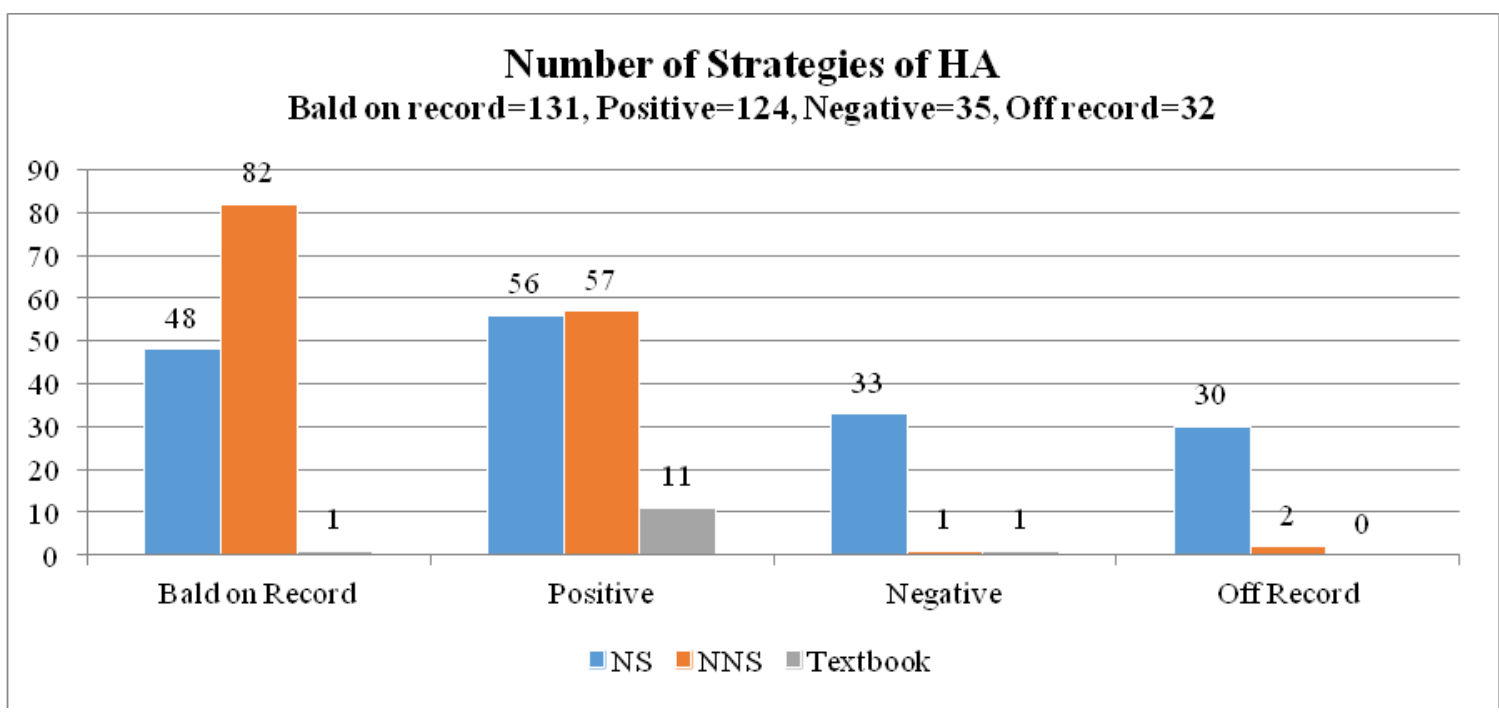

Figure 5.10 Number of Strategy Used

\begin{tabular}{|c|c|c|}
\hline $\begin{array}{c}\text { Politeness Strategies } \\
\text { of } \mathrm{HA} \\
\mathrm{NS}=167\end{array}$ & $\begin{array}{c}\text { Politeness Strategies } \\
\text { of } \mathrm{HA} \\
\mathrm{NNS}=142\end{array}$ & $\begin{array}{c}\text { Politeness Strategies } \\
\text { of } \mathrm{HA} \\
\text { TX }=13\end{array}$ \\
\hline$-33 \%$ & $40 \%$ & $8 \% 0 \%-8 \%$ \\
\hline Bald on Record & Bald on Record & Bald on Record \\
\hline Positive & Positive & Positive \\
\hline Negative & $\square$ Negative & $\square$ Negative \\
\hline$\square$ Off Record & $\square$ Off Record & Off Record \\
\hline
\end{tabular}

Figure 5.11 Proportion of Strategies by Group 


\section{Bald on Record Strategy}

Criteria for Specifying Expressions of Bald on Record Strategy

- Using the te-form of verbs denoting a request, and the sentence ends. For example, Misete [Show me].

- Using the te-form of verbs denoting a request, to which is added Kudasai/onegai shimasu [Please] to be polite. For example, Misete kudasai [Please show me].

- Using the te-form of verbs denoting a request, to which is added the verb Tanomu [Ask, Beg] to emphasise the requester's seriousness. For example, Misete, tanomu [I beg you to show me].

- Using the te-form of verbs denoting a request, to which is added the verb Hoshii [Want, Wish] to emphasise the requester's seriousness. For example, Misete hoshii [I want you to show me].

- A requester expresses a wish with a limitation, or names what he/she wants. For example, Miru dake [Only just look at it], Kopī dake [Let me only make a copy], or Hinto [Give me a hint].

\section{Number of Expressions of Bald on Record Strategy}

Bald on record strategy was uttered in 48 utterances ( 0.6 times per NS/discourse) and in 82 utterances (once per NNS/discourse). ${ }^{160}$ This implies that the NNSs used bald on record strategy around twice as frequently as the NSs in proportion to the total number of utterances in each group. ${ }^{161}$ There were 13 model exemplars in the TXs; however, only one presented bald on record strategy.

\footnotetext{
16048 (utterances) $/ 80$ (participants) $=0.6$ times (NS), $82 / 80 \doteqdot 1.0$ times (NNS)

$16158 \%(\mathrm{NNS}) / 29 \%(\mathrm{NS})=2$
} 


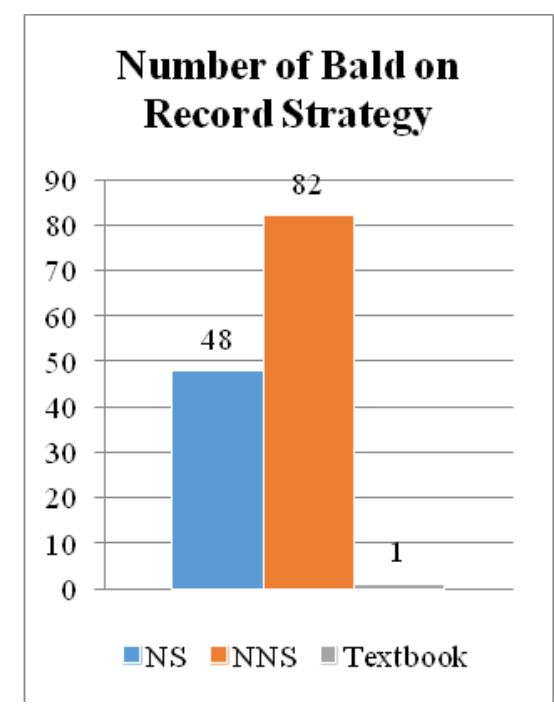

Figure 5.12 Number of Bald on Record Strategy Used by Group

As for expressions, the te-form such as, Misete [Show me], Oshiete [Tell me] as well as variants, for example, adding the word kudasai [please], were widely used by the NNSs. The total was 62 utterances $(76 \%)$ by the NNSs for bald on record strategy; while the NSs used the te-form 20 times (42\%) in bald on record strategy.

Other than the $t e$-form, bald on record strategy includes the following expressions: Onegai shimasu [Please] and V-te hoshii [I want you to V], directly revealing the request by the requester, and Mitai [I want to see (your homework)] and its variations, which expose the requester's thought. In the case of Onegai (shimasu) [Please], there were reverse utterances, such as misete, onegai [Show me, please] and the other was Onegai, misete [Please show me].

The $t e$-form was overwhelmingly used in bald on record strategy. Among the NSs, various kinds of expressions were applied: Onegai shimasu [Please], Tanomu [I beg of you] 14 times (29\%), and V-te hoshii, such as Oshiete hoshii, Misete hoshii 10 times (21\%). The native speakers used Mitai [I want to see], Miru dake [I'll only look (at it)], Kopī [I want a copy], and Hinto [Give me a hint], through which the requester plainly articulated his/her wish. However, this type of expression was not employed by the NNSs.

One NNS used Yomitai desu [I want to read], which is straightforwardly pronouncing the speaker's wish. It conforms to Japanese grammar; nevertheless, taking the setting of 
request into consideration, the use of this expression in real life is questionable. This is because it does not seem to contain the customary disposition of humility towards the other party when making a request. It sounds rather like an order, because finishing the sentence with desu makes it formal and clearly states the speaker's wish very explicitly. ${ }^{162}$

Table 5.1 Typical Expressions of Bald on Record Strategy

\begin{tabular}{|l|l|}
\hline Japanese in the Romanised alphabet & English translation \\
\hline Misete/tasukete/oshiete/kashite kudasai & Please show/help/tell/lend me. \\
\hline Onegai shitai/shimasu, tanomu & Please, I beg you. \\
\hline Oshiete/misete hoshii n' da & I want you to show/tell me. \\
\hline Mitai, miru dake, kopi, hinto & Show me/just look at it/copy it/give me a hint. \\
\hline Yomitai desu & I want to read it. \\
\hline
\end{tabular}

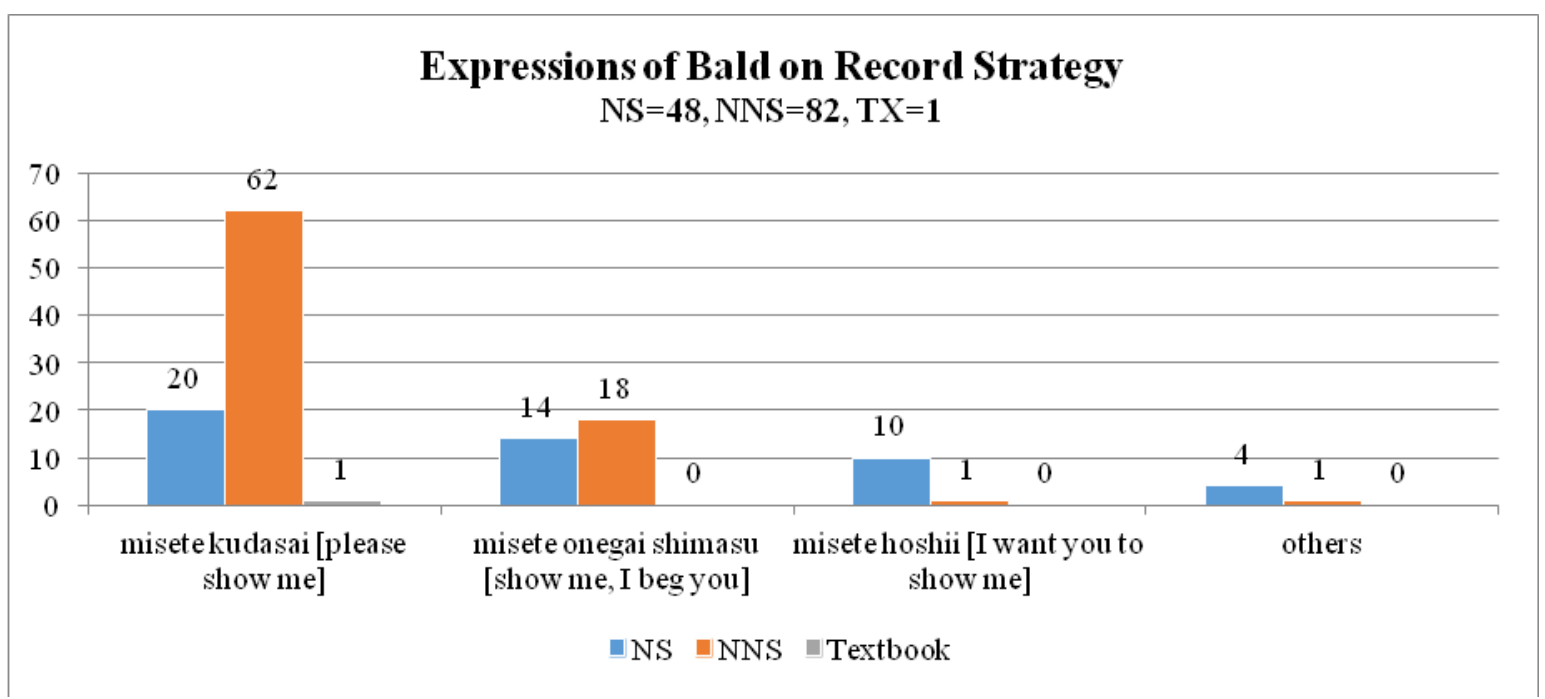

Figure 5.13 Expressions of Bald on Record Strategy

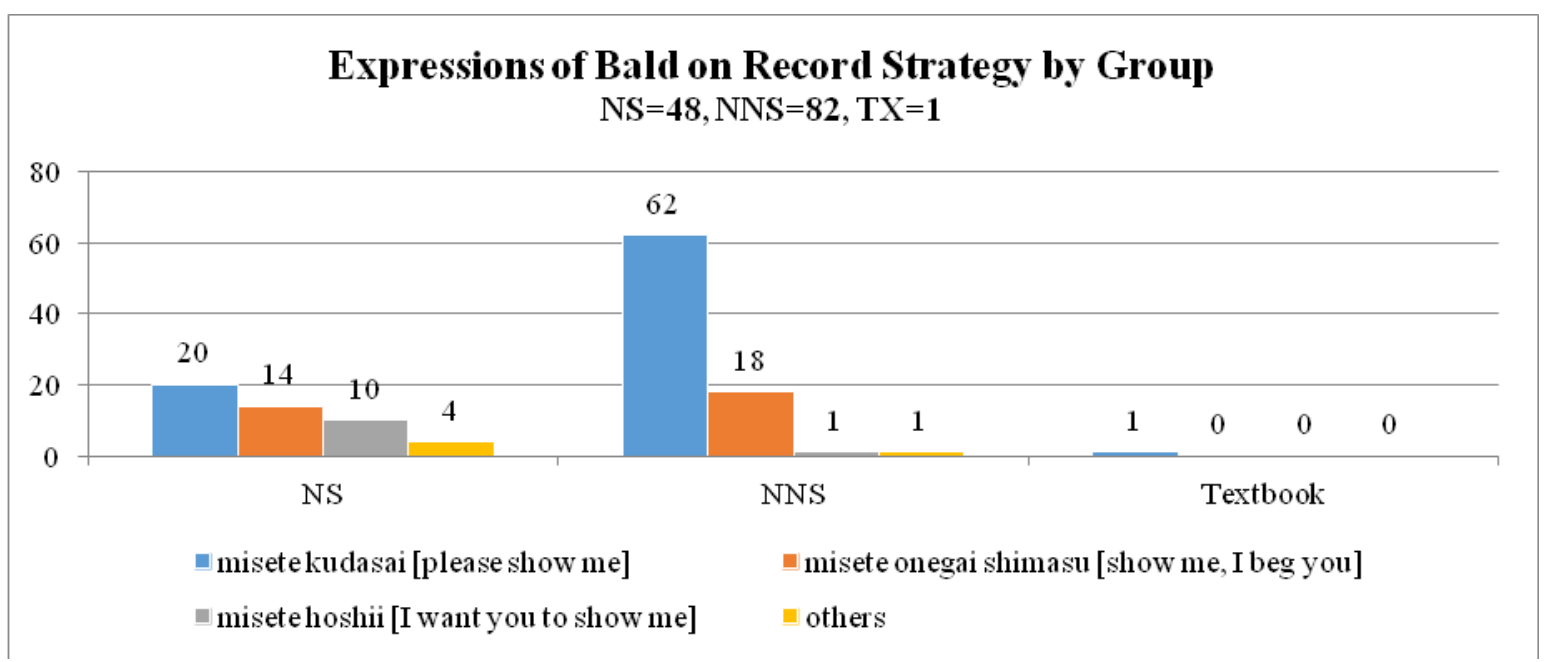

Figure 5.14 Expressons of Bald on Record Strategy by Group

162 The sentence Yomitai desu [I want to read] is, therefore, categorised as a bald on record strategy. 


\begin{tabular}{|c|c|c|}
\hline $\begin{array}{c}\text { Expressions of } \\
\text { Bald on Record } \\
\text { NS }=48\end{array}$ & $\begin{array}{c}\text { Expressions of } \\
\text { Bald on Record } \\
\text { NNS }=82\end{array}$ & $\begin{array}{c}\text { Expressions of } \\
\text { Bald on Record } \\
\text { TX }=1\end{array}$ \\
\hline $21 \%$ & $1 \% \frac{1 \%}{\square}$ & $\begin{array}{l}0 \% \\
100 \\
\%\end{array}$ \\
\hline $\begin{array}{l}\text { misete kudasai [please show } \\
\text { me] }\end{array}$ & $\begin{array}{l}\text { misete kudasai [please } \\
\text { show me] }\end{array}$ & $\begin{array}{l}\text { misete kudasai [please } \\
\text { show me] }\end{array}$ \\
\hline $\begin{array}{l}\text { misete onegai shimasu } \\
\text { [show me, I beg you] }\end{array}$ & $\begin{array}{l}\text { misete onegai shimasu } \\
\text { [show me, I beg you] }\end{array}$ & $\begin{array}{l}\text { misete on egai shimasu } \\
\text { [show me, I beg you] }\end{array}$ \\
\hline $\begin{array}{l}\text { misete hoshii [I want you to } \\
\text { show me] }\end{array}$ & $\begin{array}{l}\text { misete hoshii [I want you } \\
\text { to show me] }\end{array}$ & $\begin{array}{l}\text { misete hoshii [I want you } \\
\text { to show me] }\end{array}$ \\
\hline$\square$ others & others & others \\
\hline
\end{tabular}

Figure 5.15 Proportion of Expressions of Bald on Record Strategy

\section{Positive Politeness Strategy}

Criteria for Specifying Expressions of Positive Politeness Strategy

- The requester takes an interrogative form to the interlocutor when making a request. ${ }^{163}$ Alternatively, although it is not a direct requisitive expression, the requester includes his/her wish in the utterance and asks the interlocutor's intention.

- A requisitive sentence includes juju dōshi [verbs of giving and receiving], such as kureru/kurenai [give/not give] and morau/moraenai [receive/cannot receive]. In some cases either ii [ok, all right] or ka na [(in context) all right?, maybe?] or both are added at the end of the requisitive sentence, for example, Misete moratte mo ii? [Can I look at it, ok?].

163 By contrast, in bald on record strategy the speaker clearly states his/her request in an affirmative sentence. 
- The requester utters the main verb and adds $i i$ (and some cases $k a n a$, too) after the verb, such as Kiite mo ii? [May I ask?]. The definition of the main verb does not include juju dōshi here.

- Other than the above, the requester utters his/her wish using other verbs, such as Onegai dekiru? [Can I request?] and Onegai dekinai? [Cannot I request?], etc. At the end of the requisitive sentence, sometimes $k a n a$ is added, Onegai dekiru ka na? [Can I request, maybe?]

\section{Number of Expressions of Positive Politeness Strategy}

There is minimal difference between the 56 utterances by the NSs and 57 utterances by the NNSs in the number of uses of positive politeness strategy. However, the percentage in the total number of HA utterances by language group differs: 33 per cent for the NSs and 40 per cent for the NNSs. In the model exemplars of the TXs, the majority of requisitive sentences employed positive strategy, being 11 out of $13(84 \%)$ in this category.

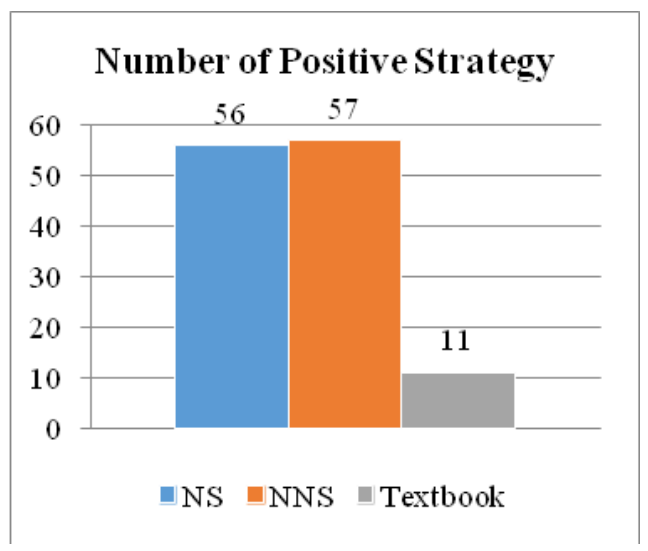

Figure 5.16 Number of Positive Politeness Strategy

The frequent use of juju dōshi was prominent since these verbs commonly appeared in the utterances and exemplars. ${ }^{164}$ In addition, expressions forming an interrogative sentence

164 Few utterances and exemplars employed itadaku/itadake nai [(lit.) the polite form of receive].

These were categorised under morau/morae nai in the present study. 
in order to sound out the other's intention, such as Ii ka na? [Is it all right, I wonder?], Mite mo ii? [Is it all right to look?] were also uttered, especially by the NNSs.

A similar proportion of usages of kureru/kurenai [give/not give] was observed: 52 per cent by the NSs, 60 per cent by the NNSs, and 46 per cent by the TXs. Particularly, among the three groups, the proportion of usage of kurenai appeared very similar to each other as 47 per cent by the NSs, 48 per cent by the NNS, and 46 per cent by the TXs. On the other hand, the use of morau/moraenai [receive/cannot receive] was different according to group. While 32 per cent of the NSs used it, and 14 per cent of the NSs did so, only nine per cent of the TXs used it in the politeness strategy. The NSs' usage of morau/moraenai was three times that of the TXs. Morau/moraenai is regarded as more polite than kureru/kurenai, indicating that the NSs tended to use more polite expressions of positive politeness strategy than the other two. In other words, the differences in usage among the three groups derived from the NSs frequent use of morau/moraenai, not kureru/kuranai. Furthermore, the TXs did not employ affirmative forms of juju dōshi at all, but only negative forms, such as as kuranai and moraenai.

The total percentage of the two types of juju doshi reached 84 per cent by the NSs and 74 per cent by the NNSs respectively, but for the TXs it was only 55 per cent. In other words, when requesting something of someone, the majority of the NSs and the NNSs appeared to have thought that utilising juju dōshi was most appropriate, at least when the interlocutor's status was socially equal to the requester's.

It was noteworthy that, instead of using morau/moraenai, the NNSs very much preferred the word $i i$ [ok]. This accounted for 26 per cent of the politeness strategies of the NNSs; but the proportion of its use by the NSs was only seven per cent, just above a quarter of that of the NNSs, and only one TX used it. Ii must have been easily acquired by the NNSs and is an easy-to-use word, although the TXs barely presented it. 


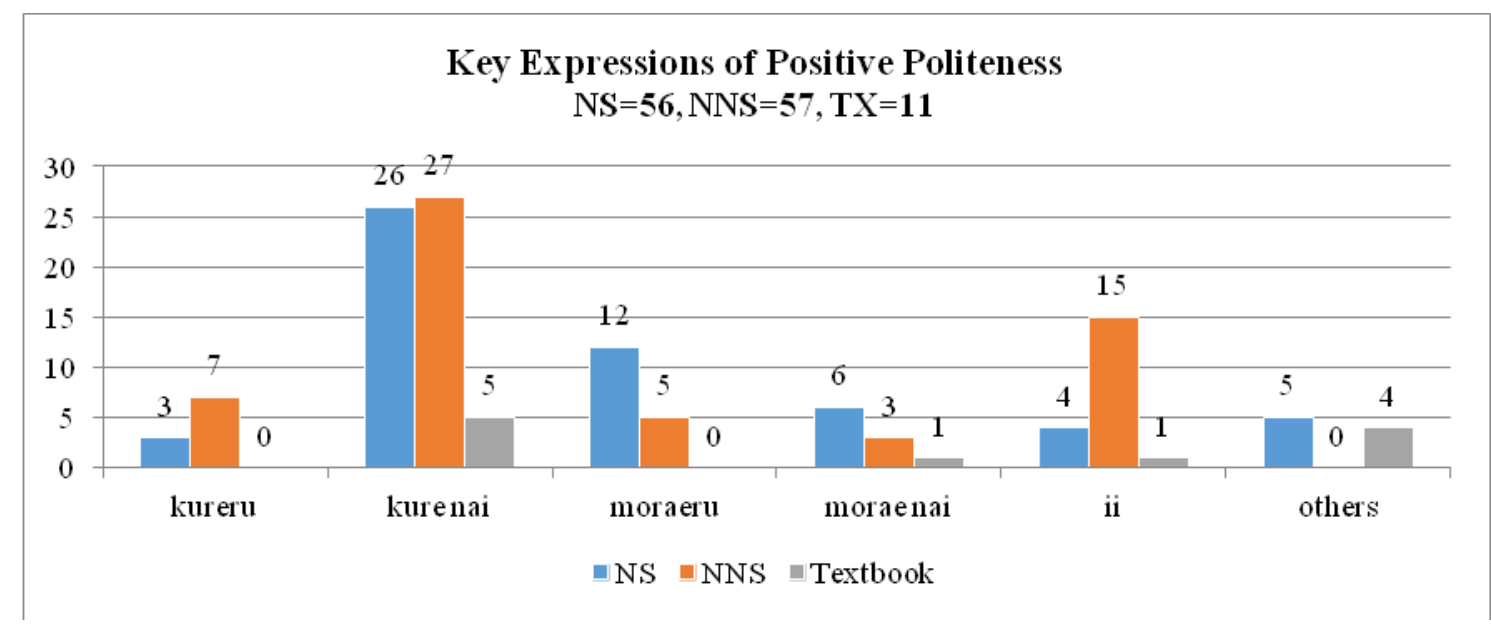

Figure 5.17 Key Expressions of Positive Politeness Strategy

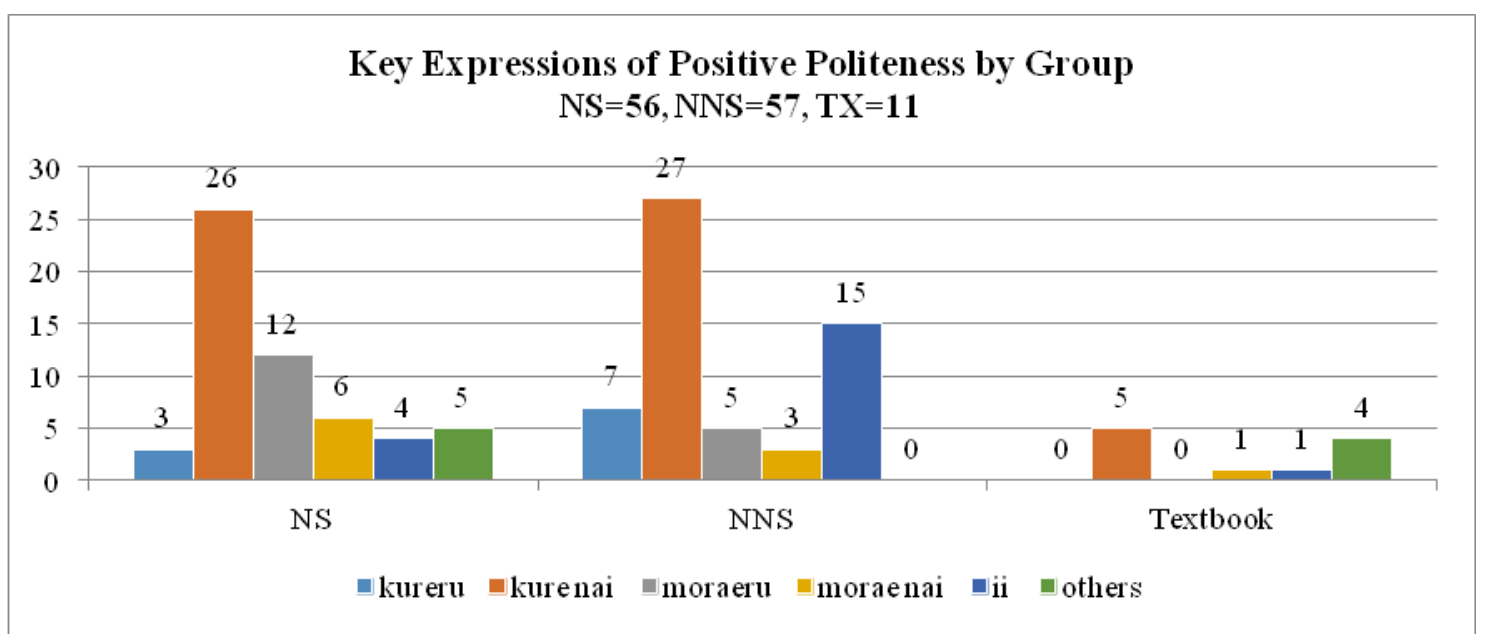

Figure 5.18 Key Expressions of Positive Politeness Strategy by Group

\begin{tabular}{|c|c|c|}
\hline $\begin{array}{c}\text { Expressions of } \\
\text { Positive Politenses } \\
\text { NS }=56\end{array}$ & $\begin{array}{c}\text { Expressions of } \\
\text { Positive Politeness } \\
\text { NNS }=\mathbf{5 7}\end{array}$ & $\begin{array}{c}\text { Expressions of } \\
\text { Positive Politeness } \\
\text { TX }=11\end{array}$ \\
\hline & $0 \%$ & $0 \%$ \\
\hline $\begin{array}{l}\square \text { kureru } \\
\text { kurenai } \\
\text { moraeru } \\
\text { moraenai } \\
\square \text { ii } \\
\square \text { others }\end{array}$ & $\begin{array}{l}\square \text { kureru } \\
\square \text { kurenai } \\
\square \text { moraeru } \\
\square \text { moraenai } \\
\square \text { ii } \\
\square \text { others }\end{array}$ & $\begin{array}{l}\text { kureru } \\
- \text { kurenai } \\
\text { moraeru } \\
\text { moraenai } \\
\text { mii } \\
\text { others }\end{array}$ \\
\hline
\end{tabular}

Figure 5.19 Proportion of Key Expressions of Positive Politeness Strategy 
Table 5.2 Typical Expressions of Positive Politeness Strategy

\begin{tabular}{|l|l|}
\hline Japanese in the Romanised alphabet & English translation \\
\hline $\begin{array}{l}\text { Misete kureru?/ Kuremasu ka? } \\
\text { Tetsudatte kureru? (ka na?) }\end{array}$ & $\begin{array}{l}\text { Please will you show me? } \\
\text { Please will you help me? }\end{array}$ \\
\hline Misete kurenai? (ka na?) & Would you let me see it? \\
\hline Kashite moraeru? (ka na?) & Could you lend it to me? \\
\hline Kashite moraenai? (ka na?) & Would you lend it to me? \\
\hline Mite (mo) ii? & Is it ok if I read it? \\
Kiite (mo) ii? (ka na?) & Is it ok I ask? \\
\hline Onegai dekiru? & Can I ask a favour of you? \\
Onegai dekinai? (ka na?) & Can't I ask a favour of you? \\
\hline
\end{tabular}

Characteristics of Expressions of Positive Politeness Strategy

- Use of nai

Another distinguishing feature of positive politeness strategy is the frequent use of the word nai [(auxiliary verb) denial, not $x]$ in requisitive sentences of juju dōshi. One of the functions of nai is to sound out and induce someone to do something when it is uttered at the end of a sentence with a rising tone, according to Nihon kokugo daijiten [Complete Japanese Language Dictionary] (1980: 130).

Use of the word nai with juju dōshi was very common across the groups, as around 70 per cent of both the NSs and the NNSs, and 100 per cent of exemplars did so. Thirty-two out of 47 utterances with juju dōshi $(68 \%)$ by the NSs, 30 out of $42(71 \%)$ by the NNSs, and all six exemplars $(100 \%)$ in the TXs utilised nai to form a negative interrogative sentence when requesting. Typical examples of using nai with Misete kurenai? [Won't you show me it?] were 26 NSs, 27 NNSs, and five exemplars of TXs; and those with Kashite moraenai? [Won't you lend me it?] were six NSs, three NNSs, and one TX, including their variations. The requesters/personae predominantly used the form of $V$-te + kure + nai in positive strategy.

Although there is another expression of positive politeness strategy using the juju dōshi of moraenai, the number of times it was used was far less than kurenai, and another use of nai was in two utterances of dekinai [cannot] by the NSs. 


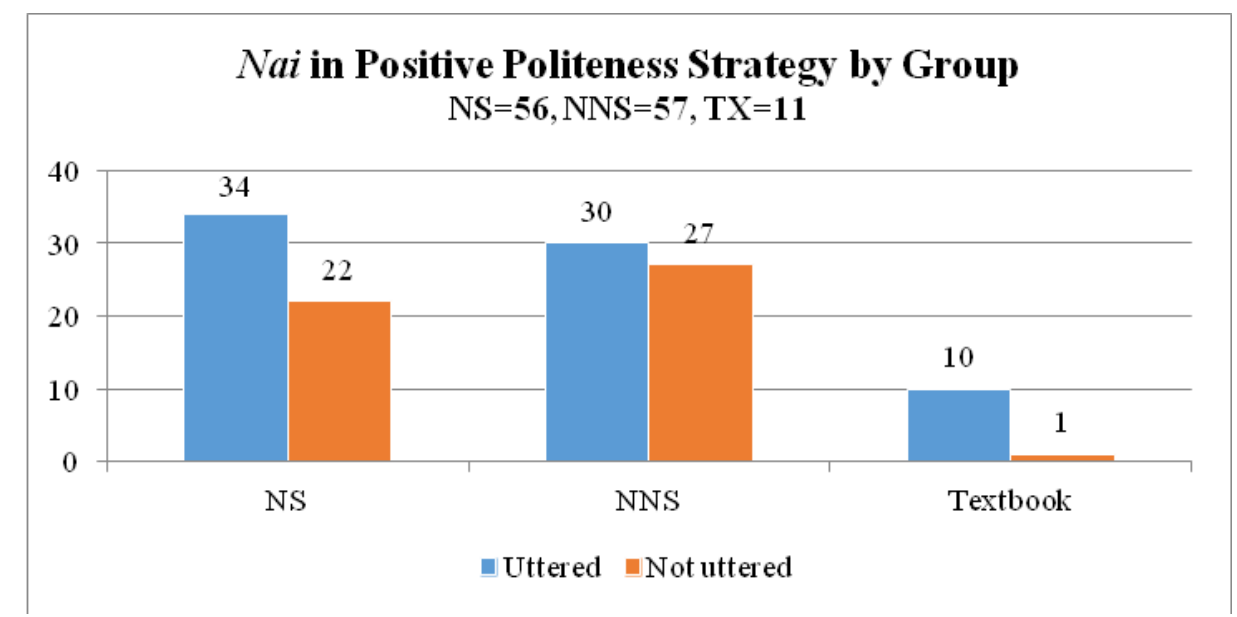

Figure 5.20 Nai in Positive Politeness Strategy by Group

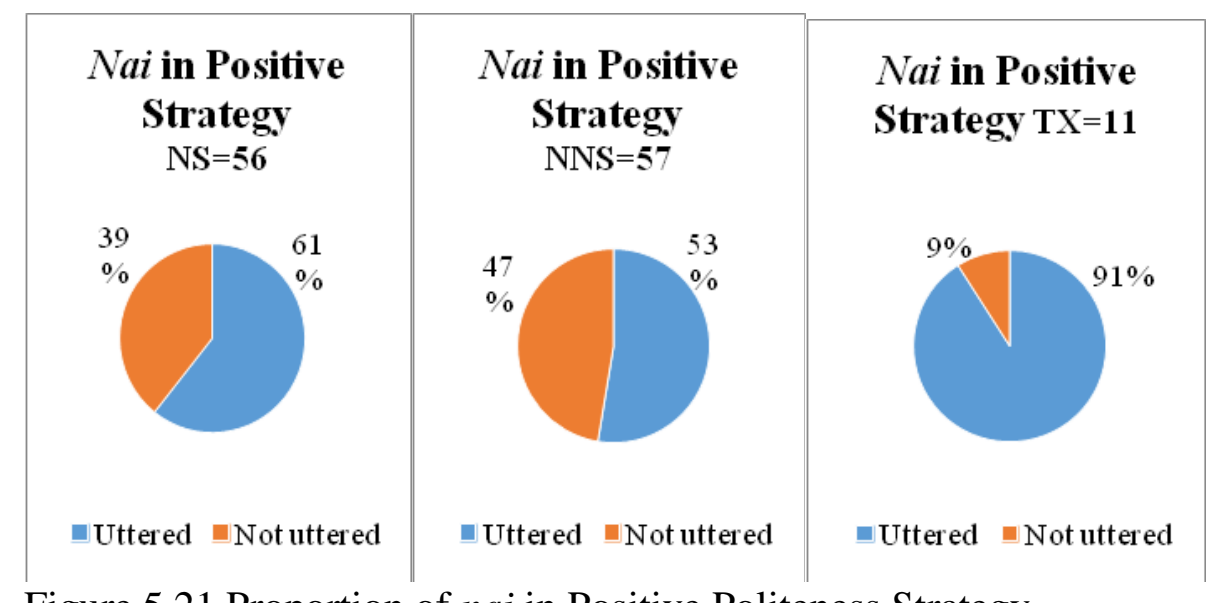

Figure 5.21 Proportion of nai in Positive Politeness Strategy

When making a request, the participants and the writers of the TXs tended to become more polite. Both sentences Misete kureru? [Would you show me?] and Misete kurenai? [Wouldn't you show me?], take the form of interrogative sentences. However, the latter, being negative, sounds more polite than the former. When a question is asked in the negative form, it makes it easier for an interlocutor to decline owing to the fact that the reply in Japanese is 'yes' either way-either 'Yes, I shall' or 'Yes, that's right, I shall not,' thereby allowing the interlocutor who replies with negative intention to lose less face and minimise the loss of face to the requester, too. By contrast, if someone is asked something in an affirmative interrogative sentence (can, would, etc.), and the hearer wants to decline it, he/she has to generate a semantically negative sentence in response, thereby inducing loss of face to 
both parties. While this is very subtle, nevertheless it can be a decisive factor as to whether it sounds more polite or not.

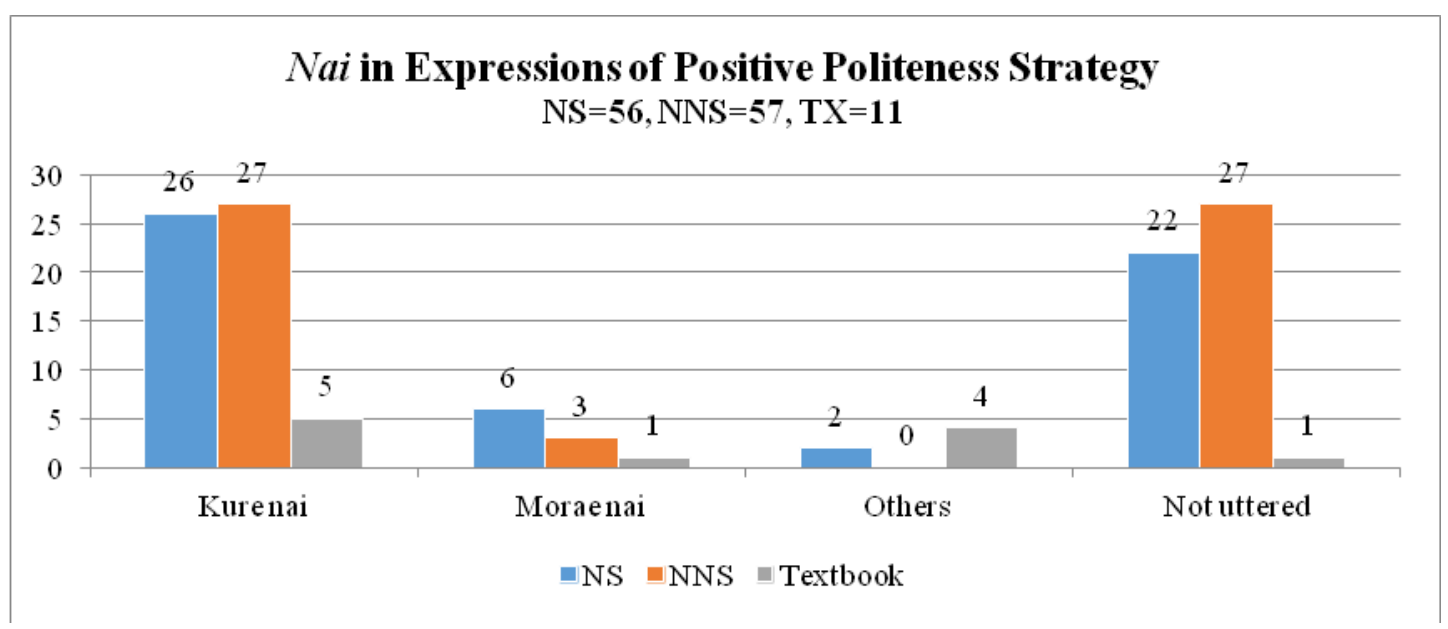

Figure 5.22 Nai in Expressions of Positive Politeness Starategy

N.B. Others in TXs include three occurrences of itadakenai [(lit.) not receive].

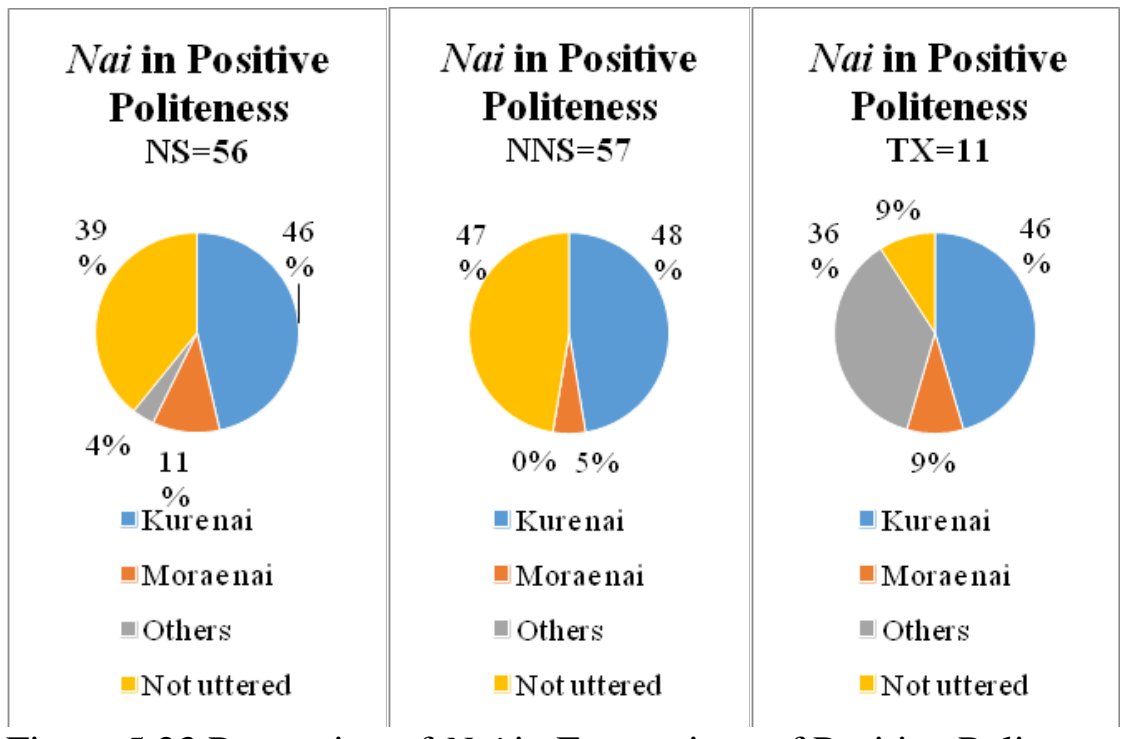

Figure 5.23 Proportion of Nai in Expressions of Positive Politeness Starategy

- $\quad$ Use of ka na

In positive politeness strategy, the NSs often used $k a$ na (a sentence-ending compound particle) expressing a speaker's emotion or impression, such as Can I expect, I wonder? The particle $k a$ is normally used at the end of interrogative sentences (like a verbalized question mark) and the other particle $n a$ functions to reduce the nuance of assertiveness by the speaker. In other words, it works to lessen the demands of a question, in 
particular when making a request. ${ }^{165}$ Twenty-two out of 56 utterances (39\%) of positive politeness strategy by the NSs ended with $k a$ na. The native speakers' utterances, for example, included Mite mo ii ka na? [Am I allowed to look, I wonder?], Kashite moraeru ka na? [Can you lend it to me, I wonder?], or Onegai dekiru ka na? [Can I ask you something, maybe?], and so on.

On the other hand, the compound word ka na was used by only one participant out of 57 NNSs. One exemplar in the TX presented $k a n a$, which consisted of 9 per cent of all exemplars of their positive politeness strategy. The native speakers seemed to minimise the request as much as possible by using $\mathrm{ka} \mathrm{na}$, at the same time watching the interlocutor's response, although their request was clearly uttered. The use of $k a n a$ might be difficult for (or may not be taught to) NNSs. In the present experiments, the use of $k a n a$ was a distinguishing feature of the NSs.

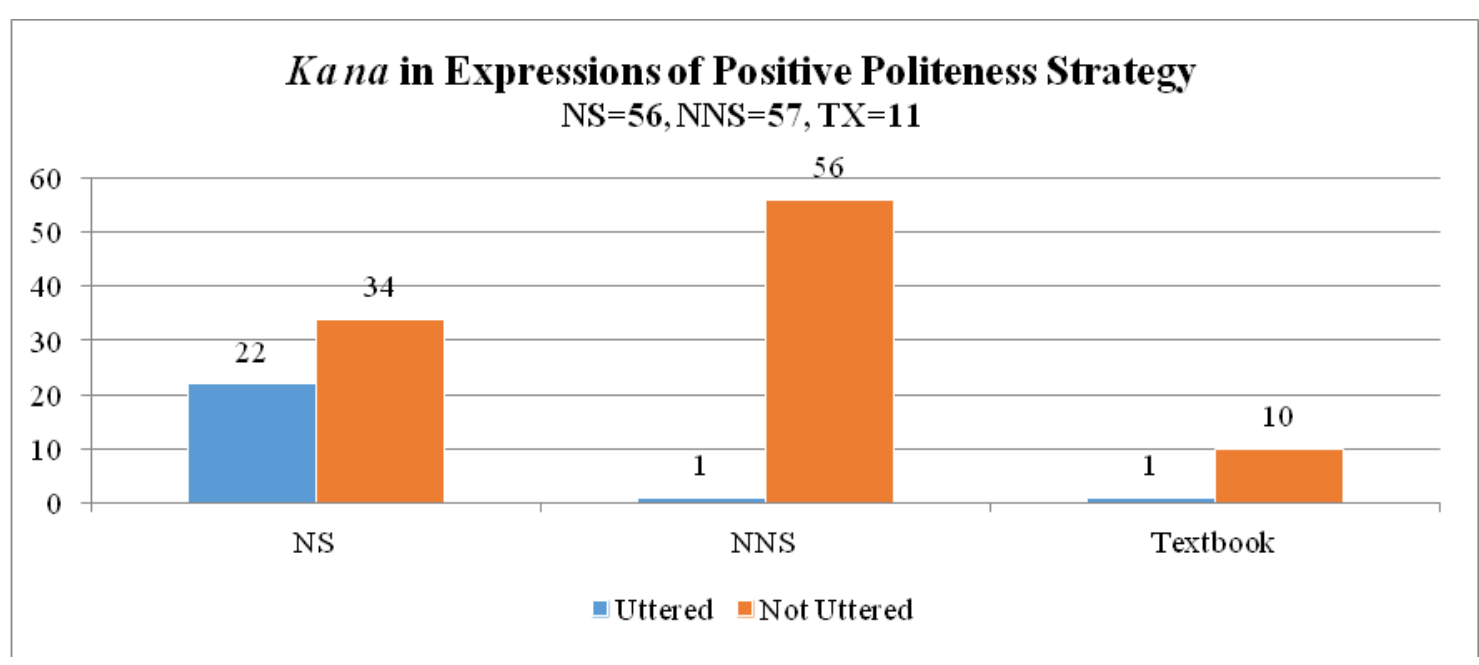

Figure 5.24 Ka na in Expressions of Positive Politeness Strategy by Group

165 Kenbo, Hidetoshi, et al. 1994. Sanseido kokugo jiten [Sanseido Japanese language dictionary].

Dai 4-han. ed. Tokyo: Sanseido (207). 


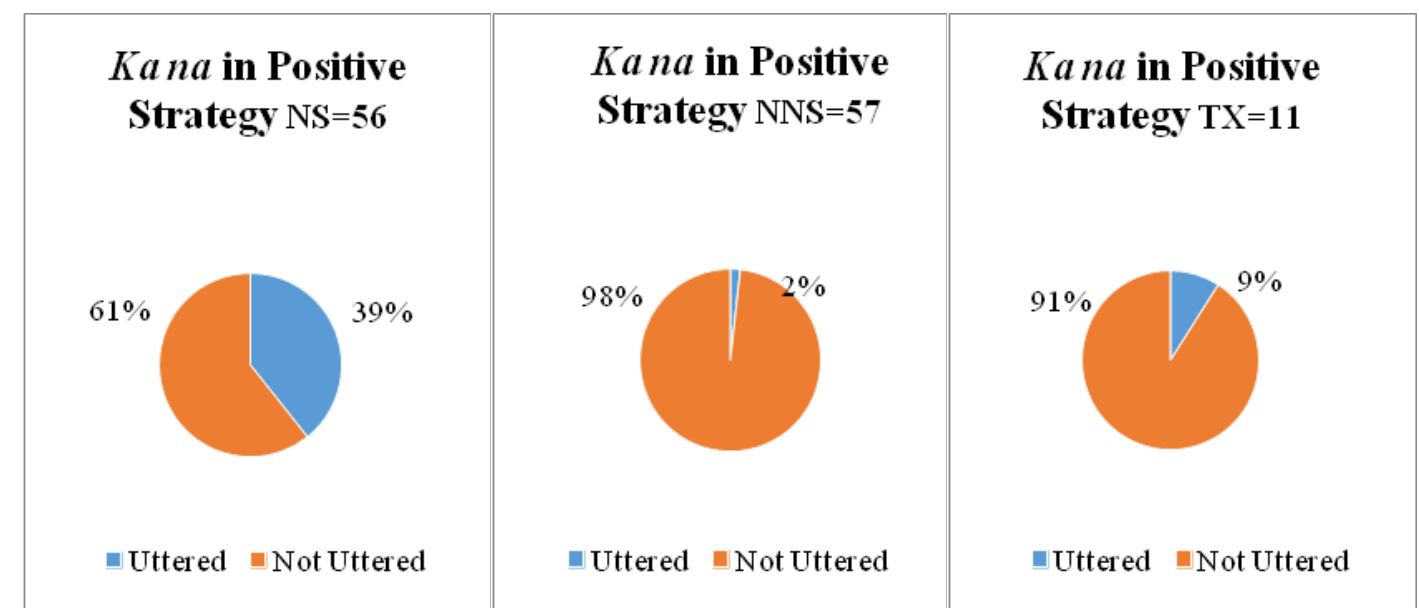

Figure 5.25 Proportion of $k a n a$ in Expressions of Positive Politeness Strategy

\section{Negative Politeness Strategy}

Criteria for Specifying Expressions of Negative Politeness Strategy

- The requisitive sentence employs aposiopesis, and it ends with a particle or the te-form, such as kedo [but, however], to omotte [I think], tara [if]. The utterance therefore does not form a complete sentence but is left hanging.

- If the main verb expresses the speaker's request or wish directly, for example, hoshii [want], kureru [give], or morau [receive], and if the requisitive sentence takes the form of aposiopesis by ending with kedo, to omotte, or tara, etc., it is regarded as negative politeness strategy.

\section{Number of Expressions of Negative Politeness Strategy}

There was a huge discrepancy in the number of usages of negative politeness strategy. While 33 negative politeness sentences were uttered by the NSs, only one utterance each by the NNS and in the TX were recognised as negative politeness sentences. The proportion of use of negative politeness strategy by the NSs was 20 per cent for their HAs, and eight per cent for the TXs, but it was only one per cent for the NNSs. 


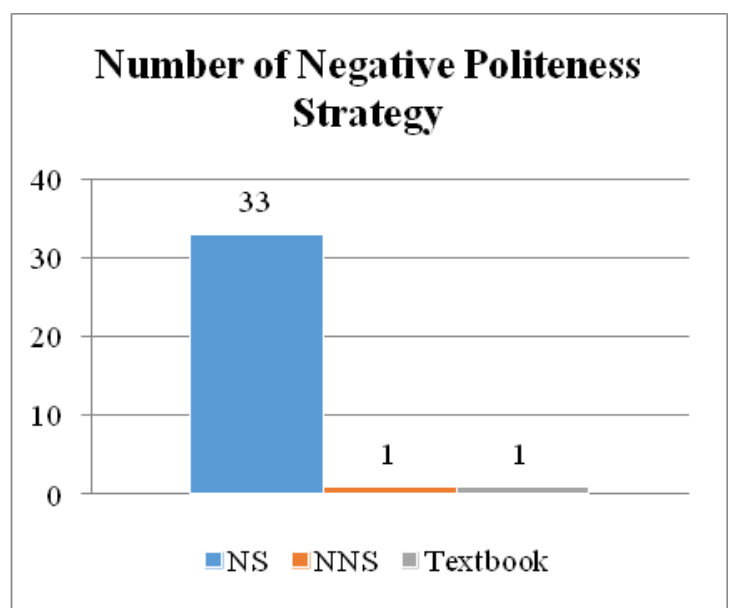

Figure 5.26 Number of Negative Politeness Strategy

Many NSs used the te-form of the main verb together with the word kedo [(lit.) but, however, although]. ${ }^{166}$ For instance, Oshiete hoshii n' da kedo... [I want you to tell/teach me...]. This type of expression was used 14 times (43\%) by the NSs, followed by 10 utterances for Misete kure nai ka naa to omotte... [I'm wondering whether you'd show me...] (30\%), and four utterances for Moshi oshiete moraetara... [If you'd tell me...] (12\%), in the subjunctive.

166 The word kedo is a conjunctive particle which implies a speaker's hesitant feeling when it is used at the end of the sentence as a kind of sentence final particle. (Kenbo, Hidetoshi, et al. 1994. Sanseido kokugo jiten [Sanseidoo Japanese language dictionary]. Dai 4-han. ed. Tokyo: Sanseido. 342). Shirakawa (2009: 16) claims that there is the case that even if the sentence ends with kedo, the intention of a speaker is conveyed. 


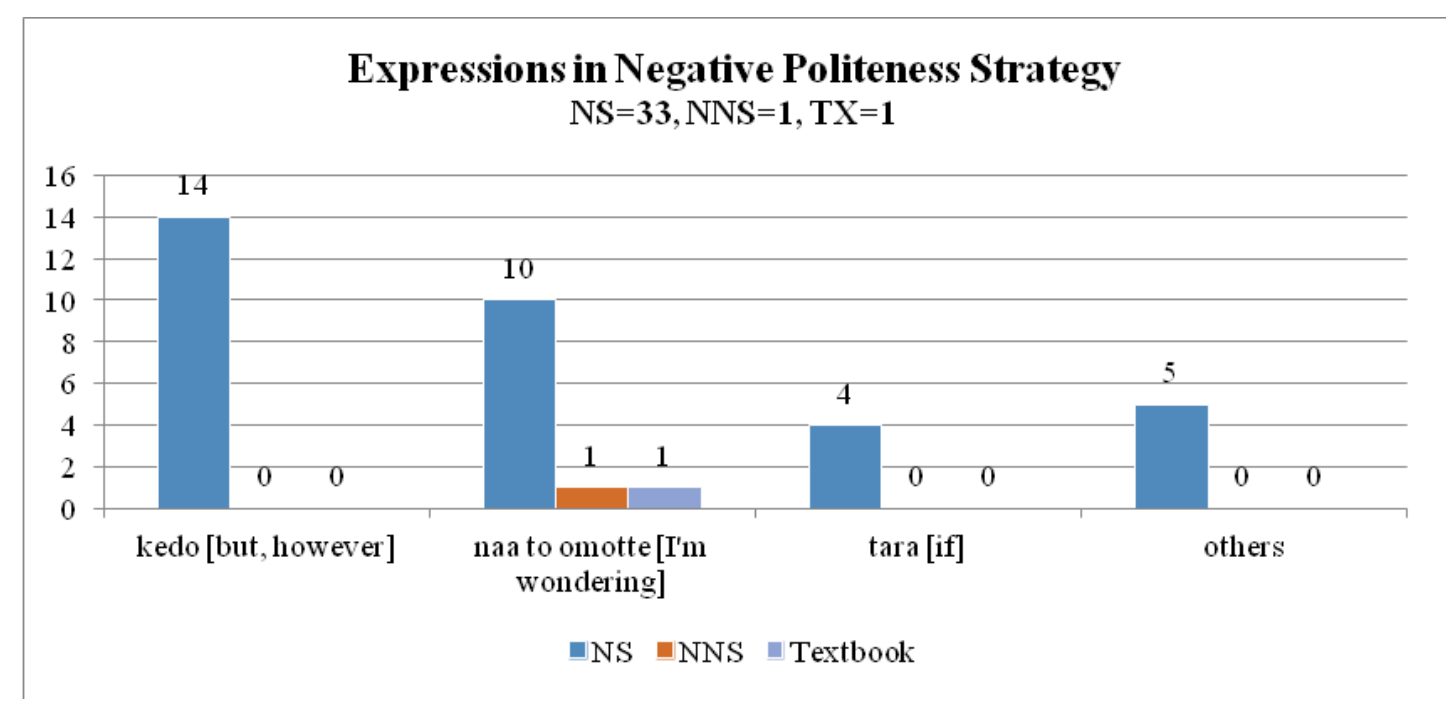

Figure 5.27 Expressions in Negative Politeness Strategy

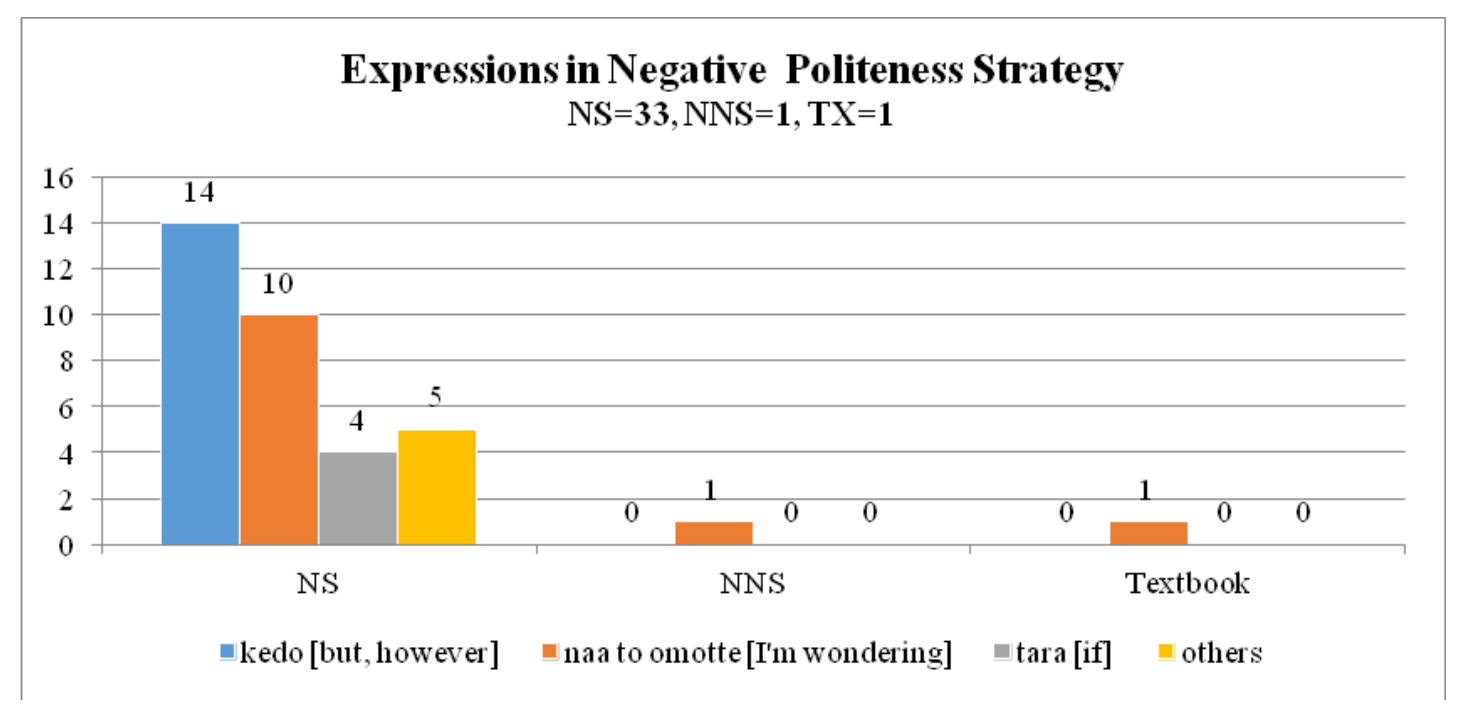

Figure 5.28 Expressions in Negative Politeness Strategy by Group

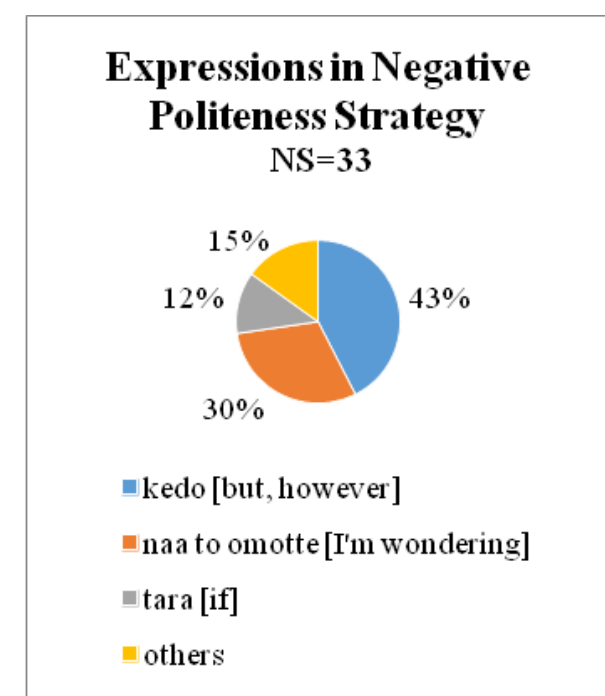

Figure 5.29 Proportion of Expressions in Negative Politeness Strategy 
Table 5.3 Typical Expressions of Negative Politeness Strategy

\begin{tabular}{|l|l|}
\hline Japanese in the Romanised alphabet & English translation \\
\hline Mitai $n$ 'da kedo... & I want to see it. \\
\hline Misete moraenai ka naa to omotte... & I'm wondering whether you'd mind showing me. \\
\hline Moshi oshiete moraetara... & I'd be grateful if you could tell/show me. \\
\hline
\end{tabular}

Characteristics of Expressions of Negative Politeness Strategy

- Use of Aposiopesis

One of the characteristics of negative politeness strategies in the present experiments is that the utterance is not grammatically complete but ends in aposiopesis. This works to keep a distance between the speaker and the interlocutor, which corresponds to the concept of negative politeness strategy. By leaving the end of a sentence open, the speaker's feeling will be recognised as politely hesitant compared with the completed sentence which may convey an air of inappropriate assertiveness by the speaker.

In particular, the conjunctive particle kedo implies the speaker's feeling of hesitancy when it is used at the end of the sentence. Unlike kedo, however, neither naa to omotte nor tara hold hesitant implications of themselves. A sentence left unfinished in this way indicates that the speaker (requester) leaves the right of decision to the interlocutor in a setting of request, because hesitant expressions tend to convey a sense that the speaker is politely reserved and restrained.

\section{Difficulty in Generating Aposiopesis}

Unlike bald on record and positive politeness strategies which only need to generate grammatically uncomplicated and simple sentences, negative politeness strategy seemed to be difficult for the NNSs to create since they rarely utilised this form. The reason might be that unfinished sentences, in general, tended to be longer than bald on record or positive 
politeness strategies in the present experiments, even though they did not contain a main clause.

For example, one NS uttered Mishite moraetari nanka suru to arigatai n' da kedo [I'd be grateful if you'd show me, or something], which ended with the word kedo. Although the sentence utilised verbs of mishite [(col.) misete, show] together with morau [receive] in suggesting his hope, this was a circumlocutory sentence. Another was Misete kurenai ka naa to omotte [I'm wondering whether you might show me], where naa to omotte emphasises the indirectness of the speaker's request misete. The other was Moshi oshiete moraetara [I'd be grateful if you could tell/show me]. The last example used a combination of moshi [if] and tara, which also acknowledges the distance that the speaker thinks exists between the interlocutor and him/her.

Furthermore, since the TXs present no model exemplars of negative politeness strategy, there may be little (or no) opportunity for NNSs to recognise the message of a requester which does not contain a main clause. The crucial thing is that the NNSs are expected to understand the implicature of these kinds of modest expressions when uttered by the NSs because the requester believes his/her utterance is more polite than bald on record or positive politeness strategies.

\section{Off Record Strategy}

Criteria for Specifying Expressions of Off Record Strategy

Owing to the nature of off record strategy, the requester does not utter any direct requisitive expressions nor use a verb implying a request. The individual sentences are, as a consequence, very vague, and whether or not the utterance is a request can only be identified through the context. Various types of expressions of off record strategy were practised. 
- $\quad$ Type 1. Asking an interlocutor's availability, such as Kono ato jikan aru? [Do you have time later?].

- $\quad$ Type 2. Mentioning a speaker's own weak point, such as Donna koto kaitara ii ka (wakaranai) [(I don't know) what should I write?].

- $\quad$ Type 3. Asking what an interlocutor did, such as Dono kurai jikan kakatta? [How long did you take to finish it?].

N.B. Whereas positive and negative politeness strategies reveal a tendency to use several frequently repeated phrases or words, expressions of bald on record strategy and off record strategy are much more diverse in the present experiment.

\section{Number of Expressions of Off Record Strategy}

There were 30 utterances of off record strategy by the NSs and two utterances by the NNSs. Similarly to negative politeness strategy, only a few NNSs employed off record strategy. The percentage of the total number of utterances is 18 per cent for the NSs and a mere one per cent for the NNSs. There were no examples of off record strategy in the TXs.

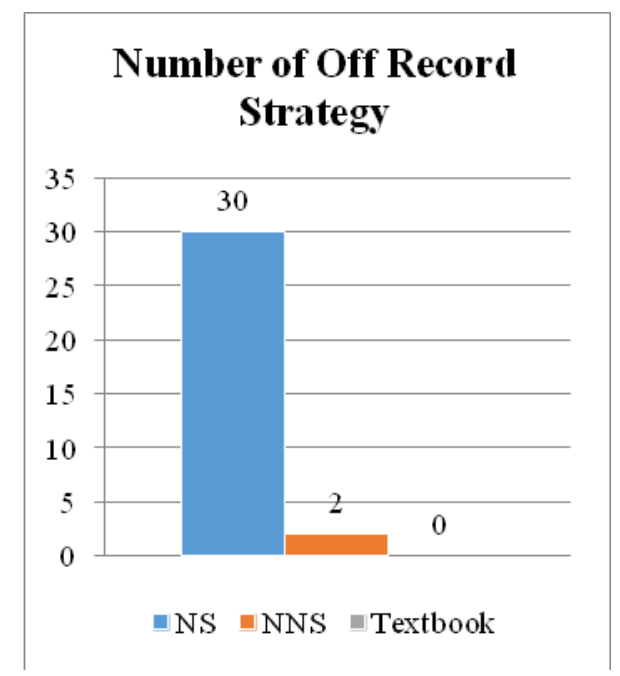

Figure 5.30 Number of Off Record Strategy 


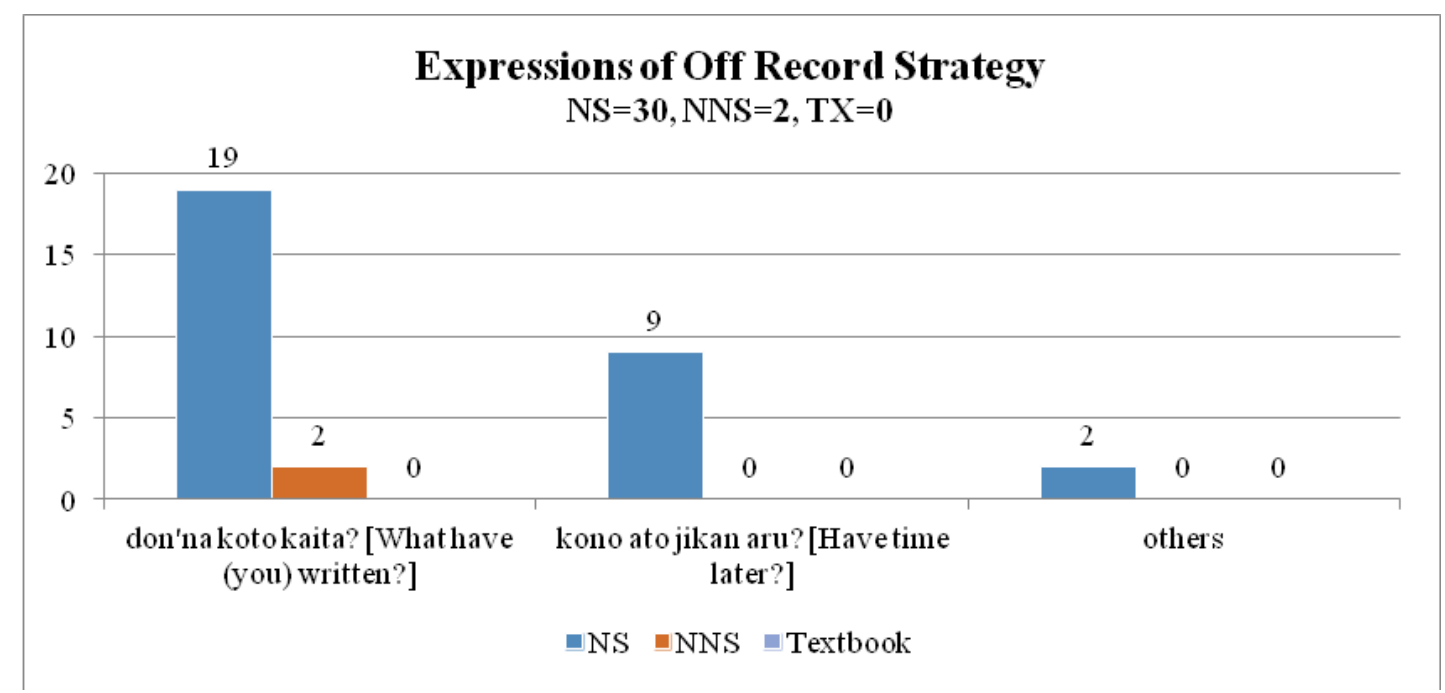

Figure 5.31 Expressions of Off Record Strategy

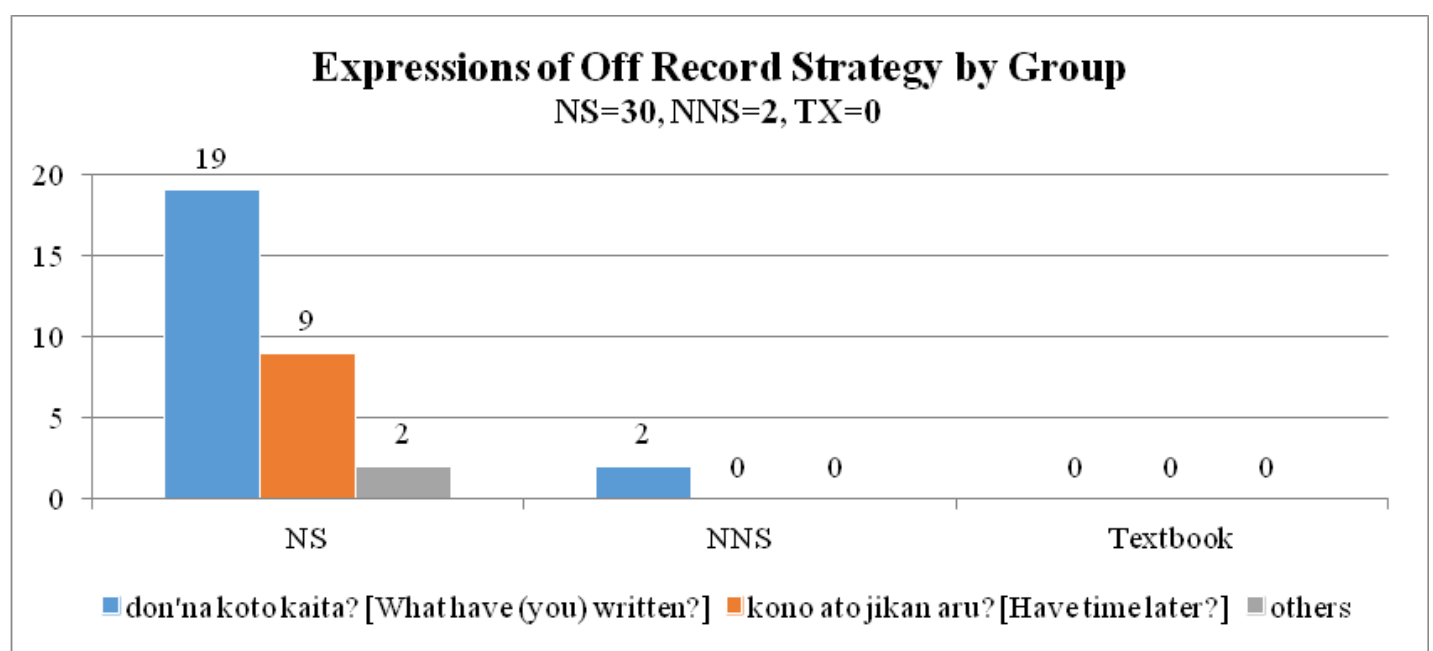

Figure 5.32 Expressions of Off Record Strategy by Group

\begin{tabular}{|c|c|}
\hline $\begin{array}{c}\text { Expressions of Off } \\
\text { Record Strategy } \\
\text { NS }=\mathbf{3 0}\end{array}$ & $\begin{array}{c}\text { Expressions of Off } \\
\text { Record Strategy } \\
\text { NNS }=2\end{array}$ \\
\hline $30 \%$ & $0 \% \quad 0 \%$ \\
\hline $\begin{array}{l}\text { don'na koto kaita? [What have } \\
\text { (you) written?] }\end{array}$ & $\begin{array}{l}\text { don'na koto kaita? [What have } \\
\text { (you) written?] }\end{array}$ \\
\hline $\begin{array}{l}\text { kono ato jikan aru? [Have time } \\
\text { later?] }\end{array}$ & $\begin{array}{l}\text { kono ato jikan aru? [Have time } \\
\text { later?] }\end{array}$ \\
\hline$\square$ others & others \\
\hline
\end{tabular}

Figure 5.33 Proportion of Expressions of Off Record Strategy 
Table 5.4 Typical Expressions of Off Record Strategy

\begin{tabular}{|l|l|}
\hline Japanese in the Romanised alphabet & English translation \\
\hline Donna koto kaita? & What have you written? \\
Donna koto kaitara ii ka? & What should I write? \\
Dono kurai jikan kakatta? & How long did you take? \\
Dō yatte kaku no? & What websites did you check? \\
\hline Kono ato jikan aru? & How did you write it? \\
\hline $\begin{array}{l}\text { Dareka tetsudatte kureru hito inai ka? } \\
\text { Ima nanka motteru, kami toka? }\end{array}$ & Have time later? \\
\hline
\end{tabular}

It was obvious that expressions of off record strategy were vague and ambiguous. The NNSs might not have noticed that there were expressions other than bald on record or positive (and negative) politeness strategies when making a request, which were regarded as very polite and reserved. The majority of off record expressions that the NSs uttered were phrases starting with donna [what] or dono kurai [how]. The interrogative sentences using what or how were not very difficult for the NNS participants. However, judging from the fact that only one NNS used this strategy, the rest of them presumably did not think of this. As none of the TXs either used off record strategy, this appears to have been beyond the scope of what was taught.

Textbooks would be enhanced by the inclusion of such indirect expressions uttered by NSs in model exemplars, or at least an appropriate explanation about off record strategy, in order to prevent the NNSs being embarrassed (or losing face) when not understanding what a NS implies.

\subsubsection{Frequent Utterances of Head Acts}

Fifty-three NSs and 35 NNSs uttered HAs more than once. Two-thirds of the NSs and over 40 per cent of the NNSs repeated requisitive sentences, regardless of the types of politeness strategies. Of these, it became apparent that 18 out of $53 \mathrm{NSs}(34 \%)$ and 11 out of 
35 NNSs (31\%) utilised different types of politeness strategies. Seven NSs (13\%) utilised negative and positive politeness strategies, ${ }^{167}$ six NSs (11\%) used negative and bald on record politeness strategies, ${ }^{168}$ and five NSs (10\%) used off record and positive politeness strategies. ${ }^{169}$ Eleven NNSs, who mostly employed bald on record as a group compared with the others, utilised only one combination of bald on record and positive politeness strategies. 170

Further investigation shows that the approaches of the NSs tended to begin with rather inexplicit (circumlocutory) expressions of request followed by more direct ones. In other words, the first HA of the NSs was less straightforward, and they gradually seemed to use more direct expressions. However, the NNSs seemed not to change their strategy of approach, even if they uttered HAs more than once. Since the model discourses in the TXs examined in this study all presented only one HA per discourse, none of them exemplified this tendency.

167 For example, Hinto hoshii naa to omotte... [I think I'd like to have a hint] (Negative)+misete kureru [Would you show me?] (Positive).

168 For example, Oshiete hoshii kedo... [I'd like you to teach me,...] (Negative)+onegai [Please] (Bald).

169 For example, Jikan aru [Have time?] (Off Record)+mite mo ii? [Can I see it?] (Positive).

170 For example: Misete [Show me] (Bald)+tetsudatte kurenai? [Wouldn't you help me?] (Positive). 


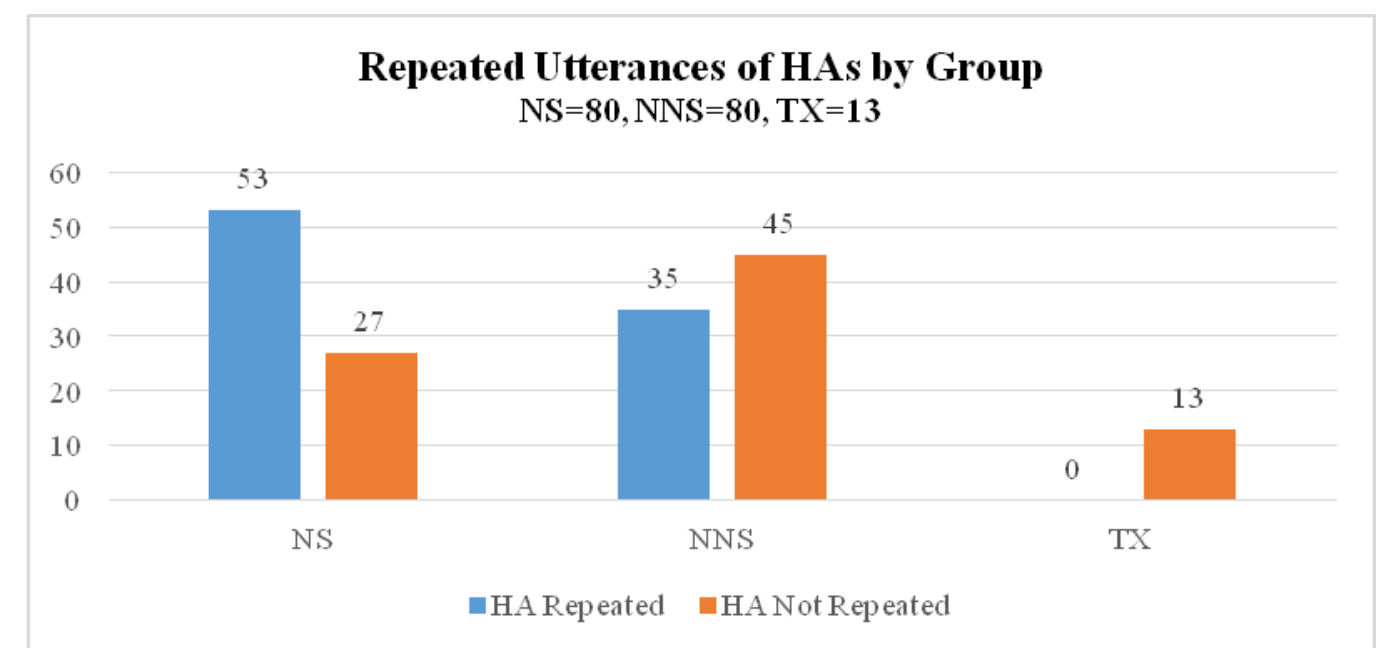

Figure 5.34 Repeated Utterances of Head Acts by Group

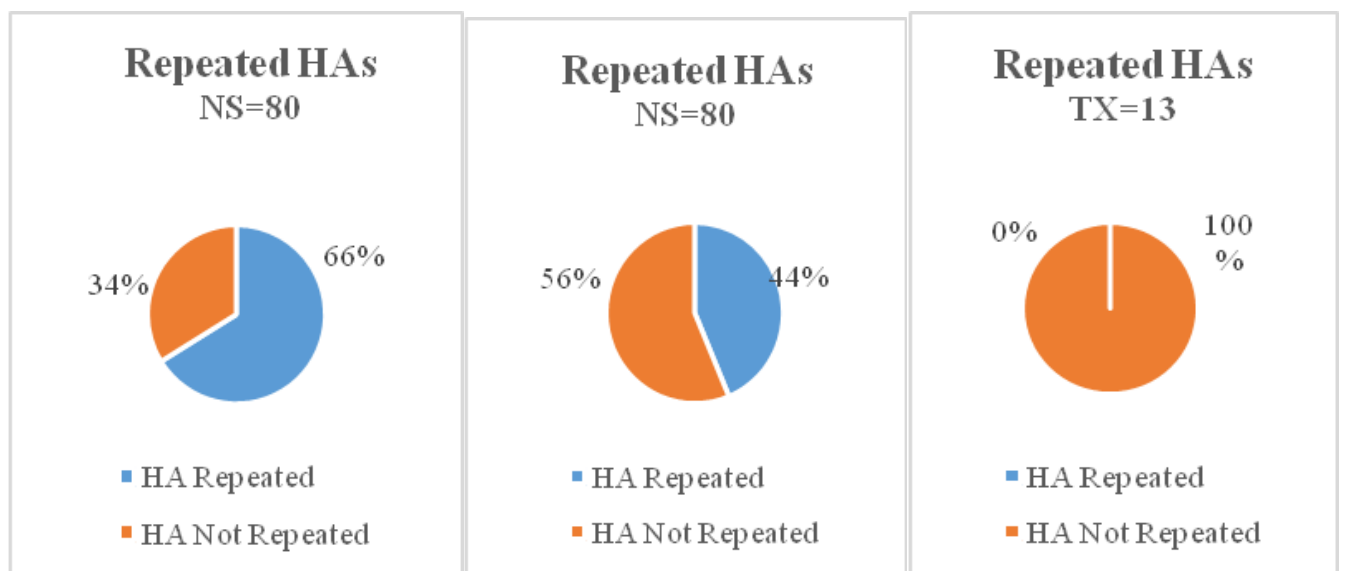

Figure 5.35 Proportion of Repeated Utterances of Head Acts

Table 5.5 Combination of Politeness Strategies

\begin{tabular}{|l|r|r|}
\hline Combination of Strategies & \multicolumn{1}{|c|}{ NS } & \multicolumn{1}{|c|}{ NNS } \\
\hline Negative+Positive & $7(13 \%)$ & 0 \\
\hline Negative+Bald on Record & $6(11 \%)$ & 0 \\
\hline Off Record+Positive & $5(10 \%)$ & 0 \\
\hline Bald on Record+Positive & 0 & $11(31 \%)$ \\
\hline Subtotal (who used different politeness strategies) & $\mathbf{1 8 ( 3 4 \% )}$ & $\mathbf{1 1 ( 3 1 \% )}$ \\
\hline Used the same politeness strategy & $35(66 \%)$ & $24(69 \%)$ \\
\hline Total & $\mathbf{5 3 ( 1 0 0 \% )}$ & $\mathbf{3 5 ( 1 0 0 \% )}$ \\
\hline
\end{tabular}




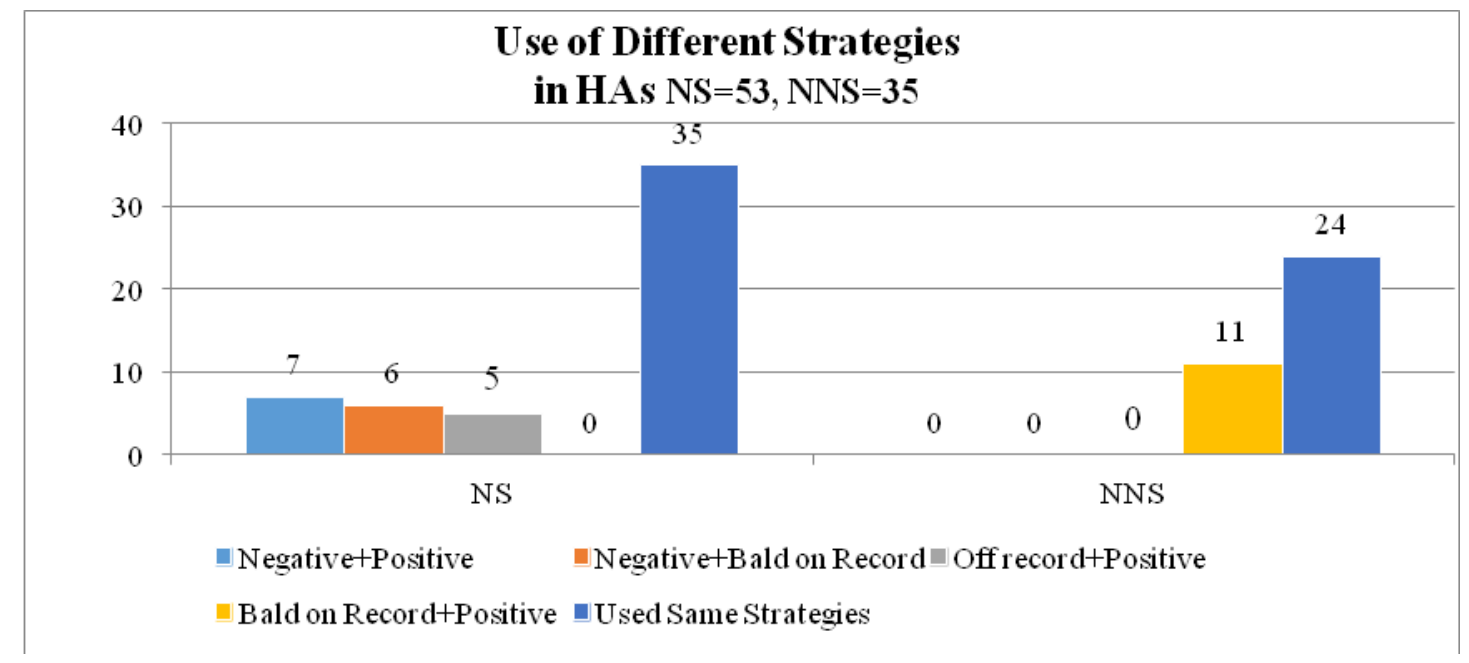

Figure 5.36 Use of Different Strategies in Head Acts by Group
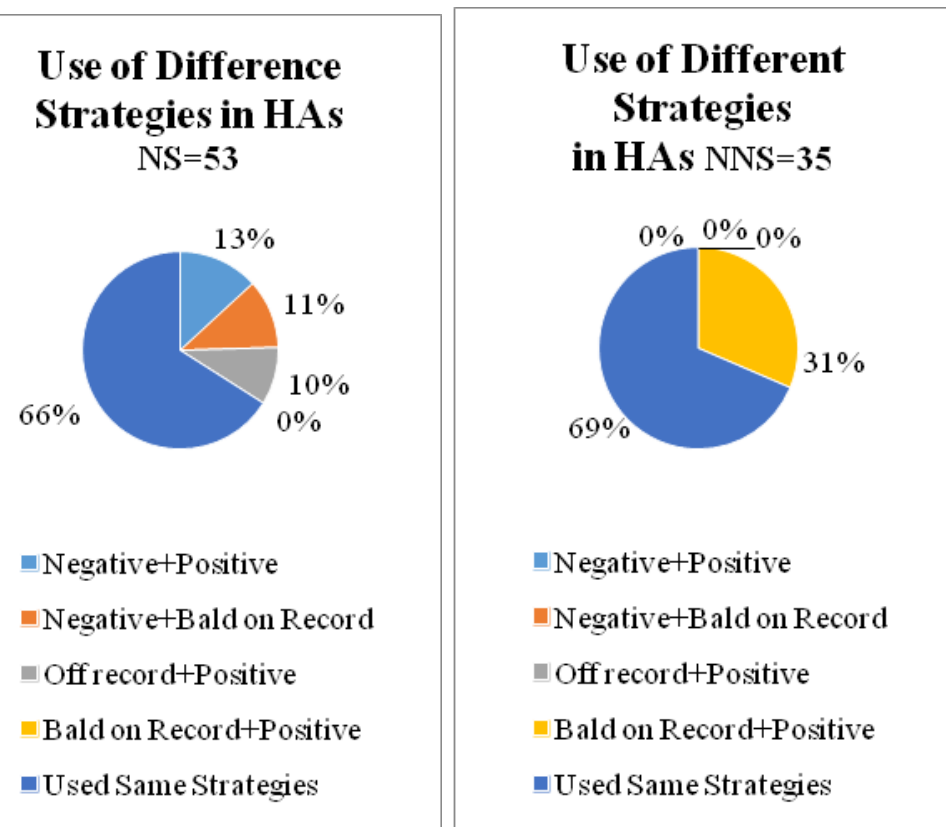

Figure 5.37 Proportion of Use of Different Strategies in Head Acts

\subsubsection{Supportive Moves of Request}

Supportive Moves (SMs) are speech acts to aggravate or mitigate a speaker's request, and they appear externally to the Head Act, either before or after it (Blum-Kulka et al. 1989: 287). In this study, the collected data were analysed based on five different types of SMs 
from the CCSARP. These are Preparator, Grounder, Disarmer, Promise of Reward (Reward), and Imposition Minimiser (Minimiser). ${ }^{171}$

Items analysed were:

(1) Number and proportion of utterances of $\mathrm{SMs}^{172}$

(2) Order of utterances of SMs

\subsubsection{Number of Supportive Moves}

The total number of the five different supportive moves uttered by the NSs was 405 (an average of 5.1 times per participant), 253 by the NNSs (an average of 3.1 times per participant), and 32 by the TXs (an average of 2.5 times per exemplar). The number of SMs uttered by the NSs was 1.6 times more than that of the NNSs, and twice as many as that in the TXs respectively.

171 Hereafter the names of the supportive moves in parentheses are used in the present research instead of those introduced in the CCSARP.

172 Pie charts and bar graphs were drawn based on the following concept. When drawing a pie chart/bar graph of a supportive move, if the number of utterances by either the NSs or the NNSs was larger than 80, or the number of exemplars presented in the TXs was larger than 13, the pie chart and bar graphs were drawn based on the numbers of utterances and examples as they were. If the number of utterances by either the NSs or the NNSs, or both, was smaller than 80 , or the number of exemplars presented in the TXs was smaller than 13, the pie chart and bar graphs were drawn based on the numbers of utterances and exemplars as they were together with the numbers of the un-uttered by the participants and/or un-presented in the exemplars. In other words, if none of utterances by the NSs or the NNSs was larger than 80 , or no exemplars were larger than 13 , the pie chart/bar graphs include those un-uttered/un-resented portion in order to reach $80 \mathrm{and} /$ or 13 respectively. Otherwise, the pie chart/bar graphs would mislead the readers, although they still reflect the status quo. 


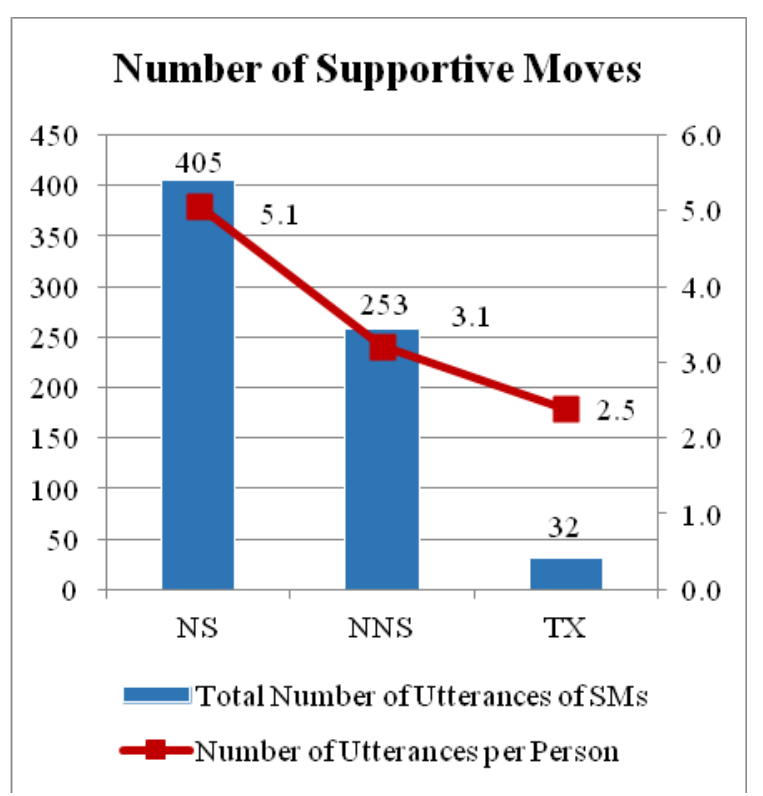

Figure 5.38 Number of Supportive Moves

The analyses show that the NSs and the NNSs used five SMs but the TXs used only four SMs, none of which included rewards in the present study. There were some noteworthy similarities and differences in the choices of SMs by participants and the writers of the TXs.

As for similarities, a grounder was the most favoured supportive move across the groups. The NSs' frequency of use was 2.3 times in a discourse, the NNSs used it on average 1.6 times and the TXs 0.8 times, and although the TXs showed it less than once, it was still one of the most frequently used SMs exemplified in the TXs. While a minimiser was in the second place as the favoured SM for the NSs, it was the third favourite for the NNSs, and in the TXs it tied in first place with the grounder.

The average utterance per requester or persona of the other remaining three SMspreparator, reward, and disarmer-was once or less than one utterance per discourse. By and large, the proportions of SMs by the NSs and the NNSs were similar in terms of grounder, preparator, reward, and disarmer. As for minimiser, however, the use by the NNSs was only 40 per cent of that of the NSs, which differentiates the NNSs from the NSs.

The proportion of use of SMs in the TXs was significantly different from that of the NSs and the NNSs. Although disarmers and rewards might be tactful supportive moves to 
induce the interlocutor to accept the request, the proportion of disarmers in the TXs was nine per cent, but their use among the NSs and the NNSs was negligible. By contrast, no TX presented a reward, whereas three per cent of each of the NSs and NNSs uttered rewards.

The characteristics of rewards demand good negotiation skills and the ability to read the interlocutor's feelings. Ideally, the TXs should exemplify as many supportive moves as possible, but this seemed unrealistic. The authors probably tended to choose items that they thought to be more relevant for learners to acquire as a basic conversational structure of requests. This could be one of the reasons for not presenting rewards in the TXs. Unlike the results of the role-plays performed by the NSs and the NNSs where some SMs were repeatedly employed, the authors seem to have simply presented a plausible story but they did not seem to be concerned about the frequency of pertinent utterances. For instance, grounders should have been uttered by personae more often than appeared in the results of the present study (0.8 times/personae) because the NSs and the NNSs did so more than once in a discourse. In other words, when authors create the scenarios, they appear to be concerned about the types of SMs but not about their frequency.

\section{Grounders (Positive politeness)}

Grounders, which inform a requestee (interlocutor) of the reasons, explanations, or justifications for a request by a requester, ${ }^{173}$ occupied a significantly large part of the utterances in this present study. There were 182 grounders by the NSs, 126 by the NNSs, and ten exemplars in the TXs. This means that the NSs uttered grounders 2.3 times per discourse, the NNSs did so 1.6 times, and the exemplars presented them 0.8 times in a discourse.

173 The definition of grounder is described as: "The speaker gives reasons, explanations, or justifications for his or her request, which may either precede or follow it" in the CCSARP (1989: 287). 
One hundred and fifty-four out of $182(85 \%)$ utterances of grounders by the NSs were reasons for not completing the assignment, and for the NNSs it was 76 out of $126(60 \%)$ utterances, for the same reason. Their expressions included, for example, Mada yatte nai [I haven't started it yet], Owatte nai [I haven't finished], and so on. Another reason was time constraints, such as being too busy or having no time. Eleven NSs $(6 \%)$ chose this reason related to time, as did 24 of the NNSs (19\%), which was three times more than the NSs. The typical explanations were Isogashikatta [I was too busy] and Jikan ga nakute [I didn't have enough time]. Another reason which naturally only NNSs could make use of was the difficulty of the Japanese language. Fourteen utterances (11\%) accounted for the difficulty of the Japanese grammar, vocabulary or the requester's lack of proficiency in the Japanese language. The typical expression was Nihongo wa muzukashii [Japanese is difficult]. ${ }^{174}$

There was a tendency for the requester to repeat the same reason. Forty-four NSs $(56 \%)$ repeated the same reason, and so did 20 NNSs $(28 \%) .{ }^{175}$ One NS and eight NNSs, however, did not provide the requestee with the reason for the request. While the direct (primary) reason for requesting, Mada yatte nai [I haven't finished], occupied 85 per cent for the NSs and the indirect (secondary) reason, Isogashii [I'm too busy], accounted for only six per cent; for the NNSs the indirect (secondary) reason was 19 per cent, which was three times more than for the NSs, although the order of frequency was the same in the two groups. ${ }^{176}$

174 The researcher of the present experiments did not tell the participants what type of assignment/homework it was that they needed some help with. However, 14 NNSs seem to have thought spontaneously that it was something related to the Japanese language.

175 The calculations of these are: $44 / 79=56 \%$ (NS) and 20/72=28\% (NNS). These are effective numbers. In the case of nominal numbers, they will be: $44 / 80=55 \%$ (NS) and $20 / 80=25 \%$ (NNS)

176 The definition of primary and secondary reasons here is as follows: although both I was busy and I have not started yet are both issues related to the speakers (requesters), 'being busy' of itself is not a direct cause of the problem, so it is a secondary reason; but the speaker's not having started yet is the direct problem here, so it is the primary reason. In other words, it may have been possible for others to have overcome this situation of 'being too busy'. 
The native speakers tended to use more direct explanations which would appeal to the requester's great need of the requestee. By contrast, use of being too busy as a reason or excuse might run the risk of giving the interlocutor the opportunity of questioning the speaker as to why he/she had left it unfinished until then, so the degree of appeal to the interlocutor was less than the explanation of the requester's current situation.

All scenarios in the exemplars in the TXs were unrelated to a homework/assignment, so, owing to the differences in the settings of the model discourses, all reasons given as grounders were categorised as "others". ${ }^{177}$

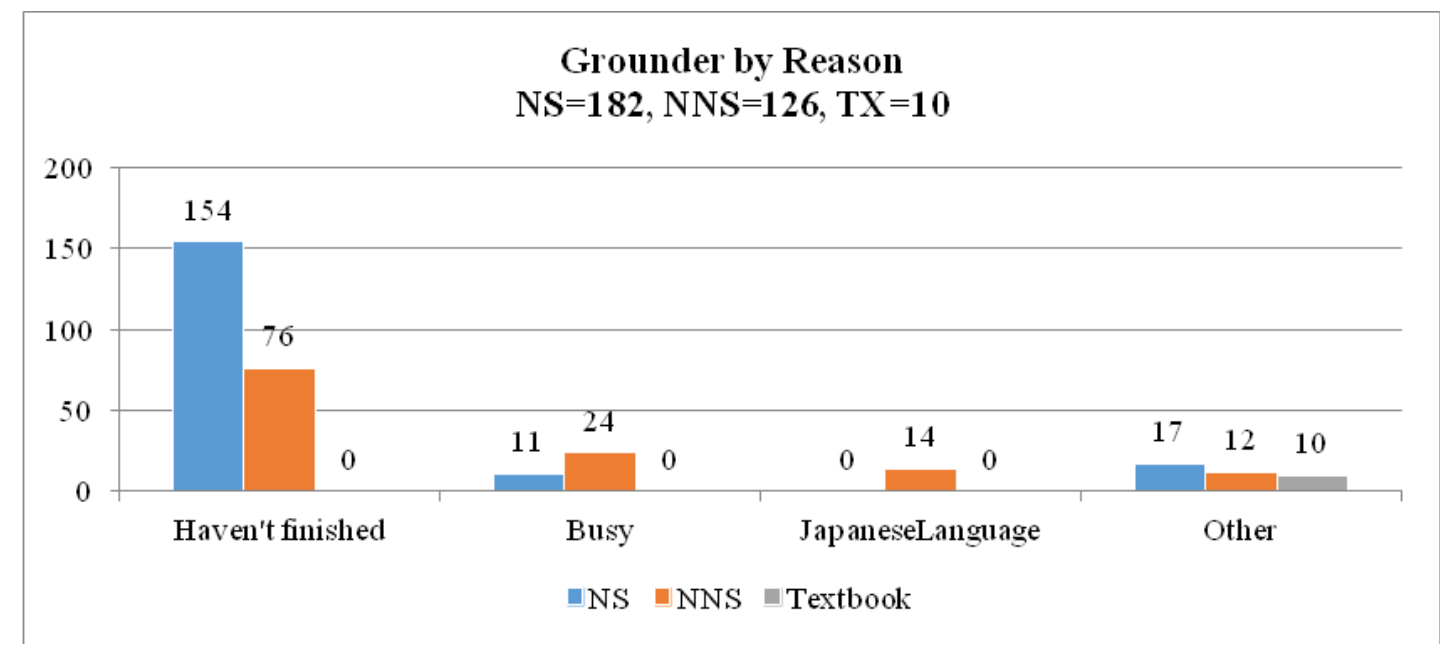

Figure 5.39 Grounder by Reason

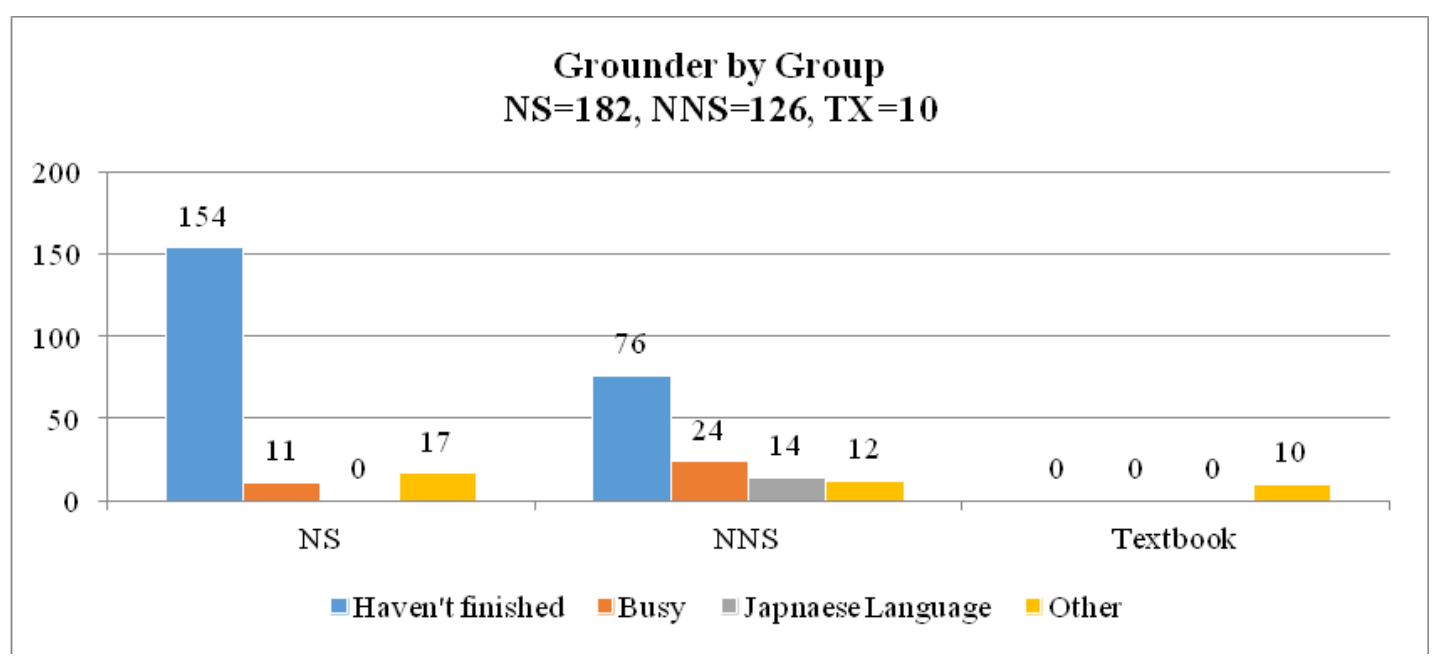

Figure 5.40 Grounder by Group

177 Three exemplars do not present reasons for the request. 


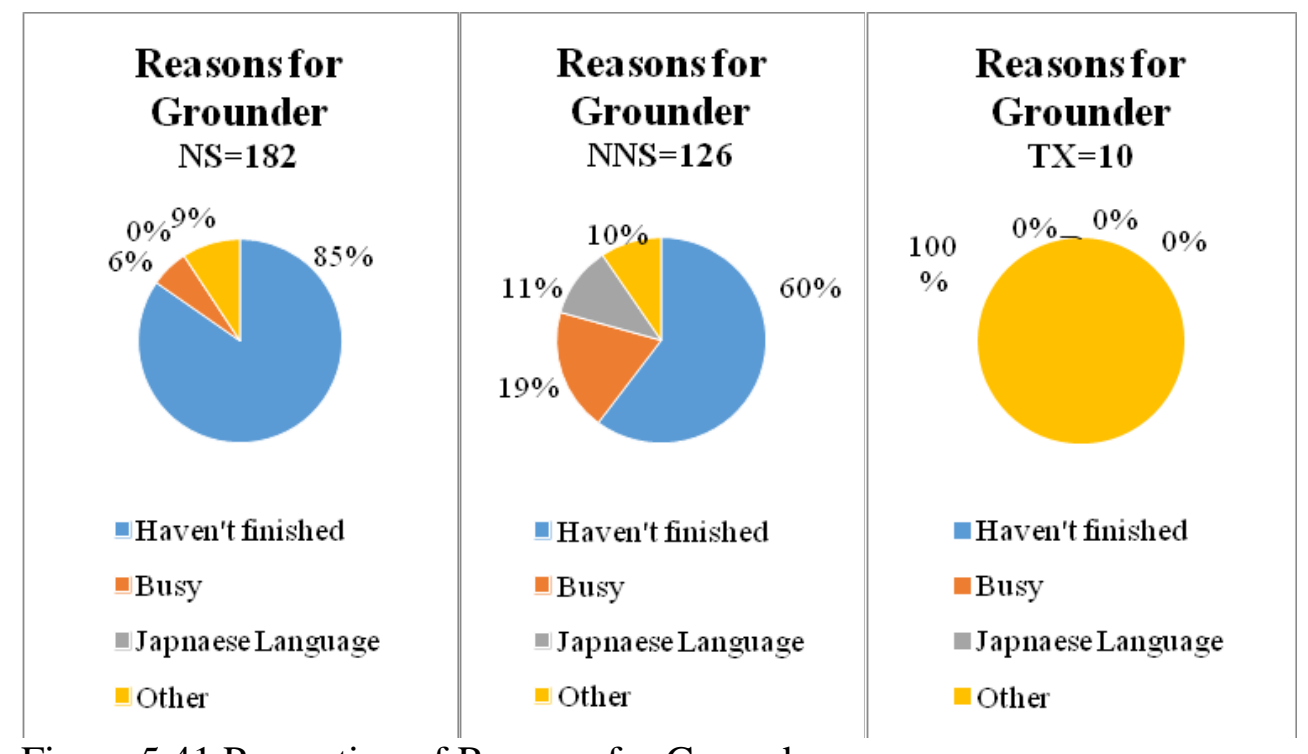

Figure 5.41 Proportion of Reasons for Grounder

\section{Minimisers (Negative politeness)}

The definition of an imposition minimiser by the CCSARP is a situation in which the speaker tries to reduce the imposition placed on the hearer by the request. ${ }^{178}$ This was the second most frequently used SM by the NSs and in the TXs after grounders, and the third for the NNSs following grounders and preparators. One hundred and twenty-two such utterances were delivered by the NSs $(21 \%)$ and ten exemplars $(22 \%)$ in the TXs, whereas the NNSs utilised minimisers only 43 times $(11 \%)$ in their entire SMs. This means that the NSs used them more than once in a discourse (1.3 times), whereas the NNSs did so 0.5 times, and for the TXs it was 0.8 times.

The most frequent expression of minimisers was chotto [a little bit]. This word was dominant among minimisers and accounted for 66 per cent of the NSs' and 45 per cent of the NNSs' minimising utterances; for the TXs it reached 69 per cent. Others were Moshi dekireba [If you can] or Moshi kanō nara [If it is possible], and variations on these were frequently uttered by the NSs. In such cases, the requester is explicitly indicating dependence

178 In the CCSARP (288). 
on the requestee's judgement (condescension) as to whether he/she will accept the request. Although this was uttered by 17 NSs (14\%), only two NNSs did so $(2 \%)$ and there was no exemplar in the TXs.

Other expressions were used to downplay the imposition of the request by using the word dake [only], such as Saisho dake [Only the beginning], or to restrict a requester's conduct, such as Sankō ni suru dake [Only to refer to it], Kopī suru dake [Only to photocopy it]. The number of uses of dake by the NSs was $12(10 \%)$ and that of the NNSs was $2(3 \%)$.

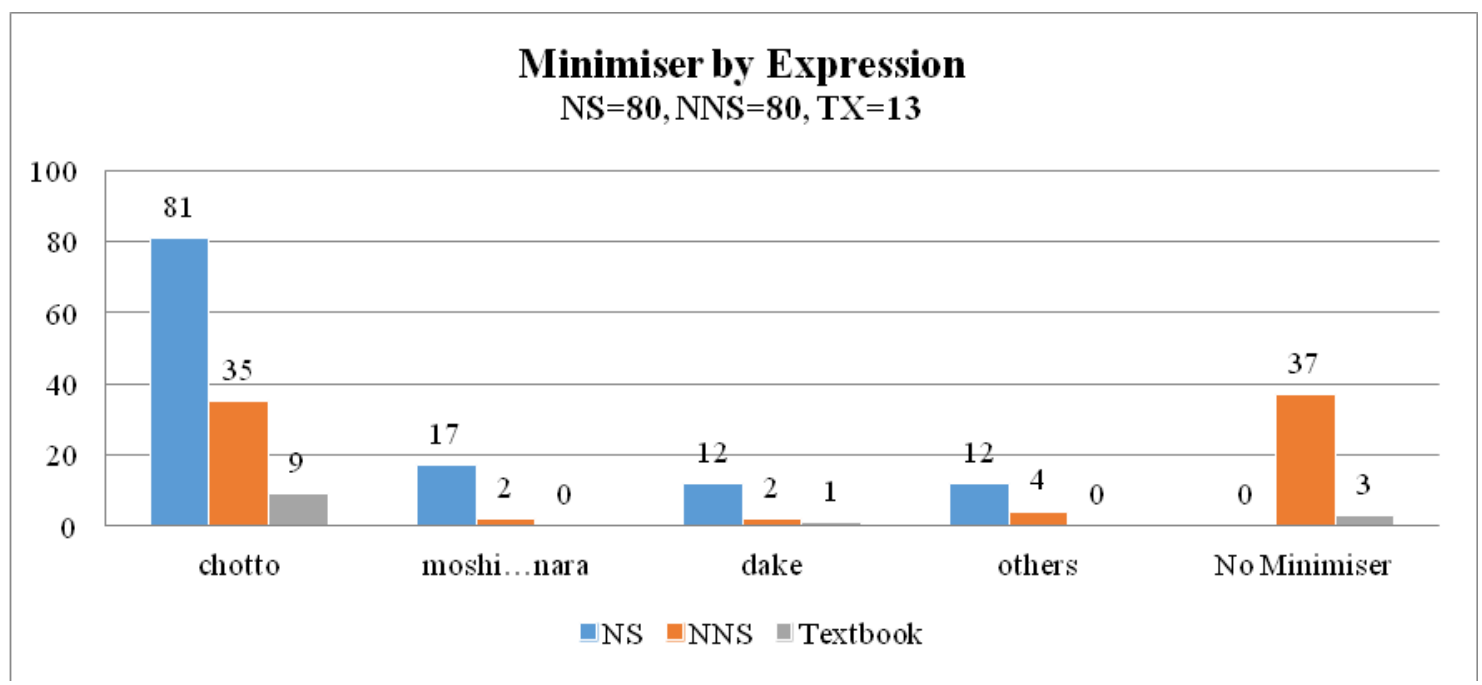

Figure 5.42 Expressions of Minimiser

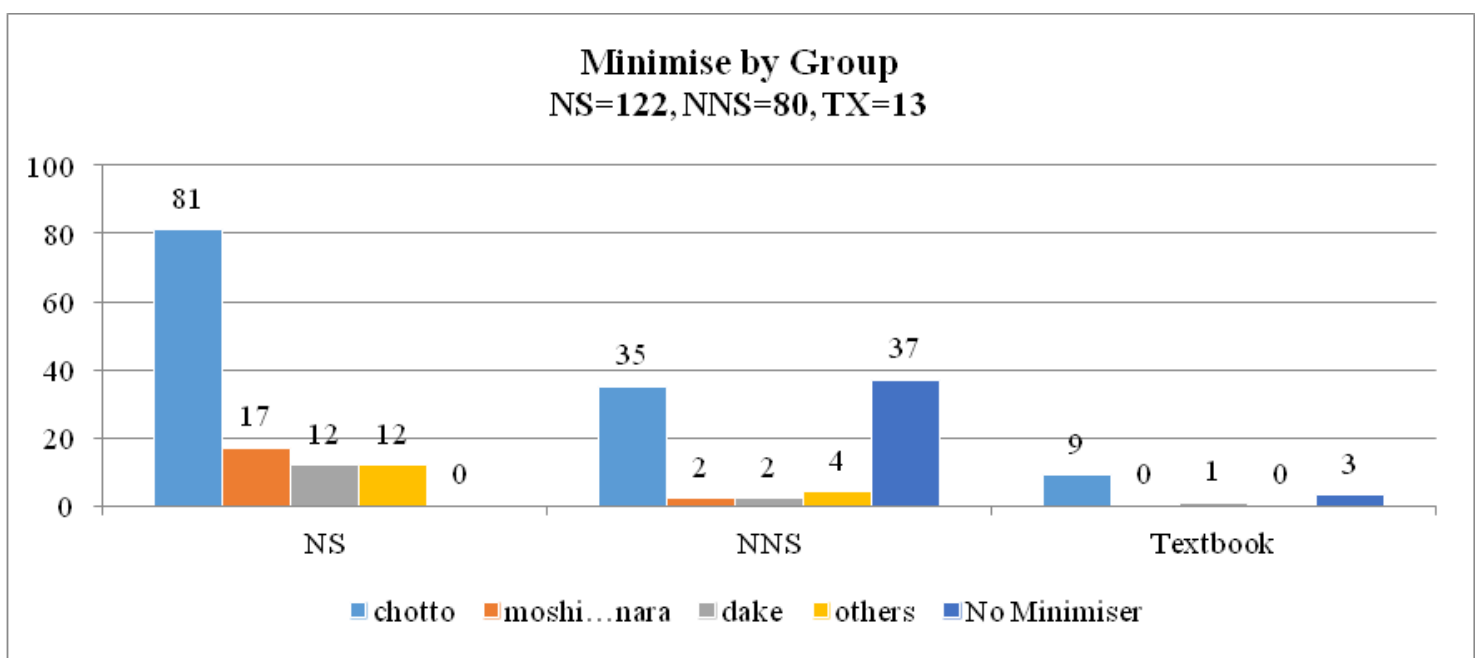

Figure 5.43 Minimiser by Group 


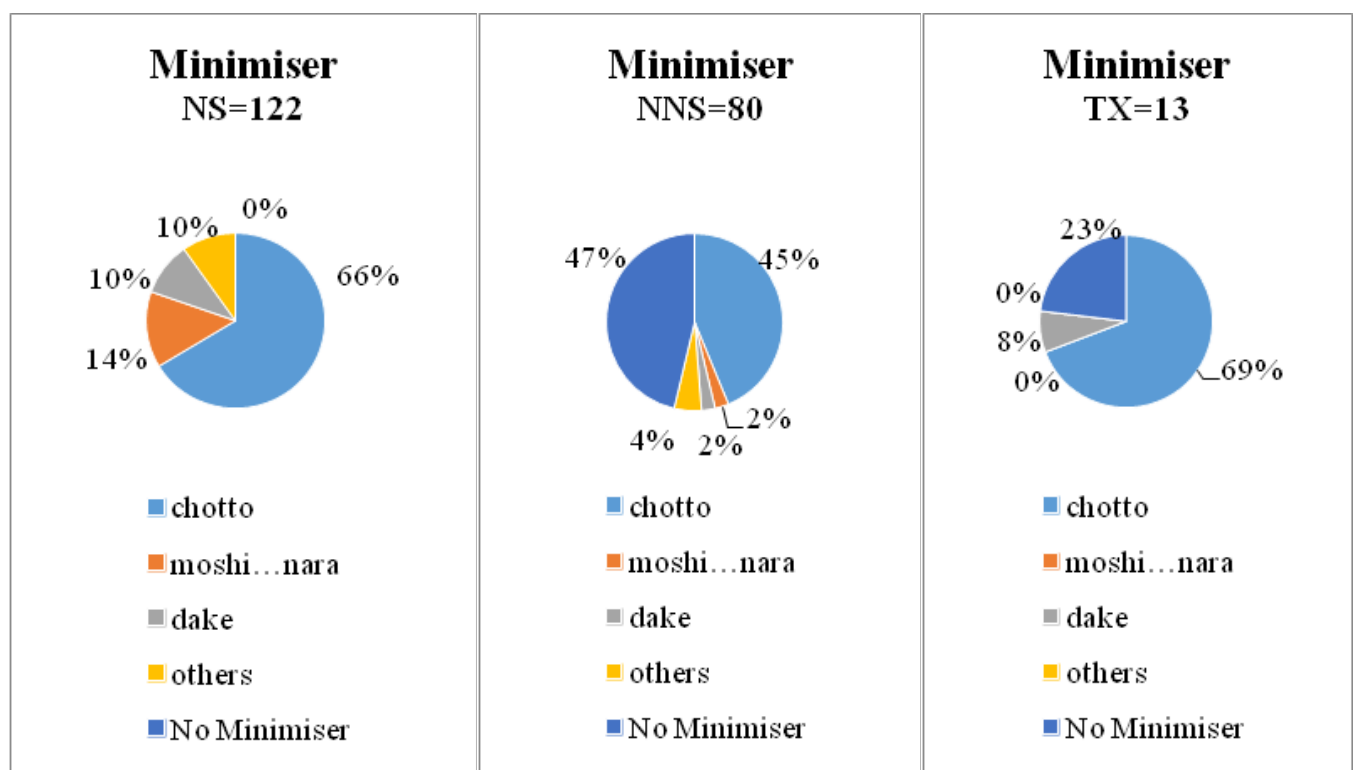

Figure 5.44 Proportion of Minimiser

\section{Preparators (Negative politeness)}

In most cases the preparators were the first utterance by a requester in this experiment, presumably so as to ascertain the potential availability of the hearer to carry out the request. Eighty NSs (100\%) and 68 NNSs $(85 \%)$ uttered some preparatory expressions, and eight (62\%) out of 13 exemplars presented preparators in the TXs.

The absolute majority of preparators were used in sentences asking casually whether the other had finished the assignment, such as Mö yatta/owatta? [Have you done yet/finished it yet?]. This expression amounted to 64 utterances by the NSs $(80 \%)$ and 44 by the NNSs $(55 \%)$. One requester of the NS group asks a friend about note-taking in order to check whether the interlocutor is likely to comply with a request by saying, Senshū no jugyō deta? Nōto toka totteru yo ne [Did you attend the class last week? You took notes or something, didn't you?]. This utterance does not directly ask whether the interlocutor has finished the assignment; however, it implies that the requester believes in the requestee's capability to help with the assignment. 
Onegai ga aru [I have something to ask] and its variants were used by three NSs, nine NNSs, and seven exemplars. Another expression, Tanomitai/kikitai koto ga aru n' da/arimasu [I'd like to ask you something] was employed by one NS and six NNSs. The preparatory nature of these two sentences is very similar to each other, and the former was most often presented in the TXs. Consequently, the proportion of Onegai ga aru in the TXs was as high as 54 per cent because of the small number of examples. Onegai ga aru can be applied to any requests at the start of the discourse, so this might be the reason for the high percentage in the TXs. On the other hand, its low usage by the NSs and NNSs would be that they prefered to ask the interlocutor's capability to help with the assignment straightforwardly by uttering Mō yatta/owatta?, particularly since their interlocutors were close friends.

Another expression of preparator, Dōshitara ii ka [What shall I do?] and its variations, was utilised by four NSs and 11 NNSs. This implies the speaker's expectation of some help by revealing his/her inner thoughts indirectly. Behind the utterance of this expression, there was a common understanding for the NSs and the NNSs (but not presented in the TXs) that the speaker was asking for some help from their interlocutors about the assignment/homework. That is perhaps the reason why the TXs did not have this expression. ${ }^{179}$

Four NSs, two NNSs and one exemplar uttered the widely used expression, Jikan aru? [Do you have time?], to ask whether or not it was convenient for the interlocutor to help them. Another inquiry took the form of asking how the interlocutor wrote the assignment, such as Doyatte kaita? [How did you write it?], which was uttered by five NSs and two NNSs.

179 In the exemplars of the TXs, requesters asked for a mobile phone number and making copies, etc. Therefore, the expression, dōshitara ii ka, does not suit some model discourses. 
While none of the NSs omitted preparators, 12 NNSs (15\%) and five TXs (38\%) did not use any preparatory sentences. The main reason about one-third of TXs did not present preparators might be that they were focusing on how to utter a requisitive sentence rather than considering the way of approaching the interlocutor. However, judging from the fact that all the NSs and 85 per cent of the NNSs employed preparators, the TXs should take preparators into consideration.

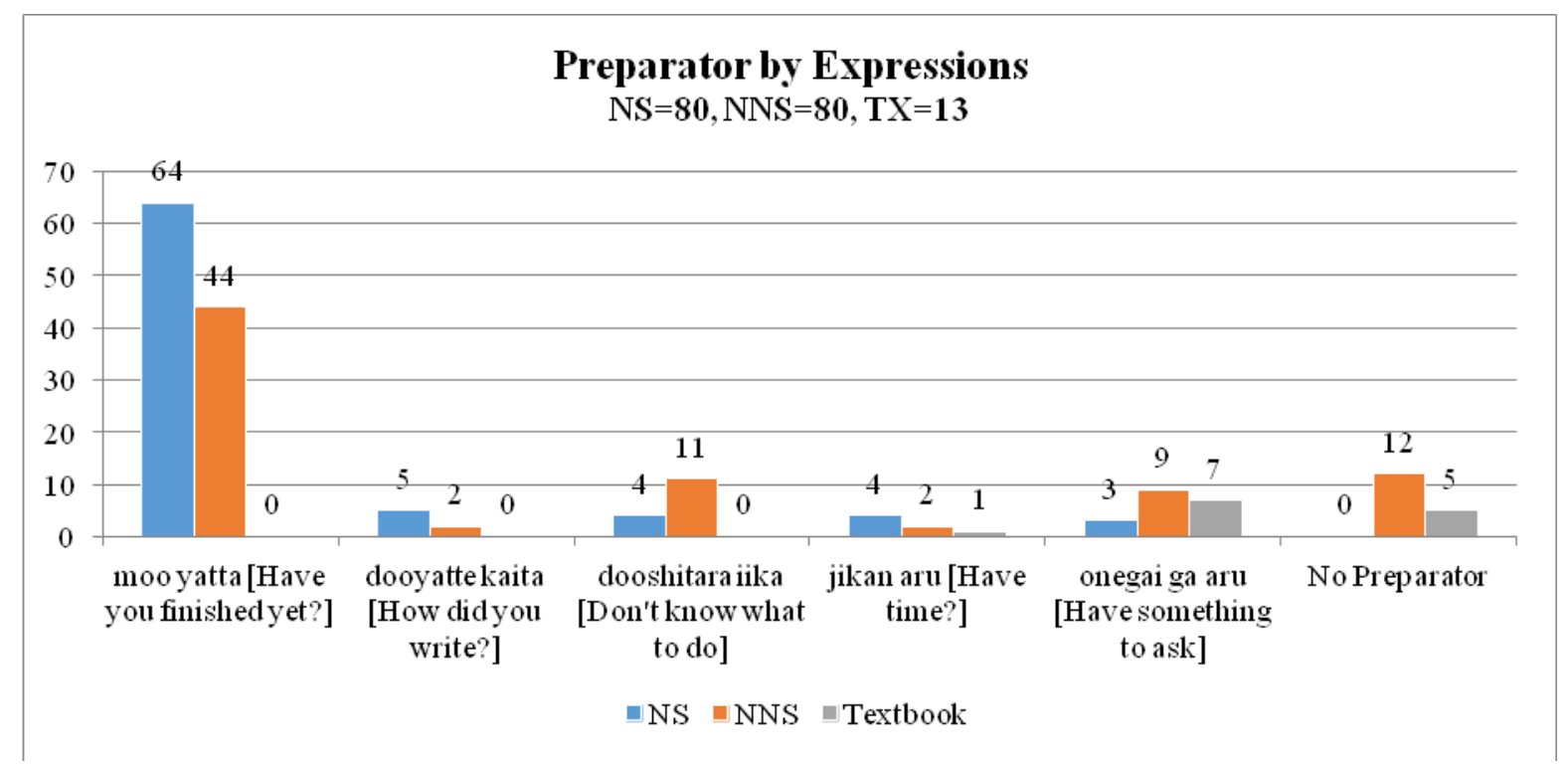

Figure 5.45 Expressions of Preparator

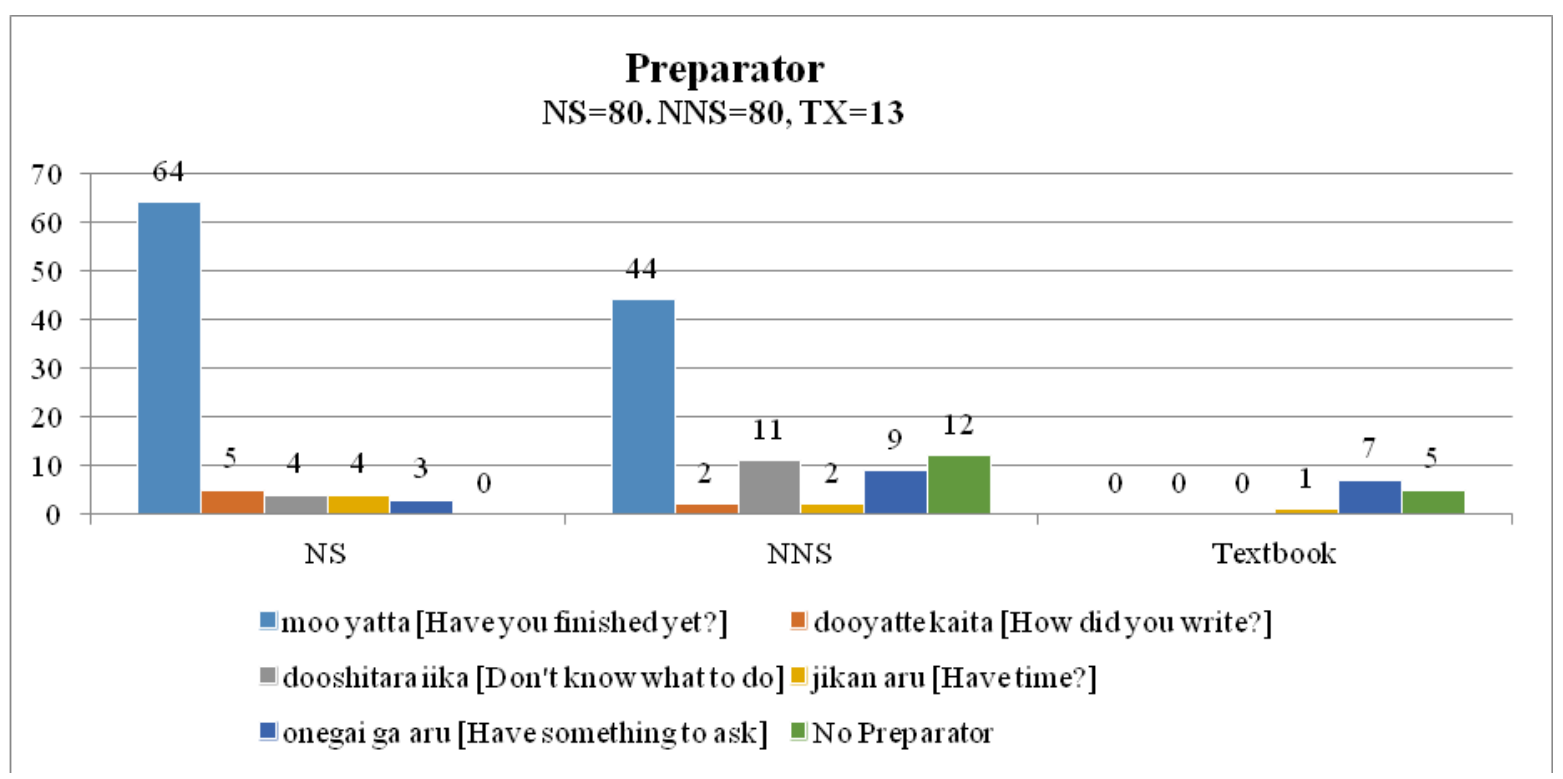

Figure 5.46 Preparator by Group 


\begin{tabular}{|c|c|c|}
\hline $\begin{array}{c}\text { Preparator } \\
\mathrm{NS}=\mathbf{8 0}\end{array}$ & $\begin{array}{c}\text { Preparator } \\
\text { NNS }=80\end{array}$ & $\begin{array}{c}\text { Preparator } \\
\mathrm{TX}=13\end{array}$ \\
\hline$-0 \%$ & $\begin{array}{l}11 \% \\
3 \% \\
14 \% \\
\end{array}$ & $38 \%$ \\
\hline $\begin{array}{l}\text { moo yatta [Haveyou finished } \\
\text { yet?] }\end{array}$ & $\begin{array}{l}\text { moo yatta [Have you finished } \\
\text { yet?] }\end{array}$ & $\begin{array}{l}\text { moo yatta [Haveyou finished } \\
\text { yet?] }\end{array}$ \\
\hline $\begin{array}{l}\text { dooyatte kaita [How didyou } \\
\text { write?] }\end{array}$ & $\begin{array}{l}\text { dooyattekaita [How didyou } \\
\text { write?] }\end{array}$ & $\begin{array}{l}\text { dooyatte kaita [How didyou } \\
\text { write?] }\end{array}$ \\
\hline $\begin{array}{l}\text { dooshitara iika [D on't know } \\
\text { what to do] }\end{array}$ & $\begin{array}{l}\text { dooshitara iika [Don't know } \\
\text { what to do] }\end{array}$ & $\begin{array}{l}\text { dooshitara iika [D on't know } \\
\text { what to do] }\end{array}$ \\
\hline -jikan aru [Have time?] & 口jikan aru [Have time?] & Đikan aru [Have time?] \\
\hline $\begin{array}{l}\text { on egai ga aru [Have something } \\
\text { to ask] }\end{array}$ & $\begin{array}{l}\text { on egai ga aru [Have } \\
\text { something to ask] }\end{array}$ & $\begin{array}{l}\text { - onegai ga aru [Have } \\
\text { something to ask] }\end{array}$ \\
\hline No Preparator & $\square$ No Preparator & No Preparator \\
\hline
\end{tabular}

Figure 5.47 Proportion of Preparator

\section{Rewards (Positive politeness)}

Thirty-nine discourses by the NSs (49\%) entailed a reward when negotiating between the requester and the requestee, and so did 22 discourses by the NNSs (28\%). By contrast, none of the TXs presented a reward in the exemplars of the setting of request.

While 17 out of 39 NSs' discourses offered a reward from the requester-for example, treating the interlocutor to lunch or a cup of coffee - the other remaining 22 out of $39 \mathrm{NSs}$ ' discourses triggered a reward by the requestee. Their utterances included Kadai dashi owattara nanka ogoru shi [As soon as I submit the assignment, I'll treat you, so...] by a requester, and Jaa ashita no hirugohan ogotte kureru nara [Well, if you'll buy me lunch tomorrow] by a requestee. 
As for the NNSs, 11 discourses included a reward uttered by the requesters and another 11 discourses uttered by the requestees respectively. The content of the reward was similar to that of the NSs, mostly providing a treat to the other, yet their expressions were simpler or more direct. For example, Kōhī katte ageru kara [I'll buy you a cup of coffee] by a requester of the NSs group.

Not all responses of either the requestee (if the requester offered the reward) or the requester (if the requestee offered a counterproposal) were accepted immediately. Sometimes negotiations occurred; however, in the end the reward worked effectively, as none of them was rejected, and finally the requesters' wishes were taken into account by the requestees in the present study. The percentage of presenting a reward was still below 50 per cent - even in the case of the NSs, although they were very close to it - but it functioned satisfactorily from the viewpoint of the requester. ${ }^{180}$ Twenty-one per cent of the NSs offered a reward to requestees. This strategy, rewards, should have been listed in the TXs as one of the effective actions when making a request in Japanese as long as the model dialogue was not too aggressive.

180 No rewards in this experiment were rejected by any interlocutor, although there were some negotiations between the two parties, such as a requestee counter-proposing lunch instead of the cup of coffee which was offered at first by the requester. 


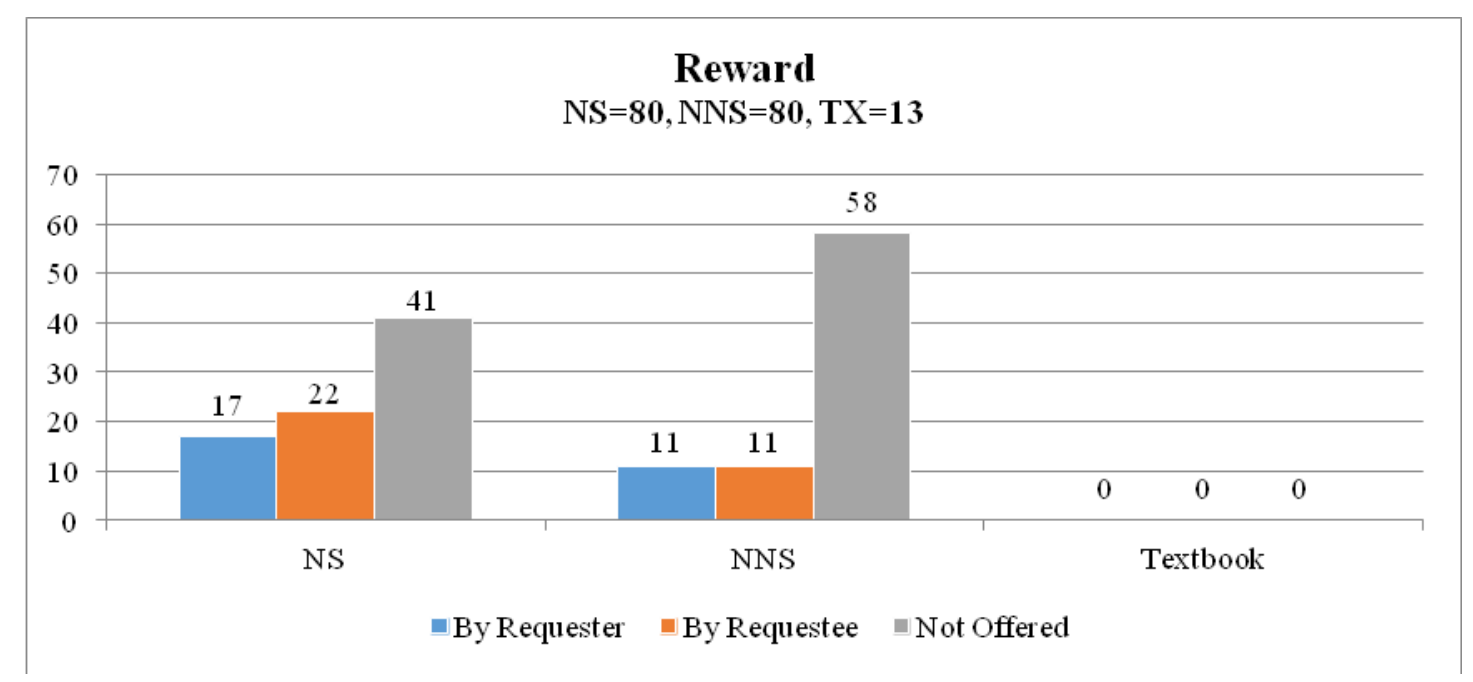

Figure 5.48 Reward by Group

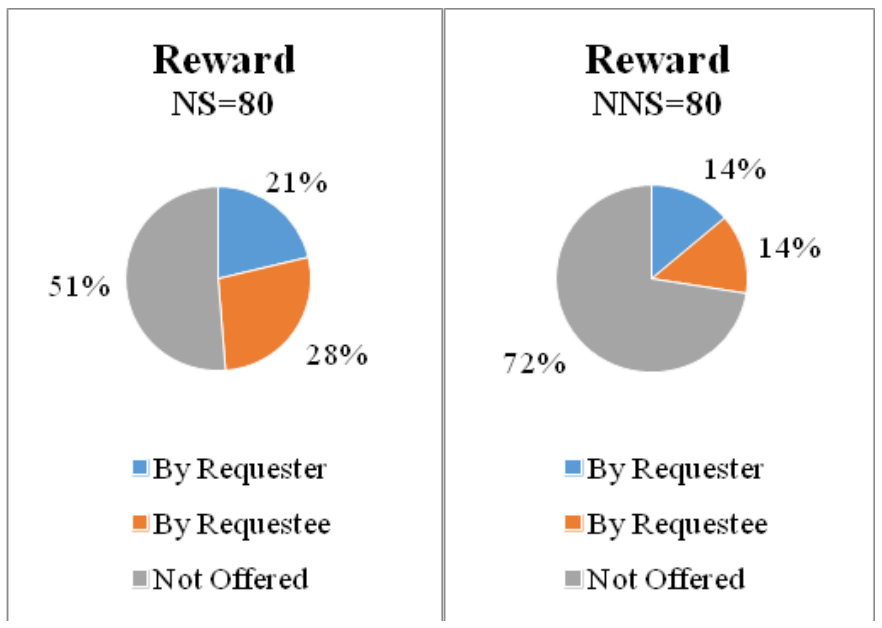

Figure 5.49 Initiator of Reward

N.B. There were no exemplars of reward presented in the TXs.

\section{Disarmers (Positive politeness)}

Disarmers were rarely uttered by the NSs, the NNSs, and exemplars in the present study. There were only four utterances by the NSs, five by the NNSs, and four in the TXs. Comparatively, the TXs, with disarmers taking nine per cent of all utterances in the TXs, showed a higher proportio, compared to only one per cent each of the NSs and the NNSs.

With the expression Tomodachi darō? [You're my friend, aren't you?], the requester tries to emphasise their relationship: two NSs and five NNSs used this. Also, Warui kedo... [It's wrong of me, but...] was used by two NSs and three exemplars in the TXs. The other was presented only in the TXs, Tsugō no ii toki de ii n' desu [It's ok whenever you're 
available], which shows the requesters' apologetic feelings in making the request, and at the same time it was understood to be a burden on the requestee. The latter two, Warui kedo [Sorry, but] and Tsugō no ii toki de ii n' desu, being employed by none of the NNSs, must have been difficult for them to think of uttering, although Warui kedo could have been used if they recognised that the function of this phrase is to reveal that the speaker is considering the feelings of the other.

On the other hand, the rather straight expression Tomodachi darō? tended to be favoured by the NNSs rather than the NSs. In general, the authors of the TXs seem to have opted for more modest sentences than the others.

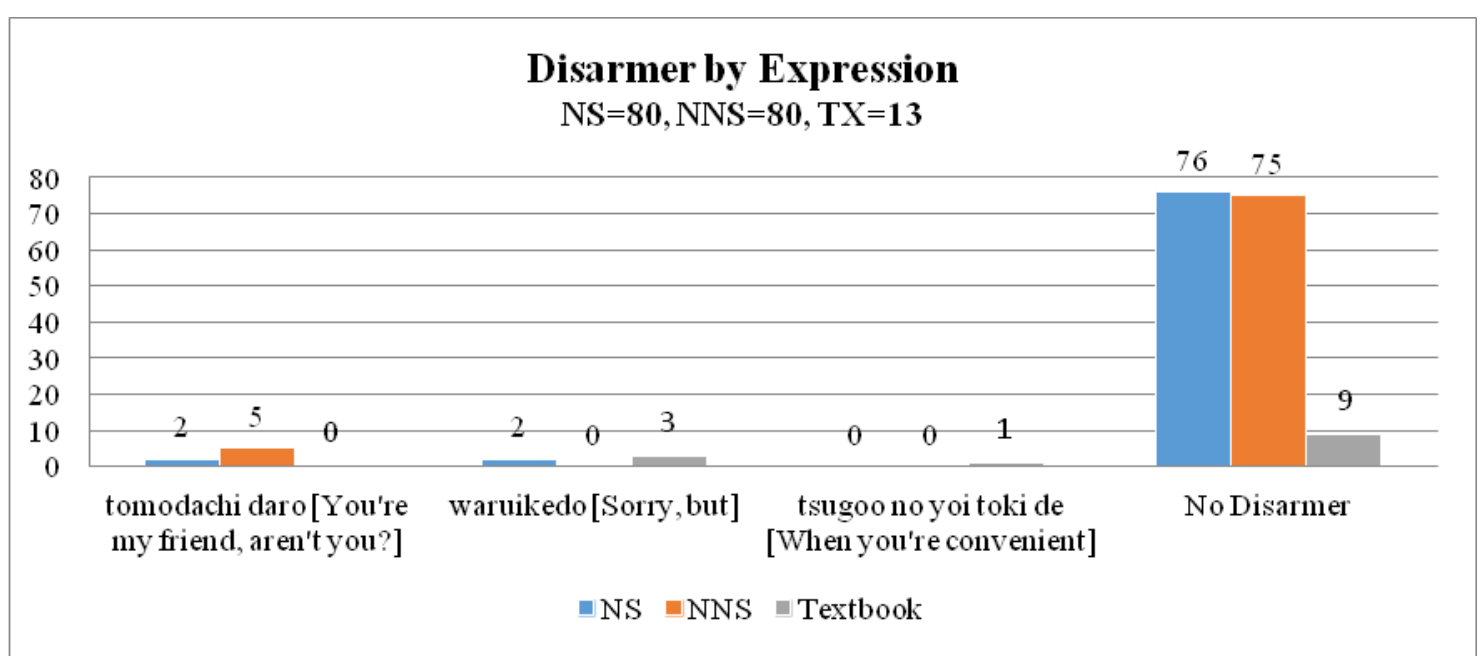

Figure 5.50 Expressions of Disarmer

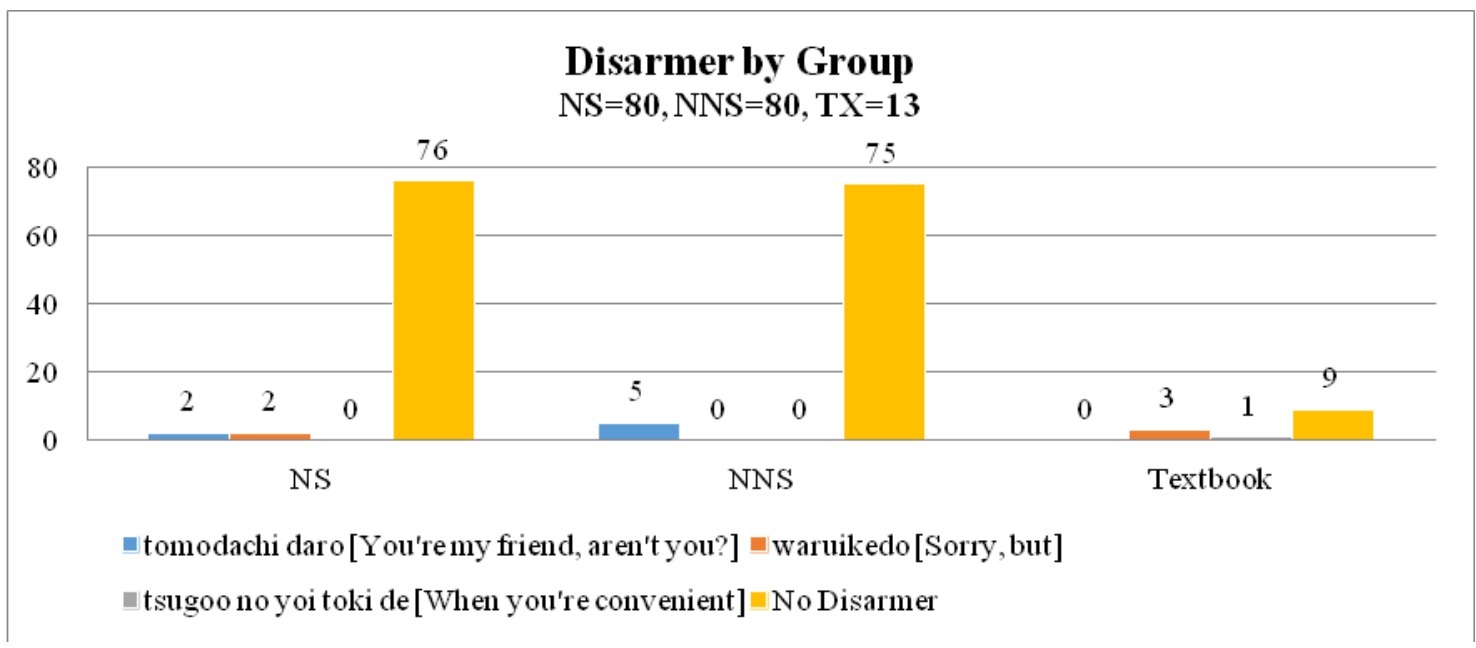

Figure 5.51 Expressions of Disarmer by Group 


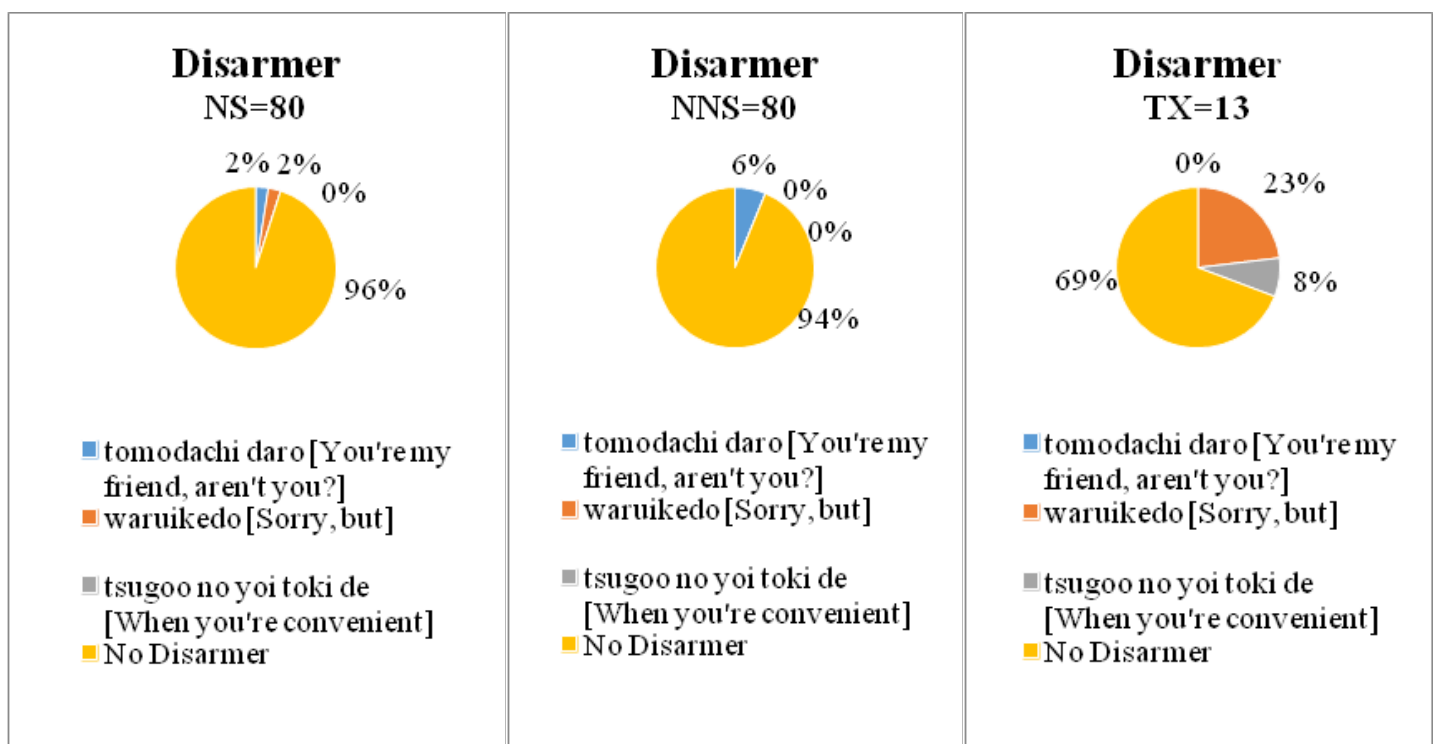

Figure 5.52 Proportion of Disarmer by Expression

\subsubsection{Structure of Discourse of Request}

\subsubsection{Order of Utterances}

By analysing the order of utterances until a speaker utters the first HA, ${ }^{181}$ it is possible to understand the strategy of approach in order to elicit assent from an interlocutor. The order of utterances of SMs was largely concentrated in the following three groups which comprised a combination of preparators, grounders, and minimisers.

These are:

(1) Preparator $>$ Grounder $>\mathrm{HA}^{182}$

(2) Preparator $>$ Minimiser $>\mathrm{HA}$

(3) Grounder $>\mathrm{HA}$

Seventy-seven NSs out of $80(96 \%)$ took one of the above three orders of utterance of SMs before uttering the first HA, as did 53 NNSs (66\%), and seven exemplars out of 13 TXs $(54 \%)$.

181 The order of SMs before the first utterance of HA.

182 "Preparator $>$ Grounder $>$ HA" indicate the order of utterance, starting from prepartor followed by grounder and then HA. 
Forty-four, more than half of the NSs (55\%), employed the order of preparator>grounder $>\mathrm{HA}$, followed by $21 \mathrm{NSs}(26 \%)$ who uttered SMs in the order of preparator $>$ minimiser $>\mathrm{HA}$, and $12 \mathrm{NSs}(15 \%)$ with the order of grounder $>\mathrm{HA}$. The order of utterances by the NNSs showed a wider range of combinations than that of the NSs and the TXs. The most frequently used combination by the NNSs was, like that of the NSs, preparator>grounder>HA, which was used by 25 NNSs (31\%), followed by 14 NNSs with preparator $>$ minimiser $>\mathrm{HA}(18 \%)$ and another $14 \mathrm{NNSs}$ with grounder $>\mathrm{HA}(18 \%)$. Among the noteworthy characteristics of the NNSs was that 11 of them (14\%) abruptly uttered the HA - the requisitive sentence-without prior SMs, which meant that they did not utter any SMs. This approach only appeared in utterances of the NNSs, and not in those of the NSs or the TXs.

The authors of the TXs seemed to be comfortable with adopting the order of grounder $>\mathrm{HA}$, as five out of 13 exemplars (38\%) employed this, and two TXs used preparators >grounder (16\%). However, another five applied a different order. As exceptional cases, three NSs and one NNS did not utter HAs. The breakdown of those who did not utter HAs was that two NSs employed the order of preparator>grounder, one NS uttered only a grounder, and one NNS used a preparator only. Their order was categorised according to the order of SMs, since it is useful to comprehend their approach, even though there was no HA. 


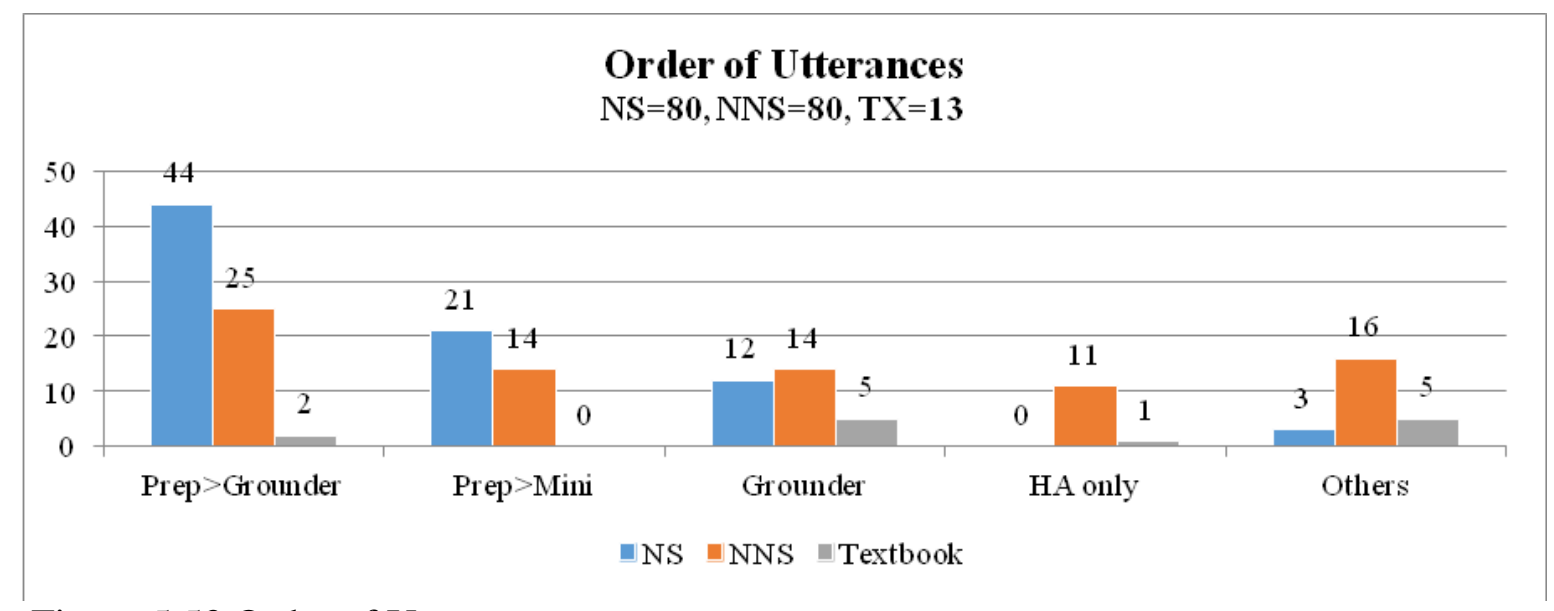

Figure 5.53 Order of Utterances

Abbreviations: Prep (Preparator), Mini (Minimiser)

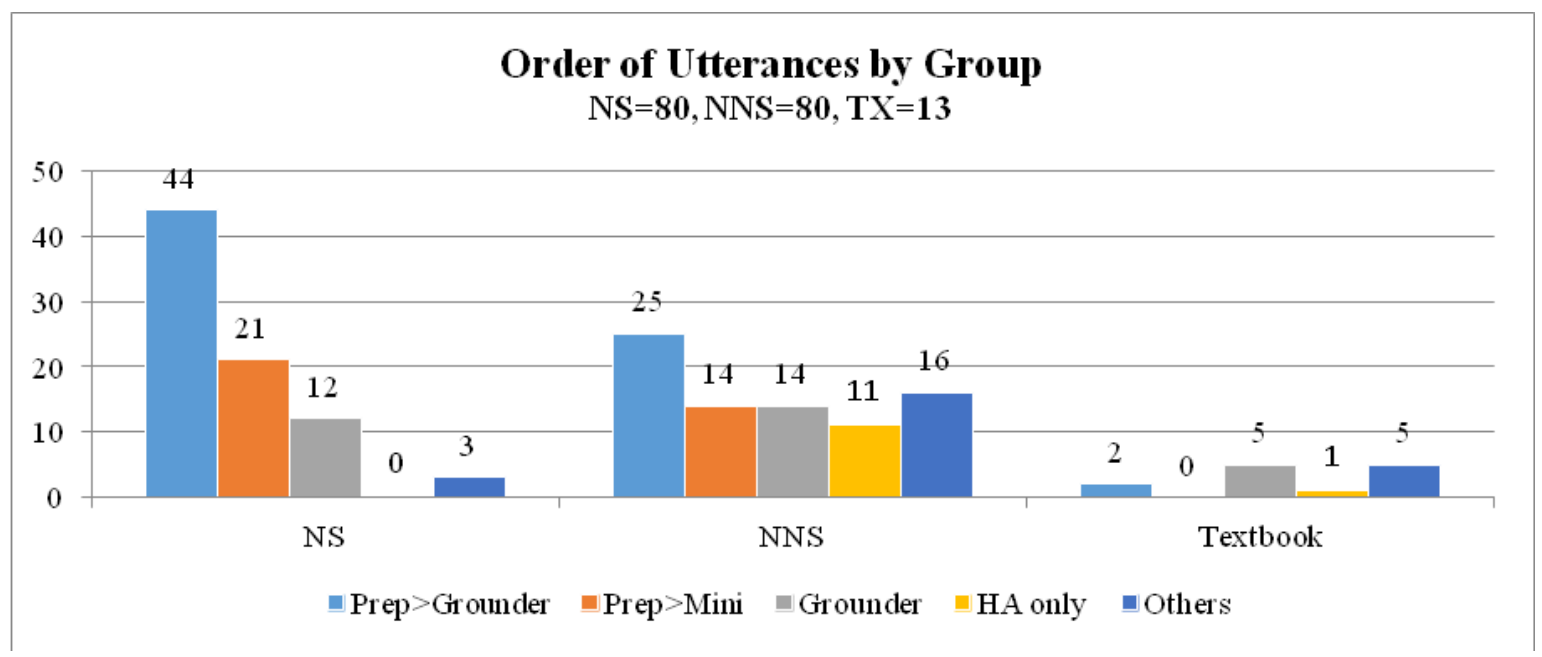

Figure 5.54 Order of Utterances by Group

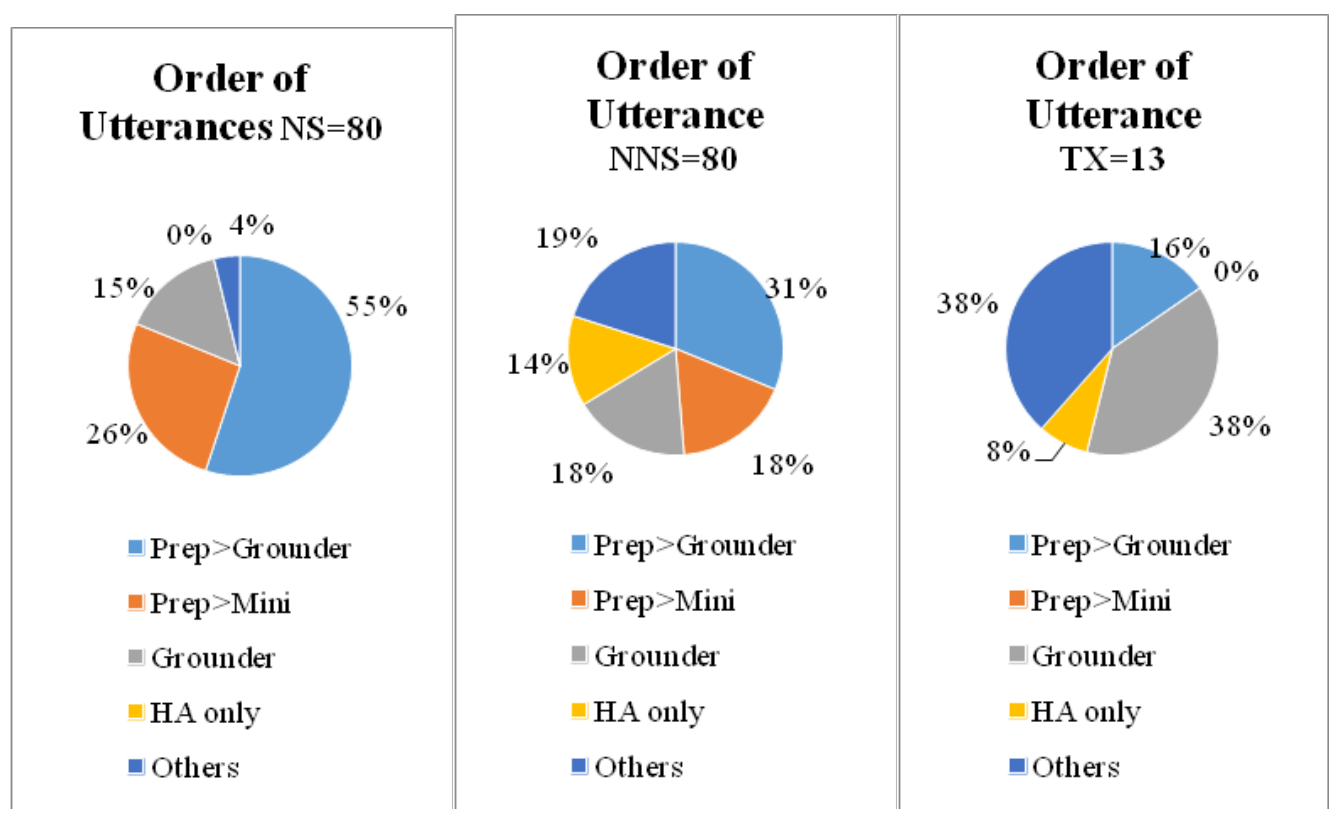

Figure 5.55 Proportion of Order of Utterances 


\subsubsection{Usages of Different Supportive Moves}

Sixty-five NSs (81\%) made use of two different SMs before uttering the first HA, ${ }^{183}$ while the combination of two SMs+HA was the most often used strategy by 39 NNSs (49\%), similarly to the NSs. In the case of the TXs, both one SM+HA and three SMs+HA were the most popular exemplars of five each (38\% respectively).

In contrast to the NNSs, 12 native speakers (15\%) tended not to use a rather plain combination of one SM+HA. For the NSs, to make use of only one SM was apparently inadequate, as the majority of them (81\%) adopted two SMs. In other words, they chose to elaborate using different types of SMs. By comparison, the NNSs used only one SM (18\%) or only HA (14\%), so that in total half of the NNSs used either one SM or no SM before the HA.

The TXs appeared to have bipolar characteristics. Five of them elaborated three SMs+HA (38\%), whereas another five utilised only one SM+HA (38\%). As long as they appeared to be only focusing on 'how to make a request', the length of the model exemplars was short; but when they presented a lengthier model discourse, it introduced various types of SMs. This is understandable, as the authors of the TXs would presumably have focused on presenting the most relevant sentence when making a request, and probably owing to space constraints, about forty per cent of them used only one SM. On the other hand, when there was no (or little) space limitation, they seemed to use more supportive moves.

183 This is the number of combinations of SMs whereby a speaker used different types of SMs together with HAs. If a speaker exercised the same SMs more than once in a discourse, it was counted as one SM no matter how many times the speaker uttered the same SM. 


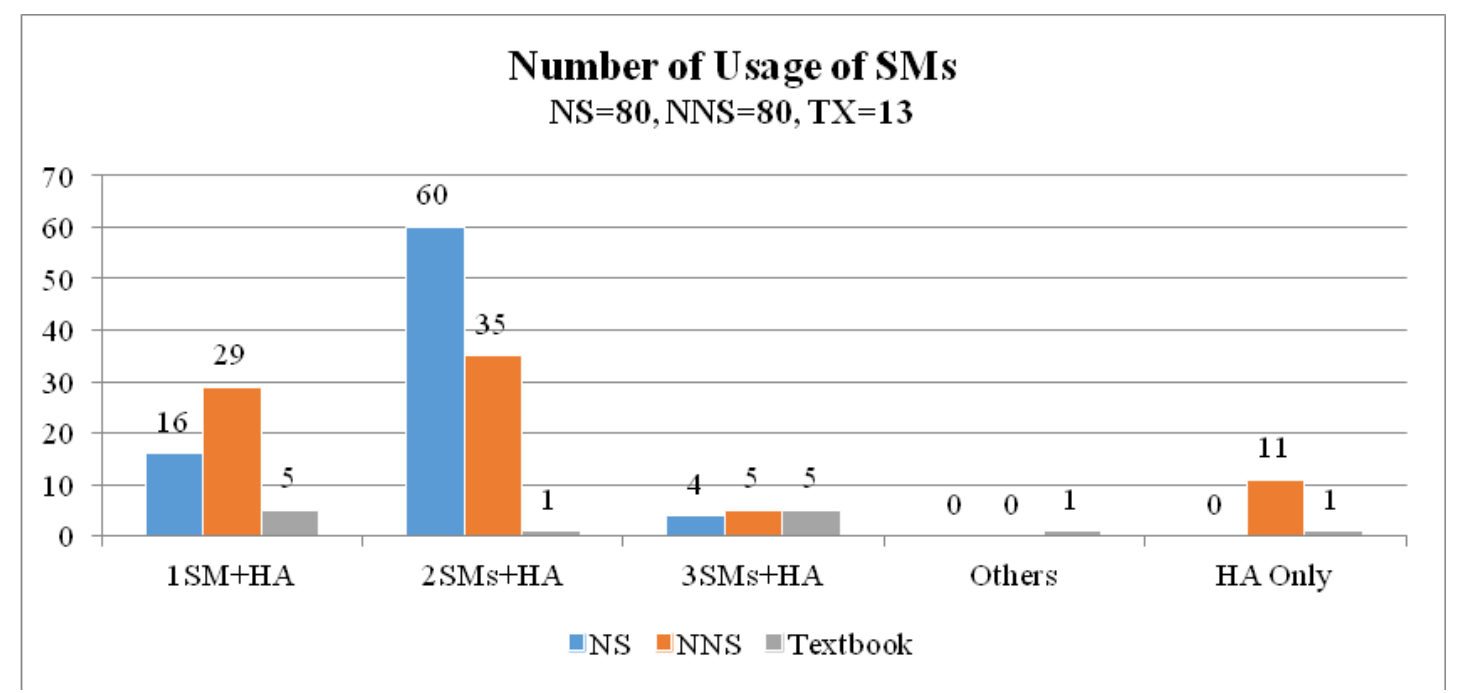

Figure 5.56 Number of Usage of Supportive Moves

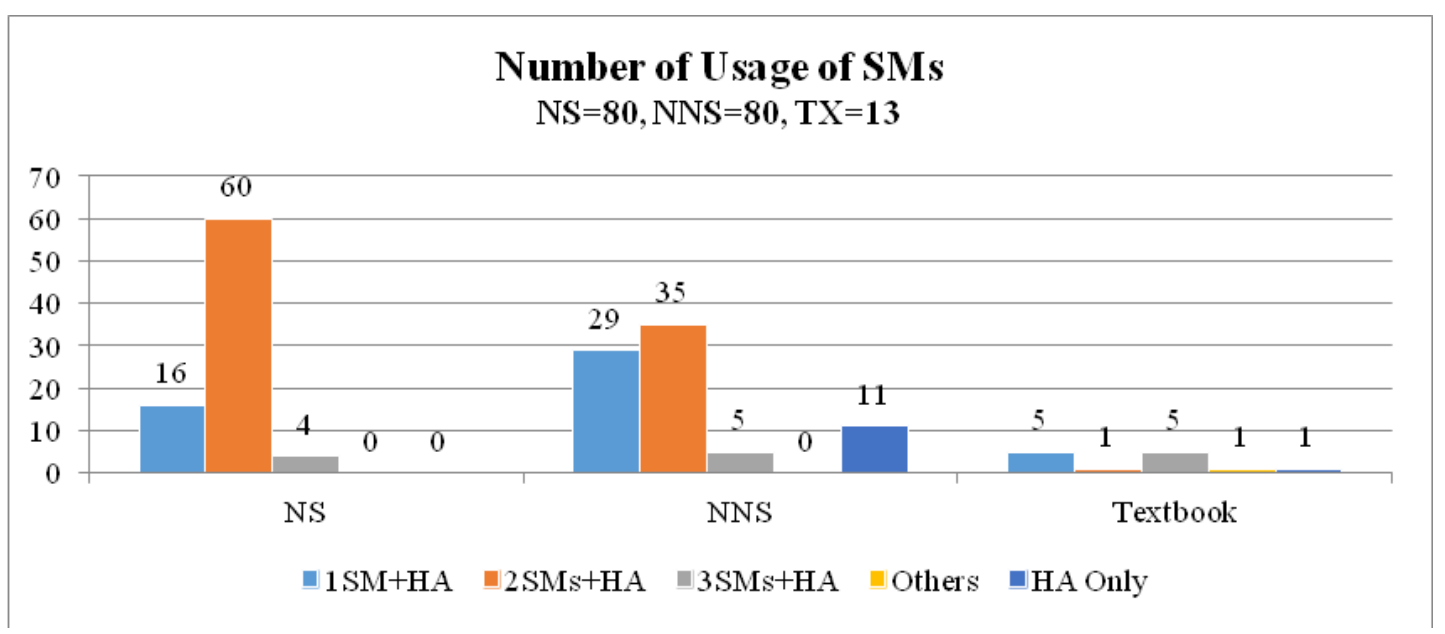

Figure 5.57 Number of Usage of Supportive Moves by Group 


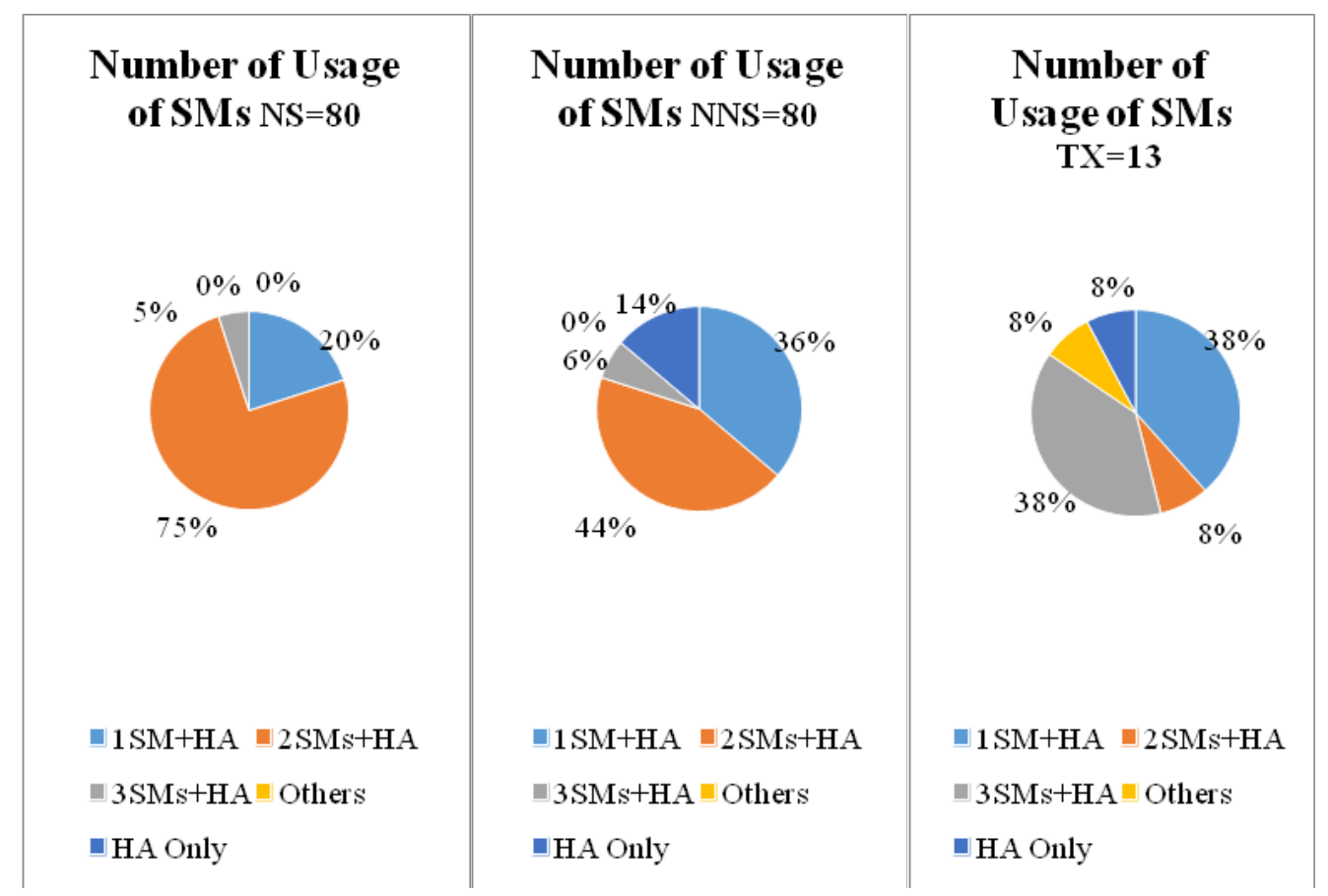

Figure 5.58 Proportion of Usage of Supportive Move

\subsection{Findings and Discussion}

In this section, like the previous section, the results of analyses are discussed from the aspect of the similarities and differences in the experimental data of native speakers of Japanese (NSs), Japanese language learners (NNSs), and exemplars of the textbooks (TXs) based on Brown and Levinson's politeness theory.

\section{Research questions}

To remind the reader, the research questions addressed are:

1. What is the structure of the discourses of settings of apology and request employed by an apologiser and a requester who are (a) native speakers of Japanese, (b) Japanese language learners, or (c) exemplars in Japanese language TXs? 
2. What particular expressions/phrases in the settings of apology and request are employed by an apologiser and a requester who are (a) native speakers of Japanese, (b) Japanese language learners, or (c) exemplars in the Japanese language TXs?

3. In what way and/or how should Japanese language TXs be improved in order to more closely reflect "authentic" native speaker discourse?

\subsubsection{Structure of Discourse of Request}

The present study investigated which of the four politeness strategies were used in each of the three groups, and to what degree. It was recognised that the proportion of uses of HAs, the core element of request, and SMs differed little among the three groups, for the ratio of HAs and SMs is around one-third in each group. ${ }^{184}$ Nevertheless, a discrepancy in the use of HAs appeared at the level of individual strategies. While the NSs used four strategies rather equally, which fell between five per cent for off record strategy and ten per cent for positive politeness strategy in the discourses, those of the NNSs were between zero (negative politeness and off record strategies) and 21 per cent (bald on record strategy) and, in the case of the TXs, between zero (off record strategy) and 25 per cent (positive politeness strategy). Given that the NNSs and the model discourses of the TXs rarely used off record strategy, it appears to have been the most difficult for them to employ. ${ }^{185}$

\footnotetext{
184 The percentage of HAs to SMs of the NSs and the TXs is 29:71, and that of the NNSs is 35:65.

185 In the pie graphs, the percentage shows 0 (zero) for the NNSs and the TXs because the numeral value was rounded off, although there was one utterance/exemplar of negative strategy by both the NNSs and the TXs, and two utterances of off record strategy by the NNSs.
} 
Table 5.6 Number of Politeness Strategies Used by Group

\begin{tabular}{|l|r|r|r|}
\hline Number of Strategies & \multicolumn{1}{|c|}{ NS } & NNS & \multicolumn{1}{|c|}{ TX } \\
\hline HA Bald on Record & 48 & 82 & 1 \\
\hline HA Positive & 56 & 57 & 11 \\
\hline HA Negative & 33 & 1 & 1 \\
\hline HA Off Record & 30 & 2 & 0 \\
\hline SM Positive & 203 & 142 & 14 \\
\hline SM Negative & 202 & 111 & 18 \\
\hline Total & $\mathbf{5 7 2}$ & $\mathbf{3 9 5}$ & $\mathbf{4 5}$ \\
\hline
\end{tabular}
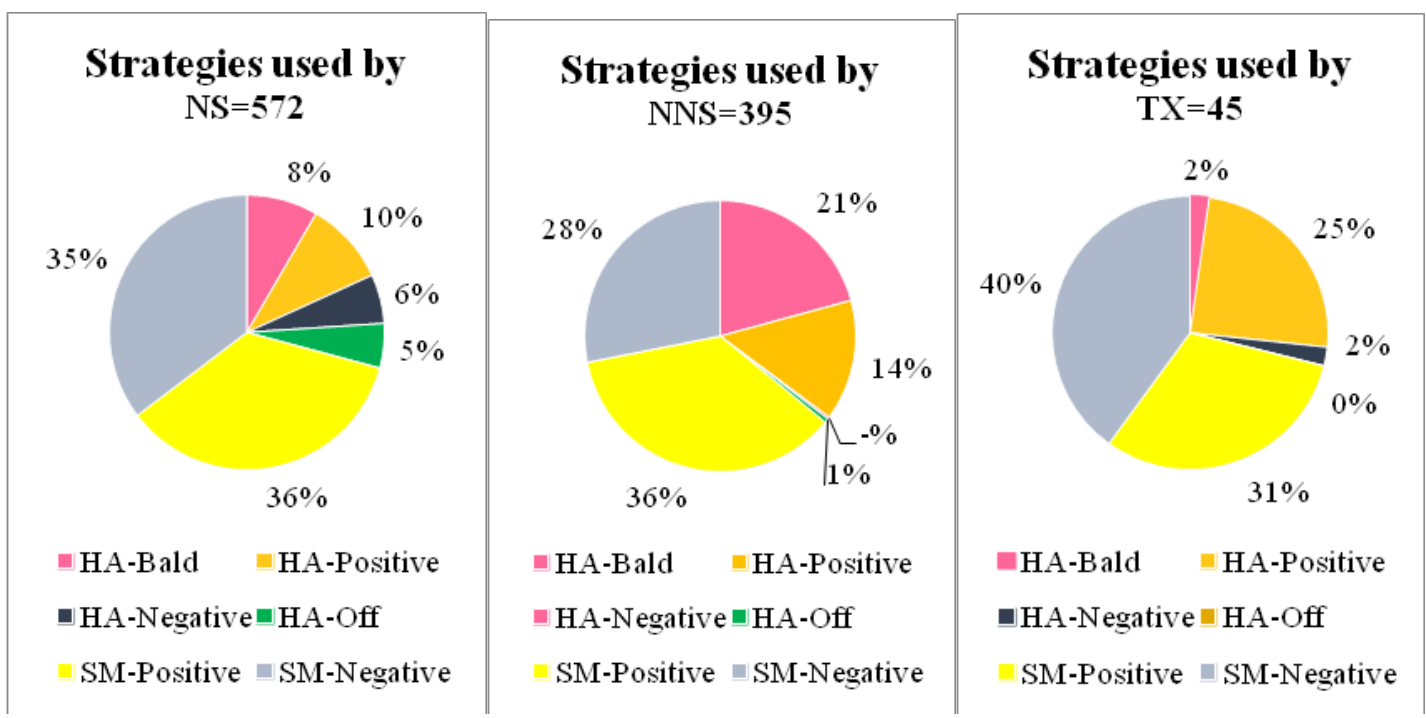

Figure 5.59 Proportion of Discourses by Politeness Strategy

The differences in the three groups were found in the rather delicate and intractable off record strategy, and ticklish and blunt bald on record strategy. This is so because for the former it is necessary that students be provided with a thorough explanation of how to use it, but the latter seems to be rather easy for learners to acquire because the sentence patterns are simple and short. It may be surmised that the authors might have thought that off record strategy was too demanding to acquire and bald on record was too plain-spoken and might easily cause offence. Hence, the appearance of both strategies in the exemplars was minimal.

The differences in the usage of SMs were not as great as with the HAs, but some were still observed. Positive politeness strategy was used more frequently by the NSs and the NNSs than negative politeness. Thirty-six per cent of both the NSs and the NNSs uttered it, 
and it occurred in 31 per cent of the TXs. Hence, negative politeness was used secondarily by the NSs and the NNSs which was the opposite to the result of the TXs. The TXs' use of negative politeness strategy in SMs was as high as 40 per cent, which exceeds their use of positive politeness strategy in SMs. The results from the experiments revealed that the TXs demonstrated frequent use of the negative politeness strategy in SMs. It can be deduced that the authors of the TXs might have been concerned about courteousness and hesitationinsinuating indirectness - in the speech acts more than the other two. By contrast, the NNSs did not favour negative politeness very much, which was the lowest among the three at 28 per cent. The NSs were in between the two, and their all-around use of the four strategies in the discourses was notable.

The proportion of HAs to SMs in the three groups was somewhat similar, although that to SMs by the NNSs was slightly higher than for the other two. Twenty-nine per cent and thirty per cent each were HAs by the NSs and the TXs, with 36 per cent by the NNSs. While all TXs presented only one HA in their model discourses, the NSs uttered HAs 2.1 times and the NNSs did so 1.8 times on average in their discourses. This might be because the NNSs seemed to make more effort to use HAs rather than SMs compared with the others. In other words, they were probably not proficient enough to be concerned about SMs but focused on the requests.

Table 5.7 Number of Utterance per Discourse

\begin{tabular}{|c|c|c|c|}
\hline Number of Utterance per Discourse & NS & NNS & TX \\
\hline HA (times) & $2.1 \quad(29 \%)$ & $1.8 \quad(36 \%)$ & $1.0 \quad(30 \%)$ \\
\hline SM (times) & $5.1 \quad(71 \%)$ & $3.1 \quad(64 \%)$ & $2.5 \quad(70 \%)$ \\
\hline Total & $7.2(100 \%)$ & $4.9(100 \%)$ & $3.5(100 \%)$ \\
\hline
\end{tabular}

The results of the analysis of the whole discourses are as follows. In general, the positive strategy was the most frequently used by all the groups, followed by the negative strategy because of the high proportion of SMs in the discourses. As a tendency, the NSs and 
the TXs included a relatively high ratio of the negative strategy, but this was not so much the case for the NNSs. Although the proportion of bald on record and off record strategies of the NSs was not very high, they still appeared to use the four strategies rather all-round compared with the other two. In the case of the NNSs, overuse of bald on record stood out, and the model discourses presented in the TXs mostly comprised only positive and negative strategies.

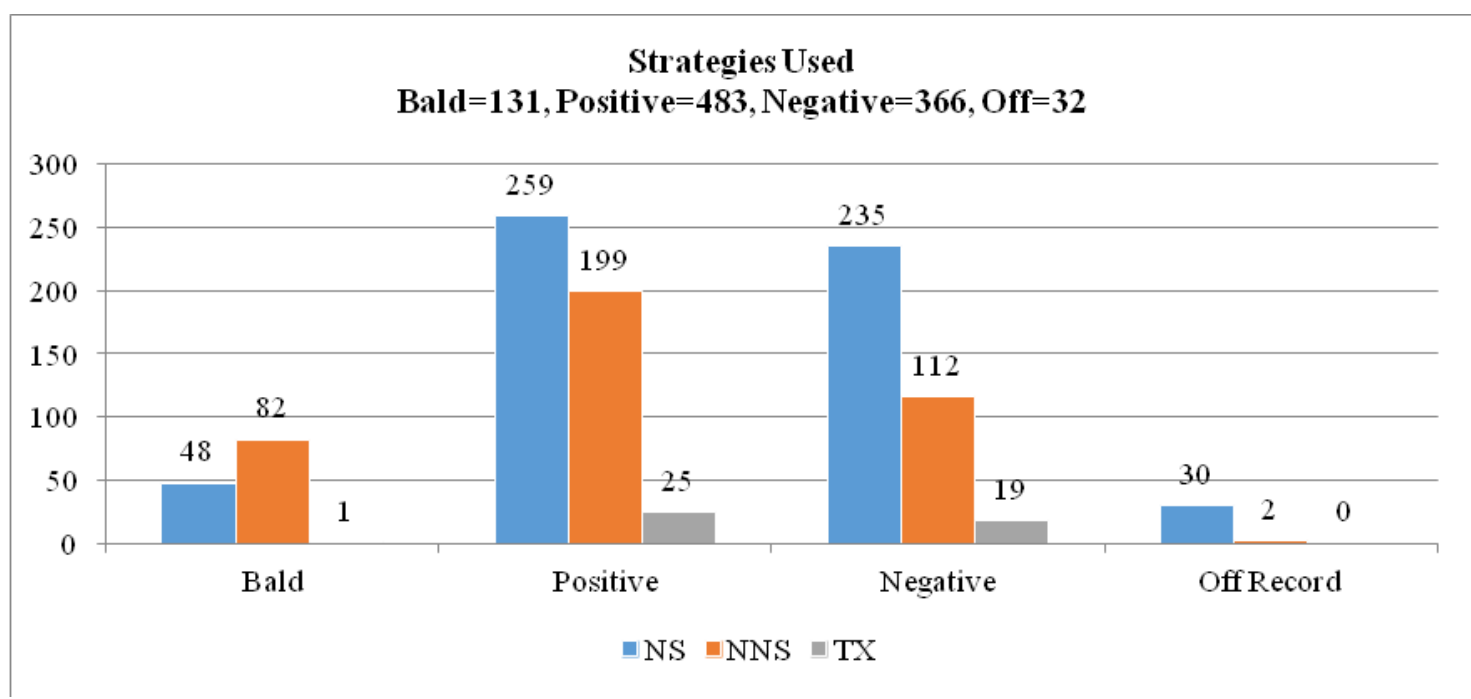

Figure 5.60 Politeness Strategies Used

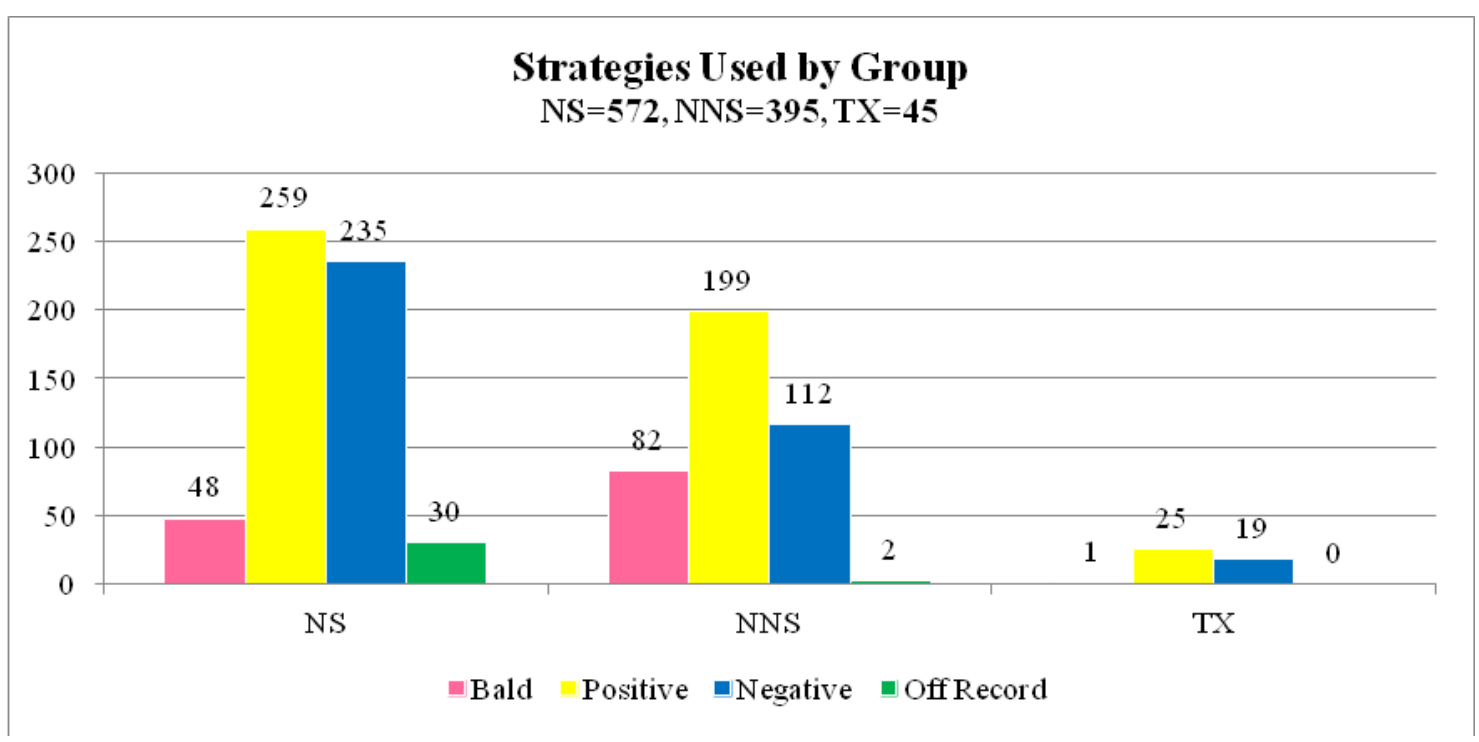

Figure 5.61 Politeness Strategies Used by Group 


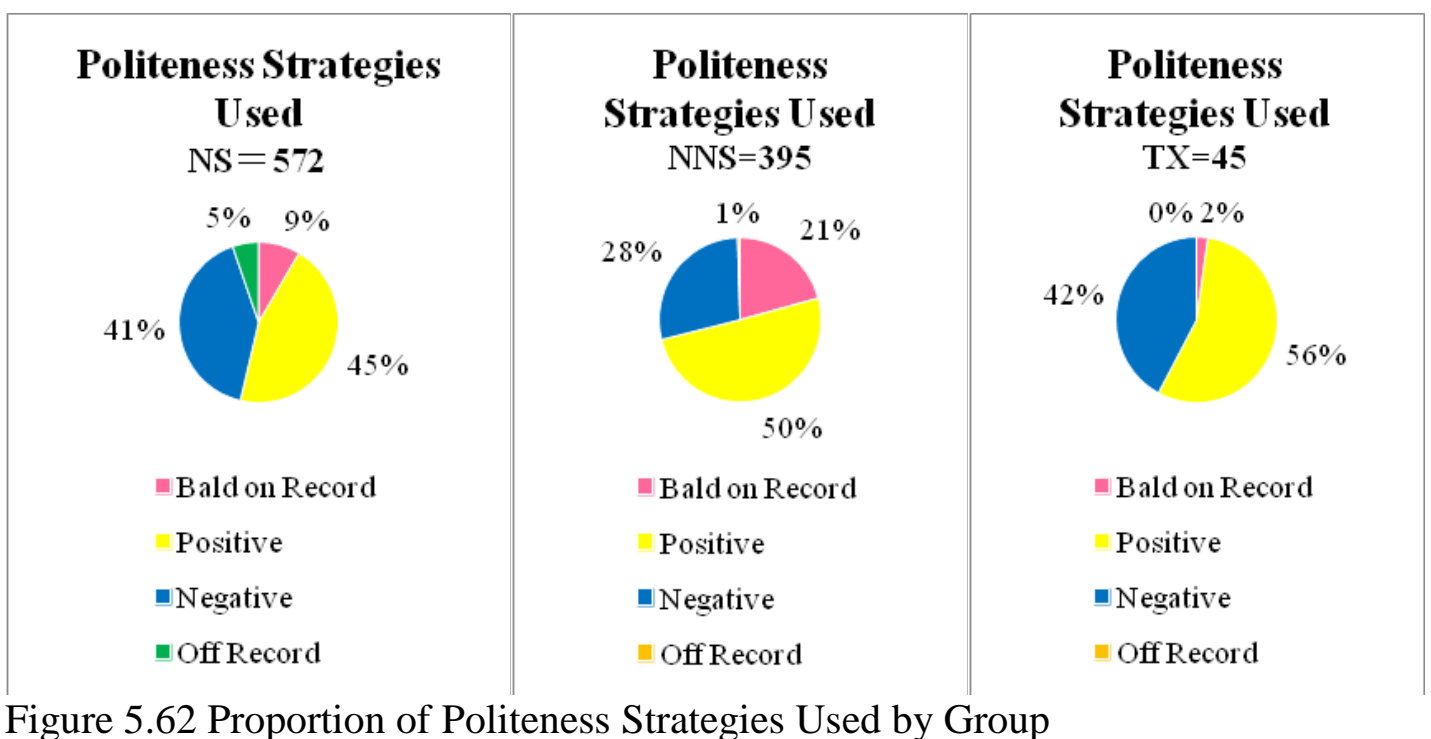

Figure 5.62 Proportion of Politeness Strategies Used by Group

\subsubsection{Structure of Head Acts}

Brown and Levinson categorised the factors influencing the choice of strategies: i.e., the four politeness strategies and when a speaker chooses (utters) them. ${ }^{186}$ The NSs applied the four strategies, and as they were native speakers of Japanese, there were no obstacles preventing them from utilising whatever strategies were available. In their case, even though the condition of the setting was the same (in the present experiments), the four strategies were used, which may suggest that the strategy to be used depends on the speaker (requester)'s

186 Brown and Levinson (1987: 71-2) explained the four strategies as follows. By using bald on record strategy, S[peaker] can avoid the danger of being misunderstood; for positive strategy $\mathrm{S}$ can minimize the face-threatening aspects of an act by assuring the addressee that $\mathrm{S}$ considers himself to be 'of the same kind'; for negative strategy S can maintain social distance, and avoid the threat of advancing familiarity towards the addressee; he can give a real 'out' to the addressee by making it clear that he doesn't really expect H[earer] to say, "yes" unless he wants to, thereby minimizing the mutual face loss incurred if $\mathrm{H}$ has to say, "no"; for off record strategy he can avoid responsibility for the potentially face-damaging interpretation. The Japanese expressions of ...kurenai? [Can't you...?] and applying aposiopesis, for example, ..na n' da kedo [V, (I presume...)] can correspond to positive and negative strategies respectively. 
discretion. Unlike in a setting of apology, there are fewer fixed formulaic set phrases in Japanese in the case of request, allowing more freedom of personal choice. ${ }^{187}$

The following four sentences were uttered by the participants of the present experiments. The utterances of bald on record strategy Misete [Show me], of positive politeness strategy Misete moraenai? [Can't you show me?], of negative politeness strategy Misete hoshii n' da kedo... [I was wondering if you could show me...], and of off record strategy Donna koto kaita? [What have you written?] were enunciated by the NSs in spite of the fact that the distance between the speaker and the hearer, and the imposition, were the same. This can be interpreted to mean that all politeness strategies are pragmatically permitted in the real world when making a request in Japanese.

Some people employ strong expressions of request and others do not when approaching the hearer. Sasaki (1995) affirms that even though the contents and means of requisitive expressions may vary, the requester's goal is the same. The results of the present experiments, in particular for the NSs, corroborate Sasaki's because they utilised not one or two politeness strategies but all four types. All of the participants used mostly the same limited verbs, such as misete [the te-form of miseru, show], tetsudau [help], or oshieru [teach/tell] except in off record strategy. According to Kumagai and Shinozaki (2006, see Ch. 2, Sec. 5), there is a belief in 'the more the better' when making a request. Hence, if a speaker (requester) wants to be seen as polite by a hearer (requestee), the expression becomes long and complex, and various strategies are applied. This was also corroborated by the present

187 The phrase Yoroshiku onegai shimasu [Please (I hope you to do appropriately)] and its variations, such as onegai [Please] are very often used after the requisitive sentence. However, this phrase needs another sentence of request. It does not make sense if used alone, because it does not contain a word(s) as to what the speaker wants the hearer to do. On the other hand, it can be used baldly, for example, when a husband leaves home for the office in the morning and asks his wife if she wants him to buy something on the way back in the evening, Nanika iru? [Need something?], then the wife may answer Tamago onegai! [Eggs, please!]. 
study, as the shorter sentences, such as Misete in bald on record strategy, were the less polite, whereas the longer sentences like Misete hoshii n' da kedo... in negative politeness strategy were the more polite and contained even some hesitancy on the part of the requester.

Interestingly, Sasaki (1995: 62-3) states that indirect expressions in English were used by English speakers in Australia when requesting. If that is so, the reason for using bald on record or positive strategies more than negative or off record strategies by the NNSs in the present experiments may suggest that their limited competency in the Japanese language prevented them from applying the more sensitive expressions needed for negative and off record strategies than for bald on record strategy.

In contrast to the NSs, the different characteristics in usage of HAs by the NNSs and the TXs became apparent. As Kumatoridani (1995, see Ch. 2, Sec. 5) argues, indirect expressions which do not contain the vocabulary of request ${ }^{188}$ can nevertheless be regarded as a request in employing off record strategy. While 30 utterances (18\%) of the HAs by the NSs employed these kinds of sentences, there were only two (1\%) uttered by the NNSs, and none was presented in the TXs. From the findings of the present study, it can be deduced that for the NNSs generating a request using off record strategy seemed to be very hard.

As scholars such as Jinnouchi (2006), Kawakami (1993), Makino (1996), and Yasui (1983) (see Ch.2, Sec. 2) argue, when a speaker employs an indirect expression, the speaker expects the hearer's guesswork to perceive the speaker's unspoken intention through the context (implicature). It might not be realistic to expect this type of manoeuvre from NNSs, who were not at an advanced level of the Japanese language in the present study. Given that the NSs use them - albeit probably unconsciously, and NSs would employ the off record

188 For example, the sentence Kono ato jikan aru? [Do you have time later?] contains the speaker's implicit intention I want to ask you something later. Nine NSs uttered this expression in the present study. 
strategy even if their conversational partner was a Japanese language learner-therefore, the authors of textbooks need to consider including examples of indirect expressions.

In addition, mention should be made of Sato's (1997, see Ch. 2, Sec. 5) findings, in which she claims that the fathers' generation ${ }^{189}$ of university students used more indirect expressions than the young. The NSs in the present experiments were, however, using indirect expressions, although they are younger than Sato's subjects. This suggests that even for young Japanese people today, making a request indirectly doubtlessly comes naturally to them.

The TXs are inclined towards positive politeness strategy, because there was only one exemplar each of both bald on record strategy and negative politeness strategy, and no exemplar of off record strategy. Their tendency towards positive politeness strategy stands out among the three groups. This comes from the high usage of juju dōshi in their model expressions. Regardless of who the interlocutor (requestee) is in the TXs, whether friends or workplace colleagues, the authors of the TXs seem to have thought that using juju dōshi was appropriate for requisitive sentences because 11 exemplars out of $13(84 \%)$ used them in their HAs.

It may be said that Japanese people are generally reserved and do not say things explicitly, so they often choose the negative or off record strategies (Hori 2006, see Ch.2, Sec. 3). Nevertheless, this is not necessarily applicable when they are in-group members, as the result of the present study shows that the NSs utilised all four politeness strategies respectively, with not much tendency to one particular strategy. This is similar to the findings of Haugh (2003, see Ch. 2, Sec. 2) and Unosawa (2005, see Ch. 2, Sec. 5) that if the distance between the two parties is short, their utterances are straightforward, and Fukushima (1996: 686) discovers that young Japanese spoke more directly than the British subjects and even

189 The fathers, who participated in a Sato's research, were between 40 and 60 years old. 
baldly when the relationship with their interlocutor was close. Thus, from the results of the present study it can be surmised that even if the social setting is the same (close friends), the personality of the speakers might be the determinant in selecting the strategy because four different politeness strategies were chosen by the NSs.

With regard to what and how HAs were used, some participants employed different types of politeness strategies in a discourse. While the NSs uttered HAs 2.1 times on average and the NNSs 1.8 times in a discourse, of the participants who uttered HAs more than once, there were 53 NSs $(66 \%)$ out of 80, and 35 NNSs (44\%). The numbers of those who repeated the same politeness strategy of HAs were $35(66 \%)$ out of 53 NSs, and $24(69 \%)$ out of 35 NNSs. The remaining 18 NSs (34\%) out of 53 NSs and 11 (31\%) out of 35 NNSs employed different politeness strategies.

The combinations of the different strategies used were, (1) negative+positive politeness strategies, (2) negative+bald on record strategies, or (3) off record+positive politeness strategies. All of the NNSs who changed the strategy used a combination of bald on record+positive strategies. Since most NNSs used either bald on record and positive politeness strategies, there was in practice only one possibility in this combination unless they repeated the same strategy.

As for the order of appearance of different strategies, the expression with the greater sense of distance was mostly uttered first, followed by a less-distanced expression. In other words, the more direct expressions did not appear in the first utterance. This indicates that the requester was sounding out the requestee. Nevertheless, not many participants changed their strategy, and if they uttered HAs more than once, they seemed to repeat the same strategy, as mentioned above. This means that once the participants determined what strategy to use, they did not seem to deviate much from it. In the TXs, the HA was only used once in all model discourses, so the order of appearance of different strategies does not apply to them. 


\subsubsection{Structure of Supportive Moves}

The present study categorised the collected data into five supportive moves: grounder, reward, disarmer, minimiser and preparator. Based on Brown and Levinson's politeness theory, the first three were regarded as positive strategies and the last two as negative strategies. ${ }^{190}$ It is expeditious for foreign language learners and the authors of TXs to comprehend which SMs are employed by native speakers and to what extent. The types of politeness strategy including the number of SMs to be employed depend on the degree of the relationship with the other(s) and the level of burden with which a requester asks a requestee. The requester may make an abrupt request of a close friend because there is a common understanding between them. However, when a person makes a request to someone, but the two parties do not share a common understanding of the topic, the requester's development of conversation might differ, even if their relationship is close. For example, if the requester knows that the requestee does not share the topic which he/she is going to raise, the requester might ask, Shukudai wasurechatta n'da, warui kedo, chotto tetsudatte kurenai ka na?

190 According to Brown and Levinson's politeness theory, Strategy 13 of positive politeness reads that S[peaker] gives reasons as to why he wants what he wants, which corresponds to 'grounder' (128). Likewise, Strategy 15 of Positive politeness fits 'reward' because it explains giving gifts to $\mathrm{H}$ [earer] (goods, ...) by satisfying some of H's wants (129). Strategy 7 and Strategy 9 of positive politeness suit 'disarmer' because the politeness theory describes Strategy 7 as asserting common grounds, such as familiarity in the S-H relationship, and Strategy 9 asserts or presupposes S's knowledge of and concern for H's wants (123-5). With regard to negative politeness strategy, 'minimiser' comes under both Strategy 2 of question, hedge? (145); and Strategy 4 of minimizing the imposition, Rx (176). 'Preparator' is assigned also to Strategy 2 of question, hedge, in which Brown and Levinson depicted that hedges on illocutionary force are the most important linguistic means of satisfying the speaker's want. They raised examples of expressions, such as $I$ wonder if..., and Do me a favour, will you? (145-7). 
[Forgot my homework. Sorry to say, but, can't you help me, please?], which comprises three SMs and one HA: grounder, disarmer, minimiser, and request. ${ }^{191}$

The proportion of positive and negative strategies among the three groups did not differ significantly: it was just on 50 per cent each for the NSs, and the NNSs' positive politeness strategy was slightly higher than negative politeness strategy with 55 per cent to 45 per cent. For the TXs, on the contrary, negative politeness strategy was higher than positive politeness strategy, at 55 per cent to 45 per cent.

This differs from the ratio of positive and negative strategies in the apologetic discourses among the three groups, because in the case of apology, positive politeness in SMs accounted for a considerably large part of the three groups, at around two-thirds of the SMs. In other words, the negative politeness strategy in the discourses of request was utilised more frequently than in those of apology.

In general, the number and type of SMs of the politeness strategies adopted in discourses depended on which strategy(ies) the speaker decided to use. This may be surmised from the fact that the NSs uttered 5.1 SMs (71\%) on average out of 7.2 of all utterances in a discourse of requests, while the NNSs uttered 3.1 SMs (64\%) out of 4.9 for all discourses, and the TXs used 2.5 SMs (71\%) out of 3.5 for all utterances. In short, SMs in the discourse

191 The following utterances illustrate a person who wants a ride because of rain as he/she did not bring an umbrella, but the requestee does not already know this. Since the requester knows that the friend (requestee) does not know the background of the request (no umbrella), the requester may apply five different types of SMs along with the HA at a stretch in order to persuade the friend. For instance, Nee, kyō kuruma de kita? Ame ga furidashita n' da kedo, kasa motte konakatta n'da yo ne. Ashita ohiru ogoru kara sa, moshi mō sugu kaeru nara, chotto eki made nosete 'tte moraenai ka na? [Hey, did you come by car today? It's started raining, but I didn't bring an umbrella. If you're going home soon, could you just give me a ride to the station? I'll buy you lunch tomorrow.] These examples comprise preparator (Did you come by car today?), grounder (It's started raining, but I didn't bring an umbrella), reward (I'll buy you lunch tomorrow), minimiser (just), disarmer (If you're going home soon), (five SMs), and request (one HA) (Could you give me a ride to the station?). 
of requests are given considerably more weight, and the requester must have been concerned about using types and number of SMs while developing the discourse, especially compared with apology.

Table 5.8 Ratio of Supportive Moves by Group

\begin{tabular}{|l|r|r|r|}
\hline Supportive Moves & \multicolumn{1}{|c|}{ NS } & \multicolumn{1}{c|}{ NNS } & \multicolumn{1}{c|}{ TX } \\
\hline Ratio of SMs in a discourse of request & $\begin{array}{r}71 \% \\
(405 / 572)\end{array}$ & $\begin{array}{r}64 \% \\
(253 / 395)\end{array}$ & $\begin{array}{r}71 \% \\
(32 / 45)\end{array}$ \\
\hline $\begin{array}{l}\text { Average number of times SMs occurred } \\
\text { in discourse of request }\end{array}$ & 5.1 times & 3.1 times & 2.5 times \\
\hline
\end{tabular}

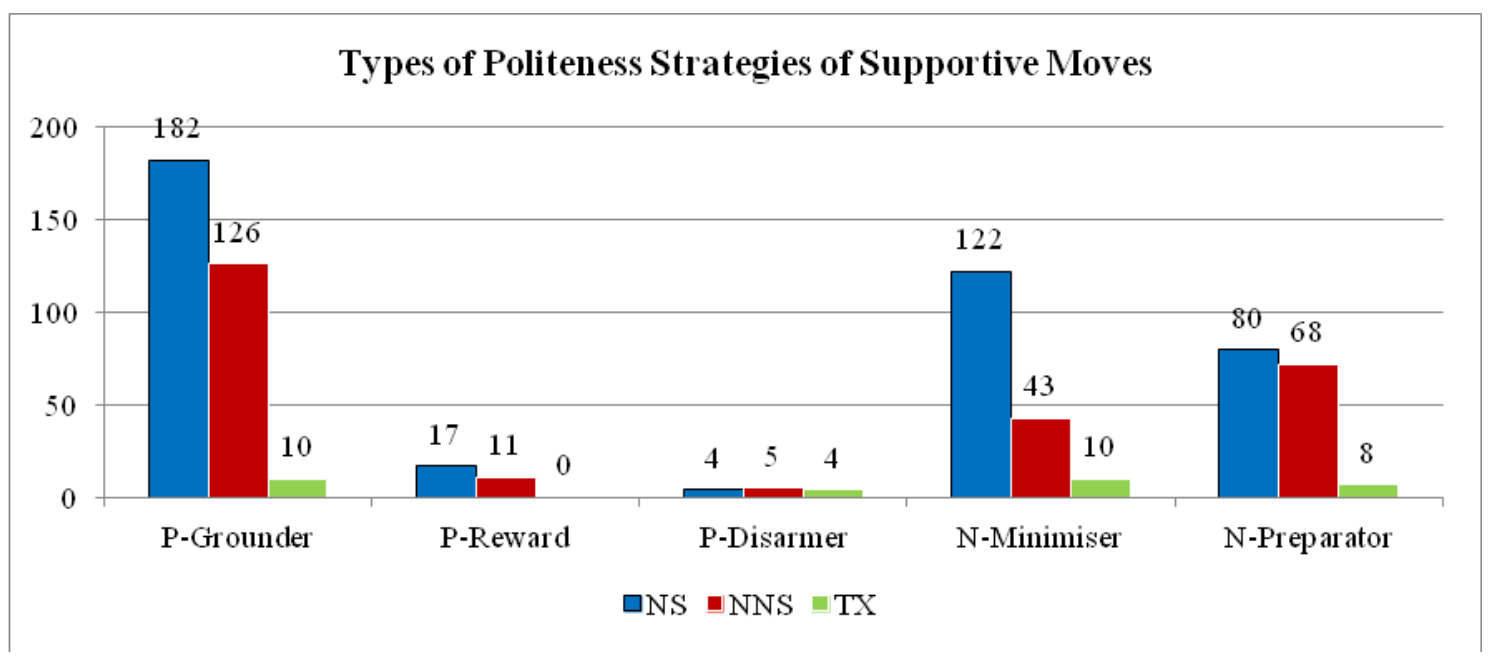

Figure 5.63 Types of Politeness Strategies of Supportive Moves

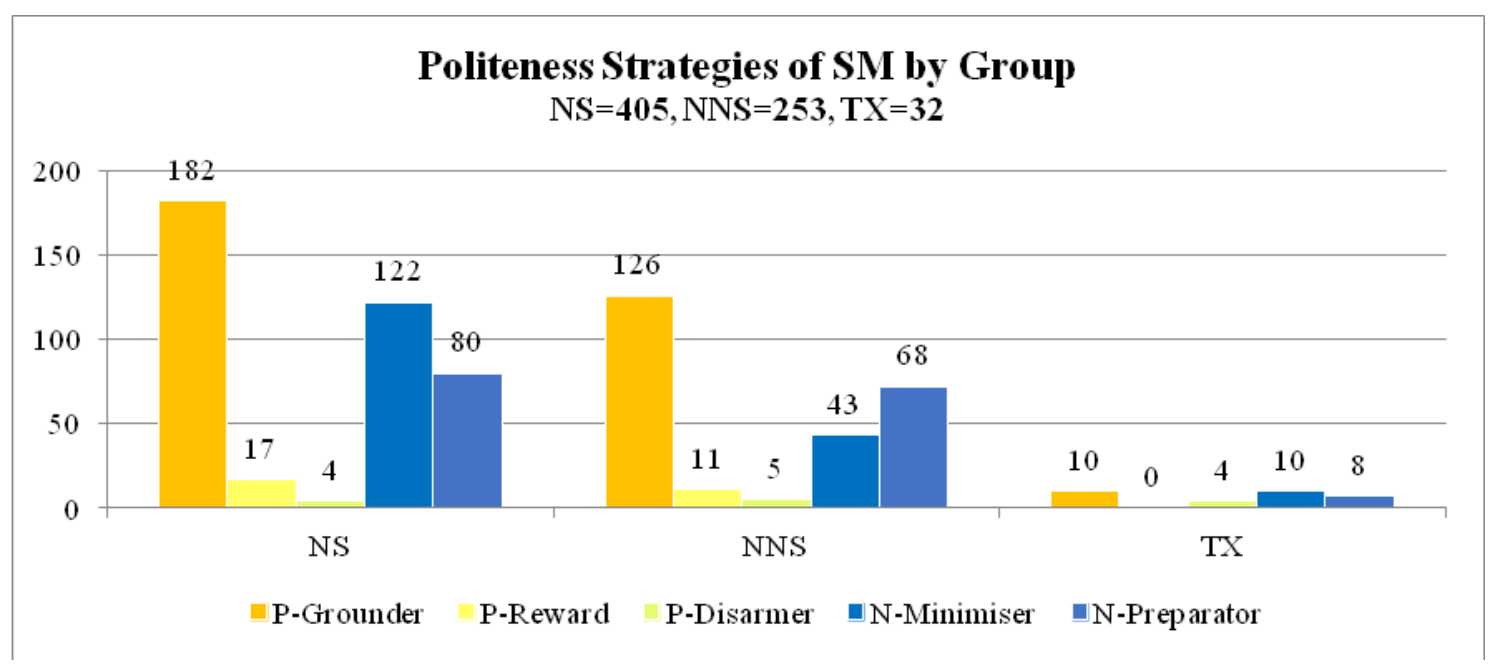

Figure 5.64 Politeness Strategies of Supportive Moves by Group 


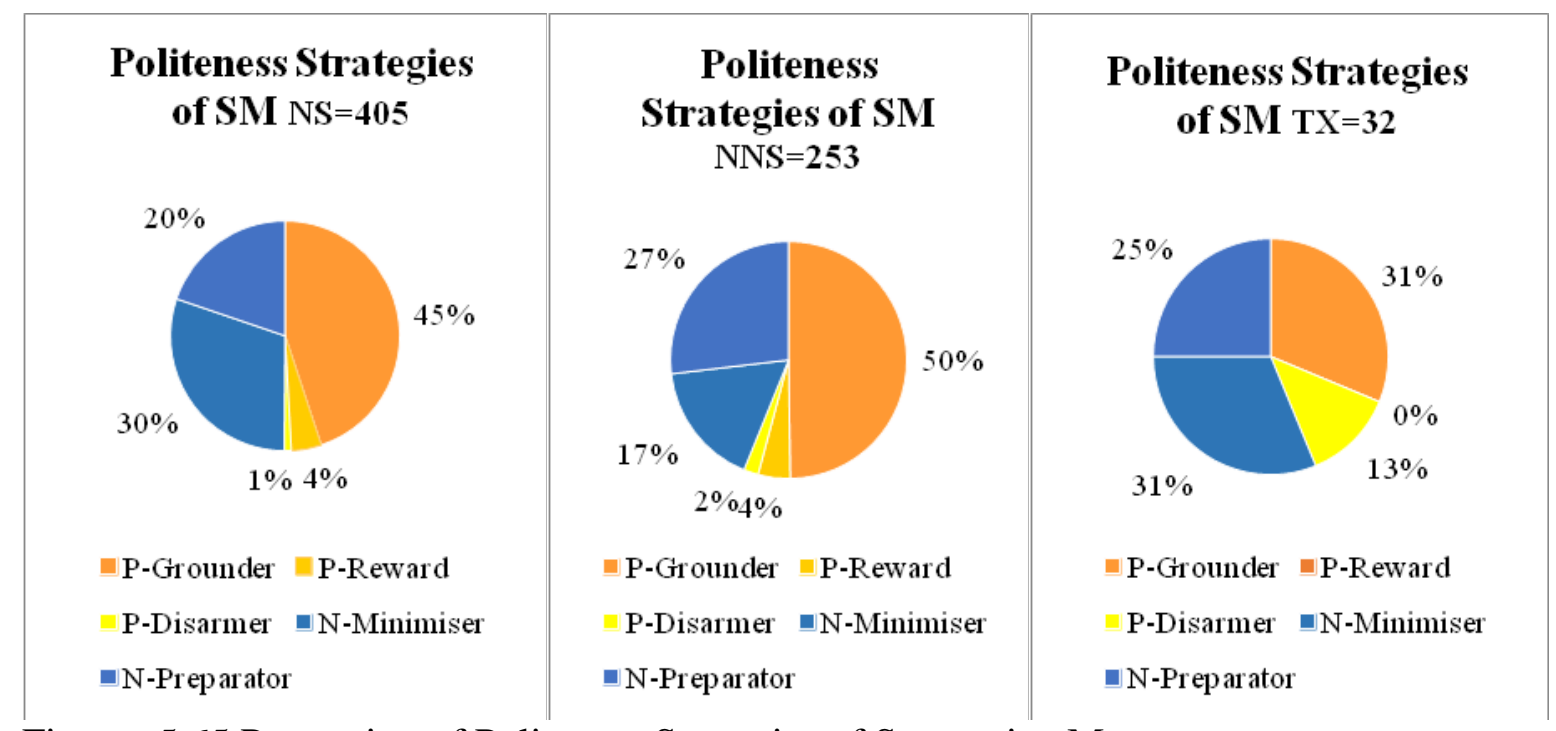

Figures 5.65 Proportion of Politeness Strategies of Supportive Moves

\section{Grounders (Positive politeness)}

Among the three groups, the most widely uttered SM was a grounder: 45 per cent out of all SMs uttered by the NSs, 50 per cent by the NNSs, and 31 per cent in the TXs. There were few differences in terms of the frequency of grounders among the three groups. The grounder was the only SM utilised more frequently than the HA (the request) by the NSs. This means that one grounder was uttered per 0.9 utterances of HAs by the NSs. For the NNSs and the TXs, it was the second most often employed category following HAs, which implies that one grounder was uttered per 1.1 HAs by the NNSs and per 1.3 HAs in the TXs.

Moreover, the proximity of HA and grounder is worth noting. Grounders and HAs were typically uttered in close succession, as can be seen. It may be speculated that this might derive from the notion on the part of the requester that a request was negative in itself, and, therefore, it was necessary to do a 'background briefing' of the request - the grounder of positive politeness strategy-before actually broaching the request, and that this grounder needed to be closely followed by the request itself in order to retain its efficacy. The CCSARP by Blum-Kulka et al. did not mention proximity, but this factor became clear in the present experiments. 


\section{Rewards (Positive politeness)}

Rewards was not utilised very much by the participants and the exemplars. Only four per cent each of the NSs and the NNSs out of the total number of utterances were rewards, and it was not exemplified in the TXs. Curiously, a reward was uttered not only by the requesters but also by the requestees (interlocutors) in the present study, such that 22 of the NSs interlocutors (role-players of requestees) asked for a reward prior to the acceptance of the request, as did 11 of the NNSs interlocutors. ${ }^{192}$ It seems that the Japanese native speakers who played the role of requestee did not hesitate to ask for something in return, such as a cup of coffee, as long as the relationship was close and the degree of imposition was not heavy as in the case of the present experiments.

An offer of a reward is one of the tactics used by a requester to realise his/her wish, but none of the TXs presented it. Presumably, the authors did not think that a reward was a good tactic, nor was it of high priority to be acquired by language learners, in spite of the fact that the sentences of rewards were relatively simple, for example using juju dosshi, such as Kōhī katte ageru yo [I'll buy you a cup of coffee]. Offer of a reward is a blunter and pointblank strategy. This could be the reason for rewards not being included in TXs.

\section{Disarmers (Positive politeness)}

Unlike rewards, the NSs and the NNSs used disarmers in very small numbers, for only one per cent and two per cent respectively. On the other hand, the proportion of disarmers was 13 per cent for the TXs. Both rewards and disarmers were categorised as positive strategies; however, it became clear that the preferred strategy for approaching a requestee by a requester in the TXs differed from that of the NSs and the NNSs.

192 The analysis of the hearers' data was outside the scope of the present study. 


\section{Minimisers (Negative politeness)}

There are two SMs categorised as negative politeness strategy: minimisers and preparators. Both played important roles in the present study.

For the NSs, minimisers were the second most regularly used SM (30\%); and for the TXs, they were equal to grounders as the most frequently uttered SM (31\%). By contrast, the NNSs used it in only 18 per cent of their total utterances of SMs, so that its use was only around one-third of that of the most used SM, grounders, in the NNSs.

A typical word used as a minimiser, chotto, was quite frequently uttered by the NSs and presented in the TXs, but was not used as much by the NNSs. While minimisers were employed once per 1.4 HAs by the NSs and 1.3 HAs in the TXs, the NNSs uttered them only once per 3.3 HAs. It can be said that when a requester of the NSs and the TXs uttered the request (HA), a minimiser was mostly uttered together with it. In strong contrast to this, the fewer utterances of a minimiser by the NNSs was a striking difference. The function of a minimiser had probably not been taught explicitly in class, even though the TXs presented it as frequently as the NSs did.

\section{Preparators (Negative politeness)}

Preparators function to alert the requestee to his/her situation, and the requstee's response which alert the requester to the requestee's situation, namely, preparators may work both ways between the two parties. ${ }^{193}$

Preparators were uttered 80 times in total by the NSs, 68 times by the NNSs, and there were eight exemplars presented in the TXs. The proportion of preparators out of the total

193 For example, a conversation of two friends:

A: Onegai ga aru n'da kedo, ima ii? [I like to ask you, but are you ok, now?]

B: Ima chotto isogashii n'da kedo, nani? [I'm a bit busy now, but what is it?] 
number of utterances was between 14 per cent and 18 per cent among the groups. It formed the second most frequent SMs of the NNSs, and a third of the NSs and the TXs. This implies that almost all participants used a preparator: 1.0 time by the NS, 0.9 times by the NNSs, and 0.6 times in the exemplars. ${ }^{194}$

The number of preparators per persona in the TXs was notably lower than the other two. Owing to the inherent nature of preparators, it should be uttered before the HA in order for the requester to check the requestee's availability and/or capability. The textbook authors perhaps omitted it in order to focus more on the HAs together with the primary SMs of grounder and minimiser, but it cannot be said that the TXs reflect authentic conversations.

\subsubsection{Expressions of Discourse of Request}

It was discovered that the discourses of request - in particular, their Head Acts (HAs) contained a greater variety of expressions than the discourses of apology. The politeness strategies used when making a request varied from the bald on record strategy to the off record strategy, and the expressions/phrases preferred by each group also became clear. In the HAs, respondents chose the verb misete [show] as a kind of core verb in the present experiments, and variations of misete were also constantly utilised, such as misete+kudasai [Please show me]. It goes without saying that the off record strategy did not fall under this because the main verbs of the off record strategy were not directly related to the actual request.

In discourses of request overall, positive politeness strategy occupied about 45 per cent of the entire utterances of the NSs, 50 per cent of the NNSs, and 56 per cent of the TXs,

194 NS: 80 preparators/80 NSs=1.0 time/person

NNS: $68 / 80 \risingdotseq 0.9$ times/person

TX: $8 / 13 \risingdotseq 0.6$ times/example 
followed by negative politeness strategy in 41 per cent of the NSs, 28 per cent of the NNSs, and 42 per cent of the TXs. Speakers used various strategies and sometimes combined different strategies when making a request. However, the positive and negative strategies were the two primary ones employed in the requisitive discourses in the present experiments.

The substantial commonality of requistive expressions among HAs in the three groups was that all of them used juju dōshi for positive politeness strategy as Okamoto (1988) and Ueno (1983) observe (see Ch. 2, Sec. 5). Since juju dōshi are basic vocabulary for learners at elementary and intermediate levels, they are likely to have some familiarity with their use. However, their usage is so different from European languages that, although many NNSs might learn it in class, and although they might understand it when heard, they tend to take much longer to use it felicitously themselves. It is appropriate, therefore, for these sentence patterns to appear in the model discourses of the TXs.

Other strategies, i.e., negative and off record strategies, were to some extent employed in the present study, but they were mostly used only by the NSs. As Nakamura et al. (2004, see Ch. 2, Sec. 5) observe, it is a characteristic of Japanese culture to express concern about others, including when the conversational partner is a close friend. Some NSs would have chosen strategies that needed more attentiveness, such as negative and off record strategies rather than positive politeness strategy, let alone the bald on record strategy.

According to Brown and Levinson's politeness theory, negative politeness and off record strategies demand more distance between the two parties than positive and bald on record strategies. Kumai (1992: 77-8) argues that a requester does not have the right of decision-making in a request. ${ }^{195}$ Therefore, the requester should avoid repeated requests or utterances to justify the request in Japanese. In addition, expressions of the bald on record

195 The example presented in Chapter 2, Section 5 is that a requester says, doyō kurai wa kashita hō ga ii desu [At least on Saturday, you'd better lend me the book]. Uttering the word V ho ga ii [had better $V$ ], the requester judges the requestee's act which is not performed yet. 
strategy sometimes sound almost like an order. ${ }^{196}$ These reasons may account for why the bald on record strategy was rarely preferred by the NSs.

\subsubsection{Expressions of Head Acts}

The Head Acts (requests) were uttered on average 2.1 times per discourse by the NSs, 1.8 times by the NNSs, and by 1.0 exemplar in the model discourses in the TXs. This means that the NSs uttered 1.2 times more HAs than the NNSs.

Comparing the apologetic utterances collected in the present study with those of requests, it was found that the difference of apologies between the NSs and the NNSs was larger than that of requests. This result was the same as the case between the NSs and the TXs.

From this, it can be surmised that when one misbehaves, repeated apologies are effective in Japanese culture in repairing the victim's damaged feelings, because the apologiser threatens his/her own positive face. On the other hand, there is no such tacit understanding when making a request. Repeated requests do not necessarily help the requester to have the request fulfilled. The requester might even run the risk of being regarded as too aggressive becuse the requester intrudes upon the requestee's negative face.

The total utterances per model discourse being around half that of the NSs shows that the number of requests in the TXs seems appropriate. This is presumably a compromise on the part of authors between presenting vital requisitive expressions and lack of space in the TX.

196 For example, Nōto misete (yo) [Show me your notes] or Mado o akete (yo) [Open the window]. Although it depends on the requester's intonation or the relationship of the two parties, if the intonation is strong and the relationship is not very close, the examples above can be perceived as not really a request but an order. 
Meanwhile, some NSs used the negative and off record strategies, which are regarded as indirect (negative politeness) and ambiguous (off record). Only two NNSs and none of the TXs uttered these. This reveals the characteristics of conversations by the Japanese native speakers, and it corroborates the findings of Hashimoto 2002, Jinnouchi 2006, Torikai 2005, Tsuda 1999 (see Ch. 2, Sec. 5).

Table 5.9 Number of Head Acts per Discourse

\begin{tabular}{|l|l|l|l|}
\hline Head Acts & NS & NNS & TX \\
\hline Number of HA/discourse (times) & 2.1 & 1.8 & 1.0 \\
\hline
\end{tabular}

\section{Bald on Record Strategy}

The bald on record strategy appeared in the utterances of the three groups, but this included only one utterance in the TXs. The constituent mostly used in the bald on record strategy was the verb misete [show], and its variations, such as Misete kudasai and Misete onegai shimasu, etc., as explained in this chapter. In 44 out of 48 utterances of the bald on record strategy by the NSs, and 81 out of 82 by the NNSs, misete was used. It sounds natural in Japanese for misete to be used by both sets of participants because it directly denotes the feeling and/or wish of the speaker who wants the hearer to comply with the request. Among the three groups, the NNSs used the bald on record strategy in as many as 21 per cent of the total number of discourses (or 58 per cent of the HAs), followed by the NSs' eight per cent (or 29 per cent of the HAs), and two per cent of all exemplars (or eight per cent of the HAs) in the TXs.

In the case of native Japanese speaker students, if the relationship between the two parties is intimate and the imposition is not heavy, the requester's speech may be blunt (Fukushima 1996, see Ch. 2, Sec. 5). Fukushima explains the reason for speaking in an abrupt manner as deriving from the solidarity among in-group members. Although the proportion of the bald on record strategy in HAs by the NSs was not high in the present study, it was still 
29 per cent, so the present experiment corroborates their findings. The only exemplar of the bald on record strategy in the TX also employed the word misete, like the NSs and the NNSs.

The order of frequency of use of the expressions by the NSs was misete+kudasai [please] in 42 per cent, misete+onegai shimasu [I beg] in 29 per cent, and misete+hoshii [want] in 21 per cent, whereas for the NNSs as high as 76 per cent of bald on record expressions was misete+kudasai followed by misete+onegai shimasu in 22 per cent. Only two of these expressions uttered by the NNSs accounted for 98 per cent. ${ }^{197}$ In other words, using hoshii appears to have been difficult for the NNSs, presumably because the range of application of kudasai and onegai shimasu is wider than for hoshii, so they were more likely to be familiar with these phrases.

\section{Positive Politeness Strategy}

Juju dōshi are a key element when requesting in Japanese as observed by Ueno (1983) and Okamoto (1988), and this has been verified in the present study (see Ch. 2, Sec. 5). Corresponding to Brown and Levinson's Strategy 11, "Be optimistic" of positive politeness, the sentence Misete kurenai? [Won't you show me?] was the one most frequently uttered by both the NSs with 26 utterances and the NNSs with 27 utterances. ${ }^{198}$ This was the most frequently uttered sentence by the NSs, and the second most frequent by the NNSs following the bald on record strategy Misete (+kudasai) [(Please) show me]. In fact, affirmative and negative forms of juju dōshi appeared in the experiments, and ka na [Can I expect? I wonder] was added at the end of the sentence with some juju dōshi expressions.

197 The remaining two per cent of expressions were one utterance each of Misete hoshii [I want you to show me] and the other.

198 Some examples of "optimistic" positive politeness presented in the book by Brown and Levinson are, You'll lend me your lawnmower for the weekend, I hope/won't you/I imagine [Shümatsu shibakariki o kashite kureru yo ne] and I've come to get a little salt [Chotto shio o morai ni kita n'da] (126). 
The requester chooses the most appropriate expression in order for the request to be accepted by the hearer and to accomplish the (requester's) aim of having the request be carried out. The reason that the use of juju dōshi is regarded as positive politeness strategy is that the speakers want to be favoured by the hearer (requestee), so they try to get into the interlocutor's psychological territory through appealing by the use of kureru [(you) givelfavour (me)] or morau [(I) receive]. For example, the sentence Misete kurenai? [Won't you show me?] denotes "(lit.) Won't you give me a showing?" and therefore reinforces recognition that the requestee will be doing the requester a favour by accepting the request. Hence, this approach falls under positive politeness.

The reason morau is more polite than kureru is that the subject of the predicate kureru is you (requestee), whereas the subject of the predicate morau is I (requester) (Yamada 2011). What this means is that the viewpoint of the speaker (requester) is different: in the former the emphasis is on the second person giving a benefit to the speaker (requester), while in the case of the latter the emphasis is on the speaker's receipt of the benefit (Shimizu 2009). According to Kim (2000), kurenai (the negative form of kureru) is used to someone of equal rank in an in-group environment of Japanese native speakers more than kureru (affirmative form), in frequency followed by the morau group. The present experiments corroborate Kim's findings regarding the order of frequency of usage of juju dōshi (see Ch. 2, Sec. 5).

Juju dōshi were employed in the majority of utterances of positive politeness: in 84 per cent out of 56 utterances by the NS, 74 per cent out of 57 utterances by the NNSs, and 55 per cent of 11 exemplars. Furthermore, 61 per cent of the NSs, 53 per cent of the NNSs, and 91 per cent of the exemplars used the negative form. The TXs presented requisitive sentences showing only the negative form.

What is evident from this study is that the participants and the exemplars tended to use the negative form kurenai rather than the affirmative form kureru, while in the case of the 
morau group, the opposite occurred, i.e., the affirmative form moraeru ('can receive') was used more often than the negative form moraenai. Iori (2011: 55-6) argues that the order of politeness when asking is always that the negative form of a verb is more polite than the affirmative form. In general, there is understanding that negative sentences are more polite than affirmative sentences in Japanese (Kabaya et al. (1993), Kato (2004), Okamoto (1988), Ueno (1983), see Ch. 2, Sec. 4 and Sec. 5). In the present experiments, the order of frequency of use by the NSs was kurenai, moraeru, moraenai, and kureru, which only partially agrees with Iori. In addition, the frequent use of the word $i i$ by the NNSs is noteworthy. It accounted for 26 per cent of the positive politeness by the NNSs, so $i i$ was an easy word for them to apply when requesting. By contrast, this appeared in only seven per cent and nine per cent respectively the NSs and the TXs.

It was also found in the present study that the NSs' usage heavily concentrated on kurenai and moraenai, which eliminated the lightest word kureru and the heaviest (the most polite) word moraenai among the four different expressions. ${ }^{199}$ When taking Iori's assertion into consideration and the conditions of the present experiments, it seems that the NSs judged the expressions of kurenai (negative of kureru) and moraeru (affirmative of morau)not kureru (affirmative) nor moraenai (negative) — as most appropriate for making a request to a close friend. At the same time, perhaps kureru was too blunt for the NSs, even when their interlocutor was a close friend.

Table 5.10 Nai Used in Positive Politeness Strategy

\begin{tabular}{|l|cc|c|c|}
\hline Negative form (Nai) & \multicolumn{2}{|c|}{ NS } & NNS & TX \\
\hline Nai used & $34(61 \%)$ & $30(53 \%)$ & $10(91 \%)$ \\
\hline Nai not used & $22(39 \%)$ & $27(47 \%)$ & $1(9 \%)$ \\
\hline Total & $\mathbf{5 6}(\mathbf{1 0 0 \%})$ & $\mathbf{5 7 ( 1 0 0 \% )}$ & $\mathbf{1 1 ( 1 0 0 \% )}$ \\
\hline
\end{tabular}

The phrase ka na anticipates an answer from the other (Mihara 1995), and implies a speaker's sense of asking for confirmation from the interlocutor (Kenbo et al. 1994). The

199 The four expressions are kureru, kurenai, morau, moraenai. 
speaker is anxious about the other's reaction (Kumagai 1995). The biggest difference between the NSs and the NNSs/the TXs is that users of $k a n a$ were almost exclusively the NSs, as there was only one utterance by the NNS and one TX exemplar that used this phrase. From this, it may be inferred that the NNSs had not been taught appropriate use of $\mathrm{ka} \mathrm{na}$ because over 90 per cent of the TXs did not present it, such that learners could not (did not) use it at the end of the sentence.

Table 5.11 Ka na Uttered in Positive Politeness Strategy

\begin{tabular}{|l|c|c|c|}
\hline$K a$ na used in positive politeness & NS & NNS & TX \\
\hline$K a$ na uttered & $22(39 \%)$ & $1(2 \%)$ & $1(9 \%)$ \\
\hline$K a$ na not uttered & $34(61 \%)$ & $56(98 \%)$ & $10(91 \%)$ \\
\hline Total & $\mathbf{5 6 ( 1 0 0 \% )}$ & $\mathbf{5 7 ( 1 0 0 \% )}$ & $\mathbf{1 1 ( 1 0 0 \% )}$ \\
\hline
\end{tabular}

\section{Negative Politeness Strategy}

With regard to negative politeness strategy of HAs, the discussion here will be focused only on the NSs' expressions because there was only one such utterance each by the NNSs and in the TXs. As Kumai (1992) claims, aposiopesis is difficult for the Japanese language learners to understand, and her findings were confirmed in this study (see Ch. 2, Sec. 5). ${ }^{200}$ Fourteen out of 33 utterances $(43 \%)$ of negative politeness strategy of the present experiments ended with the word kedo... [however, but...], followed in frequency by ten utterances of to omotte... [I wonder, I suppose], and four utterances of tara... [if...]. Thereby, the end of such the sentence implicitly reveals the speaker's (unspoken) intention (Morita 1996).

Libra (1978) points out that it is effective for listeners of Japanese to hear the speaker's hesitation in ending to omotte... [I wonder, I suppose], because the hearer can tell that the speaker is humble and reserved as this is implicit in such words. The attitude of the

200 In Kumai's research, all Japanese native speakers utilised aposiopesis, but only two out of 14 Japanese language learners did not. 
speakers, in which they lower themselves to the hearer in order to place themselves at a disadvantage, results in greater imbalance in the relationship, which may prompt a feeling of obligation in the hearer to do something for the requester. By co-operating with the requester the hearer restores the balance of the relationship.

By means of aposiopesis i.e., stopping the sentence in the middle of the utterance, it means that although the sentence is incomplete grammatically, it is complete semantically (Shirakawa 2009). Japanese scholars assert that aposiopesis is one of the typical characteristics of the Japanese language, and it is used for various purposes: in order to have smooth communication with others (Kashiwazaki 1993, Ikeda 1995), to mitigate the speaker's giving too strong an impression (Cho 2000), to convey a feeling of hesitancy (Mizutani 1983, Mihara 1995, Kanamori 2008), to be more polite and less pushy than with a completed sentence (Shimizu 2009), and to lower the speaker's position in order to keep a distance from the other (Kumagai 2008). We may assume that in the present experiments the NSs used aposiopesis intuitively, rather than because they consciously knew the effect of it.

The issue here is that although aposiopesis is a typical characteristic of the Japanese language, only one NNSs used it, and only one out of 13 model discourses in the TXs exemplified it. We may speculate that the authors of the TXs thought that it was cognitively too difficult for learners at the intermediate level, since it demands a certain degree of "native speaker intuition" in order to comprehend the unspoken ending of the sentence. Whatever the reason, it is inadequate if the authors do not include the use of aposiopesis in their model dialogues, considering native speakers' constant use of it. Unless textbooks clearly present aposiopesis, including explanations of its usage, learners' acquisition of it may be delayed. 
Table 5.12 Usages of Aposiopesis

\begin{tabular}{|l|r|r|}
\hline Type of Aposiopesis & Number of utterance \\
\hline kedo... & 14 & $43 \%$ \\
\hline naa to omotte... & 10 & $30 \%$ \\
\hline Tara ... & 4 & $12 \%$ \\
\hline Others & 5 & $15 \%$ \\
\hline Total (by the NSs) & $\mathbf{3 3}$ & $\mathbf{1 0 0 \%}$ \\
\hline
\end{tabular}

\section{Off Record Strategy}

An indisputable fact exposed through the experiments is that the off record strategy was employed only by the NSs, who used it in 30 utterances, with only two such utterances by the NNSs, and no examples appeared in the TXs. The off record strategy means that the speaker (requester) does not speak directly, and therefore does not use a requesting verb such as misete [Show (me)] or tasukete [Help (me)]. As off record strategy does not use verbs of request, the speakers' expressions were widely varied. The most utilised expressions were Donna koto kaita? [What have you written?] in 19 utterances (63\%), followed by Kono ato jikan aru [Do you have time later?] in nine utterances (30\%).

As Miyake (1993: 65-6) and Nakamura and Akui (2004: 104) affirm, the more indirect expressions that are used, the more polite the utterance becomes to the hearer. This is especially so if the speaker's real intention in the off record strategy remains unspoken, yet the speaker expects the hearer to guess somehow what he/she has implied (Grice's implicature). Therefore, commencing with Donna koto kaita? uttered by 19 NSs, for example, the speakers can develop the conversation upon hearing the response from their interlocutor, which is usually specific. For instance, the following were examples of responses in the present experiments: Eigo no rekishi da yo [About the history of the English language] or Toshokan no hon o sankō ni shite kaita yo [I wrote it by referring to books in the library]. The requester does not use direct requisitive items of vocabulary; therefore sometimes it 
prompts the interlocutor voluntarily to offer his/her support, saying, for instance, Miru? [Wanna see mine?]. Such a way of developing the conversation using off record strategy might derive from the common ground of the two parties in terms of competency in the language, and in the setting of the present study where both are students with an assignment.

It appears that off record strategy is difficult for learners of Japanese to acquire, not only because of the lack of exemplars presented in the TXs, but also because off record strategy itself demands a high competency in the Japanese language both linguistically and culturally. Therefore, it is hard to develop a smooth conversation using the off record strategy if the circumstances of the two parties are not shared in common or if the other party shares the same circumstances but is somehow insensitive. Hence, the possibility of the use of the off record strategy is limited, depending on the conversational partner. It is self-evident that it will be more difficult if the partner's cultural background is different from that of the requester, as in the case of many NNS learners.

\subsubsection{Expressions of Supportive Moves}

\section{Grounders (Positive politeness)}

The main expression uttered as a grounder by both the NSs and the NNSs was Mada yattenai [I haven't started yet] (the primary reason), which describes the requester's present situation. Isogashikatta [I was busy] (a secondary reason), followed Mada yattenai in frequency. Both expressions formed the majority of grounders; however, the NSs predominantly uttered the former, 14 times more frequently than the latter. For the NNSs, although Mada yattenai was uttered more than Isogashikatta, it was only three times more often than the latter. The two groups differed thus in the degree of preference for the two expressions. It was clear that, for both the NSs and the NNSs, the expression preferred in order to persuade the requestee was one that explained the present situation, such as Mada 
yattenai, rather than to explain the reason, as in Isogashikatte [sic] yattenai. This is probably because providing the primary reason for the request was deemed to sound more persuasive when asking for the requestee's help. ${ }^{201}$

Another expression of grounder was Nihongo ga/wa muzukashii [Japanese is difficult], which was uttered obviously only by the NNSs, in spite of the fact that the requestees of the present study were also NNSs whose proficiency level was the same as the requestes. It can be said that this reveals the honest feelings of the NNSs requesters. In the case of the TXs, there were no settings asking for help with an assignment, so the expressions of grounder which were used by the NSs and the NNSs in the present experiments did not appear in them.

\section{Minimisers (Negative politeness)}

Minimisers were frequently used by almost all participants, as well as being presented in the model discourses: they were employed as many as 1.5 times per discourse by the NSs; 0.5 times by the NNSs, and 0.8 times by the TXs. Among the various minimisers, chotto was overwhelmingly used in 66 per cent of all attenuation words by the NSs, 81 per cent by the NNSs, and 90 per cent in the TXs. ${ }^{202}$ The word chotto was employed in 81 utterances by 80 NSs (100\%) and in 9 out of 13 examples (69\%), but in only 35 utterances by 80 NNSs (45\%). The word chotto is effective and useful for learners not only because of how easy it is to use, but also its functionality in decreasing the impression of the speaker's imposition. The fact was that the learners' use of it was less than half that of the NSs and the TXs. This might be due to a lack of teachers' informing the NNSs of its effectiveness.

\footnotetext{
201 Because Isogashikatte [sic] was the reason for not having started yet. See Footnote 174 above.

202 The NSs: $66 \%=81$ utterances of chotto/122 utterances of all words/phrases of attenuation. The NNSs: $81 \%=35 / 43$. Thirty-seven NNSs did not use attenuation. The TXs: $90 \%=9 / 10$. Three model discourses did not use attenuation.
} 
The tendency to use chotto was similar to the case of apology, for both the NSs and the TXs used it quite often, whereas only around half of the NNSs did so. It can be said that the NSs and the TXs were trying to lessen the weighty impression of the request more than the NNSs, as Matsumoto (1985), Spees (1994), Hidasi (2007) argue (see Ch. 2, Sec. 2). If an attenuating word like chotto is used in the sentence, it becomes more courteous as Kimura claims (see Ch. 2, Sec. 5). There are other words of attenuation, such as dake [only], which can limit the content of a request or emphasise the triviality of it, but these attenuations were not often uttered when compared with chotto.

Chotto was normally included in the HA, for example Chotto misete kurenai ka na? [Why don't you just show me?]. This is essential knowledge and a required keyword for the NNSs. The small usage of attenuation by the NNSs in both settings of apology and request suggests that this form of communication among Japanese native speakers is inadequately explained to learners in the classroom.

\section{Preparators (Negative politeness)}

Preparators work by the requester's sounding out the hearer's availability before uttering a HA (Kyi 2004). Preparators are categorised by Blum-Kulka et al. as an independent element of discourse, and they fulfil an important function prior to exercising the HA, because whether or not the requester decides to make a request depends on the hearer's response to the preparators. Hashiuchi (1988) and Izaki (2000) observe that the procedure of request by Japanese native speakers is first to attempt to prepare the hearer for what the speaker is going to tell them.

Preparators were uttered once by the NSs per discourse, and by around 90 per cent of the NNSs. Although it may depend on the distance between the requester and the requestee, the core sentence(s) of request, i.e., the HA, did not seem to be abruptly broached to the 
requestee, especially by the NSs. Around 60 per cent of the TXs utilised preparators even though about half of the requesters' topics were not the same as that of the NSs and NNSs. ${ }^{203}$

One notable preparatory expression was Onegai ga aru [I have something to ask], which was especially presented in the TXs, presumably because it can be utilised in a wider range of occasions than Mō yatta [Have you finished yet?].

\subsubsection{Order of Utterance}

A significant difference in order of utterance was discovered in the present experiments. Over eighty per cent of the NSs uttered a preparator before the first requisitive sentence. This result was quite similar to that of Izaki's study (2000, see Ch. 2, Sec. 5), which revealed different approaches by the Japanese and the French when requesting. While almost all Japanese native speakers uttered a preparator (the word yokoku [prior notice] is used in Izaki's research), the number of the French who employed yokoku was extremely small. And while the Japanese added some explanation after yokoku which is corroborated by the present study, the French did so less than the Japanese (Izaki 2000, 86-7).

Less than half of the NNSs uttered a preparator prior to the first HA, and eleven NNSs (14\%) did not employ a preparator at all; in other words, they started the HA from the beginning of the discourse. This was in strong contrast to the results of the NSs, because no NSs uttered the HA at the very beginning. There was one scenario in the TXs (8\%) in which the HA was uttered first, and only two scenarios applying a preparator first (16\%), unlike the discourses by the NSs.

One of the reasons for the difference between the NSs/theTXs and the NNSs in the present experiments may be that the majority of the NNSs have not yet fully understood the

203 For example, there were scenes where a requester asked a hearer to correct his/her writing. 
Japanese native speakers' way of approach when requesting. Furthermore, the authors of the TXs probably need to reconsider the scenario because the percentage of the personae in the exemplars who uttered a preparator prior to the first request was only 20 per cent of the NSs. From this, it is obvious that the exemplars do not fully reflect authentic discourses.

\subsection{Summary}

When the individual utterances of requests are examined closely, they reveal that certain expressions and linguistic features are employed almost exclusively by a certain group. These are subtle and sophisticated communication devices, but can make the utterances and/or model exemplars sound distinctive.

As for the number of utterances, the NSs uttered more sentences than the other two groups, which was the same result as that of apology, and this was extremely close to the average length per sentence of the setting of apology. ${ }^{204}$ On average NNSs took about 1.6 times longer to utter one sentence than the NSs.

When the proportions of HAs and SMs of the three groups are compared, they are found to be not largely different: around 3 (HA) to 7 (SM). This result suggests that the SMs were by and large more substantial than the HAs in the discourses of request. This outcome was the converse of that of the discourses of apology. It is evident that when developing a conversation, the proportion of HAs and SMs varies depending on the given setting.

Regarding the expressions, it became clear that the $t e$-form was the centre of the requisitive sentence (HA). Bald on record was preferred almost always by the NNSs. Some of the NNSs straightforwardly uttered their wish by using the unmodified desu-form. Despite being grammatically correct, the $d e s u$-form sounds demanding and it may be surmised that this was the reason for its non-use by the NSs/TXs. A significant difference between the NSs

204 The NNSs took 1.7 times longer than the NSs (see Chapter 4, Section 4.1). 
and the NNS/TXs appeared in the usage of off record strategy because there were only two utterances of off record strategy by the NNS in the latter group. Other noteworthy features of the HAs were the use of juju dōshi together with the negative word nai [no, not], aposiopesis, and attenuation.

The three SMs - grounders, minimisers, and preparators - were mainly and almost similarly used by the three groups. The grounder used by the NSs was the only SM that exceeded the number of utterances of the HA. The minimiser was another commonly used SM but differences in usage were recognised. Preparators were mostly uttered prior to the HA. Both the NS and the NNS uttered these 1.0 and 0.9 times respectively on average, so it was something almost all participants had acquired well. By contrast, there were only seven exemplars in the TXs, so that they were conspicuous by being few in number. The usage of the other two SMs, rewards and disarmers, was limited to one group each: rewards were used by the NSs and disarmers by the TXs.

Concerning the procedure of development of the conversation, three-quarters of the NSs and over 40 per cent of the NNSs employed two different SMs before the first HA, but the TXs applied either one SM or three SMs followed by the HA, with both occupying 38 per cent each. It was revealed that the TXs do not actually very closely reflect native speakers' discourse in this respect.

These are summaries of the findings of the similarities and differences of the discourses of requests.

In Chapter 5, I analysed the data of requisitive discourses. From the results, similarities and differences among the three groups were identified; in particular, the usage of politeness strategies of HAs differed from each other. Also, the individual group was apt to 
choose a certain supportive move(s). In the following chapter, Chapter 6, I will summarise all findings obtained through the experiments, and demonstrate how textbooks should be improved. 


\section{CHAPTER 6 SUMMARY}

\subsection{Introduction}

In this chapter, I will summarise all results of the experiments of apologies and requests in terms of structures and expressions of the NSs, the NNSs, and the TXs. Furthermore, I will design four examples of model discourses to be as natural as possible with remarks by referring to the results of the present study. These comprise two different sets of social relationships (equals in apology and senior-junior in request) in the settings.

Brown and Levinson's politeness theory sorted utterances by analysis based on the concept of face-threatening acts (FTAs), but they did not investigate in detail what kinds of politeness strategy(ies) were utilised by a speaker in order to develop a conversation. Thus, it is more instructive to investigate the utterances collected in the present experiments at the discourse level by first adopting Blum-Kulka et al.s' CCSARP to categorise the individual utterances, followed by Brown and Levinson's politeness theory. The present study revealed the tendency of the apologiser and the requester to apply certain politeness strategy(ies) at the level of the entire discourse.

\subsection{Apology}

It has become apparent that a speaker's competency level and probably cultural background influence their preferred speech acts. There were differences discovered regarding the frequency of use of apologetic expressions and the types of expressions; and usages of the past tense, attenuation, and aposiopesis were quite different among the three groups. From the viewpoint of politeness theory, all three groups used negative politeness 
strategy more often than positive politeness strategy. None of them used either bald on record or off record strategy.

What was found here is that negative politeness strategy was applied in over twothirds of all the utterances of discourses among the three groups; and positive politeness strategy was employed in about one-third. Although the proportions of the strategies were similar, the difference of expressions used became distinct in a broad overview of the results. While the authors of the TXs applied expressions that were more polite than the other two groups, the NNSs opted to use a simple and less formal degree of apology. What this implies is that the NNSs did not seem to be influenced very much by the TXs as to the appropriateness of an utterance when apologising.

The apologetic expressions of the three groups were epitomised in three items of vocabulary respectively. There were clear preferences in each group: sumimasen was used by the NSs, gomen nasai was mostly used by the NNSs, and mōshiwake arimasen by the TXs. Hence, the most polite expression was preferred by the TXs, and the plainest word by the NNSs. The frequency of utterance of the apology was also different in each group, in that the NSs uttered an apology more than four times per discourse on average, the NNSs did so 2.6 times, and even the TXs presented it 1.8 times.

The predominant SMs of apology were explanation (positive politeness) and admission of the facts (negative politeness), and the other two SMs were promise (positive) and placation (positive). The NSs and the NNSs used all four of the SMs, while the TXs presented both explanation and admission of the facts; but, the other two SMs, promise and placation, were presented only once each among the 13 model exemplars.

There were three major differences in terms of linguistic usage among the three groups, namely the use of the past tense, aposiopesis, and attenuation. As for the past tense, the TXs used it in HAs in a similar proportion to the NSs, but the NNSs rarely used it. The NNSs 
would have heard or learned the past tense of apologetic expressions, such as sumimasen deshita or mōshiwake arimasen deshita, yet few of them actually uttered these at the end of the discourse. This is not a grammatical issue but a pragmatic one. It may also well reflect that it feels unnatural to native speakers of English and they will need to consciously fight aginst this perception until it is embedded in their linguistic usage. At least, one of the reasons for the non-use of the past tense by the NNSs may be because there is no clear explanation of its usage provided in the TXs, nor were they perhaps explicitely taught in textbooks or by teachers.

Aposiopesis was quite often used by the NSs and the TXs, with around three quarters of the NSs and two-thirds of the given exemplars using aposiopesis in explanations for the late arrival. But, aposiopesis was much less used by the NNSs-only 11 per cent. This suggests that aposiopesis is also not adequately explained to the NNSs in spite of the examples presented in the TXs, such that learners are slow in turning it into natural practice.

When explaining the cause of offence, the NSs extremely frequently used attenuation, for example, employing the word chotto. But while this occurred most frequently in the utterances of the NSs, few NNSs used it, and there was only one example in the TXs. The authors of the TXs overlooked the efficacy of chotto, and obviously included no explanation of this usage of the word. The above three elements - using the past tense, attenuation, and aposiopesis-somewhat distinguished the NNSs from the NSs in terms of the expressions uttered.

The order of utterances in the discourses, too, differed between the NSs and the TXs. The NSs uttered an apologetic expression as soon as they saw the victim, whereas the exemplars of the TXs gave a bold statement, admission of the facts. The number of the NNSs who uttered apologetic expressions was not as high as that of the NSs; yet, they were inclined to utter it first like the NSs did. 
In Japanese, it is more acceptable to use the apologetic expression at the very beginning of the conversation because the seriousness and earnestness of the apologiser can be better sensed by the victim compared to when admission of the facts (or any other utterance) comes first. In brief, the order of utterance is either the apologetic expression followed by admission of the facts or the converse. The NSs and the NNSs tended to use the former, while the TXs leaned towards the latter. Since the purpose of apology is to narrow the distance from the victim, the order of utterance, especially starting from an apology, is vital. The authors of the TXs need to consider what should be uttered when the apologiser first meets the victim.

From the present experiments, therefore, it became clear that there were differences in the discourses of apologies in the three groups in their ways of adopting vocabulary and approaching the interlocutor, a victim.

\subsection{Request}

In the discourses of requests, overall usage of the politeness strategies among the three groups did not appear to be noticeably different, but when HAs alone were examined, little commonality was seen among them. While the NSs used four strategies with no strong preponderance of a particular strategy, the NNSs focused mostly on bald on record and positive politeness strategies and the other two strategies were scarcely used. As for the TXs, almost all exemplars applied positive politeness strategy. In brief, the strategies used for HAs varied according to the groups. 
Brown and Levinson claimed that four politeness strategies are used when requesting ${ }^{205}$ and this differentiates requests from apologies because the latter only appeared in Strategy 6: Apologize in Negative politeness in Politeness (1987: 187). This would be because the way of approaching a requestee by a requester depends on the requester's discretion based on the distance and social rank of the two parties along with the level of imposition. $^{206}$

The similarities of the three groups were identified as follows. The proportions of HAs in the total number of discourses by the three groups were not very different at around 30 per cent by all groups. Hence, the proportion of SMs (70\%) is large in the discourses, so that they had an influence on the proportion of politeness strategies of the discourses of request.

Among various SMs, the grounders (explanations) used by the NSs were the only supportive move which exceeded the number of HAs. This implies that the NSs placed great importance on explaining the reason for the request. Only HAs and grounders by the NSs were uttered more than twice per discourse on average. This implies that the attitude of the NSs is that a grounder is indispensable when requesting. Other SMs used by the three groups were less frequently used than the HAs.

The three primary SMs—grounders, minimisers, and preparators-were between 60 per cent and 67 per cent of the discourses in each group; ${ }^{207}$ and the secondary SMs-rewards and disarmers - displayed recognisable differences in their use. Although both the NSs and

205 Brown and Levinson demonstrate in their book (1987) that speech act of requests fall into four different strategies: bald on record, positive politeness, negative politeness, and off record strategies (pp. 69, 81, 227).

206 (Brown and Levinson, 1987: 68-9).

207 NS $(67 \%)=$ Grounder $(32 \%)+$ Minimiser $(21 \%)+$ Preparator $(14 \%)$

NNS $(60 \%)=$ Grounder $(31 \%)+$ Minimiser $(11 \%)+$ Preparator $(18 \%)$

TX $(62 \%)=$ Grounder $(23 \%)+$ Minimiser $(23 \%)+$ Preparator $(16 \%)$ 
the NNSs utilised these secondary SMs, their use was insignificant. By contrast, the TXs appeared considerably different in the use of subsequent SMs, albeit small in number. For example, disarmer was the SM that was most utilised by the TXs, and while the NSs and the NNSs uttered it to some degree, they did not use it as much as the TXs did.

It is understandable that the TXs would cover the various strategies in order to be model exemplars. Thus, they should also have considered rewards, because the NSs especially made use of them in order to obtain consent from the requestee. The nature of rewards demands skill in negotiation as a tactic, therefore learners may need more explicit instruction in this, and not only from the model exemplars presented in the TXs.

Concerning the expressions of HAs, juju dōshi had a central function in expressing the requisitive sentences. They were often used together with the auxiliary verb with the negative meaning nai, especially by the NSs. The most frequently and commonly used expression of request among the three groups was $V$-te+kurenai. Many textbook examples as well as more than half of the NSs and the NNSs employed this negative form. It can be said that $V$-te+kurenai was employed widely by the all three groups as a typical requisitive expression.

By contrast, the phrase $k a$ na was used only by the NSs, and not uttered by the NNSs nor presented in the TXs. Likewise, it was mostly only the NSs who used aposiopesis, which was barely utilised by the NNSs and the TXs. Use of aposiopesis thus showed a marked difference between the NSs and the NNSs/the TXs. As mentioned in Chapter 5, aposiopesis functioned not only in requests but also in the explanations for being late when apologising. It is understandable, therefore, that since the TXs do not present the phrase ka na or aposiopesis, the NNSs could not (or did not) appropriately apply them. This is evidence that the TXs do not reflect authentic speech. In a nutshell, sentences and phrases expressing the hesitant and 
reserved feeling of a requester did not seem to have been acquired by the NNSs in the present experiments.

Differences in the use of SMs by the three groups appeared in minimisers and disarmers, too. The minimisers occupied 30 per cent of the SMs for the NSs, and 32 per cent for the TXs. In particular, among various words and phrases of minimisers, the frequent use of chotto stands out. It is absolutely certain that the NNSs who participated in the role-plays knew the word chotto, but similar to the discourses of apology, they did not utter this mitigating word as often as the others. It is not very clear why the NNSs did not utter in requisitive sentence, but clearly they did not think to emply it in such a context, perhaps because this usage had not been explicitly explained.

Other distinctive characteristics of the discourse of requests were that, unlike apologies, the first HA was generally not uttered without prior SMs. ${ }^{208}$ A widely adopted procedure by the NSs and the NNSs was that the requesters asked about the interlocutor's availability in order to check if he/she would be a possible requestee, followed by an explanation about the reason(s) for the request. Conversely, the TXs started with the grounder (explanation) followed by the HA. The very first utterance when a requester intends to make a request is crucial in order to gauge whether or not the requester will actually raise the request, and if so, the timing of it. Therefore, confirming the interlocutor's availability before explaining the requester's wish is a sort of discernment [wakimae].

The number of SMs to be uttered before the first HA seems to depend on the relationship between the speakers and the content of the request. In the present experiments, a combination of two SMs followed by the HA was mostly used by the NSs and the NNSs.

208 There were 11 NNSs who uttered only HAs. This occurred with one exemplar of the TX, but with none of the NSs. 
Ninety-five per cent of the $\mathrm{NSs}^{209}$ as well as 80 per cent of the NNSs ${ }^{210}$ uttered more than one SM before the first utterance of the HA; but, in the case of the TXs, their usages were polarised at either three SMs or one SM, 38 per cent each. It seems unnecessarily complex and inappropriate for TXs to present three SMs before the first HA. This revealed that the TXs did not reflect authentic conversations.

\subsection{Similarities and Differences in the Settings of Apology and Request}

From a comparison of the data of the three groups, five kinds of similarities and differences were discovered. These were (1) the NNSs were different but the TXs and the NSs were similar, (2) the TXs were different but the NSs and the NNSs were similar, (3) the NSs were different but the NNSs and the TX were similar, (4) no similarity was found among the three groups, and (5) the three groups were the same or very similar. The following table summarises points of similarities and difference and the countermeasures of the latter.

209 NS: $75 \%$ of the NSs used two SMs and so did $20 \%$ of them utter one SM.

210 NNS: $44 \%$ of them used two SMs and so did $36 \%$ of them utter one SM. 
Table 6.1 Summary of Findings of Major Differences among the Three Groups

\begin{tabular}{|c|c|c|c|}
\hline Findings & Points of Difference & $\begin{array}{c}\text { Type of } \\
\text { Difference }\end{array}$ & Countermeasures \\
\hline (1) $\mathrm{NNS} \neq \mathrm{NS} / \mathrm{TX}$ & $\begin{array}{l}\text { - Use of the past tense (A) } \\
\text { - Aposiopesis (A) } \\
\text { - } \text { Minimiser chotto (R) }\end{array}$ & Expression & $\begin{array}{l}\text { The NNSs need } \\
\text { more explanation. }\end{array}$ \\
\hline (2) $\mathrm{TX} \neq \mathrm{NS} / \mathrm{NNS}$ & $\begin{array}{l}\text { - The type of the first } \\
\text { utterance (A) } \\
\text { - Use of disarmer (R) } \\
\text { - Number and order of SMs } \\
\text { before the first HA (R) }\end{array}$ & Structure & $\begin{array}{l}\text { The authors need to } \\
\text { better understand } \\
\text { authentic } \\
\text { conversations more. }\end{array}$ \\
\hline (3) $\mathrm{NS} \neq \mathrm{NNS} / \mathrm{TX}$ & $\begin{array}{l}\text { - Attenuation chotto (A) } \\
\text { - Use of ka na (R) } \\
\text { - Aposiopesis (R) }\end{array}$ & Expression & -ditto- \\
\hline (4) $\mathrm{NS} \neq \mathrm{NNS} \neq \mathrm{TX}$ & $\begin{array}{l}\text { - Apologetic expressions (A) } \\
\text { - } \quad \text { Type of Requisitive } \\
\text { sentence, HA (R) }\end{array}$ & $\begin{array}{l}\text { Expression } \\
\text { Structure }\end{array}$ & -ditto- \\
\hline (5) $\mathrm{NS}=\mathrm{NNS}=\mathrm{TX}$ & $\begin{array}{l}\text { - Ratio of usage of HA vs. } \\
\text { SM (A,R) } \\
\text { - Use of negative form in } \\
\text { HA (R) }\end{array}$ & $\begin{array}{l}\text { Structure } \\
\text { Expression }\end{array}$ & - \\
\hline
\end{tabular}

(A): in the discourses of apologies, (R): in the discourses of requests

Other than the above, it was recognised that the utterance speed of the pairs of the NSs and those of the NNSs was different. The NSs spoke roughly 40 per cent faster than did the NNSs in the two settings. In addition, both the NSs and the NNSs took shorter time and used fewer sentences in discourses of apologies than those of requests. This latter tendency, with the setting of a request necessitating longer and more numerous utterances, also appeared in the model exemplars of the TXs.

The following tables show distinctive features of discourses of apology and request respectively. 
Table 6.2 Features of Discourse of Apology

\begin{tabular}{|l|l|l|l|}
\hline Characteristics & \multicolumn{1}{|c|}{ NS } & \multicolumn{1}{c|}{ NNS } & \multicolumn{1}{c|}{ TX } \\
\hline Frequency of HAs & 4.2 times/discourse & 2.6 times & 1.8 times \\
\hline Types of HAs & Sumimasen & Gomen nasai & Mōshiwake ariasen \\
\hline Strategy of HAs & Negative & Negative & Negative \\
\hline Major strategies of SMs & $\begin{array}{l}\text { Explanation and } \\
\text { Admission of the facts }\end{array}$ & $\begin{array}{l}\text { Explanation and } \\
\text { Admission of the } \\
\text { facts }\end{array}$ & $\begin{array}{l}\text { Explanation and } \\
\text { Admission of the } \\
\text { facts }\end{array}$ \\
\hline
\end{tabular}

Table 6.3 Features of Discourse of Request

\begin{tabular}{|c|c|c|c|}
\hline Characteristics & NS & NNS & TX \\
\hline Frequency of HAs & 2.1 times/discourse & 1.8 times & 1.0 time \\
\hline Types of HAs & $\begin{array}{l}\text { Tasukete yo } \\
\text { [Help me.] } \\
\text { Misete kurenai ka na? } \\
\text { [Can't you show me?] } \\
\text { Mitai n' da kedo... } \\
\text { [I'd like to see it...] } \\
\text { Kono ato jikan aru? } \\
\text { [Do you have time } \\
\text { later?] }\end{array}$ & $\begin{array}{l}\text { Misete } \\
\text { [Show me.] } \\
\text { Misete kurenai? } \\
\text { [Can't you show } \\
\text { me?] }\end{array}$ & $\begin{array}{l}\text { Misete moraenai? } \\
\text { [Couldn't you show } \\
\text { me?] }\end{array}$ \\
\hline $\begin{array}{l}\text { Main strategies used for } \\
\text { HAs }\end{array}$ & $\begin{array}{l}\text { Bald on record } \\
\text { Positive politeness } \\
\text { Negative politeness } \\
\text { Off record }\end{array}$ & $\begin{array}{l}\text { Bald on record } \\
\text { Positive politeness }\end{array}$ & Positive politeness \\
\hline $\begin{array}{l}\text { Main strategies used for } \\
\text { SMs }\end{array}$ & $\begin{array}{l}\text { Grounder and } \\
\text { Minimiser }\end{array}$ & $\begin{array}{l}\text { Grounder and } \\
\text { Preparator }\end{array}$ & $\begin{array}{l}\text { Grounder and } \\
\text { Minimiser }\end{array}$ \\
\hline
\end{tabular}

\subsection{Textbooks}

The results of the present experiments show that the contents of the TXs do not adequately reflect the native speaker's speech acts. This corroborates the findings of Liddicoat (2000, see Ch. 2, Sec. 1), who argues that model conversations in the textbooks are too simplified, and also Ishida (1998, see Ch. 2, Sec. 1), who claims that no textbooks can satisfy all students. The results of the present study, too, demonstrate that several points concerning the contents of textbooks, especially the structure and expressions of the discourses, could be improved. 


\subsubsection{Structure}

When introducing new sentence patterns or expressions, ${ }^{211}$ they should be presented in the form of a discourse so that the learners may visualise a scene where they are likely to use these new sentence patterns in real life. Thus, it will not be difficult for learners to imagine a possible scenario of when, where and how to use these items.

A key point in devising such a scenario is to explicitly clarify the scene and the relationship(s) between the personae, and maintain the natural flow of discourse. In other words, no matter how relevant a certain "new" formulaic expression, grammatical point, or sentence pattern is (for the learners), the authors should avoid unnatural conversation. The present study identified that this is not yet always satisfactorily achieved in textbooks for learners of Japanese.

It became clear that the TXs used in the present experiments did not explicitly indicate the speakers' intention in the utterance. In indicating the intention of a speaker (semantic meaning), which is more or less equivalent to the individual categories of the CCSARP, what they are learning, and the kind of scene the utterance is appropriate for, becomes clear to students. A discourse may be divided into three structural parts: commencement, development, and ending of the conversation, which explain the mission of individual utterances as a simplified example. In this way, learners with a different cultural background can become aware of the purpose and meaning of each utterance.

The order of utterances for apologies is more culturally predetermined in Japanese than with requests. The flow of a discourse of apology that reflects the speaker's intentions is normatively as follows.

(1) Sumimasen [Sorry.] - to apologise (Apology)

211 For example, sumimasen or gomen nasai in a scene of apology, and $V$-te kuremasen $k a$ in a scene of request. 
(2) Osokunarimashita [I was late.] - to admit what he/she did (Admission of the Facts)

(3) Nebō shite... [I overslept...] - to explain (Explanation)

(4) Kyō hayaku nemasu [I will go to bed early tonight.] - to express resolve that the transgression will not happen again (Pledge)

Repeated apologies were a characteristic of the NSs. It may be unnecessary, however, to show apologies many times in the limited space of textbooks, but this should at least be pointed out as a commentary note. Creating a plausible model discourse using the conventional fixed forms may not be difficult. Based on the results of the present experiments, the core structure of requests in Japanese can be presented in textbooks as follows.

(1) Mò yatta? [Have you finished yet?] — to confirm the other's situation (Preparator)

(2) Mada yatte nai n' da yo nee [I haven't even started yet.] - to explain one's own situation (Grounder)

(3) Chotto misete kurenai ka na? [Can't you just show me (yours)?] - a request with hesitation and which avoids being too pushy (Request and Minimiser)

(4) Ashita ranchi ogoru kara sa [I'll treat you to lunch tomorrow.] - a reward, if necessary (Reward)

The significance of these sentences is that they are uttered together with the speaker's intention. In the utterances by the NNSs in this study, many of them bluntly uttered Misete [Show me], while the NSs did not: the latter mostly asked the other parties' availability before making a request. By elaborating explicitly on the intention of each speaker's utterances through a typical example, learners will be able to comprehend more easily the Japanese native speakers' way of developing the conversation. 


\subsubsection{Expressions}

The appropriate expression in Japanese varies according to the setting and the relationship with the interlocutor. It may be unrealistic to present examples in textbooks based on all possible combinations of relationship between speaker and hearer. At the least, however, examples for cases in which the interlocutors are senior-junior and socially equal could cover the majority of situations. ${ }^{212}$ Three apologetic expressions occurred in the present experiments, suggesting that textbooks for those who are studying Japanese should at least explain the different usages of gomen nasai, sumimasen, and mōshiwake arimasen, together with when to use the past tense with the last two of these. As these were not explained or written in the textbooks, learners used only one of them in this experiment, gomen nasai. None of the textbooks explained their respective usages clearly.

Aposiopesis was employed quite often by the NSs in both settings. For example, Isoide kita n' desu kedo... [I hurried here, but...] was uttered when an apologiser who explained the reason for his/her delay, and Shukudai mitai n' da kedo... [I'd like to see your homework...] in the HA of request. It is noteworthy that both the NSs and the TXs uttered/presented aposiopesis, but the NNSs rarely used it. Even though there were examples of aposiopesis in the textbooks, the learners could not/did not utilise it. It is understandable that aposiopesis is not easy to acquire; and this is a noteworthy case of its being inadequate to present a model discourse without explanation as to how to create or use the difficult linguistic forms. Needless to say, it is essential to provide a thorough explanation.

212 For the case of a subordinate, the model example of an equal can be utilised. 


\subsubsection{Model Discourses Created Based on Data Collected from the Present Experiments}

The following is recommended as an example of the flow of model discourses for learners, in which the marked point is the statement of the speaker's intention (underlined here).

Table 6.4 Apology to a Senior Party: The apologiser came to a working place 30 minutes late

\begin{tabular}{|l|l|l|}
\hline Expression & Apologiser's Intention & Remarks \\
\hline $\begin{array}{l}\text { Sumimasen. } \\
\text { [Sorry.] }\end{array}$ & Apologising to a victim. & $\begin{array}{l}\text { Apologise first before the } \\
\text { explanation. }\end{array}$ \\
\hline $\begin{array}{l}\text { Chotto osoku } \\
\text { narimashita. } \\
\text { [I was late.] }\end{array}$ & $\begin{array}{l}\text { Admission of the fact-(of } \\
\text { the cause of the trouble) } \\
\text { with attenuation. }\end{array}$ & Clarify the fact. \\
\hline $\begin{array}{l}\text { Jitsu wa densha ga } \\
\text { konakute... } \\
\text { [Actually, the train didn't } \\
\text { come on time...] }\end{array}$ & $\begin{array}{l}\text { Explaining the cause } \\
\text { using a form of } \\
\text { aposiopesis. }\end{array}$ & $\begin{array}{l}\text { Explain the cause of the trouble. } \\
\text { The aposiopesis functions to } \\
\text { expose the speaker's apologetic } \\
\text { feeling. }\end{array}$ \\
\hline $\begin{array}{l}\text { Ashita wa motto hayaku } \\
\text { uchi o demasu. } \\
\text { [I'll leave earlier } \\
\text { tomorrow.] }\end{array}$ & $\begin{array}{l}\text { Expressing the speaker's } \\
\text { intention (not to repeat } \\
\text { the misdemeanour } \\
\text { again). . }\end{array}$ & $\begin{array}{l}\text { Express the apologiser's firm } \\
\text { resolve not to repeat the } \\
\text { misdemeanour. }\end{array}$ \\
\hline $\begin{array}{l}\text { DI amo sumimasen deshita. } . \\
\text { Closing remark }\end{array}$ & $\begin{array}{l}\text { (including a feeling of } \\
\text { appreciation for the } \\
\text { victim's acceptance of } \\
\text { the apology). }\end{array}$ & $\begin{array}{l}\text { At the end of the conversation, } \\
\text { once again, utter the apology. This } \\
\text { time the apology should be in the } \\
\text { past tense, such as sumimasen } \\
\text { deshita or mōshiwake arimasen } \\
\text { deshita } \text { However, gomen nasai } \\
\text { does not have a past tense. }\end{array}$ \\
\hline
\end{tabular}

Table 6.5 Apology to an Equal Party: The apologiser promised to go to a movie but failed to show up on time

\begin{tabular}{|l|l|l|}
\hline Expression & Apologiser's Intention & Remarks \\
\hline $\begin{array}{l}\text { Gomen (ne). } \\
\text { [Sorry.] }\end{array}$ & $\begin{array}{l}\text { Apologising to the } \\
\text { victim. }\end{array}$ & $\begin{array}{l}\text { Gomen is more casual than } \\
\text { sumimasen. (Appropriate for use to } \\
\text { an equal.) }\end{array}$ \\
\hline $\begin{array}{l}\text { Choto osoku natte. } \\
\text { [I was late.] }\end{array}$ & $\begin{array}{l}\text { Describing the fact (of } \\
\text { the cause of the trouble) } \\
\text { with attenuation. }\end{array}$ & $\begin{array}{l}\text { Using the te-form is more casual } \\
\text { than the masu-form (mashita). }\end{array}$ \\
\hline $\begin{array}{l}\text { Shigoto ga } \\
\text { owaranakutte... } \\
\text { [I was so busy that I } \\
\text { couldn't finish work... }\end{array}$ & $\begin{array}{l}\text { Explaining the cause } \\
\text { using a form of } \\
\text { aposiopesis. }\end{array}$ & $\begin{array}{l}\text { Using a form of aposiopesis } \\
\text { reveals the speaker's apologetic } \\
\text { feeling. }\end{array}$ \\
\hline $\begin{array}{l}\text { Kondo wa motto hayaku } \\
\text { deru yo ni suru yo. } \\
\text { [I'll make sure I leave } \\
\text { earlier next time.] }\end{array}$ & $\begin{array}{l}\text { Expressing the speaker's } \\
\text { intention (not to repeat } \\
\text { the misdemeanour } \\
\text { again). }\end{array}$ & $\begin{array}{l}\text { Express the apologiser's firm } \\
\text { resolve. }\end{array}$ \\
\hline Hontō ni gomen ne. & $\underline{\text { Closing remark }}$ & At the end of the conversation, \\
\hline
\end{tabular}




\begin{tabular}{|l|l|l|}
\hline [I am really sorry.] & $\begin{array}{l}\text { (including a feeling of } \\
\text { appreciation for the } \\
\text { victim's acceptance of } \\
\text { the apology). }\end{array}$ & $\begin{array}{l}\text { once again, utter the apology. } \\
\text { Gomen (nasai) does not have a } \\
\text { past tense, so in order to emphasise } \\
\end{array}$ \\
& $\begin{array}{l}\text { it, honto ni [really] is added } \\
\text { instead. Alternatively, warukatta } \\
\text { (ne) < the past tense of warui }[I \\
\text { was sorry] can be an appropriate } \\
\text { apology among intimates. }\end{array}$ \\
\hline
\end{tabular}

Table 6.6 Request to a Senior Party: The requester wants the senior party to check his/her draft written in the senior's native language

\begin{tabular}{|c|c|c|}
\hline Expression & Requester's Intention & Remarks \\
\hline $\begin{array}{l}\text { Ima chotto yoroshii deshō } \\
\text { ka? } \\
\text { [May I bother you a bit } \\
\text { please?] }\end{array}$ & $\begin{array}{l}\text { Asking about the other's } \\
\text { availability with } \\
\text { minimiser. }\end{array}$ & $\begin{array}{l}\text { Ask about the other party's } \\
\text { availability with a word of } \\
\text { conjecture. }\end{array}$ \\
\hline $\begin{array}{l}\text { Jitsu wa eigo de tegami o } \\
\text { kaita no de, ojikan no aru } \\
\text { toki ni sukoshi mite } \\
\text { itadakenai deshō ka? } \\
\text { [Actually, I wrote a letter } \\
\text { in Japanese, so when you } \\
\text { have time, could you } \\
\text { please look it over for } \\
\text { me?] }\end{array}$ & $\begin{array}{l}\text { Explaining the reason } \\
\text { for his/her situation, and } \\
\text { making the request. }\end{array}$ & $\begin{array}{l}\text { Explain the reason for the request } \\
\text { before the request. In order to avoid } \\
\text { the impression of being pushy, the } \\
\text { requester leaves the requestee with } \\
\text { discretion over when to deal with } \\
\text { it. The requisitive expression is } \\
\text { used, a negative form of the word } \\
\text { itadaku [humble form of 'receive']. }\end{array}$ \\
\hline $\begin{array}{l}\text { Dōmo arigatō gozaimasu. } \\
\text { [Thank you very much.] }\end{array}$ & $\frac{\text { Remarks of }}{\text { appreciation. }}$ & $\begin{array}{l}\text { Appreciate the requestee's } \\
\text { acceptance. While the present form } \\
\text { is used, the request has only been } \\
\text { accepted by the requestee, and the } \\
\text { favour has not yet commenced } \\
\text { (been accomplished). }\end{array}$ \\
\hline $\begin{array}{l}\text { Dōmo airgatō } \\
\text { gozaimashita. [Thank you } \\
\text { very much.] }\end{array}$ & (Later) Closing remark. & $\begin{array}{l}\text { Once the request has been fulfilled } \\
\text { (accomplished), the past tense } \\
\text { should be uttered. }\end{array}$ \\
\hline
\end{tabular}

Table 6.7 Request to an Equal Party: The requester wants help from an equal party for his/her Assignment

\begin{tabular}{|l|l|l|}
\hline Expression & Requester's Intention & Remarks \\
\hline $\begin{array}{l}\text { Ima chotto ii? } \\
\text { [Are you free now?] }\end{array}$ & $\begin{array}{l}\text { Asking about the other's } \\
\text { availability with } \\
\text { minimiser. }\end{array}$ & $\begin{array}{l}\text { Ask about the other party's } \\
\text { availability. Do not make a request } \\
\text { abruptly. }\end{array}$ \\
\hline $\begin{array}{l}\text { Shukudai yatta? } \\
\text { [Have you finished the } \\
\text { assignment?] }\end{array}$ & $\begin{array}{l}\text { Checking the other's } \\
\text { situation as to whether } \\
\text { he/she will be able to } \\
\text { help. }\end{array}$ & $\begin{array}{l}\text { Need to confirm the other's } \\
\text { situation before making a request. }\end{array}$ \\
$\begin{array}{l}\text { Jitsu wa mada yatte } \text { nai } \\
n \text { 'da. } \\
\text { [As a matter of fact, I }\end{array}$ & $\begin{array}{l}\text { Explaining the reason } \\
\text { for his/her situation. }\end{array}$ & $\begin{array}{l}\text { Explain the reason for the request } \\
\text { before making the request. }\end{array}$ \\
\hline
\end{tabular}




\begin{tabular}{|c|c|c|}
\hline haven't started it yet.] & & \\
\hline $\begin{array}{l}\text { Chotto tetsudatte kurenai } \\
\text { ka na? } \\
\text { [Can't you just give me a } \\
\text { bit of help me?] }\end{array}$ & Making the request. & $\begin{array}{l}\text { When making the request, the word } \\
\text { chotto can function to mitigate the } \\
\text { imposition of the request. Using a } \\
\text { negative form conveys a more } \\
\text { polite impression than a positive } \\
\text { form. } \\
\text { Ka na effectively asks for the } \\
\text { other's reply and also lessens the } \\
\text { imposition. }\end{array}$ \\
\hline $\begin{array}{l}\text { Ashita ranchi ogoru kara } \\
\text { sa. } \\
\text { [I'll buy you lunch } \\
\text { tomorrow.] }\end{array}$ & Offering a reward. & $\begin{array}{l}\text { A reward can sometimes work in } \\
\text { favour of the requester. }\end{array}$ \\
\hline $\begin{array}{l}\text { Warui ne. } \\
\text { [Sorry for troubling } \\
\text { you.] }\end{array}$ & Closing remark. & $\begin{array}{l}\text { Acknowledge the requestee's } \\
\text { acceptance. }\end{array}$ \\
\hline
\end{tabular}

In Chapter 6, various aspects of structures and expressions of the discourses of apologies and requests became apparent including the similarities and differences between them. In addition, the points of improvement for the textbooks were also discussed. The conclusion of the present study is described in the final chapter, Chapter 7. 


\section{CHAPTER 7 CONCLUSION}

\subsection{Introduction}

Chapter 7 presents the overall findings of the present study, which includes a summary of the results of experiments, pedagogical implications, and recommendations from the standpoint of Japanese language teaching and learning.

Numerous comparative studies have been carried out, either on interactions between native speakers and non-native speakers or comparisons between native speakers and model discourses in the textbooks of any language. However, no comparative studies have been carried out using the following three different sources of information: the conversational data of native speakers of Japanese, of non-native speakers of Japanese, and the model exemplars presented in Japanese language textbooks.

The present study aimed to fill this gap in the research literature, identify the characteristics of the speech acts, and the similarities and differences in these three groups of data in order to contribute to improvement in the content of textbooks for the teaching and learning of Japanese as a second or other language. Conversational data from role-plays carried out by native speakers of Japanese and by Japanese language learners, and model exemplars presented in widely available Japanese language textbooks were collected. The three sets of data were analysed combining two kinds of frameworks: Brown and Levinson's politeness theory and Blum-Kulka et al.'s CCSARP. The findings obtained provide useful and relevant information for the authors of textbooks for Japanese as a second or other language.

It became clear through the present study that, by and large, textbooks presented more polite expressions than those used by native speakers of Japanese and Japanese language 
learners. Although the model exemplars were natural, on the whole, presenting natural discourse models does not mean that they are explicit enough for learners - and perhaps neither for teachers. Authors of textbooks should provide detailed explanations on which examples may be used, along with the limitations of usage such as inappropriate settings, so that learners can avoid both impoliteness and being misunderstood by Japanese nativespeakers. As the findings indicate, the lack of appropriate explanations or instructions in the textbooks undermines the development of the competency of the learners. Below are the findings from the three research questions.

\subsection{Overview of the Study}

There have been comparative studies conducted by many scholars who examined native speakers and non-native speakers or native speakers and textbooks; however, to the best of my knowledge, no other study has compared all three parties.

Before conducting the experiments, it was assumed that most examples of non-native speech would differ from both native speakers and the textbooks. However, the extent of similarities and differences were not known; nor how the model exemplars might differ from authentic native-speaker speech.

Furthermore, it may be assumed that example sentences in the textbooks are natural and resemble those of the native speakers. Because the authors of the textbooks in the present study are Japanese native speakers, they were expected to have employed natural structures and expressions while taking into account the level of the learners. If the sentences are not

natural, then questions should be raised as to how they differed from the authentic speech of native speakers. 
The results from the present experiments can be said to be new findings since the experiments contrasted the data of three different groups. The following are specific results in terms of similarities and differences of them. The broad likenesses here imply that all groups showed similar outcomes through the experiments, and differences indicate that either none of the three groups showed the same results or one group differed from the other two.

Research question 1: What similarities and differences in discourse structures are revealed among the three groups: native speakers of Japanese, non-native speakers and model exemplars presented in the textbooks?

The discourse structure among the three groups was found to display similarities in politeness strategies as well as the proportion of Head Acts and Supportive Moves. Nevertheless, differences were also discovered, particularly in the order and frequency of utterances, because the data from three groups showed different tendencies.

\section{Finding 1A: Similarities in Structure of Discourses of Apologies}

- The relative proportion among the four politeness strategies used in the entire discourse differed little among the three groups; namely, the ratio of negative politeness to positive politeness was 7 to 3 .

- By and large, the proportion of HA and SM was also alike, at around 50 per cent each, although the NSs utilised HAs the most (59\%), whereas the textbooks did so the least $(48 \%)$

\section{Finding 1B: Differences in Structure of Discourses of Apologies}

- The type of strategy chosen upon first utterance by an apologiser when the two parties met was different between the native/non-native speakers and the textbooks. While 
the native/non-native participants uttered the apology (HA), such as sumimasen [I'm sorry], at the very beginning of the discourse, the textbooks presented admission of the facts (SM) first, for example, osokunarimashita [I was late] followed by the apology.

- The frequency of utterance of apologies was different. The native speakers apologised more often and repeatedly than the other two, with the textbooks demonstrating the lowest frequency.

- The frequency of usage of SMs was different regarding promise and placation. The native speakers uttered them most, and were likely to make a promise. By contrast, the textbooks barely presented these strategies in their model exemplars. ${ }^{213}$

\section{Finding 1C: Similarities in Structure of Discourses of Requests}

- The entire discourses were primarily comprised of positive politeness strategy among all three groups: it exceeded the other three politeness strategies in both HA and SM.

- Positive and negative politeness strategies accounted for the majority of discourses involving requests, altho ugh their characteristics were reversed. The other two politeness strategies, bald on record and off record strategies, were limited in use.

- Supportive Moves of positive politeness strategy occupied approximately two-thirds of the entire discourse utterances of the three groups.

213 Although the native speakers uttered apologies (negative politeness strategy) most often among the three groups, the reason for no significant differences appearing in the proportion of politeness strategies was that the native speakers employed promise and placation (positive politeness strategies) more frequently than the other two groups. This revealed that while the native speakers apologised more often than the non-native speakers/textbooks, their proportion of positive to negative politeness remained almost the same as the others, as explained the above similarities. 
- The proportion of HA (request) to SMs (grounder, minimiser, preparator, reward and disarmer) among each of the three groups was 3 to 7 , so their usage in their focus on the "request" did not differ very much. The ratio of HAs by the non-native speakers, however, appeared slightly higher than that of the others. Yet, it can be said that no substantial differences emerged in the structure of the discourses.

\section{Finding 1D: Differences in Structure of Request}

- The three groups had different inclinations in using politeness strategy in the HA (request). The native speakers were rather balanced without much preponderance of any particular politeness strategies, while the non-native speakers and the textbooks each seemed to concentrate on certain ones of the four politeness strategies: the nonnative speakers tended to use bald on record and positive politeness, while the textbooks used mostly positive politeness along with bald on record and negative politeness.

- Different ways of approaching the personae in the model discourses were revealed. The textbooks presented a grounder first, whereas the majority of the native speakers and about half of the non-native speakers started with a preparator before the first HA (request).

- The number of SMs before the first HA differed in the textbooks from that of the native and the non-native speakers. The native/non-native speakers uttered mostly one or two SMs before the first HAs; and they rarely uttered three SMs. However, more than one-third of the textbooks presented three SMs in their examples.

- Another noticeable difference in the textbooks was the frequent use of disarmers, which were not utilised as often by the other two groups. 


\section{Research question 2: What similarities and differences in expressions are revealed among the three groups: native speakers of Japanese, non-native speakers and model exemplars presented in the textbooks?}

No similarities of expressions appeared in the setting of apology, but one particular negative form of requisitive sentence was commonly used by all three groups. On the other hand, each group displayed its own characteristic expressions.

\section{Finding 2A: Similarity in Expression of Discourses of Apologies}

- The expression sumimasen was uttered in common by the three groups.

\section{Finding 2B: Differences in Expression of Discourses of Apologies}

- Preferred expressions of apology (HA) differed remarkably from one another, with predominantly sumimasen used by native speakers, gomen nasai the non-native speakers, and mōshiwake arimasen in textbooks.

- The use of the past tense of apology (HA) was prominent with native speakers and the textbooks, and it would appear in a last apologetic sentence close to the end of the discourse. The apology of the past tense was concentrated on the expression sumimasen deshita [I was sorry]. Conversely, the non-native speakers' infrequent use of the past tense stood out.

- Another difference between the native speakers/textbooks and the non-native speakers was noticeable also at the end of the sentence. Aposiopesis was quite often employed by the former group when explaining the reason for the cause of a trouble, such as chotto nebō shite... [I overslept a bit ...], but aposiopesis wasnot uttered by the nonnative speakers very much. 
- The word chotto [a little bit] was very frequently uttered by native speakers in an explanation of the reason for the trouble. This worked to minimise the apologiser's misconduct/error to a victim. This word, however, was not utilised much by either the non-native speakers or the textbooks.

\section{Finding 2C: Similarity in Expression of Discourses of Requests}

- The negative form of juju dōshi, such as V-te kurenai, was commonly employed in a requisitive sentence (HA) among the three groups.

\section{Finding 2D: Differences in Expression of Discourses of Requests}

- The frequent utterance of a minimiser, typically the word chotto [a little bit], separated the native speakers/textbooks from the non-native speakers. In the case of requests, chotto functions to minimise the impression of a burden when a requester is going to ask an interlocutor for help. As raised in above Findings 2B: Differences of Discourses of Apologies, chotto was not utilised by the non-native speakers very much, but the native speakers used it often in both apologies and requests.

- Conspicuous differences appeared at the end of requisitive sentences. One involves aposiopesis, such as misete hoshii n'da kedo... [I'd like you to show me (your note), but...], and another involves the phrase ka na [(in context) all right?, maybe?]. Both were often applied by the native speakers, but not by the non-native speakers or the textbooks. 


\section{Research question 3: In what way can Japanese language textbooks be improved in order to more closely reflect "authentic" native speaker discourse?}

\section{Finding 3:}

It is arguable that the structures of the discourses between the native speakers and in the textbooks in the two different settings of apology and requests were relatively similar; but the experiments of this study demonstrated that there were also several noteworthy differences in discourse between them. Thus, while the framework for the model discourses can remain broadly unchanged, a few important points should be revised at a broad level in order to become closer to native-speaker discourse.

In the discourse of apologies, for example, the first utterance of an apologiser should be an apologetic expression (HA), not admission of the facts. In the case of request, prior to the first utterance of the request (HA), a preparator should be used rather than a grounder so as to secure the availability of the addressee. Furthermore, in order to further improve the quality of textbooks, it is imperative to refer to native speakers' expressions, such as the forms of the ends of sentences and attenuation, etc.

The results were that the proportions of HAs to SMs of both apology and request were similar to some extent. This implies that from the viewpoint of politeness, too, differences were slight. By contrast, differences appeared in the use of vocabulary among the three groups: namely, their expressions when apologising and requesting were not the same, and the way of approaching the hearer differed. As mentioned earlier in this chapter, it is reasonable to assume that the discourses of the non-native speakers would be different from those of the native speakers. What non-native speakers have to do is to recognise and overcome these differences.

On the other hand, given that the textbooks were all designed and written by native speakers, we might have expected their model discourses to reflect authentic native speaker 
speech; however, this did not necessarily turn out to be the case. There were cases where the model exemplars differed from the native speakers' discourse, and this difference was crucial. For example, while the native speakers uttered the apologetic expression at the very beginning in the setting, the textbooks did not present the apology first in their scenarios but admission of the facts. In the case of requests, the native speakers utilised several SMs before the first request, while the textbooks used the SM either more or less than the native speakers. These are just a few of the findings.

Those who are studying Japanese, especially outside Japan, might be very dependent on a teacher and a textbook. Sometimes, even teachers too rely on the textbooks, which indicates that textbooks have a big influence on learners, particularly those who are overseas. That the contents of textbooks do not seem to accurately reflect native speakers' speech acts cannot be ignored, and the results of the present study should be taken into consideration by authors when they design or revise textbooks in the future.

\subsection{Pedagogical Implications}

The results of the present research raise various pedagogical implications. How would Japanese textbook writers use the findings to improve the texts they write? And how would language teachers utilise the findings to improve their teaching? My analysis has demonstrated that textbooks do not provide sufficient explanation, from which it may be deduced that the writers themselves are not sufficiently aware of the norms of Japanese discourse progress. Firstly, therefore, it is important that writers of Japanese textbooks better familiarise themselves with Japanese discourse structure, and provide sufficient explication for both teachers and learners alike. 
Secondly, Japanese textbook writers and Japanese language teachers should emphasise more strongly to their students that language and culture are inseparable; and the result of that in the case of Japanese is an exceptionally strong connection between social hierarchy and the appropriate words and phrases used in discourse, depending on the setting.

Thirdly, previous research suggests that there are both advantages and disadvantages to explicit and implicit instruction in second language acquisition. Nonetheless, the present study finds that explicit instruction particularly for the purpose of learning grammar, vocabulary and cultural linguistic praxis seems to be more effective than the other.

Fourthly, before starting to teach based on a model discourse in a textbook (or similar teaching materials), teachers should check whether or not the background of the discourse is explicitly described and whether it can be understood easily by the students. If teachers regard the explanation is insufficient, they should add supplementary information, such as the reasons for choosing a particular word or phrase by a model persona in the given setting.

Fifthly, the experiments in the present research revealed native speakers' high usage of attenuation in apologies and minimisers in requests. The word chotto may be included in both settings. At least in the sample textbooks used for the present experiments, chotto was presented in the discourses of requests; but merely presenting it in the model discourses without explanation had been insufficient for the learners to understand and internalise its usage. Vocabulary such as chotto, for instance, were items which could easily be introduced and understood early by the students, in order to encourage more natural-sounding Japanese discourse with relatively ease. Even if there is little or no explanation about these in the textbooks used, as long as the teachers provide reasons for when and how to utilise them, the students' competency levels may be expected to rise. 
Sixthly, likewise, if description of the salient usage of the past tense in settings of apology (and presumably thanks) were presented in the textbooks with adequate explanation, non-native speakers could probably use it more appropriately.

The present researcher acknowledges that it might be difficult for authors of textbooks to incorporate all the discrepancies between the native speakers' dialogues and those of the model discourses, because there are limitations to learners' competency as well as pressure on publication space. For example, this study identified that it seems not to be easy for learners to acquire the phrase $k a n a$, because it should not be used on all occasions from the viewpoint of pragmatics, such as when speaking to a superior. Aposiopesis, too, requires very detailed grammatical and pragmatic explanations, such that one cannot expect it to be easily acquired by learners without sufficient explanation.

In conclusion, it is here recommended that designers of Japanese language textbooks, and teachers of Japanese language who use them, should pay far greater attention in future to explaining in detail the cultural and social settings of model discourses. For learners from different cultural and language backgrounds, the degree of difficulty of the newly introduced vocabulary, etc., varies depending on the learners. The authors of textbooks and Japanese language teachers need to be aware that merely presenting sentences is inadequate even though their conversations may be natural. It is highly desirable to provide explanations about native speaker usage in textbooks: in particular, when and on what occasion to use the vocabulary, phatic expressions and structures presented in model discourses. 


\subsection{Recommendations}

The following recommendations based on the results of the present study may be useful for authors of Japanese language textbooks and teachers of Japanese.

- A model exemplar of discourses should be presented not as a single isolated sentence but in the form of discourse. This means that there should be at least two utterances by two people.

- The relationship between the personae in the model exemplars must be described clearly because the level of speech acts of Japanese conversation depends on that relationship. Without description, it is difficult for students to grasp the basis for putting them into practice in order to apply the appropriate speech acts in various settings.

- At least two different types of model discourses should be prepared for the same context, because the speech acts vary depending on the relationship between the two parties. In one, the two parties should be equal in social rank, ${ }^{214}$ and in the other, the relationship should involve a senior-junior pair. ${ }^{215}$

- Certain linguistic features of discourses in Japanese, such as using the past tense when apologising at the end of the discourse, are indispensable in order to make the model exemplars sound natural. It should be explained why the past tense has been presented. It is not enough to present a "natural" discourse, without explaining its salient features.

214 Covering all the various relationships is ideal, but unrealistic. Textbooks should show the scenarios which are likely to occur regularly (e.g., those involving different ages and/or social ranks). A detailed explanation of the relationships is also indispensable, because the expressions used in the case of a pair of middle-aged women would differ from those used by male university classmates, even though the relationship in both settings is equal.

215 The case of different social relationship is easier than that of equals, because the junior party is expected to employ the polite form, including a use of keigo primarily, no matter the age and gender of the junior speaker. 
- Include the speaker's intention explicitly together with a scenario. This will allow learners to grasp the flow of a speaker's speech act objectively.

- Specific description of the semantic meaning of a speaker's intention will enable learners to grasp the flow of a speaker's speech act objectively.

\subsection{Considerations for Further Research}

The present experiments were designed with two settings: apologies involving a senior-junior relationship, and requests carried out between partners in an equal relationship. The research did not consider converse settings, namely apologies to an equal partner or requests to a senior partner, and the results suggest that these are avenues of research that might reward further enquiry. Furthermore, possibilities exist for future study by considering other scenarios, such as invitation, declining an invitation, thanking of various generations, and so forth.

From an aspect of students' competency progress, it would be fruitful to study longterm effects over a longer period of time. Pustjens et al. (2007: 435-6) studied the short-term and the long-term effect of primary level students' Dutch language achievement scores. They conclude that the long-term effects of primary education were small. Their results might not simply apply to university students or older generations of language learners, and the longertem studies of adult learners, such as university students, do not yet seem to be available. Thus further research focusing on the longer-term effect of instructed Japanese language acquisition would be a worthwhile study. 


\subsection{Concluding Remarks}

Brown and Levinson's politeness theory has been very influential since its introduction in 1978, and they advocated it as being universal in their book Politeness: some universals in language usage (1987). In this study, the data collected through role-plays by the participants, native speakers of Japanese and non-native speakers of Japanese, and the model discourses presented in the textbooks, revealed that the proportion of use of politeness strategies in the three groups was little different in similar settings/scenarios, and this finding corroborates Brown and Levinson's claim of universality for their politeness theory.

The native speakers of Japanese inclined toward negative politeness in order to keep or maintain a distance from an interlocutor in both settings of apologies and requests. Notwithstanding the disposition of the Japanese people, there was no significant difference in applying politeness strategies between native speakers and non-native speakers of Japanese whose cultural and linguistic backgrounds were not the same, as mentioned above. It is arguable, therefore, that the present study supports the universality of Brown and Levinson's politeness theory.

In addition, the present study has identified some linguistic features of the Japanese language that learners need to be taught explicitly, i.e., when and to whom one should employ certain Japanese expressions. Some appear to have been overlooked by the authors of the textbooks hitherto, even though they are native speakers of Japanese. Perhaps precisely because they are native speakers, they had simply failed to recognise that several relevant points of praxis were missing and that these are as important as other items of vocabulary, phrases and grammar.

The influence of the textbooks at the macro-level upon learners is prominent to a certain extent, and this study shows that the lack of genuine reflection in the textbooks of authentic utterances, such as choosing suitable expressions for the settings of apology and 
request at the micro-level, appears to have a detrimental effect on the development of the conversational skills of learners, especially from the viewpoint of pragmatics.

It is essential, therefore, to point out such shortcomings individually. Therefore the findings of the present study can offer valuable insights for improving the quality of textbook contents. The authors are expected to simulate authentic conversations of native speakers of Japanese when creating model discourses. Refinement of the presentation of exemplars in Japanese as a second or other language textbooks in accordance with the recommendations of the present study will benefit students of the Japanese language.

Overall, these findings reveal that, at the macro- and micro-level aspects of communication, textbooks are crucially influential in the learners' acquisition of ability to hold natural conversations with native speakers of Japanese. I hope that the findings of this study may become a basis for creating improved teaching materials. 


\section{Today's experiment}

Thank you for participating in the experiment (role-playing) today. The content of the experiment is written as follows. If there are any questions, please let me know.

I would like you to please imagine the scene and perform a role-play freely as you wish. Any kind of ending to the story is fine.

\section{(1) Apology}

Role: One of you is a part-time worker in a café, and the other is the 40 years old owner of the café

Scene: It is now 10.30 a.m. and the café is crowded. A part-time worker, who is a university student and supposed to work from 10 a.m., has just arrived. The student has not informed the owner that he/she would be late. The owner is very dissatisfied with the university student.

\section{(2) Request}

Role: Both of you are university students, and know each other well.

Scene: You have an assignment to present by tomorrow. While one already finished it, the other has not yet started it. Neither of you knows whether the other has finished it. The one who has not yet written it wants some help. The other student does not easily feel he/she should help the friend; however, whether or not the friend's request is accepted depends on the content and development of the story of the friend. 


\section{実験内容}

本日は実験(ロールプレイ)に参加していただきまして、ありがとうございます。 以下は実験の内容です。内容に関してご質問がおありでしたら、お知らせください。

皆様には以下の場面を想定し、会話を自由におこなってください。どのようなスト ーリーの展開になっても構いません。

（1）謝罪の場面

役割：一人は 40 歳のカフェオーナー。もう一人はカフェでバイトをしている大学生。 場面：午前 10 時半でカフェは混雑している。本来大学生のバイト開始時間は 10 時 からであるが、10 時半になってようやく到着した。到着するまで、連絡をいれてい なかった。オーナーは、大学生に対して大いに不満である。

\section{（2）依頼の場面}

役割：二人は同じ授業を受けている大学生。

場面：明日までの宿題がある。一人は既に書き上げたが、もう一人は未着手。お互 い、相手が書いたかどうかは知らない。未だ書いていない学生は、相手に手伝って もらいたいと思っている。一方依頼された学生は、そう簡単に手伝う気持ちにはな らない。しかし相手の話す内容によって最終的に受諾するか否かを決める。

以上 


\section{Intermediate-level Japanese Language Students}

\section{日 本 語 会 話 ?}

Do you want to earn $\$ 15$ by speaking in Japanese?

Who:

Intermediate-level Japanese language students who stayed in Japan less than one year.

What:

To perform four different role-plays with your friend in Japanese. Less than 30 minutes!

When:

Whenever you and your friend are available.

Purpose:

This is a part of a PhD research study.

Contact:

Nobuko (Noelle) KATO, Room VZ 711

nobuko.kato@vuw.ac.nz

TX 0277860936
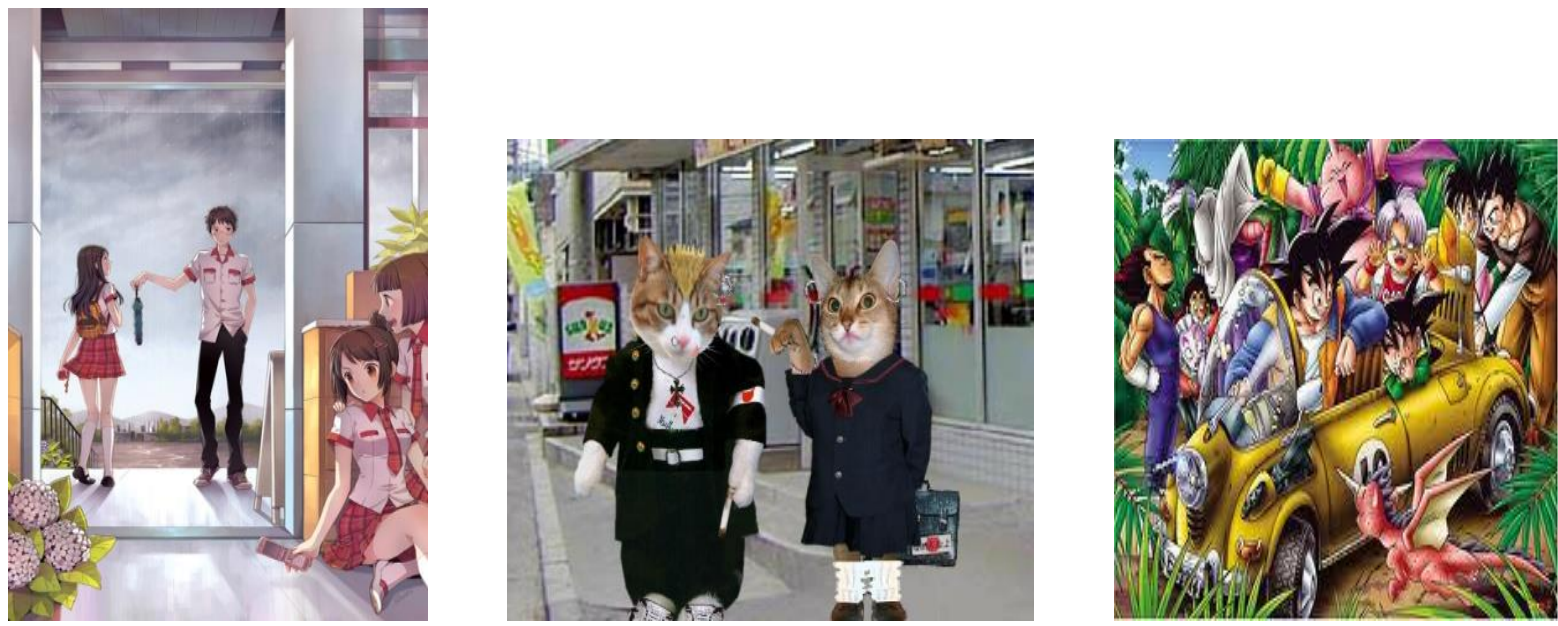


\section{List of textbooks (Apology)}

Banno, Eri, Yutaka Ohno, Yoko Sakane, Chikako Shinagawa, and Kyoko Tokashiki. 1999. Genki: An Integrated Course in Elementary Japanese II. Tokyo: The Japan Times.

Bunka Gaikokugo Senmon Gakkō. 2000. Shin bunka shokyū nihongo II [New Bunka beginners Japanese II]. Tokyo: Bonjinsha.

Kaigai Gijutsusha Kenshū Kyōkai [The Association for Overseas Technical Scholarship]. 2000. Shin nihongo no chükyū, Honsatsu [New Japanese for intermediate level, Main text]. Tokyo: Surīei Nettowāku.

Kokusai Gakuyūkai Nihongo Gakkō [Japan Student Services Organization, Japanese Language School]. 1994. Ryūgakusei no nihongo kaiwa [Japanese language conversation for international students]. 3 ed. Tokyo: Kokusai Gakuyūkai [Japan Student Services Organization].

Kuma, Yumiko, Tomoko Suzuki, Junko Nakayama, and Akiko Yuhara. 2003. Shin jitsuyō nihongo I [New practical Japanese I]. Tokyo: Ezoe Gakuen Shinjuku Nihongo Gakkō.

Nagara, Susumu. 1990. Japanese for everyone: A functional approach to daily communication. Tokyo: Gakken Co. Ltd.

Sasaki, Mizue, and Masami Kadokura. 2007. Kaiwa no nihongo: Japanese through dialogues for intermediate learners [Japanese language for conversation]. Tokyo: The Japan Times.

Surīei nettowāku. 1998. Minna no nihongo shokyū II Honsatsu [Japanese for Everyone, Beginners Level II, Main textbook]. Tokyo: Surīei Nettowāku.

Surīei nettowāku. 2008. Minna no nihongo chūkyū I Honsatsu [Japanese for Everyone, Intermediate level I, Main textbook]. Tokyo: Surīei Nettowāku.

Takushoku Daigaku Ryūgakusei Bekka, Nihongo Kenshū Sentā [Takushoku University Japanese Language Center for Foreign Students, Japanese Language Learning Center]. 2001. Hirake nihongo ge [Open Japanese, Vol. 2]. Tokyo: Takushoku Daigaku [Takushoku University].

Tomisaka, Yoko. 1997. Nameraka nihongo kaiwa [Smooth communication in Japanese]. Tokyo: Aruku.

Tsukuba Language Group. 1992. Situational Functional Japanese: Volume Two. Tokyo: Bonjinsha. 


\section{List of textbooks (Request)}

Bunka Gaikokugo Senmon Gakkō. 2000. Shin bunka shokyū nihongo II [New Bunka beginners Japanese II]. Tokyo: Bonjinsha.

Bunka Gaikokugo Senmon Gakkō. 2004. Bunka chūkyū nihongo I [Bunka intermediate Japanese I]. Tokyo: Bunka Gaikokugo Senmon Gakkō.

Kaigai Gijutsusha Kenshū Kyōkai [The Association for Overseas Technical Scholarship]. 2000. Shin nihongo no chūkyū, Honsatsu [New Japanese for intermediate level, Main text]. Tokyo: Surīei Nettowāku.

Kobayashi, Hitomi. 2008. Nihongo kaiwa torēningu [Conversational training in Japanese]. Tokyo: Asuku Shuppan.

Kokusai Gakuyūkai Nihongo Gakkō [Japan Student Services Organization, Japanese Language School]. 1994. Ryūgakusei no nihongo kaiwa [Japanese language conversation for international students]. 3 ed. Tokyo: Kokusai Gakuyūkai [Japan Student Services Organization].

Kuma, Yumiko, Tomoko Suzuki, Junko Nakayama, and Akiko Yuhara. 2003. Shin jitsuyō nihongo I [New practical Japanese I]. Tokyo: Ezoe Gakuen Shinjuku Nihongo Gakkō.

Miura, Akira, and Naomi Hanaoka McGloin. 2008. An Integrated Approach to Intermediate Japanese. Tokyo: The Japan Times.

Ohso, Mieko, and Yoko Koyama. 1988. Japanese for You. Tokyo: Taishukan Shoten.

Surīei nettowāku. 1998. Minna no nihongo shokyū II Honsatsu [Japanese for Everyone, Beginners Level II, Main textbook]. Tokyo: Surīei Nettowāku.

Yamauchi, Shigekatsu. 2006. Step Up Nihongo 3. Tokyo: Aishīai.

Yoneda, Ryusuke, Kazuko Fujii, Mie Shigeno, and Hiroko Ikeda. 1998. Bijinesu no tame no nihongo [Getting down to business: Japanese for business people]. Tokyo: Surīei Nettowāku. 


\section{Sample Transcription of an Apology Role-play by a Pair of NSs}

$\mathrm{JaA}$ : Apologiser who is a Japanese native speaker.

JaB: Café owner who is a Japanese native speaker.

The parentheses indicate the utterances of an interlocutor which occurred simultaneously.

\begin{tabular}{|c|c|c|}
\hline & Dialogue (41 seconds) & $\begin{array}{l}\text { Semantic } \\
\text { Intention of } \\
\text { Speaker }\end{array}$ \\
\hline $\mathrm{JaB} 1$ & あれ、ちよつと今日羊 んじゃあない の? & 事実陳述 \\
\hline JaA1 & $\begin{array}{l}\text { 店長 すいません。ちよつと一 (ん) 昨日からなんとなく具合が } \\
(\text { あ一) よくなくって。 }\end{array}$ & 謝罪 理由陳述 \\
\hline $\mathrm{JaB} 2$ & そーなんだ一。 & 応答 \\
\hline $\mathrm{JaA} 2$ & $\begin{array}{l}\text { はい：朝ちよつと起きれなからたんですけど、なんとか } \\
\text { 来たんですけど（あー ほんと）すいません。 }\end{array}$ & 状况説明、謝罪 \\
\hline $\mathrm{JaB3}$ & 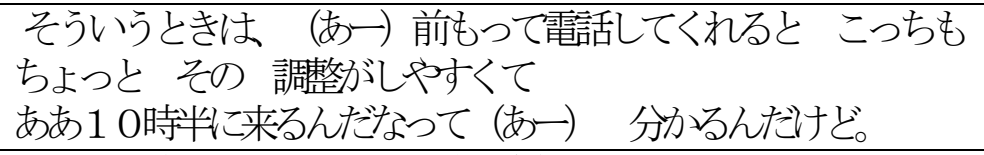 & 注意、状洗帨月 \\
\hline $\mathrm{JaA} 3$ & $\begin{array}{l}\text { あ一そんなんですけど、しようと思ったんですけけどー } \\
\text { (うん）ちよつと 番号が分んなからたんでー。 }\end{array}$ & 言訳 \\
\hline $\mathrm{JaB} 4$ & 君、何ケ月ここで働 ているの ここで。 & 疑問 \\
\hline $\mathrm{JaA} 4$ & $\begin{array}{l}\text { すいません、本当に すいません。 あの一 あれ } \\
\text { あの一、今日はちよつと遅くまで働くので。 }\end{array}$ & 㛛罪 補償 \\
\hline $\mathrm{JaB5}$ & いッよ早く帰つても。 & 却下 \\
\hline $\mathrm{JaA} 5$ & すいませんでした。 & 謝罪 \\
\hline JaB6 & $\begin{array}{l}\text { 分かりました。じゃあ、 ちよつと 今日忙しいから とにかく } \\
\text { 早く着替えて (あ一) 早く出てきてくれるかなあ。 }\end{array}$ & 承知、作業指示 \\
\hline JaA6 & あ一、分かりました。すいませんでした。 & 承知、謝罪 \\
\hline $\mathrm{JaB7}$ & は! & 応答 \\
\hline
\end{tabular}

\begin{tabular}{|l|l|l|}
\hline & Dialogue: English translation & $\begin{array}{l}\text { Semantic } \\
\text { Intention of } \\
\text { Speaker }\end{array}$ \\
\hline JaB1 & Oh? You're a bit late today, aren't you? & $\begin{array}{l}\text { Recognition and } \\
\text { statement of the } \\
\text { fact }\end{array}$ \\
\hline JaA1 & $\begin{array}{l}\text { Manager, sorry, I'm a little... (um). Since yesterday I haven't } \\
\text { felt (ah) well. }\end{array}$ & $\begin{array}{l}\text { Apology and } \\
\text { explanation }\end{array}$ \\
\hline JaB2 & I see. & Response \\
\hline JaA2 & $\begin{array}{l}\text { Yes, I couldn't get up this morning, but I somehow managed } \\
\text { to come here, (oh, really?) sorry. }\end{array}$ & $\begin{array}{l}\text { Description of the } \\
\text { fact and apology }\end{array}$ \\
\hline JaB3 & $\begin{array}{l}\text { In that case, (ah) if you'd called us beforehand, we'd have } \\
\text { been able to make adjustments easily, and could understand } \\
\text { (ah) that you'd come at 10.30. }\end{array}$ & \begin{tabular}{l} 
Admonition \\
\hline JaA3
\end{tabular} Yes, you're right..., and I'd like to have called you (um), but \\
\hline
\end{tabular}




\begin{tabular}{|l|l|l|}
\hline & I don't know the number... & \\
\hline JaB4 & Look here, , how long have you been working here?! & Question \\
\hline JaA4 & I'm sorry, indeed sorry. Well, ah, err, I'll work late today. & $\begin{array}{l}\text { Apology and } \\
\text { reparation }\end{array}$ \\
\hline JaB5 & Don't worry, you can go home early. & Rejection \\
\hline JaA5 & I am sorry. & Apology \\
\hline JaB6 & $\begin{array}{l}\text { Okay, well, it's busy today, so go in anyway and change your } \\
\text { clothes (ah), and get to work immediately, will you? }\end{array}$ & $\begin{array}{l}\text { Acknowledgemen } \\
\text { t and instruction }\end{array}$ \\
\hline JaA6 & Yes, I understand. I am sorry. & $\begin{array}{l}\text { Acknowledgemen } \\
\text { t and apology }\end{array}$ \\
\hline JaB7 & Right! & Response \\
\hline
\end{tabular}




\section{Sample Transcription of an Apology Role-play by a Pair of NNSs}

NaA: Apologiser who is a non-native speaker of Japanese.

NaB: Café owner who is a non-native speaker of Japanese.

\begin{tabular}{|c|c|c|}
\hline & Dialogue: English translation & $\begin{array}{l}\text { Semantic Intention } \\
\text { of Speaker }\end{array}$ \\
\hline $\mathrm{NaA} 1$ & 遅くなって、本当にすみません。 & 事実提示、謝罪 \\
\hline $\mathrm{NaB} 1$ & 何で羊刻してんだよ一。 & 不満表明 \\
\hline $\mathrm{NaA} 2$ & ちよつと電車が 遅くなりました。 & 理由提示 \\
\hline $\mathrm{NaB} 2$ & え、何でちやんと起きなからたの。 & 詳細質問 \\
\hline $\mathrm{NaA} 3$ & らん、起きたけど、ウェリントンの電車はいらも遅れています。 & 理由（言い訳） \\
\hline $\mathrm{NaB3}$ & えーマそれー理由になり なり ならないんだよ。 & 注意 \\
\hline $\mathrm{NaA} 4$ & あ一本当にすみません。 & 謝罪 \\
\hline $\mathrm{NaB} 4$ & まあ、いいよ。でも 今度遅刻しないようにね。 & 了解、忠告 \\
\hline $\mathrm{NaA} 5$ & らんはい、次ちよつともつと早、電車に乗るつもりです。 & 宣言 \\
\hline $\mathrm{NaB5}$ & い、考えだ。 & 応答 \\
\hline $\mathrm{NaA6}$ & はいすすません。 & 謝罪 \\
\hline NaB6 & じやあ、働け。 & 作業指示 \\
\hline
\end{tabular}

\begin{tabular}{|l|l|l|}
\hline & Dialogue: English translation & $\begin{array}{l}\text { Semantic Intention } \\
\text { of Speaker }\end{array}$ \\
\hline NaA1 & I'm late, I'm very sorry. & $\begin{array}{l}\text { Admission of the } \\
\text { fact and apology }\end{array}$ \\
\hline NaB1 & Why are you so late? & $\begin{array}{l}\text { Question about the } \\
\text { reason }\end{array}$ \\
\hline NaA2 & Well, the train was late. & $\begin{array}{l}\text { Explanation of the } \\
\text { cause }\end{array}$ \\
\hline NaB2 & What? Why didn't you get up on time? & Suggestion \\
\hline NaA3 & $\begin{array}{l}\text { Yeah, I did get up, but the trains in Wellington are always } \\
\text { late. }\end{array}$ & Further explanation \\
\hline NaB3 & Hmm, that's no reason. No, it can't be. & Disagreement \\
\hline NaA4 & Um..., I'm really sorry. & Apology \\
\hline NaB4 & Well, okay, but you won't be late next time, right? & $\begin{array}{l}\text { Acceptance the } \\
\text { apology and warning }\end{array}$ \\
\hline NaA5 & Um, yes, I intend to take an earlier train next time. & Promise \\
\hline NaB5 & That's a good idea. & Response \\
\hline NaA6 & Yes, sorry. & Apology \\
\hline NaB6 & Alright, get to work. & Instruction \\
\hline
\end{tabular}




\section{Sample Transcription of a Request Role-play by a Pair of NSs}

$\mathrm{JrA}$ : Requester is a Japanese native speaker. JrB: Requestee is a Japanese native speaker.

The parentheses indicate the utterances of an interlocutor which occurred simultaneously.

\begin{tabular}{|c|c|c|}
\hline & Dialogue (58 seconds) & $\begin{array}{l}\text { Semantic } \\
\text { Intention of } \\
\text { Speaker }\end{array}$ \\
\hline JrA1 & 宿題 やった??課題 なんかセミナーの トピック決めてー。 & $\begin{array}{l}\text { 相手の状况質問 } \\
\text { 、詳絊提示 }\end{array}$ \\
\hline JrB1 & あ一やつたやった。 めつちや 大変だったよ。 & 応答、状況報告 \\
\hline JrA2 & 終わつた一? & 質問 \\
\hline $\mathrm{JrB2}$ & 終わつた。 やってないの? & 回答、質問 \\
\hline JrA3 & 明日までやね。 & 詳兹回確忍 \\
\hline $\mathrm{JrB3}$ & うん そうそうそう。 & 応答 \\
\hline JrA4 & なんか やろうと思っとったんやけどー 時間なくてー。 & 事情陳述 \\
\hline $\mathrm{JrB} 4$ & あ一 そうなんやー。 & 応答 \\
\hline JrA5 & 何にした一? & 詳細質問 \\
\hline JrB5 & えー、私なん力言語習得的な一。（あ一） & 回答 \\
\hline JrA6 & どうしようかなーと思ってー。難しい? 時間卦からた? & 詳細質問 \\
\hline JrB6 & 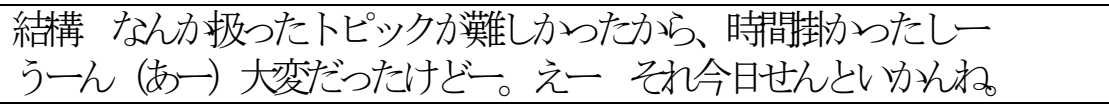 & 回答、質問 \\
\hline JrA7 & うん、今日放課後やろうかねてと思ってたん。 & 回答 \\
\hline JrB7 & あ、放課俊力口。 & 応答 \\
\hline JrA8 & 今日時間女る? & 相手の事情質問 \\
\hline $\mathrm{JrB} 8$ & あ一、今日 うん 時間ある。 & 回答 \\
\hline JrA9 & えつ、ほんまに? 緒こ やってもらってもいい? & 確忍 依頼 \\
\hline JrB9 & 一緒こ一? & 応答 (躊谓) \\
\hline JrA10 & うん、緒こ手伝つてもらってもいい? & 更なる依頼 \\
\hline $\mathrm{JrB} 10$ & $\begin{array}{l}\text { あ、手伝う一。 えーと、えーらん、まあ 今日時間あるし一 } \\
\text { う一ん、まあ真理子 うーん ま この前 遅水たん 許してくれたしー。 }\end{array}$ & $\begin{array}{l}\text { 事情提示 前回 } \\
\text { の負 目ゆえ受 } \\
\text { 諾 }\end{array}$ \\
\hline JrA11 & ありがとう。 & 感謝 \\
\hline $\mathrm{JrB11}$ & 緒こ「ゆうきやん」の放課後。 & 具体的な提案 \\
\hline $\mathrm{JrA} 12$ & ありがとう。 & 感謝 \\
\hline $\mathrm{JrB12}$ & その代わり ジュース奢つて。 & 条件提示 \\
\hline JrA13 & あ、いいよ いいよ 全然いよ。 & 承諾 \\
\hline
\end{tabular}




\begin{tabular}{|c|c|c|}
\hline & Dialogue: English translation & $\begin{array}{l}\text { Semantic } \\
\text { Intention of } \\
\text { Speaker }\end{array}$ \\
\hline JrA1 & $\begin{array}{l}\text { Finished the homework yet? The topic of the seminar, to } \\
\text { decide on the topic... }\end{array}$ & $\begin{array}{l}\text { Question about the } \\
\text { other party's } \\
\text { situation }\end{array}$ \\
\hline $\mathrm{JrB1}$ & Yeah, yeah, I've done it already. It was really hard. & Response \\
\hline $\mathrm{JrA2}$ & You've done it!? & Surprise \\
\hline $\mathrm{JrB} 2$ & I've done it. . Haven't you done it yet? & $\begin{array}{l}\text { Confirmation and } \\
\text { question }\end{array}$ \\
\hline JrA3 & We've got till tomorrow, right? & Confirm the detail \\
\hline $\mathrm{JrB3}$ & Yeah, right. & Response \\
\hline JrA4 & $\begin{array}{l}\text { Well, I thought I'd get to work on it, but... I've run out of } \\
\text { time. }\end{array}$ & $\begin{array}{l}\text { State the situation } \\
\text { of own }\end{array}$ \\
\hline $\mathrm{JrB} 4$ & Oh, I see. & Response \\
\hline JrA5 & What did you decide on? & Question \\
\hline JrB5 & Er, about language acquisition (ah) & Response \\
\hline JrA6 & $\begin{array}{l}\text { I'm wondering what to do..., was it difficult? Did it take a } \\
\text { lot of time? }\end{array}$ & Question in detail \\
\hline JrB6 & $\begin{array}{l}\text { Pretty much, it took a lot of time "'cause my topic was } \\
\text { hard, , hmm (ah) it was really difficult..., Err, you need to } \\
\text { get it done today, don't you? }\end{array}$ & $\begin{array}{l}\text { Response and } \\
\text { question }\end{array}$ \\
\hline JrA7 & Yeah, I thought I'd do it after school. & $\begin{array}{l}\text { Response and } \\
\text { explanation about a } \\
\text { plan }\end{array}$ \\
\hline JrB7 & Oh, after school... & Response \\
\hline JrA8 & Do you have any time, today? & $\begin{array}{l}\text { Question about the } \\
\text { other's availability } \\
\text { (request) }\end{array}$ \\
\hline JrB8 & Err, today, yeah, I've got time. & Response \\
\hline JrA9 & Really? Can I ask you to do it with me? & $\begin{array}{l}\text { Confirmation and } \\
\text { request }\end{array}$ \\
\hline JrB9 & With you? & $\begin{array}{l}\text { Response } \\
\text { (hesitation) }\end{array}$ \\
\hline JrA10 & Yeah, would you mind helping me? & Further request \\
\hline $\mathrm{JrB} 10$ & $\begin{array}{l}\text { Ah, help..., er, uh, well, I have time today, hmn, well } \\
\text { Mariko, hmm, well, you forgave me when I was late the } \\
\text { other day... }\end{array}$ & $\begin{array}{l}\text { State the current } \\
\text { situation and } \\
\text { indebtedness }\end{array}$ \\
\hline JrA11 & Thank you! & Appreciation \\
\hline $\mathrm{JrB11}$ & After the class of "You can". & Specific proposal \\
\hline JrA12 & Thank you. & Appreciation \\
\hline $\mathrm{JrB} 12$ & For that, you can treat me to juice. & Request a reward \\
\hline JrA13 & Ah, ok, ok, absolutely no problem. & Acceptance \\
\hline
\end{tabular}




\section{Sample Transcription of a Request Role-play by a Pair of NNSs}

$\mathrm{NrA}$ : Requester is a non-native speaker of Japanese.

$\mathrm{NrB}$ : Requestee is a non-native speaker of Japanese.

The parentheses indicate the utterances of an interlocutor which occurred simultaneously.

\begin{tabular}{|c|c|c|}
\hline & Dialogue (63 seconds) & $\begin{array}{l}\text { Semantic } \\
\text { Intention of } \\
\text { Speaker }\end{array}$ \\
\hline NrA1 & ジョアンさん、（うん）宿題もうおおつた? & 状況質問 \\
\hline $\mathrm{NrB} 1$ & 終わつた、どうして。 & 応答、疑問 \\
\hline $\mathrm{NrA} 2$ & $\begin{array}{l}\text { あ一実は 私は一 あの一 はじ はじめ はじめな からた } \\
\text { です。あ一 わたし 手伝って くれる。216 }\end{array}$ & 理由陳述、依頼 \\
\hline $\mathrm{NrB} 2$ & 締め切りは 明日でしようー。 & 実情陳述 \\
\hline $\mathrm{NrA3}$ & あ一 はい!でも私時間がない、一人でなにも なともできー。 & 言い訳 \\
\hline $\mathrm{NrB3}$ & 今まで何をしてるの一。 & 詳細質問 \\
\hline $\mathrm{NrA4}$ & あ一、今まで あの一 忙しかわた & 回答 \\
\hline NrB4 & 何がー。 & 詳細質問 \\
\hline $\mathrm{NrA5}$ & あ一手伝つてくれー。 & 依頼 \\
\hline NrB5 & 今度だナ。 & 受諾 \\
\hline NrA6 & サンキュー。 & 感謝 \\
\hline
\end{tabular}

\begin{tabular}{|c|c|c|}
\hline & Dialogue: English translation & $\begin{array}{l}\text { Semantic } \\
\text { Intention of } \\
\text { Speaker }\end{array}$ \\
\hline $\mathrm{NrA1}$ & Hi, Joanne. (Hi) Have you done the homework yet? & $\begin{array}{l}\text { Question on the } \\
\text { situation of the } \\
\text { interlocutor }\end{array}$ \\
\hline NrB1 & Yeah, I've done it . Why? & $\begin{array}{l}\text { Respond and } \\
\text { question about the } \\
\text { motivation of the } \\
\text { question }\end{array}$ \\
\hline $\mathrm{NrA} 2$ & $\begin{array}{l}\text { Ah, in fact, I, well, have not star...start, started it yet. Aah, } \\
\text { I, Can you help me? }\end{array}$ & $\begin{array}{l}\text { Explain own } \\
\text { situation and } \\
\text { request }\end{array}$ \\
\hline $\mathrm{NrB} 2$ & It's due tomorrow, isn't it? & $\begin{array}{l}\text { Clarification of the } \\
\text { condition }\end{array}$ \\
\hline $\mathrm{NrA3}$ & Ah, yes. But, I have no time. I can't do any, anything alone. & Excuse and request \\
\hline $\mathrm{NrB3}$ & What have you been doing till now? & Critical comment \\
\hline $\mathrm{NrA} 4$ & Ah, well, I've been busy till now. & $\begin{array}{l}\text { Explanation } \\
\text { (excuse) }\end{array}$ \\
\hline
\end{tabular}

216 The utterance is incorrectly applied in this context. 


\begin{tabular}{|l|l|l|}
\hline NrB4 & With what? & Criticism \\
\hline NrA5 & Aah, help me! & Entreaty \\
\hline NrB5 & Only this time. & $\begin{array}{l}\text { Acceptance with a } \\
\text { condition }\end{array}$ \\
\hline NrA6 & Thank you. & Appreciation \\
\hline
\end{tabular}




\section{GLOSSARY}

\begin{tabular}{|c|c|}
\hline $\begin{array}{l}\text { Admission } \\
\text { of the fact }\end{array}$ & $\begin{array}{l}\text { A supportive move, whereby apologisers confess their involvement in the } \\
\text { offensive act. }\end{array}$ \\
\hline Aposiopesis & $\begin{array}{l}\text { A speech act of leaving a sentence unfinished (incomplete), whereby speakers } \\
\text { attempt to imply their feeling to a hearer, for example, hesitation or reluctance, etc. }\end{array}$ \\
\hline $\begin{array}{l}\text { Back- } \\
\text { channelling }\end{array}$ & $\begin{array}{l}\text { A sign showing that a hearer is listening to what a speaker says attentively. It is } \\
\text { caleed aizuchi in Japanese. }\end{array}$ \\
\hline CCSARP & The Cross-Cultural Speech Act Realization Project by Blum-Kulka et al. \\
\hline Disarmer & $\begin{array}{l}\text { A supportive move, whereby requesters try to remove any potential objection the } \\
\text { hearer might raise upon being confronted with the request. }\end{array}$ \\
\hline Explanation & $\begin{array}{l}\text { A supportive move whereby apologisers utter the cause which brought about the } \\
\text { violation. }\end{array}$ \\
\hline FTA & $\begin{array}{l}\text { Face, in this study, is defined as an individual's self-esteem and their public self- } \\
\text { image. Thus, a face-threatening act is an utterance that intrude upon the hearers' } \\
\text { face (self-esteem). }\end{array}$ \\
\hline Grounder & A supportive move, whereby requesters give reasons for their request. \\
\hline HA & $\begin{array}{l}\text { Head Acts. The apology and the request proper in discourses respectively. The HA } \\
\text { is part of the sequence which serves to realise the act independently of any other } \\
\text { elements (SMs) in discourses. }\end{array}$ \\
\hline JSOL & Japanese as second or other language \\
\hline Juju dōshi & Verbs of giving and receiving, such as kureru [to give] and morau [to receive] \\
\hline Keigo & $\begin{array}{l}\text { Honorific expressions/words used, in particular, if the interlocutors or the third } \\
\text { persons are seniors of the speaker. }\end{array}$ \\
\hline Minin & $\begin{array}{l}\text { A supportive move, whereby requesters try to reduce the imposition placed on the } \\
\text { hearer by their request. }\end{array}$ \\
\hline NS & Native speaker \\
\hline NNS & speaker \\
\hline Placation & $\begin{array}{l}\text { A supportive move, whereby apologisers express one, or more than one, of the } \\
\text { following: pledge, repair, responsibility, and beseeching, in order to beg for } \\
\text { forgiveness of a victim. }\end{array}$ \\
\hline Preparator & $\begin{array}{l}\text { A supportive move, whereby requesters announce that they are about to make a } \\
\text { request, by asking about the potential availability of the hearer for carrying out the } \\
\text { request. }\end{array}$ \\
\hline Promise & $\begin{array}{l}\text { A supportive move, whereby apologisers feel the need to promise forbearance or } \\
\text { reparation. }\end{array}$ \\
\hline Reward & $\begin{array}{l}\text { A supportive move, whereby requesters offer some compensation in order to } \\
\text { increase the likelihood of the hearer's compliance with the speakers' request. }\end{array}$ \\
\hline SFP & Sentence-final particle \\
\hline SM & $\begin{array}{l}\text { Supportive Move. A supportive move is a unit external to the HA (apology or } \\
\text { request) modifying the HA's impact by either aggravating or mitigating its force, } \\
\text { occurring either before or after the HA. For discourses of apology, there are four } \\
\text { SMs: explanation, admission of the fact, promise, and placation. For discourses of } \\
\text { request, there are five SMs: grounder, minimiser, preparator, reward, and disarmer. }\end{array}$ \\
\hline Tameguchi & Casual speech (usually) among peers \\
\hline TX & Textbook \\
\hline Wakimae & $\begin{array}{l}\text { Discernment or awareness of the appropriate level of polite language (e.g. } \\
\text { honorifics) that should be socially acceptable in the given context. }\end{array}$ \\
\hline
\end{tabular}




\section{REFERENCES}

Akahori, Naoko. 2000. "Amerika jin no irai hyōgen ni okeru danjo sa: Seibetu to shinmitsu sa no hijū [Gender Differences in the Use of Expressions of Request of Americans]."

Eibei bunka [English and American Cultures] no. 30:33-47.

Andersen, Peter A. 2012. "The Basis of Cultural Differences in Nonverbal Communication." In Intercultural communication: a reader, edited by Larry A. Samovar, Richard E. Porter and Edwin R. McDaniel, 293-312. Boston, Mass.: Wadsworth Cengage Learning.

Angouri, Jo. 2010. "Using textbook and real-life data to teach turn taking in business meetings." In English language teaching materials: theory and practice, edited by Nigel Harwood, 373-394. New York: Cambridge University Press.

Arundale, Robert B. 2010. "Constituting face in conversation: Face, facework, and interactional achievement." Journal of Pragamatics no. 42:2078-2105.

Austin, J. L. 1962. How to do things with words: the William James Lectures delivered at Harvard University in 1955. Oxford: Clarendon Press.

Azuma, Shoji. 1997. Shakai gengogaku nyümon [Introduction to sociolinguistics]. Tokyo: Kenkyusha.

2001. "Poraitonesu sutoratejī no nichibei hikaku - Jōshi to buka no aida de no fuman hyōmei to sono kaiketsu kōshō [A Comparison of politeness strategies between Japanese and Americans: Expressing complaint between superior and subordinate and the method of resolving issues]." In Danwa no poraitonesu [Discourse politeness], 59-73. Tokyo: Kokuritsu Kokugo Kenkūjo [The National Institute for Japanese Language and Linguistics].

Barnlund, Dean C, and Miho Yoshioka. 1990. "Apologies: Japanese and American." International Journal of Intercultural Relations no. 14:193-206.

Banno, Eri, Yutaka Ohno, Yoko Sakane, Chikako Shinagawa, and Kyoko Tokashiki. 1999. Genki: An Integrated Course in Elementary Japanese II. Tokyo: The Japan Times.

Bataineh, Ruba Fahmi, and Rula Fahmi Bataineh. 2006. "Apology strategies of Jordanian EFL university students." Journal of Pragamatics no. 38:1901-1927.

Blum-Kulka, Shoshana, Juliane House, and Gabriele Kasper. 1989. Cross-Cultural Pragmatics: Requests and Apologies, Advances in discourse processes. Norwood, N.J: Ablex Publishing Corporation.

Blum-Kulka, Shoshana, and Elite Olshtain. 1984. "Requests and Apologies: A Cross-Cultural Study of Speech Act Realization Patterns (CCSARP)." Applied Linguistics no. 5 (3):196-213.

Boku, Senka. 2010. "OPI dēta ni miru nihongo gakushūsha to nihongo bogo washa ni yoru bunmatsu hyōgen no shiyō: Setsujoku joshi de owaru iisashi hyōgen o chūshin ni [Usage of unfinished sentences by Japanese language learners and Japanese native speakers: Based on the unfinished sentences ending with conjunctive particles]." Nagoya Daigaku Daigakuin Kokusai Gengo Bunka Kenkyūka Nihongo Gengo Bunka Senkō [Japanese Language and Culture Programme, Studies of Foreign Languages and Cultures, Graduate School, Nagoya University] no. 11:217-235.

Brown, Penelope, and Stephen C. Levinson. 1987. Politeness: some universals in language usage, Studies in interactional sociolinguistics 4. Cambridge [Cambridgeshire] ; New York: Cambridge University Press.

Buckingham, B.R., Hollis L. Caswell, Donald D. Durrell, E.R. Jobe, William Kottmeyer, Ullin W. Leavell, Evan Lodge, and John C. Whitnnery. 1952. "What Are Textbooks for?" The Phi Delta Kappan no. 33 (5):241-247. 
Bunka Gaikokugo Senmon Gakkō. 2000. Shin bunka shokyū nihongo II [New Bunka beginners Japanese II]. Tokyo: Bonjinsha.

- 2004. Bunka chūkyū nihongo I [Bunka intermediate Japanese I]. Tokyo: Bunka Gaikokugo Senmon Gakkō.

Chang, Wei-Lin Melody, and Michael Haugh. 2011. "Evaluations of im/politeness of an intercultural apology." Intercultural Pragmatics no. 8 (3):411-442.

Cho, Youngnam. 2000. "Kedo de owaru hatsuwa no goyōron teki kenkyū: Iiowari no kedo o chūshin ni [Pragmatic study of utterances of kedo: Focusing on kedo at the end of the sentence]." Gengo Bunka to Nihongo Kyoiku [Language, culture and Japanese language pedagogy], Departmental Bulletin Paper of Ochanomizu Women's University no. 19:89-100.

2000. "Yōken no kaiwa ni okeru iisashi hyōgen ni tsuite [Unfinished sentences in business conversations]." Gengo Bunka to Nihongo Kyōiku [Language, Culture and Japanese Language Education], Departmental Bulletin Paper of Ochanomizu Women's University no. 20:27-38.

Cohen, Andrew D., and Elite Olshtain. 1981. "Developing a Measure of Socilcultural Competence: The Case of Apology." Language Learning no. 31 (1):114-134.

Coulmas, Florian. 1981. "Conversational routine: explorations in standardized communication situations and prepatterned speech." In Rasmus Rask studies in pragmatic linguistics v. 2, edited by Florian Coulmas, 1-18. The Hague; New York: Mouton.

2005. "Linguistic etiquette in Japanese society." In Politeness in language, edited by Richard J. Watts, Sachiko Ide and Konrad Ehlich, 299-323. Berlin, New York: Mouton de Gruyter.

Culman, Hillah, Nicholas Henry, and Henry VanPatten. 2009. "The Role of Explicit Information in Instructed SLA: An On-Line Study with Processing Instruction and German Accusative Case Inflections." Die Unterrichtspraxis / Teaching German no. 42 (1):19-31.

Davis, Jr., O.L. 1962. "Textbooks and Other Printed Materials." Review of Educational Research no. 32 (2 (April)):127-140.

Dekeyser, Robert. 1994. "Implicit and Explicit Learning of L2 Grammar: A Pilot Study." TESOL Quarterly no. 28 (1):188-194.

Dobeta, Nobuko. 2001. "Nihongo ni okeru poraitonesu no araware kata: Danwa sankasha no jōhō ryō o chūshin ni [Expressions of politeness in the Japanese language: Based primarily on the quantity of information held by participants in a conversation]." Keiai Daigaku Kokusai Kenkyū [Bulletin of International Studies, Keiai University] no. 8:53-79.

Duffy, Thomas M, Lorraine Higgins, Brad Mehlenbacher, Cynthia Cochran, David Wallace, Charles Hill, Diane Haugen, Margaret McCaffrey, Rebecca Burnett, Sarah Sloan, and Suzanne Smith. 1989. "Models for the Design of Instructional Text." Reading Research Quarterly no. 24 (4):434-457.

Eelen, Gino. 1999. "Politeness and ideology: A critical review." Pragmatics no. 9 (1):163173

Ellis, Rod. 2009. Implicit and explicit knowledge in second language learning, testing and teaching, Second language acquisition. Bristol, UK ; Buffalo [N.Y.]: Multilingual Matters. .2012. Language teaching research and language pedagogy. Chichester, West Sussex ; Malden, MA: Wiley-Blackwell. 
Ellis, Rod, and Natsuko Shintani. 2014. Exploring language pedagogy through second language acquisition research, Routledge introductions to applied linguistics.Milton Park, Abington Oxon: Routledge.

Evi, Rusiana, Miki Yamashita, and Yukako Morimoto. 2006. "Indoneshia e yōkoso sakusei hōkoku [A report on production of Indonesia e yōkoso]." Nihongo Kyōiku Kiyō [Bulletin of Japanese Language Education] no. 2:121-126.

Frantzen, Diana. 1998. "Focusing on form while conveying a cultural message." Hispania no. 81 (1):134-145.

Fraser, Bruce. 1981. "On Apologizing." In Conversational routine: explorations in standardized communication situations and prepatterned speech, edited by Florian Coulmas, 259-272. The Hague; New York: Mouton. . 1990. "Perspectives on Politeness." Journal of Pragamatics no. 14:219-236. 2005. "Whither politeness." In Broadening the horizon of linguistic politeness, edited by Robin Tolmach Lakoff and Sachiko Ide, 65-83. Amsterdam; Philadelphia, PA: John Benjamins Publishing.

Fujii, Noriko. 2005. "Learning from learner discourse: Rethinking grammar instruction." Japanese Language and Literature no. 39 (2):291-337.

Fukushima, Saeko. 1996. "Request Strategies in British English and Japanese." Language Sciences no. 18 (3/4):671-688.

Furubeppu, Hizuru. 2009. "Nihongo kyōiku jisshūsei to sakusei shita nihongo kyōkasho wa jisshū de ikani katsuyō sareta ka: Setsumei kōdō no shiten de jisshū kyōiku o kangaeru [How is the Japanese language textbook used by students of the JAFL course, during teaching practice: Learning how to explain language in action." Kokusai Bunkagakubu Kiyō, Yamaguchi Kenritsu Daigaku [Bulletin, Department of International Studies, Yamaguchi Prefectural University] no. 2:1-15.

Furukawa, Yoshiko, and Kaoru Fujinaga. 2007. "Indoneshia no chūtō kyōiku muke nihongo kyōzai sakusei purojekuto [Project for developing textbooks for high schools in Indonesia]." Kokusai kyōryū Kikin Nihongo Kyōiku Kiyō [Bulletin, Japanese Languge Education, The Japan Foundation] no. 3:45-62.

Gagné, Nana Okura. 2010. "Reexamining the notion of negative face in the Japanese sociolinguistic politeness of request." Language and Communication no. 30:123-138.

Gass, Susan M., and Noël Houck. 1999. Interlanguage refusals: a cross-cultural study of Japanese-English, Studies on language acquisition. Berlin; New York: Mouton de Gruyter.

Goffman, Erving. 1967. Interaction ritual: essays on face-to-face behaviour. London,: Allen Lane.

1971. Relations in Public. Harmondsworth, Middlesex, England: Penguin Books Ltd.

Gondo, Sachiyo, and Hiromi Iryo. 2006. "Nihongo chūkyū kaiwa jugyō no hōkoku: Kyōzai oyobi shirabasu no sakusei ni mukete [Report on Japanese conversation classes for intermediate learners: Development of materials and syllabus based on communicative teaching]." Kurume Daigaku Ryügakusei Bekka [Bulletin of the Institute of Foreign Language Education, Kurume University] no. 13:151-161.

Grice, Paul. 1989. Studies of the way of words. Cambridge, MA: Harvard University Press.

Gu, Yueguo. 1990. "Politeness phenomena in modern Chinese." Journal of Pragamatics no. 14 (2):237-257.

Hadley, Alice Omaggio. 1993. Teaching Language in Context: Proficiency Oriented Instruction. Second ed. Boston: Heinle \& Heinle.

Hall, Edward. 1976. Beyond Culture. Garden City, New York: Anchor Press / Doubleday. Hashimoto, Yoshiaki. 2000. "Komyunikēshon ron kara mita echiketto [Etiquette from a Viewpoint of Theory of Communication]." Gengo [Language] no. 29 (4):22-29. 
2001. "Hairyo to kōritsu: Poraitonesu riron to Guraisu no setten [Consideration and efficiency: A point of tangency of theories of politeness and Grice's cooperative principle]." Gengo [Language] no. 3 (12):44-51.

Hashiuchi, Takeshi. 1988. "Kaiwa no shikumi o saguru [Exploring the scheme of conversation]." Nihongogaku [Japanese Language] no. 7 (March):43-55. 1999. Disukōsu: danwa no orinasu sekai [Discourse: The world of conversations]. Tokyo: Kuroshio Shuppan.

Hata, Hiromi. 1988. "Gaikokujin no tame no nihongo kaiwa sutoratejī to sono kyōiku [Japanese conversational strategies for foreigners and education about them]." Nihongogaku [Japanese Language] no. 7 (3):100-117.

Haugh, Michael. 2003. "Japanese and non-Japanese perceptions of Japanese." New Zealand Journal of Asian Studies no. 5 (1):156-177.

_. 2008. "Utterance-Final Conjunctive Particles and Implicature in Japanese Conversation." Pragmatics no. 18 (3):425-451.

Hayashi, Oki, and Nihongo Kyōiku Gakkai. 1996. Nihongo kyōiku handobukku [A handbook of Japanese language education]. Tokyo: Taishukan Shoten.

Hayashi, Takuo. 2008. Danwa bunseki no apurōchi: Riron to jissen [Approach of discourse analysis: Theory and practice]. Tokyo: Kenkyusha.

Hernández, Todd. 2008. "The Effect of Explicit Instruction and Input Flood on Students' Use of Spanish Discourse Markers on a Simulated Oral Proficiency Interview." Hispania no. 91 (3):665-675.

Hidasi, Judit. 2007. "Meaning-gaps as a Means of Communication Strategy in Japanese." In Japanese linguistics: European Chapter, edited by Yoshihiko Ikegami, Viktoria Eschbach-Szabo and Andrâe Wlodarczyk, 79-88. Tokyo: Kurosio Shuppan.

Hill, Beverly, Sachiko Ide, Shoko Ikuta, Akiko Kawasaki, and Tsunao Ogino. 1986. "Universals of Linguistic Politeness: Quantitative Evidence from Japanese and American English." Journal of Pragamatics no. 10:347-371.

Hinata, Shigeo, and Junko Hibiya. 1988. Danwa no kōzō [Discourse structure]. Tokyo: Aratake Shuppan.

Hofstede, Geert H., Gert Jan Hofstede, and Michael Minkov. 2010. Cultures and organizations: software of the mind: intercultural cooperation and its importance for survival. 3rd ed. New York: McGraw-Hill.

Holmes, Janet. 1989. "Sex differences and apologies." Applied Linguistics no. 10 (2):194-212. . 1990. "Apologies in New Zealand English." Language in Society no. 19 (2):155-199.

Hori, Motoko. 2006. "Nihon shakai no ningen kankei to gengo e no hyōshutsu [Appearance of human relationship and language of Japanese society] " In Poraitonesu to engo kyōiku [Politeness and English language education], edited by Motoko Hori, Sanae Tsuda, Yoko Ohtsuka, Yasumi Murata, Yuka Shigemitsu, Mami Ohtani and Kazuyo Murata, 233-242. Tokyo: Hituzi Syobo.

Horiguchi, Sumiko. 1997. Nihogo kyōiku no kaiwa bunseki [Discourse analysis for Japanese language education]. Tokyo: Kuroshio Shuppan.

Hou, Kokuyaku. 2005. "Gendai nihongo no shazai hatsuwa kōi no ruikei to kinō [Similar patterns and functions of apologies of the modern Japanese]." Nihongogaku [Japanese Language] (April):78-90.

Hughes, Rebecca. 2010. "Materials to develop the speaking skill." In English language teaching materials: theory and practice, edited by Nigel Harwood, 207-224. New York: Cambridge University Press.

Ide, Risako. 1998. "'Sorry for your kindness': Japanese interactional ritual in public discourse." Journal of Pragmatics no. 29:509-529. 
Ide, Sachiko. 1989. "Formal forms and discernment: two neglected aspects of universals of linguistic politeness." Multilingua no. 8 (2/3):223-248. . 2001. "Kokusaika shakai no nakano keii hyōgen [Polite expressions in international society]." Nihongogaku [Japanese Language] no. 20 (4):4-13. .2005. "Ibunka to komyunikēshon gaku [The study of intercultural communication]." In Kōza shakai gengo kagaku: Ibunka to komyunikēshon [Lecture: sociolinguistics science; Cross-cultural communication], edited by Sachiko Ide and Masako Hiraga, 2-23. Tokyo: Hituzi Syobo.

- 2006. Wakimae no goyōron [Pragmatics of discernment]. Tokyo: Taishukan shoten.

Ide, Sachiko, Beverly Hill, Yukiko M Carnes, Tsunao Ogino, and Akiko Kawasaki. 2005. "The concept of politeness: An empirical study of American English and Japanese." In Politeness in language, edited by Richard J. Watts, Sachiko Ide and Konrad Ehlich, 281-297. Berlin, New York: Mouton de Gruyter.

Ideno, Rei. 2011. "Japanese Culture" in the Japanese language textbooks published abroad." Ryūkoku Daigaku Daigakuin Kokusai Bunka Kenkyū Ronshū [Departmental Bulletin Paper, Ryūkoku University Institutional Repository] no. 8:67-84.

Ijuin, Ikuko. 2004. "Bogo washa ni yoru bamen ni öjita supīchi sutairu no tsukai wake: Bogo bamen to sesshoku bamen no sōi [A comparison of speech style adaptation between native situations and contact situations: Similarities and differences between native speakers and non-native speakers]." Shakai Gengo Kagaku [The Japanese Journal of Language in Society] no. 6 (2):12-26.

Ikeda, Hiroshi. 1995. "Bunmatsu hyōgen no jūyōsei [Importance of sentence ending expressions]." Gekkan Gengo [Monthly Journal of Languages] no. 12:128-129.

Ikeda, Rieko. 1993. "Shazai no taishō kenkyū: Nichibei taishō kenkyū. Feisu to iu shiten kara no kōsatsu [The comparative study of Japanese and American: From the veiwpoint of face]." Nihongogaku [Japanese Language] no. 12 (November):13-21.

Ikuta, Shoko. 1997. "Poraitonesu no riron [Theory of politeness]." Gengo [Language] no. 26 (6):66-71.

Iori, Isao. 2011. "Nihongo kyōiku kara mita yari morai hyōgen [Expressions of Giving and Receiving from a Viewpoint of the Japanese Language Education]." Nihongogaku [Japanese Language] no. 30 (11):50-58.

Ishida, Toshiko. 1998. Nihongo kyōjuhō [Methodology of Japanese language teaching]. Revised ed. Tokyo: Taishukan Shoten.

Itoi, Ritsu, Kenichi Ohbuchi, and Mitsuteru Fukuno. 1996. "A Cross-Cultural Study of Preference of Accounts: Relationship Closeness, Harm Severity, and Motives of Account Making." Journal of Applied Social Psychology no. 26 (10):913-934.

Izaki, Yasuko. 2000. "Irai kaiwa ni mirareru yūsen taikei no bunkateki sōi to kitai no zure: Nihonjin to furansujin nihongo gakushūsha no sesshoku bamen no kenkyū [Cultural differences of preference and deviations from expectations in requesting: A study of Japanese and French learners of Japanese in contact situations]." Nihongo kyōiku [Journal of Japanese Language Teaching] no. 104:79-88.

Jary, Mark. 1998. "Relevance theory and the communication of politeness." Journal of Pragamatics no. 30 (1):1-19.

Jinnouchi, Masataka. 2006. "Bokashi hyōgen no nimensei [Dichotomy of ambiguous expressions]." In Gengo kōdō ni okeru hairyo no shosō [Consideration in linguistic behaviours], 115-131. Tokyo: Kokuritsu Kokugo Kenkyūjo [The National Institute for Japanese Language and Linguistics]. 
Jones, Kimberly. 1993. "Nihonjin no konfurikuto ji no hanashiai: Amerikajin kenkyuusha kara mita baai [Japanese Conversational Style when in Conflict: From the Viewpoint of an American Researcher]." Nihongogaku [Japanese Language] no. 12 (April):6874.

Jones, Kimberly, and Tsuyoshi Ono. 2005. "Discourse-centered approaches to Japanese language pedagogy." Japanese Language and Literature no. 39 (2):237-254.

Jucker, Andreas H. 1988. "The relevance of politeness." Multilingua no. 7 (4):375-384.

Kabaya, Hiroshi. 2011. "Taigū komyunikēshon kyōiku kara mita nihongo nōryoku no ikusei [Japanese proficiency based on the teaching and learning of interactive communication]." Waseda Daigaku Nihongo Kenkyū Kyōiku Sentā Kiyō [Bulletin of the Center for Japanese Language, Graduate school of Japanese Applied Linguistics, Waseda University] no. 9:9-14.

Kabaya, Hiroshi, Yoshikazu Kawaguchi, and Megumi Sakamoto. 1993. "Irai hyōgen hōryaku no bunseki to kijutsu [Analysis and description of expressional strategies of request]." Waseda Daigaku Nihongo Kenkyū Kyōiku Sentā Kiyō [Bulletin of the Center for Japanese Language, Waseda University] no. 5:53-69. 1998. Keigo hyōgen [Honorific expressions]. Tokyo: Taishukan Shoten.

Kaigai Gijutsusha Kenshū Kyōkai [The Association for Overseas Technical Scholarship]. 2000. Shin nihongo no chūkyū, Honsatsu [New Japanese for intermediate level, Main text]. Tokyo: Surīei Nettowāku.

—. 2003. Shin nihongo no chūkyū, Bunsatsu eigo yaku [New Japanese for intermediate level, Separate volume, English version]. ed. Tokyo: Surīei Nettowāku.

Kamio, Akio. 2002. Zoku jōhō no nawabari riron [Theory of territory of information: Volume 2]. Tokyo: Taishukan Shoten.

Kanasugi, Takao. 2008. "Gengo sutoratejī no yakuwari [The role of linguistic strategies]." Taisei Gakuin Daigaku Kiyō [Bulletin of Taisei Gakuin University] no. 10:49-62.

Kano, Michihito. 2002. "Nihongo kyōkasho to kyōjuhō e no eikyō - Chūgoku kōkō nihongo kyōkasho sakusei o tōshite [The influence of JSOL textbooks on teaching methods, with reference to Japanese language textbooks used at high schools in China: The Influence on methods of instruction]." Bungakubu Kiyō, Bunkyō Daigaku [Bulletin of the Faculty of Language and Literature, Bunkyō University] no. 15 (2):40-52.

Kashiwazaki, Hideko. 1993. "Hanashikake kōdō no danwa bunseki: Irai/yōkyū hyōgen no jissai o chūshin ni [Discourse Analysis of Requests with Phatic Communication]." Nihongo kyooiku [Journal of Japanese Language Teaching] no. 79 (March):53-63. . 2010. "Bunshō no rikai/sanshutsu no ninchi katei o fumaeta kyōiku e: Dentatsu mokuteki de no dokkai to sakubun no jikken to tomo ni [Education based on the cognitive process of understanding and producing texts: In conjunction with an experiment in reading and composition for communication]." Nihongo kyoiku [Journal of Japanese Language Teaching] no. 146:34-48.

—. 2011. "Gengoryoku ikusei o sasaeru bunshō rikai, sanshutu no kenkyū [Study of discourse understanding and producing toward linguistic competencies]." Jissen Joshi Daigaku, Bungakubu Kiyō [The faculty of Letters, Jissen Women's College Annual Reports of Studies] no. 53 (69-80).

Kataoka, Kuniyoshi, and Sachiko Ide. 2002. Bunka intā-akushon gengo [Culture, interaction, and language]. Tokyo: Hituzi Syobo.

Katayama, Kiyomi, Fumiko Uemura, Masako Matsui, and Eriko Yanagida. 2008. "Komyunikatibu na shokyū nihongo kyōzai no kaihatsu o mezashite [Creating communicative textbooks for beginners of Japanese]." Kumamoto Daigaku Ryūgakusei Sentā Kiyō [Bulletin of the International Students Center, Kumamoto University] no. 12:39-64. 
Kato, Shigehiro. 2004. Nihongo goyōron no shikumi [Mechanism of Japanese pragmatics]. Edited by Ken Machida, Shirīzu, Nihongo no shikumi o saguru 6 [Series, Exploring the structure of Japanese language 6]. Tokyo: Kenkyusha.

Kawaguchi, Sachiko. 2005. "Nihongo kyōkasho ni okeru kamoshirenai - Bunmyaku to kinō ni yoru kyōzai bunseki [Kamoshirenai in Textbooks of Japanese as a Second Language: Textbook Analysis through Context and Function]." Seigakuin daigaku ronsoo [Bulletin, Seigakuin University] no. 17 (3):37-48.

Kawaguchi, Yoshikazu. 2005. "Nihongo kyōkasho ni okeru kaiwa to wa nanika: Aru honbun kaiwa hihan [What does discourse mean in a Japanese textbook?: Critique] "Waseda Daigaku Nihongo Kyōiku Kenkyü Kiyō [Bulletin of the Graduate School of Japanese Applied Linguistics, Waseda University] no. 6:1-13.

Kawakami, Kyoko. 1993. "Hanashiai no gutaiteki bunseki - Kyōkan to hanpatsu [Detailed analysis of discourse: Sympathy and disagreement]." Nihongogaku [Japanese Language] no. 12 (April):43-57.

Kawarazaki, Mikio. 1997. "Nihongo kyōkasho [Japanese language textbooks]." Nihongo kyōiku [Journal of Japanese Language Teaching] no. 94:115-122.

Kawase, Ikuo. 2007. "Nihongo kyōzai kaihatsu: Kyōkasho sakusei ni kansuru kadai [Development of teaching materials: Issues in creating textbooks]." Nihongo kyōiku [Journal of Japanese language teaching] no. 135:23-28.

Kenbo, Hidetoshi, et al. 1994. Sanseido kokugo jiten [Sanseidoo Japanese Language Dictionary]. Dai 4-han. ed. Tokyo: Sanseido.

Kim, Changnam. 2000. "Nihongo bogo washa ni okeru irai hyōgen no shiyō jittai ni tsuite [Japanese Native Speakers' Real Use of Expressions of Request]." Journal of Chiba University Eurasian Society no. 3:30-43.

Kimura, Hideki. 1987. "Irai hyōgen no nicchū tasihō kenkyū [A Comparative Study of Expressions of Request between Japanese and Chinese]." Nihongogaku [Japanese Language] no. 6 (10):58-66.

Kindaichi, Hideho. 1987. "Orei to owabi no kotoba [Words of appreciation and apology]." Gengo [Language] no. 16 (4):75-82.

Kindaichi, Haruhiko, Oki Hayashi, and Takeshi Shibata. 1989. Nihongo hyakka daijiten [Encyclopaedia of the Japanese language]. Tokyo: Taishukan Shoten.

Kitao, Kenji, and Kathleen S. Kitao. 1988. "Poraitonesu: Ningen kankei o iji suru komyunikēshon shudan [Politeness: Communicative measures in maintaining relationships]." Nihongogaku [Japanese language] no. 7 (3):52-63.

Kobayashi, Hitomi. 2008. Nihongo kaiwa torēningu [Conversational training in Japanese]. Tokyo: Asuku Shuppan.

Koike, Mari. 2000. "Nihongo bogo washa ga shitsurei to kanjiru no wa gakushūsha no donna hatsuwa ka: Irai no bamen ni okeru bogo washa no hatsuwa to hikaku shite [What utterances do native speakers of Japanese feel impolite?: A comparative study of Japanese native speakers with learners of Japanese in a setting of request]." Hokkaido daigaku ryūgakusei sentā kiyō [Journal of international student centre, Hokkaido university] no. 4 (58-80).

Kokuritsu Kokugo Kenkyūjo [The National Institute for Japanese Language and Linguistics]. 1981. Nihongo no bunpō (ge) [Japanese Grammar Vol. 2]. Tokyo: Kokuritsu Kokugo Kenkyūjo [The National Institute for Japanese Language and Linguistics]. 1989. Danwa no kenkyū to kyōiku II [Study of discourses and pedagogy II]. Tokyo: Kokuritsu Kokugo Kenkyūjo [The National Institute for Japanese Language and Linguistics]. 
Kokusai Gakuyūkai Nihongo Gakkō [Japan Student Services Organization, Japanese Language School]. 1994. Ryūgakusei no nihongo kaiwa [Japanese language conversation for international students]. 3 ed. Tokyo: Kokusai Gakuyūkai [Japan Student Services Organization].

Kokusai Kōryū Kikin [The Japan Foundation]. 2013. Kaigai no nihongo kyōiku no genjō [Survey report on Japanese]. Tokyo: Kuroshio shuppan.

Kuma, Yumiko, Tomoko Suzuki, Junko Nakayama, and Akiko Yuhara. 2003. Shin jitsuyō nihongo I [New practical Japanese I]. Tokyo: Ezoe Gakuen Shinjuku Nihongo Gakkō.

Kumagai, Tomoko. 1993. "Kenkyū taishō to shite no shazai: Ikutsuka no kirikuchi ni tsuite [Apologies as a Research Objective: About Some Aspects]." Nihongogaku [Japanese Language] no. 12 (November):4-12. 1995. "Irai no shikata: Kokugo kenkyūjo Okazaki chōsa no dēta kara [Ways of requesting: Based on data of the Okazaki City study by the National Institute for Japanese Language and Linguistics]." Nihongogaku [Japanese Language] no. 14 (October):22-32.

2008. "Irai to shazai ni okeru hatarakikake no sutairu [Interactional styles in settings of request and apology]." Gengo [Language] no. 37 (1):26-33.

Kumagai, Tomoko, and Koichi Shinozaki. 2006. "Irai bamen de no hatarakikakekata ni okeru sedaisa, chiikisa [Generational and Regional Differences in Approach for Requesting]." In Gengo kōdō ni okeru hairyo no shosō [Consideration in Linguistic Behaviours], 19-54. Tokyo: Kokuritsu Kokugo Kenkyūjo [The National Institute for Japanese Language and Linguistics].

Kumai, Hiroko. 1992. "Ryūgakusei ni mirareru danwa kōdō jō no mondaiten to sono haikei [Problems in foreign students' discourses and the background to them]." Nihongogaku [Japanese Language] no. 11 (December):72-80.

Kumar, Krishna. 1986. "Textbooks and Educational Culture." Economic and Political Weekly no. 21 (July):1309-1311.

Kumatoridani, Tetsuo. 1988. "Hatsuwa kōi riron to danwa kōdō kara mita nihongo no wabi to kansha [Apology and appreciation from view poins of the theory of speech acts and discourses]." Hiroshima daigaku kyooiku gakubu kiyoo [Bulletin of the Department of Education, Hiroshima University] no. 2 (37):223-234.

. 1993. "Hatsuwa kōi taishō kenkyū no tame no sōgōteki apurōchi: Nichi ei go no wabi o rei ni [An integrative approach to contrastive speech-act analysis: A Case of apologies in Japanese and English] " Nihongo kyōiku [Journal of Japanese Language Teaching] no. 79:26-40.

1995. "Hatsuwa kōi riron kara mita irai hyōgen [Expressions of request from the viewpoint of speech acts theory] " Nihongogaku [Japanese Language] no. 14 (October):12-21.

Kwarciak, B.J. 1993. "The acquisition of linguistic politeness and Brown and Levinson's theory." Multilingua no. 12 (1):51-68.

Kyi, Tida. 2004. "Irai shinikui baai no irai hyoogen [Expressions of Request When It Is Difficult to Make a Request]." Waseda Daigaku Nihongo Kenkyuu Kyooiku Sentaa Kiyoo [Bulletin of Center for the Japanese Language, Waseda University] no. 17:7193.

Laohaburanakit, Kanokwan. 1995. "Nihongo ni okeru kotowari: Nihongo kyōkasho to jissai no kaiwa to no hikaku [Refusal in Japanese: A comparison of Japanese textbooks and authentic conversational data]." Nihongo kyöiku [Journal of Japanese Language Teaching] no. 87 (November):25-40.

Lebra, Takie Sugiyama. 1976. Japanese patterns of behavior. Honolulu: University Press of Hawaii. 
Leech, Geoffrey N. 1983. Principles of Pragmatics, Longman linguistics library. 30. London, New York: Longman.

Leng, Limin. 2004. "Chūgoku chūtō kyōiku ni okeru nihongo kyōkasho no bunseki: Renshū o tōshite mita kyōkasho no henka [Analysis of Japanese language textbooks in Chinese secondary education: Changes in textbooks from the viewpoint of drills]." Gengo Bunka to Nihongo Kyōiku [Language, Culture, and Japanese Language Education] no. 28:95-98.

Lester, Julie H., and Earl H. Cheek Jr. 1997/1998. "The "Real" Experts Address Textbook Issues." Journal of Adolescent \& Adult Literacy no. 41 (4):282-291.

Li, Ye. 2005. "Danwateki kanten kara mita irai hyoogen [Expressions of Request from the Viewpoint of Discourse]." Shinshu Daigaku Nihongo Kyooiku Kenkyuu [Bulletin, Japanese Language Education, Shinshu University] no. 5:2-17.

Liddicoat, Anthony J. 2000. "Everyday speech as culture: Implications for language teaching." In teaching languages, teaching cultures, edited by Anthony J. Liddicoat and Chantal Crozet, 51-64. Melbourne: Language Australia.

2008. "Pedagogical Practice for Integrating the Intercultural in Language Teaching and Learning." Japanese Studies no. 28 (3):277-290.

Lin, Lin. 2010. "Nichijō kaiwa ni mirareru aru/Iru kōbun keishiki [A study of aru and Iru employed in conversational style]." Research Review, Kaetsu University no. 53 (1):27-45.

Locher, Miriam A, and Richard J. Watts. 2005. "Politeness theory and relational work." Journal of Politeness Research no. 1:9-33.

Long, Christopher. 2010. "Apology in Japanese gratitude situations: The negotiation of interlocutor role-relations." Journal of Pragamatics no. 42:1060-1075.

Makino, Seiichi. 1996. Uchi to soto no gengo bunkagaku [Sociocultural and sociolinguistics studies on uchi 'inner circle' and soto 'outer circle']. Tokyo: Aruku.

Mao, Lu Ming Robert. 1994. "Beyond politeness theory: 'Face' revisited and renewed." Journal of Pragmatics no. 21:451-486.

Matsuda, Yuichi, Yonghee Kim, Jueun Lee, and Eunnan Park. 2008. "Kankokujin nihongo gakushūsha no irai hyōgen: Irai kōi o hanashite to kikite ga tomoni okonau baai [Expressions of requests in Korean learners of Japanese: When both speaker and hearer do the requestive act]." Ibaraki daigaku ryūgakusei sentā kiyō [Research bulletin of internaqtional student centre, Ibaraki university] no. 6:47-60.

Matsui, Tomoko. 2001. "Kanrensei riron kara mita poraitonesu - Ito dentatsu sei no mondai ni tsuite [Politeness from the viewpoint of Relevance Theory: Issues concerning conveyance of intention]." Gengo [Language] no. 30 (12):52-59.

Matsumoto, Shusuke, Masako Yonezawa, and Sayaka Irie. 2005. "Chūkyū nihongo kyōzai ni kansuru ichi shian [An attempt on intermediate level teaching materials]" Dōshisha Daigaku Ryūgakusei Bekka Kiyō [Bulletin, the Center for Japanese Language, Dōshisha University] no. 5:73-90.

Matsumoto, Yoshiko. 1985. "A Sort of Speech Act Qualification in Japanese: Chotto [a little bit]." Journal of Asian Culture no. IX (143-159). . 1989. "Politeness and conversational universals." Multilingua no. 8 (2/3):207-222. - 2001. "Tyotto: Speech Act Qualification in Japanese Revisited." Japanese Language and Literature no. 35 (1):1-16.

Matsumoto, Shusuke, Masako Yonezawa, and Sayaka Irie. 2005. "Chūkyū nihongo kyōzai ni kansuru ichi shian [An attempt on intermediate level teaching materials]" Dōshisha Daigaku Ryūgakusei Bekka Kiyō [Bulletin, the Center for Japanese Language, Dōshisha University] no. 5:73-90. 
Matsushima, Midori. 2003. "Chūgoku no bijinesu nihongo kyōzai ni okeru taigū hyōgen no araware kata [Polite expressions in Japanese textbooks for business purposes in China] " Bekka Nihongo Kyōiku, Daito Bunka Daigaku, Bekka Ronshū [Bulletin of the Special Course for Japanese Language Education, Daito Bunka University] no. 5:55-66.

Maynard, Senko K. 1997. Danwa bunseki no kanōsei: Riron, hōhō, nihongo no hyōgensei [Discourse analysis: Theory, methodology, and Japanese expressions]. Tokyo: Kuroshio Shuppan.

—. 2004. Danwa gengogaku: Nihongo no disukōsu o sōzō suru kōsei retorikku sutoratejū no kenkyū [Discourse Linguistics: A study of personality, rhetoric, and strategy in creating Japanese language discourse]. Tokyo: Kuroshio Shuppan.

McCarthy, Michael. 1991. Discourse analysis for language teachers, Cambridge language teaching library. Cambridge: Cambridge University Press.

Mihara, Yoshiko. 1995. "Setsuzoku joshi keredomo no sūjoshiteki yōhō ni kansuru ichi kōsatsu [A study of the sentence which ends with keredomo]." Yokohama Kokuritsu Daigaku Ryūgakusei Sentā Kiyō [Bulletin of the International Student Centre, Yokohama National University] no. 2:79-89.

Minami, Fujio, and Nozomi Tanaka. 1983. "Hajimeni: Danwa no kenkyū to kyōiku no hitsuyōsei [Introduction: The necessity for study of discourse and education]." In Danwa no kenkyuu to kyooiku I [Study of Discourse and Education I], edited by Kikuo Nomoto, 1-6. Tokyo: Ōkurashō Insatsukyoku [Ministry of Finance Press].

Mine, Fuyuki. 1995. "Nihongo gakushūsha no kaiwa ni okeru bunmatsu hyōgen no shutoku katei ni kansuru kenkyū [Study of Japanese language acquisition: How learners acquire sentence endings]." Nihongo kyoiku [Journal of Japanese Language Teaching] no. 86:65-80.

Miura, Akira, and Naomi Hanaoka McGloin. 2008. An Integrated Approach to Intermediate Japanese. Tokyo: The Japan Times.

Miyachi, Asako, and Haisei Sai. 2009. "Neitibu to non-neitibu no gassaku ni yoru nihongo kyōkasho [A Japanese language textbook produced by collaboration between Japanese native speakers and non-native speakers]." Nihongogaku [Japanese language] no. 28 (11):48-63.

Miyahira, Katsuyuki. 1998. "Facework in intercultural directive discourse: From crosscultural comparisons to intercultural coordination (II)." Gengo Bunka Kenkyū Kiyō, Ryukyu Daigaku [Bulletin of the Languages and Cultural Researches, University of the Ryukyus] no. 7:21-45.

Miyaji, Yutaka. 1995. "Irai hyōgen no ichi [A study of expressions of request]." Nihongogaku [Japanese Language] no. 14 (October):4-11.

Miyake, Kazuko. 2011. Nihongo no taijin kankei haaku to hairyo gengo kōdō [Understanding of relationship with others and consideration in language action in Japanese]. Tokyo: Hituzi Syobo.

Miyake, Tomohiro. 1993. "Haseiteki imi ni tsuite: Nihongo shitumonbun no ichi sokumen [On Delivative Meaning: Some Observations on Japanese Interrogative Sentences]." Nihongo kyooiku [Journal of Japanese Language Teaching] no. 79:64-75.

Miyazaki, Satoshi. 2005. "Nihongo kyōkasho no kaiwa disukōsu to meijiteki anjiteki na chōsei kōdō: Kyōkasho danwa kara manaberu koto manabenai koto [Discourses in Japanese textbooks and explicit and implicit speech-acts: What can and cannot be learned from discourses presented in Japanese textbooks] " Waseda Daigaku Nihongo Kyōiku Kenkyū Kiyō [Bulletin of the Graduate School of Japanese Applied Linguistics, Waseda University no. 7:1-25. 
Mizutani, Nobuko. 1988. "Hanashikotoba no hikaku taishō [Comparison and contrast of spoken language]." In Hanashikotoba no komyunikēshon [Spoken language in communication], 85-108. Tokyo: Bonjinsha.

1991. "Taigū hyōgen shidō no hōhō [Teaching Politeness in Japanese]." Nihongo kyōiku [Journal of Japanese Language Teaching] no. 69:24-35.

1993. "Kyōwa kara taiwa e [From cooperative language to discourse]." Nihongogaku [Japanese Language] no. 12 (April):4-20.

—. 2001. Zoku nichi-ei hikaku hanashi kotoba no bunpō [Grammar of spoken language: Comparative study of Japanese and English]. Tokyo: Kuroshio Shuppan.

Mizutani, Osamu. 1986. "Kyōkasho ni arawareta gengo kōdō [Language behaviour in Japanese textbooks]." Nihongo kyōiku [Journal of Japanese Language Teaching] no. 59:62-75.

Mizutani, Osamu, and Nobuko Mizutani. 1983. Nihongo Notes 5: Studying Japanese in Context. Tokyo: The Japan Times.

1984. Nihongo notes 6: Situational Japanese 1. Tokyo: The Japan Times. 1987. How to be polite in Japanese. Tokyo: The Japan Times.

Mori, Junko. 2005. "Why not why? The teaching of grammar, discourse, sociolinguistic and cross-cultural perspectives." Japanese Language and Literature no. 39 (2):255-289.

Morita, Yoshiyuki. 1996. Imi bunseki no hōhō: Riron to jissen [Method of semantic formulas: Theory and practice]. Tokyo: Hituzi Syobo.

Moro, Yuji. 1999. "Hirakareta disukōsu gainen no tame ni [For the concept of wider discourse]." Gengo [Language] no. 28 (1):20-28.

Nagara, Susumu et al. 1990. Japanese for everyone. Tokyo: Gakken Co. Ltd.

Nagasaka, Miaki, and Mari Kida. 2011. "Chūgoku no daigaku no nihongo jugyō ni okeru kaiwa shidō ni kansuru chōsa: Chū, Jō kyū reberu o taishō to shita kyōshitsu katsudō no jittai to kyōshi no ishiki [Research on the teaching of Japanese conversation classes at Chinese universities: Class activities at the intermediate and advanced Levels and teachers' perceptions]." Kokusai Kōryū Kikin, Nihongo Kōiku Kiyō [Bulletin of Japanese Language Education, The Japan Foundation] no. 7:43-57.

Nakada, Tomoko. 1989. "Hatsuwa kōi to shite no chinsha to kansha [Apology and thanks in Japanese and English]." Nihongo kyōiku [Journal of Japanese Language Teaching] no. 68:191-203.

Nakamichi, Makio, and Mami Doi. 1993. "Nihongo kyōiku ni okeru shazai no atsukai [Dealing with apology in Japanese language education]." Nihongogaku [Japanese Language] no. 12 (November):66-74.

1995. "Nihongo kyōiku ni okeru irai no atsukai [Ways of dealing with requests in Japanese Language Education]." Nihongogaku [Japanese Language] no. 14 (October):84-93.

Nakamura, Shin, and Kaori Akui. 2004. "Irai hyōgen no kansetsusei ni kansuru kenkyū: Hiiraisha no shiten kara [Study of indirect expressions of requests: From the viewpoint of the requestee]." Kawamura Gakuen Joshi Daigaku Kenkyü Kiyō [Bulletin, Kawamura Women's College] no. 15 (1):99-115.

Nihongo kyōzai risuto sakusei iinkai [Committee of creating a list of Japanese language teaching materials]. 2011. Nihogo kyōai risuto No. 41 [Guide to Japanese Language Teaching Materials No. 41]. Tokyo: Bonjinsha.

Nishimura, Fumiko. 2011. Lying: Strategies to Manage Undesirable Communicative Situations in Japan and New Zealand. Doctor of Philosophy, Management Communication, The University of Waikato. 
Noda, Hisashi. 2009. "Kokusaika jidai ni okeru nihongo komyunikēshon nōryoku no ikusei: Nihongo kyōiku no shiten kara [Raising Japanese Communicative Competence in an Era of Internationalisation: From the Viewpoint of Japanese Language Education]." Kochi Daigaku Sōgō Kyōiku Sentā Shūgaku Ryūgakusei Shien Bumon Kiyō [Bulletin, Support Center of Domestic and International Students, Kochi University] no. 3:2943.

Nunan, David. 1988. The learner-centred curriculum: a study in second language teaching. Cambridge [England]; New York: Cambridge University Press.

1991. Language teaching methodology: A textbook for teachers. Hemel Hempstead, Hertfordshire: Prentice Hall International (UK) Ltd. 1999. Second language teaching \& learning. Boston, Mass.: Heinle \& Heinle Publishers.

Ogawa, Haruko. 1995. "Kansha to wabi no teishiki hyōgen [Formulaic expressions of appreciation and apology]." Nihongo kyōiku [Journal of Japanese Language Teaching] no. 85:38-52.

Ogiwara, Chikako. 2000. "Nihonjin no iisashi hyōgen ni taisuru sasshi no arawarekata [Perceptiveness of the Japanese about unfinished sentences]." Waseda Daigaku Nihongo Kenkyū Kyōiku Sentā [Bulletin of the Center for Japanese Language, Waseda University] no. 36:69-85. 2008. Iisashi hatsuwa no kaishaku riron [Theory of interpretation of utterances of unfinished sentences]. Yokohama: Shunpūsha.

Ohbuchi, Ken'ichi, Sachiko Chiba, and Osamu Fukushima. 1996. "Mitigation of interpersonal conflicts: Politeness and time pressure." Personality and Social Psychology Bulletin no. 22 (10):1035-1042.

Ohbuchi, Ken'ichi, Masuyo Kamada, and Nariyuki Agarie. 1989. "Apology as aggression control: Its role in mediating appraisal of and response to harm." Journal of Personality and Social Psychology no. 56:219-27.

Ohso, Mieko, and Yoko Koyama. 1988. Japanese for You. Tokyo: Taishukan Shoten.

Ohta, Amy Snyder. 2001. "A longitudinal study of the development of expression of alignment in Japanese as a foreign language." In Pragmatics in Language Teaching, edited by Kenneth R. Rose and Gabriele Kasper, 103-120. Cambridge, UK: Cambridge University Press.

Ohtani, Mami. 1999. "Shazai ni taisuru hentō no sutoratejī: Feisu o tegakari ni [Strategies of response to apologies: Based on face]." Gengo Bunka to Nihongo Kyōiku, Ochanomizu Joshi Daigaku [Language, Culture and Japanese Language Education, Departmental Bulletin of Ochanomizu Women's University] no. 18:51-54. 2000. "Shazai teikei hyōgen no nichi-eigo tiashō kenkyū [A comparative study of apologetic expressions]." Gengo Bunka to Nihongo Kyöiku, Ochanomizu Joshi Daigaku [Language, Culture and Japanese Language Education, Departmental Bulletin of Ochanomizu Women's University] no. 20:90-97.

Okabe, Etsuko. 2004. "Chū jōkyū nihongo kyōkasho ni okeru kōtō hyōgen katsudō: Sōgō kyōkasho o chūshin to shite [Speech-acts in Japanese textbooks for intermediate and advanced levels: The case of textbooks for general purposes] "Nagasaki Gaikokugo Daigaku Ronsō [Journal of the International Student Centre, Nagasaki University of Foreign Studies] no. 8 (37-48).

Okamoto, Shigeko. 1999. "Situated politeness: Manipulating honorific and non-honorific expressions in Japanese conversations." Pragmatics no. 9 (1):51-74.

Okamoto, Shin'ichiro. 1988. "Irai hyōn no tsukaiwake no kiteiin [Ruling factors of using requisitive expressions]." Aichi Gakuin Daigaku, Bungakubu Kiyō [Bulletin, Department of Literature, Aichi Gakuin University] no. 18:7-14. 
Okamoto, Shin'ichiro. 2002. "Politeness and the perceptions of irony: Honorifics in Japanese." Metaphor and Symbol no. 17 (2):119-139.

Okano, Kimiko. 1990. "Kokusaibu yō nihongo kyōkasho no hensan no kihon hōshin [Principles of creating Japanese language textbooks for the international department]." Kōza nihongo kenkyū, Waseda Daigaku Nihongo Kyōiku Kenkyū Sentā [Bulletin of the Centre for Japanese Language, Waseda University] no. 25:18-27.

Okazaki, Toshio. 1987. "Danwa no shidō [Instruction on discourses]." Nihongo kyōiku [Journal of Japanese Language Teaching] no. 62:165-178.

—. 1989. Nihongo kyōiku no kyōza: Bunseki, shiyō, sakusei [Teaching materials for Japanese language education: Analysis, usage, and creation]. Tokyo: Aruku.

Oki, Hiroko. 2006. Nihongo danwaron [Study of Japanese language discourse]. Osaka: Izumi Shoin.

Olshtain, Elite. 1989. "Apologies across Languages." In Cross-Cultural Pragmatics: Requests and Apologies, edited by Shoshana Blum-Kulka, Juliane House and Gabriele Kasper, 155-172. Norwood, NJ: Ablex Publishing Corporation.

Porter, Richard E., and Larry A. Samovar. 1997. "An Introduction to Intercultural Communication." In Intercultural communication: a reader, edited by Larry A. Samovar and Richard E. Porter, 5-26. Belmont: Wadsworth Pub. Co.

Pustjens, Heidi, Eva Van de gaer, Jan Van Damme, Patric Onghena, and Georges Van Landeghem. 2007. "The Short-Term and the Long-Term Effect of Primary Schools and Classes on Mathematics and Language Achievement Scores." British Educational Research Journal no. 33 (3):419-440.

Rai, Mirei. 2005. "Irai ni okeru 'owabi shazai gata' hyōgen ni kansuru kōsatsu: Nihongo bogo washa to taiwanjin nihongo gakushūsha o taishō ni [Study of apologetic-style expressions in requests: Comparing Japanese native speakers and Taiwanese learners of Japanese language]." Nihongo Kyōiku Kenkyū, Waseda Daigaku [Japanese Language Educational Study, Waseda University] no. 6:63-77.

Saito, Shuichi. 1986. "Kyōkasho ron [Theory of textbook]." Nihongo kyooiku [Journal of Japanese Language Teaching] no. 59:1-12.

Sakamoto, Megumi. 2001. "Keigo to keii hyōgen [Honorifics and honorific expressions]." Nihongogaku [Japanese Language] no. 20 (April):14-21.

Sakuma, Katsuhiko. 1983. "Kansha to wabi [Appreciation and apology]." In Hanashi kotoba no hyōgen [Expressions of Spoken Language], edited by Osamu Mizutani. Tokyo: Chikuma Shobo.

Samejima, Shigeki. 1998. "Komyunikēshon tasuku ni okeru nihongo gakusūsha no teikei hyōgen/bunmatsu hyōgen no shūtoku katei [Learning processes of standard forms and sentence final expressions by Japanese language learners in communication tasks]." Nihongo kyōiku [Journal of Japanese Language Teaching] no. 98:73-84.

Samovar, Larry A., and Richard E. Porter. 1997. Intercultural communication: a reader. 8th ed, Wadsworth series in communication studies. Belmont: Wadsworth Pub. Co.

Sanada, Shinji, Katsumi Shibuya, Masataka Jinnouchi, and Seiju Sugito. 1992. Shakai gengogaku [Sociolinguistics]. Tokyo: Ōfü Shuppan.

Sao, Chitose. 2004. "Dōshisha daigaku ryūgakusei bekka jōkyū kurasu Sōgō kyōkasho kōsō [Design of the textbook General Japanese for advanced level at the Center for Japanese Language, Dōshisha University]." Dōshisha Daigaku Ryūgakusei Bekka Kiyō [Bulletin of the Centre for Japanese Language, Dōshisha University] no. 4:75101.

Sasaki, Michiko. 1995. "Irai hyōgen no taishō kenkyū [A comparative study of expressions of request: The case of English] " Nihongogaku [Japanese language] no. 14 (October):61-68. 
Sasaki, Mizue, and Masami Kadokura. 2007. Kaiwa no nihongo: Japanese through dialogues for intermediate learners [Japanese language for conversation]. Tokyo: The Japan Times.

Sato, Hiroki. 2011. "Gendai nihongo no shazai kotoba ni kansuru kenkyū [A study of apologetic words of the modern Japanese]." Iwate Daigaku Daigakuin Jinbun Shakai Kagaku Kenkyūka Kiyō [Bulletin of the Graduate School, Humanities and Social Science Department, Iwate University] no. 20 (June):21-38.

Sato, Keiko. 1997. "A Gender-Difference of Request Realization in Pragmatic Strategies: The Case of Japanese Speakers." Nagoya Joshi Daigaku Kiyō [Bulletin of Nagoya Women's University] no. 43:225-232.

Sato, Yutaka. 2000. "Chūkyū kyōkasho han katsudō hōkoku [Progress report by the development team of the ICU intermediate level Japanese textbook]." ICU Nihongo Kyōiku Kenkyū Sentā kiyō [Bulletin of ICU Japanese Language Research Center] no. 9:9-26.

Sawada, Tazuko. 2001. "Nihongo chūkyū gakushūsha no tame no nihon jijō shirabasu [The syllabus of Japan-related content for intermediate level Japanese language learners " Nara Kyōiku Daigaku Kiyō [Bulletin of Nara University of Education] no. 50 (1):147157.

Schlenker, Barry R., and Bruce W. Darby. 1981. "The Use of Apologies in Social Predicaments." Social Psychology Quarterly no. 44 (3):271-278.

Schmidt, Richard W., and Silvia Nagem Frota. 1986. "Developing Basic Conversational Ability in a Second Language: A Case Study of an Adult Learner of Portuguese." In Talking to learn: conversation in second language acquisition, edited by Richard $\mathrm{R}$. Day, 237-326. Rowley, MA: Newbury House.

Schmitt, Norbert. 2008. "Review article: Instructed second language vocabulary learning." Language teaching research no. 12 (3):329-363.

Scott, Virginia M. 1990. "Explicit and Implict Grammar Teaching Strategies: New Empirical Data." The French Review no. 63 (5):779-789.

Searle, John R. 1969. Speech acts: an essay in the philosophy of language. London: Cambridge U.P.

Sei, Rumi. 2003. "'Tsumaranai mono desu ga' kō-Jittai chōsa to nihongo kyōkasho to no hikaku kara [A consideration of the use of the phrase tsumaranai mono desuga '[this] is a trifling thing, but': Comparison of the contents of Japanese textbooks and the results of actual surveys]." Ibunka Komyunikēshon Kenkyū, Kanda Gaikokugo Daigaku [Bulletin of Cross-Cultural Studies, Kanda University of International Studies] no. 15:17-39.

Seki, Masaaki, Satoshi Toki, Fumiya Hirataka, Akito Ozaki, Yukiko Tsubaki, and Yoko Nakai. 2010. Kaiwa kyōzai o tsukuru [Creating Teching Materials of Conversation]. Tokyo: Surīei Nettowāku.

Shibuya, Katsumi. 2008. "Sutairu no tsukaiwake to komyunikēshon [Differentiation of usage of speech style and communication]." Gengo [Language] no. 37 (1):18-25.

Shigemitsu, Yuka. 2005. "Nani o kokochi yoi to kanjiru ka: Kaiwa no sutairu to ibunka kan komyunikēshon [What feels comfortable?: Speech style and cross-cultural communication]." In Kōza Shakai Gengo Kagaku: Ibunka to Komyunikēshon [Lecture: Sociolinguistics Science; Cross-Cultural Communication] edited by Sachiko Ide and Masako Hiraga, 218-237. Tokyo: Hituzi Syobo. 
Shimada, Noriko, and Tomoyo Shibahara. 2005. "Nihongo kyōzai sakusei no tame no mittsu no shiten: Kyōju sekkei ron no tekiyō, gakushū katei e no chūmoku, kyōshitu katsudō no bunseki shihyō [Three viewpoints of creating Japanese teaching materials: Applying the theory of teaching design, focusing on procedure of learning, and analytical indicators of classroom activities]." Kokusai Kōryū Kikin Nihongo Kyōiku Kiyō [Bulletin, Japanese Language Education, The Japan Foundation] no. 1:53-67.

Shimada, Noriko, Tomoyo Shibahara, and Kokusai Kōryū Kikin. 2008. Kyōzai kaihatsu [Creating teaching materials], Kokusai Kyōryoku Kikin Nihongo Kyōjuhō Shirīzu, 14 [The Japan Foundation, Series 14, Methodology of Teaching Japanese]. Tokyo: Hituzi Syobo.

Shimizu, Yukichi. 2009. "Irai hyōgen ni miru poraitonesu: Seisa no kakawari o chūshin ni [Politeness expressions appearing in requests: Focusing on gender differences]." Tokushima Daigaku Kokugo Kokubun Gakkai [Bulletin of the Society of the Japanese Language and Literature, Tokushima University] no. 22 (35-53).

Shinmura, Izuru. 1991. Kōjien 4ed. Tokyo: Iwanami Shoten.

Shinozaki, Setsuko, Reiko Iino, and Liyun Zeng. 2004. "Chūgoku ryūyō shō no shōgakusei yō nihongo kyōzai seisaku ni tuite: Kaigai de no nihongo kyōzai seisaku no arikata [The project of creating Japanese language teaching materials for elementary schools in Liaoning Province, China: The ideal way of creating Japanese language teaching materials overseas]." Kokusai kōryū kikin nihongo kyōiku kiyō [Bulletin, Japanese languge education, The Japane Foundation] no. 14:87-104.

Shirakawa, Hiroyuki. 1991. "Te-kei ni yoru iisashi no bun ni tsuite [About unfinished sentences in te-form]." Hiroshima daigaku Nihongo Kyōiku Gakka Kiyō [Bulletin of the Department of Teaching Japanese as a Second Language, Hiroshima University] no. 1:39-48.

1996. "Kedo de owaru bun [Sentences ending with kedo]." Hiroshima Daigaku Daigakuin Kyōikugaku Kenkyūka Kiyō, Dai 2 bu [Bulletin Part 2, Arts and Science Education, The Graduate School of Eduction, Hiroshima University] no. 6:9-17.

- 2009. Iisashibun no kenkyū [Study of unfinished sentences]. Tokyo: Kuroshio Shuppan.

Shōgakukan (ed.). 1988. Kokugo daijiten [Great dictionary of the Japanese language]. Tokyo: Shogakukan. . 1988. Kokugo daijiten [Great dictionary of the Japanese language]. Tokyo: Shogakukan.

Soanes, Catherine, and Angus Stevenson. 2008. Concise Oxford English dictionary. 11th ed. Oxford [England]; New York: Oxford University Press.

Spees, Hiroko. 1994. "A Cross-Cultural Study of Indirectness." Issues in Applied Linguistics no. 5 (2):231-252.

Sugimoto, Naomi. 1997. "A Japan-U.S. Comparison of Apology Styles." Communication Research no. 24:349-370. . 1998. "Norms of apology depicted in U.S. American and Japanese literature on manners and etiquette." International Journal of Intercultural Relations no. 22 (3):251-276.

. 2001. "Evaluation of Apology Episodes in Japan and the U.S." Ferris Studies no. 36:35-59.

Surīei nettowāku. 1998. Minna no Nihongo shokyū II Honsatsu [Japanese for Everyone, Beginners Level II, Main textbook]. Tokyo: Surīei Nettowāku. . 2008. Minna no Nihongo chūkyū I Honsatsu [Japanese for Everyone, Intermediate level I, Main textbook]. Tokyo: Surīei Nettowāku. 
Takagi, Miyoshi. 2011. "Kyōkasho ni nai nihongo nōryoku [Japanese language competency not presented in Japanese language textbooks]." Waseda Daigaku Nihongo Kyōiku Kenkyu Kiyō [Bulletin of the Graduate School of Japanese Applied Linguistics, Waseda University] no. 8,9: 51-57.

Takasaki, Midori, Kazumi Tachikawa, Rie Osawa, and Yuko Hoshino. 2008. Nichijō kaiwa no bunseki [Analysis of daily conversation]. Edited by Midori Takasaki and Kazumi Tachikawa, Koko kara hajimaru bunshō, danwa [The point where sentences and discourse start]. Tokyo: Hituzi Syobo.

Takiura, Masato. 2001. "Keii no hokorobi [Open seams of polite expression]." Gengo [Language] no. 30 (12):26-33. 2008. Poraitonesu nyūmon [An introduction to politeness]. Tokyo: Kenkyusha.

Takushoku Daigaku Ryūgakusei Bekka, Nihongo Kenshū Sentā [Takushoku University Japanese Language Center for Foreign Students, Japanese Language Learning Center], 2001. Hirake Nihongo Ge [Open Japanese, Vol. 2]. Tokyo: Takushoku Daigaku [Takushoku University].

Tamamura, Fumio, and Kaigai Gijutsusha Kenshū Kyōkai [The Association for Overseas Technical Scholarship]. 1993. Nihongo jitsuyō jiten [The AOTS Nihongo dictionary for practical use]: Surīei Nettowāku.

Tanaka, Harumi, and Sachiko Tanaka. 1996. Shakai gengogaku e no shōtai [An invitation to sociolinguistics]. Kyoto: Mineruba Shobo.

Tanaka, Michihiko, Ryusuke Yamaguchi, and Yumiko Nasu. 2007. "Ryūgakusei no nīzu to reberu ni awaseta nihongo kyōzai no kaihatsu (1) [Development of Japanese teaching materials according to the needs and competency levels of international students (1)]." Seisen Ronsō [Bulletin of Seisen University] no. 15:283-292.

. 2008. "Ryūgakusei no nīzu to reberu ni awaseta nihongo kyōzai no kaihatsu (2)

[Development of Japanese teaching materials according to the needs and competency levels of international students (2)]." Seisen ronsō [Bulletin of Seisen University] no. 16:1-11.

. 2009. "Ryūgakusei no nīzu to reberu ni awaseta nihongo kyōzai no kaihatsu (3)

[Development of Japanese teaching materials according to the needs and competency levels of international students (3)]." Seisen ronsō [Bulletin of Seisen University] no. 17:95-101.

Tanaka, Nozomi. 1988. Nihongo kyōiku no hōhō: Kōsu dezain no jissai [The methodology of Japanese language education: Designing a course programme]. Tokyo: Taishukan Shoten.

1996. "Gaikokugo kyōiku to shite no nihongo kyōiku [Japanese language education as a foreign language]." In Nihongo Kyōiku Handobukku [Handbook of Japanese Language Education], edited by Oki Hayashi and Nihongo Kyōiku Gakkai [Society of Japanese Language Education], 20-55. Tokyo: Taishukan Shoten.

. 1996. "Gaikokugo kyōiku to shite no nihongo kyōiku [Japanese language education as a foreign language]." In Nihongo Kyōiku Handobukku [Handbook of Japanese Language Education], edited by Oki Hayashi and Nihongo Kyōiku Gakkai [Society of Japanese Language Education], 20-55. Tokyo: Taishukan Shoten.

Terao, Yuko. 2000. "Nihongo kyōiku kyōzai ron [Materials for teaching Japanese as a foreign/second language]." Gakkō Kyōikugaku Kenkyū, Hyogo Kyōiku Daigaku [Bulletin of Study of Pedagogy, Hyogo University of Education] no. 12:1-6. . 2001. "Gurōbaru jidai no nihongo kyōiku to nihongo kyōkasho [Teaching Japanese as a second and/or foreign language and textbooks used for non-native learners in an age of globalisation] " Gengo kenkyū, Hyogo Kyōiku Daigaku [Bulletin of Language Studies, Hyogo University of Education] no. 17:155-166. 
Thomson Kinoshita, Chihiro, and Emi Otsuji. 2009. "Bijinesu nihongo kyōkasho to jendā no tamenteki kōsatsu [Multidimensional study of the textbooks of business Japanese and gender]." Sekai no Nihongo Kyōiku [Japanese Language Eduation around the Globe] no. 19:49-67.

Tomisaka, Yoko. 1997. Nameraka nihongo kaiwa [Smooth communication in Japanese]. Tokyo: Aruku.

Tomlinson, Brian. 2010. Principles of effective materials development. Edited by Nigel Harwood, English language teaching materials: theory and practice. New York: Cambridge University Press.

Torikai, Kumiko. 2005. "Tsūyaku ni okeru ibunka komyunikēshon gaku [Study of crosscultural communication in interpreting]." In Kōza Shakai Gengo Kkagaku: Ibunka to komyunikēshon [Lecture: Sociolinguistics Science; Cross-Cultural Communication], edited by Sachiko Ide and Masako Hiraga, 24-39. Tokyo: Hituzi Syobo.

Trosborg, Anna. 1987. "Apology strategies in natives/non-natives." Journal of Pragamatics no. 11:147-167.

Tsuda, Sanae. 1999. Danwa bunseki to bunka hikaku [Discourse analyses and comparison of cultures]. Tokyo: Rīberu Shuppan.

Tsukuba Language Group. 1992. Situational Functional Japanese: Volume Three. Tokyo: Bonjinsha

Ueno, Tazuko. 1983. "Meirei to irai [Order and Request]." In Hanashikotoba no hyōgen [Expressions of Spoken Language], edited by Osamu Mizutani, 45-53. Tokyo: Chikuma Shobo.

Unosawa, Kazuko. 2005. "Irai no kikō: Nichi ei ni okeru hatsuwa jijō no taishō kenkyū [Structure of requests: Comparative study of English and Japanese utterances]." Kokusai Keiei Bunka Kenkyū [Studies of International Management and Culture] no. 10 (1):143-174.

Usami, Mayumi. 1999. "Shiten to shite no nihongo kyōikugaku [Japanese Language Pedagogy as the Viewpoint]." Gengo [Language] no. 28 (4):34-43. . 2001a. "Danwa no poraitonesu [Discourse Politeness]." Dai 7 kai, Kokuritsu Kokugo Kenkyūjo Kokusai Shinpojium, Dai 4 kai Senmon bukai [The National Institute of Japanese Language and Linguistics, Seventh International Symposium, Session 4]:958. 2001b. "Disukōsu poraitonesu to iu kanten kara mita keigo shiyō no kinō [The function of the usagae of honorifics in "discourse politeness" in Japanese conversation]." Gogaku kenkyūjo ronshū, Tokyo Gaikokugo Daigaku [Journal of the Institute of Language Research, Tokyo University of Foreign Studies] no. 6:1-29. 2002a. Discourse politeness in Japanese conversation: Some implications for a universal theory of politeness. Tokyo: Hituzi Syobo. . 2002b. "Disukōsu poraitonesu riron kōsō (4) [Theoretical Structure of Discourse Politeness (4)]." Gengo [Language] no. 31 (10):98-103. . 2002c. "Poraitonesu riron no tenkai (3) [Development of theories of politeness 3]." Gengo [Language] no. 31 (3):108-112.

. 2003. "Ibunka sesshoku to poraitonesu: Disukōsu poraitonesu riron no kanten kara [Cross-cultural contact and politeness: From the viewpoint of discourse politenss theory]." Kokugogaku [Japanese National Language] no. 54 (3):117-132.

Watts, Richard J. 2003. Politeness. Cambridge, UK; New York: Cambridge University Press. Winitz, Harris. 1996. "Grammaticality Judgment as a Function of Explicit and Implicit Instruction in Spanish." The Modern Language Journal no. 80 (1):32-46. 
Werkhofer, Konrad T. 2005. "Traditional and modern views: The social constitution and power of politeness." In Politeness in Language, edited by Richard Watts, Sachiko Ide and Konrad Ehlich, 155-202. Berlin, New York: Mouton de Gruyter.

Wolfson, Nessa. 1988. "The Bulge: A Theory of Speech Behavior and Social Distance." In Second Language Discourse: A Textbook of Current Research, edited by Jonathan Fine. Norwood, NJ: Ablex Publishing Corporation.

$\mathrm{Xu}$, Yiping, Gwan Choi, Jiin Chiueh Lai, Cao Dafeng, Masami Kadokura, and Chihiro Kinoshita Thomson. 2012. "Nihon kenkyū to nihongo kyōiku no kurosu rōdo: Nihongo kyōzai ni okeru nihon bunka rikai [Crossroads of Japanese Studies and Japanese Language Education: Japanese Cultural Understanding in Japanese Teaching Materials]." Nihongo kyōiku [Journal of Japanese Language Teaching] no. 151:21-33.

Yabuuchi, Akio. 2004. "Poraitonesu ryō no ibunka kan hikaku: Shazai, puraibashī, jisshitsu kōi [Cross-cultural comparison of politeness levels: Apology, privacy, substantive acts]." Wakayama Kenritsu Ika Daigaku Igakubu, Kyōyō Igaku Kyōiku Daikōza [Bulletin of Liberal Arts and Sciences, Wakayama Medical University] no. 34 (27-52).

Yamada, Toshihiro. 2011. "Ruikeiron teki ni mita nihongo no yari morai hyōgen [Expressions of "giving and receiving" in Japanese from the viewpoint of typology]." Nihongogaku [Japanese Language] no. 30 (11):4-14.

Yamaguchi, Toshiko. 2008. "Komyunikatibu gengo kyōiku no jidai ni okeru nihongo kyōkasho no datōsei [The adequacy of Japanese language textbooks in an era of communicative language teaching]." Kokusai Kirisutokyō Daigaku Gakuhō, Kyōiku Kenkyū [Bulletin of Educational Studies, International Christian University] no. 50:89-95.

Yamamoto, Motoko. 2004. "Shakaiteki sōgo kōi to shite no shazai hyōgen: Gengo hyōgen sentaku no haikei niwa nani ga aru no ka [Apologetic expressions as socialinteractional behaviour: What is the Motivation for the selection of apologetic expressions?]." Shinshu Daigaku Ryūgakusei Sentā Kiyō [Journal of International Student Center, Shinshu University] no. 5:19-32.

Yamauchi, Shigekatsu. 2006. Step Up Nihongo 3. Tokyo: Aishīai.

Yanabu, Akira. 1984. "Bunmatsu no hyōgen [Expression of the end of the sentence]." In Nihongo no hataraki [Functions of the Japanese Language], edited by Masaaki Nomura, 223-244. Tokyo: Chikuma Shobo.

Yasui, Minoru. 1983. "Chokusetsu hyōgen to kansetsu hyōgen [Direct expression and indirect expression]." In Nihongo no retorikku [Rhetoric of the Japanese Language], edited by Akira Nakamura, 88-105. Tokyo: Chikuma Shobo.

Yokoyama, Sugiko. 1995. "Kyōjuhō to kōsu dezain [Teaching method and course design]." In Gaisetsu Nihongo Kyōiku [Outline of Japanese Language Pedagogy], edited by Orie Endo, 91-115. Tokyo: Sanshūsha.

Yoneda, Ryusuke, Kazuko Fujii, Mie Shigeno, and Hiroko Ikeda. 1998. Bijinesu no tame no nihongo [Getting down to business: Japanese for business people]. Tokyo: Surīei Nettowāku.

Žegarac, Vladimir. 2008. "Culture and communication." In Culturally speaking : Culture, Communication and Politeness Theory, edited by Helen Spencer-Oatey, 48-70. London, New York: Continuum. 